\title{
ENVIRONMENTAL HEALING ARCHITECTURE: A PREVENTATIVE APPROACH TO URBAN WELLNESS
}

\author{
by \\ William G. Harispuru \\ Bachelor of Architectural Science, Ryerson University, Toronto, 2005
}

\author{
A design thesis|project \\ presented to Ryerson University \\ in partial fulfillment of the \\ requirements for the degree of \\ Master of Architecture
}

Toronto, Ontario, Canada, 2009

๑William G. Harispuru 2009

PROPERTY OF 


\section{Author's Declaration}

I hereby declare that I am the sole author of this thesis|project.

I authorize Ryerson University to lend this thesis|project to other institutions or individuals for the purpose of scholarly research.

William G. Harisouru

I further authorize Ryerson University to reproduce this thesis|project by photocopying or by other means, in total or in part, at the request of other institutions or individuals for the purpose of scholarly research.

William G. Harispu 


\title{
ENVIRONMENTAL HEALING ARCHITECTURE: A PREVENTATIVE APPROACH TO URBAN WELLNESS
}

\author{
Master of Architecture Degree 2009 \\ William G. Harispuru \\ Master of Architecture \\ Ryerson University
}

\begin{abstract}
The contents of this thesis|project summarize thirteen months of research and design into the architecture of human health. Specifically, it examines the problems in Canada's health culture and offers a more sustainable solution with respect to the design of the physical environment. Research includes the interconnected issues of a changing population demographic, alternative therapy, ancient civilizations and their approach to preventative health, as well as the role that evidence based design plays in the modern healing environment. Research methods include literature reviews, case studies, site visits, field research, personal interviews and design experimentation. This thesis|project examines the healthy attributes inherent in nature, and their inclusion in the design of existing corrective health facilities. This thesis|project investigates how these natural features can be incorporated into a proposed Toronto community. The West Donlands Health and Wellness Centre will promote an alternative preventative health lifestyle for its users. Community programming is offered through a holistic definition of health, this includes traditional allopathic health services, education, physical fitness and healing.
\end{abstract}




\section{Acknowledgments}

A large number of people influenced the contents of this thesis/project. Chief amongst them is my supervisor, Prof. Yew Thong Leong, who was abundantly helpful and offered invaluable assistance, support and guidance throughout my research. His enthusiasm during my thesis|project development granted me the strength to execute a fully coherent design proposal, and fulfill all of my aspirations. This thesis|project would not have been possible without his supervision.

Deepest gratitude is owed to the additional members of my advisory committee, both Chair and Assoc. Prof. Kendra Schank Smith, and practicing architect Gregory Colucci. Kendra's insight offered clarifying direction during my design research. As well, her support reinforced my confidence and ambitions with regards to the next stages of my professional career. I would like to extend thanks to my acting external committee member, Gregory Colucci. His professional criticism included stressing the importance that phenomenological experiences play in architecture, and how they outweigh the simple aesthetic design of space, with respect to the health of the human occupant.

I would also like to thank those involved in my interim critique, which became a pivotal point during my thesis|project development. This includes Dir. and Assoc. Prof. Colin Ripley, and Instr. Cheryl Atkinson. I thank Colin for continuing to emphasize the critical importance that architecture plays today as a vehicle for renewed social consciousness, all the while demonstrating it as being very much an act of instinctual experimentation. I thank Cheryl for her continual input into this and many other works ranging far beyond the scope of this thesis|project. Her ability to see the pure essence of a proposition and apply extreme rigor to the design process is highly admirable.

Furthermore, I would like to thank the Department of Architecture for my Graduate Award, which supported me during my two years of study at Ryerson. I also thank the Department for a travel grant award, which enabled me to conduct field research in both Seattle and San Francisco.

I cannot end without thanking my family and friends, on whose constant encouragement and love I have relied throughout my time of studies-your thoughts have kept me grounded. I am indebted to many of my colleagues whom I respect tremendously and have grown together with. And finally, I am grateful to the examples of my mother, Maria Del Carmen, my father, Domingo Mario, and very importantly, Daniela. Their courage and conviction will always inspire me, and I hope to continue, in my own small way, the noble way in which they give themselves to others. 
This thesis|project is dedicated to my loving parents,

for their ever-present patience, understanding and support. 


\section{Table of Contents}

List of Figures xiii

List of Appendices xvi

Introduction 1

Research Methodologies 7

\section{Evolution to an Unhealthy Culture}

1.1 Current Health Issues in Canada 11

1.2 A Sedentary Life in the Suburbs: An Inactive Generation 12

1.3 An Aging Population 13

\section{Preventative Cultural Approaches to Well-Being}

2.1 Old Systems of Holistic Healing 15

2.2 Hippocratic Medicine in Ancient Greece 16

2.3 Roman Advances in Natural Therapy and Infrastructures 17

2.4 Learning from East Asia 19

2.5 Current Day Systems of Holistic Healing 21

2.6 Current Japanese Society and Preventative Health $\mathbf{2 1}$

2.7 Alternative Therapies of the Future in Canada $\mathbf{2 2}$

\section{Current Researches into Natural Systems of Environmental Healing}

3.1 Evidence Based Design 25

3.2 Field Research $\mathbf{2 7}$

3.2 The Healing Powers of Light 29

3.3 Experiencing Nature $\mathbf{3 3}$

3.4 Sound, Music and Art Therapy $\mathbf{3 7}$

3.5 Transfer of Nosocomial Infection 39

\section{Theoretical Discourse}

4.1 Process vs. Place 44

4.1.1 Argument: The Need to Follow the Grain of Nature $\mathbf{4 4}$

4.1.2 $19^{\text {th }}$ and $20^{\text {th }}$ Century Examples of Environmental Importance 44

4.1.3 Trends in Healthcare Today: Moving to a More Natural Way of Healing 46

4.1.4 CASE STUDY: Apotex Centre, Baycrest Centre for Geriatric 47

4.1.4.1 Memory Loss 49

4.1.4.2 The 'Home-Like' Environment 50

4.1.4.3 Creating Places to Heal 52 
4. 2 Adaptability and Flexibility $\mathbf{5 3}$

4.2.1 Argument: The Need for Adaptable and Flexible Buildings $\mathbf{5 3}$

4.2.2 Factors in Health Design: Changing technology, the Spread of infection \& Cost $\mathbf{5 3}$

4.2.3 Modernism and Patient Care: Exploring the idea of Standardization 55

4.2.4 The Neutral Grid and Later Attempts at Flexibility $\mathbf{5 7}$

4.2.5 The Modern Single Patient Room 58

4.2.6 CASE STUDY: The New Women's College Hospital Addition $\mathbf{5 9}$

4. 3 The Economic Value of Design 61

4.3.1 Argument: Good Design Pays for Itself $\mathbf{6 1}$

4.3.2 The Difficulties Involved in Assigning Value to Design 62

4.3.3 The Current North American Hospital Boom: Rising Care Standards 62

4.3.4 CASE STUDY: Fable Hospital 63

4.3.4.1 Analysis of Investment Breakdown 64

4.3.4.2 Is Evidence Based Design Worth the Extremely High Capital? 65

4.3.4.3 Where Was Money Saved? 66

4.3.4.4 What Evidence Should I Buy? 67

\section{Design Hypotheses}

A Preventative Approach to Urban Wellness 69

\section{A General Reader}

Mind and Nature: Health and well-being in the modern built environment $\mathbf{7 3}$

\section{Design Project}

7.1 Program and Vision 95

7.2 Background Site Analysis $\mathbf{1 0 0}$

7.3 Contextual Linkages 104

7.4 Design Language and Principles 109

7.5 The West Donlands Wellness Centre: Experiencing Community Health 114

\section{Summary 137}

\section{Appendices 141}

10 Reference List 237 


\section{List of Figures}

Figure 1.1 View of Levittown in post-war America. Image credit: TESSELLAR

Figure 1.2 Major issues in Canadian health

Figure 2.1 Hippocratic medicines in ancient Greece. Image credit: Medinfo

Figure 2.2 Roman public baths. Image credit: Intermedia

Figure 2.3 The human body as seen in eastern and western medicine. Image credit: Chinese Medicine Works

Figure 2.4 Connection between alternative therapies in Canada today and ancient civilizations approach to health

Figure 3.1 Evidence based design process. Image credit: Hamilton and Watkins

Figure 3.2 'Over London by Rail,' Gustave Dore (1872) London Slums during the Industrial Revolution. Image credit: the Valve

Figure 3.3 Rickets in Children. Image credit: Talk Origins

Figure 3.4 Controlled views of nature for post surgery patients. Image credit: Roger Ulrich

Figure 3.5 Hospital Garden Pedestrian aggregations within an eight hour period. Image credit:

Marcus and Barnes

Figure 3.6 Oriente Station, Lisbon, Santiago Calatrava. Image credit: Flickr

Figure 3.7 Evidence based design centres itself around the idea of patient focused care

Figure 4.1 The Nightingale Pavilion Ward. Image credit: Royal Herbert Pavilion

Figure 4.2 Baycrest Apotex Centre Hospital, Site Plan. Image credit: Diamond + Schmitt Architects

Figure 4.3 Apotex Centre Ground Plan, indicating indoor and outdoor courtyards. Image credit:

Diamond + Schmitt Architects

Figure 4.4 Apotex Centre, Resident Unit Plan Indicating varying furniture layout. Image credit:

Diamond + Schmitt Architects

Figure 4.5 Family clusters creating the larger Apotex community. Image credit: Diamond + Schmitt Architects

Figure 4.6 Dining room at the Apotex Centre. Image credit: Diamond + Schmitt Architects

Figure 4.7 Kitchen common area at the Apotex Centre. Image credit: Diamond + Schmitt

Architects

Figure 4.8 Venice Hospital, plan and city connection. Image credit: CASE

Figure 4.9 Venice Hospital, Section of Care Unit Cell. Image credit: CASE

Figure 4.10 McMaster Hospital, Traffic Segregation in plan. Image credit: Zeidler

Figure 4.11 McMaster Hospital, Section indicting Interstitial Space. Image credit: Zeidler

Figure 4.12 The Green Patient Room. Image credit: Anshen + Allen Architects

Figure 4.13 The Standardized Patient Wing. Johnny Bean Engineers 
Figure 4.14 Women's College Hospital, Site plan. Image credit: Diamond + Schmitt Architects

Figure 4.15 Women's College Hospital, Clinical Neighbourhood. Image credit: Diamond + Schmitt Architects

Figure 4.16 Factors Inherent in varying levels of evidence based design. Image credit: The Centre for Health Design

Figure 4.17 Fable Hospital, Incremental Cost to achieve a better building. Image credit: The Centre for Health Design

Figure 4.18 Fable Hospital, Financial Impact of Design Decisions. Image credit: The Centre for Health Design

Figure 5.1 Design hypothesis

Figure 6.1 The planet earth as a well-balanced ecosystem. Image credit: Lifeboat Foundation Figure 6.2 The baths of Caracalla in ancient Rome. Image credit: Leland M. Roth Figure 6.3 The Chauvet cave paintings. Image credit: Coquina Figure 6.4 The Industrial Revolution. Image credit: Environmental History Resources Figure 6.5 The glass skyscrapers of our modern city. Image credit: Wikimedia Figure 6.6 The multi-sensory experience of walking through a forest. Image credit: Corbis Figure 6.7 Views to nature in a hospital ward. Image credit: Anshen + Allen Architects Figure 6.8 The French sidewalk café. Image credit: Corbis

Figure 6.9 Weekly breakdown of where the average North American in urban conditions spends their time. Image credit: Lawrence E. Hinkle

Figure 6.10 Key areas that mediate between the built environment and mental well being. Image credit: Jane Wernick

Figure 6.11 Factors that were found to be associated with significantly worse mental well being. Image credit: Jane Werncik

Figure 6.12 Factors that were not found to be associated with significantly worse mental well being. Image credit: Jane Werncik

Figure 6.13 The thermal baths at Vals blend in to the natural surroundings. Image credit: Peter Zumthor.

Figure 6.14 The processional caverns of the Vals thermal baths. Image credit: Peter Zumthor Figure 6.15 The sun deck at the Vals thermal baths. Image credit Peter Zumthor

Figure 6.16 The primary experience and therapy of bathing oneself. Image credit: Peter Zumthor Figure 7.1 Dream of Arcadia (1838). Image credit: Thomas Cole

Figure 7.2 Robert Chitham, Drawing reconstruction of the baths of Caracalla. Image credit: John Dawes

Figure 7.3 Wellness Centre: Programmatic diagram

Figure 7.4 Wellness Centre: Organizational service structure

Figure 7.5 Toronto Waterfront: Communities Masterplan 
Figure 7.6 West Donlands bounding conditions

Figure 7.7 West Donlands Masterplan

Figure 7.8 West Donlands site circulations

Figure 7.9 West Donlands Community. Image credit: Waterfront Toronto

Figure 7.10 Wellness Centre critical site linkages.

Figure 7.11 Wellness Centre critical site linkages. Image credit: Waterfront Toronto and Metrolinx Figure 7.12 West Donlands Masterplan: Section through contextual linkages

Figure 7.13 Forest canopy filtering light and shadow. Image credit: Eikonograpia

Figure 7.14 Industrial railand corridor. Image credit: Comstock

Figure 7.15 The emergence of the natural in the unnatural

Figure 7.16 The linear geometry of the rail lines. Image credit: GO Transit

Figure 7.17 The idea of 'porosity in architecture' was executed during the design experimentation process.

Figure 7.18 The West Donlands Wellness Centre: View from Don River Park

Figure 7.19 Critical Site Circulations

Figure 7.20 Site Deriving Lines

Figure 7.21 Final Site Circulations

Figure 7.22 Porous Landscape Integration

Figure 7.23 Site Plan

Figure 7.24 Ground Level Plan: Health and Education

Figure 7.25 Lower Level Plan: Swimming Centre and SPA

Figure 7.26 Second Level Plan: Fitness

Figure 7.27 Gatekeeper and entry Reception

Figure 7.28 Medical Examination Rooms

Figure 7.29 Digital Information Kiosks

Figure 7.30 Lower Entry Courtyard

Figure 7.31 Main Recreational Pool

Figure 7.32 Entry into SPA

Figure 7.33 Calidarium

Figure 7.34 Frigidarium

Figure 7.35 Contextual landscape connections along railway

Figure 7.36 Section through recreational pool

Figure 7.37 Section through swimming centre

Figure 7.38 Section through community circulation

Figure 7.39 The composition of space, light and ephemeral materials was found to create successful architectural experiences 


\section{List of Appendices}

Appendix 1: Evidence based design approach to Wellness Centre design

Appendix 2: Field research: Evidence based design, San Francisco CA

Appendix 3: Fable Hospital: Incremental costs to achieve a better building. Image credit: The Centre for Health Design

Appendix 4: Fable Hospital: Financial impact on design decisions. Image credit: The Centre for Health Design

Appendix 5: 'Third Places' of nature reconnection in the modern urban environment. Image credits: Claude Cormier, West 8, HOK life, Torontoist, JRA Associates, GH3, Waterfront Toronto and Weissmanfredi

Appendix 6: Design Project: Programmatic Analysis

Appendix 7: West Donlands existing conditions. Image credits: Waterfront Toronto

Appendix 8: West Donlands infrastructure development. Image credit: Ontario Realty Corporation

Appendix 9: Don River Park Proposal. Image credit: Michael Van Valkenburgh and Associates

Appendix 10: Steven Holl Architects: District Energy Heating/Cooling Plant. Image credits:

Waterfront Toronto

Appendix 11: Railway Disturbance Mediation

Appendix 12: Physical modeling exercises

Appendix 13: Physical lighting studies 


\section{ENVIRONMENTAL HEALING ARCHITECTURE: \\ A PREVENTATIVE APPROACH TO URBAN WELLNESS}

Health depends on a state of equilibrium among the various factors that govern the operation of the body and the mind; this equilibrium in turn is reached only when man lives in harmony with his external environment.

Hippocrates (Purves, 2002, p.xiii)

\section{Introduction}

Canadians face many difficult issues that are constraining our future as a healthy and viable society. These include climate change, an aging population, pollution, food safety security and building a sustainable economy (Martel \& Caron, 2006; Lister, 2008). Many of these issues are health related. Today there is an alarming increase in adult, and childhood, obesity- as well as in diabetes (Hobday, 2006; Shields \& Tjepkema, 2006). These shifts will place significant burden on our future health care system. There is growing public dissatisfaction with traditional modes of healing and a move towards more natural ones (Crenlin, Andersen \& Connor, 1997). Alternative therapists from homeopaths, naturopaths, herbalists and nutritionists offer more sensitive approaches to healing. Traditional allopathic medicine has been categorized as being more curative than preventative and more chemical then natural (Low, 2004). This leads to the public search for more preventative modes of well-being. The question that needs to be asked is: how can preventative measures be introduced today in order to promote good health and offer a sustainable solution to our Canadian health issues?

Running parallel to the idea of preventative health is the debate on quality of life and its relation to design of our physical environment (Purves, 2002). What role do architects play in offering answers to these problems? Are there design solutions that can address many of these issues? Architects play a pivotal role in the way we shape our cities, homes and working environments. These are the spaces that we occupy and the environments that influence our health. The time we spend indoors today is increasing, and deprives Canadians of the physical and mental benefits associated with the outdoor environment. Studies into the psychological benefits of exposure to nature exist in a growing body of research literature. Implementing these results into the design of our 
physical environment can contribute as a form of preventative medicine through design. This evidence based approach to design plays an essential role in the sensitive development of our future healthy environments.

This thesis|project will take a deeper research approach than that of strictly healthcare design, and find that by integrating nature's healthy attributes into the design of our built environment, we can foster community wellness in both a holistic and preventative manner. This in turn reduces our dependency on corrective health approaches and promotes a lifestyle that ultimately allows individuals to control their own levels of health in a sustainable manner. This thesis|project will take a look at the natural human system of health and will allow us to identify the reasons for our problems. To be looked at more closely will be the paradigm shift regarding the issues of a changing population demographic, human health, alternative therapies and the role that evidence based design plays in the design of our healing environments. Connections will be made to ancient civilizations and their practice of natural and preventative healing techniques before the advent of technology. Further connections will demonstrate how the field of evidence based design has proven the benefits of these ancient principles, and can be applied to the design of our modern care environments. Research analysis will be further focused in the following three areas: (i) care environments today causing more harm than good and bringing forth the debate of process vs. place, (ii) adaptable and flexible buildings that conform to future change, and (iii) placing a cost value on healthy design.

To begin with, connections can be made between evidence based design and the way ancient societies practiced preventative health through a deep attachment to nature. The industrial revolution changed humans into a mechanical indoor species (Hobday, 2006). It is now estimated that the average Canadian spends 90 per cent of their time indoors (Canada Green Building Council, 2004). Before technology and chemical medicine were adopted by our culture, ancient civilizations lived in balance with nature. The ancient Greeks lived in harmonic balance with nature regarding internal bodily fluids and the external environment (Bergdolt, 1999). The ancient Romans adopted alternative therapies as daily routine, making public baths a social norm. These baths were places to enjoy art, therapeutic retreat and even undergo medical procedures (Cruse, 2004). Early East Asian cultures viewed the body as a microcosm of nature. Within the body, mountains, rivers, forests and even villages exist (Bivins, 2007). Sickness is similar to 
unwanted forces of nature in the real world. Adopting these ancient ideals today seems viable when looking at today's level of public health. As stated by Low (2004), alternative medicine is on the rise in Canada, perhaps because of the negative aspects of traditional chemical medicine in North America. There is a growing level of public distrust of such medicine.

A growing body of research today is in the field of environmental psychology. The psychological benefits associated with exposure to the natural environment have been proven with critical research studies. The area of study that attempts to incorporate these benefits into the design of our built environments is known as evidence based design. Evidence based design is design that is "based on the best available information from credible research and evaluations of projects" (Hamilton, 2004, p.1). Studies have demonstrated the psychological benefits of exposure to nature, retaining healthy levels of natural day lighting, and exposure to the therapeutic qualities of art and music (Hobday, 2006; Joseph \& Ulrich, 2007; Marcus \& Barnes, 1995; Ulrich, 1984). Research studies have also stressed the importance of minimizing the spread of hospital infections when designing our modern care facilities (Joseph, 2006).

A major focus of research analysis is associated with process vs. place. Over the past century, technology has turned the care environment into an institutionalized setting, causing more harm than good. A new trend has emerged today and is classified as patient focused care (Purves, 2002). By implementing therapeutic practices into the built environment, these spaces can be environments of natural healing for the patient. The psychological benefits derived from employing evidence based design research lead to a new generation of design around human occupants. This approach to design places the patient as the priority in the healing process, not the medical procedure itself (Prasad, 2008). Care design results in the creation of a place of healing instead of a building whose architecture is dominated by functional medical processes. Creating the built environment using evidence based design can result in many benefits. Some of these benefits include a quicker patient recovery time after surgery and reductions in patient stress, the need for pain medication and medical error by staff (Berry, Parker, Colie \& Hamilton, 2004). This approach also offers a sensitive method of care design and deinstitutionalizes the outdated notions of hospital design used in the early $20^{\text {th }}$ century. 
Another major focus of research is adaptability and flexibility. The constant change in medicine and technology supports the need for a responsible approach to designing adaptable and flexible buildings. As well, changes in disease must not outpace the services made available by the facility. This would have negative effects on both the planning and cost of healthcare facilities. Designing buildings that are adaptable and flexible regarding the changes in medical advances remains a priority today (Zeidler, 2008). Healthcare is moving towards an outpatient basis; however the inpatient care setting is still needed for high-risk patients (The Globe and Mail, 2008). These opposing modes of delivering healthcare must be balanced by planners and designers. Having the ability to adapt with changing time and technology will enhance the longevity of buildings. Designing a care facility with a longer life expectancy is beneficial; however, healthcare planners are reluctant to adopt a strategy that requires high initial capital. Evidence based design can be employed as a strategy that will allow a facility to save unnecessary costs in the long run because of its ability to adapt to future service needs.

The final major focus of research is the economical value of design. The cost of designing a facility that is focused around the patient could be high. Putting a value on good design seems difficult. Very few studies exist showing that evidence based design can demonstrate cost savings over time (Purves, 2002). One study amalgamated evidence from various hospital settings over several years of operation (Berry, Parker, Colie \& Hamilton, 2004). This study was known as the Fable Hospital, and employed strategies that included acuity-adaptable rooms, outdoor gardens, larger windows, artwork and varying levels of occupant safety. These design features had added a substantial percentage to the initial project investment; however, substantial cost savings were demonstrated. These features saved the hospital unnecessary costs in an impressively short payback period.

In summary, this thesis|project indicates that the design of our future built environment should be based on the idea of achieving successful levels of occupant health. It will examine the approach of using natural therapies found in a wide body of literature to promote healthy building design. Benefits from using this approach have been been experienced by all building occupants including patients, staff and visiting families. There exists great strength in applying these findings to healthcare environments; however the design portion of this thesis|project will consider how these principles can be 
extrapolated to other building types. This thesis|project will attempt to offer a proposed Toronto neighbourhood an alternative lifestyle of preventative health. The aim is to achieve a more sustainable solution to the problems and issues involved in the culture of Canadian health. 


\section{Research Methodologies}

Research methods in this thesis|project include literature reviews (medical journals, print and electronic databases), case studies, site visits, field research, telephone interviews, personal interviews and design experimentation. The following is a breakdown of the telephone, in-person interviews, site visits and field research components of the methodology.

\section{List of telephone interviews}

- February 26, 2009: Diane Silver, Planner, City of Toronto, Toronto ON

\section{List of in person interviews}

- September 26, 2008: Gary W. Collins, Associate Principal, Anshen + Allen Architects, San Francisco CA

- October 2, 2008: Trevelee Goodwin, Finance and Administration Officer, East End Community Health Centre, Toronto ON

- November 07, 2008: Gregory Colucci, Principal, Diamond and Schmitt Architects Ltd, Toronto ON

- November 22, 2008: lan Trotter, Environmental Service Manager, Wellesley Central Place, Toronto ON

- December 05, 2008: Rob Devitt, President and CEO, Toronto East General Hospital, Toronto ON

- December 05, 2008: Ralph Fernando, Vice President of Finance, Toronto East General Hospital, Toronto ON

- January 13, 2009: Naveen Sarin, Founder and President, Harbourfront Health and Wellness Centre, Toronto ON 
- March 3, 2009: Martin W. Waldron, Senior Medical Architect, Principal, Anshen + Allen Architects, San Francisco, CA

- March 6, 2009: Margaret D. Timothy, Executive Assistant to the Dean, Stanford University, Stanford CA

- May 17, 2009: Daryl J. Barnett, Director of Rail Corridor Infrastructure, GO Transit, Toronto ON

- May 18, 2009: Ramani Ramakrishnan, Professional Engineer; Acoustic Specialist, Ryerson University, Toronto ON

\section{List of site visits}

- October 2, 2008: Coxwell/Queen Community Health Centre, 1619 Queen Street East, Scarborough ON

- October 2, 2008: Hospital for Sick Kids, 555 University Ave, Toronto ON

- October 2, 2008: Toronto General Hospital, 200 Elizabeth Street, Toronto On

- October 2, 2008: Princess Margaret Hospital, 610 University Ave, Toronto ON

- October 2, 2008: Mount Sinai Hospital, 600 University Ave, Toronto ON

- October 9, 2008: McMaster University Health Sciences Centre, 1280 Main Street West, Hamilton ON

- October 9, 2008: Credit Valley Hospital, 2200 Eglington Ave West, Mississauga ON

- October 24, 2008: Apotex Centre for Geriatric Care, 3560 Bathurst Street, Toronto ON 
- November 22, 2008: Wellesley Central Place, 160 Wellesley Street East, Toronto ON

- November 24, 2008: Wellesley Community Centre/Public Library, 495 Sherbourne Street, Toronto ON

\section{List of field research}

- March 3, 2009: Anshen + Allen Architects, 901 Market Street, San Francisco, CA

- March 4, 2009: Lucille Packard Children's Hospital, 725 Welch Road, Palo Alto, CA

- March 4, 2009: Stanford University Green Earth Sciences Building, 397 Panama Mall, Stanford CA

- March 4, 2009: Kaiser Permanente Hospital, 700 Lawrence Expressway, Santa Clara CA

- March 4, 2009: Santa Clara Valley Medical Centre, 751 S. Bascom Avenue, San Jose, CA

- March 6, 2009: Olympic Sculpture Park, 2901 Western Avenue Seattle, WA

- March 6, 2009: Seattle Public Library, 1000 4th Ave, Seattle, WA 


\section{Chapter 1: The Evolution to an Unhealthy Culture}

The Canadian way of life and its system of health have been compromised. This deterioration of public health has varying causes. One factor is that we are less physically active than we were fifty years ago. This inactivity has led to obesity among both children and adults, and is a growing concern within North American culture. The adoption of the suburbs combined with technology has weakened our level of human health, and decreased activity levels within our society. Our unhealthy reliance on the automobile has been caused by modern urban planning design. These factors have made us an indoor species, and made us increasingly susceptible to diseases associated with the interior environment. Combined with an ever increasing aged population, these stresses may make our national health care system unsustainable. These general issues inherent in our culture support the need for change.

\subsection{Current Health Issues in Canadian Culture}

Today, our western culture reduces our standard of living and life expectancy. Smoking is the number one preventable cause of death in Canadians; obesity is a close second (Felepchuk, 2008). Obesity increases adverse health risks such as diabetes, heart attack, stroke, high blood pressure, colon cancer, osteoarthritis and early mortality (Hobday, 2006). In a study conducted by the World Health Organization (2000), (as cited in Dunai, Novak, Chung, Kayumov, Keszei, Levitan, \& Shapiro, 2007) it was found that obesity has become a global epidemic that demands greater attention in all countries. Research conducted by Statistics Canada found that $23 \%$ of Canadians in 2004 were obese, and another $36 \%$ were overweight (Shields \& Tjepkema, 2006). This means that $59 \%$ of Canadians had excessive amounts of body weight. Katzmarzyk and Janssen's (2004) study, (as cited in Dunai et al., 2007) showed that the economic burden of these factors on our healthcare system is astounding; close to $\$ 10$ billion annually, nearly $5 \%$ of the entire healthcare budget, is invested in treating obese and overweight people. These statistics seem daunting when obesity is considered a preventable disease. Prevention offers advantages such as the reduced need for westernized medical treatments and lower healthcare costs. Moreover, treating the adverse health risks associated with obesity through western medical practices can be considered harmful because of chemically induced side-effects (Low, 2004). Obesity and malnutrition due to poor diet are becoming an extremely important issue in Canada today. 


\subsection{A Sedentary Life in the Suburbs: An Inactive Generation}

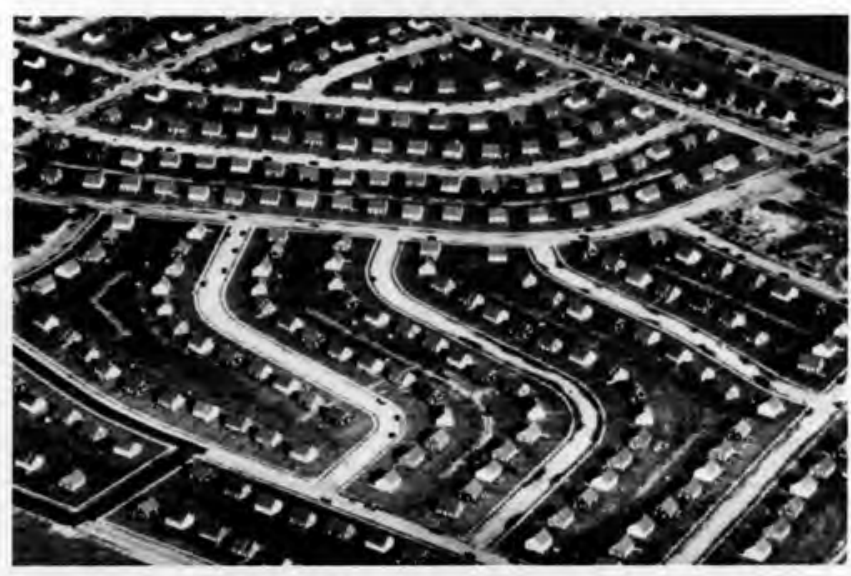

Figure 1.1 View of Levittown in post-war America Image Credit: TESSELLAR
The Canadian middle class has become a sedentary population due to lack of exercise and reduced mobility (Farr, 2008). The design of our modern western cities has allowed this culture to evolve. Farr stated that, prior to the twentieth century compact cities were pedestrian-friendly areas of human occupation. People inhabited areas

locally, and lived their entire lives close to their places of birth. Today, the use of the automobile has had profound effects on the way we have planned our cities.

Suburbanization has made us an auto-dependent species. In post-war America the single family dwelling became an affordable mode to raise a new family as a result of the North America adoption of this widely criticized development pattern (fig.1.1). Additionally, this design has resulted in populations choosing to live their lives predominantly indoors. It is estimated that the average Canadian spends 90 per cent of their lives indoors (Canada Green Building Council, 2004). Hobday (2006) states that the psychological effects associated with this living pattern include depression, vitamin deficiencies and low levels of energy, not to mention decreasing the effectiveness of the immune system. Today, depression has been found to occur in one in six North American adults, a direct effect from living an indoor lifestyle (Canadian Press, 2007).

Studies have shown that a connection exists between urban sprawl and obesity. One study reports that residents living the farthest distances from urban city centres are the most likely group to be overweight or obese (Shields \& Tjepkema, 2006). Evidence suggests that an increased reliance on vehicles has had a direct effect on our motivation to walk specific distances. The study also points to the healthier state of individuals who live in urban city centres because early immigrants populated these areas and brought their healthier cultures with them. Immigrants have always been traditionally less likely to be obese then resident Canadians. Shields and Tjepkema (2006) also found that the percentage of residents who commute to census metropolitan areas (CMA) correlates with obesity. In zones that had the fewest number of residents commuting to CMA's, 
obesity was found as being significantly higher, that of $28 \%$. Residents who lived in completely non-influenced zones (less then $40 \%$ or less residents commuting to a CMA) were seen as being twice as likely to be obese $(44 \%)$ compared to the national Canadian average.

\subsection{An Aging Population}

Canada's health care system is also being burdened by a rapidly aging population. According to Canadian census data for 2006, the number of Canadians aged 55-64 and approaching retirement is up to 3.7 million people, a jump of over $28 \%$ in the past 5 years (Martel \& Caron, 2006). Baby boomers were individuals born between 1946 and 1965. This post-war generation accounts for approximately one in every three Canadians. The proportion of seniors aged 65 and over is increasing steadily in all provinces, while the percentage of children under the age of 15 is decreasing. Fewer children and more aged persons in Canada will affect the market place in education and in health.

This demographic trend will have a major impact on the labour force, health insurance plans and public pensions. It will further the economic instability of Canada. Rosemary Bender is the director general of social and demographic statistics at Statistics Canada; in an interview with $\mathrm{CBC}$ stated that "baby boomers have hit the tail end of the workingage population" (CBC News, June 17, 2007). She goes on to state that "they're in the pre-retirement age so there's an awful lot of interest in terms of ... the need for knowledge transfer or potential skills shortages." A point will be reached where more people will be attempting to leave the work force than enter it. Employers are starting to redesign pension plans, extend the allowable working age as well as create more flexible hours.

In final, these trends in Canada will threaten our national healthcare system (fig.1.2). A rapidly aging population, a growing number of inactive people who rely on the automobile and an increasingly obese population are just a few of many developments. Many adverse health risks occur because of our culture's adoption of an indoor lifestyle. Our detachment from the natural environment over the past century has decreased the strength of our immune system, and has made us more prone to acquiring infections. It also increases depression and vitamin deficiencies. It is important to state that cultures 
evolve over time and cannot change their unhealthy ways overnight. These trends will continue, and if change is to occur, the built environment must be part of the change process. It is the responsibility of the architect to design a built environment that enhances human health. The goals are the prevention of sickness and healing the already sick. Many connections can be made between these goals and the way ancient cultures lived with a preventative approach to human health.

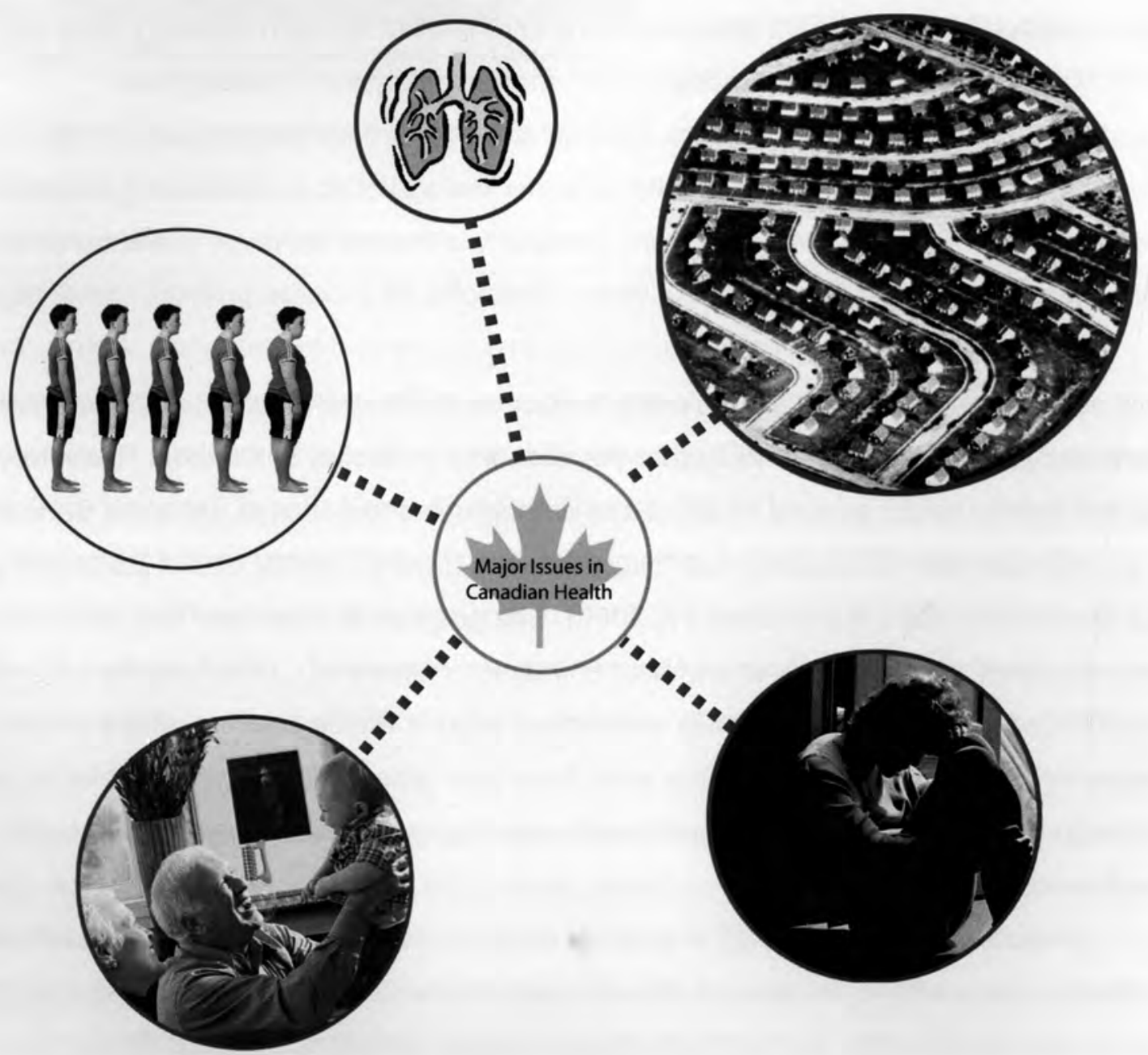

Figure 2.2 Major issues in Canadian health today include lung cancer, an increased aging population, obesity in adults and children as well as depression 


\section{Chapter 2: Preventative Cultural Approaches to Well-Being}

The problems faced today regarding the state of our national health are culturally driven. Living indoors with minimal exposure to nature and remaining minimally mobile are growing trends in westernized society. The health related risks involved in this lifestyle are grave, and spending for our public healthcare needs to increase in order to counteract these issues. Many current health problems can be cured; however complex medical methods are required. We have increasingly relied on modern allopathic western medicine as a primary cure for the majority of our ailments. This method is proving to be increasingly costly. One way of minimizing the cost of this system of health is to take a preventative approach to health and well being. Preventative medicine is dedicated to the prevention of disease, injury, disability and the promotion of health (Medical Dictionary, 2008). Ancient civilizations adopted holistic, preventative approaches to living. Partly because of minimal levels of technology, and also because of the important role that spirituality played in daily life. Interestingly, many eastern cultures have continued to approach human health in this manner, and some western populations now seem to be adopting these natural systems of healing.

\subsection{Old Systems of Holistic Healing}

Before the advances in modern day technology, many civilizations lived with a high and intimate regard for nature, approaching health in a preventative manner. The Hippocratic Oath of the ancient Greeks was based on on a holistic system of healing. Purves (2002) states, that these early ideals outlined a well-balanced life with healthy levels of exposure to nature, good diet, exercise, music and poetry. Similarly, Bivins (2007) states that East Asian cultures illustrated the human body as a microcosm of nature composed of canals and forests- a natural system in itself. Yet almost overnight, in mid-nineteenth century Europe, these ideas were converted into a mechanical model. Today, the degradation of our natural environment has increased infectious diseases (Bergdolt, 1999). Our health care system today is an increasingly complex policy driven network, and our reliance on chemical medicine remains the dominant force in our culture. However, a growing number of individuals are resisting this trend, dissatisfied with traditional allopathic medicine. Low (2004) states that alternative and holistic approaches to medicine and therapy are regaining popularity today. These forms of therapy have occurred throughout history and in various cultures. Much can be learned from old 
systems of holistic healing, including these of the ancient Greeks, Romans, and early East Asian cultures.

\subsection{Hippocratic Medicine in Ancient Greece}

Hippocratic medicine was the established mode of medicine in ancient Greek society, depicted in the writings of the main Corpus Hippocraticum. Written between 430-322 BCE, this text consists of fifty-two treatises by various authors. However, the Corpus (main body of the text) was written by Hippocrates of Kos (460-370 BCE). Bergdolt (1999) states this text was the dominant literature in health for almost two millennia. Many of the Presocratic ideas which had evolved centuries before were modified into what was known as Hippocratic medicine (fig.2.1). Although different from its earlier counterpart, Hippocratic medicine focused on the individual and not the illness itself (Cruse, 2004). Hippocratics

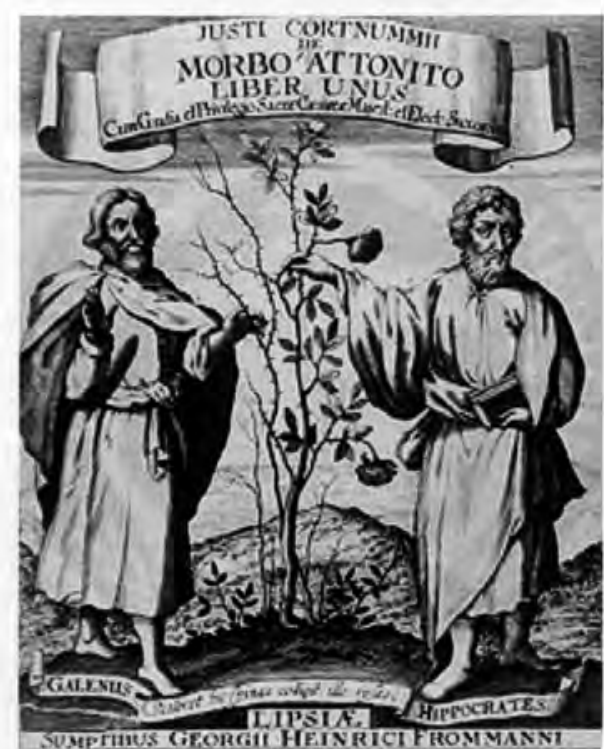

Figure 3.1 Hippocratic medicines in ancient Greece Image credit: Medinfo differed from previous medical practitioners because he attempted to define the specific reasons for disease, and the processes by which it affected the human body. They were also significantly different in how he used application and theory in the medical process, instead of blaming those who caused the disease- the gods (Cruse).

The humoral theory was the key to Hippocratic medicine; it characterized the body as having internal fluids which were known as the four humours (Cruse, 2004). These humours consisted of blood, phlegm, yellow bile and black bile. These humours determined your state of health and well-being; the 'pneuma', or air, was thought to be the source of consciousness and thought. Polybos was the famous son-in-law of Hippocrates, and wrote in the tract De Natura hominus (Nature of Man), that optimal health was the perfect mixture or combinations of these fluids. He believed that a specific way of living allowed for this normal pattern of health to develop. Disease would occur when fluids were unbalanced through imbalances in nature. Bergdolt (1999) states that these imbalances were portrayed with characteristics associated with male and 
females. The male sex was characterized by factors of warmth and dryness, while the female sex had traits of being cold and moist.

Hippocratic health was not solely interested in the balance of internal bodily measures. External factors related to the environment were also held to be extremely important. As Bergdolt (1999) states, the unknown author of the tract Peri diates provides one of the first comprehensive accounts of health and diet. Producing good health included factors associated with age, sex, sleeping and walking, climatic characteristics of the region, as well as levels of physical exercise and diet of the individual. Spiritual factors also determined how one would live a healthy and prosperous life. The seasons of the year, the heavenly star constellations and even dreams played important roles. The correct combination of these factors, most notably emphasized through physical exercise, nutrition and rest, was seen as critical to balance and thus well-being.

In addition to these factors outlined in the Peri diates, King (2001) shows the importance of a harmonious balance existing and emphasized between man and nature itself. An individual conducting himself as a medical practitioner would need to abide by the rules of nature, and therefore become independent from any godly intervention. This was an important point in human history- the point at which medicine completely detached itself from the ultimate prognosis from the gods. The idea of a natural type of healing in medicine was encouraged in the Hippocratic concept of health. Bergdolt (1999) states that individuals having a bad diet and false lifestyles could ruin their body and mind respectively. Hippocratic medicine freed itself from superstition, and placed the patient within the realm of the natural environment.

\subsection{Roman Advances in Natural Therapy and Infrastructures}

Centuries later, the Romans adopted much of the same principles as the Greeks. King (2001) states that the early Romans placed faith in naturalism as did early Greek medicine. Naturalism was study of nature through plants and animals. It was from this area of study that came one of their largest works of writings to survive until modern day. A text on naturalism was written by the famous writer and philosopher Pliny the Elder, and entitled the Naturalis Historia. According to Pliny, doctors were not present in Rome for over 600 years until the arrival of the Greek doctor named Archagathus in 219BC. Pliny had diverging thoughts on health, and had believed that the practice of medicine 
was not necessary. He believed that the natural environment provided all that one would need to cure oneself when sick. His view was expressed in some of the writing from the Naturalis Historia text:

But Earth is kind and gentle and bountiful, ever a handmaiden in the service of mortals, producing by our forcing her, or pouring out spontaneously, what scents and flavours, what juices, what tactile surfaces what colours! ...she produces medicinal herbs, and is always productive on behalf of humankind. (King, 2001, p.34)

The Romans made their advances in medicine and health along these lines of natural healing and therapeutic practices. They progressed well past the ideas of the early Greek societies, through the process of building hygienic infrastructures in the major

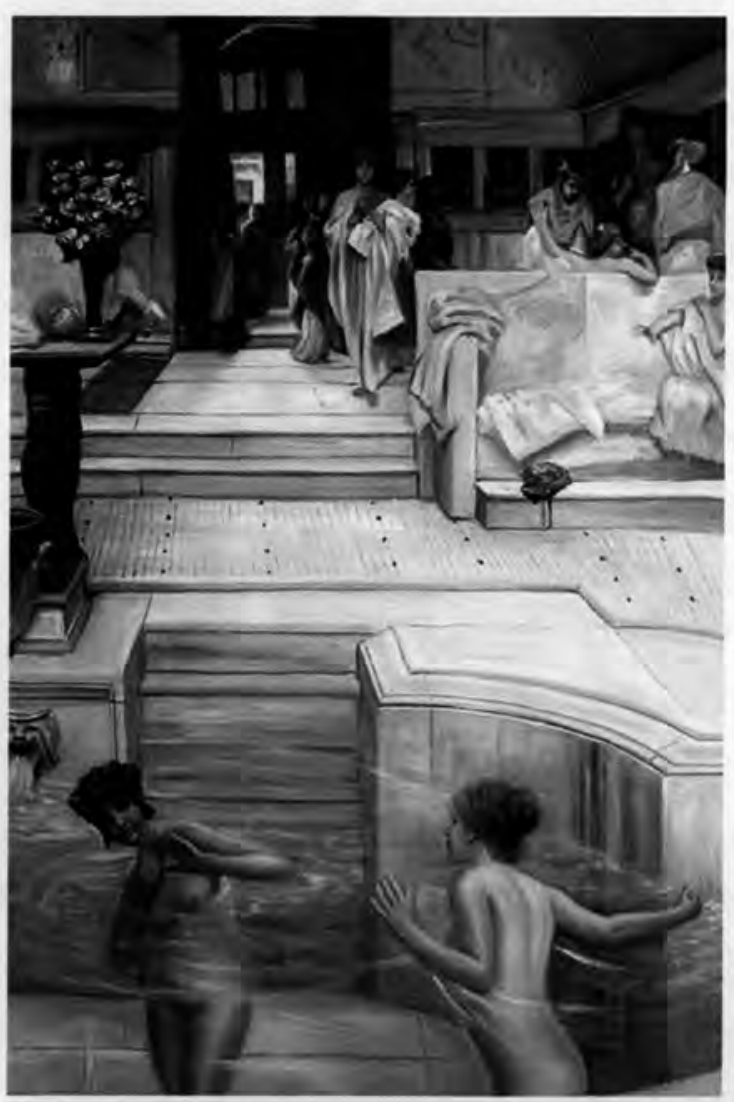

Figure 2.2 Roman public baths Image credit: Intermedia cities that were part of the Roman Empire. They built advanced road systems, aqueducts and even sewers systems for human cleanliness. Cruse (2004) states that Roman engineering allowed for a safer mode of life, introducing hygiene and the drainage of stale water sources, rivers and the buildings they occupied. With this came the adoption of both public and private baths for all levels of society. Originally intended for the utilitarian purposes of personal cleansing, public baths evolved into therapeutic forms of personal medicine. They became integral to a person's sense of well being. Public baths became a place to socialize, relax and enjoy artwork- a practice in medicine that no culture until then had ever seen (fig. 2.2). Therapies of oil massage became common, and other health procedures would take place in these new medical buildings. Cruse reports that these public baths became associated with the liking of a place of health (2004). 
Because water and bathing were important themes within religion and human health, these buildings became increasingly important to Classical Roman medicine. In addition to maintaining a person's state of well-being, baths were an important tool for alleviating symptoms of disease, such as inflammation of the skin, joints and muscles.

\subsection{Learning from East Asia}

Ancient East Asian cultures have practiced natural healing methods similar to those of the ancient Greeks and Romans. "Much of East Asian medical theory emerges from a more ancient narrative of the creation of the universe and of the natural world" (Bivins, 2007, p.20). These ancient beliefs are embodied in various ordering systems, systems that hold the common underlying ideas of man's unity with nature. The Yin and Yang are the most common type of ordering system. In this system, the status of a person is described by a set of principles with opposing characteristics. The Yin and Yang are opposing states, and may be defined as having varying levels of masculine or feminine traits. Similar to the humoral theory of Hippocratic medicine, the points describe health levels as cold or wet, and hot or dry, and used to diagnose illness. Once diagnosed, these illnesses were cured with the opposing forces-wet cured with dry, and hot cured with cold. Herbal treatments and acupuncture were some examples of treatment therapies. Although connections can be made with preceding forms of ancient medicine, the $Y$ in and Yang theories differed because the division of the traits was not strictly one sided. Every diagnosis was seen to hold a duality of both sides of the Yin and Yang traits.

Man's unity with the environment was a core principal in the Five Element Theory written in 200BCE. This theory was adopted in the Neijing medical texts of ancient China. In it, man "considers the human body as a microcosm of the universe" (Bivins, 2007, p.21). This included the natural environment, Chinese culture and even the country state itself. The body was seen as an entity that was run like a country, with its own ruler, officials, ministers and communication systems. A harmonious balance was needed in order for the system to successfully orchestrate itself. As in the natural environment, the body's fluids were transported via channels of twelve waterways connected to the body's vital organs. Figure 2.3 shows the differences between early East Asian ideas of the body and the modern western approach. Western theories developed much later in the technological era interpreted the body as a mechanical system, operating through a 

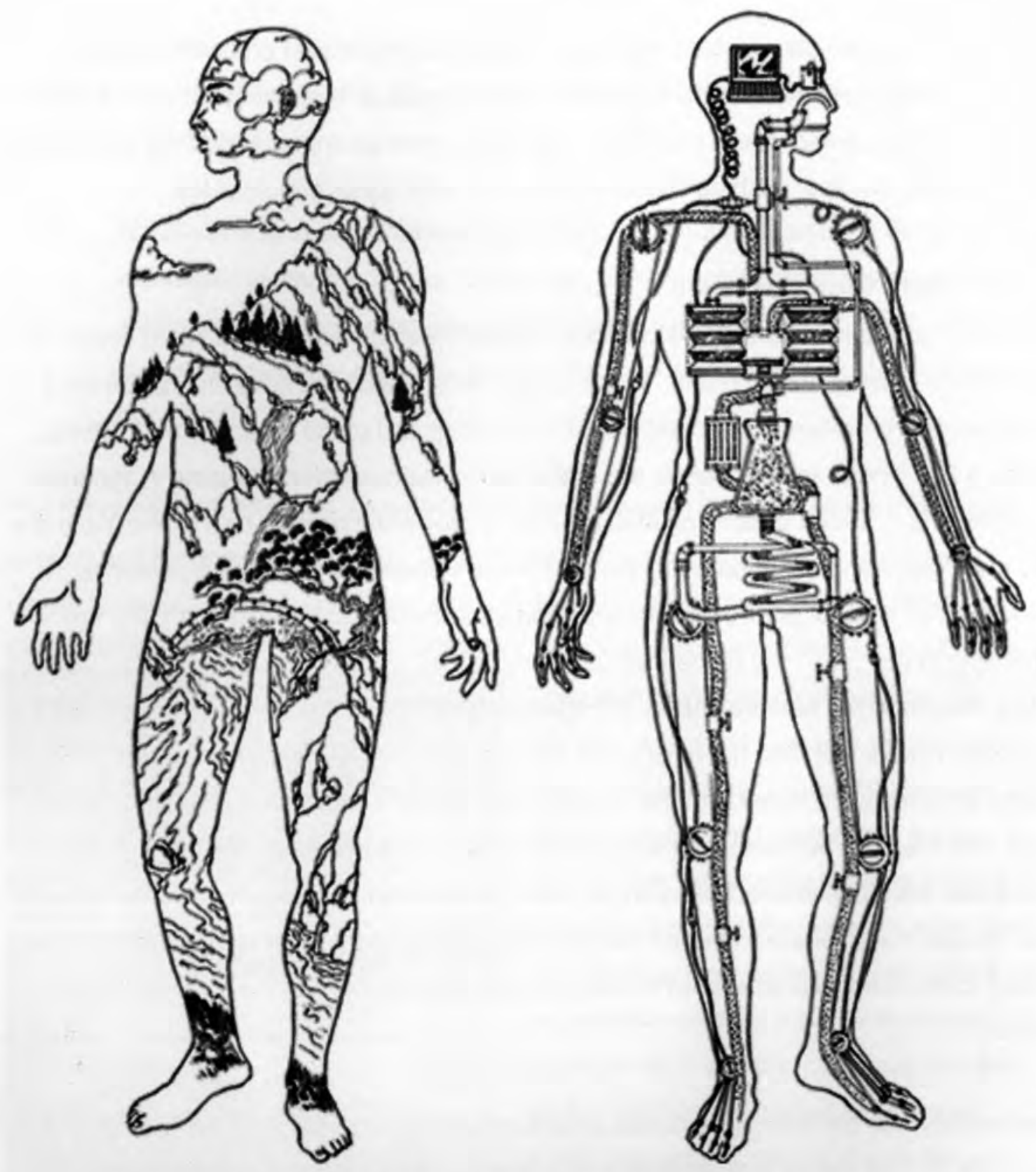

Figure 2.3 The human body as seen in eastern and western medicine Image credit: Chinese Medicine Works

complex system of wires and piping. This approach takes a curative approach to human health, in contrast to the East Asian ideals of taking preventative measures that induce a harmonious balance. This model of the body was seen as an important contribution to East Asian medicine, focusing on therapies, disease and the overarching idea of preventative medicine. This model was widely accepted and is still common within Asian culture today. 


\subsection{Current Day Systems of Holistic Healing}

Adopting a preventative lifestyle has benefits and has been practiced by many different cultures throughout history. Our western culture is the polar opposite to this proactive rationale of healthy living. We have grown accustomed to the chemical way of life, and are a pill-popping nation. Fighting the complex diseases of our modern time with medication seems discouraging, considering the drug induced side-effects associated with chemical medicine. The existence of chronic illnesses across cultures cannot be avoided, and is managed through high-technology medical procedures worldwide. The human species has evolved as a product of the natural environment, and our general level of health reflects the health of our natural environment. Today, the environment is more chemically polluted then ever. Thus, it seems logical that advanced and complex diseases have evolved. There are, however, certain cultures today that resist the concept of western chemical wellness and the associated Big-Pharma industry. Jinks, Lassey and Lassey (1997), state that these cultures have retained their natural systems of preventative healing throughout history. Low (2004) further demonstrates that other cultures, including Canada's, see the validity of adopting these proactive approaches to health and well-being.

\subsection{Current Japanese Society and Preventative Health}

In Japanese society, a preventative approach to living continues to be the way in which health is practiced and is recognized as both a social and cultural norm. The results of this approach are both astounding and impressive. The World Fact Book (2008) states that Japan had a population of 127.2 million people. The statistics regarding its public health are impressive. Jinks, Lassey and Lassey state that Japan leads the world with the highest life expectancy (1997). Japan's life expectancy in 1991 was 82.8 years for women, and 76.4 years for men. In 1991, approximately 12.6 percent of Japan's national population was 65 years of age or older. It is estimated that by 2025 , that number will increase to over 24 percent. Compared to other countries, Japan also has the lowest rate of infant mortality, 4.3 per 1000 live births. These successes constitute a healthy nation, and are particularly impressive when the proportion of GDP devoted to health care is examined; approximately 6.9 percent, the lowest amongst the most advanced industrial nations. 
Jinks, Lassey and Lassey (1997) state that these successes come from a public/private mixture of care, supplemented by the instilled preventative measures of the country's traditional culture. Preventative care is seen as a cultural norm in Japan. Proper health amongst individuals can be attributed to specific cultural characteristics, such as having a generally low-fat and high fiber diet. This largely vegetarian diet can be attributed to the high costs of seafood and meats. The level of health can also be seen through the intimate regard for nature that the Japanese hold, followed by their general enjoyment of the outdoors. Much effort has gone into the protection of the natural environment, which can be seen in the reduction of water and air pollution. Jinks and colleagues (1997) also describe the importance of exercise to the Japanese. Exercise is widely practiced at work, where a holistic approach to the body and health are sewn into the cultural fabric. The number of diagnosed cases of cancer is nonetheless increasing in Japan, as in any other industrialized nation. However, the ideals of preventative care and the promotion of good health have been given top priority today more than ever (Jinks, Lassey and Lassey 1997). This proactive set of cultural ideals is concurrent with the benefits outlined through the Japanese system of health-a system that is regarded as one of the most successful in the world.

\subsection{Alternative Therapies of the Future in Canada}

The holistic approach to well-being and living a lifestyle of preventative health has been successful in various cultures throughout history. Alternative medicine is an emerging market in Canada. In a report published by the Fraser Institute in 1997, it was estimated that Canadians spent approximately $\$ 1.8$ billion annually out of pocket on complementary and alternative medical practices, while spending an additional $\$ 2$ billion on vitamins, herbs, books and diet plans (Canada Business, 2008). Alternative therapies include acupuncture, aromatherapy, herbal medicine, homeopathy, massage, meditation, reiki, and yoga.

Alternative therapies vary greatly from traditional (allopathic) forms of medicine. Low (2004) describes several fundamental differences between the two approaches to healing. Alternative medicine is natural, preventative and "slow and gentle;" allopathic medicine is chemical, curative and "fast and brutal" (Low, 2004, p.55). Contrasting views exist regarding why people are moving away from traditional modes of allopathic healthcare in Canada, and towards alternative modes of medicine. Low describes two 
main reasons for this trend. The first is that people seek to take control of their own health and healing for ideological reasons. They believe that health is a holistic system that includes not only the physical, but also the psychological environment to which one is exposed - especially how one approaches the idea of being sick. The second is that people are generally unsatisfied with allopathic medicine, because they dislike negative medical prognoses from medical professionals. Specific reasons to why people truly choose to move to alternative therapies remains argued among health professionals. Minimal studies exist indicating why this move is so great today.

A recent questionnaire however of over 2055 Quebecers provided quantitative data regarding Canadians' choices in moving to more natural forms of healing. Crenlin, Andersen and Connor (1997) report that, the most influential factors in choosing alternative medicines were word of mouth ( 32 per cent) followed by the fear of pharmaceutical drug side-effects (21 per cent). Dissatisfaction with conventional medicine was not as sufficient an issue (14 per cent), as it was in Low's study (2004). The study asked for the medical reasons that those questioned had for using alternative modes. The top reasons were respiratory illness ( 27 per cent), followed by ear, nose and throat infections ( 24 per cent). The third highest medical reason for the switch to alternative medicines occurred with musculoskeletal problems (15 per cent). Prevention of infection was not on the list; however, 5 percent of respondents made the switch for this reason. Although prevention of illness is not a top concern among those Canadians in this study, there exist a few individuals for whom it was important.

In summary, the unhealthy state of our general population is culturally driven. As research has demonstrated, many present day cultures look back to how ancient civilizations had practiced a preventative mode of health, which involves a lifestyle that holds intimate regard for the natural environment (fig. 2.4). Due to the complex diseases of today, chemical medicine will always be needed. This method, however, is becoming increasingly costly and causes chemical induced side-effects. Preventative health takes a proactive approach to reducing the chances of becoming ill. In ancient civilizations, this included exposure to the beneficial qualities of nature. If these natural therapies are not speculative and scientific studies demonstrate their true worth today, then why can't we introduce these natural elements into our interior lifestyles to obtain their benefits? Can these elements prove beneficial in attempting to heal the sick by implementing them in 


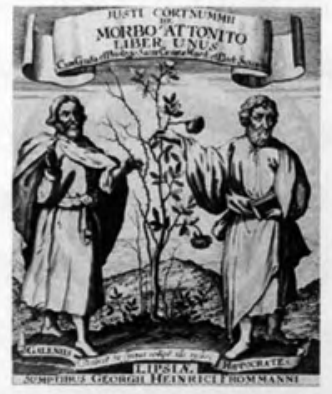

Ancient Greece

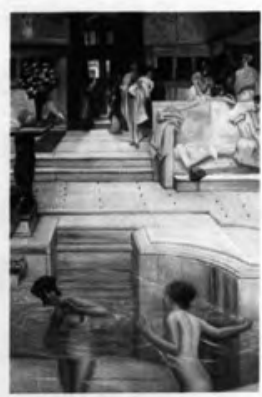

Romans Therapies

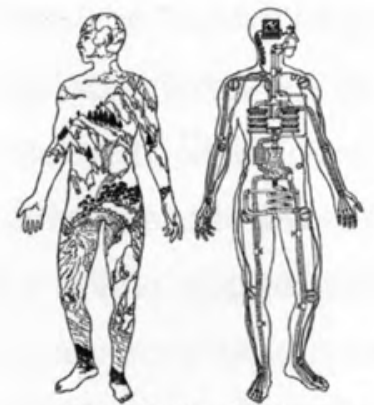

East Asia vs. West

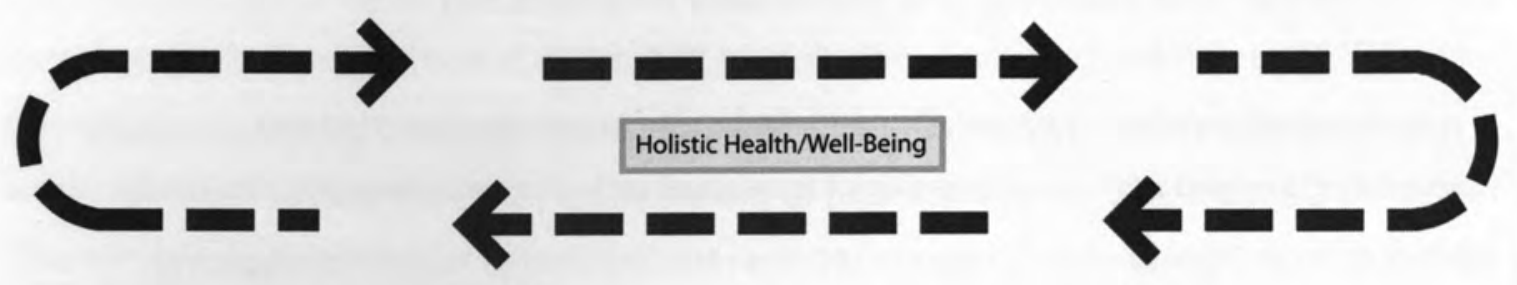

Alternative Therapies in Canada
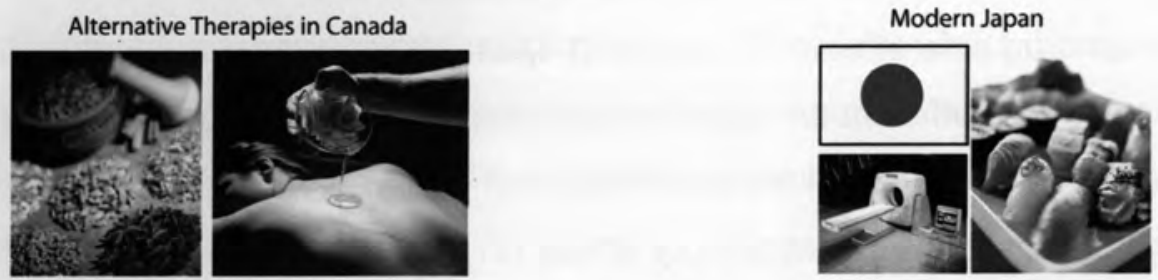

Figure 2.4 There is a cyclical connection in how alternative medicine/therapies in Canada today are returning to the same ideals found to be important in ancient cultures' approach to health

the design of our buildings? Can well designed spaces prevent sickness from occurring altogether? The following chapter will answer these questions, and will look at the wide body of research that attempts to prove the beneficial qualities of natural therapies known as evidence based design. 


\section{Chapter 3: Current Research into Natural Systems of Environmental Healing}

North America today is experiencing one of the largest healthcare building booms in history. In both the United States and Canada, an aging population, urban sprawl and the sedentary lifestyle that people are living increases the need for added health care services. These trends are driving the expansion of all aspects of medical care. According to a report published by the Ontario Hospital Association (2003) (as cited in the Globe and Mail, 2008), the average hospital in Toronto is over 40 years old; it will cost $\$ 8.4$ billion to modernize these facilities. In the United States, the figures are even more staggering. Zimring and Bosch (2008) estimate that $\$ 76$ billion a year will be spent on healthcare construction, with over 86 per cent of all hospitals in the country proposing additional buildings. Parallel with this massive investment in hospital infrastructures appears the realization that healthcare environments are unnecessarily dangerous, and that hospitals generally increase levels of anxiety, adding little help to those seeking an environment to better themselves in. Weinstein's (1998) study (as cited in Joseph, 2006) estimated that 88000 people die of hospital-acquired infections a year in the United States, and that their treatment costs over $\$ 4.5$ billion each year. Zimring and Bosch also estimated that 98000 people die annually of preventable medical errors while in hospital (2008). A more preventative and safely designed environment for patients to heal in will become increasingly important in the near and present future. One way to foster such an environment is to return to more natural healing systems. A growing body of research regarding natural therapies that are implemented into the design of our care environments is categorized as evidence based design.

\subsection{Evidence Based Design}

Hospital administrators and facility planners see the negative impact that poorly designed and planned facilities have on resultant patient outcomes, staff error and increased costs. An ever growing body of research is showing that well designed environments can dramatically increase the quality and safety of healthcare settings. Measures include a reduction in hospital-acquired infections, reduced patient falls, reduced medical errors, reduced patient transfers, reduced nurse turnover, a reduction in medication, quicker hospital discharge rates and added patient satisfaction (Zimring \& Bosch, 2008; Mobach, 2004). This approach of taking various types of health researches 
and implementing the findings into the design of the interior environments for the purpose of occupant health and safety is known as the field of evidence based design.

Evidence based design is design that is "based on the best available information from credible research and evaluations of projects;" "these designs ultimately should improve the organization's clinical, economic, productivity, satisfaction, and cultural measures" (Hamilton, 2004, p.1). Ultimately, this approach creates high quality healing environments for the individual occupant. Evidence based design is rooted in the approach of evidence based medicine, where "clinical protocols are based on systematic reviews of the research literature that evaluate the quality and quantity of research supporting the efficacy of specific clinical decisions" (Zimring \& Bosch, 2008, p.147). The evidence based design process was set forth by Kirk Hamilton, a practicing architect and leader in the design of healthcare facilities in North America (fig. 3.1). This systemic process consists of identifying key issues in the design project, gathering applicable research studies, interpreting these studies into design concepts, developing a hypothesis about what the outcome will be and then measuring the outcome upon project completion. This evidence based design approach was used in the preliminary design portion of this thesis|project, and EVIDENCE-BASED DESIGN PROCESS

\begin{tabular}{|c|c|c|}
\hline & Task & Activity \\
\hline 1 & Identify the Client's Coals & $\begin{array}{l}\text { Note most important and facility-related } \\
\text { global and project-based goals }\end{array}$ \\
\hline 2 & Identify the Firm's Goals & $\begin{array}{l}\text { Understand the firm's strategic, project } \\
\text { and evidence-based design objectives }\end{array}$ \\
\hline 3 & Identify the Top 3-5 Key Design Issues & $\begin{array}{l}\text { Narrow the possible choices; work on } \\
\text { high impact decisions }\end{array}$ \\
\hline 4 & Convert Design Issues to Research Questions & $\begin{array}{l}\text { Reframe statement of design issues to } \\
\text { become research topics }\end{array}$ \\
\hline 5 & $\begin{array}{l}\text { Gather Information (Benchmark Examples, Literature } \\
\text { Sources, Internal Studies) }\end{array}$ & $\begin{array}{l}\text { Infinite possibilities must be narrowed; } \\
\text { limited perspectives must be expanded }\end{array}$ \\
\hline 6 & Critical Interpretation of the Evidence & $\begin{array}{l}\text { No direct answers; requires open-minded } \\
\text { creativity, balance, and critical thinking }\end{array}$ \\
\hline 7 & Create Evidence-Based Design Concepts & $\begin{array}{l}\text { Based on creative interpretation of the } \\
\text { implications of research findings }\end{array}$ \\
\hline 8 & Develop Hypotheses & $\begin{array}{l}\text { Predict the expected results of the } \\
\text { implementation of your design }\end{array}$ \\
\hline 9 & Select Measures & $\begin{array}{l}\text { Determine whether your hypothesis is } \\
\text { supported }\end{array}$ \\
\hline
\end{tabular}

Figure 4.1 Evidence based design process Image credit: Hamilton \& Watkins

focused on many of the issues relating to both the program and siting of a proposed Wellness Centre in Toronto (appendix 1). By supporting the healthy design of our interior environments with medical evidence, using an evidence based design approach allows 
future care settings to evolve into high quality healing environments that offer a multitude of therapeutic advantages. Mobach (2004) outlines some design concepts that are being implemented into the healing environment; these include views to nature, maximized day lighting in patient rooms, and varying levels of music therapy and artwork within the common areas of hospitals.

Connections between evidence based design and the preventative lifestyle carried out by early civilizations can be seen. These ancient cultures approached a day to day lifestyle in a holistic manner, ones that held an intimate relationship with the healthy attributes inherent in nature. They considered their hospital facilities to be true places of hospitality, where "healthcare was also associated with religion, music, poetry, the arts and good food" (Purves, 2002, p.3). This empirical evidence demonstrates this as a successful approach to health before the time of complex chemical medicine and disease brought forth during the industrial revolution. The advances in manufacturing and production brought with it illness due to high levels of pollution, overcrowded living conditions and plagues of infectious disease. The approach of health taken by ancient cultures before this era offers empirical health data that scientists have used for the design of our healthy environments today.

Grounding design in both empirical and scientific evidence has been recognized as a promising initiative from several not-for-profit advocacy health groups. These groups supply this body of research knowledge to hospital administrators interested in implementing the latest design findings to their facilities. The Centre for Health Design (California) and the International Academy for Design and Health, (Stockholm, Sweden) for example, advocate designing a physical environment of therapeutic environments for all its occupants, including patients, their families and the healthcare staff (Centre for Health Design, 2008; International Academy for Design and Health, 2008). Major areas of research include the healing powers of light, the exposure of patients to nature, sound, music and art therapy, as well as the transfer of nosocomial infections. The research into these areas will be looked at in more depth in the preceding sections.

\section{Field Research}

Several architecture firms in North America have used the evidence based design approach as a primary approach to their design practice. Anshen + Allen Architects are 
one of the pioneers of this adaptive process. Located in San Francisco California, Anshen + Allen have focused their client list to that of healthcare, academic and research buildings. Field research included visiting many of the Anshen + Allen projects in the San Francisco area, a meeting with a Principal architect of the firm, as well as visiting many other design projects in the Seattle area (appendix 2).

In a meeting with a senior medical architect and Principal of Anshen + Allen Architects, Martin Waldron described how their approach was different from that of previous practitioners in the field of study, in that "individuals like Kirk Hamilton had advanced the research culture, and Anshen + Allen has gone in another direction" (M. Waldron, personal communication, March 3, 2009). The new direction came when the practice realized that there was a missing piece to the puzzle, one that was embodied in William McDonough's theories on eco-effectiveness and the entire notion of sustainability (McDonough \& Braungart, 2002). Anshen + Allen saw the dangerous use of specific materials in the healing environment, and started "questioning the culture that made us originally design with materials that were harmful" (Waldron, 2009). Thus, importance was placed on the ideas of healing the sick, but also the idea of occupant well-being. This complicated network of issues became a more holistic and sustainable approach to design, one that would involve various peoples input from the early stages of a project. An Integrated Design Process (IDP) would be adopted by the practice, one that involved architects, interior designers and medical practitioners in the hopes of truly designing environments of healing.

Another area of specialized approach by Anshen + Allen was looking at the building's design from perspective of the end user. Using healthy materials as well as incorporating research studies was very much a part of a successful design outcome using this process. However, what was just as important was the experience the occupant felt in that specific space. As a technique for design, 'narratives of experience' were written from the perspective of occupant. Narratives were seen as a "train of words and images" taken from the perspective of children, the elderly, visiting family and even medical staff (Waldron, 2009). These narratives aided in the design process by demonstrating specific experiences including feelings of emotion, pain, happiness and the potential misuse of offending colours and materials. 
What the research into evidence based design found was that much of the original ideas involved in this design process demonstrated the strictly quantitative nature of designing with scientific research studies. The field research on the other hand showed the approach of designing spaces with more qualitative methods, such as the tool of 'narratives of experience.' This had reinforced how different occupant's experience space differently, which the singular action of designing with research studies does not make concession to. Architecture exists as a combined field of both art and science and from the perspective of the writer, should be approached in an equal manner.

Phenomenological architectures for example, place major importance on the user's experience, and draws connections to the sensorial and psychological aspects of the built environment (Pallasmaa, 2005; Sharr, 2007). This artful approach should not be overshadowed by the scientific approach of designing healthy and safe environments of healing. Thus, the design of our future healthy environments should take both of these areas into consideration, both the quantitative and the qualitative approaches to design.

\subsection{The Healing Powers of Light}

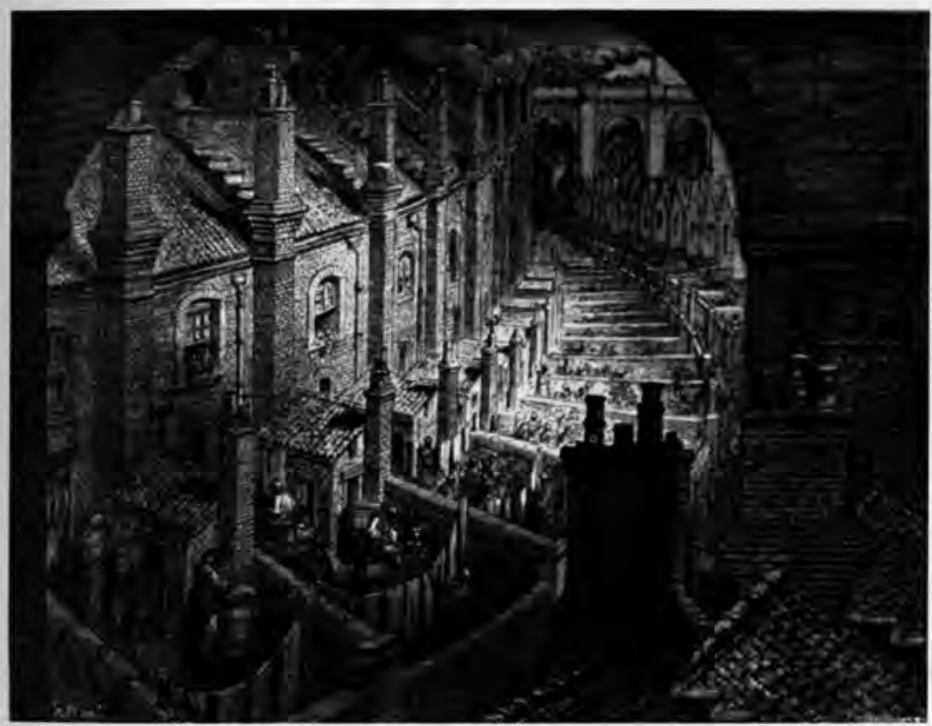

Figure 5.2 'Over London by Rail,' Gustave Dore (1872) London Slums during the Industrial Revolution Image credit: the Valve
Biological organisms, including the human species, need natural light to grow, reproduce and behave positively. Hobday noted that, historically entire civilizations have been centered on the sun (2006). Williams (1991) states, however, that the industrial revolution disrupted our previous exposure to acceptable levels of natural light within our built environment. History has a habit of repeating itself, especially in architecture and health. Today, the positive effects associated with patient exposure to healthy levels of natural light have been documented through evidence based research studies (Joseph, 2006; Hobday, 2006). The benefits include decreased levels of patient depression, agitation, stress and pain, as well as vitamin deficiencies (Joseph, 2006). This evidence is now allowing us to include natural light in 
our healing environments. This evidence supports the need for designs to maximize the amount of natural light.

The connection between light and health has been made throughout history. Early civilizations had seen the importance of the sun, and had centered entire societies on its existence. As Hobday states, the great Egyptian polymath Imhotep had made the historical connection between the sun, architecture and health (2006). Hobday also described Roman civilizations in the third century AD that created laws on the right to light, and meticulously oriented their villas, public baths and cities around the sun. Hobday indicates, however, that during the Industrial Revolution of the $19^{\text {th }}$ and $20^{\text {th }}$ century, housing conditions in England were appalling, with

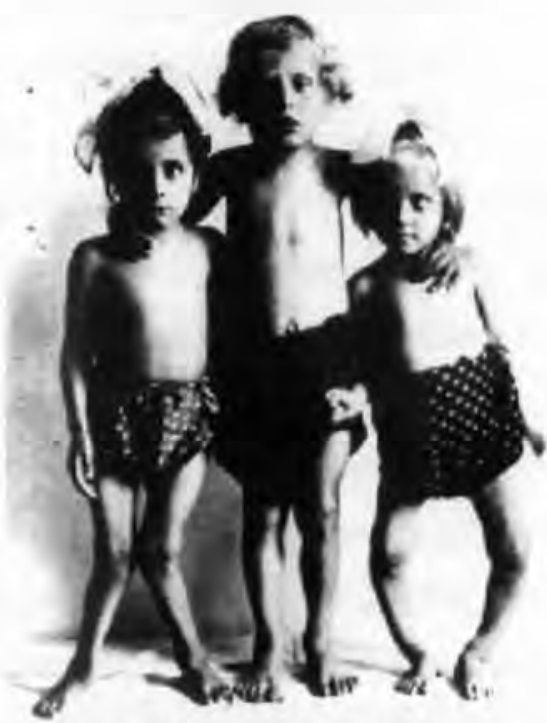

Figure 3.3 Rickets in Children Image credit: Talk Origins skies being covered in an everlasting mantle of smoke (fig.3.2). The smoke contributed to the plagues of both rickets in children (fig.3.3) and tuberculosis in adults. In 1921, scientists established that rickets in children was due to a lack of vitamin D, caused by minimal exposure to the sun.

Following this discovery, exposing oneself to the sun was considered a form of preventative medicine, and natural forms of therapy such as sunbathing and heliotherapy were introduced into modern societies. Architects therefore incorporated these new discoveries into the building forms of the modern architectural movement (Hobday, 2006). However, the invention of the electric light bulb in the 1930's created a cheap and efficient way to light our internal environments (Nield, 2008). Hospitals and homes gradually relied less and less on natural lighting. Today, it is estimated that $90 \%$ of our time as Canadians is spent indoors (Canada Green Building Council, 2004). This has led to increased incedences of diseases that are regarded as preventable, such as depression, heart disease, and diabetes (Hobday, 2006).

Our largest attachment towards the sun and healthy levels of natural light occurs within our innate and internal biological processes. Sunlight enters the eye and sends electrical 
signals that travel to the visual cortex and on to the rear of the brain (Hobday, 2006). It is here that our internal biological clocks respond to the daily and nightly cycles of the sun, and to the variation of the seasons. A chemical reaction produces melatonin, a hormone that tells us that we, and all organs of the body, are awake. This master clock gives the body what is known as a circadian rhythm, the 24 hour cycle our bodies and systems need to adapt to the external environment. Lewy, Nurnberger, Wehr, Pack, Becker and Powell's (1985) study, (as cited in Joseph, 2006) stated that when levels of natural or artificial light are minimal during the day, the reduced level of melatonin contributes to sleeplessness and depression, as well as other related health issues. This has profound effects on individuals, especially those impacted by the institutionalized setting of modern hospitals and the healing process.

By minimizing the amount of natural light that patients are exposed to, the body's circadian rhythm becomes compromised. Studies have been published demonstrating that increasing natural and artificial light helps to reduce depression and seasonal affective disorder (SAD) within patients. Depression is a great problem in today's industrialized world. Hobday (2006) states that it is estimated that 340 million people suffer from depression, and that 40-80 per cent of people suffering from depression do not seek any form of treatment. Benedetti, Colombo, Barbini, Campori, and Smeraldi's (2001) study (as cited in Joseph, 2006) allocated depressed bipolar patients into two sections of a hospital. In the study, half of the patients had rooms facing east (exposed to bright light during the morning hours) and the other half had rooms facing west (exposed to minimal levels of bright light all day). Those patients receiving higher levels of natural morning light were in hospital 3.67 days less then those patients in the westward facing rooms. In an American Journal of Psychiatry (2005) study, (as cited in Hobday, 2006) it was found that bright light therapy was just as effective as medication in the treatment of mood disorders and depression. In a study by Golden (2005), (as cited in Hobday, 2006) a team of researchers conducted a statistical analysis of 20 studies in bright light therapy over a 20 year period. These amalgamated studies demonstrated that bright light therapy was an extremely effective method for the reduction of depression.

Hobday also links minimal light exposure levels to the global rise in obesity (2006). For example, depressed patients retain low levels of serotonin in the brain, which causes 
them to eat meals that are high in carbohydrates and contributes to weight gain. As well, sunlight stimulates the production of a strong appetite suppressant known as alphamelanocyte. Vitamin D normalizes food intake and levels of blood sugar, a chemical that is directly provided by exposure to the sun. The connection between living an indoor lifestyle and related depressiveness could be another facet to the global rise in child and adult obesity levels.

Natural levels of light have also been seen to affect people's mood and satisfaction at work. Alimoglu and Donmez (2005) study, (as cited in Joseph, 2006) studied 141 nurses in a Turkish hospital. Evidence found that those receiving at least 3 hours of natural daylight had a higher level of satisfaction at work and lower levels of stress. Heerwagen and Heerwagen's (1986) study, (as cited in Joseph, 2006) found that people at work preferred daylight as opposed to artificial lighting. Several factors that contributed to this preference included visual health, work performance, office pleasantness, psychological comfort and a level of general health. Mroczek, Mikitarian, Vieira, and Rotarius (2005) study, (as cited in Joseph, 2006) showed the positive impact of supplementary levels of natural light on job satisfaction. When atriums and windows were added to majority of rooms of a hospital, 70 percent of the staff had positive increases in their work experiences. Exposure to lighting can not only benefit the patients of a health facility, but also the staff who work there. In an age where staff error and burnout are rising, designing for the hospital staff is just as important as designing for the patient.

Another important effect of exposure to natural light relates to the synthesis of vital chemical reactions in the skin. McColl and Veitch's (2001) study (as cited in Joseph, 2006) found that to the majority of vitamin $D$ in the blood of the human body can only be synthesized by exposure to sunlight. They reported that while most people are able to synthesize vitamin D. Some people such as shift workers, the elderly and the institutionalized chronically ill have difficulty with this process. Liu's (2006) study (as cited in Hobday, 2006) found that vitamin D was essential to the body's ability to fight against tuberculosis. This is extremely important since it is estimated that over one third of the global population die from tuberculosis, more so now then at any other point in history (Hobday). The World Health Organization (as cited in Hobday, 2006) states that there are 2 million deaths each year and, that by 2020, 36 million people will die if infection controls do not improve. This is a direct result of lack of exposure to proper 
lighting. Vitamin D deficiency cannot be fixed by any form of medication. Care environments must be sensitive to these factors, and hospital design must look at the implications that a poorly lit space has on both the staff and patient.

\subsection{Experiencing Nature}

The presence of the natural environment has been documented as having a direct effect on people recovering from illnesses. Studies have shown that individuals admitted to hospitals who are exposed to nature through views or direct engagement have experienced health benefits. These include quicker recovery times, reduced levels of stress and blood pressure, and reduced physical discomfort and levels of depression (Schweitzer, Gilpin \& Frampton, 2004; Ulrich, 1984). Hospital staffs exposed to nature have a general feeling of rejuvenation and return to work feeling more relaxed and refreshed (Marcus \& Barnes, 1995). Research also points to the beneficial psychological effects that naturally shaped built form has on our mental health (Joye, 2007).

Studies have shown that patients recovering from surgeries recover more quickly when given a room with a view to nature, as opposed to a room with minimally stimulating views. In a famous study conducted by Roger Ulrich (1984), patients admitted for gall bladder removal surgery in a suburban Pennsylvania hospital were researched. Patients recovering from this type of surgery typically experienced considerable levels of anxiety; their engagement with the outside world was restricted to views

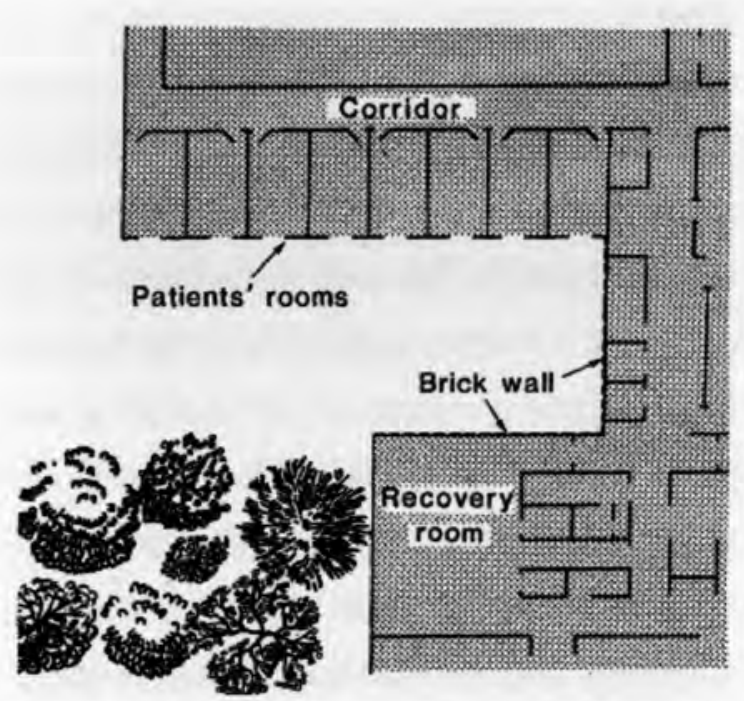

Figure 3.4 Controlled views of nature for post surgery patients Image credit: Roger Ulrich through a window. The study used two types of patient rooms: those with a view of a small stand of deciduous trees, and those with a view of a monotonous brown brick wall (fig.3.4). Data were recorded on 46 patients grouped into 23 pairs by experienced surgical nurses. 
Ulrich's study found that patients who were exposed to a view of a natural setting with tall standing trees spent less time in the hospital recovering from their surgeries than those patients with views of the brick wall (1984). The former spent an average 7.96 days recovering in comparison to the latter group spending 8.70 days. Also recorded were the comments about the patients' condition during this post surgical period. The data showed that patients with views of nature made on average only 1.13 negative complaints, while the patients with the brick wall view made 3.96 complaints. Finally, the amounts of painkillers administered to the patients showed a similar trend. The wall group needed significantly higher amounts of analgesic medication then did the nature group. Ulrich's study showed that views of nature during the recovery process have therapeutic qualities; however, it remained subjective to what each individual patient feels as therapeutic in his/her own eyes (1984). It was suggested that perhaps a view of a lively urban street may be advantageous to certain patients. Nevertheless, the study concluded that hospital orientation regarding patient views was an important factor affecting patient recovery.

This early evidence had sparked a number of subsequent studies regarding environmental psychology and the effects nature has on individuals involved in the healing process. One milestone study by Marcus and Barnes (1995) documented to the therapeutic benefits of incorporating gardens into healthcare facilities. The methodology consisted of conducting patient and staff surveys within four case study hospitals that incorporated garden programming into their facilities. Included in the analysis was attention payed to seating typology, traffic flows, levels of privacy, levels of varying vegetation, human companionship, aesthetic attractiveness, religious and spiritual connections as well as other various factors. Detailed plans were

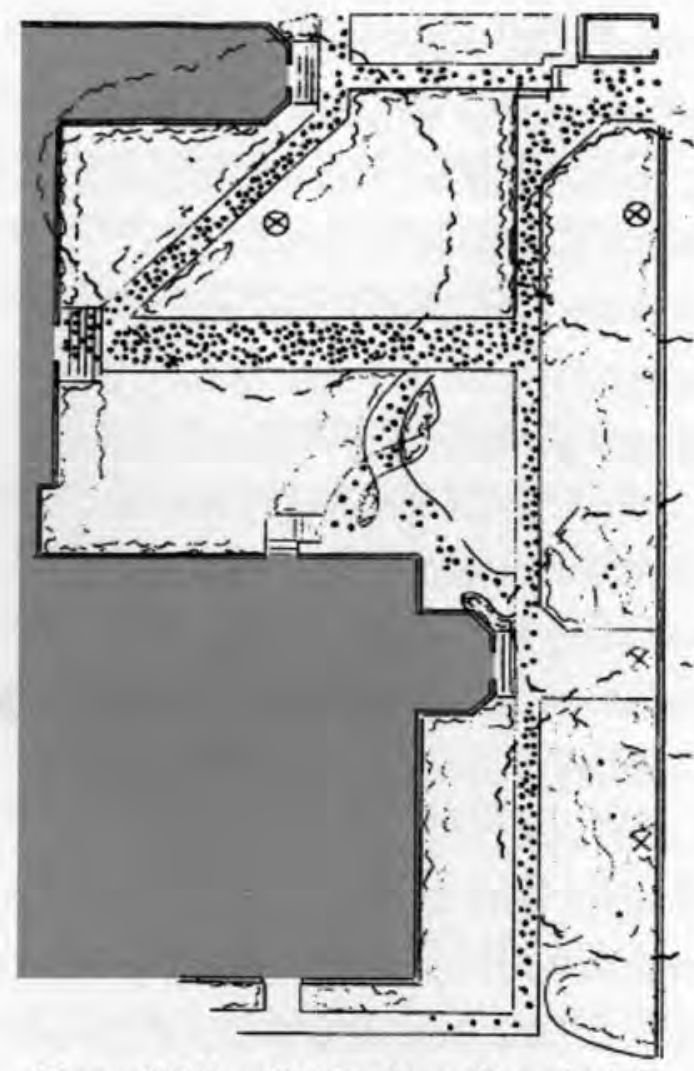

Figure 3.5 Hospital Garden with pedestrian aggregations over an 8 hour period Image credit: Marcus \& Barnes 
used to record spatial analysis of the case study gardens (fig 3.5).

In this study, Marcus and Barnes interviewed 143 garden visitors, and asked them to choose from a list of ten options of what their main purpose of frequenting the garden was (1995). Almost 94 per cent of the users stated they had visited the garden to relax, while more than half had used it to eat, talk, stroll or general reasons of outdoor therapy. Additionally, 95 per cent of the users reported that they "feel different" after visiting the garden. Levels of mood change included being less stressed, calmer, rejuvenated, stronger, able to cope, higher levels of positivity and spiritual connection. When naming the qualities that were helpful in attaining mood change, the majority claimed that trees and plants were of extreme importance. More importance were natural phenomena, such as wind/fresh air, light/sun and positive distractions like animal sounds. As well, the users enjoyed the garden because it was a peaceful escape from work and provided human companionship.

This study shows that persons in medical settings would use outdoor gardens for emotional healing and therapy. Almost 95 per cent of the patients studied reported some level of therapeutic benefit from visiting the gardens. Beneficial factors like emotional changes for patients and staff generally created a better healing environment, allowing for rejuvenation and stress relief when returning to the hospital quarters. This increase in staff morale promotes a successful clinical outcome regarding patients and the hospital community. Patients generally spoke about having higher tolerances of their medical surgeries and procedures. Family members and other visitors showed reduced levels of stress regarding the hospital visits to patient relatives. Marcus and Barnes' (1995) outcome demonstrates that designing gardens into hospital settings would benefit the entire medical community, and facilitate the patient recovery process. Their data and recommendations are relevant to the design of the next generation of therapeutic outdoor environments.

Research shows that it is due to specific evolutionary processes that people innate emotional connections to elements occurring in nature. Joye's research (2007) shows the positive connection evolved in early humans with positive levels of psychological and physiological functioning. These connections have developed despite our natural evolution as nomadic hunters and gatherers. Environmental psychology has studied this 
human/nature connection in great depth, examining how different settings can trigger different affective states in individuals. Roger Ulrich's (1983) study, (as cited in Joye, 2007) documents an automatic and unconscious response that happens rapidly regarding the immediate like or dislike of environments. This psycho-evolutionary framework can be explained through adaptation; early humans needed to quickly realize which environments would contribute to their survival or demise. This led to the actions of dislike associated with avoidance behavior, and actions of liking being associated with explorative behavior.

The fact that today we are detached from the natural environment breaks this innate connection we have had with nature in the past. Joye (2007), reports that architects have tried to recapture natural forms within their architectural expression that may only be found in nature. The art of mimicking natural forms through architecture and

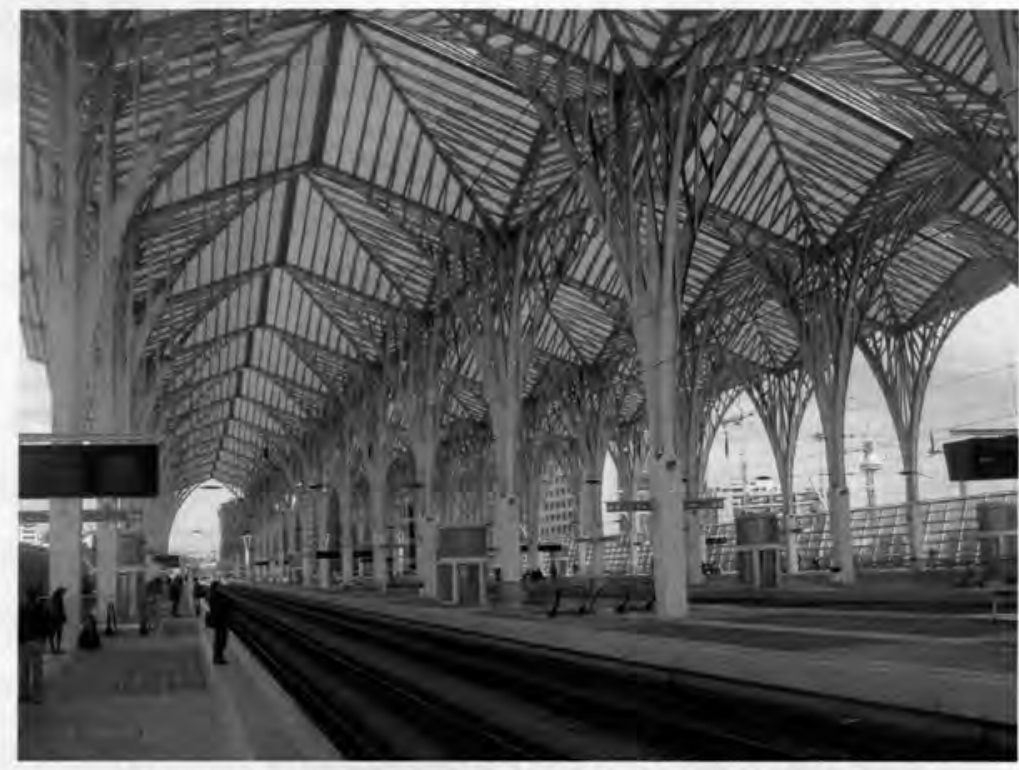

Figure 3.6 Oriente Station, Lisbon, Santiago Calatrava Image Credit: Flickr the built environment has been considered as beneficial to the human psyche. This nature-based form of expression is known as biophilic architecture, and can be seen in the works of Santiago Calatrava (fig. 3.6). Biophilic design emulates the concepts of aesthetic appeal from nature that have being hard wired into our evolutionary processes. Orian and Heerwagen's (1992) study (as cited in Joye, 2007) states that vegetative landscapes and water features have contributed to our survival and ability to procreate as early humans. The existence of flowers in a vegetated area would have signaled a food source, and future foraging sites. The savannah hypothesis came out of Van den Berg's (2004) study, (as cited in Joye, 2007), and puts forth the idea that early humans spent a majority of their time in open savannah's due to their favourable structural landscape features. This type of setting existed as low lying grassy terrain and scattered trees, 
which were easily accessible to man. The presence of trees was seen to provide protection against sun and rain by Orians and Heerwagen's (1992) study, (as cited in Joye, 2007) and acted as a potential retreat from dangerous predators. The nomadic lifestyle of early humans and their movements through savannas by hunting and avoiding predators was therefore conducive to native survival.

Another interesting theory interpreted by Ulrich's (1983) study, (as cited in Joye, 2007) came from his evolutionary framework and focused on restoration. Ulrich's theory revolved around early humans being placed into direct harm through dangerous and lifethreatening situations from predators. These scenarios would lead to "the quick onset of negatively toned affective reactions and corresponding adaptive behaviour" (Joye, 2007, p. 309). These reactions have a negative overtone and lead to stress. When the negative reaction had vanished, a need for restoration would occur in the individual. The restoration would occur through stress-reducing environmental elements in the natural savanna setting, such as vegetation and calm waters. These factors regarding the effects of the natural environment seem to have occurred inherently through evolution. This evidence points to the fact that we have an emotional affiliation with specific types of natural objects and settings. By implementing nature into our interior environments we can reduce levels of stress and anxiety amongst patients and all other individuals who occupy the space.

\subsection{Sound, Music and Art Therapy}

It has been seen that naturally occurring elements such as daylight and views of nature can be of psychological benefit to patients and staff alike in the healthcare environment. These factors facilitate healing for the occupants of the building. Other factors can be derived from man-made interventions that provide both positive and negative forms of stimulation. Sounds within the care environment play a large part in the healing process of the individual. Excessive noises in the health environment must be avoided, while playing music for healing purposes seems advantageous (Joseph \& Ulrich, 2007). Studies have shown that installing artwork can stimulate beneficial psychological effects amongst both staff and patients, acting as a form of temporary therapy (Rapp, 2008; Muir, 2008). 
Sound in healthcare settings is an important issue. Different aspects of sound impacting staff, patients and visitors are noise, speech privacy and speech intelligibility. In a paper written by the Centre for Health Design, Joseph and Ulrich (2007) state that hospitals worldwide remain extremely noisy places, reaching as high as $85 \mathrm{db}$ (A). According to the World Health Organization, background noise in hospital patient rooms should be 35 $\mathrm{dB}$ (A) during the daytime, and $30 \mathrm{db}$ (A) at night (Joseph \& Ulrich, 2007). Joseph and Ulrich state that the sound levels in hospitals are 100000 times greater in sound pressure then what is acceptable. This disturbance has direct effect on patient annoyance, blood pressure, sleep wakefulness, heart rate and respiration. Joseph and Ulrich found that unwanted noise also increased staff levels of stress, fatigue, exhaustion and burnout rates (2007). These factors commonly lead to errors in medication. Joseph and Ulrich found that noise in hospitals is caused by many factors. Some include medical equipment, sound reflecting surfaces, conversations from multiple patient rooms and other audible conversations from the nursing staff.

Sound in hospital environments can also positively impact the healing process. Music therapy has positive impacts on the building occupant (Lai, Chen, Peng, Chang, Hsieh, Huang, \& Chang, 2006) (as cited in Joseph and Ulrich, 2007). Patient heart rate, anxiety levels, respiratory rates and temperatures were reduced. Joseph and Ulrich state that music therapy has been used in many applications including intensive care, coronary care, maternity, oncology and pediatric care (2007). In Cooke, Chaboyer and Hiratos's (2005) study (as cited in Joseph and Ulrich, 2007), patients in the ambulatory setting, and more specifically the day surgery setting, indicated that music therapy reduced their anxiety levels during recovery. Similarly, Goodall and Etter's (2005) study (as cited in Joseph and Ulrich, 2007) showed that dementia patients who selected their own music saw reduced behavioural tendencies and levels of agitation decreased dramatically among dementia patients who were allowed to choose their own music.

Art in the care setting has allowed for similar conclusions as that of music therapy. Florence Nightingale saw that patients need some sort of sensory stimulation for a good quality of life (Rapp, 2008). More recently, evidence has emerged that stimulants directly affect the patient's health as well as their will to live. Rapp also states that brilliant colour and a variety of beautiful objects facilitate patient recovery. Interestingly enough, the effects are not strictly on the mind. Rapp states that little is known about the way "we are 
affected by form, by colour, and light, we do know this, that they have an actual physical effect" (Rapp, 2008, p.296). In addition to installing art into the care environment, art therapy has been adopted in many health care settings. For example, in Australia women suffering from dementia experienced positive changes occurred when participating in creative art therapy (Muir, 2008). Group psychotherapeutic programs have been started that allow for a number of individuals to be immersed in the arts under careful supervision. These creative art therapy sessions have been seen to calm patients, bring up positive levels of self-esteem and increase social interaction.

\subsection{Transfer of Nosocomial Infection}

One important area of research pertaining to the future design of healing environments addresses nosocomial infections. Also referred to as hospital-acquired infections, nosocomial infections have a tremendous impact on preventable deaths within the healthcare setting, as well as bottom line cost. Weinstein's (1998) study, (as cited in Joseph, 2006) shows that nosocomial infections are one of the leading causes of death in the United States. In 1995, hospital acquired infections contributed to more then 88 000 deaths and cost the government $\$ 4.5$ billion. Weinstein states that this amounts to more deaths than breast cancer, AIDS or car accidents. Nosocomial infection primarily affects those individuals who are immunocompromised- those individuals that are aged, already have rooted diseases, and/or have just undergone medical treatments. Joseph reports that nosocomial infections may be transmitted through the air, through water and from surfaces.

Joseph (2006) states that airborne infections can be transmitted into the hospital environment from such sources as fungal spores being released into the air from environmental reservoirs, as well as microorganisms transmitted from person to person via droplets in the air. Sources can include construction or renovation projects that are underway, as well as contaminants flowing through hospital ventilation systems.

Airborne pathogens must be carefully controlled to decrease the spread of infection. One option is HEPA filters which successfully diminish 90 per cent of air pollutants, and could be installed in most patient care areas. Another option is to isolate high-risk patients in single patient rooms in quarantined areas of the hospital. Effective planning measures can also be implemented when supervising a construction or renovation project within the hospital setting. 
Transmittance of nosocomial bacteria through water sources is also a concern. Joseph (2006) states that improperly treated water in hospitals that is deemed as potable may transmit bacteria that, when ingested, may be lethal. Recent outbreaks of Legionella have occurred in Canada and the United States, which comes from a form of water transmittance. Legionella, however, is transmitted through airborne water droplets that can be generated from faucets cooling towers, room air-purifiers and medical equipment. The risk can be minimized by maintaining and constantly inspecting the hospitals' water supply, cleaning and disinfecting water fountains and regularly cleaning faucet aerators to prevent the spread of Legionella.

Surface contamination is another way that nosocomial infections may be transferred. Joseph (2006) states, that although surfaces are reservoirs for pathogen infection, they are not the direct reason for transmittance. It is primarily through the hands of healthcare staff that cross-contamination can occur. Constant hand washing by staff is the most important preventative measure to be taken, furthered by installing automated hand washing sinks in many locations, including single patient rooms. Making sure that the staff is reducing the chances of infecting patients from dirty hands through physical contact can greatly decrease the level of hospital-acquired infections.

In summary, the unnecessary number of deaths and high costs related to poor healthcare design need to be addressed in future care settings. Evidence based design demonstrates that through applying a wide body of analytical research studies to the built form, healthier indoor environments can be created. Healing environments can implement the beneficial properties of views to natural vegetation, maximized natural day lighting, and incorporating sound, music and art therapies (fig.3.7). Additional research, however, must focus on modern day examples that have attempted to implement these ideas into the built form. Specific questions arise, including what a facility employing evidence based design look like? What is the general feedback from building occupants regarding their level of health? Have there been any cost savings from the implemented principals? Has there been a reduction in the length of patient stays, or a reduction in levels of stress? Has safety been increased? These areas will be analyzed in the next chapter. 


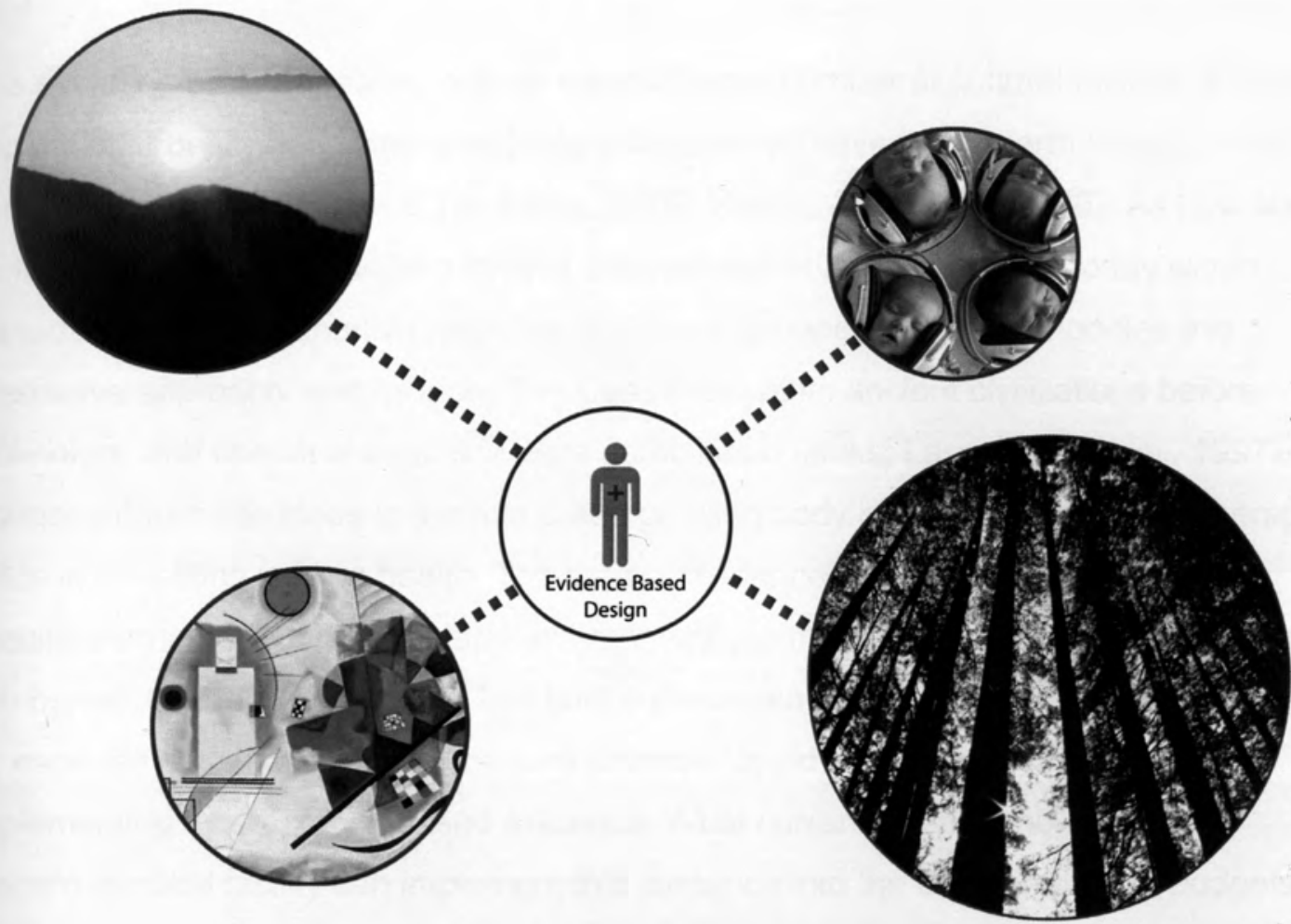

Figure 3.7 Evidence based design centres around the idea of 'patient focused care,' and can implement views to nature, maximizing natural day lighting, and incorporating sound, music and art therapies for the creation of healing environments 


\section{Chapter 4: Theoretical Discourse}

The evolution of an unhealthy culture results from a number of cultural factors. An aging population, obesity and living a sedentary lifestyle will have a long term impact on our healthcare system (Shields \& Tjepkema, 2006; Hogan, 2002; Farr, 2008). As Low states, a move towards a more holistic form of alternative healing is occurring today within Canada (2004). Preventative medicine in current Japanese culture embodies this alternative approach, and includes the ideas held within ancient civilizations before technology and chemical medicine were established (Jinks, Lassey \& Lassey, 1997). Connected to these ideas is the role that a growing body of research in natural therapies holds in benefiting human health. The area of evidence based design proves that specific elements within the natural environment (combined with alternative therapies) can benefit the healing process. The built environment that we are continually exposed to, especially those involved in the care process, could benefit tremendously from implementing this research based evidence. What currently is not known is how a modern medical facility can implement this evidence into the built form. Also, budgets in healthcare are stretched, and will leave the hospital administrator and planners asking where to invest scare resources and what the best evidence to buy is. Additionally, very few cost studies that prove the worth of implementing these design features have been published; some cost studies question if the hospital administrator should buy into evidence based design altogether. Another factor within the responsibility of the architect remains to be questioned. In designing buildings that are sensitive to human needs like care settings, what is the responsible approach with buildings that can adapt to changing trends? These areas will be looked at within the following sections, and involve research that currently exists, or is still needed.

These questions are addressed in the following three sections:

- Process vs. Place

- Adaptability and Flexibility

- The Economic Value of Design 


\section{1 Process vs. Place}

Key words: institutionalized, natural environment, making of place

\subsubsection{Argument: The Need to Follow the Grain of Nature}

Geoffrey Purves (2002), a healthcare architect in England who wrote his PHD on healthy living centres, outlines the failure of the country's National Health Service regarding the treatment of modern day cancers. He wonders why, in a country like England, where wide access to high technology is available, survival rates of such diseases of cancer seem to be much lower than those in the rest of the world. The experiences outlined by the World Health Organization indicate that throwing money at the problem does not produce a good system of human health. However, the more sustainable and holistic principles of the traditional and natural healing practices may be more affective. The importance of following the design of nature was stated by the Prince of Wales in a 2002 lecture:

\footnotetext{
Part of the problem is the prevailing approach that seeks to reduce the natural world, including ourselves, to the level of a mechanical process.....above all, we should show greater respect for the genius of nature's design, rigorously tested and refined over millions of years (Purves, 2002, p.2).
}

Purves believes that nature is the ultimate designer. The strength of his point can be seen through millions of years of evolution, concluding with what should be the healthy human species, a true product of nature (2002). These ideas outline the argument that: the institutionalized nature of the majority of our modern hospitals is a detriment to the level of our public health. In order to get optimized beneficial effects in patients, future healing environments must be designed as microcosms of nature. They should emulate as much of the external natural environment within its interiors as possible. This creates a sensitive environment of healing that benefits all building occupants.

\subsection{2 $19^{\text {th }}$ and $20^{\text {th }}$ Century Examples of Environmental Importance}

The evolutions of our places of health into institutionalized settings have increased the level of sickness throughout the age of modernism. Architecture throughout history has been seen as a representation of its era, demonstrating levels of technological 
breakthrough and design experimentation. The design of early hospitals around the turn of the $20^{\text {th }}$ century evolved around the functional processes of the clinical experience (Prasad, 2008). Despite the technical and clinical developments of the last 100 years, the idea of designing hospitals around its functional requirements is an unchanged approach even today. The modern hospital has become an institutionalized setting, not a sensitive place of healing. This may be due to the responsibility that healthcare facilities have for life and death. Prasad also states that hospitals are extremely complex organizations, and the freedom to experiment architecturally is not a primary concern to planners, staff, and builders (2002). Thus, it seems that a dichotomy has existed within hospital design throughout history: architectural design experimentation of these spaces has taken a back seat to planning functionally efficient medical spaces. Although few historical precedents of architectural experimentation into hospital design exist, some examples stress the importance between well designed spaces and patient healing.

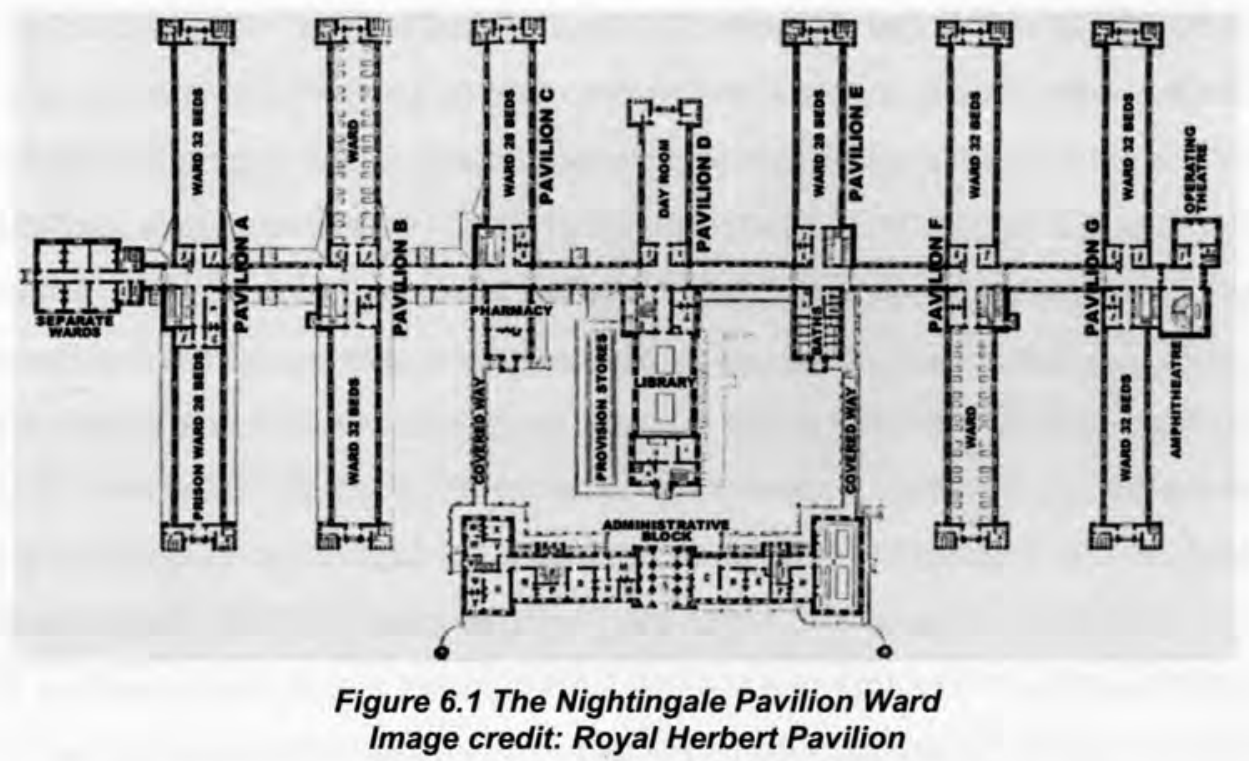

Environmental influences in hospital design emerged during the mid- $19^{\text {th }}$ Century. Florence Nightingale was one of the greatest influences in hospital architecture for over 100 years (Williams, 1991). Regarded as the founder of modern day nursing, Nightingale was a great advocate of using the environment as a form of therapy. In "Notes on Hospitals" published in 1859, Nightingale described how the first priority in facility design was that it should "do the sick no harm" (Williams, 1991, p.106). This principle was a direct response to the mortality rates of individuals within hospitals being higher than those of individuals being cared for at home. In Nightingale's view, this difference was 
due to the mismanagement of hospital ventilation systems, drainage and sanitary arrangements. Nightingale's design became known as the Pavilion Ward-a set of long and low running pavilions connected by major corridors, having large windows for purposes of cross-ventilation (fig.4.1). This design was advantageous because the rate of airborne disease was decreased. However, the size of patient populations today makes the design impractical. The low-lying plan demanded a large site; today the cost of lands directs the compact design of hospital facilities. Hospital acquired infections are just as much of a problem today as they were in the nightingale time (Joseph, 2006). Her solution of purging the infected air put others at risk; today's approach is to isolate the patient. The Modern Movement of the $20^{\text {th }}$ century would replace the Nightingale ward approach of using elements of the natural environment. Technology was to prevail as the dominant force in the future designs of our supposed healthy buildings of the 20th century.

The success of the Nightingale Pavilion Ward was overtaken with the introduction of antibiotics and construction material during the modern era. Williams states that antibiotics became the accepted form to combat bacteria, and the need for natural ventilation was reduced in the modern hospital (1991). The arrival of new methods of construction and materials allowed for the creation of the high-rise hospital, and further demise of the Pavilion Ward. Nield (2008) states that the introduction of the electric light bulb by General Electric in 1939 made hospital design susceptible to the deep-plan and the institutionalized character commonly associated with modern hospitals. The high-rise diminished the presence of the natural environment in hospitals and its inherent therapeutic qualities. Williams also notes the phenomenon of environmental docility, a theory developed by modern gerontologists. This concept states that as people age, their ability to control the environment weakens. The impact of the environment on an aged person's well-being therefore increases. This concept demonstrates how evidence based design will play an important role not only in hospital design, but also in the design of healing environments for the aged.

\subsubsection{Trends in Healthcare Today: The Move to a More Natural Way of Healing} In a forum presenting improvements for quality of care and hospital design, Roger Ulrich states that several important changes or shifts are occurring in worldwide health (2000). Whether a nation has adopted universal care, these forces remain common. These 
trends call for massive change regarding the way we administer healthcare, through the creation of better building design that is patient focused. These trends include (a) advances in mind/body medicine that indicate the relationship between stress and psychological factors affecting health, (b) reduced staff and burnout rates, (c) patient satisfaction demands, (d) change in medical technologies, (e) growing numbers of the elderly, (f) pressures to reduce cost and improve quality, (g) shifts regarding the number of in/outpatients facilities needed in the future and $(h)$ an increasing body of research demonstrating the relation between well designed environments and improved outcomes. Ulrich's points demonstrate the complex issue of future health. The architect must be aware of new trends like patient focused care, and the types of facilities that the region will need. What can be extracted from this is that evidence based design can be applied to any type of healing environment, whether it specialize in different age groups or in different types of care.

The beneficial effects of natural healing environments can be realized by adopting more holistic principles of building design. Buildings should be designed for the users in a holistic manner that promotes human well-being. The challenge and responsibility is left to the architect to put the primary focus on the person, and to design high-quality healing environments for their natural state of being. In a speech to Britain's House of Commons, Winston Churchill was once famously quoted as saying that "we shape our buildings, and afterwards our buildings shape us" (Berry, Parker, Coile \& Hamilton, 2004 , p.1). In other words, the quality of the environment directly affects the quality of our health, and its design should be given top priority, especially when it is involved in the healing process and the care setting.

\subsubsection{CASE STUDY: Apotex Centre, Baycrest Centre for Geriatric}

With existing evidence pointing to the need for more sensitive approaches regarding modern day care and the environment in which it is delivered, there are few examples of large-scale Canadian hospitals that demonstrate these holistic principles. One of the few that exists is the Apotex Centre at Baycrest hospital in North Toronto, a long-term care facility for the elderly, more specifically those who are suffering from cognitive impairment. As Franklin (2000) states, the Apotex Centre for Geriatric Care adds to the 300 chronic care beds and 200 apartments for seniors that currently exist at Baycrest 


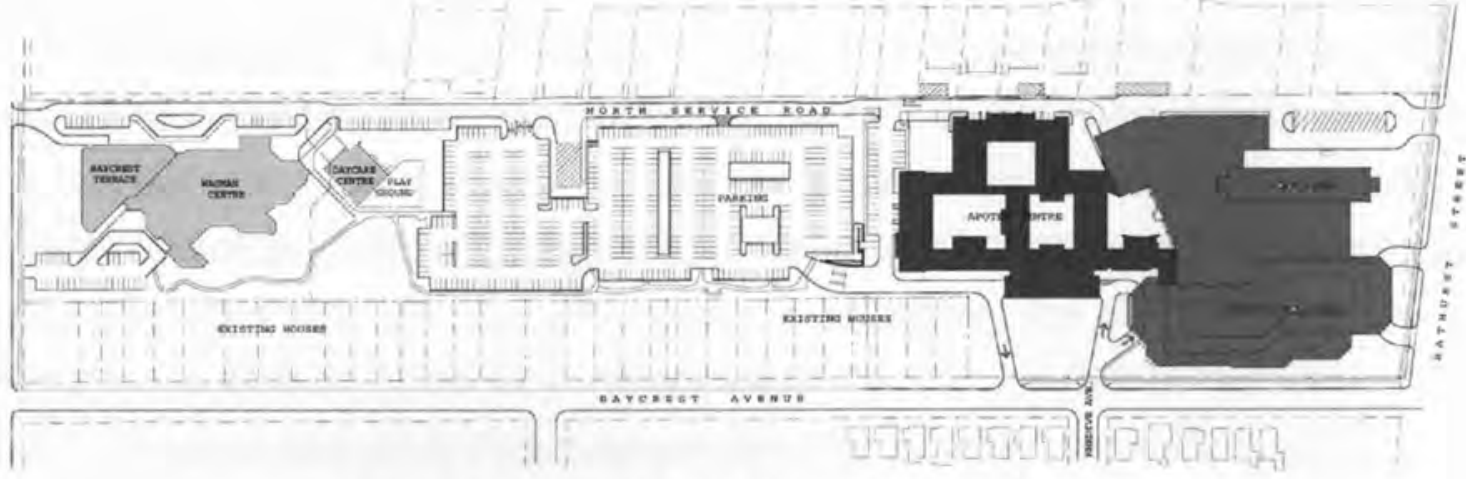

Figure 4.2 Baycrest Apotex Centre Hospital, Site Plan Image credit: Diamond + Schmitt Architects

Hospital (fig.4.2). The new Apotex facility offers 472 additional beds, and exemplifies the idea of care designed around the individual occupant. The idea of a building responding to the mental state of its patients critically addresses the occupant and their individual needs.

The overall layout of the building is similar to that of the nucleic hospital (Stow, 2008). In it the notion of solid and void exist, and it is partly planned around the massing of patient quarters and a multitude of internal and external courtyards, which are all connected through varying levels of circulation. The architect also included exposure to the natural environment through five courtyards and landscaped areas (Colucci, 2008). The front external courtyard is the only landscaped space that is publicly available (fig. 4.3). It acts as both a vehicular and pedestrian entrance to the care facility. The Northern courtyard is an exterior courtyard holding rectilinear landscaped typologies, with inspiration driven by the modern architect Carlos Scarpa. The landscape is designed with geometric functions and elements of vegetated planting and seating. The western courtyard has a bio-morphic design typology created again through elements of planting and seating; it was inspired by the surrealist sculptor Jean Arp. A Jewish Sukkot tent serves as a covered space that may be dismantled at varying points of the seasons, demonstrating a spiritual and religious connection to the space. The eastern courtyard contains varying fruit trees, and its irregular shape promotes wandering and discovery. The last courtyard, the Winter Courtyard, is a seven storey atrium. This internal courtyard serves as a focal point for the resident and staff community. Towering deciduous trees, calming sounds of water features, artwork, sculptural displays and additional biophilic structural elements 


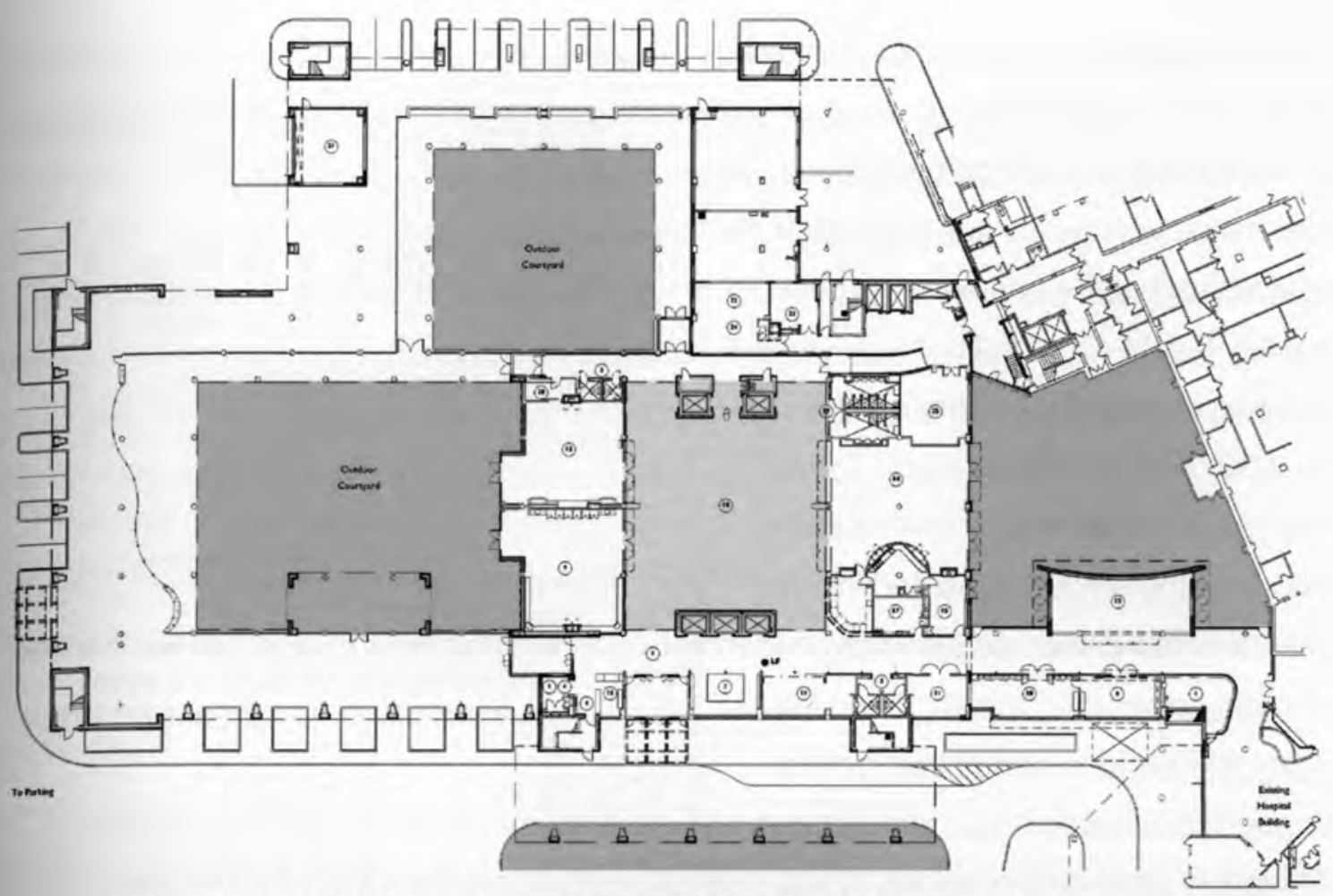

Figure 4.3 Apotex Centre Ground Plan indicating indoor \& outdoor courtyards Image credit: Diamond + Schmitt Architects

that mimic nature all exist in this space. The use of such features results in psychological benefits (Joseph, 2006).

\subsubsection{Memory Loss}

The core design principle of the Apotex Centre is patient focused care. Project architect Gregory Colucci of Diamond + Schmitt Architects described in an interview how all of the patients residing at the Apotex Centre suffer from some form of dementia or short-term memory loss. Several types of human memory allow one to interact within a space. Recognition memory "is one that effects both where you are and who you are" (G. Colucci, personal communication, November 7,2008 ). He stated that an extreme level of detail plays an essential role in the way that the architecture maximizes the patient's innate ability to comprehend their current location within the building. Cuing devices were integral to the notions of way finding in such a large building, especially when all patients suffer from memory loss.

The hospital was developed so that every instance of mental cuing was different from the next. "If two cues are similar, effort-full memory retrieval will be required to determine the meaning of each one" (G. Colucci, personal communication, November 7, 2008). 
The constantly changing masses of solid and void in the hospital layout are examples of successful way finding techniques. The alternating courtyards are the best of these devices and at the same time provide healthy levels of natural light. Courtyards differing in rectilinear or bio-morphic qualities help identify location. These changing characteristics can be seen through other architectural techniques. Corridors have been carefully given identity through colour differentiation and full scale artwork. This idea of creating identity

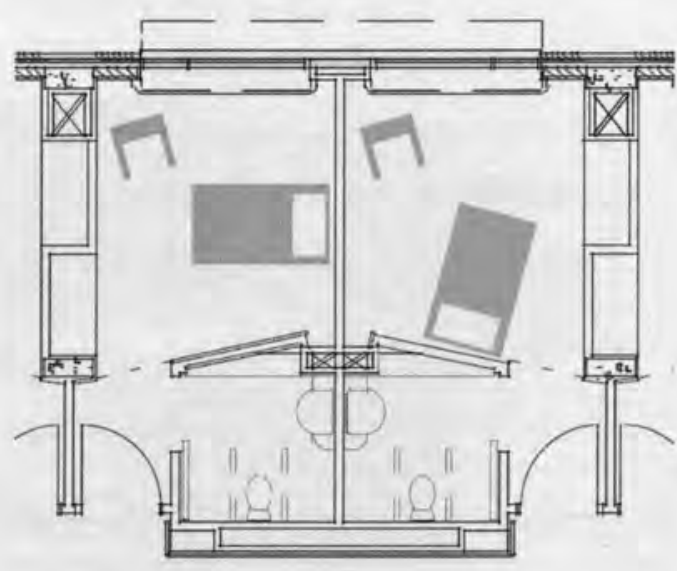

Figure 4.4 Apotex Centre, Resident Unit Plan Indicating varying furniture layout Image credit: Diamond + Schmitt Architects through circulation seems reminiscent of the naming of streets in the urban realm. Artwork is used on the portals of each home unit to strengthen a sense of location. Outside of the resident rooms, display cases are filled with memorabilia to individualize his/her personal space. These are known as memory boxes. Within the home-units, extra care was taken to support memory. The four walls within the space were differentiated through colour and material. Some walls were colourful while others remained neutral. Cherry wood paneled walls differed from window walls. The entry wall has been angled to expand the perception of the room's size, and allow for varying furniture layouts (fig. 4.4). The angled wall also helps to make the washroom visible, which hopefully reduces levels of incontinence among residents. Apotex successfully accomplishes patient focused care by designing the macro and micro environments directly around the specialized patient sickness. Dementia is a disease that continually distorts one's sense of judgment, and the way finding and cuing techniques used at Apotex Centre are specifically designed to compensate for this disease symptom

\subsubsection{The 'Home-Like' Environment}

Another core principle driving Apotex and its patient-focused care was to create as much of a home-like environment within the building as possible. The benefits of creating a home environment accrue at both the staff and patient level. During the design process, however, the design team encountered difficulties in defining the term home, as the definition of home changes from family to family (Colucci, 2008). To find the "true" 
definition of home, a "rigorous methodology of criteria" known as the integrated design process was adopted by the design team (G. Colucci, personal communication, November 7,2008 ). The personal dignity of the patients was not to be compromised, especially their understanding of what a home was. The integrated design process (I.D.P) brought together healthcare planners, engineers, architects and members of the religious community in order to come to collective agreements. The I.D.P process shows how designing healing environments based on patient focused care, this entails a multidisciplinary approach.

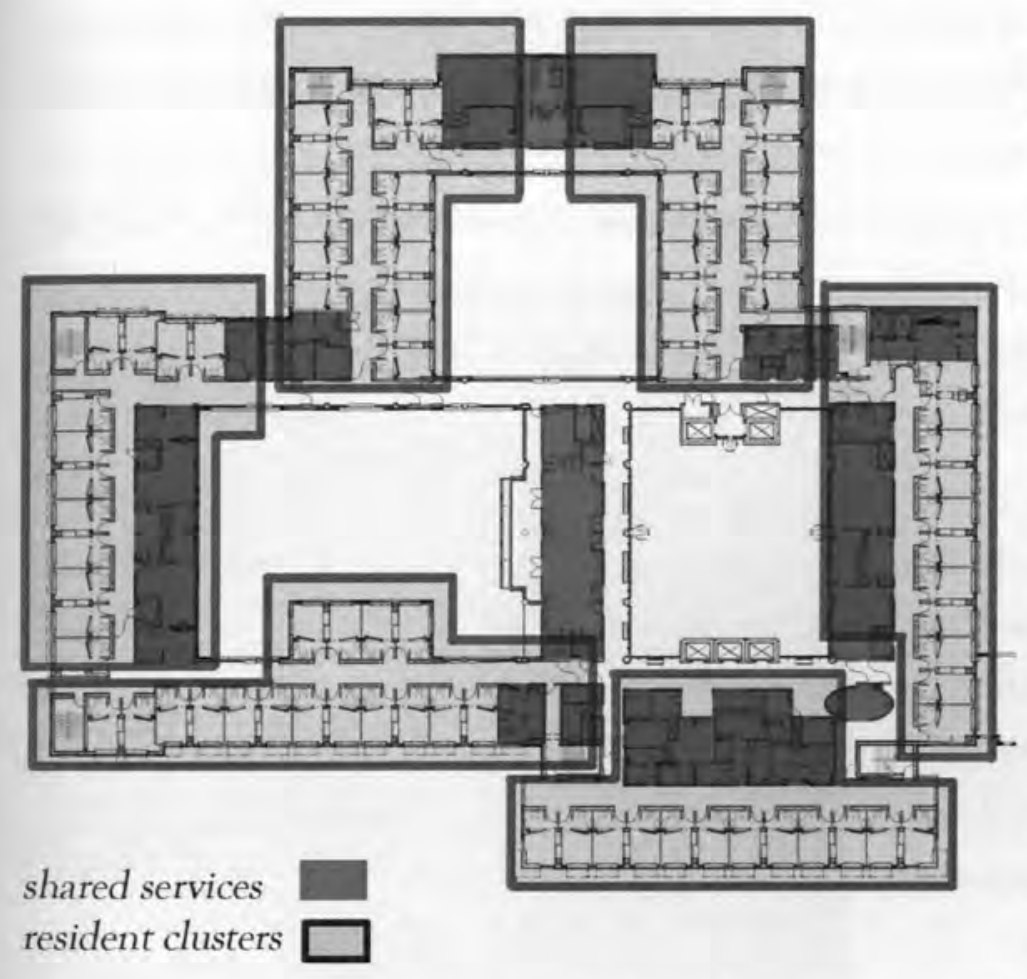

Figure 4.5 Family clusters creating the larger Apotex community Image credit: Diamond + Schmitt Architects
Defining a home-like setting for the 472 residents seems a difficult proposition. Colucci (2008) states that the building programmatically needed to function as a large community with shared services. Common services had to also function on a smaller and more repetitive scale. The building was therefore organized into six clusters of resident families (fig. 4.5). These six families made up the larger Apotex community. Each cluster

has common areas/services such as a shared living room, dining room and kitchen (fig. 4.6 \& 4.7). On a broader scale, common services included soiled utilities, nursing stations and bathing rooms. Each resident cluster would be connected to each other through the visual linking provided by the corridors and courtyards. Involving visiting family was also considered as important to each family cluster. Common areas allowed for resident visits to occur in the open or in the privacy of resident rooms. In some cases, two rooms were brought together to make one larger room, to enable couples or family to stay together. The hierarchy of family in the Apotex Centre can be seen as a success 
from the larger staff/resident community, to the smallest family created through outside visitation. Love is an attribute that all humans need to survive. Even when dementia and memory loss is great, a sense of family generated by the functional properties of the community helps with coping.

\subsubsection{Creating Places to Heal}

We are currently living in an era that is returning to more holistic methods of medicine and healing. As discussed in previous chapters, Low (2004) states that in Canada, dissatisfaction with chemical medicine is causing the gap between alternative and traditional forms of medicine to widen. Crenlin, Andersen and Connor (1997) have documented this trend through rigorous field studies and questionnaires. Before modern science and technology emerged, man lived in a symbiotic relationship with nature. Living in balance with the environment was an indicator of an individual's level of health. This was a common lifestyle one that offered daily wellness (Bergdolt, 1999; Bivins, 2007; Cruse, 2004; King, 2001). Environmental psychology research has documented that natural environments and alternative forms of therapy have undeniably beneficial characteristics. Evidence based design is the field of design based on research results such as these.

Therefore, the design of our future healing spaces should assimilate these beneficial forms of natural therapy. Future care facilities could eliminate feelings of institutionalization created by technology. They will be geared more towards our innate associations with nature, to create true places of healing.

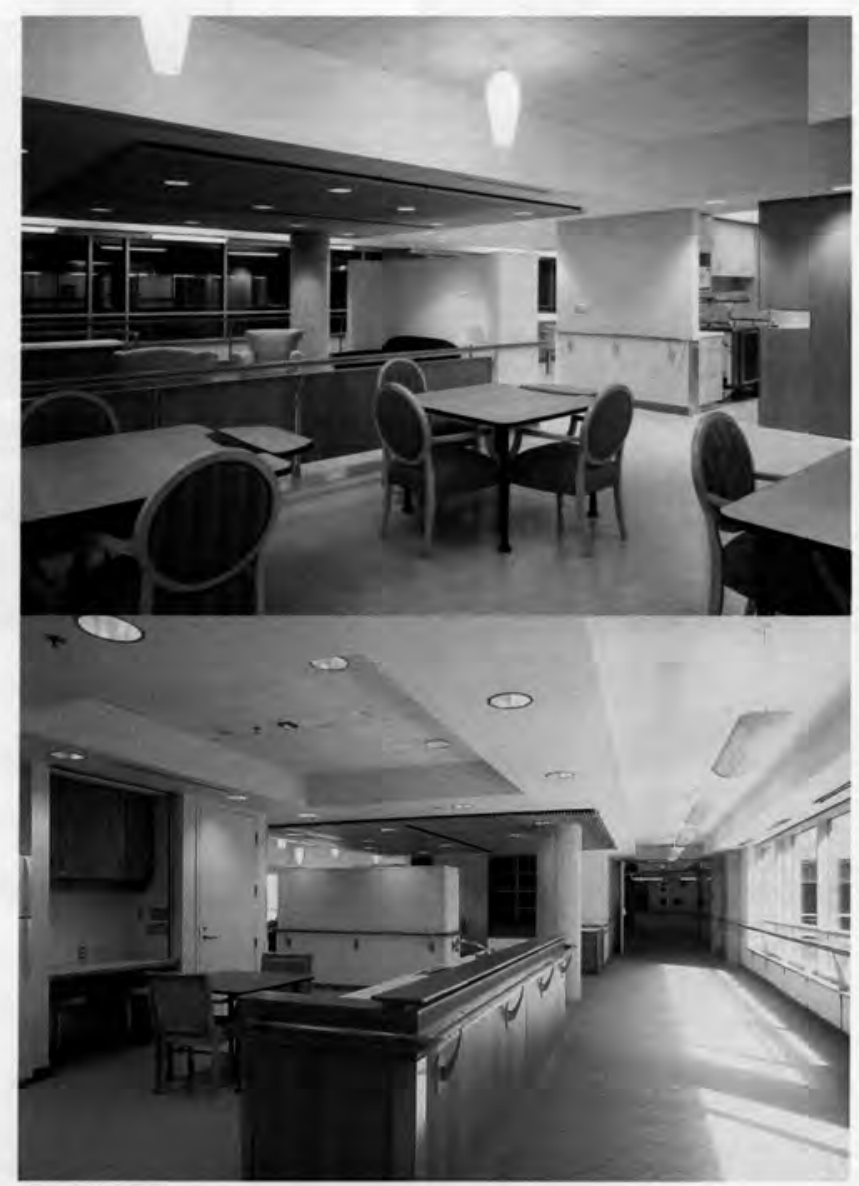

Figure 4.6 \& 4.7 Dining room \& kitchen common areas at the Apotex Centre Image credit: Diamond + Schmitt Architects 


\subsection{Adaptability and Flexibility}

Key words: technology, design flexibility, adaptability

\subsubsection{Argument: The Need for Adaptable and Flexible Buildings}

Hospitals and care facilities are a large and complex building typology, not only because of the massive programmatic responsibilities that they accommodate, but also because of the great levels of capital investment necessary for their implementation and operation. As discussed in the previous section, future care facilities will be affected by several trends regarding human healthcare that incorporates natural forms of environmental and therapeutic approaches supported by evidence. These facilities will also be affected by the technical and political factors involved in modern medicine, and the fast pace at which technology changes. Michael Davis states that to reach a more sustainable end, specific factors regarding future hospital design must be considered (Davis, 2008). Some of these factors include (a) constantly changing technology and drugs, (b) managing hospital-acquired infections, (c) the need for safer environments, (d) reduction of clinical negligence and (e) the life cycle cost of the buildings. The fact that almost every major hospital in Toronto is currently involved in renovation or expansion projects is generating debate about the sustainability of our current model of care (The Globe and Mail, 2008). The position to be taken is that: flexible care facilities will be needed to respond to the tremendous future change of the delivery of health services, the design of facilities and their successful ability to adapt. Flexibility will therefore be considered a necessity for the design of future hospital infrastructures and their sustainable mission as truly successful healing environments.

\subsubsection{Factors in Health Design: Changing Technology, Spread of Infection \& Cost} For future hospitals and care settings to be truly successful, they will need to be flexible and adaptable. The constant changes in evolving technology and drugs will drive this. The industrialization of medical processes has led us to over rely on modern chemical treatments. In an article on the design of holistic healthcare buildings, Eberhard Zeidler states that these constant changes today "are more complex" and "occurring even faster" then ever before ( 2008, p.219). He adds that if modern hospitals cannot adapt to these changes," they cannot provide the services they should". Some changes include developing technological equipment, changing the recovery room and the Intensive Care unit and changes regarding medical procedures themselves. It is understood that 
buildings that can't provide the services they should, especially hospitals, can be considered as failing at their direct purpose. It must be stated that the seductive qualities of making hospitals functional were what led to their demise and institutionalized character in the early $19^{\text {th }}$ century. When seeing the importance that functional and flexible spaces will play regarding the longevity of a hospital's life, special attention must be paid early on to creating a sensitive place for healing, as opposed to creating an efficient space for processes.

Lawrence Nield draws conclusions about changing technologies and the modern hospital similar to Zeidler's (Nield, 2008). Nield compares hospitals to modern airports and offices. Like hospitals, airports process passengers and "the management of spaces and gate lounges are controlled by sophisticated computer and information systems" (Nield, 2008, p.252). Airport terminals facilitate vast communication networks that allow their functions to operate successfully. Some of these include wireless communication, booking and ticketing, departure control and common equipment use. Changes in the modern office have also been influenced by networking systems. With the introduction of the personal computer in the 1980's, an increase in productivity and improvement to the work environment occurred. These networked spaces regained sizable floor areas, and more flexible buildings with increasing floor-to-floor heights generated more effective designs of their building services. Modern airports and offices are places where efficiency and production can occur. The proliferation of wireless technology for example can be seen in all sectors of public life; however the question of going to an airport or hospital-like setting to be healed raises questions. Human factors of privacy, family, community and love are points that efficiency cannot calculate.

Michael Davis states that one of the most important factors affecting the state of health in hospitals is the mismanagement of hospital-acquired infections (2008). These nosocomial infections are caused by the cross-contamination of patients through the spread of airborne disease resulting in vast hidden costs and the possible threats of pandemics. A modern hospital is, for the most part, a mechanically closed system, in contrast to the open sterility offered by nature. The practice of isolating high-risk patients seems to be an acceptable form of coping with infections. Patient isolation however should not disconnect the patient from the holistic therapeutic principles of evidence 
based design. New research by some architects demonstrates how an isolated single patient room can be acuity adaptable, and standardized (Ashnen + Allen, 2008).

A key challenge to the feasibility of designing future flexible and adaptable hospitals is one of cost. As Davis (2008) states, the question of how long the facility is going to serve the community is extremely important in the decision making process. The cost of a building is a primary concern to planners, and introducing higher initial costs for reasons of future flexibility may not be acceptable to the politicians making the decisions or the politicians approving the plans. Resistance to building a flexible building that carries a costly price tag is a reality; however, as Davis states, "the same factors mean that an inflexible or obsolete asset that cannot pay its way is a liability" $(2008, p .83)$. The For health providers to survive, they will need to adjust to changing conditions. Davis states that new "econometrics" will be needed so that "a value can be placed on design and quality... and measure this in a systematic way at macro and micro levels" (Davis, p.86). The economical value of design will be argued in the following section.

\subsubsection{Modernism and Patient Care: Exploring the idea of Standardization}

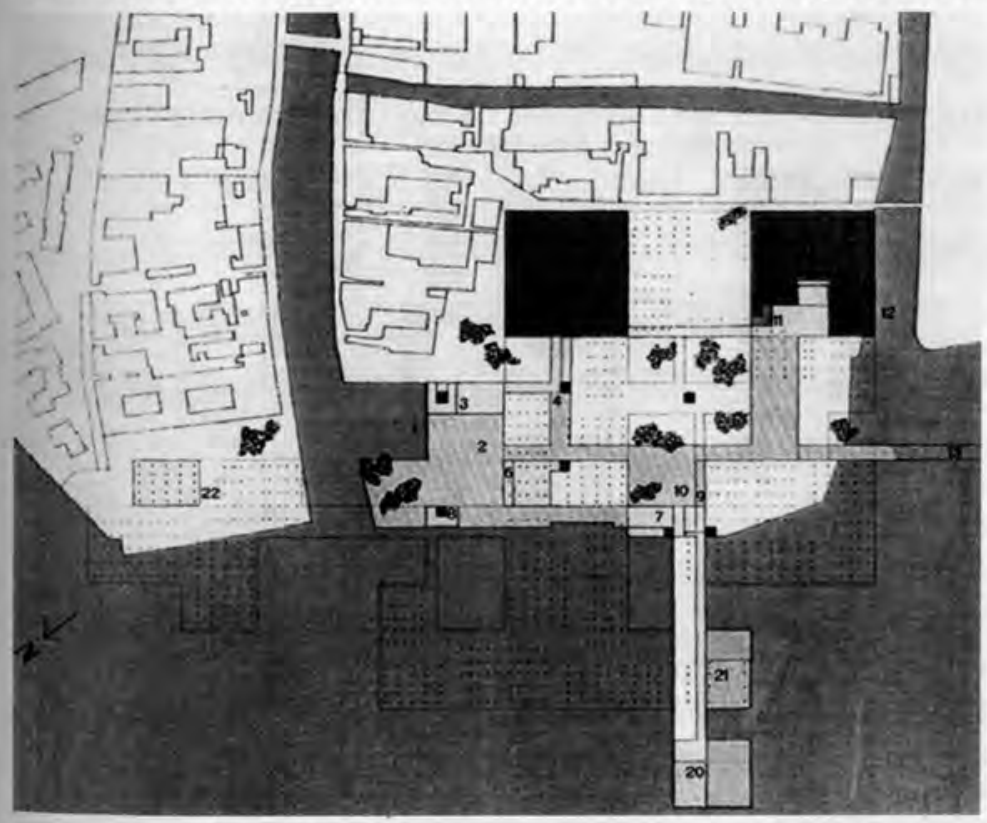

Figure 4.8 Venice Hospital, plan and city connection Image credit: CASE
The concept of the adaptable design of hospitals is not new. Le Corbusier saw that flexibility and standardized patient rooms were necessary in building a new hospital in Venice. As Addington, Kienzl and Intrachooto (2001) state, the plan for the Venice Hospital was based on two major considerations: (1) integration with the existing fabric of the city, and (2) the efficient

functioning of care to all kinds of patients. The first saw the creation of a low-lying facility that would emulate the external urban network in the surrounding neighbourhoods. Inserted courtyards and streets supposedly made the building flexible because it had the 
ability to expand and contract with the changing functions of the space (fig. 4.8). Sarkis (2001) critically states however that the buildings inner networks "acquire their shape from an external rather then programmatic source" and that the ability for the building to grow "is made in increments of the city, not in increments of the internal module" (p.86). Le Corbusier's modular construction system allowed for successful expansion of the facility; however any internal changes to its functions would require demolition and reconstruction. Realizing the second goal of designing for patients resulted in the building being divided into

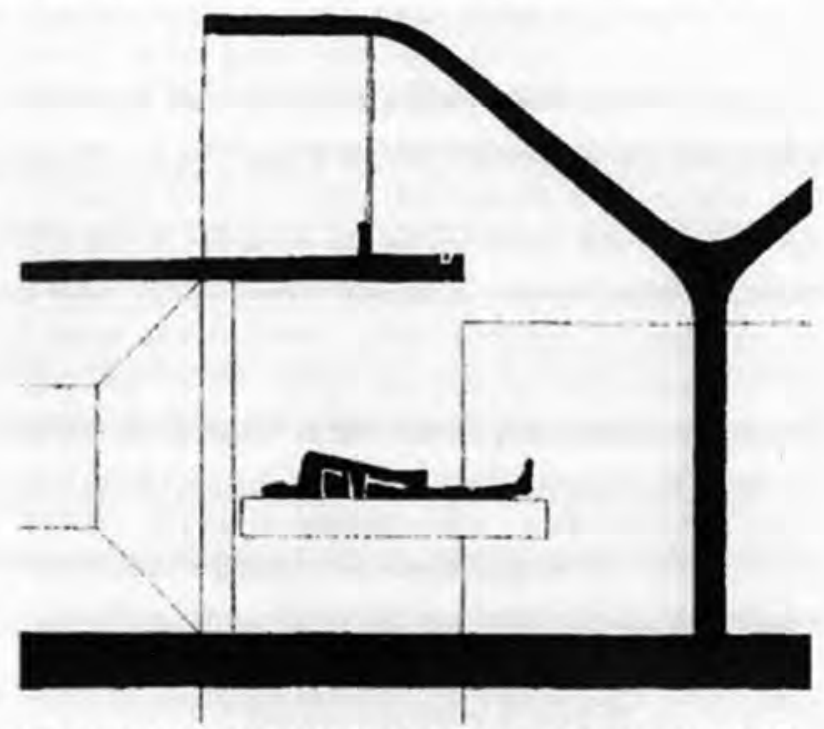

Figure 4.9 Venice Hospital, Section of Care Unit Cell Image credit: CASE three areas: (1) the general public and day patients were reconnected to the urban network at the ground level, (2) staff and services were dedicated to the second floor, (3) and acutely ill patients were located on the third floor. This vertical separation of acutely ill patients on the higher levels from the noises and stresses of the urban environment focused the hospital's design around the patient. Using all efforts to help cure the severely ill, Le Corbusier attempted to adjust the room to reduce levels of stressful noise, maximize day lighting and natural ventilation (fig. 4.9). However, he makes no effort to directly introduce nature: for example he did not provide windows in the patient cells. Subsequently, research has demonstrated the beneficial effects of views of nature on a speedy patient recovery (Ulrich, 1984). The patient rooms in the Venice Hospital were thus further isolated from the beautiful condition that is Venice. Le Corbusier demonstrates to a certain extent what our future hospitals need: for example, patient focused care that focuses on the patient condition, while attempting to make future program changes easier through a flexible and adaptable design. Very few psychological studies were available to the architect at the time; however, the spirit of healthy environmental design can be seen by Le Corbusier's early attempts even if he did not have the evidence to support his theories. 


\subsubsection{The Neutral Grid and Later Attempts at Flexibility}

A later attempt at creating a flexible and adaptable hospital was the McMaster University Health Science Centre, in Hamilton. Built in 1972, the building was formulated around

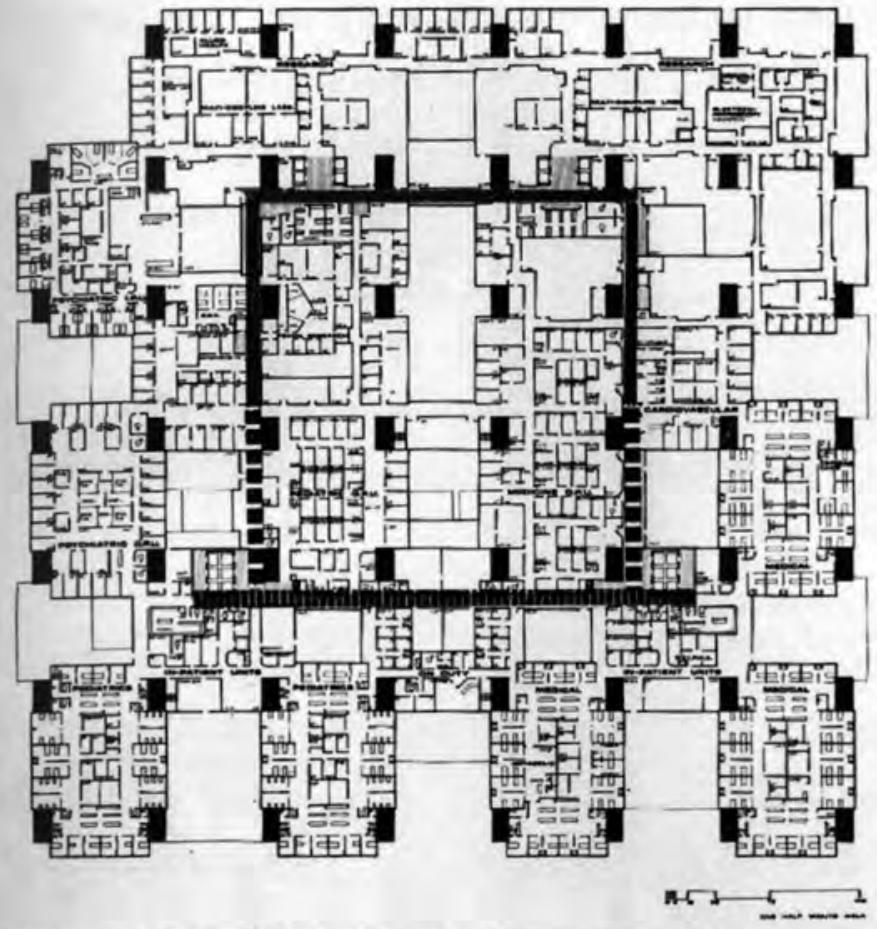

Figure 4.10 McMaster Hospital, Traffic Segregation in plan Image credit: Zeidler the concept of the neutral grid that had developed in Europe at the time. The grid emphasized circulation and the expansion of movement in a number of directions (fig.4.10). As Nield (2008) states, hospitals need a lot of "elbow room" in terms of circulation, as well as programmatic area (p. 259). The hospital implements a novel approach to service accommodation, through the inclusion of horizontal interstitial space between major floors (figure 4.11). This placement maximized

the operational efficiency of all major floors of the hospital, and facilitated flexibility. The project architect stated that implementing the interstitial space allowed for future change, and that even with major renovations, "60 per cent of the original investment could be maintained," like brick, glass, stairs and services (Zeidler, 2008, p.220). Unfortunately, hospital growth and changecannot be predicted so precisely. As Stow (2008) points out, the McMaster Hospital was a grandiose scheme that was found to "overpower the

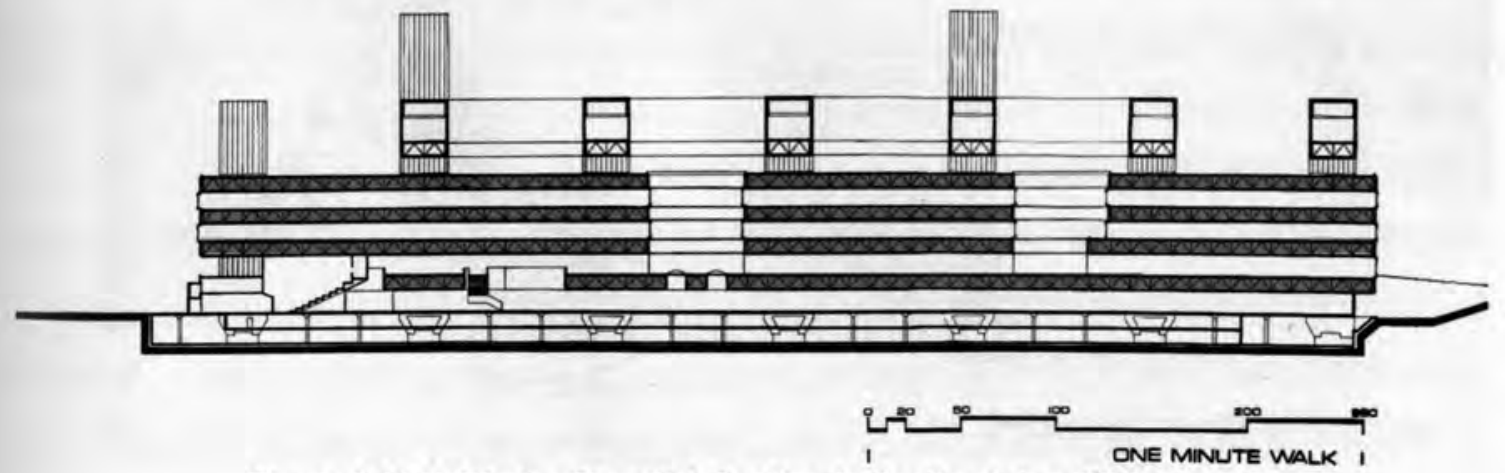

Figure 4.11 McMaster Hospital, Section indicating interstitial space Image credit: Zeidler 
community that it was built to serve" (p.21). Taken from the European formula of repetitive structural grid, the building's sheer size creates confusion. Personal site visits to the McMaster Hospital support this assertion; way finding, as much as it exists, allows orientation to be constantly compromised. The idea of the grid continues today. The rigid nature of the scheme creates wall after wall, with minimal room to adapt, thereby prescribing a tear down philosophy when change is needed. Even if $60 \%$ of the original construction can be retained, it still begs the question of whether $60 \%$ is enough.

\subsubsection{The Modern Single-Patient Room}

A recent example of combining evidence based design principles with the ideas of patient isolation and standardization occurs with the 'Green Patient Room.' Designed by Ashen + Allen architects San Francisco, the idea of the standardized patient room for inpatient facilities has been the centre of much research (fig.4.12). The room is an amalgamation of evidence based design principles, and creates an acuity-adaptable room, able to adapt to in patients who suffer from a variety of illnesses (Anshen + Allen, 2008). The room promotes the speedy recovery of the patient through various evidence based design features, with designated zones for family and staff. The family of the patient can stay overnight in a

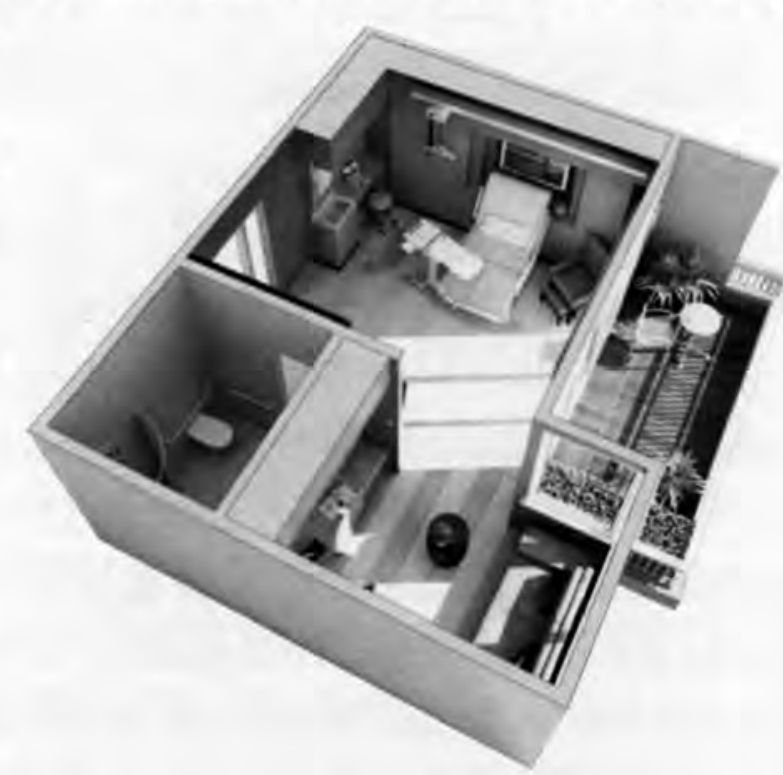

Figure 4.12 The Green Patient Room Image credit Anshen + Allen Architects separate quarter of the room, that has internet access. The room is standardized to help reduce staff error. For example, medicine is located in the same place in every patient room cabinet, and advanced computer systems digitally record and keep track of the patient's condition. Patient washrooms have double swing doors to reduce patient falls. A terrace has been created because of studies that indicate views to nature and natural day lighting aid in quicker patient recovery. The room has been an exemplar for single inpatient quarters, isolating potentially high-risk patients to reduce nosocomial infections. A report by Rogers (2005) shows how standardized patient wings are being suggested as an efficient spatial layout 
within hospitals (fig.4.13). A disadvantage of these designs (fig.4.12 \& 4.13) is that they involve walls, and are customized for only one hospital type- the inpatient facility. Both features inhibit flexibility; however, if walls are of questionable benefit for inpatient care, they are even less appropriate for outpatient models of care.

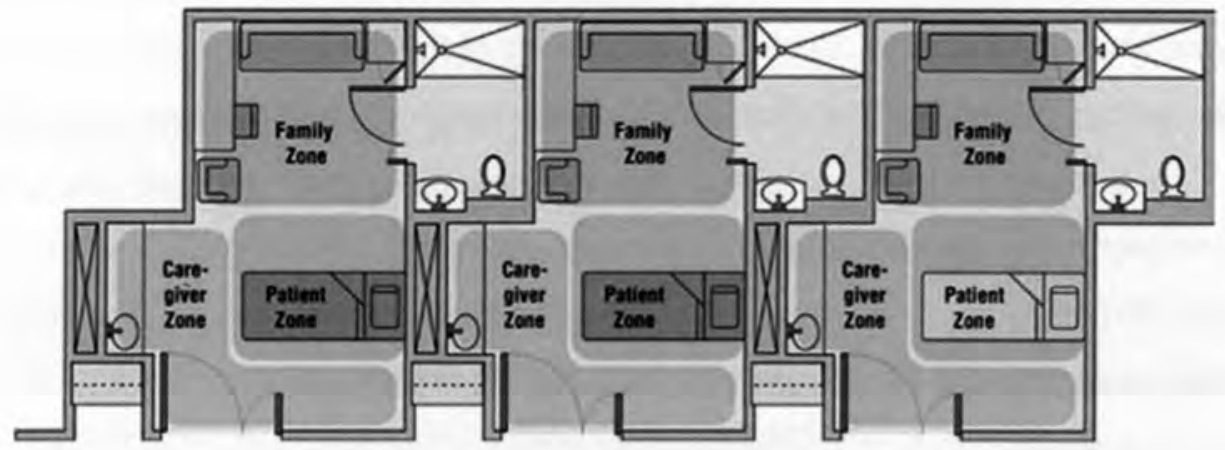

Figure 4.13 The Standardized Patient Wing Image credit: Johnny Bean Engineers

\subsubsection{CASE STUDY: The New Women's College Hospital Addition}

Gregory Colucci is project architect for the Toronto design firm Diamond + Schmitt Architects and their healthcare projects. Their portfolio consists of several successful projects in healthcare, including the Cardinal Carter Wing of St. Michael's Hospital, the Women's College Hospital of Canada and the Apotex Centre for Geriatric Care. In an

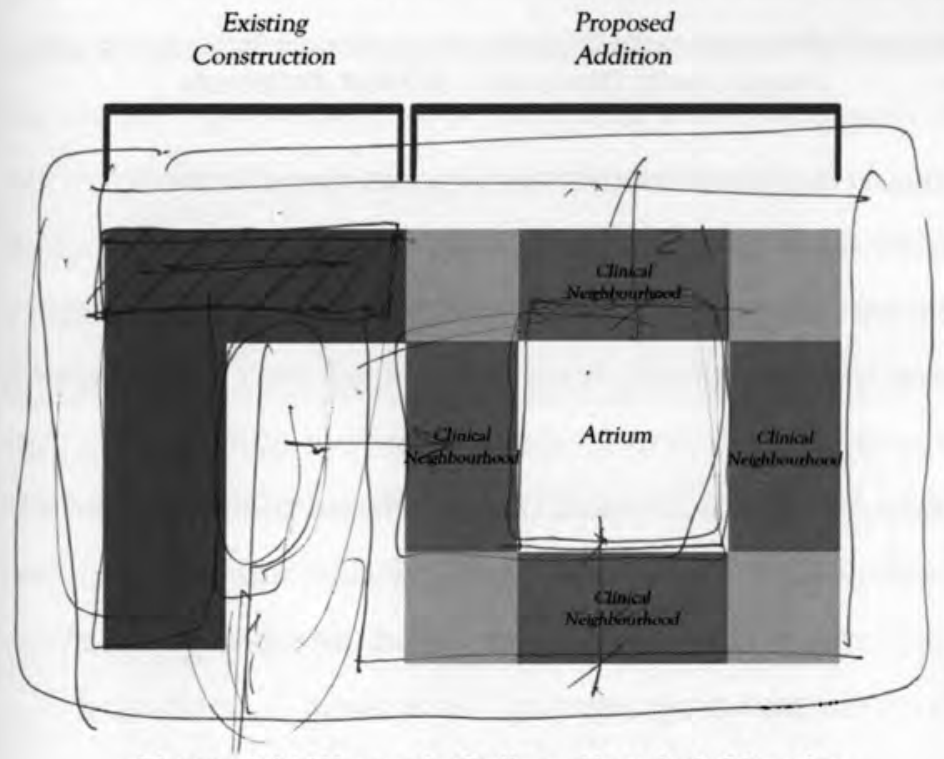

Figure 4.14 Women's College Hospital, Site plan Image credit: Diamond + Schmitt Architects interview, Colucci stated that "designing for future flexibility is fundamental to healthcare facilities," and that "flexibility should be considered within three areas...through horizontal expansion, vertical expansion and that of planning modules" (G. Colucci, personal communication, November 7 , 2008). He spoke of these factors in relationship to the renovation project for the 
Women's College Hospital in Toronto that he is currently involved in.

The project consists of a completely new outpatient wing to the hospital (fig.4.14). The project was needed because of the inflexible nature of the original hospital building.

Colucci first spoke of the need to expand the hospital's structure horizontally. His design allows for the future expansion and contraction of departments over time, as they change use and specification. Secondly, a building of this type needs to remain flexible within the vertical direction. The Women's College originally had meager floor to floor heights. These minimal ceiling heights in the Hospital did allow for future changes in information technology and medical equipment, especially MRI and CAT scan equipment. Structurally, the building would need to support this added weight, and therefore accommodation would have to be made ahead of time. The third point states that planning modules (like the clustering of Apotex Centre's resident communities) had to be modified. The future program would have to be

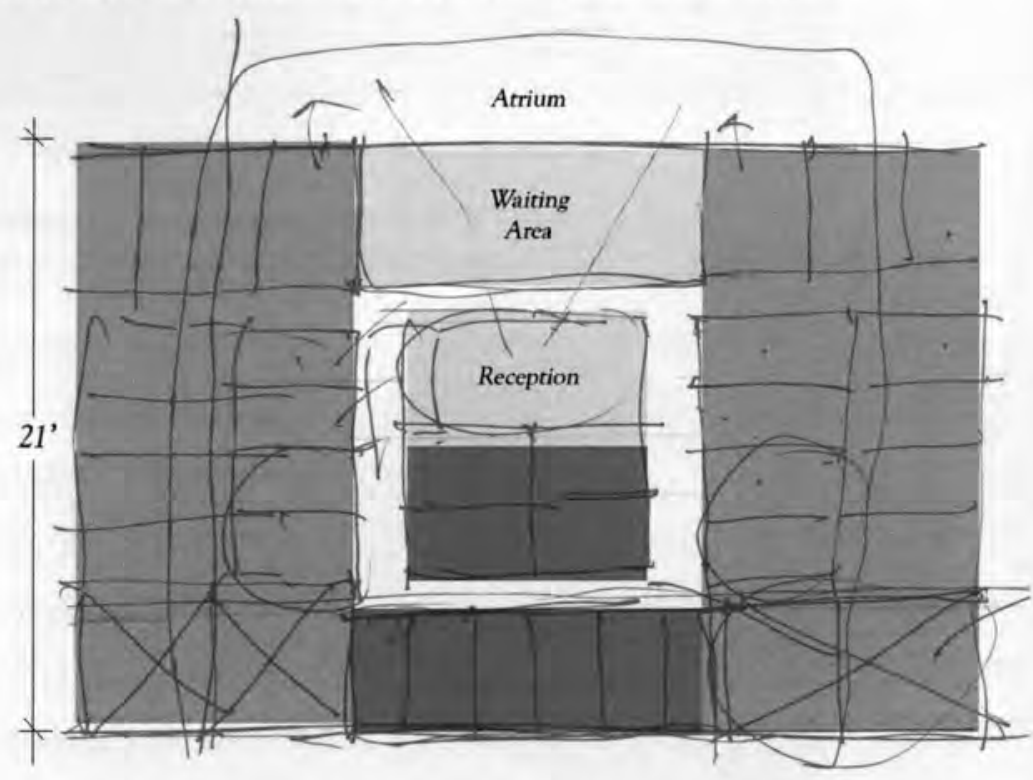

Figure 4.15 Women's College Hospital, Clinical Neighbourhood Image credit: Diamond + Schmitt Architects

designed around the idea of a clinical neighbourhood, meaning that generic modules for a wide variety of clinical practices would need to be created (fig. 4.15). The generic modules would act as common rooms and patient examination rooms that could adapt by having multiple uses at all times.

Diamond + Schmitt's approach to the Women's Hospital demonstrated the fundamental importance of adaptability in hospital design. The original building was not flexible enough to accommodate future technologies and equipment. It did not have generic properties that could support future unknown programming. As a result, a costly and timely in depth redevelopment process has been launched. Most major hospitals are currently undergoing similar renovations or additions. One factor in producing a 
sustainable building is designing for long-term use. In the case of hospitals, it must be well established early on if the facility is to be used by inpatients or outpatients, or a combination of both. This will ultimately determine the number of walls to be constructed (for standardized patient case) or not to be constructed (for flexible outpatient centre).

\section{3 The Economic Value of Design}

Key words: Initial investment, evidence based design, cost savings

\subsubsection{Argument: Good Design Pays for Itself}

A rationale for implementing holistic and therapeutic measures in the care environment has been provided in the previous sections. It must be stated that this approach, as well as designing adaptable and flexible buildings, does not come without a price tag. The benefits from these types of design are great; however convincing health care planners that these benefits outweigh the initial cost seems a difficult proposition. In addressing the United Kingdom's National Health Service, Geoffrey Purves (2002) states that the Treasury Department has moved away from the lowest cost procurement policies for delivering health, affirming that good design actually saves the health sector considerable funding. This larger initial investment, known as 'patient-focused care,' remains the new driver for all market forces, in contrast to previous emphasis on clinical procedures. Putting the patient first allows the interior environment to be designed around their own specific needs. Purves further states that building design now promotes higher standards, especially within hospitals and the delivery of primary care services. Evidence based design principles are slowly being accepted today within hospitals across North America, creating beneficial healing environments. The position to be taken for the economical value of design is: that with a higher initial capital investment in care facilities, through the inclusion of evidence based design principles, true cost savings over time can be found. This becomes evident through patient and staff stress reduction, increased safety through reduced patient transfers and falls, reduced levels of hospital acquired infections, speedier recovery times, reduction in drug costs as well as a reduction in staff turnover. 


\subsubsection{The Difficulties Involved in Assigning Value to Design}

Placing economic value on architectural design is difficult. Generating cost savings or even profits through minimal interventions of well planned design should be tested. Purves (2002) suggests that there exists "a dichotomy between empirically based scientific research and qualitative methodologies of social science research" (pg xvi). His position is directed to the minimal evidence-based research that actually demonstrates the cost benefits of what is classified as good design. As difficult as it is to calculate, Purves cannot dismiss the existing empirical data that is contributed through the humanities. These studies for example have demonstrated speedier patient recovery rates. Design elements such as a view to nature or exposure to the arts, are beneficial for the patient healing process as measured by reduced blood pressure levels and reduced levels of pain medication for patients recovering from surgeries (Ulrich, 1984). Despite these encouraging results, research is urgently needed on the question of the relationship between design and cost.

\subsubsection{The Current North American Hospital Boom: Rising Care Standards} American hospital trustees and leaders face numerous competing demands from all healthcare parties. Sadler (2006) lists some specific issues such as skyrocketing technology and pharmaceutical expenses, reduction of reimbursements, workforce shortages and increased expectations from consumers and employers. Sadler emphasizes this last point, noting that a "quality and safety revolution" has stormed the country (p.35). The consumer and employer groups are demanding reduced operating errors that unfortunately lead to harm or even death. A massive shift to both safer environments and reduced clinical negligence must occur. The reality of health care being a complex of issues can be seen here, especially when looking at the American system of health that does not offer Universal Care.

Zimring and Bosch (2008) state, that by $2011, \$ 76$ billion will be spent annually in America to create additional hospital buildings. Similarly, Sadler (2006) argues that many hospitals in the United States have seen their end, and will remain useless in the upcoming years unless they are renovated or replaced. Sadler continues to posit that, hospital leaders will need to invest more in their building design- not only for constructing new hospitals to serve for more patients, but to evolve a better healthcare typology. The biggest decision in healthcare projects is the question of what is needed and how to 
finance the project. Sadler points to the overall sustainability that a well designed building will have over time. This will not only result in a positive outcome to the patient experience, but will undoubtedly save hospital facilities significant funding in the long run. Again the argument in favour of constructing buildings that have the ability to adapt to changes reoccurs in this debate. Establishing a strategic plan from the onset of the project, which outlines the type of facility to be built, what the region needs in terms of care, and how long the building is needed for, is crucial to success and to the facilities longevity.

A similar hospital expansion is underway in Toronto. In a recent Globe and Mail article, Ivor Tosell states that Toronto is "experiencing a healthcare building boom, the likes of which it hasn't seen in decades" (2008). Over the next five years, three new research centres are slated to be built in Toronto, and over 1000 hospital beds being added in either renovation or new construction projects. There are over a dozen new hospital projects under development in the Greater Toronto Area. Some of these include Sick Children's Hospital, St. Michael's Hospital, Sunnybrook, St. Josephs, Mount Sinai, The New Women's College and the Humber River Regional Hospital at Downsview. Reasons for this include increased government funding, increased demand for services as well as increased levels of private donations. According to a report by the Ontario Hospital Association (as cited in The Globe and Mail, 2008), the average age of hospitals in Ontario is over 40 years old. The 2003 report stated that an estimated $\$ 8.4$ billion is needed to modernize and meet the need expected of Ontario hospitals. All this construction provides a promising platform for evidence based design in Toronto. It remains questionable if these facilities have or will implement research findings in their design. Nonetheless, planners need to be knowledgeable about this field of study in order to extend the longevity of their building proposals. Rob Devitt CEO of Toronto East General, states that "the whole model of care has shifted over the years" (Tosell, 2008). He states that infection control is a major issue in hospitals today. The number of procedures conducted on an outpatient basis is also increasing. This leads to reduced levels of inpatients, but they are a generally sicker population then in the past.

\subsubsection{CASE STUDY: Fable Hospital}

A few healthcare organizations have invested significant time and resources to quantify the benefits of implementing research based evidence for health facilities and their 
payback period. Such an organization is The Centre for Health Design in California. In collaboration with other research councils and healthcare organizations, this Centre has conducted multi-year assessments of healthcare buildings that incorporate varying levels of evidence based design principles. As described in The Business Case for Better Buildings, their results have been synthesized into a composite hospital scheme, appropriately named the Fable Hospital (Berry, Parker, Colie \& Hamilton, 2004). The purpose of this composite hospital case study was to examine and amalgamate the financial benefits achieved in these existing and recently renovated healthcare facilities that employ levels of evidence based design. This was done to demonstrate that higher levels of initial investment would benefit both patients and caregivers, and produce positive financial returns.

The Fable Hospital was located on an urban site and built as an addition to a 50 year old 250 -bed hospital facility. The new hospital would be renovated as a 300 bed regional medical centre, involved in the delivery of inpatient and ambulatory services, as well as a wide assortment of other medical procedures and services. The total project cost was $\$ 240$ million. The core values of the hospital design included many factors, including safety, family friendliness and patient-focused care. The design team implemented a range of design innovations, which added a total $\$ 12$ million to the construction budget (Berry et al. 2004). A list of the design features for creating a better building can be seen in Appendix 3.

\subsubsection{Analysis of Investment Breakdown}

Three types of evidence based research currently exist as defined by the Centre for Health Design: stress reduction, safety and ecological health. Figure 4.16 shows the contributing factors inherent in the varying levels of evidence based design. When applied to the cost breakdown of the Fable Hospital's additional design implementations,

\section{Stress Redcution}

connection to nature

options and choices

social support

pleasant diversions

elimination of environmental stressors
Safety

air quality
nonsocomial infections
hand hygeine
patient transfers
patient falls

Ecological Health

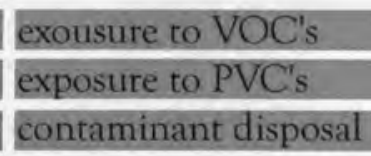

Figure 4.16 Factors Inherent in varying levels of evidence based design Image credit: The Centre for Health Design 
specific connections can be made (fig.4.17). It can be seen that a majority $9 / 14$ of the design changes were dedicated to stress reduction. The other 5 out of 14 changes were dedicated to safety. Interestingly, none of the changes could be classified as being part of ecological health. The percentages of actual dollar investment however differed considerably from the number of changes implemented. Of the additional $\$ 12$ million additionally spent in the construction budget, $69 \%(\$ 8,300,562)$ went to design changes that would increase occupant safety. Only $31 \%(\$ 3,729,238)$ went to those changes affecting levels of stress reduction (Berry et al. 2004).

\begin{tabular}{lrr}
\hline Larger Patient Rooms & $\$ 4,717,500$ & $39 \%$ \\
\hline Acuity-Adaptable Rooms & $\$ 816,000$ & $7 \%$ \\
\hline Larger Windows & $\$ 150,000$ & $1 \%$ \\
\hline Larger Patient Bathrooms with double door access & $\$ 1,509,600$ & $12 \%$ \\
\hline Hand-Hygeine Facilities & $\$ 556,800$ & $5 \%$ \\
\hline Decentralised Nursing Stations & $\$ 270,000$ & $2 \%$ \\
\hline Additional High-Efficiency particulate air filters (HEPA) & $\$ 430,000$ & $3 \%$ \\
\hline Noise-Reducation Measures & $\$ 510,000$ & $4 \%$ \\
\hline Additional family/social Spaces & $\$ 95,200$ & $1 \%$ \\
\hline Health Information Resource Centre for patient and visitors & $\$ 61,200$ & $1 \%$ \\
\hline Meditation Rooms on each Floor & $\$ 342,500$ & $3 \%$ \\
\hline Staff Gym & $\$ 450,000$ & $4 \%$ \\
\hline Art for Public Spaces and Patient Rooms & $\$ 1,050,000$ & $9 \%$ \\
\hline Healing Gardens (Interior and Exterior) & $\$ 12,029,800$ & $100 \%$ \\
\hline Total & & \\
& & \\
Categories of Benefits within Evidence Based Design & & \\
$\quad$ Stress Reduction & & \\
$\quad$ Safety & & \\
$\quad$ Ecological Health & & \\
\hline
\end{tabular}

Figure 4.17 Fable Hospital, Incremental cost to achieve a better building Image credit: The Centre for Health Design

\subsubsection{Is Evidence Based Design Worth the Extremely High Capital?}

One year after the building's full occupancy, the Fable Hospital's CEO and review panel conducted an initial financial/impact assessment. Appendix 4 shows the cost breakdown and savings after the one year period. The review panel chose to remain conservative in their analysis, attempting to strengthen the credibility of the study's message. The estimates were adjusted to reflect a larger number of patients within the facility (Berry et al., 2004). The findings were nothing short of astounding. The figures state that costs of 
$\$ 11,475,406$ were effectively recovered within a one year period. It must be stated again that these numbers were all derived from performance results measured in facilities that were a part of the Centre for Health Design's Pebble Projects. These findings successfully quantify the cost savings resulting from evidence based design. Moreover, the payback from a higher initial capital investment was very fast, one year in this example, allowing the facility to accrue financial benefits every year thereafter. Health planners questioning the viability of investing in evidence based design need not go farther than the Fable study. This case proves that the benefits of better designed spaces are not only psychological, they are also financial.

\subsubsection{Where Was Money Saved?}

Financial benefits in the Fable Hospital came out of varying types of evidence based design. Figure 4.18 shows the contributing factors related to the overall financial benefits and are described as a percentage. The majority of reduced issues and total amount of cost savings both occurred in the level of occupant safety. It was seen that 56 per cent of all reductions came from a reduction in patient transfers ( 34 per cent), a reduction in patient falls ( 21 per cent) and a smaller portion coming from reduced levels of nosocomial infections ( 1 per cent). These areas collectively saved a total of $\$ 6,426,640$ within the first year of the hospital's completion. Stress reduction played a secondary role regarding cost savings. A reduction in drug costs saved the hospital 11 per cent and a reduction in nursing turnover an additional 1 per cent. Together, these benefits saved $\$ 1,380,666$. The contributing factor of Ecological Health did not seem to impact any costs savings in this study. Additional savings of $\$ 3,668,100$ however came from an increase in market share as well as increased philanthropy, due to the overall benefits seen in the hospital's successes. These, it can be argued, are not savings but increased earnings.

\begin{tabular}{lrr} 
Reduction in Patient Falls & $\$ 2,452,800$ & $21 \%$ \\
\hline Reduction in Patient Transfers & $\$ 3,893,200$ & $34 \%$ \\
\hline Reduction in Nosocomial Infections & $\$ 80,640$ & $1 \%$ \\
\hline Reduction in Drug Costs & $\$ 1,216,666$ & $11 \%$ \\
Reduction in Nursing Turnover & $\$ 164,000$ & $1 \%$ \\
Increased Market Share & $\$ 2,168,100$ & $19 \%$ \\
Increased Philanthropy & $\$ 1,500,000$ & $13 \%$ \\
\hline Total & $\$ 11,475,406$ & $100 \%$
\end{tabular}




\subsubsection{What Evidence Should I Buy?}

The greatest benefit in this case was from improved levels of patient safety. Future health care planners will need to make these factors a top priority when asking the question: What is the best evidence to buy? The three greatest areas of cost savings came from a reduction in patient transfers ( 34 per cent savings), a reduction in patient falls ( 21 per cent savings) and a reduction in overall drug costs (11 per cent savings).

The first point of reduced patient transfers is related to several factors. Patient transfers require an additional layer of care, and therefore additional inefficiencies. Medication errors can be thus more common, additional caregivers are needed, additional equipment is needed for transport and the level of patient falls also increases. The success relating to fewer patient transfers came from the acuity-adaptable rooms, or standardized rooms. These rooms permitted for a wide variety of patient conditions and equipment to be used. Patients did not have to travel to access specific care, and therefore transfers did not occur.

The reduced number of patient falls stems from building larger patient rooms, specifically larger bathrooms with double-door access. In addition, the decentralizing of nursing stations seemed to reduce the amount of times nurses were away from their patient.

The third major area of savings was in drug costs. The connection between positive distractions relieving stresses and a diminished demand for pain-relieving drugs was made apparent by the study. Because measures like healing gardens, art for public and private spaces as well as additional family areas were implemented, patients were positively affected. Reduced medication speeded the patient recovery time as shown in previous studies (Ulrich, 1984). This saves the hospital overall costs by minimizing the number of patient days per year needed.

Other factors such as ecological health seemed to have no impact on the facility's cost savings. Ecological health is the reduction of hazardous products such as minimizing the amount of interior furnishings that emit harmful off-gases like volatile organic compounds (VOC's) or polyvinyl chloride (PVC's). Also, ecological health can be determined by the successful disposal of hazardous wastes through advanced technological equipment. No effort was made in the study to explain why ecological health measures were not 
implemented. One can speculate that specifying particular furnishings and paints that minimize the amount of VOC's emitted into the air would have a minimal impact on the overall construction budget.

The economic value of evidence based design has been established through the Fable Hospital case study. A higher initial capital investment in the design of care facilities pay's for itself, and in this case, relatively quickly. The type of evidence based design principles will vary from facility to facility; however the benefits will be common to both the individual occupant and the facility. Areas of stress reduction and safety provide the highest points of value, and indirectly convey the message that the majority of buildings and environments we are currently exposed to induce harm from added levels of stress and safety. The current massive investment in healthcare within Toronto and North America can benefit from this research.

Care environments employing evidence based design have demonstrated significant benefits regarding their long term functioning. These benefits have resulted from the creation of places and not processes, designing adaptable buildings that can adapt to changing trends and show financial cost savings over time. Hospital administrators and planners should engage with facility design at the level of the integrated design process, and should focus on the human occupant and their level of individual health. The design process must also account for the projected length of service the facility will have within the region, and the degree of design flexibility required. Budgeting priorities should not discount the fact that substantial cost savings can occur with a relatively quick period of payback. Planners should seek sufficient funding for the project to include the higher initial investment required by using evidence based design in their facility. This sounds easier than it is, however evidence based design has been shown to be beneficial in every aspect of healthcare design. 


\section{Chapter 5: Design Hypotheses}

\section{A Preventative Approach to Urban Wellness}

Evidence based design has been argued as the architectural model that can promote healing by applying a wide body of analytical research findings to the design of our built environment. Care facilities implementing these environmental design principles have demonstrated success in the following three areas: healthier patients occupying more holistically designed places of healing, buildings that are designed more sustainably that can adapt and change with time/technology, as well as facilities that can increase cost savings by reducing both safety and stress related issues.

This analysis indicates that the future of our care environments will be designed around the idea of patient focused care, and thus the individual health and well being of the building occupant. In the example of the Fable Hospital, designing around the occupant produced improved patient health while achieving cost benefits (Berry, Parker, Colie \& Hamilton, 2004). Findings demonstrated reduced levels of stress and increased safety through fewer patient transfers and falls, lower rates of hospital acquired infections, speedier recovery times, reduction in drug costs as well as a reduction in staff turnover. The Fable Study also found that one year after occupancy, the implemented evidence based design principles resulted in cost savings of almost $\$ 12$ million-thereby achieving full payback. The cost breakdown showed 56 per cent of these savings coming from safety related issues, while 12 per cent came from patient stress reduction.

This analysis demonstrates that implementing environmental research evidence into the design of our built environment ultimately increases the level of occupant health. This is critical in the design of buildings today, and more specifically the design of our care environments. Building design has typically evolved in response to factors such as: the need for efficiency regarding programmatic functions, meeting a specific construction budget or deadline, as well as attaining some level of aesthetic appeal from its architecture. The analysis in this thesis|project indicates that the design our future built environment should be based on achieving successful levels of human health. Evidence based design ultimately demonstrates healthy building design as a primary objective by preventing harm to its building occupants. Poorly designed buildings have been shown 
through time to harm human health, while buildings employing this environmental research have improved occupant's levels of both health and safety (Berry, Parker, Colie \& Hamilton, 2004).

It is important to emphasize how the majority of environmental health research findings that were analyzed in this thesis|project were found in corrective health institutions, including various types of hospital wards, mental facilities etc. Physical environmental design research is already being implemented into these types of corrective health buildings. This is being done for the purpose of bettering sick individuals and preventing any further harm. If significant architectural experimentation into health design is to occur in this thesis|project, success (or failure for that matter) must be found in what has not been charted. One obvious avenue of exploration that presents itself includes how the design of these corrective facilities can be furthered improved with the help of the available research evidence. However, a more rigorous and speculative process emerges when asking how the direct problems associated with Canadian health can be addressed to offer a truly sustainable solution.

What the preliminary historical data gathering found was that another side of human health exists: the prevention of sickness and disease. It has been found that before complex disease and chemical medicine emerged, ancient civilizations found a holistic approach to health to be successful. Living a life that was in harmony with the outdoor environment was stressed, one that would expose people to the beneficial qualities of nature, natural medicines, the arts as well as healthy levels of social interaction. Our cultural problem primarily stems from living a sedentary lifestyle. This increases the rate of obesity, diabetes, coronary problems, depression, and ultimately affects the health of an aging population. A more sustainable solution would be to offer those individuals an

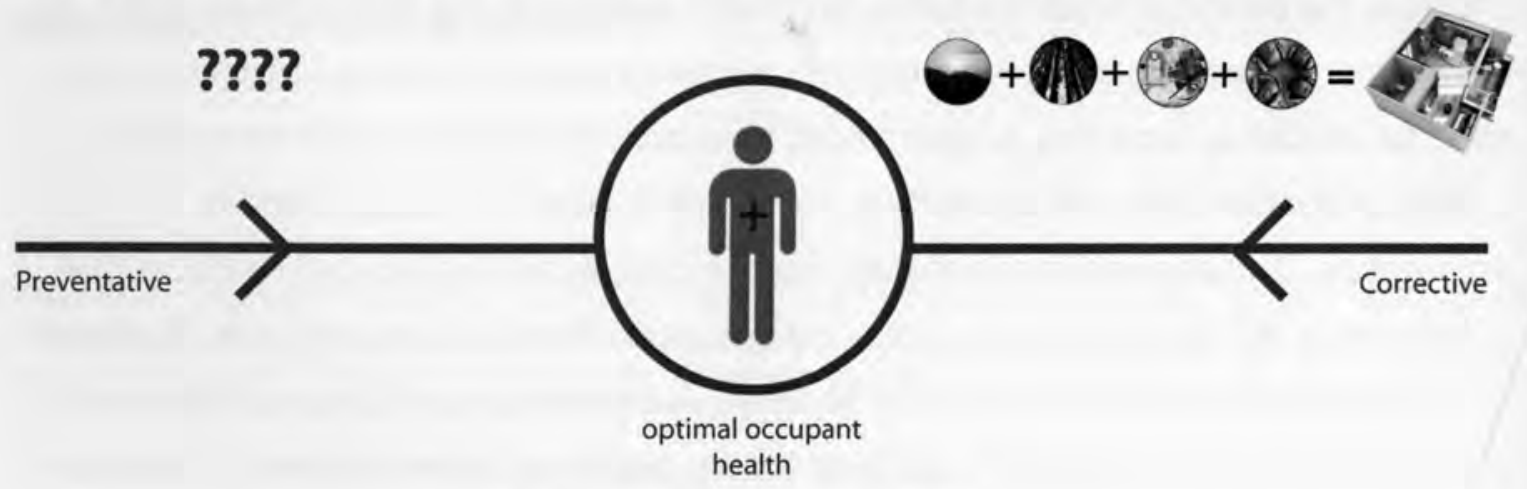

Figure 5.1 Design hypothesis 
alternative lifestyle. Offering a preventative health lifestyle at the level of the community can be used as an education tool for all members regardless of age, gender or physical impairment. Thus, the design hypothesis wishes to investigate what a healthcare facility aimed at the prevention of disease would look like. As well, the question arises of how the techniques of designing corrective facilities using an evidence based design approach can be extrapolated for their use in a preventative health community building? (figure 5.1). 


\section{Chapter 6: General Reader}

\section{Mind and Nature: Health and well-being in the modern built environment}

The modernization of the past century has seen an adverse adaptation of our places of living, learning, working and playing. They have gone from places that engaged with the beneficial qualities of nature's sensory experience, to places that administer a cold and miserable aesthetic brought forth by the machine era. We have replaced the natural properties of our environment with an easy to manufacture, sterile and pre-fabricated man made one. The questions that need to be asked are, how did humanity lose its connection to nature in the first place, and how can we possibly regain it? Does adopting a built environment that lacks the natural properties of nature have adverse health effects? We have placed Man's need for economic prosperity as a central priority in our daily lives. The obsession of leading lives with stress filled careers and the so-called comforts of home detract us from interacting with healthy 'third places'- places of sociability that remedy us from stress and further social alienation (Oldenburg, 1989). It can be said that many factors have led to our detraction from nature. Fritjof Capra (1996) argues that despite having long perceived the modern world from an anthropocentric or mechanistic view, we can now gain much from approaching the emergence of modern communities similar to that of natural ecologies. This includes the view of "seeing the world as an integrated whole rather than a disassociated collection of parts" (p.6). This paper focuses on the need to build an urban environment that makes more connections between man and nature. The beneficial psychological effects of reintroducing nature as a 'third place' in our built environment allow us to increase the health and well-being of our communities in a holistic and sustainable manner.

\section{Problems of our time today- An anthropocentric view of the world}

Capra (1996) suggests that our current view of the world has become outdated and needs to be reconceived. Within a broad cultural context, the way our modern society functions has led to the degradation of our planet's health as a successful ecosystem (figure 6.1). The uncontrolled burning of fossil fuels from industrial processes and autodependency has led to an increase in the earth's temperature. Suburban development has also led to inefficient modes of servicing ever growing populations. The primary 
concern is that with the degradation of the earth's biosphere a point of irreversibility will soon be reached, one in which Man has placed himself at the centre.

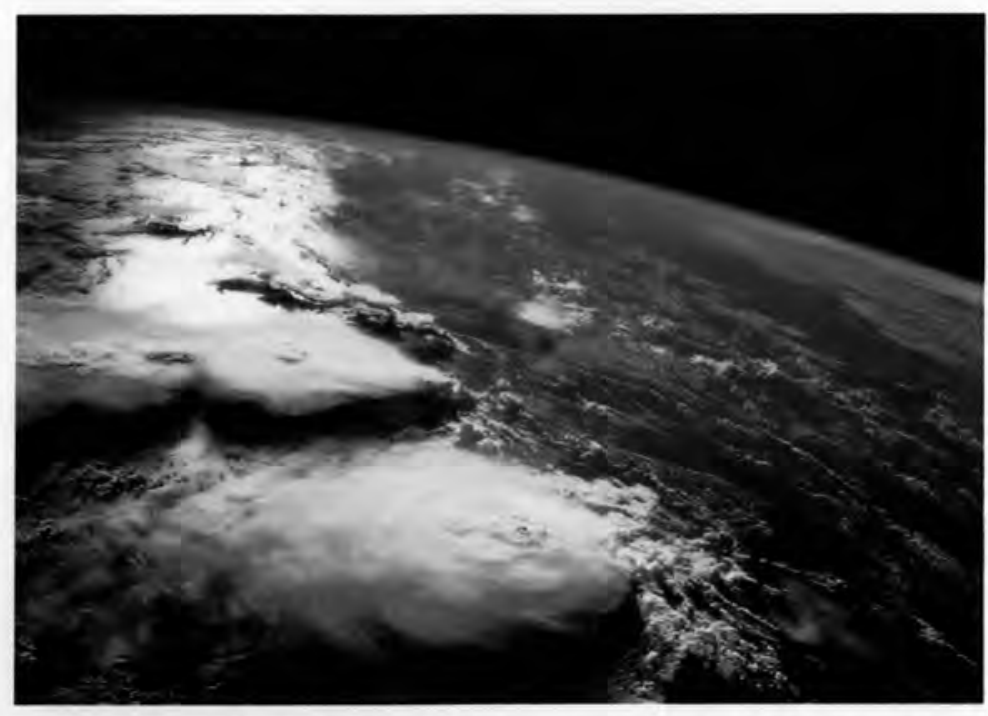

Figure 6.1 The planet earth as a well-balanced eco-system Image credit: Lifeboat Foundation
Capra states that we cannot look at our current problems in isolation from each other (1996). He states that our problems are interconnected within a larger system of issues. Whether these problems stem from our political, economic or social agendas, they must be looked at as one large crisis of human perception. Capra's position shows that only a

shift in perception will allow us to change our values and ways of thinking regarding how we operate in the larger world. His argument calls for sustainable solutions, those that will not damage the ability of future generations to have a good quality of life in exchange for our currently over-indulgent obsession with commodity.

By taking a perspective on the way that ecological systems function, Capra's (1996) position is that the anthropocentric view of the universe is outdated. By placing the human at the centre of the universe, Capra states that this "views humans as above or outside of nature, as the source of all value, and ascribes only instrumental, or use, value to nature" (p.7). His idea of deep ecology places the human species within all other living systems of the natural environment. This places man within what Capra describes as 'the web of life'-the interconnected collection of parts that embeds all objects within one balanced system. Within this, the human species exists as just one part of a larger web. Understanding all levels of life from an ecological perspective allows us to think sustainably, a mode of thought that envisions future generations and their place in the web life. 
Capra's position is not new (1996). Environmentalists have since the 1960's been attempting to bring more of a balance between man and nature to minimize environmental degradation (Naess, 1989). Predating this, ancient civilizations had also sought out a balanced position within the natural world. Their concerns were for those primarily of bodily health. The ancient Greeks, for example, saw the successes of living a life of optimal health by holding a deep relationship with nature. Attaining a positive state of health in the ancient world was crucial since the slightest

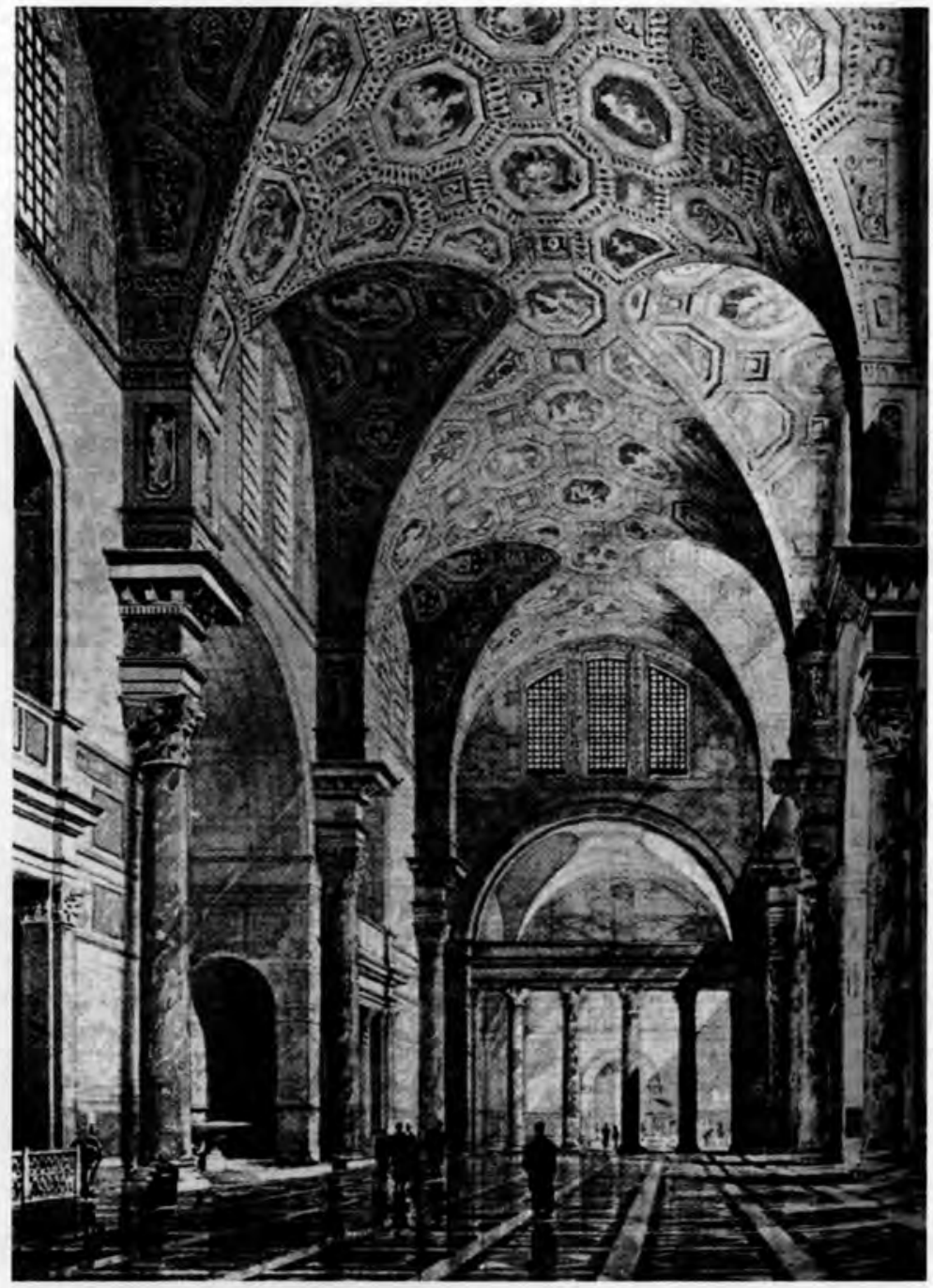

Figure 6.2 The Baths of Caracalla in Ancient Rome were places of natural therapies and places to socialize Image credit: Leland M. Roth

sickness could result in untimely death. Hippocrates stated that, "Health depends on a state of equilibrium among the various factors that govern the operation of the body and the mind; this equilibrium in turn is reached only when man lives in harmony with his external environment" (Purves, 2002, xiii). The Romans later adopted natural aquatherapies in their public baths (figure 6.2). These became integral to a person's daily sense of health and well being. Public baths became a place to socialize, relax and enjoy nature (Cruse, 2004). These practices in themselves can be considered sustainable solutions. Today, engaging in day-to-day wellness through public facilities can be classified as preventative health, a practice that is gaining popularity and is expected to reduce the burden on our healthcare system (Bryan, 2008). Living sustainably is therefore an idea that has ancient roots. The alarming state of our natural 
environment today makes it more important than ever to make the best decisions on how we function as a society. This can include more innovative ways of personal transportation, better industrial processes, new approaches to health and well being as well as to the way we build our physical environment.

\section{How are we all connected-Cognition as the process of life}

Knowing that the new view of the universe should nonetheless be one of integrative perception, mankind is now capable in Capra's view, to exist in harmony with nature (1996). Capra illustrates this concept by showing how mental process is common to all levels of natural life and binds us all together. In the theory of living systems, Capra attempts to surpass the Cartesian divide between mind and matter. He states that in the process of life, "the continual embodiment of an autopoietic pattern of organization in a dissipative structure-is identified with cognition, the process of knowing" (p.172). Life can be now understood as not an object or a thing, but as a mental process. The mind is now the very process of life itself, a process that is consistent with all living organisms, making it the organizing activity of all levels of life. Importance is placed on the interaction between living organisms and the environment as those of cognitive mental interactions-the connection between mind and nature. This may be further enforced by Capra's statement that "life and cognition become inseparably connected. Mind-or, more accurately, mental process-is imminent in matter at all levels of life" (p.172). This position places man within the realm of the natural web of life, and views Man's existence as nature as a fact. Coming from nature makes the point that we will, in one way or another, return to nature. This will be done either through either our collaboration with nature, or through its own necessity to regain ownership of itself. Changing our attitudes on how we coexist with nature is crucial for our longevity and survival. If we are then such a part of nature, the question of why we have strayed so far from our place in the web still remains. What makes us so different from plants, bacteria and other organisms that share the common trait of cognition? What is our problem?

\section{Why has the apple fallen far from the tree? Our ability to abstract}

One specialized human parameter is our ability to perceive abstractly. This is one of the key criteria to our specific level of consciousness, and the way we see things 
differentiates us from other living organisms. Animals may perceive a tree differently from humans due to their varying sensory preceptors they learned or developed through evolution. The common characteristic, however, is that through mental process and the

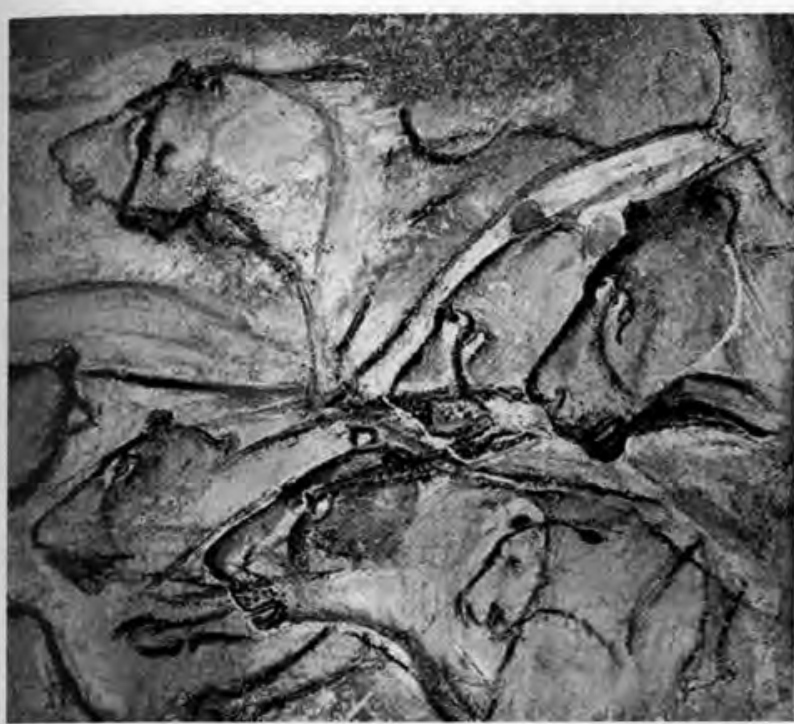

Figure 6.3 The Chauvet cave paintings demonstrate remarkable proportional and life-like accuracy and are dated to over 30000 years ago Image credit: Coquina acts of cognition organisms bring forth their own new worlds into the realm of others (Capra, 1996). We as humans, bring forth a new world through the use of abstract language-an evolutionary form of communication. We also are able to think in the abstract, as well as apply symbolic concepts to our daily lives-something that only we can do. Through this, we use symbols, mental representations and information. An example given by Capra regarding the cognitive aspect of abstraction resides in the colour red in North American city

traffic lights. We use the colour red to symbolize a warning and stopping at an intersection; however, someone from another culture may not be able to perceive its inherently abstract meaning. Information will thus not be conveyed. Many parts of our lives are abstractions of gained information, including the concepts of time, social titles and the laws of physics.

With the idea that humans are the only species that can abstract through language, a dynamic unfolding of culture had emerged over thousands of years. This included a culture that brought forth the complexities of music, art, language and scientific understanding of the universe. As Capra (1996) states:

We also developed the ability of abstract thinking, of bringing forth an inner world of concepts, objects, and images of ourselves. Gradually, as this inner world became ever more diverse and complex, we began to lose touch with nature and became ever more fragmented personalities. (p.294)

With this statement, Capra (1996) acknowledges that while mankind produced such advanced milestones as the Chauvet cave paintings and the Bhagavad Gita, we are also 
capable of creating such acts as the Holocaust and the atomic bombing of Japan (figure 6.3). This abstract and fragmented perception has in turn withdrawn us from our connection to the natural environment from which we came, and has reduced our connection to other human beings instilling hate and fear in one another. The problems of today are deep-rooted and extremely complex and must be overcome, but how does this tie into architecture? How does the detraction from the natural environment and human-nature itself affect us in the places where we work and the places where we dwell- the built environment? We seem to be a species moving away from one another because of our ability to think abstractly, which turns us away from what is truly important in life. Our health and well being, our social connection to each other and the ability to love are some of the most significant points in life. Do these factors become damaged through this incessant breaking away from nature? The perception of the world from a holistic part-to-whole relationship connects all issues into one larger problem. Perhaps our reconnection to nature can solve some of these most challenging points in life.

\section{The experiences of unhealthy modern architectures}

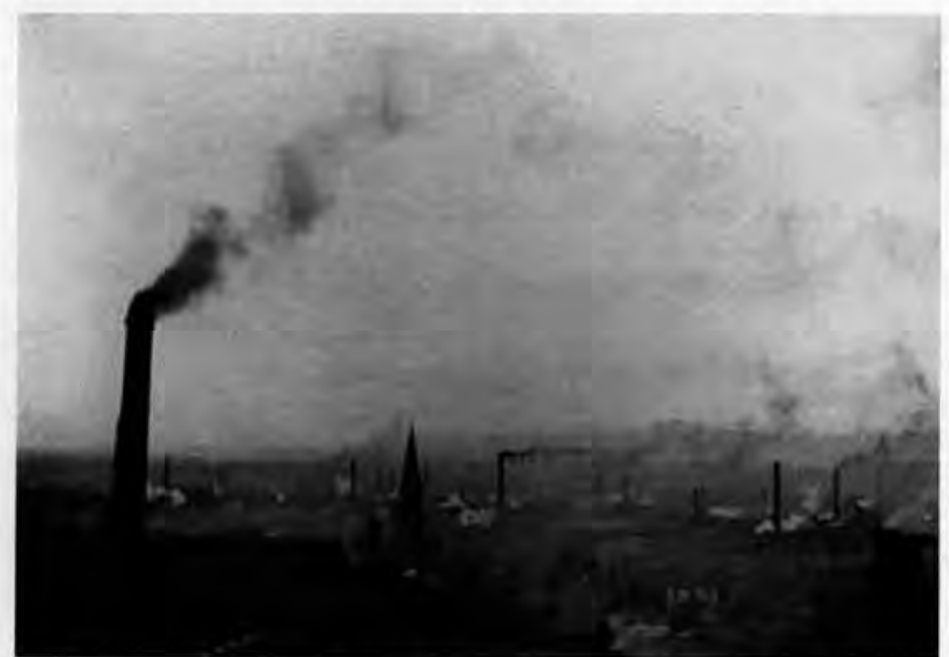

Figure 6.4 The Industrial Revolution in $17^{\text {th }}-20^{\text {th }}$ century England had detrimental effects on the level of public health Image credit: Environmental History Resources
The fragmented perception of our world has come at a great cost to our health. In architecture, we can see how the industrialized era had impacted our western societies and public health. Housing conditions in the $18^{\text {th }}, 19$ th and early $20^{\text {th }}$ century industrial England for example became appalling (figure 6.4). The skies were covered in a mantle of grey smoke and physical

health was an issue. Poor air quality led to adult tuberculosis while sunlight deprivation was later found to cause for childhood rickets (Hobday, 2006). Our detachment from nature and the introduction of industry into our modern settlements was beginning to 
have a negative impact on our health. This demonstrates a point in human history in which Man's perception of the world was now beginning to divide its shared existence with nature. Modern cities were viewed as machines of progress and economic strength. The view of sacrificing human health for the advancement of a nation's wealth demonstrates the perverse cognitive dimension of the human species.

The post-war American suburban development model had further expanded the division between Man and nature. Thirteen million veterans returning from the Second World War qualified for an allotment of life in the suburbs (Oldenburg, 1989). Military industry was now being used for the production of single family homes on an ever expanding scale. Life in a residential suburb fulfilled the aspirations of veterans

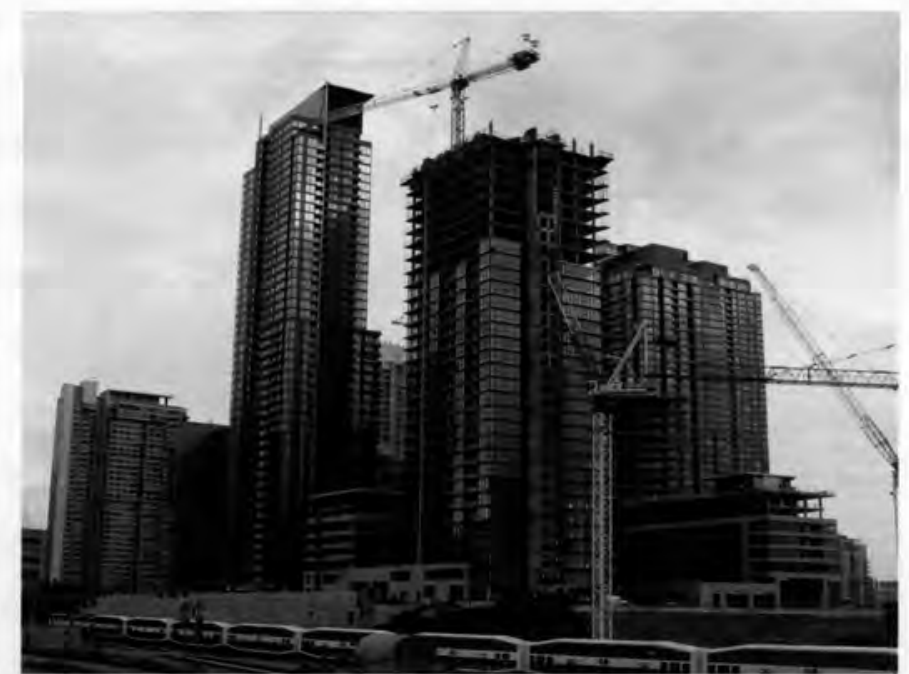

Figure 6.5 The glass skyscrapers of our modern cities lack the spatial and plastic properties embedded in nature Image credit: Wikimedia seeking a safe and quiet sanctuary from the precarious effects of war. A critique of the auto-dependent subdivision however has demonstrated the initiative as being a model that did not develop a psychologically healthy sense of place. As Oldenburg states, "Houses alone do not a community make, and the typical subdivision proved hostile to the emergence of any structure or space utilization beyond the uniform houses and streets that characterized it" (p.4). He illustrates this idea with the example of the adolescent houseguest. Much effort in the design of the suburbs was made towards obedient children or adults' place in American society. However, the teenager was seemingly overlooked:

The visiting teenager in the subdivision soon acts like an animal in a cage. He or she paces, looks unhappy and uncomfortable, and by the second day is putting heavy pressure on the parents to leave. There is no place for which they can escape and join their own kind. There is nothing for them to do on their own. There is nothing in the surroundings but the houses of strangers and nobody on the streets. (Oldenburg, p.6) 
The prefabricated suburban development saw the separation between not only man and nature, but also between people. The network of empty city streets alienated individuals from any places of social gathering. The suburbanite eventually evolved into a hermit; one that would offer minimal interaction with even his own next door neighbour. This fragmented perception of modern life reduced the natural connection between man and his environment, and in turn left the house dweller in a depressed and manic state.

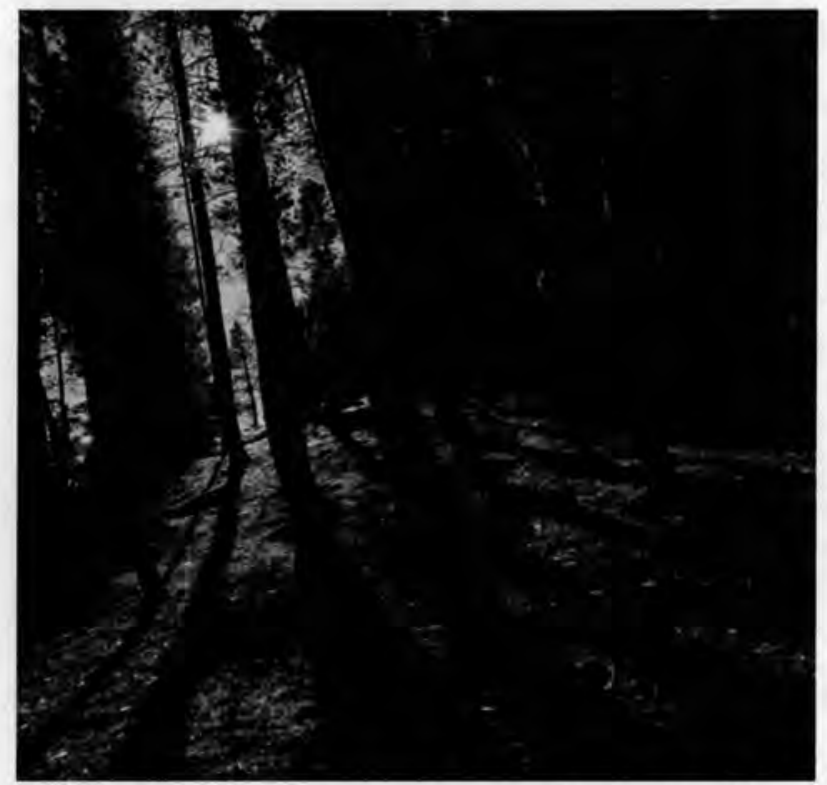

Figure 6.6 The multi-sensory experience of walking through a forest Image credit: Corbis

Besides industrialized towns and suburban developments, our modern urban cities have further evolved into sterile environments that have reduced our interaction with the natural environment. Finnish architect, Juhani Pallasmaa, argues that our modern environments have lost their phenomenological properties over time. These are the experiences that are perceived and understood from a level of human consciousness, and have a great rooting in nature. "Instead of an existentially grounded plastic and spatial experience, architecture has adopted the psychological strategy of advertising and instant persuasion; buildings have turned into image products detached from existential depth and sincerity" (Pallasmaa, p.30). He argues that in the past 30 years, our architecture has been dominated by the ocular sense, emphasized through the perspective of the camera lens. The camera's ability to flatten a three-dimensional spatial experience reduces its potential from being a physical bodily encounter of all the senses, to one of an abrupt session of rapid and seductive image exposure. Pallasmaa's idea of reduced plasticity in modern retinal architectures echoes Capra's ideas of experiencing the world from an anthropocentric perspective- one that "views humans as above or outside of nature, as the source of all value" (p.7). Our modern cities fulfill a strictly ocular experience, but cannot be felt by the rest of our senses as do experiences of nature. 
Our western technocratic and sterile world has disregarded the majority of the senses that we have naturally evolved with. We have been deprived of the properties inherent in natural materials and have replaced them with the properties of a machine aesthetic. The cold properties of sleek glass and paneled metals have become synonymous with our modern skyscrapers are strictly and are properties that are solely perceivable by the retina and none of our other senses (figure 6.5). Our fragmented level of human perception constantly seeks change and progress, and the glass towers of our modern cities represent this progress. The use of natural materials including brick, stone and wood express much different properties of time and the region from which they grow. The essence of the earth is extant within them, and their use places Man within the realm of nature. Pallasmaa argues that modern architecture can benefit from reconnecting with the experiences of nature. For example, he states that walking through a forest may be one of the richest sensorial experiences in existence. Its inherent healing properties are due to the collection and engagement of all the senses orchestrated into one singular human experience (figure 6.6). His position on the modern urban environment is demonstrated through his thoughts on architecture:

\begin{abstract}
Architecture is essentially an extension of nature into the man-made realm, providing the ground for perception and the horizon of experiencing and understanding the world. It is not an isolated and selfsufficient artifact; it directs our attention and existential experience to wider horizons. Architecture also gives a conceptual and material structure to societal institutions, as well as to the conditions of daily life. It concretises the cycle of the year, the course of the sun and the passing of the hours of the day (Pallasmaa, p.41).
\end{abstract}

\title{
A holistic view on life- Reintroducing nature
}

Capra's (1996) position is one of a necessary paradigm shift regarding our perception and our knowledge of the natural environment which he classifies as deep ecology. His view on the way we perceive our systems of life is one of a holistic worldview. This view sees "the world as an integrated whole rather than a disassociated collection of parts," one that holds "fundamental interdependence of all phenomena, and the fact that as individuals and societies, we are all embedded in (and ultimately dependent on) the cyclical processes of nature" (p.6). What can be extracted from this is that with a deep reintroduction of nature into our built environment, we have the opportunity to re-enter into a critical dialogue with nature. To become a part of the process of nature once 
again, we must re-design it back into our daily lives, which can re-establish our physical and psychological attachment to the natural world. This reattachment to the natural world is of fundamental importance to our level of public health, and can be seen through recent research studies on the topic of nature, health and architecture, which demonstrates the beneficial healthy attributes that nature has on man.

\section{Experiencing nature- Environmental psychology and Evidence Based research}

Environmental psychology research has shown the positive effect that nature can have on a person's general level of health and well-being. The new field of evidence based design attempts to apply empirical research findings to the design of our built environments. This is being done with the hopes of re-implementing

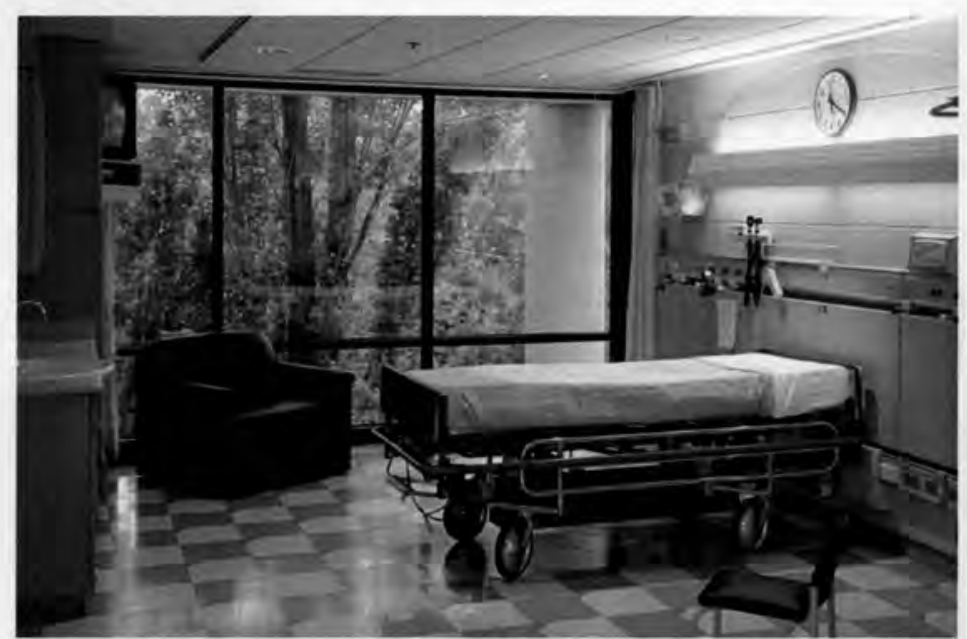

Figure 6.7 Views to nature in a hospital ward have been found to speed the recovery process of post-operative patients Image credit: Anshen + Allen Architects

environmental health and wellness into our internal spaces. A majority of these studies have emerged through the modern design of hospitals in hopes of creating a more holistic environment of natural healing. Studies have found that views to nature in a hospital ward (figure 6.7) can result in quicker recovery times, reduce levels of stress and blood pressure, and decrease physical discomfort and levels of depression among patients recovering from post-operative surgeries (Schweitzer, Gilpin \& Frampton, 2004; Ulrich, 1984). This is also consistent with hospital staff and their general feeling of rejuvenation. They return to work feeling more relaxed and refreshed through the implementation of nature within facility design (Marcus \& Barnes, 1995). Research also points to the beneficial psychological effects of Biophilic design- a practice that implements naturally shaped built form into the design of interior spaces (Joye, 2007).

The evidence for designing natural light back into our modern buildings has also been found to be critical to our state of well being. Our largest attachment towards the sun and 
healthy levels of natural light occurs within our innate and internal biological processes. Sunlight enters the eye and sends electrical signals that travel to the visual cortex and to the rear of the brain (Hobday, 2006). It is here that our internal biological clocks respond to the daily and nightly cycles of the sun, and to seasonal changes. This chemical reaction allows for the successful production of melatonin; a hormone that tells us that we, and all organs of the body, are wake. This master clock gives the body what is known as a circadian rhythm; the $24 \mathrm{hr}$ cycle our bodies and systems function with that allow us to adapt to the external environment. Lewy, Nurnberger, Wehr, Pack, Becker and Powell's (1985) study, (as cited in Joseph, 2006) stated that when levels of natural or artificial light are minimal during the day, the unhealthy reduction of melatonin creates levels of sleepiness and depression, as well as other related health issues. This has profound effects on individuals and is exemplified by the institutionalized setting of modern hospitals, offices and educational institutions.

Studies demonstrating the impact that natural light has on reduced levels of depression and seasonal affective disorder (SAD) within hospital patients have been conducted. Benedetti, Colombo, Barbini, Campori, and Smeraldi's (2001) study, (as cited in Joseph, 2006) of depressed bipolar patients allocated patients into two sections of a hospital. In the study, half had rooms facing east (exposed to bright light during the morning hours) and the other half had rooms facing west (and therefore minimal levels of bright light). Those patients receiving higher levels of natural morning light stayed in hosptial 3.67 days less than those patients in the westward facing rooms. In an American Journal of Psychiatry (2005) study, (as cited in Hobday, 2006) it was found that bright light therapy was just as effective as medication in the treatment of mood disorders and depression. In another study by Golden (2005), (as cited in Hobday, 2006) a team of researchers conducted a statistical analysis of 20 studies in bright light therapy over a 20 year period. These amalgamated studies demonstrated that bright light therapy was an extremely effective method for the reduction of depression.

The case for reintroducing nature into our built environment for the psychological benefit of building occupants has thus been demonstrated. Redesigning our communities with nature in mind can reintegrate the human component back into the larger web of life. The coexistence of man and nature can offer physical and psychological healing from designing it back into our built environment. This serves as a form of preventative health- 
diminishing the possibilities from the built environment of incurring any form of psychological or physical harm, as well as promoting health. This approach to health and well-being can be considered what Capra (1996) states as sustainable solution- one that will not damage the ability for future generations to withhold a good quality at the expense of our own.

\section{Third Places of Nature in the Community}

The importance of designing for health and well-being in our modern communities is a new emerging field of study. At the Royal Institute of British Architects a think tank is focusing on the 20-50 year future of the built environment (Wernick, 2008). Their area of interest lies in knowing whether architecture and the built environment can contribute to our happiness. Building Futures states that

In the field of economics, it has been found that increasing our wealth does not necessarily make us happier. A new science, the Economics of Happiness, has sprung up and we have found that although GDP, which measures our economic prosperity, has increased enormously since the 1950 's, our happiness has not (p.7).

Happiness can be seen as one of the most important factors of life which has a direct connection to our quality of living. The King of Bhutan has demonstrated that by measuring their country's wealth through a Gross Domestic Product (GDP), they also have a Gross National Happiness Quotient. At Warwick University academics are also researching the impacts of a Gross Happiness Level that may soon come to replace GDP in terms of measuring a country's state of wellbeing. What this shows is an increasing emphasis on providing a good quality of life or a good level of happiness is an increasingly important issue within how we design our built environments.

A reduced level of happiness in our modern communities may be due to many social and physical factors. It has been previously argued that modern urban environments are unhealthy due to the physical stresses from heavy industries, or from the reduced presence of the natural materials with their broad range of embedded sensorial qualities. A general sense of happiness and well-being within our modern urban environments may also be due to a lack of social spaces, and thus a lack of human presence-or a lack of human nature itself. Human nature is considered the psychological and social 


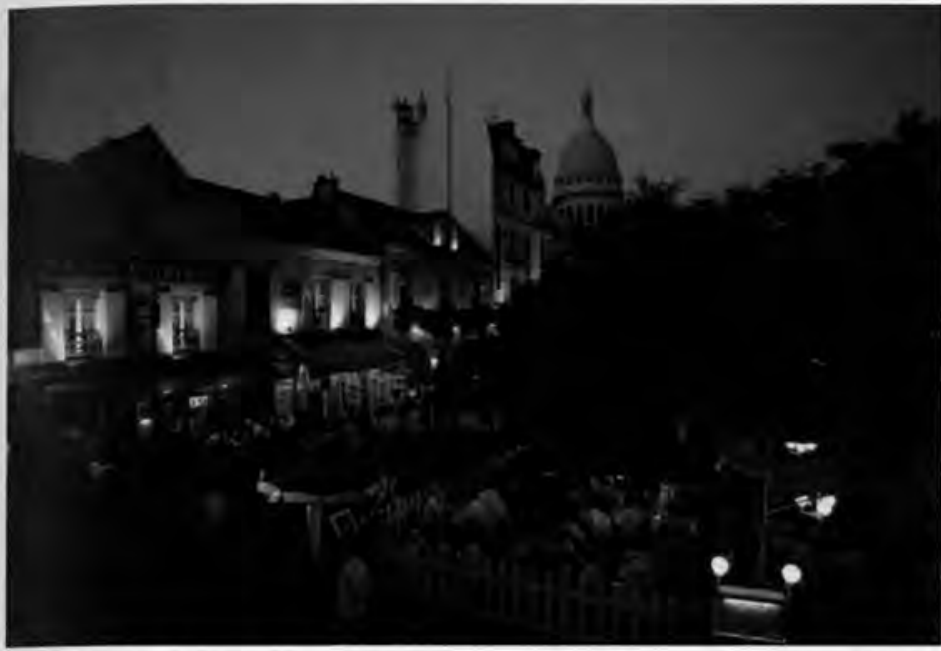

Figure 6.8 The French sidewalk café acts as a healthy third place in the city- one that offers positive stress relief and social interaction from that of the home or workplace Image credit: Corbis qualities that characterize humankind. As stated before, the modern subdivision has reduced this level of public life, or what Ray Oldenburg (1989) states as lacking 'third places'. A third place may be a place of gathering for the purposes of being social, one that exists between the homogenous world of work and the comfort of home. It is a place that acts as "peoples own remedy for

stress, loneliness and alienation" (p.20). The notion of a third place in Oldenburg's position is reminiscent of such healthy spaces such as the French café, the English pub or a public park (figure 6.8). The critique of western development includes a lack of third places integrated into the overall city fabric. This defies Man's natural tendency of being a social creature.

Empirical evidence has shown us that urbanites lack the necessary amount of healthy social interaction. Figure 6.9 shows the time North Americans spend in certain places throughout their weekly lives. This study estimated that approximately 25 per cent (or 42 hours) of a person's week was spent at work or at school, while 10 per cent was dedicated to travel. It was also seen that 50 per cent of time was spent within the home ( 84 hours) and 10 per cent (16.8 hours) was spent in playing or socializing, or what Oldenburg (1989) classifies as occupying the third place. What's interesting about this study is what can be considered socialization. Today, many aspects of social engagement involve being wired to online social networks, surfing the Web and stimulation through digital media. These activities can all occur in our homes. The culture of online socializing born in the late 90's was not yet adopted at the time of this study. With this new emergent culture comes a new breakdown of where people occupy the majority of their time. An individual's weekly life may now be seen as the following: 25 percent of time spent at work or at school (with the associated 10 per cent of travel time) followed by up to 65 per cent of time encompassing an individual's life within the 
home- through which 'remote socializing' may occur. This reduction in time spent in 'healthy' third places (such as parks and outdoor cafes) pulls people away from not only the

health benefits associated with nature, but also from the healthy

1. at home Percent

2. at work or school 50

3. travelling

4. playing or socializing

5. other activities

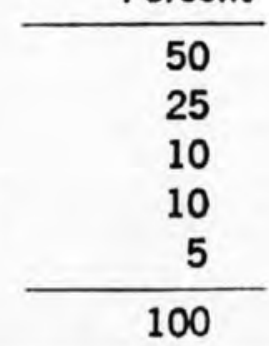

Figure 6.9 Weekly breakdown of where the average person in North American urban conditions spend their time Image credit: Lawrence E. Hinkle benefits of fellow human contact. This social alienation from the natural aspects of life (both the outdoors and human contact) cannot occur for an infinite length of time. As Patrick Goldring's position against unsocial urban sprawl states, "sooner or later there will be a check in the seemingly inexorable movement towards antlike inhumanity, organizing for organizing's sake" (Oldenburg, p.285). This check has started to occur, and researchers in England are trying to re-implement design factors that will reintroduce happiness and well being back into the built environment.

\section{Building Happiness in Greenwich England: Social and Physical Factors for Building}

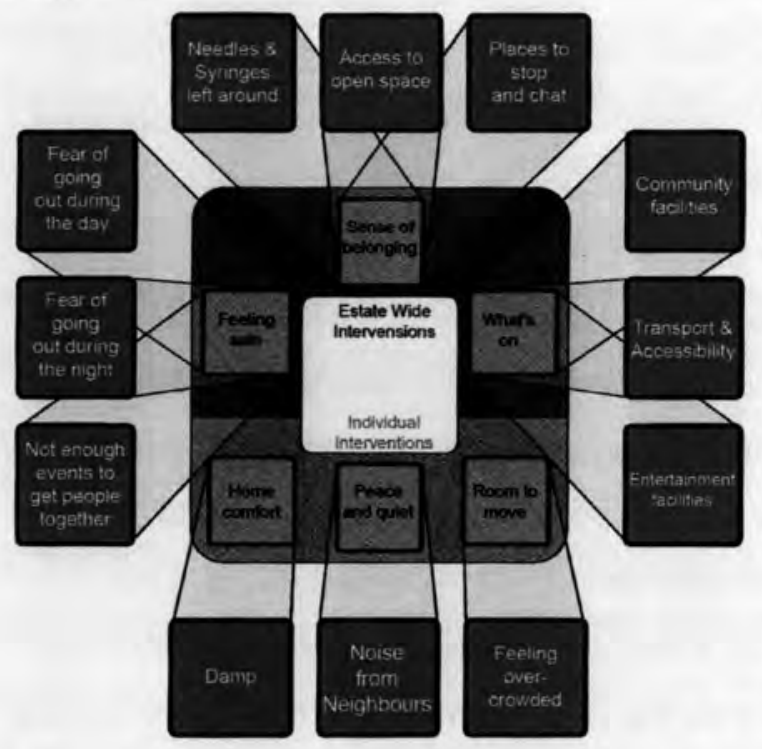

Figure $6.10 \mathrm{Key}$ areas that mediate between the built environment and mental wellbeing Image credit: Jane Wernick
Researchers have recently been interested in linking the subjective, psychological and social aspects of wellbeing and how it can impact the aesthetic design (of both form and function) of the built environment. A study in Greenwich England looked at the social and physical factors determinant in achieving happiness amongst its landowners (Guite \& Toy, 2008). Following four years of data collection, the project is now within the implementation phase. In this innovative project, simple and low-cost physical and 
social measures within the design of the built environment are being carried out to systematically to increase the positive effects of resident's mental well-being.

The study sought to find variables that both positively and negatively affected people's perception of happiness in a deprived community, in order to re-implement these components of happiness back into the fabric of Greenwich neighborhoods. The study questioned residents living throughout nine housing estates. Common variables across estates included a diverse age population and ethnically mixed populations. This study had found six domains and 13 factors that were essential in the promotion of wellbeing within the built environment of the estates. Figure 6.10 shows the relationship between the domains and the individual factors. What the research found was that both social and physical factors remained important when classifying wellbeing within the community. The domains and factors may be further classified into two more specific categories: individual interventions (those applying to interior dwelling units of the residences) and estate-wide interventions (external physical and social considerations).

Figure 6.11 shows factors that were found to be significantly associated with worse mental wellbeing.

Some of these factors included access to green spaces, places to stop and chat, feeling safe to go out at night, access to community facilities and events to get people together. With

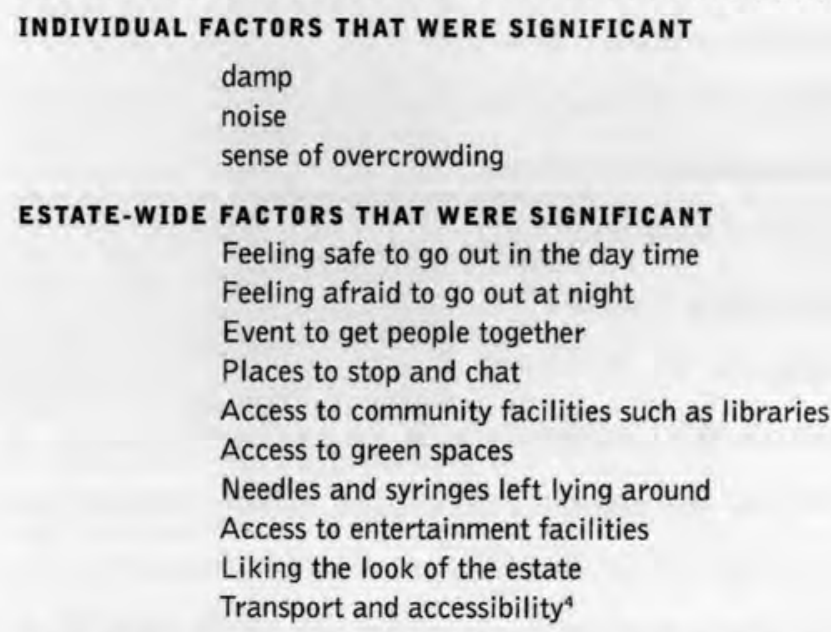

Figure 6.11 Factors that were found to be significantly associated with worse mental wellbeing Image credit: Jane Wernick

respect to designing for increased estate wide wellbeing, the researchers found it important not to pick at singular interventions for creating change. Importance was placed on looking at all factors as being part of one larger problem. A holistic approach to creating changes to the physical environment seemed more successful when all items 
can be treated as one large web of issues. Guite and Toy (2008) give the example of installing benches to increase the number of places for residents to 'stop and chat,' one of the major factors found in this study. However, without addressing the issues of drugabusers in the area, public space may be utilized in unfavourable ways. It is thus seen important to envision all issues from both the physical and social realms. The issues seem more complex than simply correcting singular physical or social factors- they, as Capra (1996) states, cannot be looked at in isolation of others and are interconnected within a large system of issues. Figure 6.12 looks at the factors that were not found to be significantly associated with worse mental wellbeing. Some of these factors included density, height of buildings, recorded crime levels and vandalism.

The findings here

indicate that mans psychological connection to the built environment and each other is associated with a community's sense of mental wellbeing.

Specific interventions that will be implemented into the Greenwich estates
INDIVIDUAL FACTORS THAT WERE NOT SIGNIFICANT

height of building

house type-house, flat, maisonette

light, heat, draughts

year of build

\section{ESTATE-WIDE FACTORS THAT WERE NOT SIGNIFICANT \\ density \\ recorded crime levels \\ sports and exercise facilities \\ shopping facilities \\ vandalism and maintenance \\ feeling that people can influence decisions}

Figure 6.12 Factors that were not found to be significantly associated with worse mental wellbeing Image credit: Jane Wernick

include gardens in front of residences, seed planting areas, events and outdoor initiatives to simply get people to socialize like communal gardens and community cafes. This study provides empirical evidence that implementing third places into communities (most notably those offering levels of nature and socializing) can foster physical and social wellbeing among residents. What is demonstrated here is how a community that was designed with such a sterile and modern machine aesthetic was found to have such a negative effect on individual's level of mental wellbeing. This shows that designing a built environment that connects man to nature and man to each other (human nature itself) offers successes in the way to design our modern built environment. As much as this scientific study has recently shown, prime architectural examples have existed over 
time that depicts the existence of 'nature as a third place' in a community- one that can foster both social interaction and acts as a sustainable approach to health and wellbeing.

The Thermal Baths of Vals

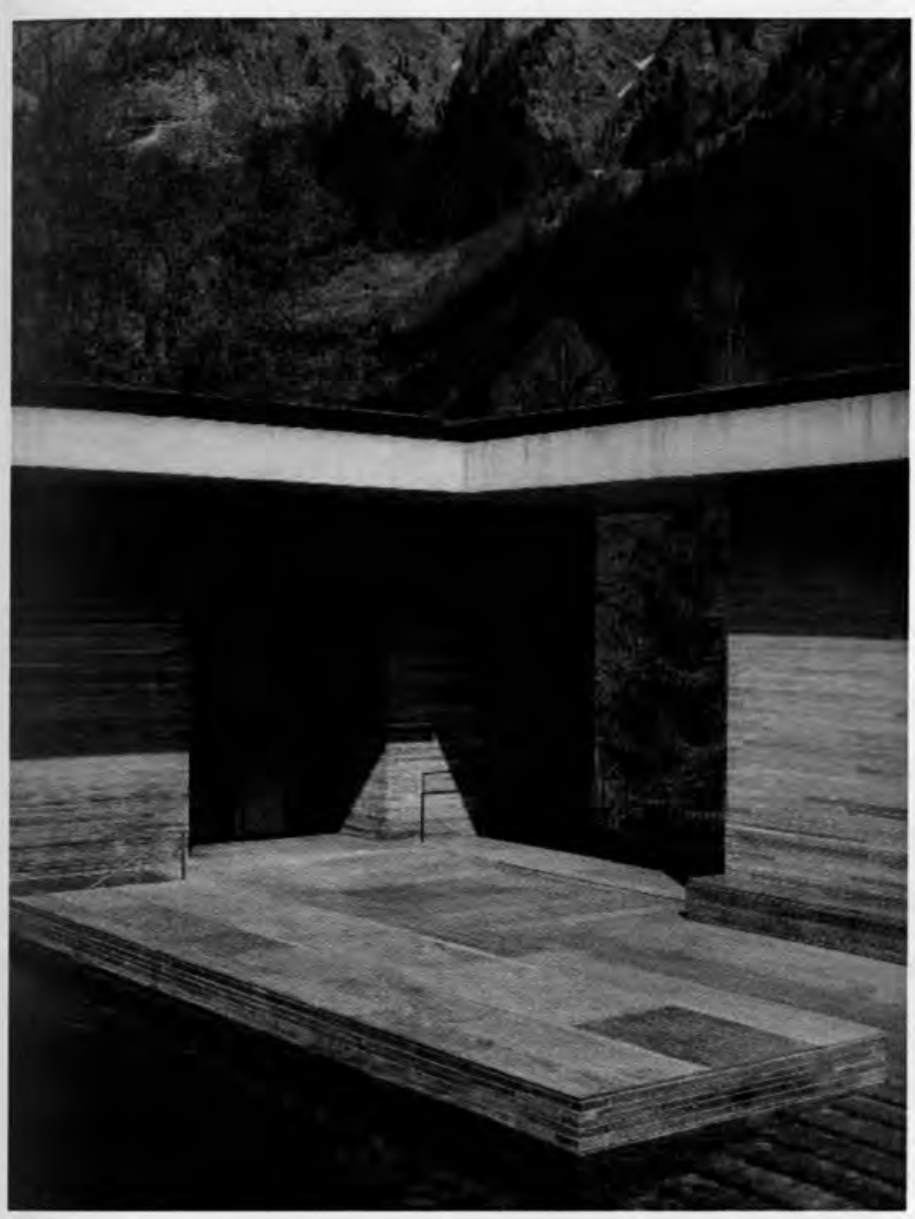

Figure 6.13 The Thermal Baths at Vals blend into the natural surroundings of the Swiss stone strata Image credit: Peter Zumthor

In the Swiss mountains of Vals, contemporary architect Peter Zumthor approached the design of a modern bathing facility as if it were a third place that emerged from the natural landscape (figure 6.13). The thermae ascribe to a Heideggerian philosophy, one that act as a "more humane expert practice of modern architecture, which was sensitive to place and people" (Sharr, 2007, p.100). Zumthor's approach to the baths make no attempt to showcase the latest developments in aqua technology, "but relies instead on the silent, primary experiences of bathing, cleansing oneself, and relaxing in the water; on the body's contact with water at different temperatures and in different kinds of spaces; on touching stone" (Zumthor, 1998 , p.156). Humanizing the architectural experience allows for the fullest experience of place, one that Zumthor attempts to characterize with the building's surroundings. The entire facility is built from the same stone strata as that of the Vals Mountains. Layer upon layer of Val's gneiss make up the stone block interior of the baths. A continuous internal space, the various rest areas and bathing opportunities blend into a singular cavernous experience. Through this, Zumthor makes the connection between person, place and experience. 
The architecture itself is an experience meant to evoke memories from the past, memories that encompass history, function, passion and aesthetics. It is also Zumthor's process to design with memory, and it is with memory that nature constantly emerges. Zumthor claims to design with a final image in mind brought forth by memory, one that attunes itself to the

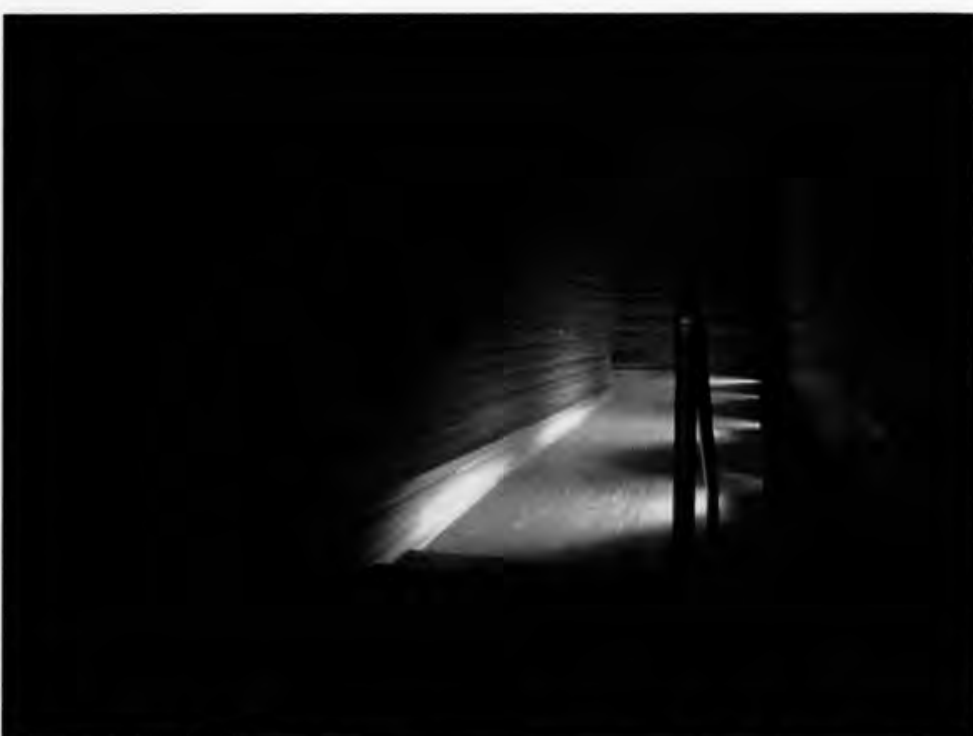

Figure 6.14 The processional caverns of the baths were conceived as atmospheres, spaces that held physical compositions that could only otherwise occur in nature Image credit: Peter Zumthor

whole of the architecture. Designing with a remembered image is a natural process that all people share as common. Envisioning all the elements of the space- walls, ceilings, floors, materials, lightness and dark- allow for a composed experience to manifest itself. Zumthor attempts to approach the built form through a holistic mind-body experience, one invoked by memories of the past.

I believe that buildings can only be accepted by their surroundings if they have the ability to appeal to our emotions and minds in various ways. Since our feelings and understanding are rooted in the past, our sensuous connections with a building must respect the process of remembering (Zumthor, 1998, p.18).

Zumthor's approach of designing with memory attempts to visualize the space as an atmosphere (figure 6.14). He compares his successes through this process as something that can only be similarly found through the mastery of nature's design. "I believe that if artistic processes strive for wholeness, they always attempt to give their creations a presence akin to that found in things of nature or in the natural environment" (Zumthor, 1998, p.30). Zumthor's obsession with natural materials and his dislike of additives/colourings is inspired by his love of experiencing nature. His objective is to inspire the human visitor with natural experiences, of time, place and material. 


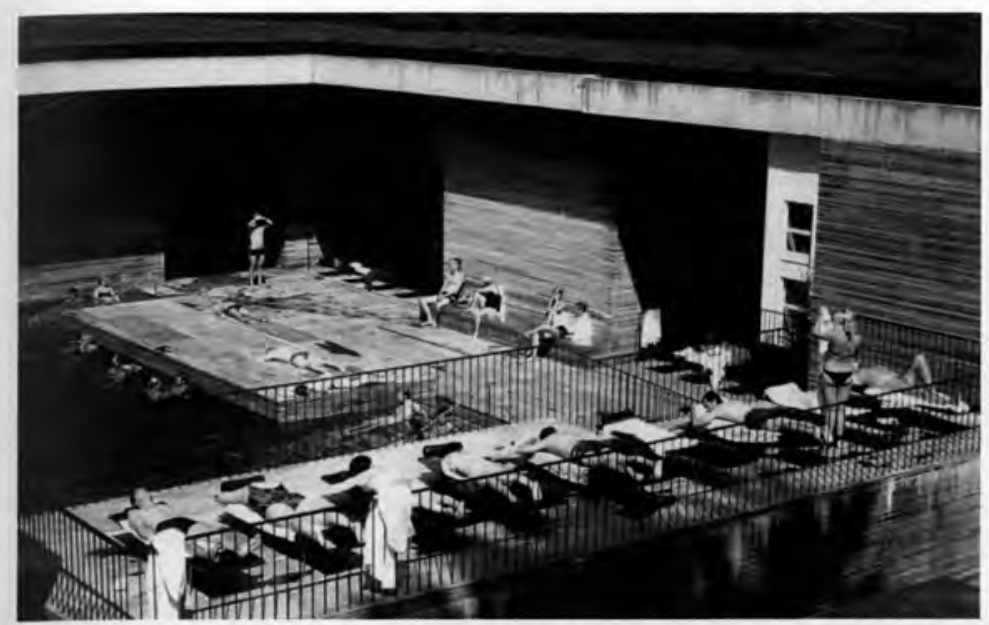

Figure 6.15 The level of social interaction on the sun deck allows the bath to be a community facility- one that offers positive levels of human connection Image credit: Peter Zumthor
The thermal baths of Vals seem to characterize the bringing together of mind and nature. Man's ultimate connection to the natural world through cognition is demonstrated here through the built form. This bath facility does not attempt to alter the perception of materials or place, and allows the human to undergo the

common liturgy of bathing with other individuals in a simple sense (figure 6.15). There is something extremely animalistic about this- walking barefoot on natural stone with ambient natural light pouring through the cavernous crevices. This experience is something almost too natural to comprehend, something that cannot be experienced in modern urban environments that we come to know so well. The connection between man and nature is therefore strong in this case (figure 6.16). This can be considered as a third place that emerges naturally from the landscape and is utilized by the community on a continual basis- one that reconnects the individual with nature as well as one another.

\section{Designing a future built environment with nature in mind}

Through the process of mental cognition, mind and nature become inseparably linked. Cognition is the common characteristic amongst all living organisms within the web of life. Man's place in the web of life thus secures his mental connection to nature. It is with the idea of 'mind and nature' which the connection of 'Man existing with nature' may emerge. Our built environment has unfortunately evolved into a sterile environment, one that lacks the sensual qualities inherent in nature. This has been proven to be unhealthy for both our physical and mental health. In order for our communities to regain their optimal health levels in a sustainable manner, we must reconnect with nature's beneficial therapies in a preventative manner. Reintroducing nature into our daily lives through the design of the built environment can allow for healthier communities to 
flourish. This can occur by offering people stress relief through views to nature. Direct views to nature can occur at highly stressful points of the day, such as in the office setting, the waiting room of a hospital or at the gym while working out.

Offering the psychological benefits of natural lighting can also occur with the confines of the indoors. Light shelves within façade design or light shafts within deep plan buildings allow for an

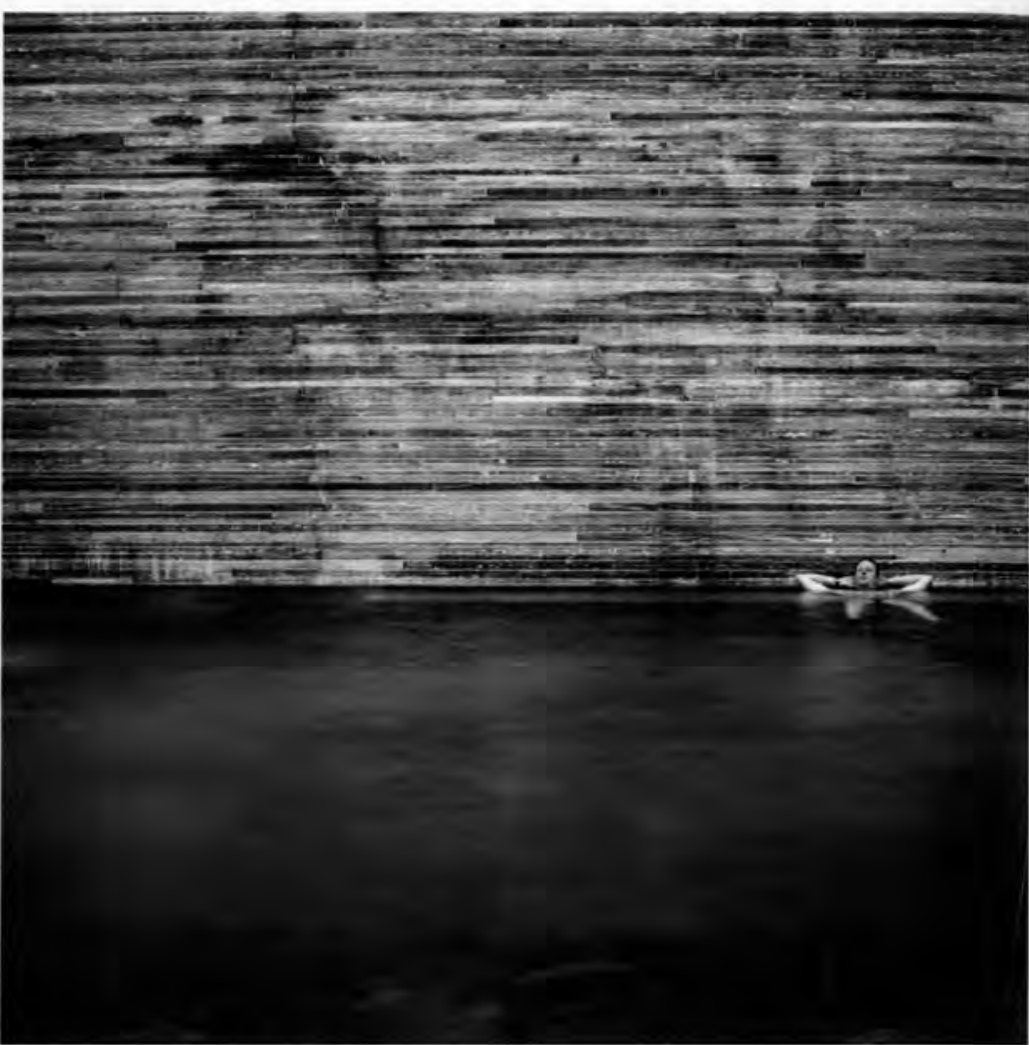

Figure 6.16 The silent primary experience and therapy of bathing by oneself Image credit: Peter Zumthor

abundance of natural day

lighting into buildings, while full spectrum lighting can be utilized for lighting our interiors at night. The use of natural materials can also offer a plethora of sensory qualities that our interiors currently lack. The use of untreated woods, natural stone and brick are all reminiscent of the time and place from which they come-earthly materials. Offering moments of phenomenal experience within architecture that mimic nature can also have profound effects on how we perceive the built environment. For example, the experience of light cadences permeating through a forest canopy or the sound offered by pools of water can offer atmospheres of experience.

These points of nature can furthermore be incorporated into the third places of our daily routines, spaces that not only reconnect us to nature, but socially to each other. Places such as community centres, cafes and general hangouts are healthy places of socializing. The healthy effects of being social can be coupled with the health effects offered through nature. This new definition of a third place that is both naturally and socially healthy can manifest itself as a public park, public bathing facilities or other 
urban open spaces. Many urban design firms have seen the strength of third places, and have used them in design proposals to reestablish a sense of community and reconnection to nature in our modern cities (Appendix 5). By encompassing the ideas of socially healthy third places that connect man to nature, the design of the future built environment can be approached in nothing less than a humanistic manner-an approach that puts the health of the human occupant as the primary concern. Designing the built environment through a humanistic approach thus ensures a sustainable approach towards healthy community building- one that ultimately reconnects us with our natural roots. 


\section{Chapter 7: Design Project}

\subsection{Program and Vision}

By integrating nature's healthy attributes into the design of our built environment, we can foster community wellness in both a holistic and preventative manner. This in turn reduces our dependency on corrective health approaches and promotes a lifestyle that ultimately allows individuals to control their own levels of health in a sustainable manner.

The architectural design experimentation of this thesis|project investigates what a healthcare facility aimed at the prevention of disease would look like. It also examines how the evidence based design techniques, proven successful in corrective facility design, could be extrapolated for their use in such a preventative approach to health. The hypothesis for design sees the successes of offering an alternative lifestyle as a possible solution to the interconnected and preventable issues involved in Canadian health. This ideal lifestyle is contained in the notion of wellness, a holistic approach to health that governs both the mind and body. The idea of shifting the emphasis from healing the sick to supporting the healthy is one that can most reasonably occur at the level of the community. Similarly to a community health centre, a community wellness

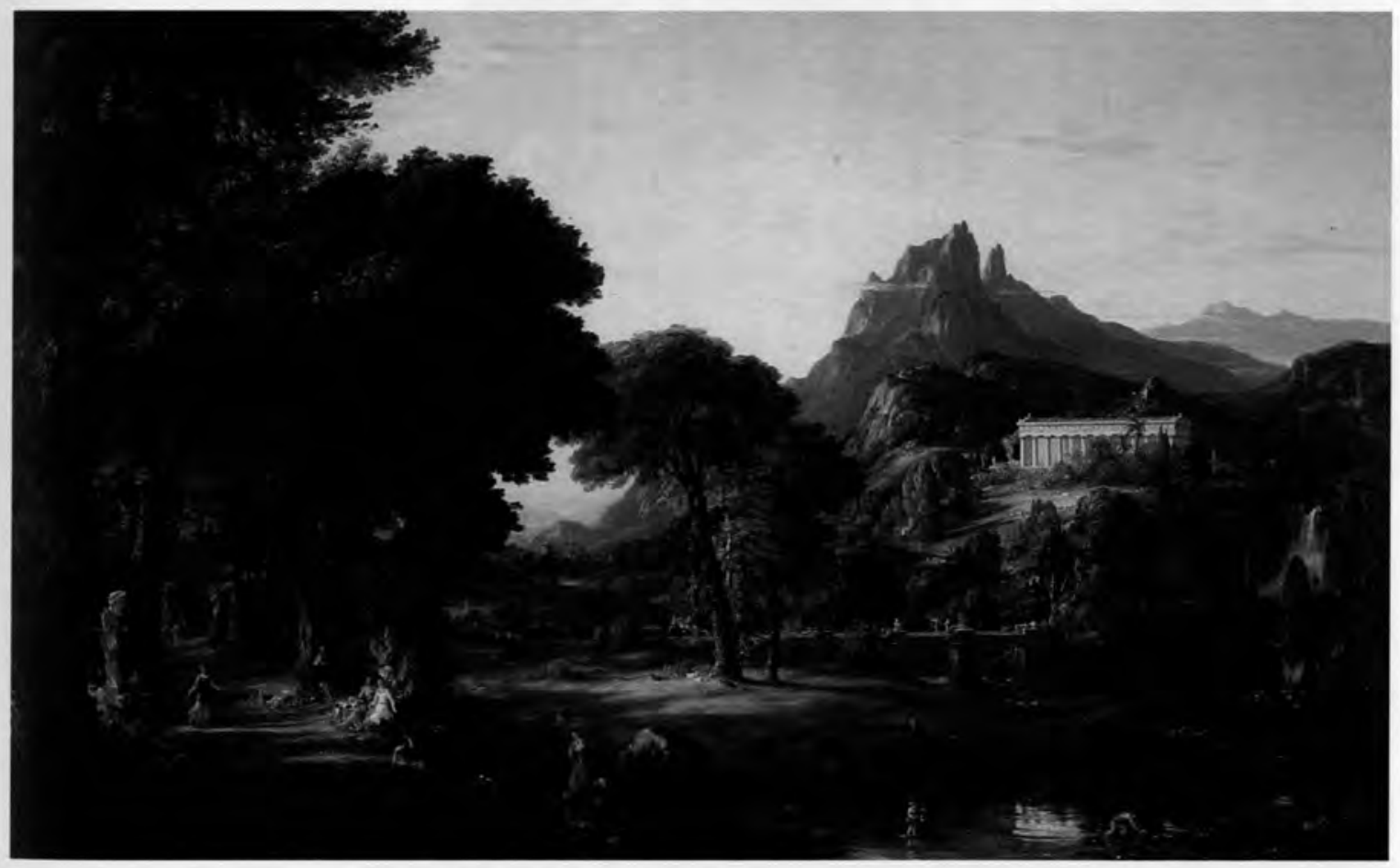

Figure 7.1 Thomas Cole: Dream of Arcadia (1838). Image Credit: Thomas Cole 
centre offers services to an extended range of users, regardless of age, gender or impairment, and offers an increased quality of life.

Prior to advances in complex disease and chemical medicine, wellness and prevention was a priority in ancient civilizations approach to health. As seen throughout traditional Arcadian mythology, wellness was the promotion of a well balanced lifestyle that included the beneficial qualities of nature, natural medicines, fitness, the arts as well as healthy levels of social interaction. This ancient Greek ideal was portrayed in Thomas

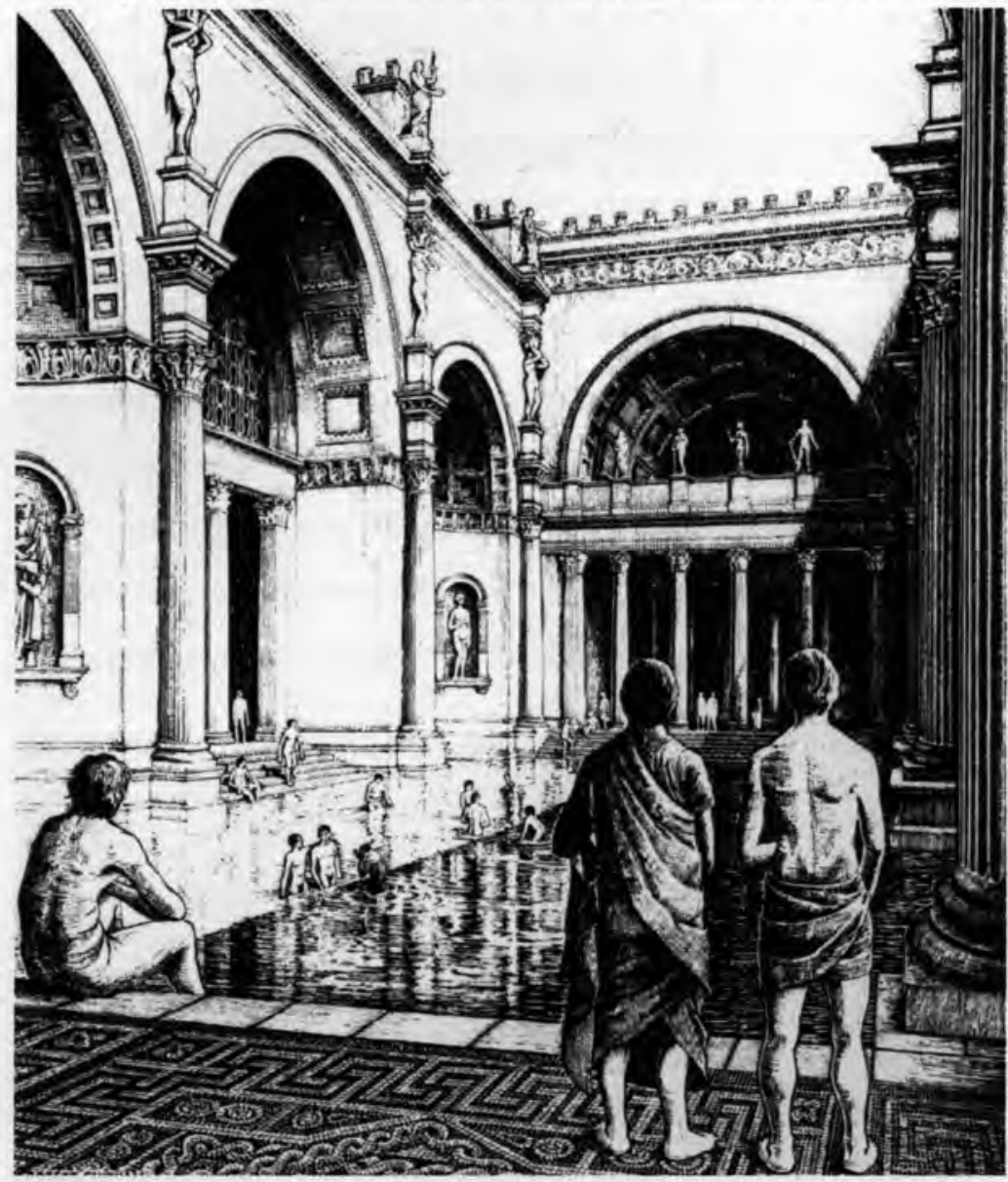

Figure 7.2 Robert Chitham: Drawing Reconstruction of the Baths of Caracalla. Imaqe Credit: John Dawes
Coles' renditions of Arcadia (fig. 7.1). Living with an intimate regard for nature was seen as pertinent to living a life of health. The early Romans also believed in this ideal, and had erected monuments to natural forms of health and community interaction. Most notable were those of their public baths (fig. 7.2). These were places where health and fitness were undertaken on a daily basis. As well, these served as places of social interaction amongst users. Preventative health was

thus practiced as a cornerstone in successful community living of these ancient cultures. Much can be learned from these ancient examples of preventative health, one whose core values exist in the ideal of community living and social interaction.

Experimentation with wellness centre design today would need to extend its services beyond merely encompassing nature and health. As seen in figure 7.3 , the emergent 
definition of wellness encompasses four major areas: health, fitness, education and healing. Programmatically, a modern day wellness centre would need to extend itself for a broad range of users including children, middle aged persons as well as the elderly.

This broad user group would show varying interests, like time of use as well as physical abilities. A breakdown of the organizational service structure of the final proposed wellness centre is shown in figure 7.4. A detailed programmatic analysis exercise was conducted throughout the design development stage, and is summarized in Appendix 6.

Health: The community wellness facility is intended to offer traditional methods of healthcare services with combined integrated services. Under the inception of a family health team, the walk-in or by appointment clinic would integrate traditional medical treatment, physical rehabilitation as well as alternative health specialists. General practitioners, nurse practitioners, and specialists would work in a team environment that would combine the alternative skills of

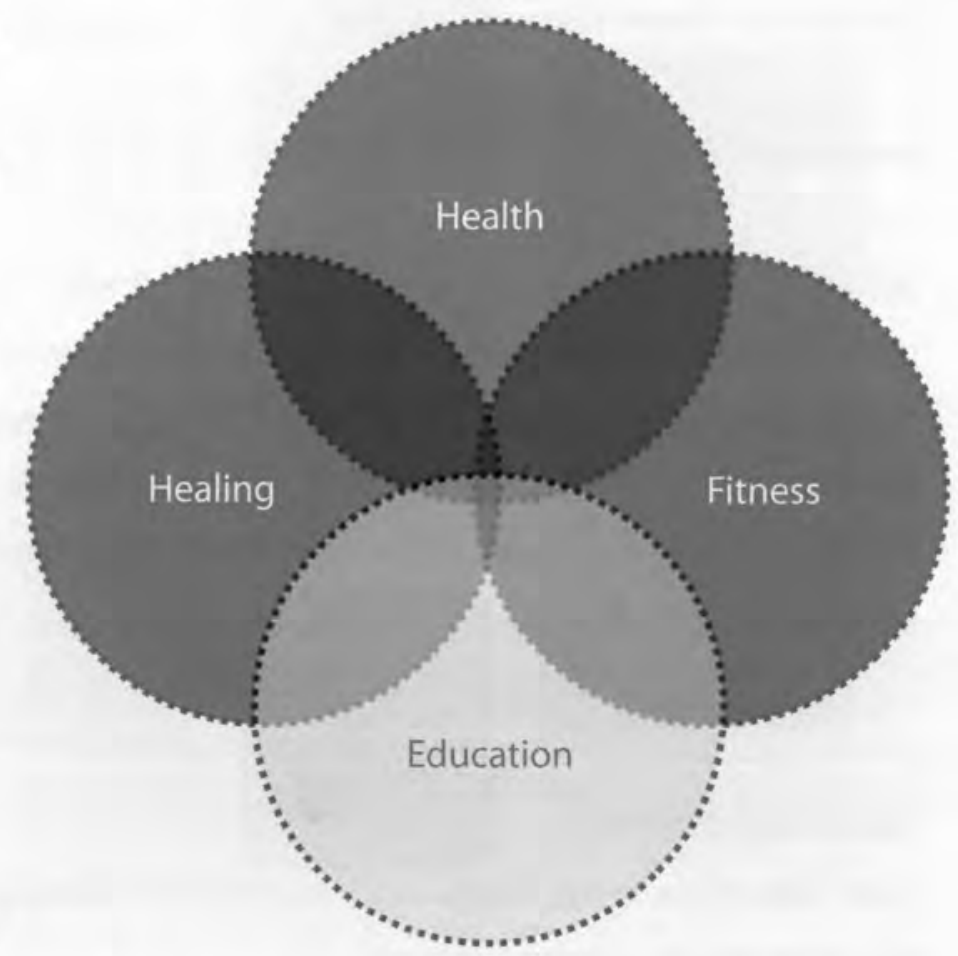

Figure 7.3 Wellness Centre: Programmatic Diagram physiotherapists, chiropractors, massage therapists, naturopaths, nutritionists and psychologists. This holistic approach to medicine is a collaborative skill trading environment demonstrating best care practices.

Education: The preventative health facility is also meant to educate. Health begins at an early age, and it is anticipated that the wellness centre would offer nutritional educational services to all users of all age. Cooking classes would educate the community on healthy foodstuffs and preparation techniques. An elementary school connection would allow for a thriving community rooftop garden that would sell goods within the community 
market café. As well, education would be provided through a digital resource database accessible throughout the building and would allow access to e-health records, prescriptions and fitness test results.

Fitness: Physical exercise would be encouraged by physicians and specialists within the wellness centre. A cardiovascular/weight room would serve the community for their fitness needs. A fitness studio would offer programs and classes such as yoga, tai chi and dance. An important portion to the fitness aspect would be the recreational swim component. Swim classes would occur in a $50 \mathrm{~m}$ short course community pool.

Healing: The final programmatic component to the facility would be the healing component. A truly holistic definition to wellness would see healing as important. This will be manifested in a spa component within the facility. The spa would consist of a number of public baths of differing temperature to aid in the exfoliation process. As well, multiple landscaped gardens are aimed at reducing environmental stressors. Primary users would include those who have undergone surgery and need remediation from stress. Also the elderly and adult population within the community would benefit from the serene setting of the spa component.

\section{Main objectives}

Main objectives in the design and vision of this thesis|project that will be discussed in the following design sections include:

Community Health: To create an urban oasis that offers the community a healing environment in an otherwise unhealthy and undesirable site

Context: Make a continuous natural landscape along the rail corridor, connecting the Don River Park in the East to the Cherry Street /Distillery Districts in the West

Design Language: Maximize the connection between architecture and the natural environment; the 'architecture of porosity' will be used as a tool for facilitating environmental healing 


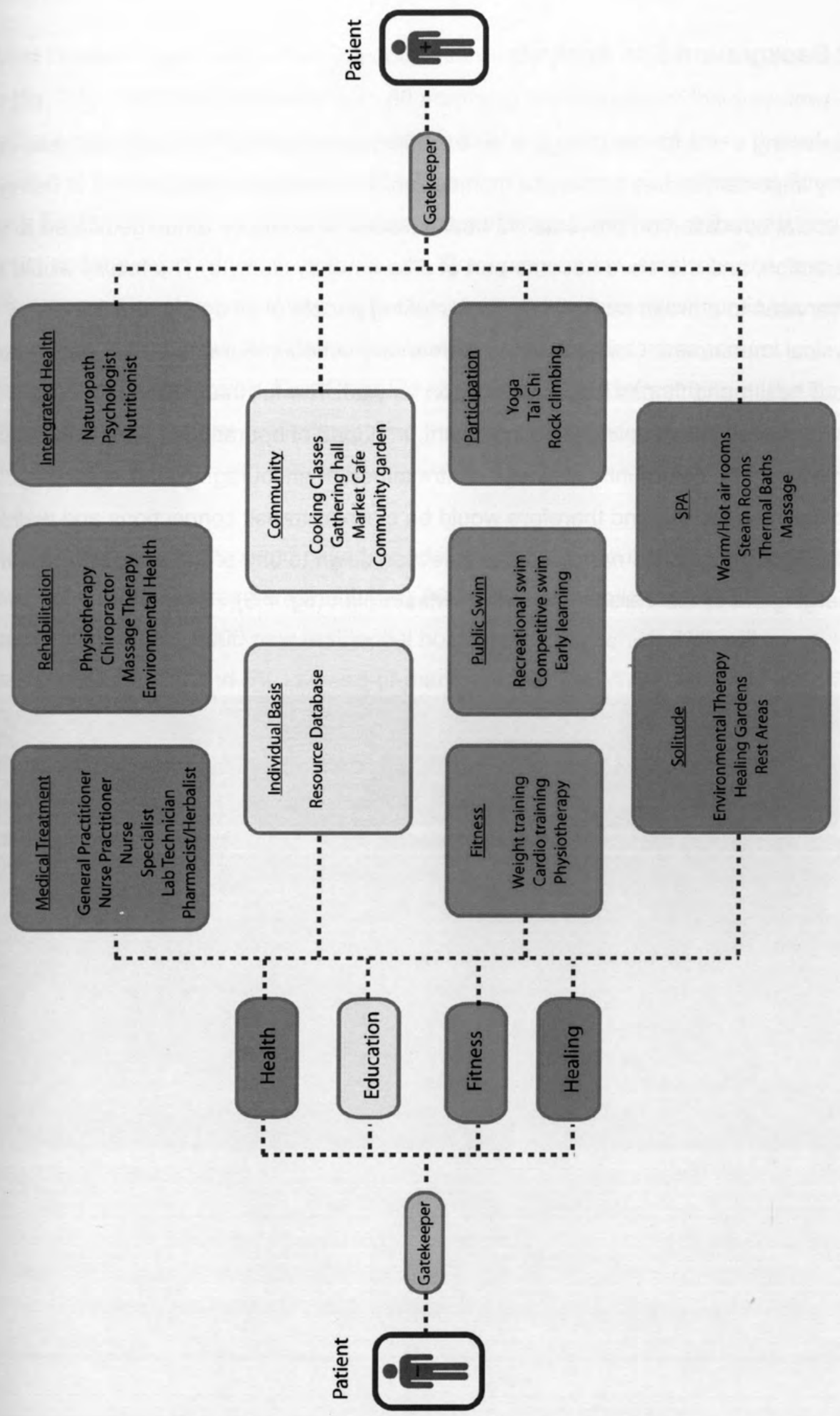

Figure 7.4 Wellness Centre: Organizational Service Structure 


\subsection{Background Site Analysis}

In selecting a site for the design of an experimental preventative health/wellness centre, many important issues presented themselves. The wellness centre aimed at being a tool for social education on preventative health issues. It would be a hub dedicated to social interaction, and therefore become part of a community lifestyle. The facility would extend its services to a broad range of users, including people of all ages, ethnicities and physical impairment. Connections to elementary school programs for the early education about health and fitness issues were seen as vital. The full integration of nature throughout all seasons was also important. In hopes of operating in a truly sustainable framework, the community wellness centre aimed at encouraging walking and cycling to and from the facility, and therefore would be close to transit connections and walking trails. These requisites narrowed site selection down to one of the several communities emerging out of the Toronto Waterfront Masterplan (fig. 7.5).

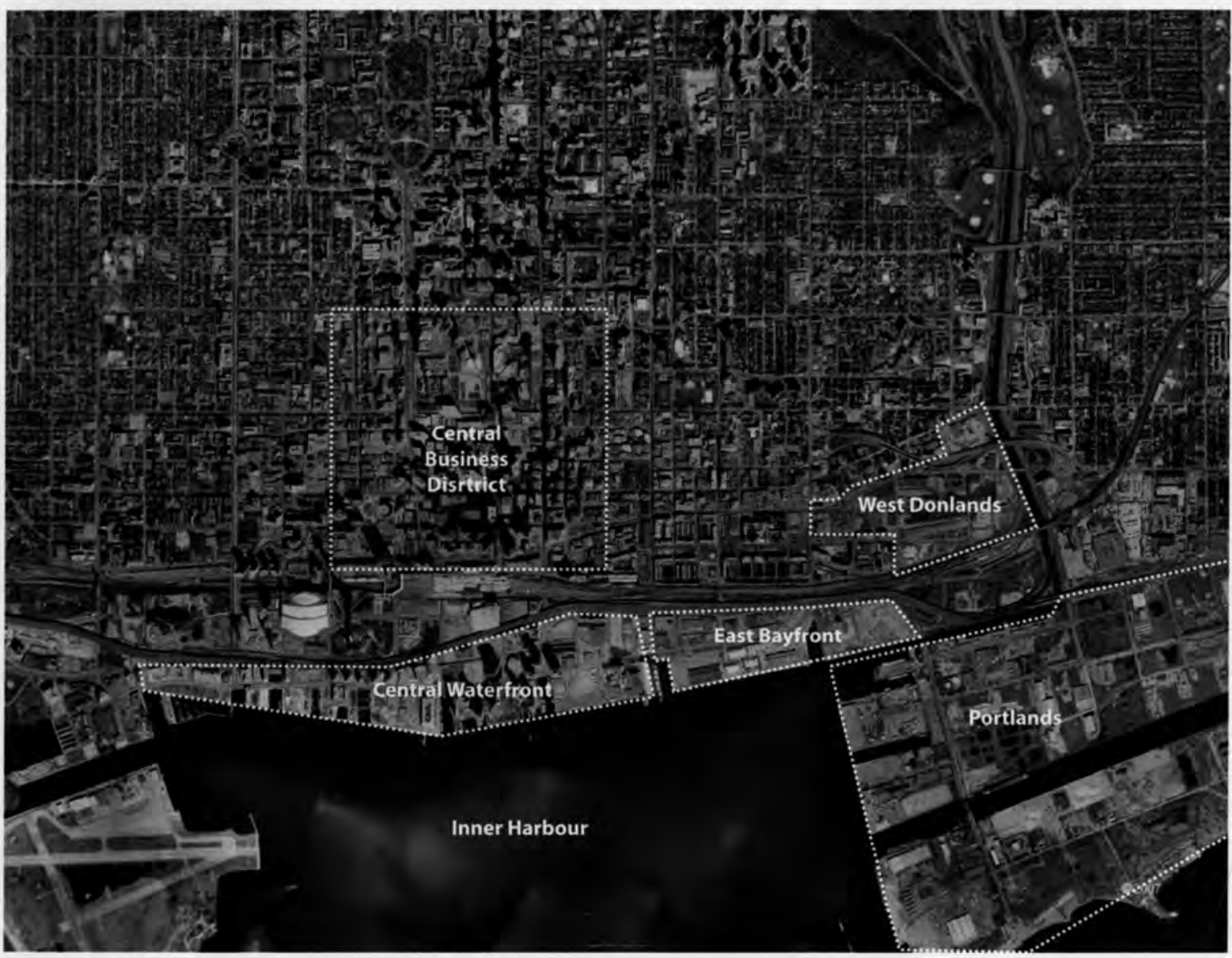

Figure 7.5 Toronto Waterfront: Communities Masterplan 
The West Donlands was selected as a model community for the experimental wellness centre (fig. 7.6). The West Donlands is an 80 acre area located east of the downtown core, adjacent to the historical Distillery District and has good connections to the existing Corktown and St.Lawrence neighbourhoods. The site is bounded by Parliament Street in the west, Eastern Avenue and King Street on the north, the Don River in the east and Mill Street and the rail corridor along the south. Previously an industrial site, the land is currently vacant. Construction of basic infrastructures and land revitalization is currently underway. This includes building demolition, soil and groundwater management systems, CN bridge expansion, Don River Flood Protection and infrastructure, the Don River Park, Bayview Avenue realignment and the River street extension (Appendix 7 and 8).

The West Donlands is a proposed mixed use development that will house a forecasted population of 11000 new residents, including 860 school age children (fig. 7.7). The development will create 6000 new residential housing units (of which $20 \%$ will be affordable housing units and $5 \%$ low-end-of-market units). The West Donlands will offer

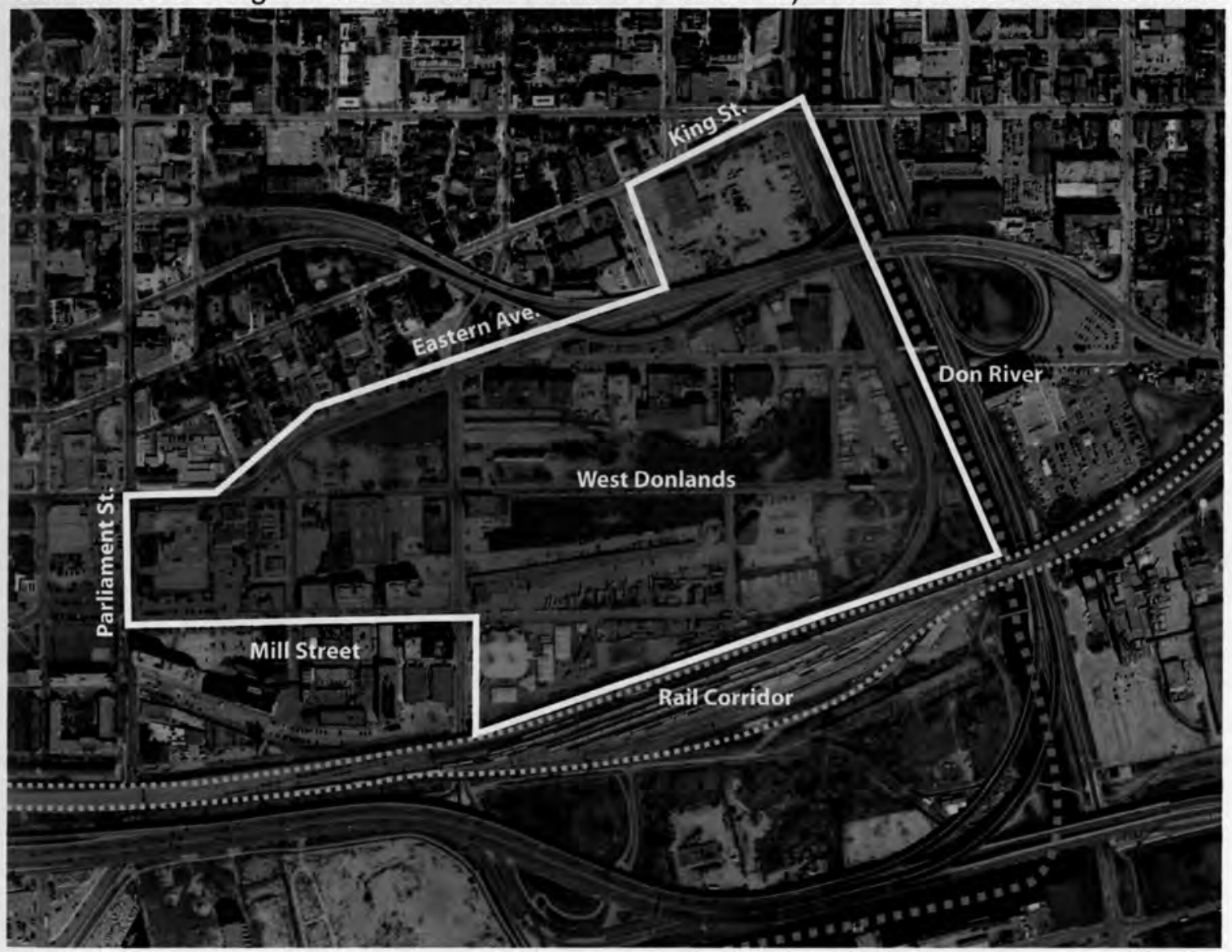

Figure 7.6 West Donlands Bounding Conditions 


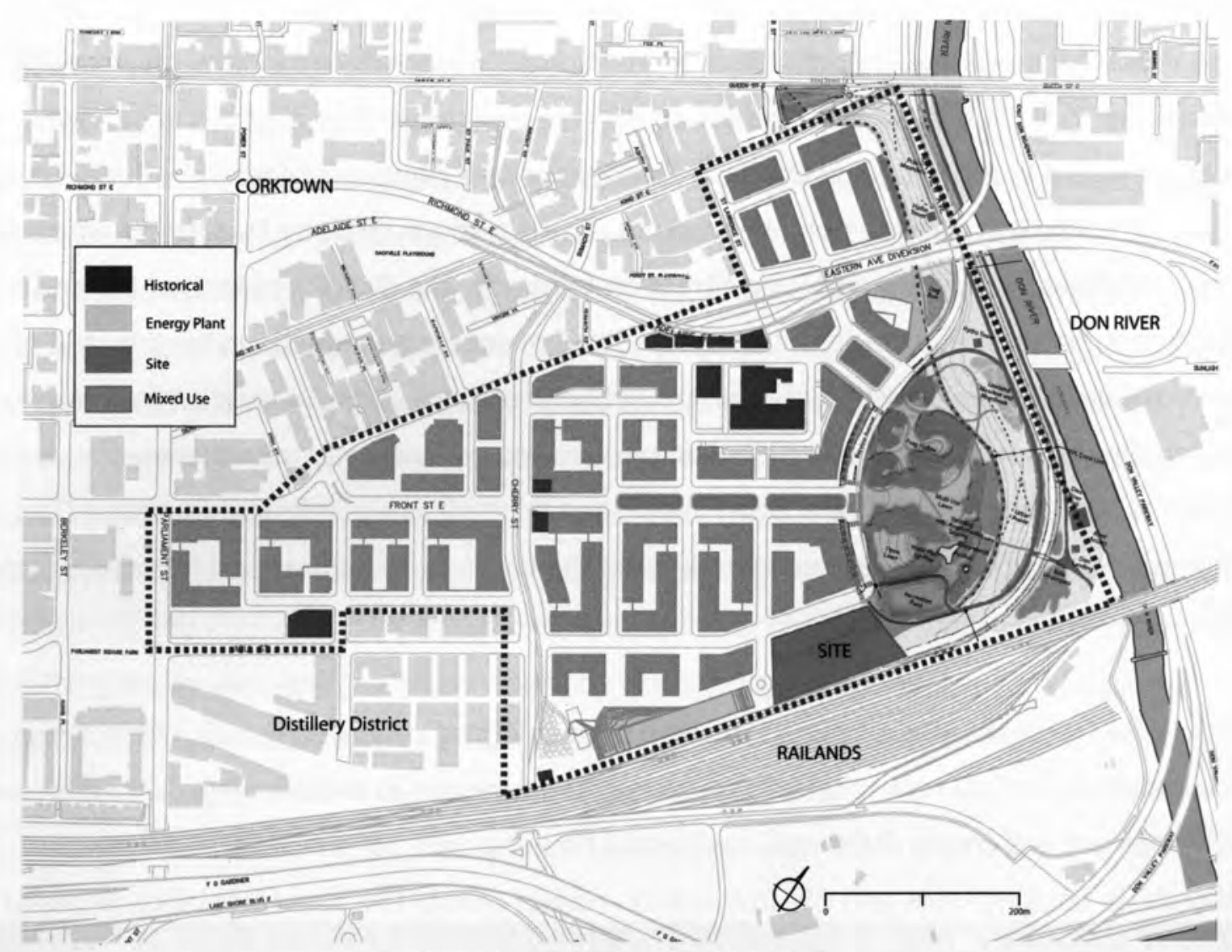

Figure 7.7 West Donlands Masterplan

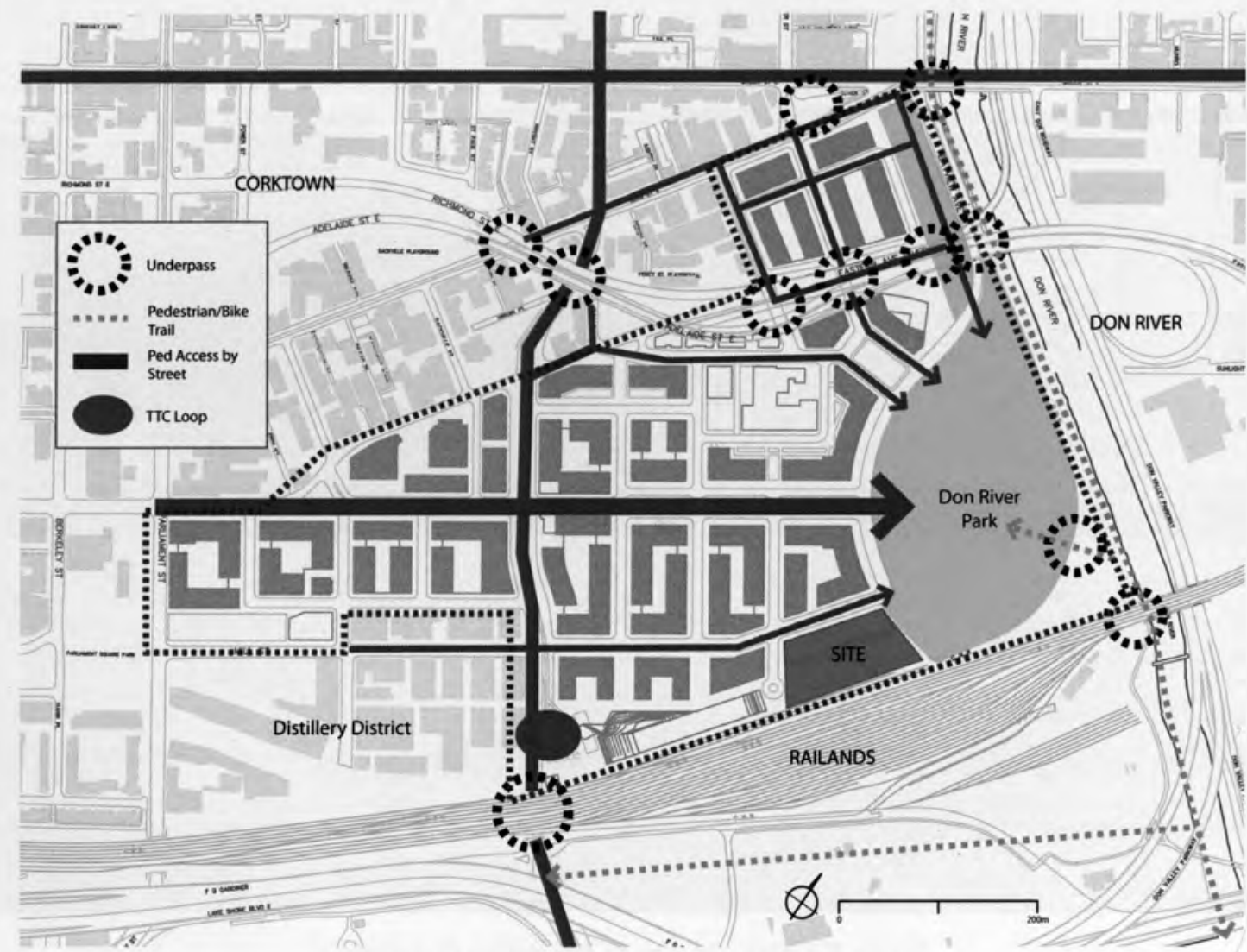

Figure 7.8 West Donlands Site Circulations 

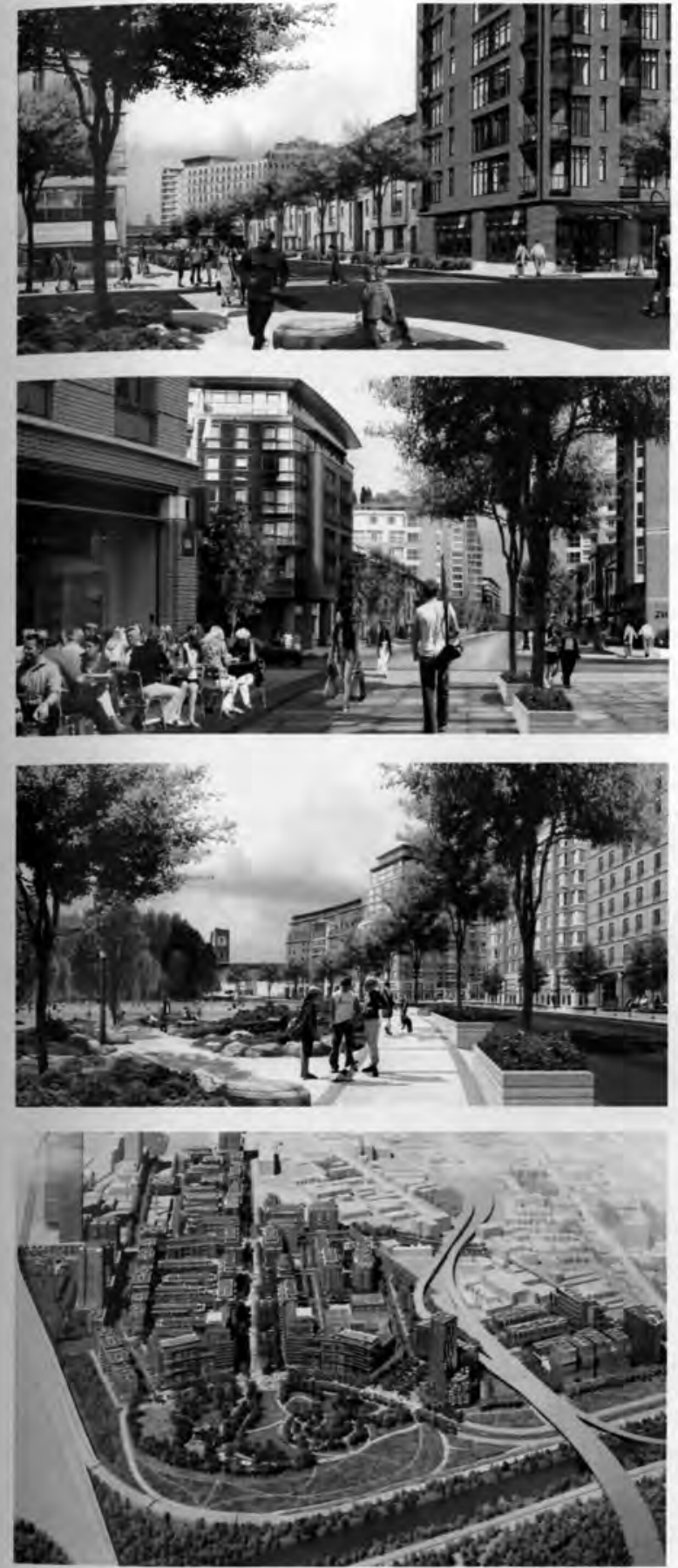

Figure 7.9 West Donlands Community Image Credits: Waterfront Toronto
750 000sf of available commercial space, and one millionsf of employment space. The development has proposed an elementary school and a recreational community facility on block 09 of the masterplan (which has been chosen as the site for this thesis|project). The development will also be serviced by a state of the art district energy heating and cooling plant. The community will contain 23 acres of public parks and spaces including an 18 acre Don River Park proposal by Michael Van Valkenburgh and Associates (Appendix 9). The Don River Park will serve as the major terminus of the eastern pedestrian access from the downtown core (fig. 7.8). The development aims to become a sustainable community that promotes pedestrian and cycling connections with adjacent neighbourhoods and the rest of the city (fig. 7.9). Public transit is located within a 5 minute radius of all residences, with a major streetcar connection at the Distillery District and Cherry Street intersection.

The West Donlands proposal serves as a model community in the downtown core

that would benefit from a community health/wellness centre. The design proposition will attempt to work with the proposed programming for the recreational community centre on block 09 of the masterplan. The design proposal will create a community hub that promotes a healthy lifestyle for its inhabitants, one that places importance on physical fitness, health education, sickness prevention as well as community healing. 


\subsection{Contextual Linkages}

The design exploration of this thesis|project attempts to work with the proposed zoning for block 09 of the West Donlands community masterplan. Currently, this site includes both the development of an elementary school of 500 pupils, as well as a community recreational facility. The end goal of this design thesis|project is to offer community users in the West Donlands specific services that would promote a healthy lifestyle and foster preventative health. In order to achieve this success, it is important to integrate the Wellness Centre into the fabric of the proposed masterplan. In order for a system to successfully operate as a balanced whole, it must be approached as an interconnected set of elements working together, rather than a system of isolated parts (Capra, 1996). His vision embodies the notion of sustainability, and in this case would result in a fully integrated and balanced community. It is therefore important to analyze the linkages that the Wellness Centre could have with the community at large.

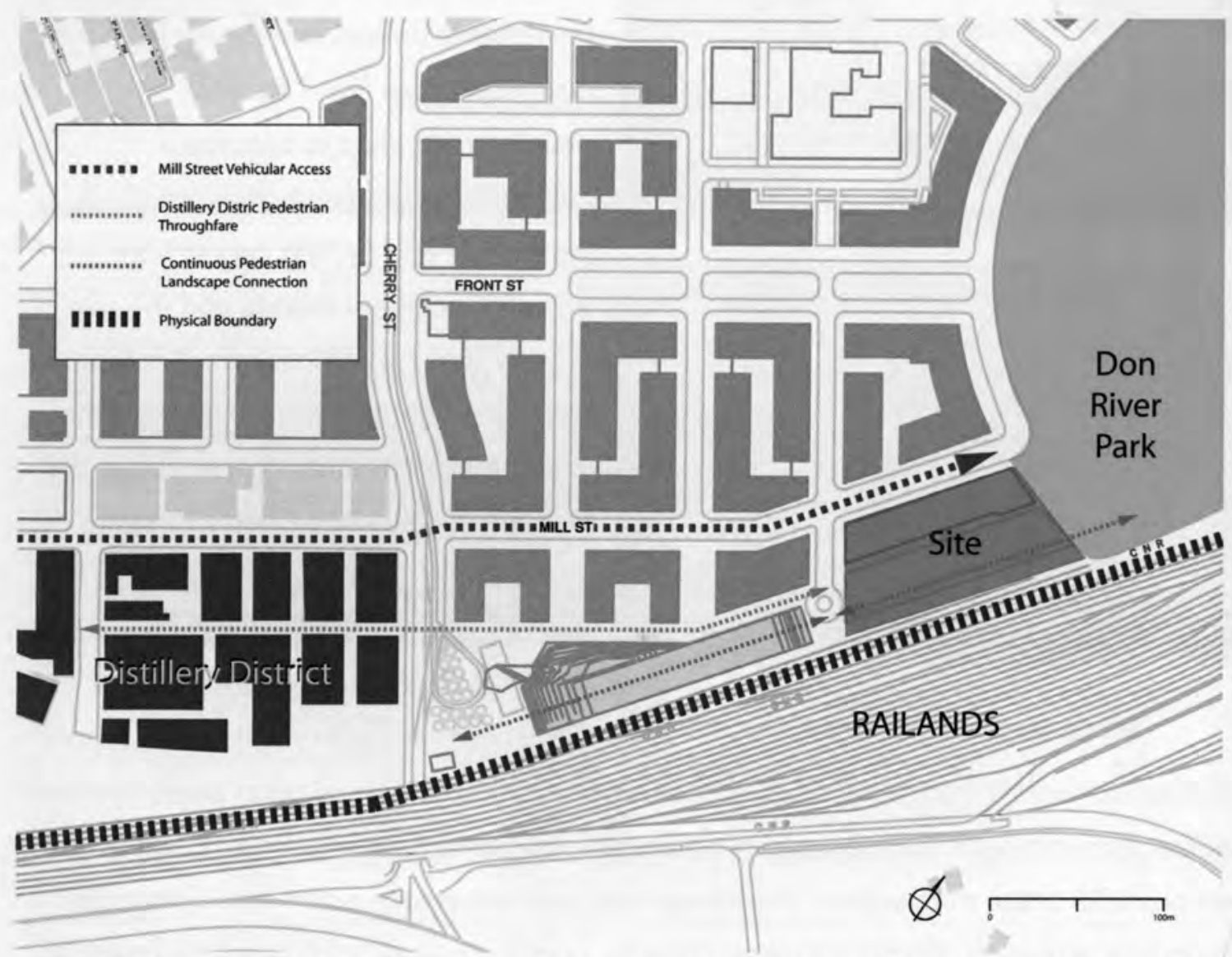

Figure 7.10 The Proposed Wellness Centre Site attempts to make critical linkages with its adjacent and neighbouring context 
The proposed site is located on the southeastern edge of the West Donlands masterplan, directly adjacent to the Don River Park proposal at Mill Street. It is also
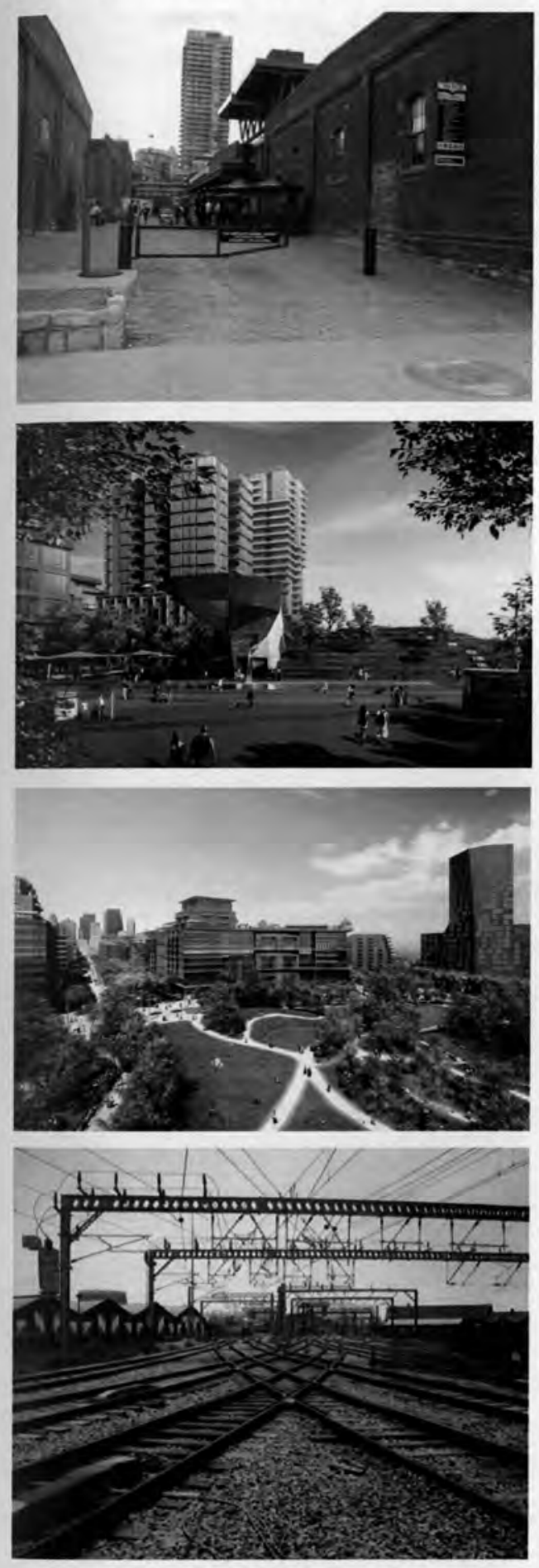

Figure 7.11 Critical Site Linkages: the Distillery District, the Energy plant, the Don River Park and the CN Railway Image Credits: Waterfront Toronto; Metrolinx adjacent to the District Energy Heating/Cooling plant proposed by Steven Holl architects (Appendix 10). Both the site and its adjacent properties all share the same physical boundary and hard edge of the southern Railands. The grain of this site truly runs along the east-west direction, alongside both the Railands and Mill Street. Figure 7.10 indicates how successful connections and linkages begin to emerge. The vehicular circulation of Mill Street flows in this direction, and terminates at the Don River Park. As well, pedestrian circulation originating from the Distillery District in the west encounters the proposed Wellness Centre site in the east via a through fare. If the Wellness Centre is proposed to be a community hub for healthy social interaction, it must capitalize on this circulation flow and bring this energy into the facility (fig. 7.11).

One major pattern that begins to emerge is the natural landscape connection between the District Energy plant and the Don River Park. The Don River Park scheme is a successful example of intensive native flora planting for the purposes of flood plain protection along the Don River. Similarly, the Steven Holl proposal attempts to educate individuals about sustainable technologies through the inclusion of an intensive green roof system. This roof is meant to be a pedestrian friendly series of platforms that brings you up from grade, to a 
height that clears the viewing plane over a railway crash wall. Integrating nature for the purposes of health into the design of the Wellness Centre is a primary objective in this thesis|project. Taking advantage of the opportunity that presents itself (by creating a continuous landscape corridor along the Railand edge) would be conducive to good neighbourhood building. Figure 7.12 shows a section through the proposed site and west Donlands Masterplan demonstrating all critical linkages attempting to be integrated with the community at large. Importance is placed on the naturalized pedestrian connection that occurs from the Distillery district in the West, to the Don River Park in the East.

With respect to the major objectives of this thesis|project, an 'oasis' of health is meant to be created for the community of the West Donlands. Placing a Wellness Centre adjacent to a railway seems counter intuitive, especially regarding negative environmental impacts. From the planning department's perspective, placing a community centre, elementary school and energy plant adjacent to a railway is a better use of the land than placing housing next to a rail line. Residences require almost $24 \times 7$ periods of quiet, whereas community centres operate for more limited time periods. Nevertheless, even a community centre requires significant mediation of railway disturbances; this thesis|project will address that fact. In meetings with the Director of Rail Infrastructure at GO transit Daryl Barnett, train derailment, in ground vibration and airbourne noise were deemed to create the greatest negative impacts on health for a community abutting a rail line (D. Barnett, personal communication, May 17, 2009). Train derailment is the most detrimental to health and safety. CN Rail predicates that a building setback of 30 metres typically be implemented to avoid damage to above grade structures. If the scope of the project demands the setback to be varied, a berm or crash wall needs to be put into place. With respect to in ground vibration, lead or rubber isolation pads typically line footings to prevent any below grade disturbances. In meetings with a Professional Engineer specializing in acoustics, it was found that depending on the mass of the building itself, a layer of insulating foam on the exterior foundation face may be sufficient (R. Ramakrishnan, personal communication, May 18, 2009). Finally, the issue of airbourne noise is typically mediated by installing a crash wall to redirect the noise. Alternatively, vegetative planting may help reduce airborne noise even further. Appendix 11 indicates the final strategies of design for railway mediation in this thesis|project. 


\subsection{Design Language and Principles}

One of the major objectives in this design thesis|project includes maximizing the connection between architecture and the natural environment. Bringing the external environment into our buildings can take advantage of nature's beneficial and healthy properties. This has been demonstrated through the wide body of analytical research involving healing through nature, as presented in the preceding chapters. Using nature in building design reduces environmental stressors and levels of depression amongst individuals, fosters quicker recovery times for patients, and improves the overall

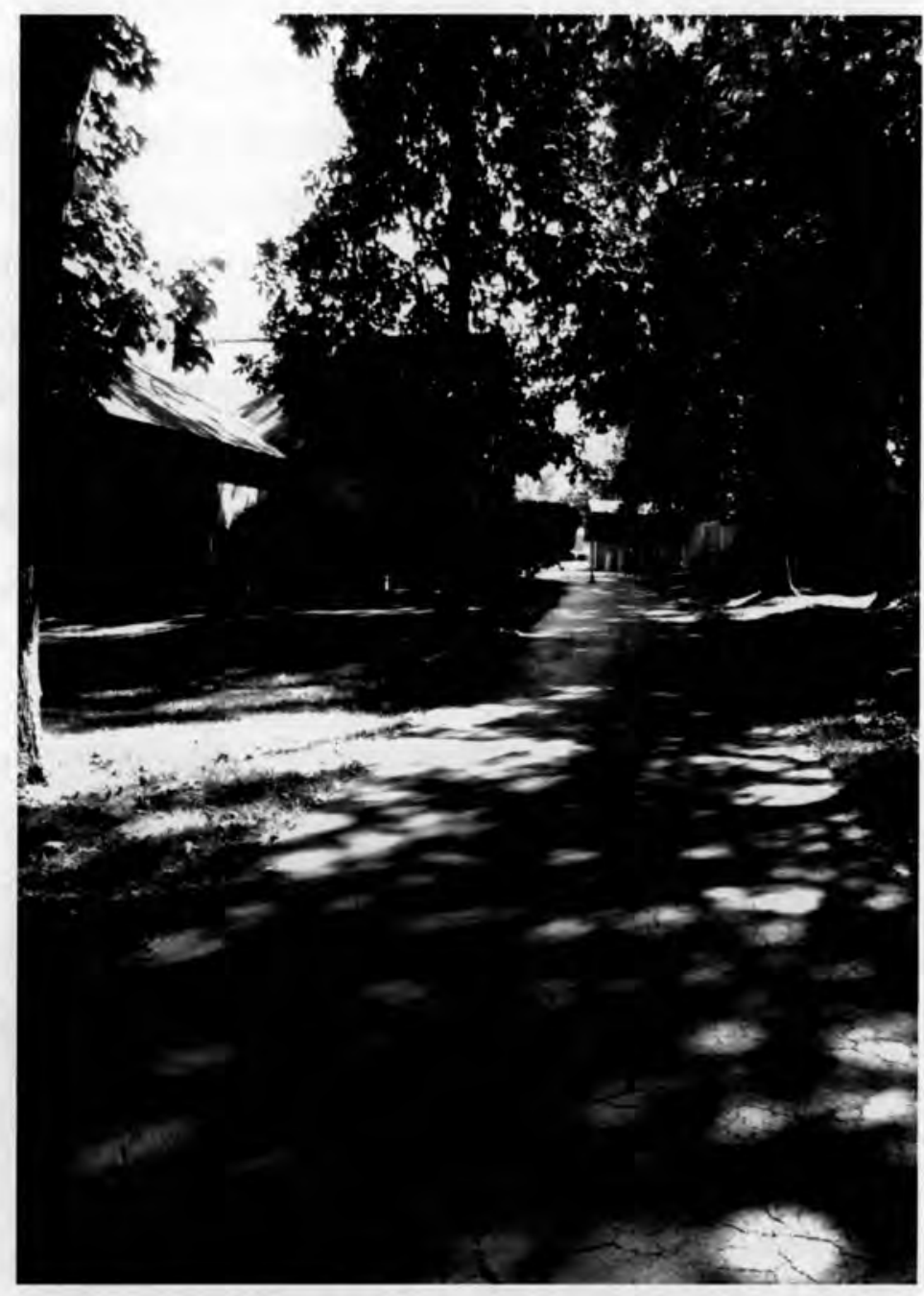

Figure 7.13 Forest canopy filtering light and shadow Image credit: Eikongraphia

psychological status of users. Finding a design language that would successfully engage the relationship between the internal and external environment would become vital to the architectural expression of this design project.

The idea of 'porosity' existing in naturally occurring phenomena became enticing during the design process. Examples were taken from the experience of walking through a forest, noticing the interaction between two frames of reference-both inside and outside of the canopy. The phenomenon of the sun's rays penetrating the dense foliage of the tree canopy was given abstract phenomenological meaning (fig. 7.13). This filtering allows a formidable environment for life to exist on the forest floor, while sustaining the 
life giving canopy itself. The fact that nature tends to be porous for the betterment of all species is important. The meaning embedded in this example can be extrapolated and "translated" into a design tool. The relationship between the inside and outside environment for reasons of occupant health can be approached through the notion of porous building design. The 'architecture of porosity' was thus adopted as a design tool that would facilitate environmental healing.

However, in the modern urban condition, the lush canopies of a natural forest do not present themselves in such a utopian manner. Many of our urban landscapes today are dominated by a sense of manufactured artificiality. Paved city surfaces, transportation corridors and a sea of ever present shadow are part of our modern industrial landscapes (fig.7.14). This is very present in the history of Toronto's waterfront. The Toronto rail

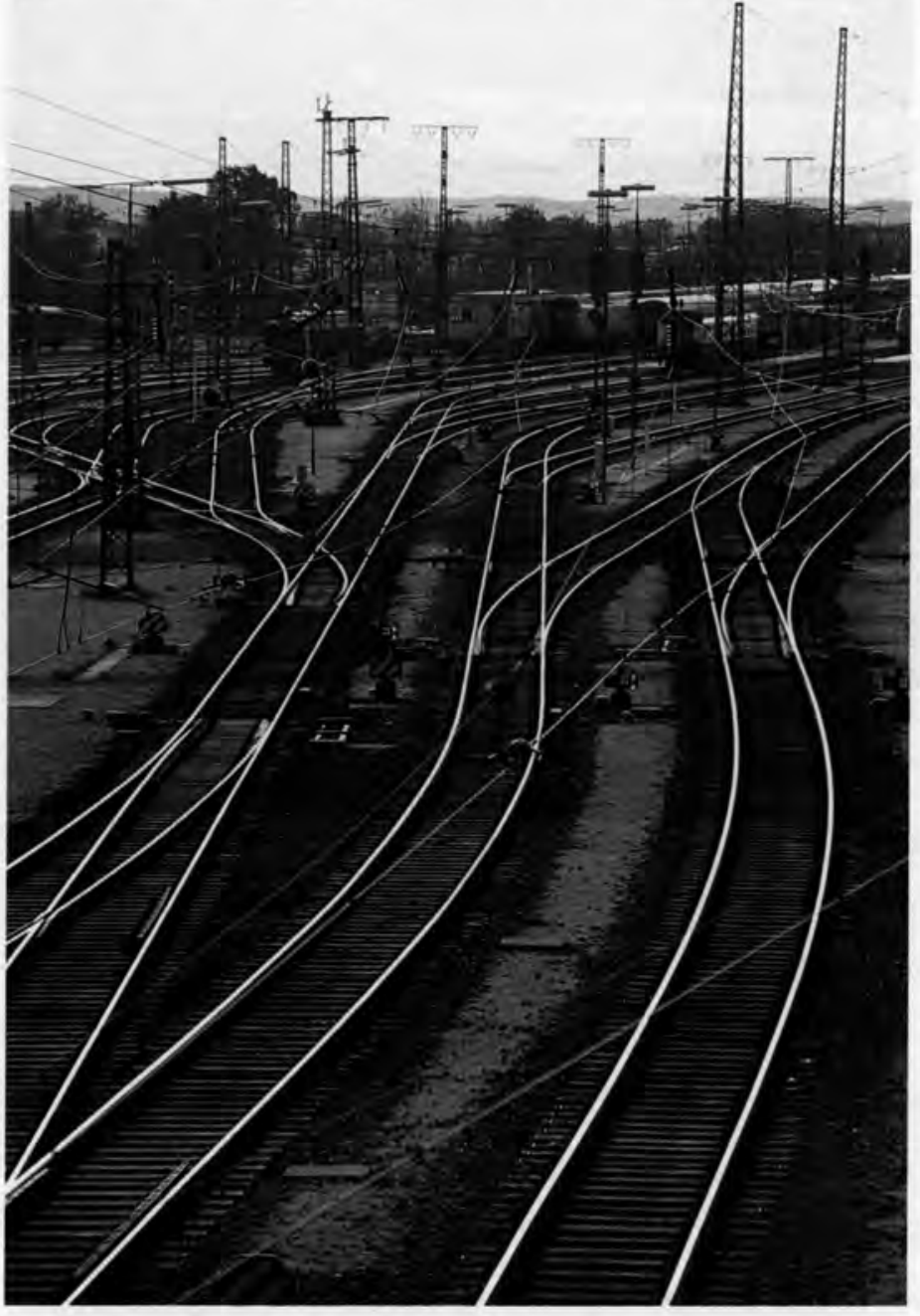

Figure 7.14 Railand corridors demonstrate the manufactured nature of our modern industrial landscapes Image Credit: ComStock corridor has been a reminder of over a century's worth of industrial transport. Much criticism had been directed towards its presence, cutting through some of the city's most important avenues. These railway corridors are by no means social spaces of healthy congregation, and are meant to be strictly utilitarian in purpose. The negative stigma associated with these industrial and dangerous places has become part of our urban repertoire. The proposal of a Wellness Centre at the West Donlands site must take these urban conditions into consideration. Creating an 'oasis of health' adjacent to a rail corridor demands that a sensitive approach be taken. 
When these railands were observed in closer detail, natural beauty was present. A series of photographic images taken along the Mimico rail lands documented emergent native flora (fig. 7.15). Historically, linear strips of nature along rail edges were trimmed back so as not to interrupt with rail traffic (a.). Along derelict portions of these tracks, tall grasses and shrubs began reclaiming important growing beds (b), as well as finding select opportunities to bud flowering plants (c). Specific cases demonstrated how the native flora engulfed entire swaths of rail tracks $(d)$. The majority of this natural landscaping occurs along railway setbacks, allowing opportunity for various grasses and sedum to grow. From a more personal level of perception, these naturally occurring native landscapes relieves the stigma involved with industrial railways. The design of a proposed Wellness Centre would benefit from making reference to these instances of natural landscaping. The idea that true architecture emerges from the qualities of its site and surroundings furthermore presents itself in this case.

a.

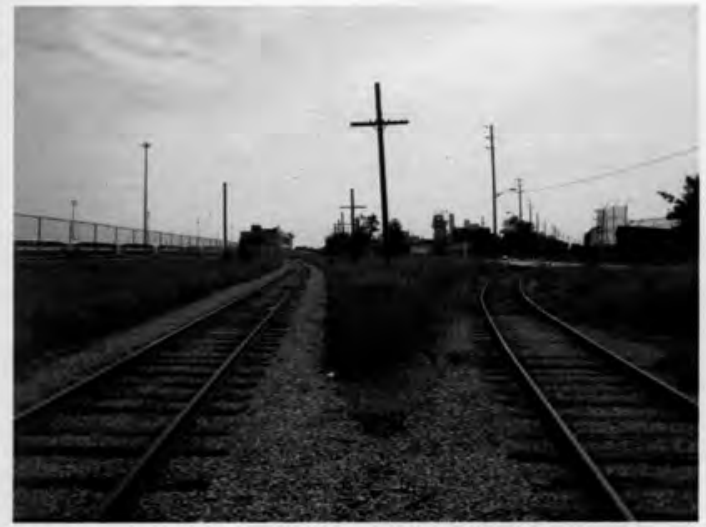

C.

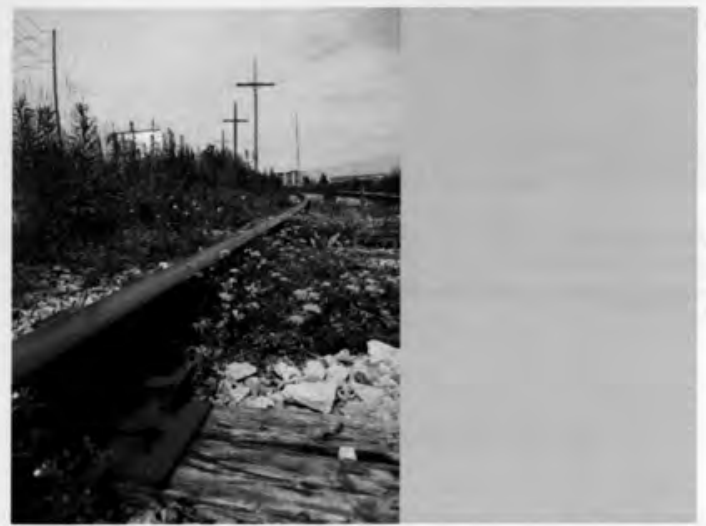

b.

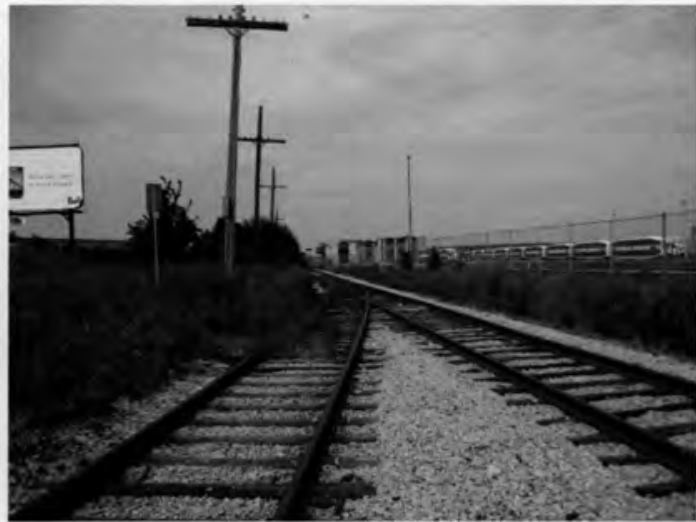

d.

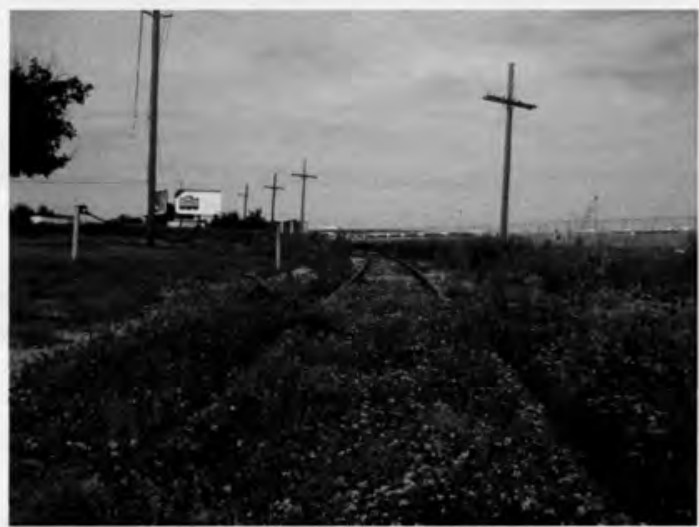

Figure 7.15 the emergence of the natural in the unnatural A photographic series taken along the Mimico rail lands illustrates the ability for nature to reclaim the industrial and artificial sense of these urban landscapes 
The site in question indicates that a natural east west flow of circulation occurs (figure 7.10). This grain is further reinforced by the linearity of the adjacent railway corridor. When approaching preliminary design exercises, the linear geometry of the rail lines were seen for their beauty and potential use as a generator of form (fig. 7.16). Form would also be guided through the importance of porosity, and thus a design language was struck from the balance of the two. This linear approach to building porosity would dictate the final design of the Wellness Centre. Figure 7.17 indicates the process that was taken to achieve a successful relationship between the internal and external environment for reasons of occupant health. This process manifested itself through intensive physical modeling exercises to express more tangible results related to materiality and sense of space (appendix $12 \& 13$ ).
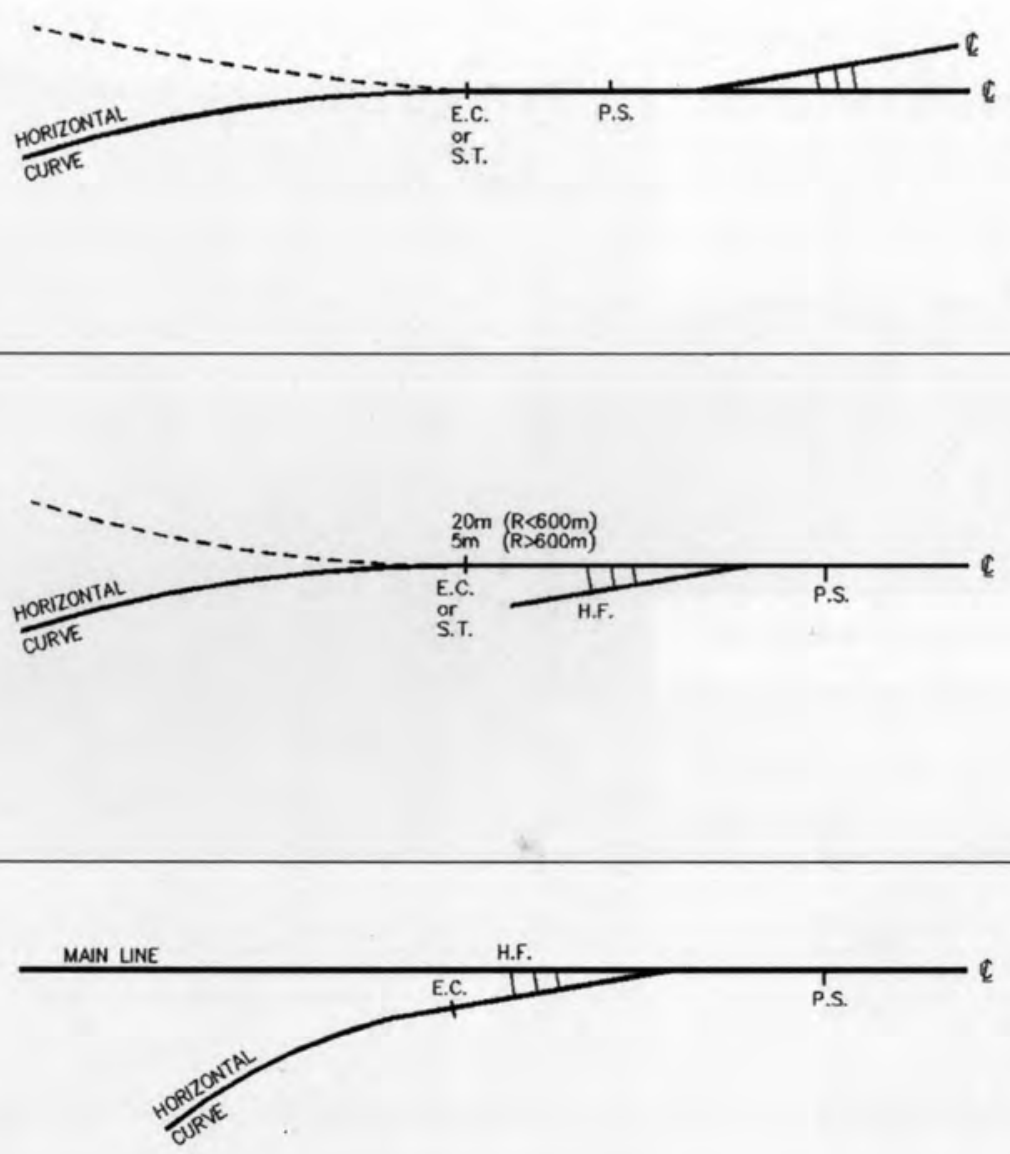

Figure 7.16 The linear geometry of rail lines were seen for their beauty and potential use as generators of form Image Credit: GO Transit 


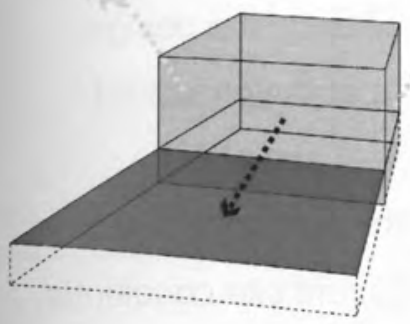

A.

1. common disjunct $w /$ site interplay (internal v.s external environment)

2. this modern approach offers minimal views to nature

3. natural daylight design is minimzed (regarding potential subterranean levels; also creates 'deep plan' building condition)

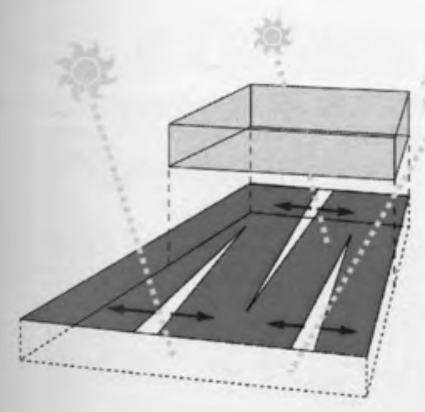

C.

1. .... and pulled apart in the horizontal

2. daylight allowed to penetrate subterranean levels

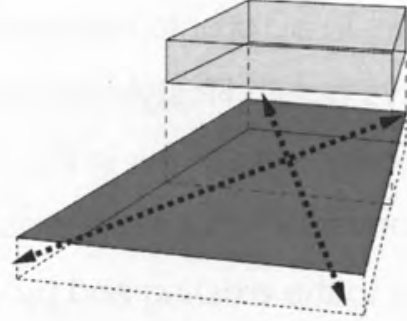

B.

1. experimentation sees potential from extracting building mass from ground surface

2. ground plane is now free and maximized in size

3. Opportunity for better interior and exteriror program integration

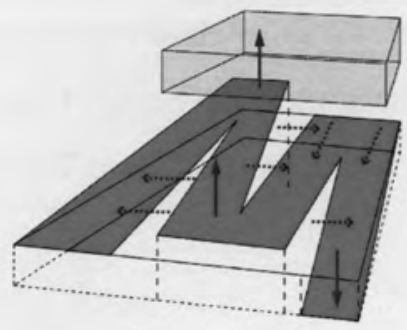

D.

1. Furthermore, ground plane is pushed and pulled in the vertical

2. maximised views to nature at all levels (due to program integration into new planar surfaces)

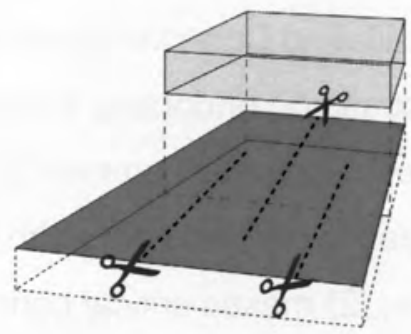

C.

1. experimentation with ground plane being sliced...

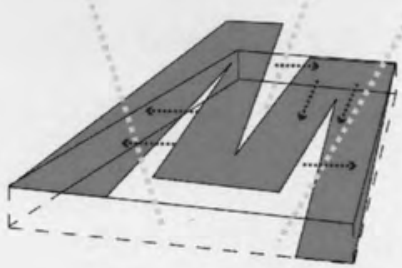

$E$.

1. Typical building mass is now therefore unecessary

2. The Landscape building is created, and allows for an extension of its natural surroundings

Figure 7.17 The idea of 'porosity in architecture' was executed during the design experimentation process; the linear geometry of rail lines were used as a tool to generate the final level of sliced porosity; this process emphasized the relationship between the internal and external environment 


\subsection{The West Donlands Wellness Centre: Experiencing Community Health}

The design experimentation of this thesis|project provides a vision that aims at facilitating Canadian health services to a Toronto community. Specifically, the design focuses on endorsing a preventative health lifestyle for the members of the proposed West Donlands community. The design objectives in this thesis|project aim at (1) creating an oasis of health for a community in an otherwise unhealthy and undesirable site, (2) makes critical connections to the existing and proposed adjacent site conditions, and (3) maximizes the connection between architecture and the natural environment through the design language of 'porosity.' In final, this thesis|project takes a critical position on the design of a future sustainable community: by integrating nature's healthy attributes into the design of our built environment, we can foster community wellness in both a holistic and preventative manner. This in turn reduces our dependency on corrective health approaches and promotes a lifestyle that ultimately allows individuals to control their own levels of health in a sustainable manner.

The following images illustrate the final outcome of design experimentation in this thesis|project. Figure 7.18 demonstrates the frontage of the Wellness Centre at Mill Street and the Don river Park, in the West Donlands community. Figures 7.19 -7.26 illustrate how the linear nature of the emerging site circulations and existing adjacent conditions allowed for the sites architecture to occur. Figures 7.27-7.35 demonstrate specific atmospheres of experience that users of the Wellness Centre will become a part of. These architectural experiences came from the unfolding research into the architecture of health in this thesis|project, a research base that emphasized the importance that light, nature, materiality and overall phenomenological experiences can play in the role of designing places that heal. In final, figures $7.36-7.38$ illustrate how the Wellness Centre's programmatic functioning links both the internal and external environment into its sectional fabric. 


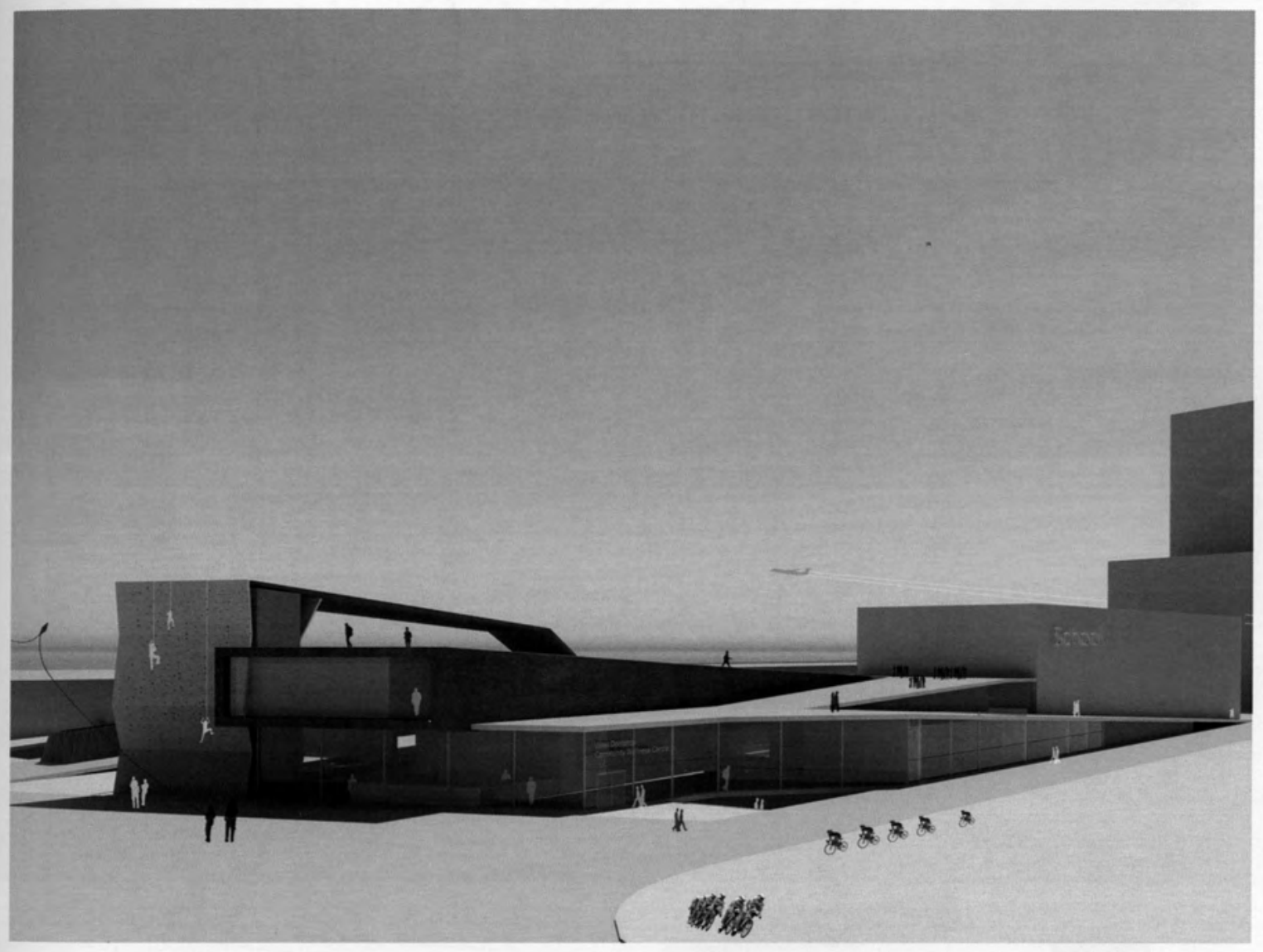

Figure 7.18 The West Donlands Wellness Centre: View from Don River park

The Wellness Centres pedestrian and green roofscape emerges from the Don River Park, making it an extension of its surroundings. The defining materiality of the buildings varying volumes makes reference to the industrial character of its abutment to the railands. 
Figure 7.19 Critical site circulations;

Figure 7.20 Site Deriving Lines
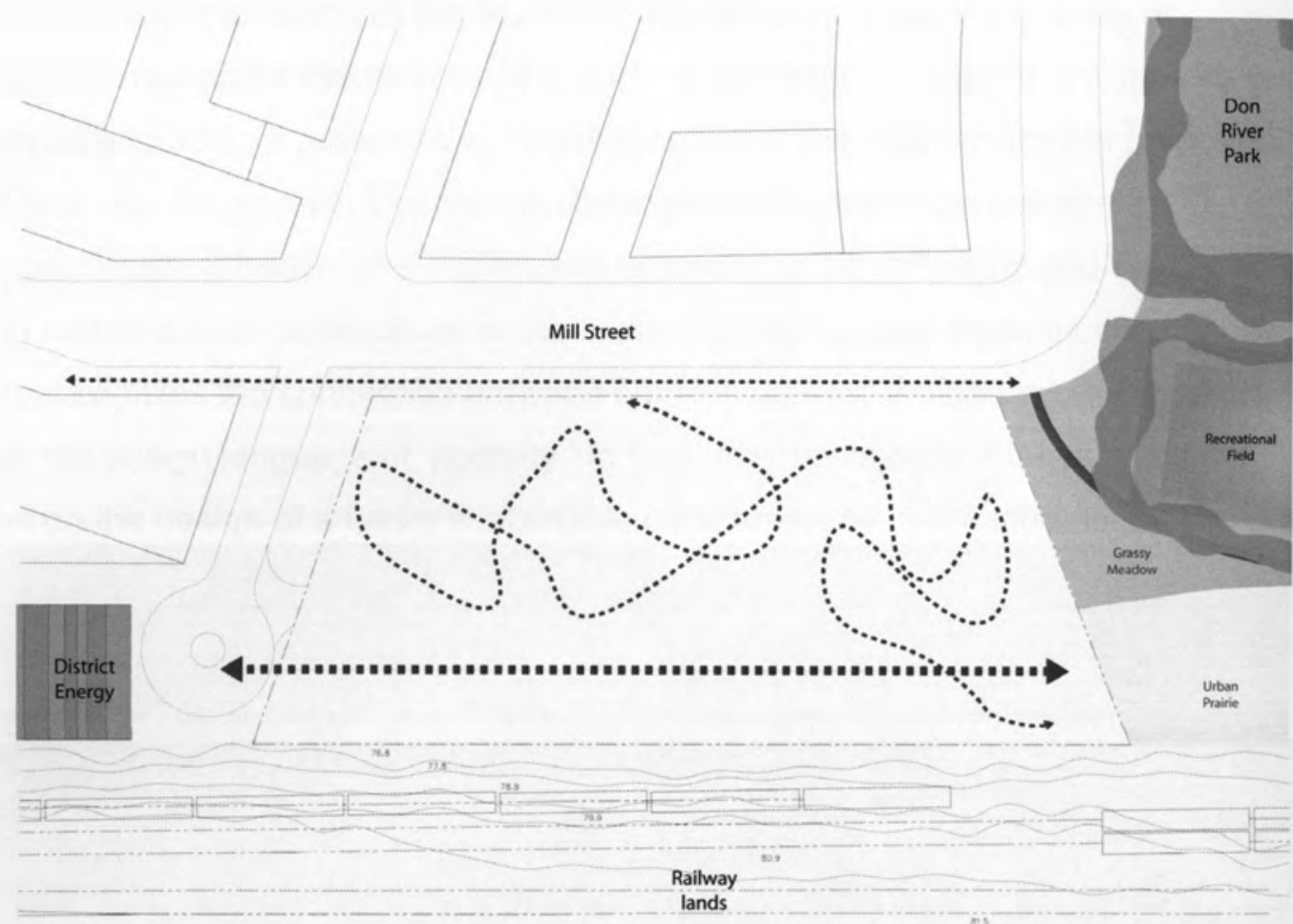

Critical Circulations
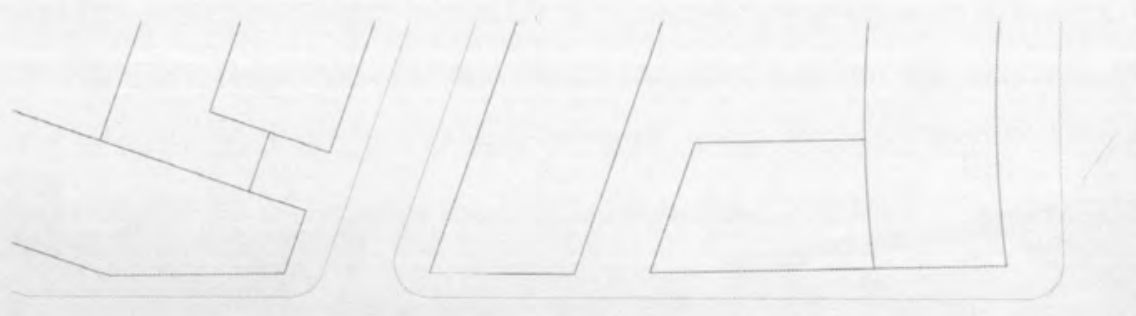

Mill Street
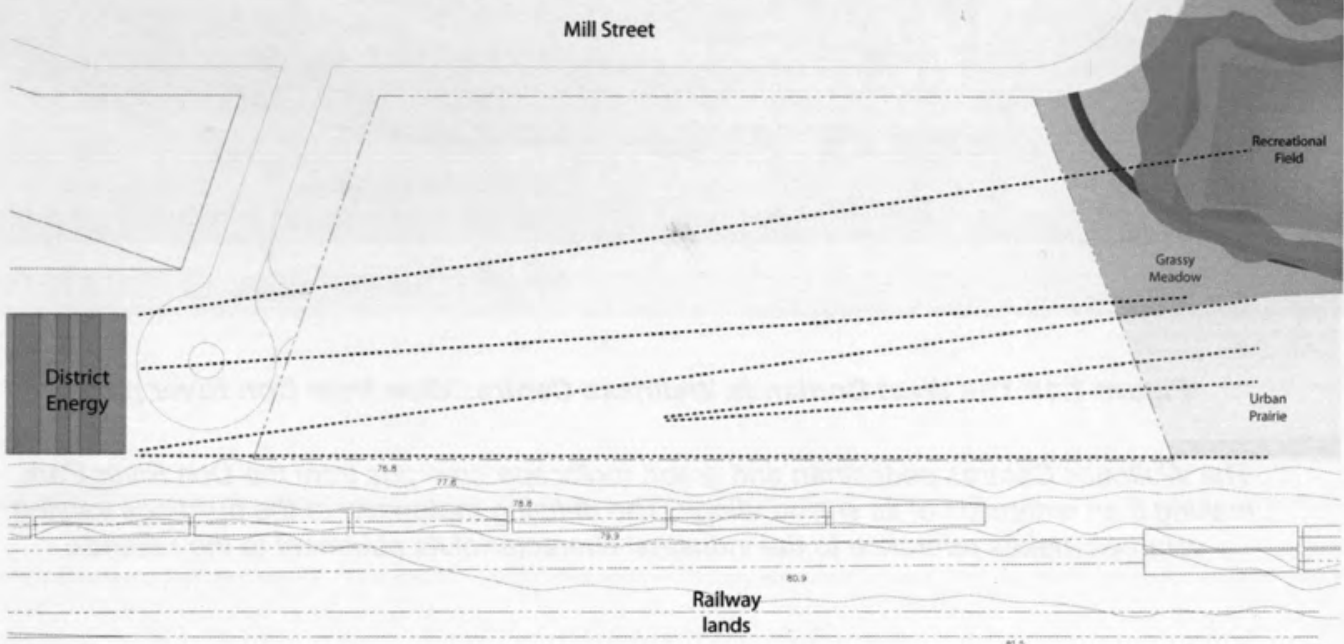
Figure 7.21 Final site circulations;

Figure 7.22 Porous landscape integration
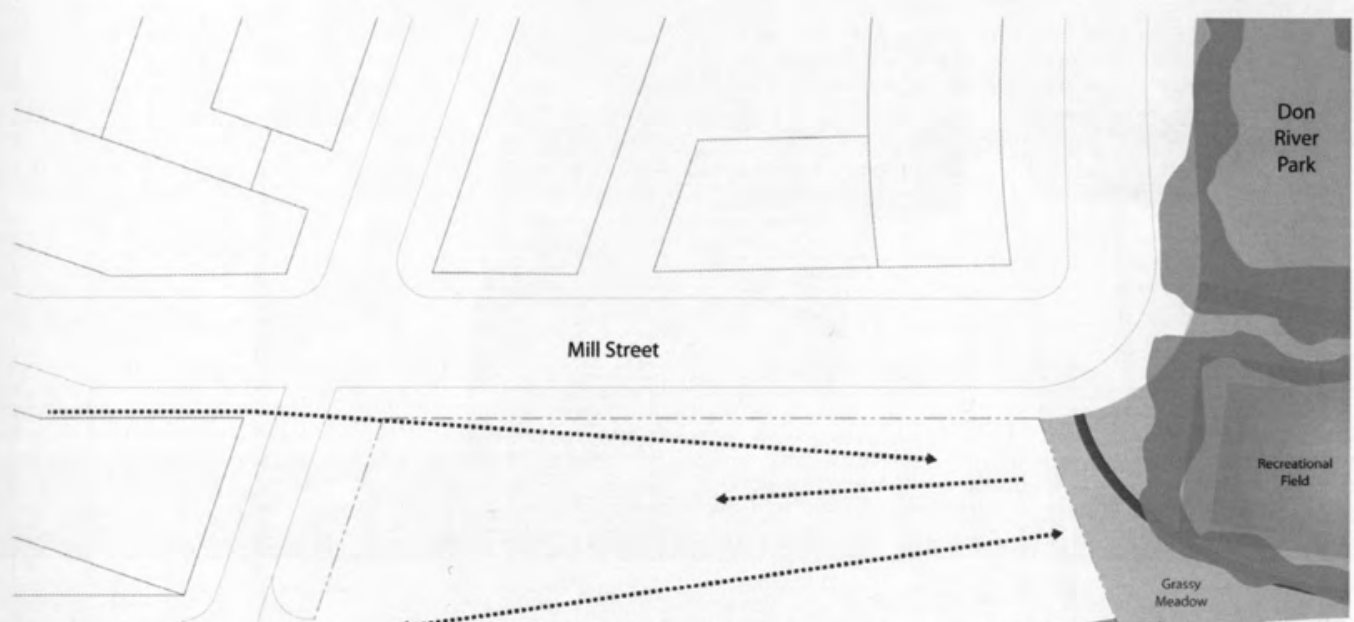

Mill Street

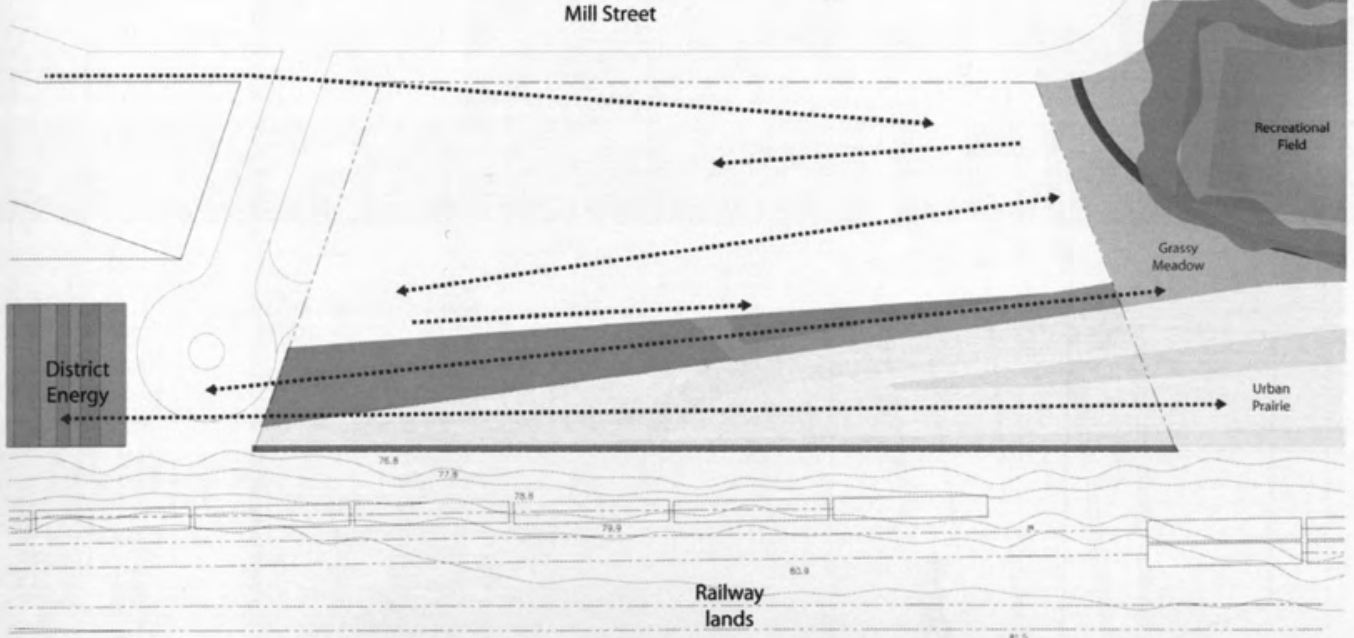

$\infty$ Final Circulations

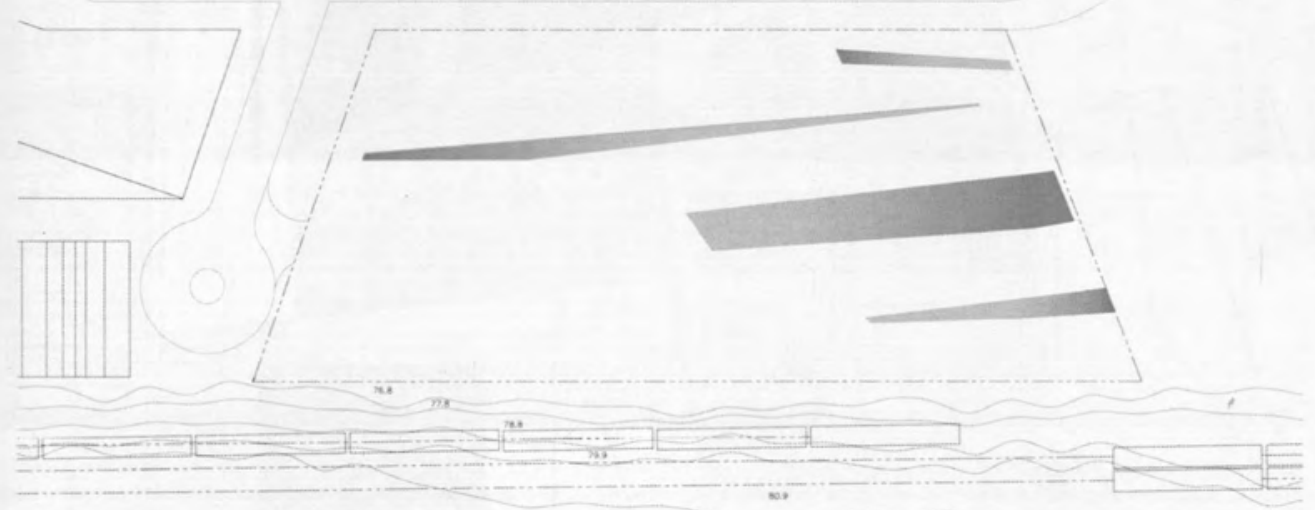



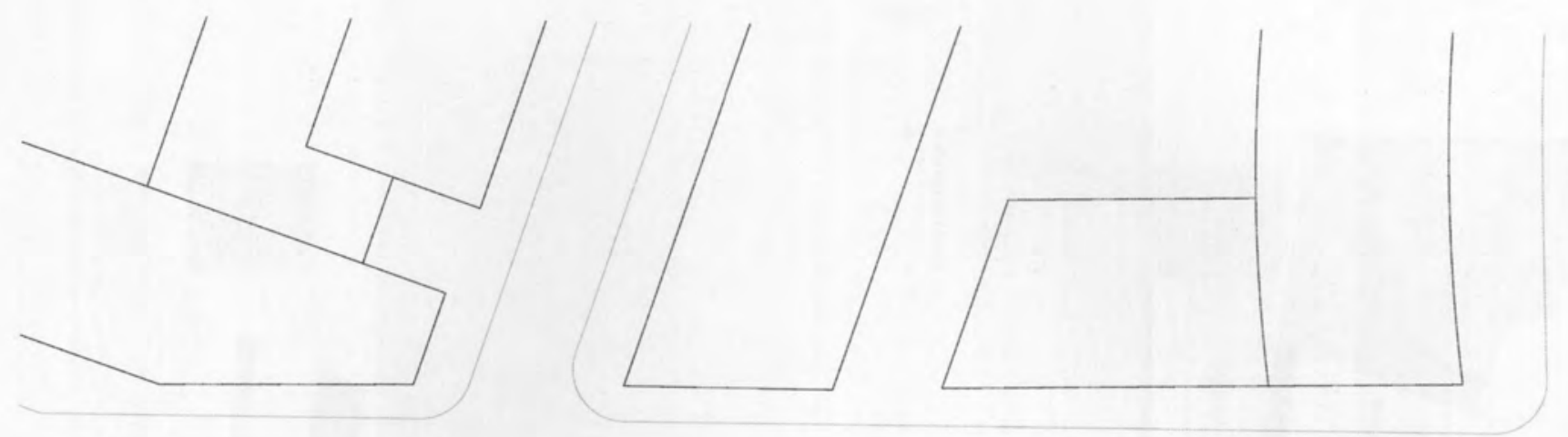

\section{Mill Street}

$\overrightarrow{\vec{\infty}}$
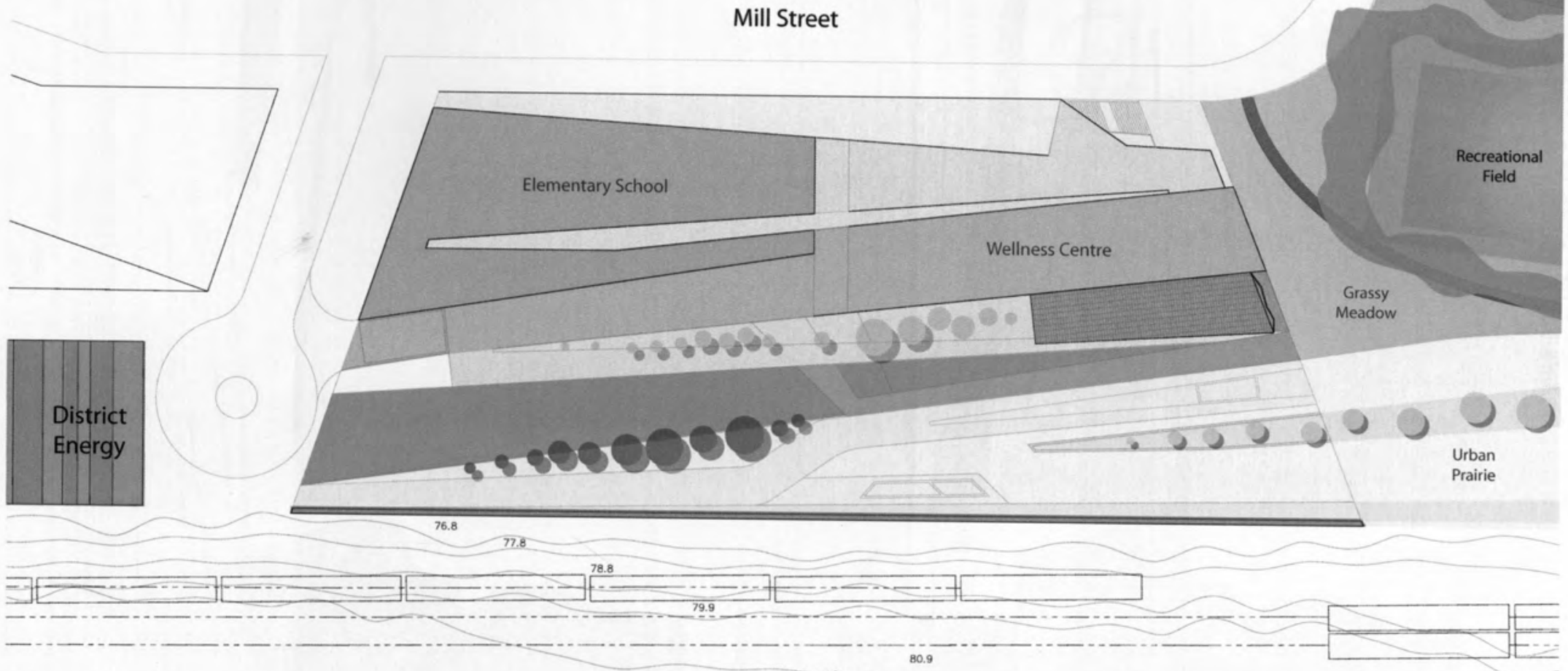

Railway

lands 


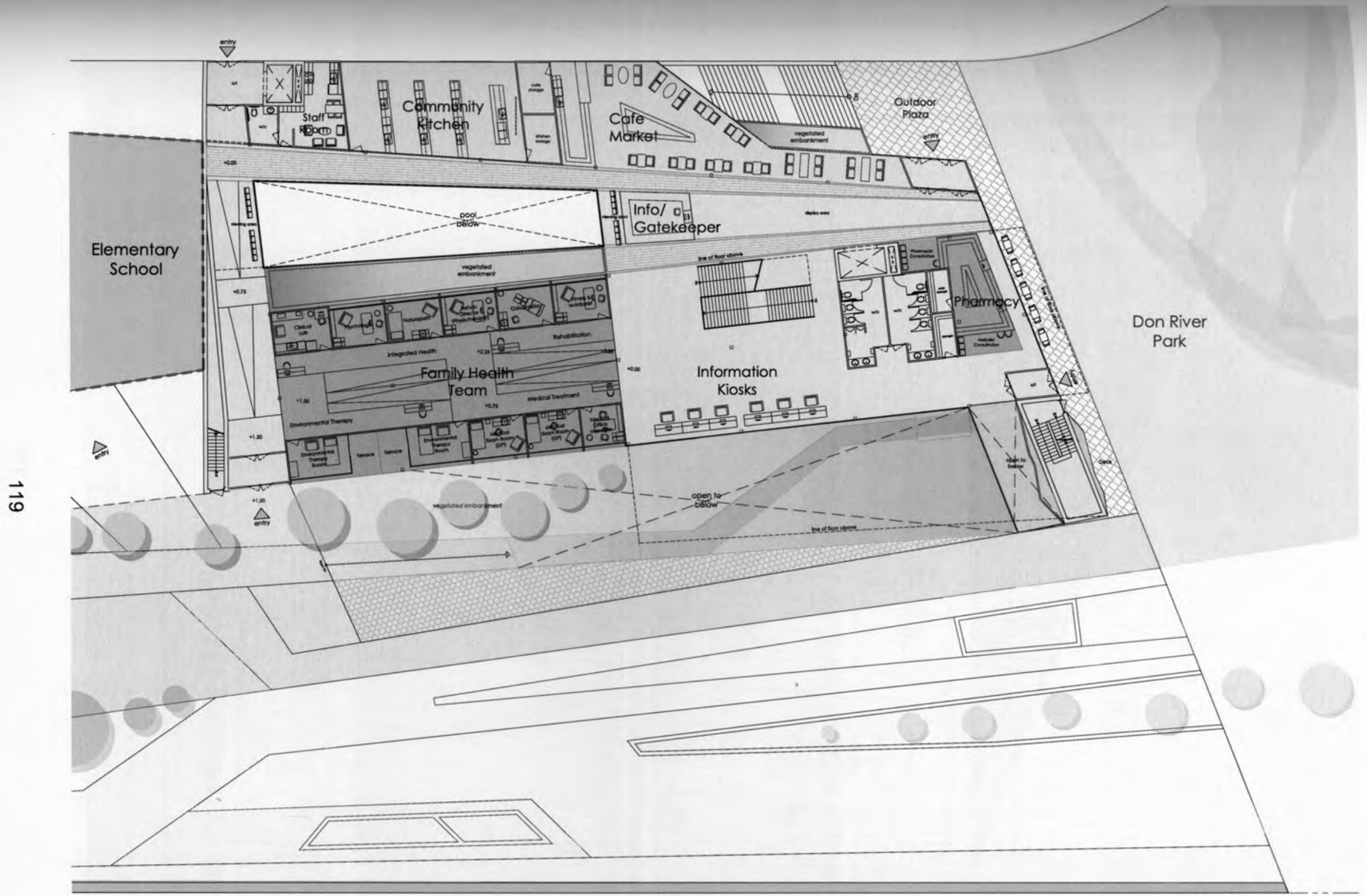

Figure 7.24 Ground level plan: health and education 


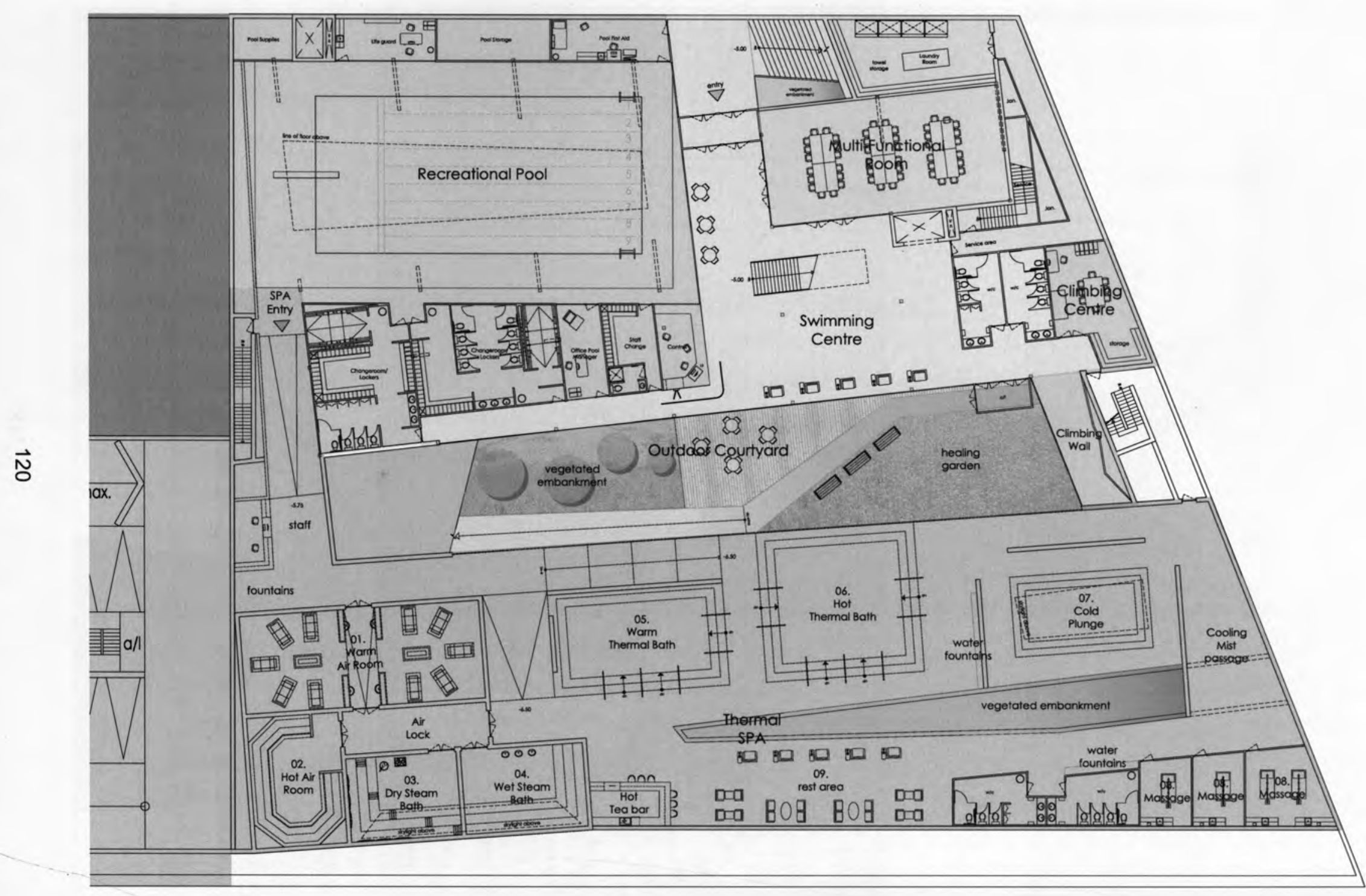

Figure 7.25 Lower level plan: swimming centre and SPA 


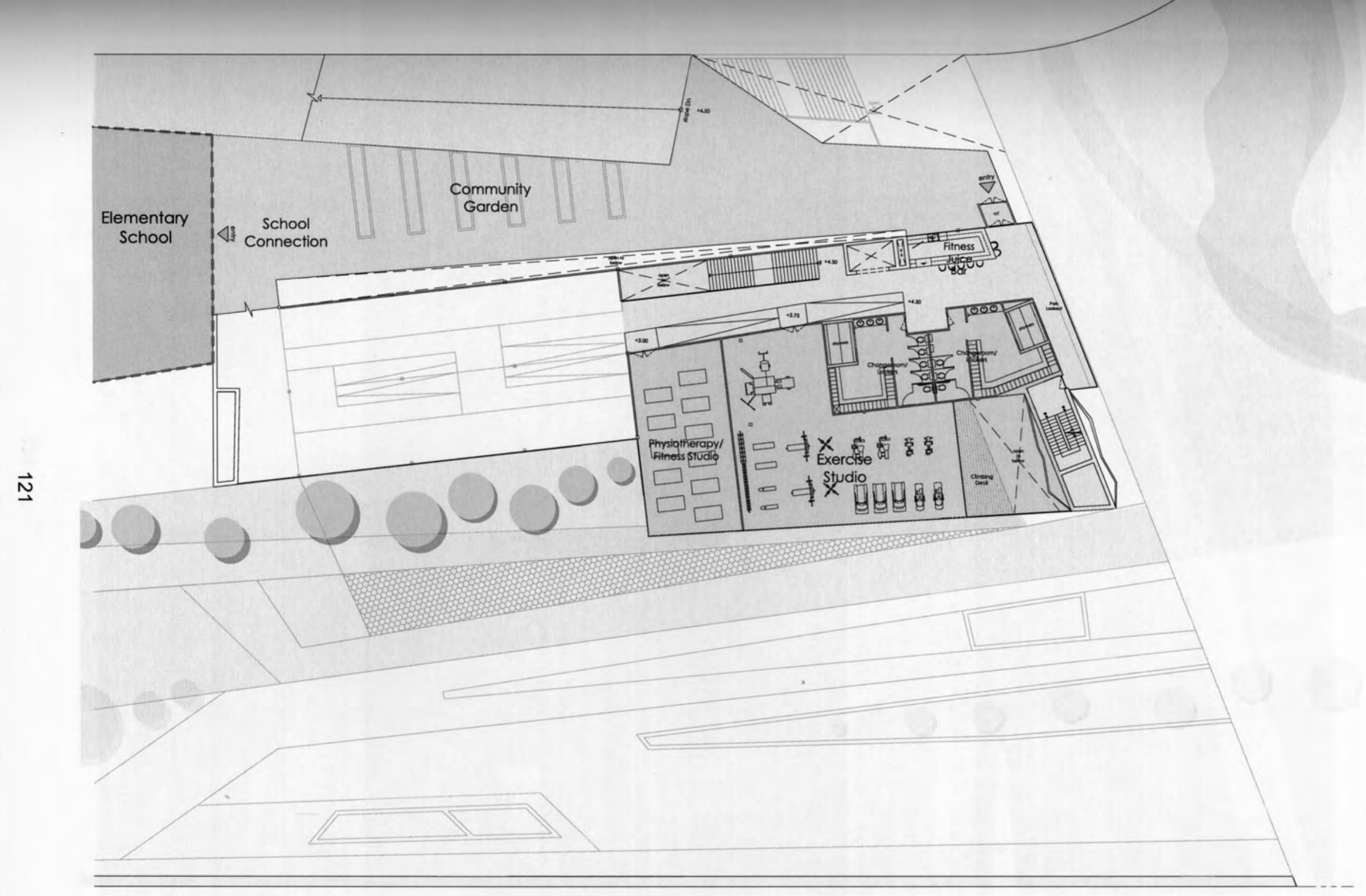




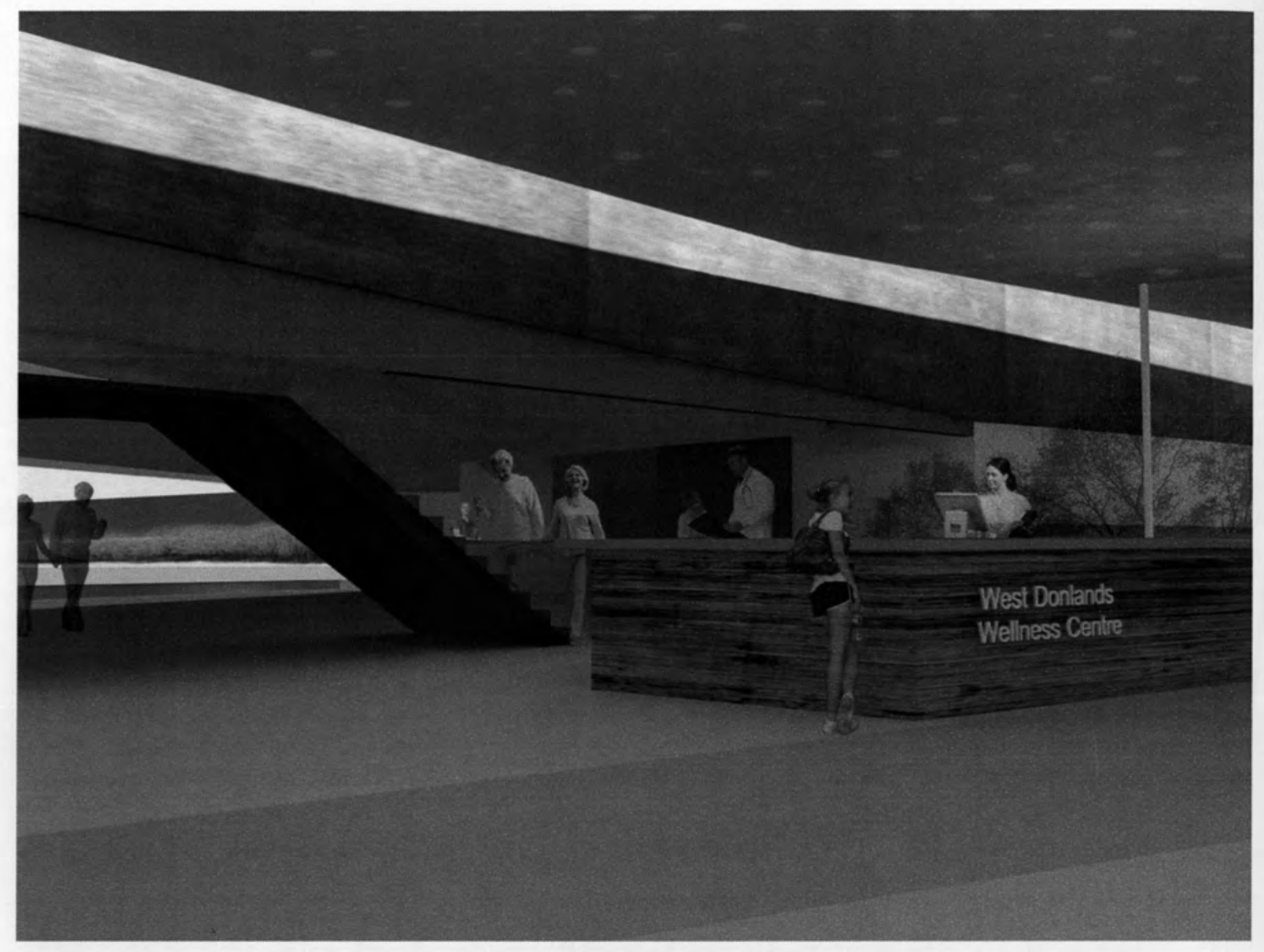

Figure 7.27 Gatekeeper and entry reception

Upon entering the Wellness Centre, the members of the community are greeted by the receptionist/gatekeeper. The 'family health team' model focuses on offering integrated health services to all of its users, and a knowledgeable practitioner directs individuals to the various services that the facility offers. This includes areas of traditional health services, education, fitness and varying modes of healing. 


\section{Figure 7.28 Medical Examination Rooms}

The 'family health team' model incorporates a collaborative work environment for the delivery of care services. This setting allows general practitioners, nurse practitioners, nurses, alternative therapists and physical therapists to work in collaborative setting. The exam rooms are designed to maximize the exposure to the beneficial effects of nature, including views, natural day lighting and materials. The exam rooms further encourage a family setting to occur, one in which comfortable furniture, artwork and a generally minimalist and clutter free design aids in the process of patient healing.

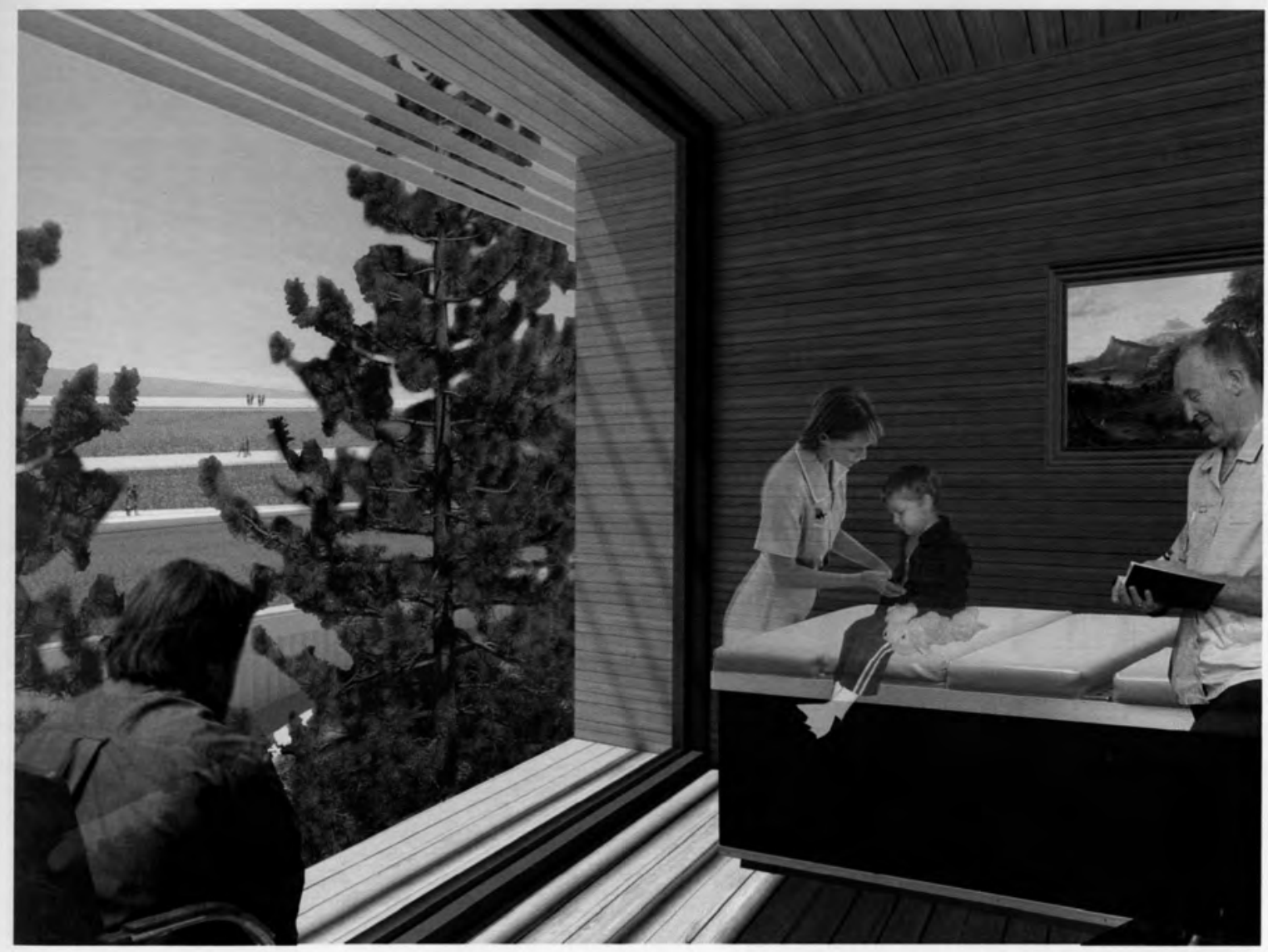


Figure 7.29 Digital Information Kiosks

An educational component to the wellness Centre includes community access to a digital resource database of health services and information. Patients and users may access e-health records, monitor their prescriptions, verify results of past fitness tests, weigh alternative forms of care and educate themselves of prevention of disease.

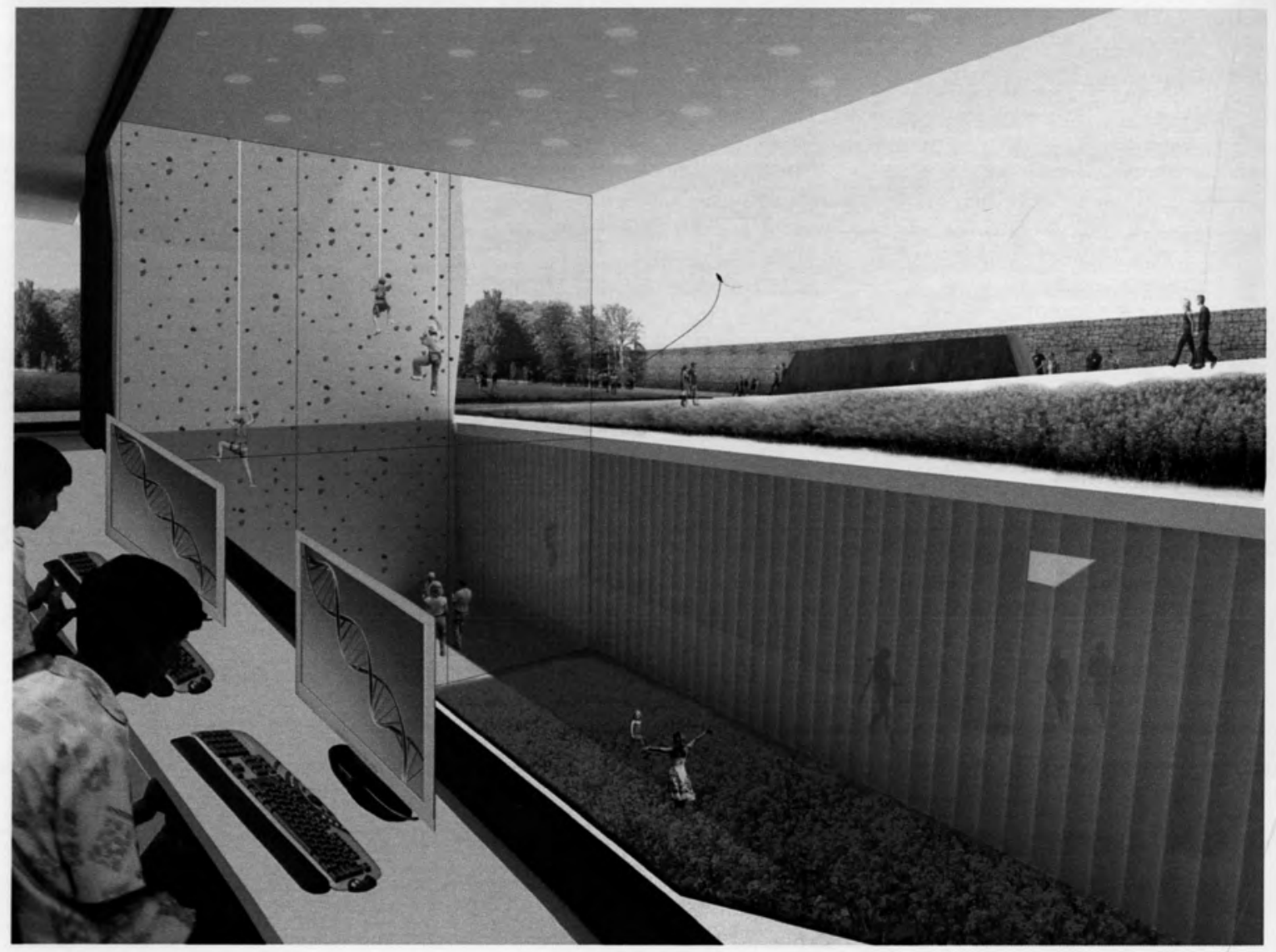




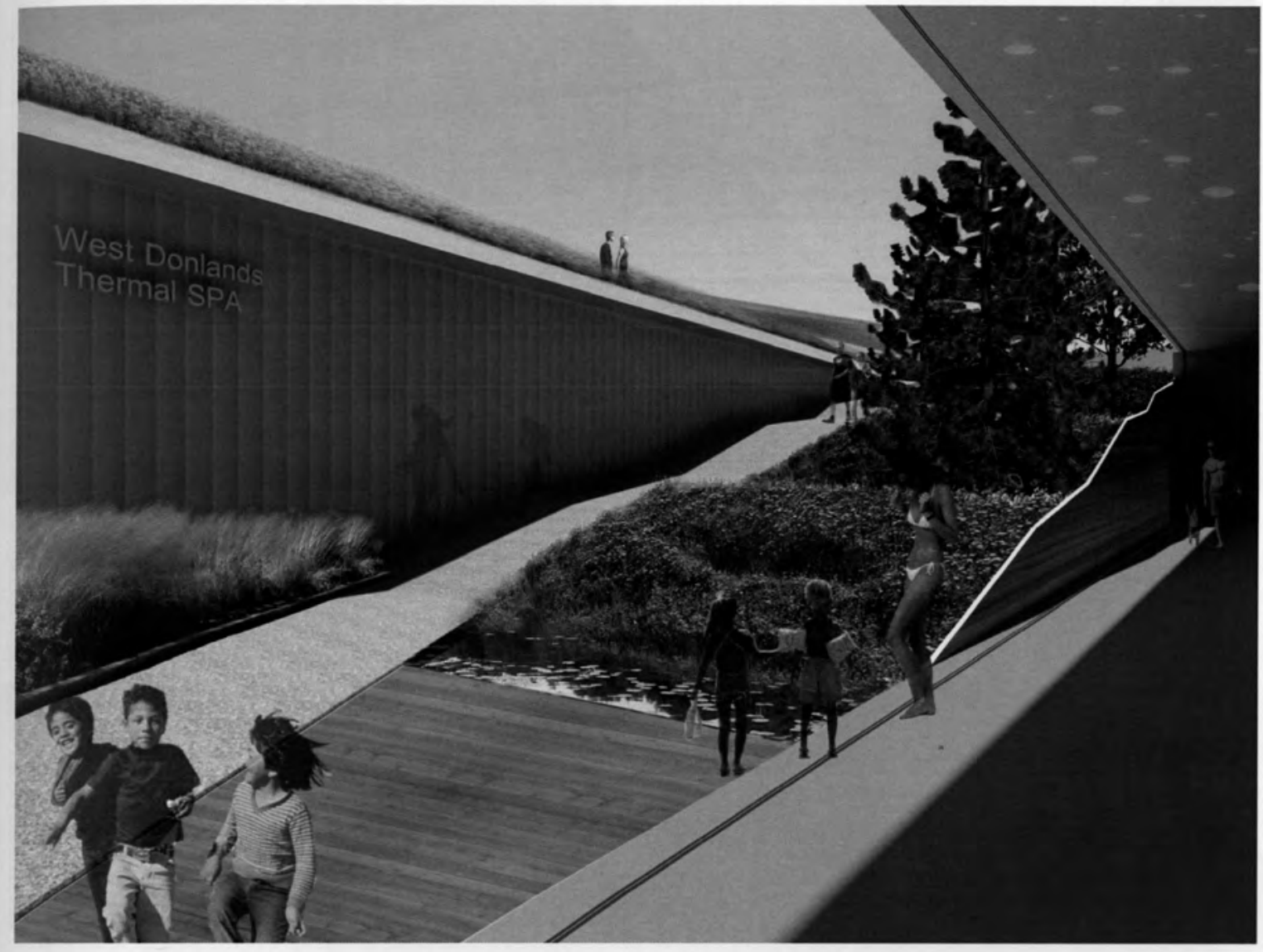

Figure 7.30 Lower entry courtyard

Direct access from the exterior landscape component of the Wellness Centre into the lower level swimming centre unveils itself through the landscape. The porous nature of the building facilitates for a great exposure to the external environment through the slicing of linear spaces. The SPA component also emerges with this approach, an area of the Wellness Centre that holds a more private and secluded atmosphere from that of the swimming centre. 


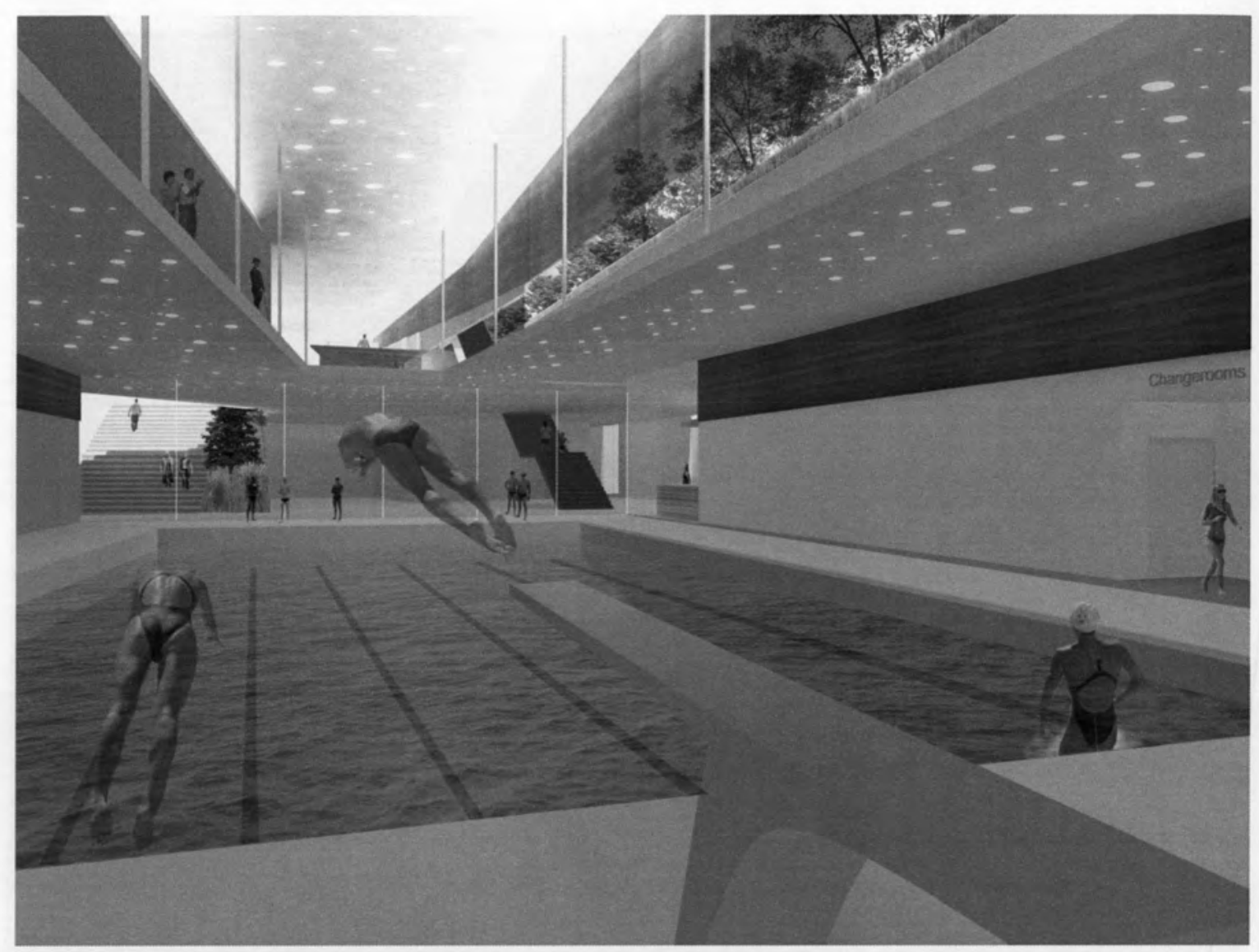

Figure 7.31 Main recreational pool

The main recreational pool acts as a dominant feature within the Wellness Centre. Acting as a crucial device of orientation and way finding, the main pool is a linear double height space that offers direct access from the main entry at grade. The space places the entry gatekeeper at its upper apex, reinforcing the importance of this as a central point of information in the facility. 


\section{Figure 7.32 Entry into SPA}

Accessed directly from the main recreational pool, the processional entry into the SPA offers a differing atmosphere of experience. With a wide and elemental material base, the user both physically and psychologically becomes separated from the noisier environment of the main recreational pool. A serene and tranquil setting begins to unfold itself. 


\section{Figure 7.33 Caldarium}

The SPA component exists as a system of thermal baths and steam rooms that offer water therapy and aid in the exfoliation of the skin process. This therapeutic environment is further reinforced through its material experience. Elemental materials consists of water, light, volcanised concrete, rammed earth and stone. This overall ambient atmosphere offers a secluded retreat of healing open to all members of the community.

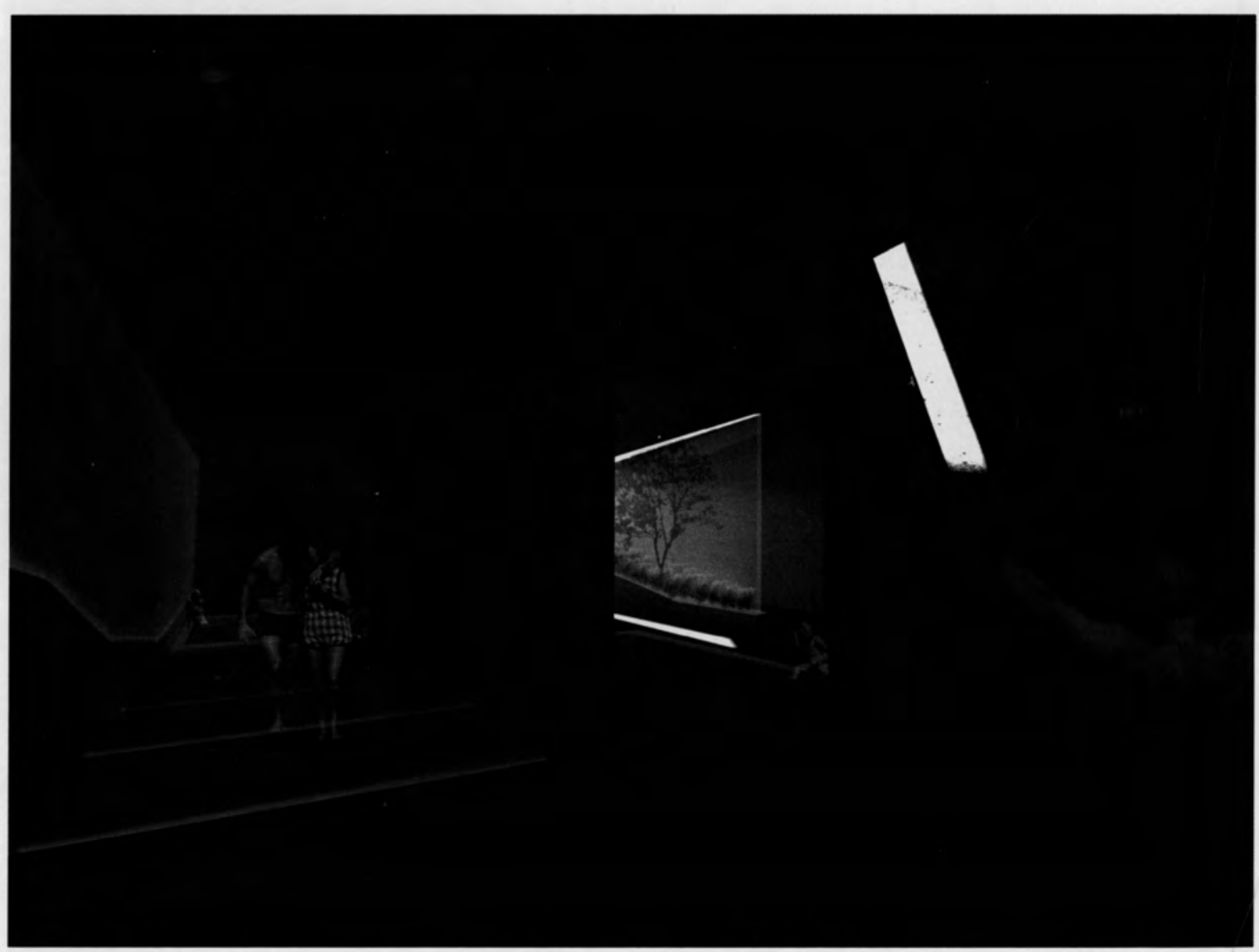




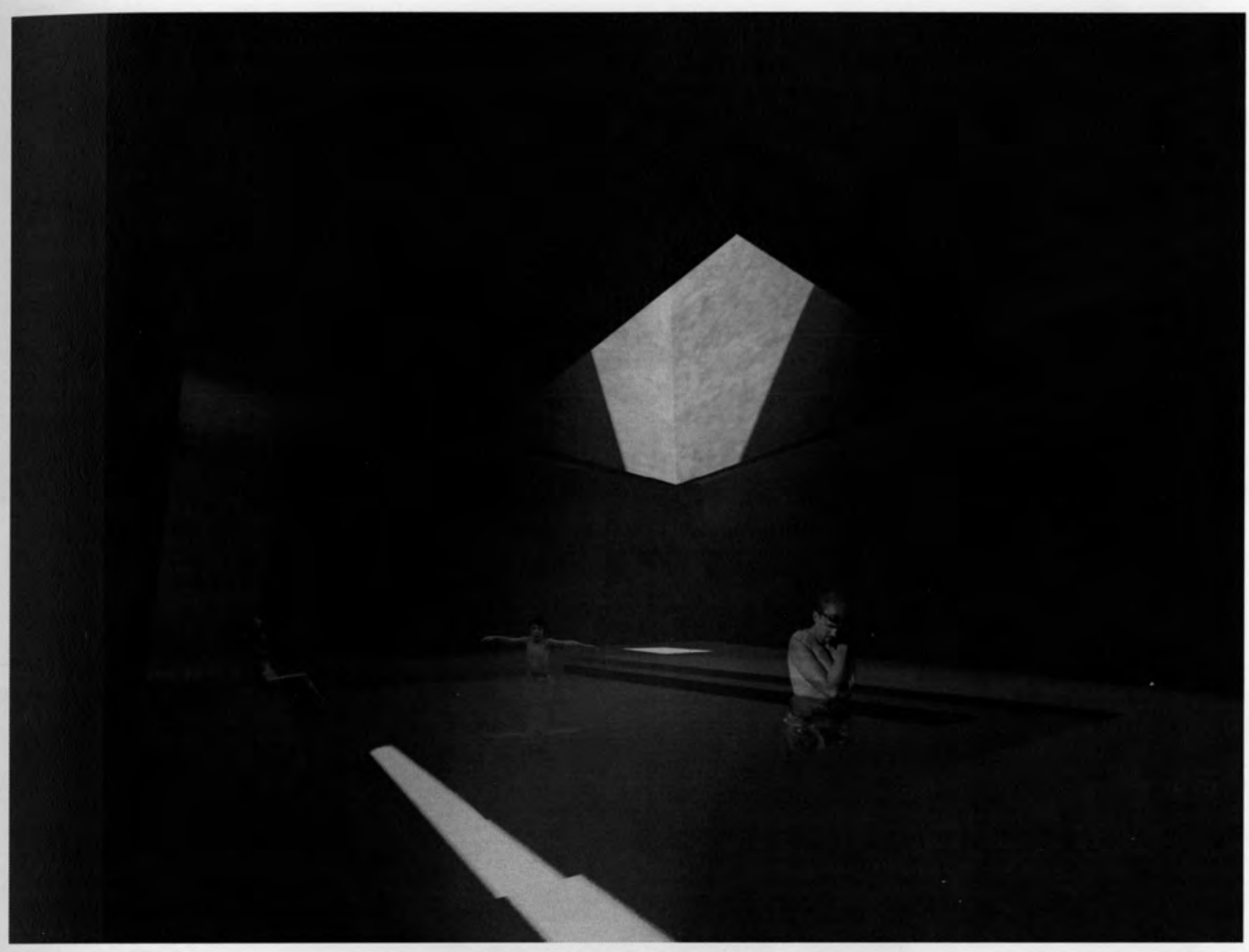

\section{Figure 7.34 Frigidarium}

The SPA experience is fully complete upon entering the frigidarium. The cold temperatures of the plunge bath close the pores and offer an invigorating termination to the process of the water therapy. This revitalizing experience creates sounds from users due to the ice cold

temperatures. Light wells serve as a method of achieving natural day lighting, and offers both visual and acoustical connections to the outdoors.

\section{Following Page: Figure 7.35 Contextual landscape connections along railway}

Working with the existing conditions of the railway and further site adjacencies, the Wellness Centre exists as an integrated portion of the larger West Donlands masterplan. Critical linkages to the District Energy Plant and the Don River Park have been made through a continuous landscaped connection along the railands. 
$\vec{\omega}$

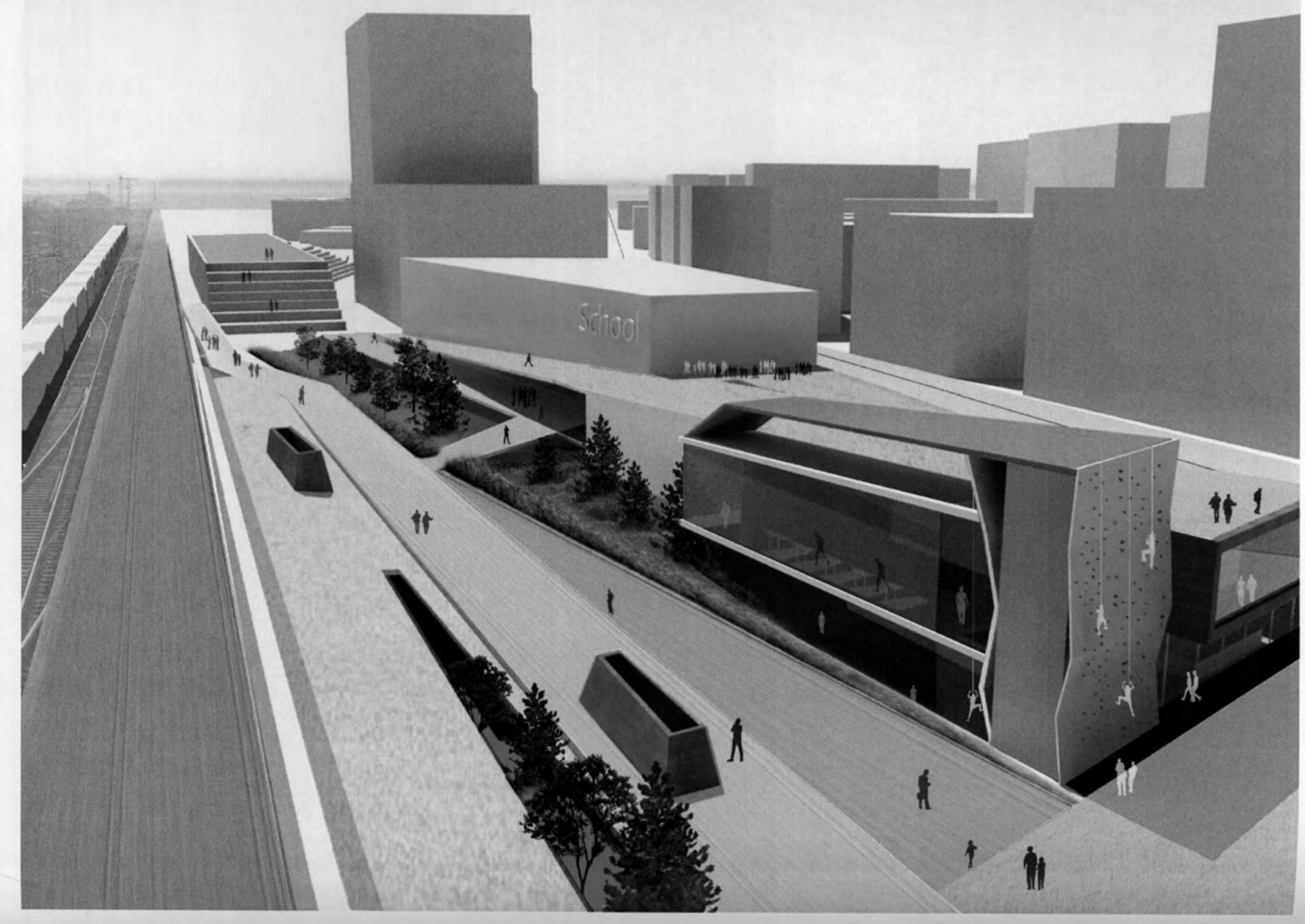




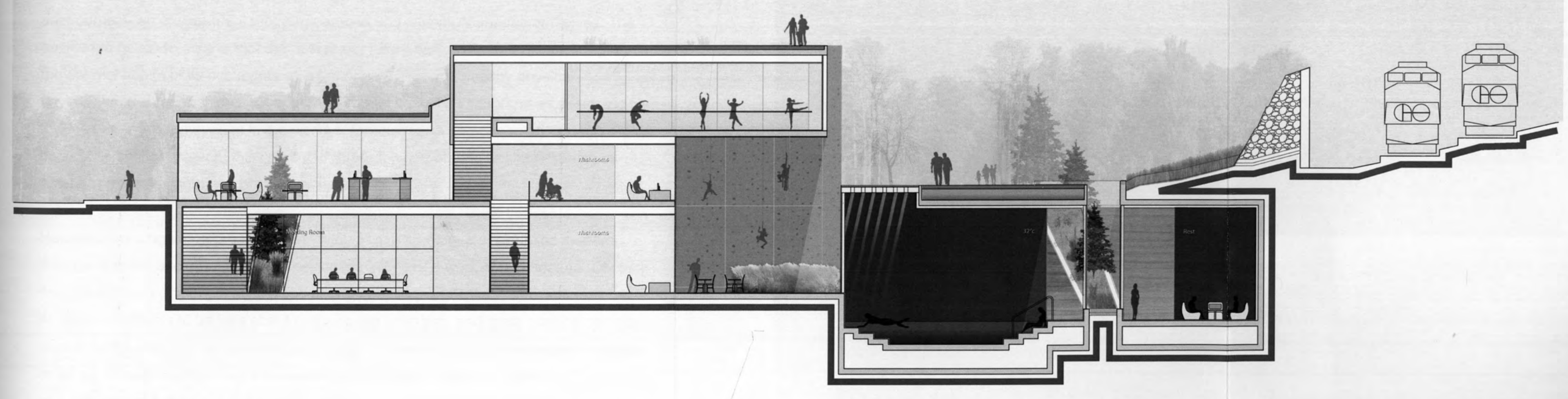




\section{Chapter 8: Summary}

This thesis|project has explored a vision for a future community lifestyle, one that places preventative health as the cornerstone to its success as a sustainable community. While being a conceptual vision, this design experiment is rooted in many concrete facts. These include addressing specific demographic shifts in Canadian health, offering alternative approaches to medical services, supplying high demand programs that are currently needed in specific Toronto communities, as well as integrating a design proposal with a larger scale masterplan. Success would not be known until a design of such caliber was to be adopted in real life; however, given the realities that this design addresses, successes could very well be granted.

On a personal level, much was learned from this thesis|project, an undertaking that was just as rewarding as it was rigorous. Taking a critical stance on what a proposed sustainable community should set out as its top priority was extremely gratifying. If the level of happiness and wellness of a community is not at its highest potential, the complexities involved in attaining the true definition of sustainability will never be reached. Architects have always played a pivotal role in shaping the physical environment- an environment that turns around and ultimately shapes ourselves. The position to be taken here is that the design our future built environments should place the optimal health of its occupants as a top priority. Building healthy physical environments as an architectural approach should be just as important as designing for reduced energy consumption in the world of tomorrow. As demonstrated through a wide body of scientific research, this can occur through incorporating healthy aspects of nature into the design of the physical environment.

However, the singular approach of designing from a catalogue of scientific research findings was not accepted in the successful definition of a work of architecture. Defining the true essence of a successful piece of architecture is undeniably subjective. Architecture seems to be less about one singular definition, and more about a personal stance on the need for critical change. Whether it is an act of technical experimentation or an act of responsible social intervention, architecture brings the innermost feelings and attitudes of a designer out into the open for all to engage with. It is the action of orchestrating the natural phenomena embedded in this world together with the 
aspirations of a specific community. All of these factors are brought together through a rigorous process of design experimentation. In this specific design process, it was found not only important for architecture to create an environment of physiological healing, but to also create an environment of healthy psychological experiences. Healthy psychological experiences have been proven to occur in the natural world, and thus are embedded in our evolutionary make up as humans. Exposing oneself to the beneficial qualities of nature has been demonstrated in this thesis|project to both correct and prevent sickness through the responsible design of the built environment. This approach to the 'architecture of healing' is most successful when it allows for individualized human experiences to occur. This offers the user their own definition of a successful (and thus healthy) space and environment. This can occur with the avoidance of designing institutionalized settings- ones that lack the beneficial properties of space, light and natural materials (fig.7.39). By using this phenomenological approach to the design of our built environment, the role of the architect creates both a healthy and sustainable environment for future generations.

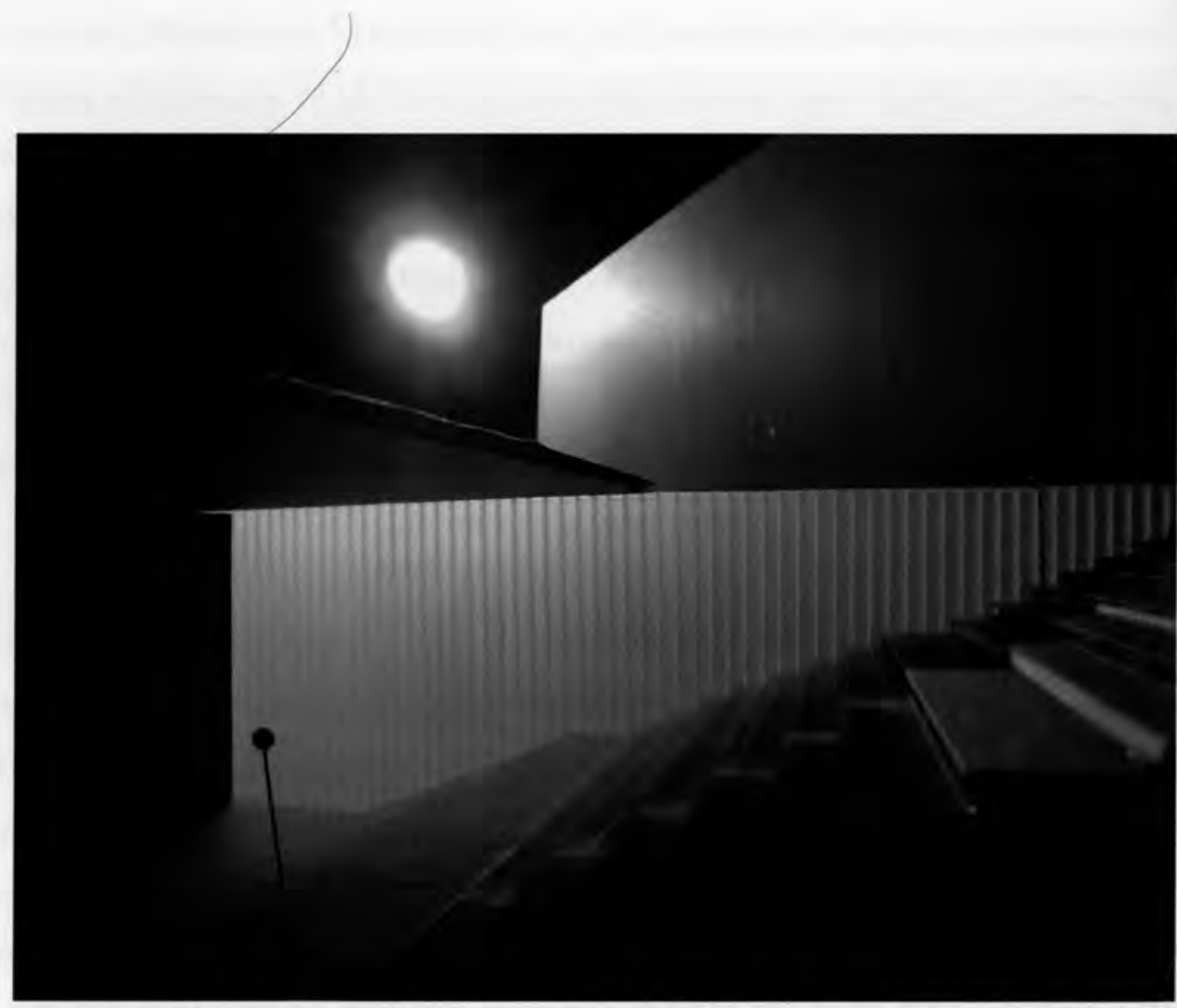

Figure 7.39 The composition of space, light and natural materials was found to create successful architectural experiences that were both physiologically and psychologically healthy for the occupant 


\begin{tabular}{|c|c|c|}
\hline & Task & Activity \\
\hline 1 & Identitfy the clients goals (West Donlands Community) & $\begin{array}{l}\text { To offer a 3rd place within the community where people can connect with a holistic approach to health and wellness: an approach that employs } \\
\text { traditional allopathic and integrated health services, allows for the human reconnection with the benificial therapies of nature, and facilitate a } \\
\text { knowldege base for a preventative health lifestyle }\end{array}$ \\
\hline 2 & Identify the firms goals (William Harispru) & $\begin{array}{l}\text { That the evidence found in specific research studies from corrective health facilities be extrapolated for their use in a preventitive health approach } \\
\text { of building; the hope is to start creating varying building typologies that are inherently 'healthy' in their quality of experience }\end{array}$ \\
\hline 3 & Identify the top 3-5 Key Design Issues & $\begin{array}{l}\text { 1. improve the quality of health within the waterfront community through extended health services } \\
\text { 2. to create a building typology that incorporates the healthy attributes of nature } \\
\text { 3. how to attract the community? } \\
\text { 4. toxic site } \\
\text { [5. water systems] }\end{array}$ \\
\hline 4 & Convert Key Design Issues to Reseach Questions & $\begin{array}{l}\text { 1. What alternative forms of health and therapy can be offered to the community that will offer a more expansive quality of life and wellness from } \\
\text { the point of health? } \\
\text { 2. What forms of natural phenomena can offer healing through building design? } \\
\text { 3. how can the proposed wellness centre appeal to the future west Donalnds community? } \\
\text { 4. how can the toxic elements of developing next to railway lands be properly mediated? }\end{array}$ \\
\hline 5 & Gather Information (Benchmark Examples, Lterature sources, Internal S & $\begin{array}{l}\text { 1. a) interview with the Harbourfront Wellness Centre, b) Caledon Centre for Recreation and Wellness c) Ministry of Health- 'Familiy Health } \\
\text { Team' Models } \\
\text { 2. a) studies on healthy views to nature, the healing powers of light, sound therapy, art therapy and facilitating for social human contact } \\
\text { 3. a) West Donlands Precinct Plan b) West Donlands Block Plan and Design Guidelines c) Feasibility study for elementary school site d)Mill } \\
\text { street rezoning application for district heating and cooling (Diane Silver Interview) } \\
\text { 4. a) controlling ground vibration b) controlling airbourne noise c) safety in case of train derailment d) controlling airborne pollution }\end{array}$ \\
\hline 6 & Critical Interpretation of the Evidence & \\
\hline 7 & Create Evidence Based design concepts & $\begin{array}{l}\text { 1. Offer a broad range of care (integrated Health Services): chiropractor, physioterapist, massage therapy, naturopath, psychologist, dietician, } \\
\text { aquatherapy, fitness, education } \\
\text { 2. create a wellness centre that makes ample connections to the outdoor environment } \\
\text { 3. integrate with the larger masterplan of the West Donlands, and the 'Don River park' scheme; } \\
\text { 4. a) install lead plated footings b) landscpae buffer c)install crash wall berm \& concrete railguard d) ??? }\end{array}$ \\
\hline 8 & Develop Hyptothesis & $\begin{array}{l}\text { 1. That these extended health services will broaden the understanding of holistic medicine through a greater interaction and attendance from } \\
\text { community members } \\
\text { 2. Greater connections to nature will facilitate for quicker healing times for post-operative patients, as well as reduced absenteism from staff, as } \\
\text { well as a more satisfied clientelle } \\
\text { 3. create a greater continuity between the park (through a continuous landscape component) and the lower precinct } \\
\text { 4. that the health and safety of the occupants does not become comprimised by being located next to railands }\end{array}$ \\
\hline 9 & Select Measures & Unable to conduct post occupancy testing due to the nature of this student project \\
\hline
\end{tabular}




\section{Appendix 2}

Field research: Evidence based design, San Francisco CA
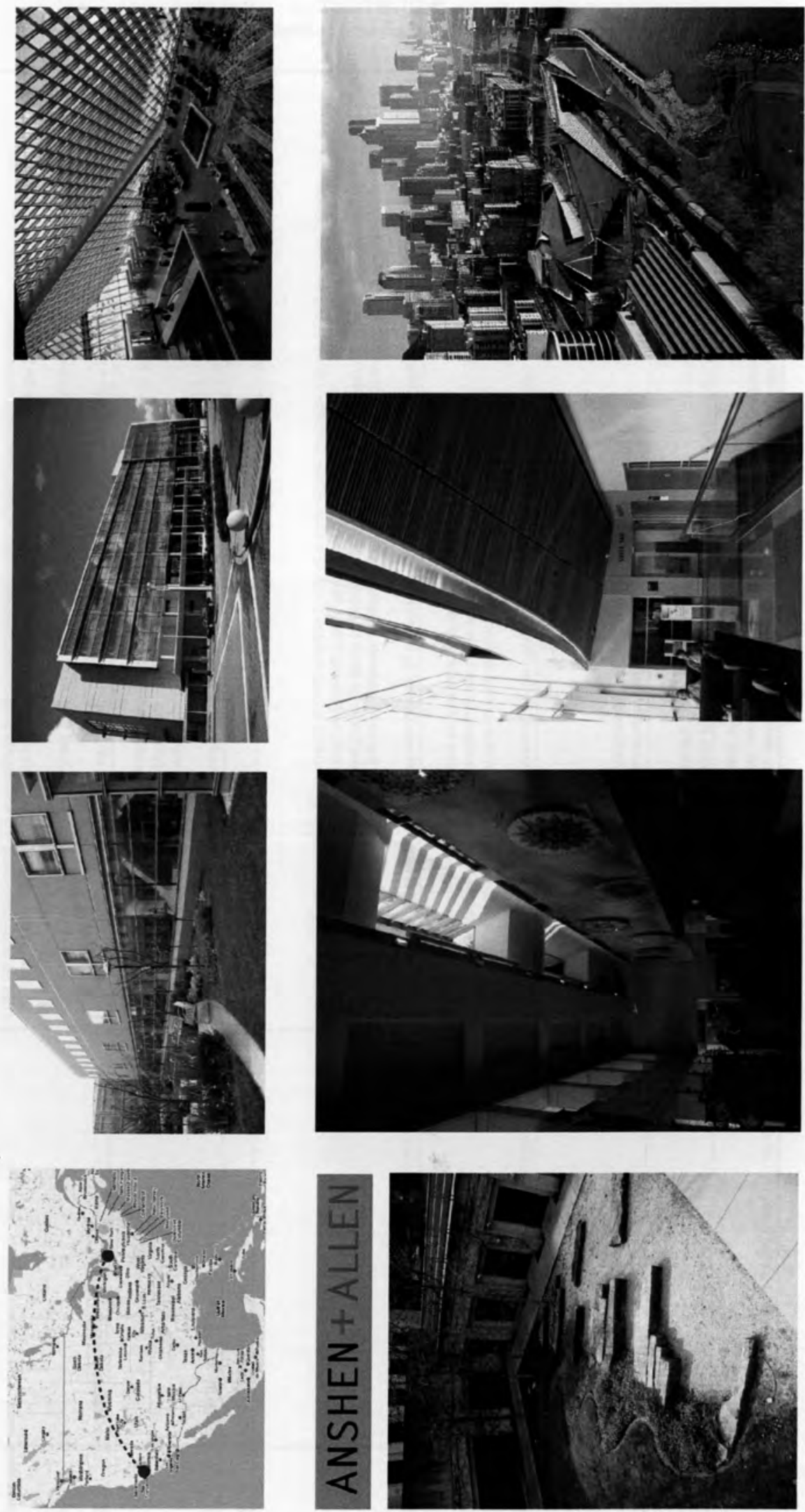


\section{Appendix 3}

Fable Hospital: Incremental costs to achieve a better building Image credit: The Centre for Health Design

INCREMENTAL COST TO ACHIEVE A BEIIER BUILDING

\section{Changes}

Larger private patient rooms

Acuity-adaptable rooms

Larger windows

Larger patient bathrooms

with double-door access

Hand-hygiene facilities

Decentralized nursing substations

Additional high-efficiency particulate air (HEPA) filters

Noise-reduction measures

Additional family/social spaces on each patient floor

Health information resource center for patients and visitors

Meditation rooms on each floor

Staff gym

Art for public spaces and patient rooms

Healing gardens (interior and exterior)

$\$ 4,717,500$

$\$ 816,000$

$\$ 150,000$

$\$ 1,509,600$

$\$ 1,071,000$

$\$ 556,800$

$\$ 270,000$

$\$ 430,000$

$\$ 95,200$

\section{Additional Cost Calculations}

Assumes an increase of 100 square feet for each of 255 single-patient rooms. Fifteen percent of the beds (45) are in an ICU configuration: 100 sq. ft. $\times 255$ beds @ \$185/sq. ft.

Assumes additional medical gases and monitor mounts in every room to provide ICU/stepdown capabilities with plug-in monitors: 255 @ $\$ 3,200 / \mathrm{rm}$.

The typical $3^{\prime} \times 5^{\prime}$ patient room window is increased to $5^{\prime} \times 8^{\prime}: 300 @ \$ 500 / e$.

The larger space allows two staff members to assist a heavy patient to the toilet, and the enlarged doorway allows patient beds to be rolled in a sitting configuration closer to the water closet: Additional 32 sq. ft./toilet $\times 255=8,160$ sq. ft. @ \$185/sq. ft.

Hand-washing sink with foot pedals at the doorway to each acute patient room. Alcoholbased hand-rub dispenser at the bedside: 255 @ \$4,200/rm.

Alcoves proximate to clusters of beds provide a charting surface, medication cassettes, supplies, alcohol-based hand-rub dispenser, and computer access to the information system: 1 per every 4 beds: 64 locations @ \$8,700/unit

HEPA $99.97 \%$ filtration installed on all AHUs serving patient areas of the hospital. Increases in motor horsepower and fan size of each AHU: 36 AHUs (25,000 CFM each) @ $\$ 7,500 /$ unit

Construction materials chosen for their sound absorption and control characteristics, and carpet was specified in most public areas. Upgraded ceiling and wall materials include additional layers of Sheetrock ${ }^{\text {IM }}$ for sound absorption and acoustical ceiling systems with higher noise reduction efficiencies.

Upgrade for acoustic materials: $\$ 430,000$

$\$ 510,000 \quad$ More public spaces added in the form of a family-style "great room" and family kitchen on each patient floor: $4 \times 750$ sq. ft. $=3,000$ added sq. ft. @ \$170/sq. ft.

Each patient floor has a resource center: $4 \times 140$ sq. ft. $=560$ sq. ft. @ $\$ 170 /$ sq. ft.

$\$ 61,200$ Quiet spaces for family and staff meditation are located on each patient floor: $4 \times 90$ sq. $\mathrm{ft}$. $=360$ sq. ft. @ $\$ 170 /$ sq. ft.

A gym with exercise machines, changing rooms, toilets, and showers is provided: 1,500 sq. $\mathrm{ft}$ @ \$175/sq.ft.+ allowance of $\$ 80,000$ for equipment

$\$ 450,000$ Assumes an additional art allowance beyond the typical budget. Fable also rotates loaned artwork from local artists and solicits donated art. Lighting enhancements to highlight selected artwork: $\$ 100,000$ Increase to art and sculpture allowance: $\$ 350,000$

$\$ 1,050,000$ Assumes additional sums above normal landscape cost for outdoor healing gardens, including a meditation garden, a strolling garden, a pond, an outdoor meeting area, outdoor dining, and a children's playground. Increase to exterior landscape allowance: $\$ 900,000$

The interior environment has been enhanced with indoor plantings, fountains, and atrium space.

Increase to interior "plantscaping" allowance: $\$ 150,000$

TOTAL

Note: All numbers are incremental increases above a typical hospital construction cost.

$\mathrm{AHU}=$ air-handling unit.

$\mathrm{ICU}=$ intensive care unit.

$\mathrm{CFM}=$ cubic feet $/$ minute. 


\section{Appendix 4}

\section{Fable Hospital: Financial impact of design decisions}

Image credit: The Centre for Health Design

\section{FINANCIAL IMPACT OF DESIGN DECISIONS}

Calculations

- Patient falls are common and can cause significant harm. Falls result from patient instability, confusion, unfamiliar surroundings, lack of assistance, poor lighting, and slippery surfaces.

- The national unlitigated average cost of a fall is $\$ 10,000$ (Hendrich, A.L. Falls, Immobility, and Restraints: A Resource Manual. St. Louis: Mosby Publishing, 1995); litigated falls can cost in the millions. Assuming that payment for care is on a case-rate basis (e.g., Medicare), the cost of patient falls goes directly to the bottom line.

- The national median rate of acute care falls is $3.5 \mathrm{falls} / 1,000$ patient days; this is the rate experienced by Fable's predecessor hospital. Fable reduced patient falls by $80 \%$ by locating toilets closer to the patient, putting double doors on bathrooms, using bed-exit features that notify a nurse when a patient is out of bed, decentralizing nursing stations, and locating supplies close by to reduce the amount of time the nurse is away from the patient. Fable's reduced patient fall rate is similar to that experienced by Pebble partner Clarian Health Partners Methodist Hospital, Indianapolis.

Savings

300 beds at $80 \%$ occupancy $=240$ beds $=87,600$ patient days $/ 1,000 \times 3.5=306$ falls $/$ year $\times \$ 10,000=\$ 3,066,000 \times 80 \%=$ savings of $\$ 2,452,800^{\circ}$

Calculations

- Transferring patients to a different room creates additional direct and indirect costs. Transfers increase the risk of medication errors and patient falls, add nursing time for transporting and assessing patients, require extra transport equipment, and contribute to hospital flow inefficiencies. Multiple transfers reduce the continuity of patient care, as more caregivers become involved in the care process.

- Including only the direct costs of additional nursing labor, laundry and linen, and equipment usage, the estimated average cost of one patient room transfer is $\$ 250$ to $\$ 300$ (Hendrich, A.L., and Lee, N., "Intra-Unit Patient Transports: Time, Motion, and Cost Impact on Hospital Efficiency," Nursing Economics, forthcoming). It is not uncommon for hospital patients to be moved three to four times during their stay. The facility Fable replaced averaged one transfer per patient.

- Because of its acuity-adaptable rooms, Fable reduced patient transfers by $80 \%$. Fable's experience is consistent with that of Clarian Health Partners Methodist Hospital, which reduced patient transfers by $90 \%$ in its redesigned, acuity-adaptable cardiac critical care unit.

Savings

19,466 patient stays $\times \$ 250=\$ 4,866,500 \times 80 \%=\$ 3,893,200$

Reduction in Nosocomial Infections

Savings: $\$ 80,640$

Calculations

- Recent estimates in the literature of the incidence of nosocomial infections in hospitals range from about $5 \%$ of patients to nearly $10 \%$ of patients. Infections are more likely in multibed rooms due to the cross-transmission of microbial pathogens between patients.

- The average cost of additional hospitalized treatment associated with nosocomial infections was estimated in one report to be in excess of $\$ 7,000$ (in 1985 dollars) (Burrington, M. Can Private Rooms Be Justified in Today's Healthcare Market? Houston: The Center for Innovation in Health Facilities, 1999). Pebble partner Bronson Methodist Hospital in Kalamazoo, Mich., estimates that each nosocomial infection averages $\$ 4,000$ in additional costs; Bronson is reimbursed for $58 \%$ of these additional costs.

- Fable reduced its nosocomial infection rate by four patients per month by using single rooms $100 \%$ of the time, HEPA filters, and increased hand-hygiene stations. Like Bronson, $58 \%$ of added infection-related costs were reimbursed.

Savings

$4 /$ month at $\$ 4,000$ unlitigated cost $=\$ 192,000 /$ year $\times 42 \%=\$ 80,640$

Reduction in Drug Costs

Savings: $\$ 1,216,666$

Calculations

- Drugs are an inevitable and expensive part of hospitalization, averaging $14.9 \%$, or $\$ 2,448$, of the overall average cost per stay of $\$ 16,438$ in 2000 (The DRG Handbook: Comparative Clinical and Financial Benchmarks. Evanston, III.: Solucient, LLC, 2002).

- Fable carefully measured pre-and postoccupancy drug usage based on literature, drawing a connection between positive distractions in the environment (such as art, music, landscape, and family involvement) and patients' reduced need for pain medication.

- Fable reduced overall per-patient pain medication use by $5 \%$, a result supported by a $16.4 \%$ drop in medication use reported for Pebble partner Karmanos Cancer Institute in Detroit for sickle-cell patients using redesigned facilities. Fifty percent of Fable's reduced drug costs were savings; the other $50 \%$ were reimbursed.

Savings

87,600 patient days $/ 4.5$ days $=19,466$ patient stays $\times \$ 2,500 /$ stay $\times 5 \%=\$ 2,433,333 / 2=\$ 1,216,666$ 
Calculations

- The healthcare industry is suffering a severe skilled-labor shortage that includes RNs. High rates of skilled labor turnover plague the industry. Because of the emotional and physical stress of healthcare work and its long hours, the design of the facility plays a particularly important role in staff attraction and retention.

- The national full-time equivalent (FTE)/occupied bed average is 5.45 staff (The 2003 Almanac of Hospital Financial \& Operating Indicators: $A$ Comprehensive Benchmark of the Nation's Hospitals, Salt Lake City: Ingenix, 2002). Fable's staff equals 1,308 FTEs, of which 30\%, or 391, are nurses. The overall appeal of Fable's facility and specific staff amenities such as break, day-care, and exercise facilities contributed to Fable's reducing RN turnover from $14 \%$ to $10 \%$. These data track the reduction in nursing turnover at Bronson Methodist Hospital after occupying its new building.

- The estimated cost of one nurse turnover varies widely in the literature. One report estimates the average cost for recruitment, orientation, and retention of a critical care nurse to be $\$ 64,000$ ("Two Hospitals, One Goal: Retaining PICU Nurses," Children's Hospitals Today, Winter 2002). Fable estimates its cost to replace one $R N$ is $\$ 20,500$, based on recruitment costs, higher registry nursing costs during recruitment, and orientation costs. Fable attributes $50 \%$ of the credit for its reduced nursing turnover to the new facility and the other $50 \%$ to salary adjustments and other retention initiatives.

Savings

39 nurses leaving $(10 \%$ of 391$)$ instead of 55 nurses $(14 \%)=\$ 328,000$ saved $(\$ 20,500$ per turnover $) / 2=\$ 164,000$

Increased Market Share

Increased Revenue: $\$ 2,168,100$

Calculations

- Fable increased its market share by $1.5 \%$, an increase consistent with that of Bronson Methodist Hospital, which increased its market share by more than $2 \%$ in 2001 and 2002 , its first two postoccupancy years.

- Fable's market share gain boosted net patient days by 1,314 ; its net patient revenue per diem is $\$ 2,200$, a figure that is consistent with Bronson's performance in its new facility. To be conservative, Fable attributes $75 \%$ of its market share gain to the new facility.

Net Revenue

1,314 additional patient days $\times \$ 2,200=\$ 2,890,800 \times 75 \%=\$ 2,168,100$

Increased Philanthropy

Increased Revenue: $\$ 1,500,000$

\section{Calculations}

- Fable's new facility played an important role in increasing philanthropic contributions from about $\$ 5$ million a year before construction of the new building to $\$ 6.5$ million during the first year of occupancy. Naming opportunities in the new facility encouraged increased giving, as did the building's tangible representation of Fable's vision for health care in the community.

- Fable's increased contributions are consistent with the experience of Pebble partner Children's Hospital and Health Center in San Diego. Children's Hospital's management believes the impact of its innovatively designed Rose Pavilion Building was instrumental in raising $\$ 5$ million during and immediately following the construction.

a. This example assumes all of Fable's acute care patient falls to be unlitigated; in actuality, because some of these falls would be litigated, Fable's costs would be significantly higher.

b. This figure, representing the estimated total reduced costs and increased revenues for Fable Hospital's first year of operation in its new facility, is on the low side. First, we sought to be conservative in our estimates to strengthen the credibility of our message. Given that Fable Hospital is built from the experiences of multiple hospitals and research streams, we wished to err on the side of underpromising rather than overpromising. Second, Fable is benefiting in ways not reflected at all in this exhibit because of insufficient data available to credibly present hard numbers that can be attributed to facility design innovation. Reduced medication errors aided by design features such as better lighting and less noise (in addition to the process improvements of bar coding and computerized order entry) is an example. 


\section{Appendix 5}

'Third Places' of nature reconnection in the modern urban environment
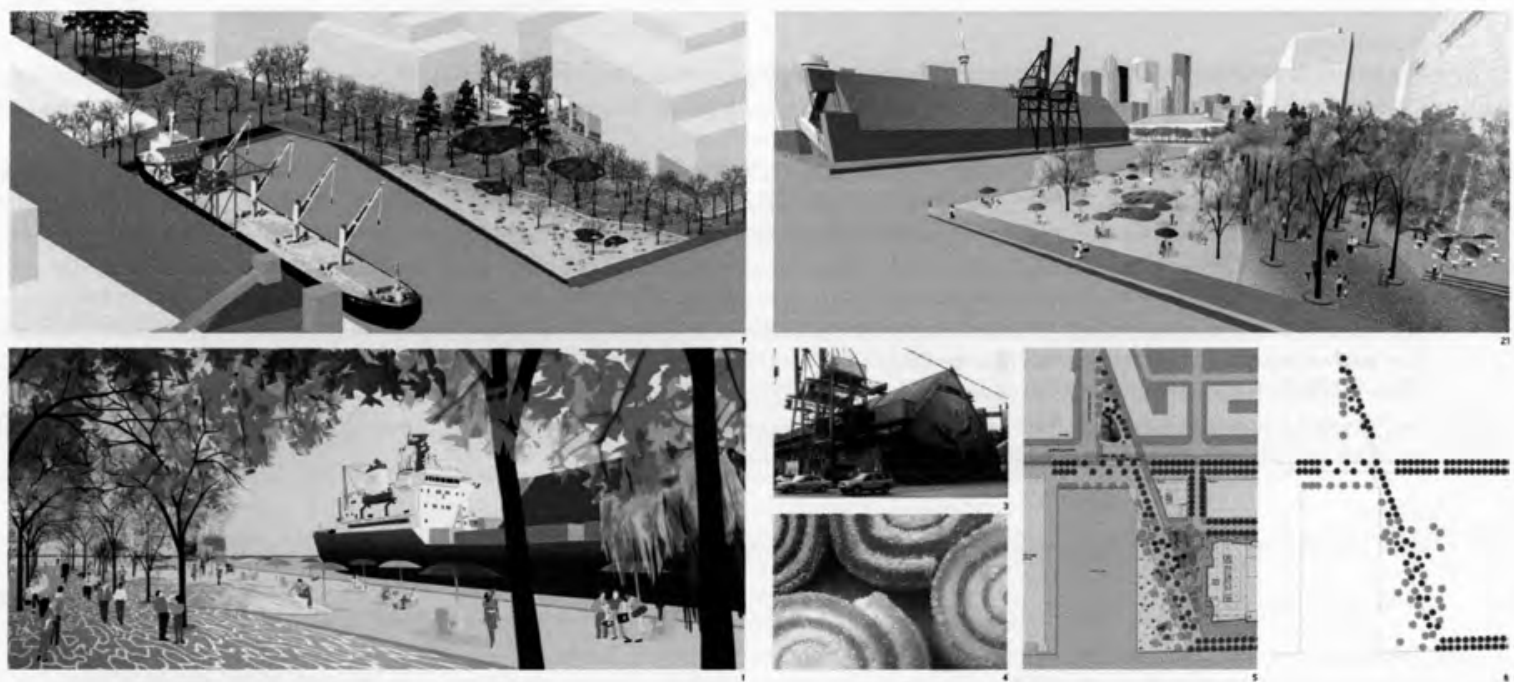

Sugar Beach Toronto- Claude Cormier Landscape Architecture + Urban Design Image Credit: Claude Cormier
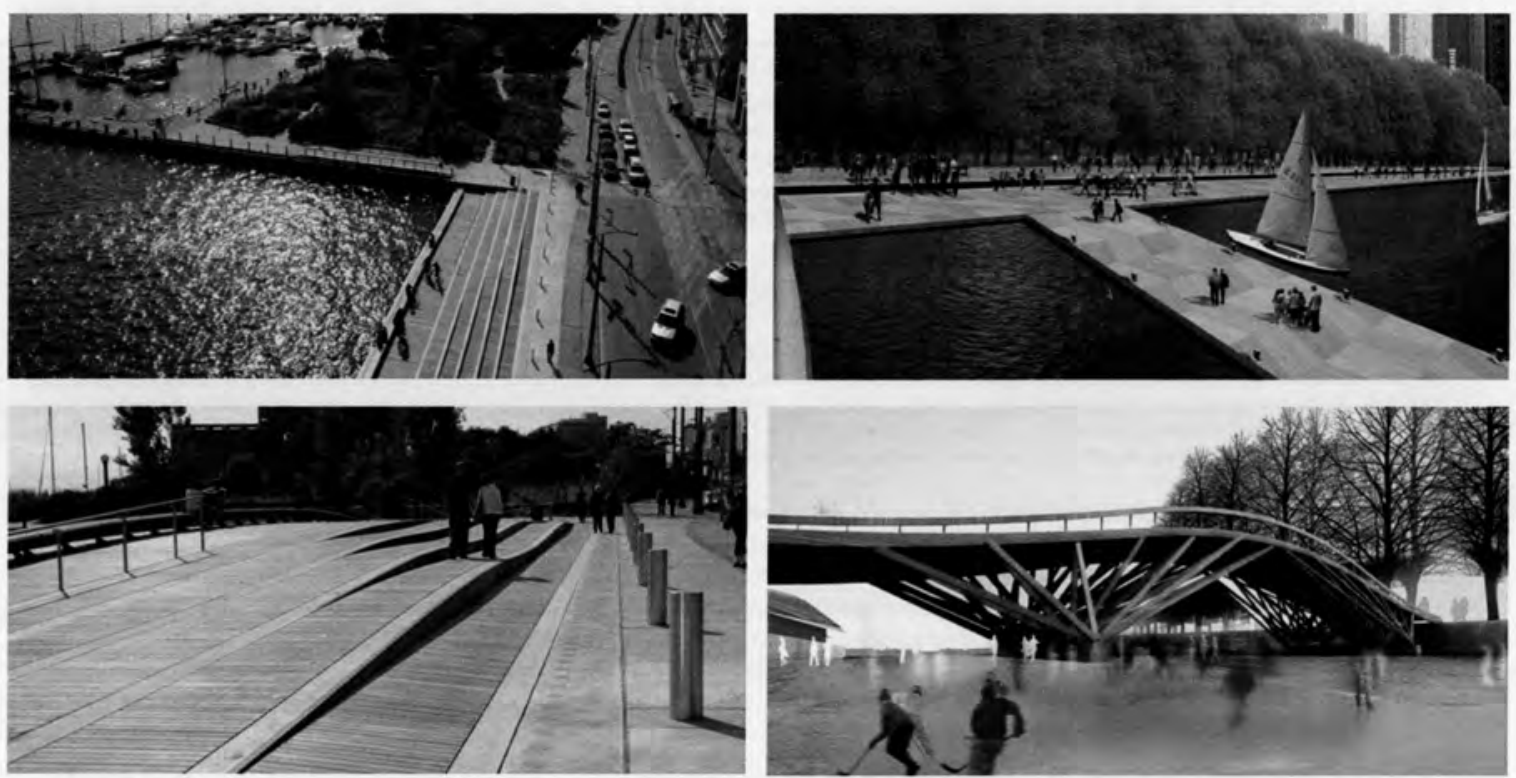

Queens Quay Wave Decks Toronto- West 8 Urban Design \& Landscape Architects Image Credit: West 8 

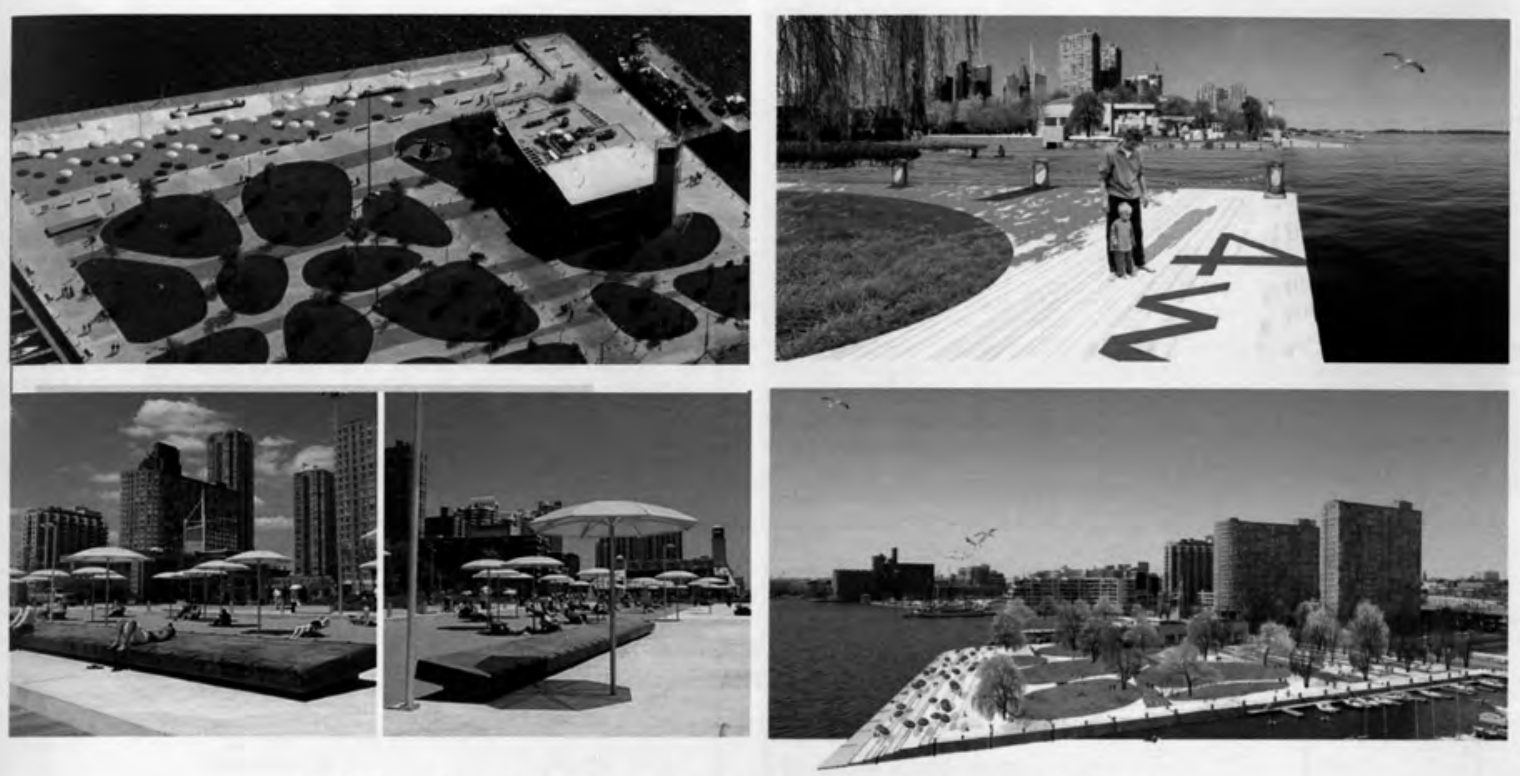

HtO Waterfront Park Slips Toronto- Janet Rosenberg and Associates

Image Credit: hoklife, Torontoist, and JRA Associates
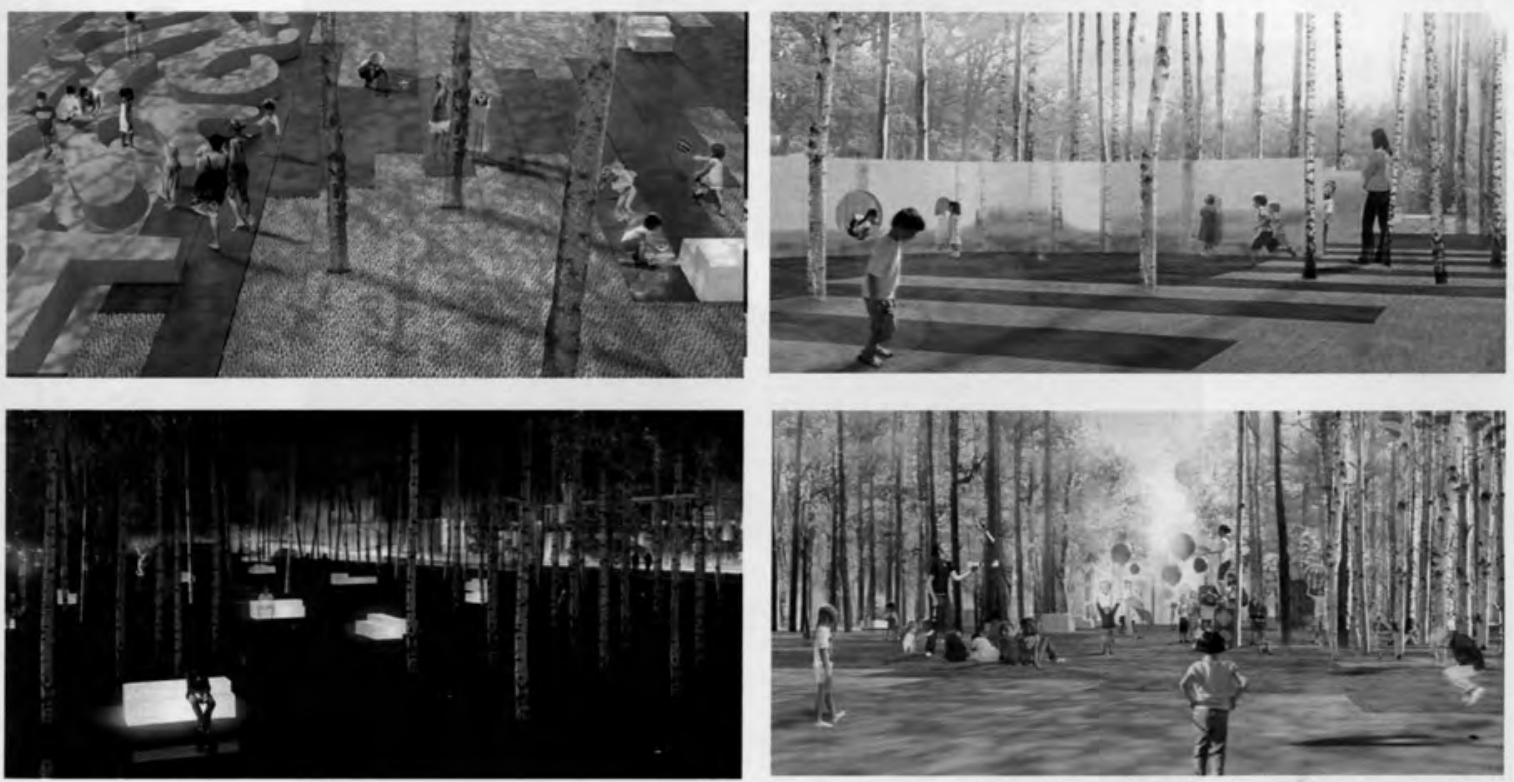

June Callwood Park Toronto- GH3

Image Credit: $\mathrm{GH} 3$ 

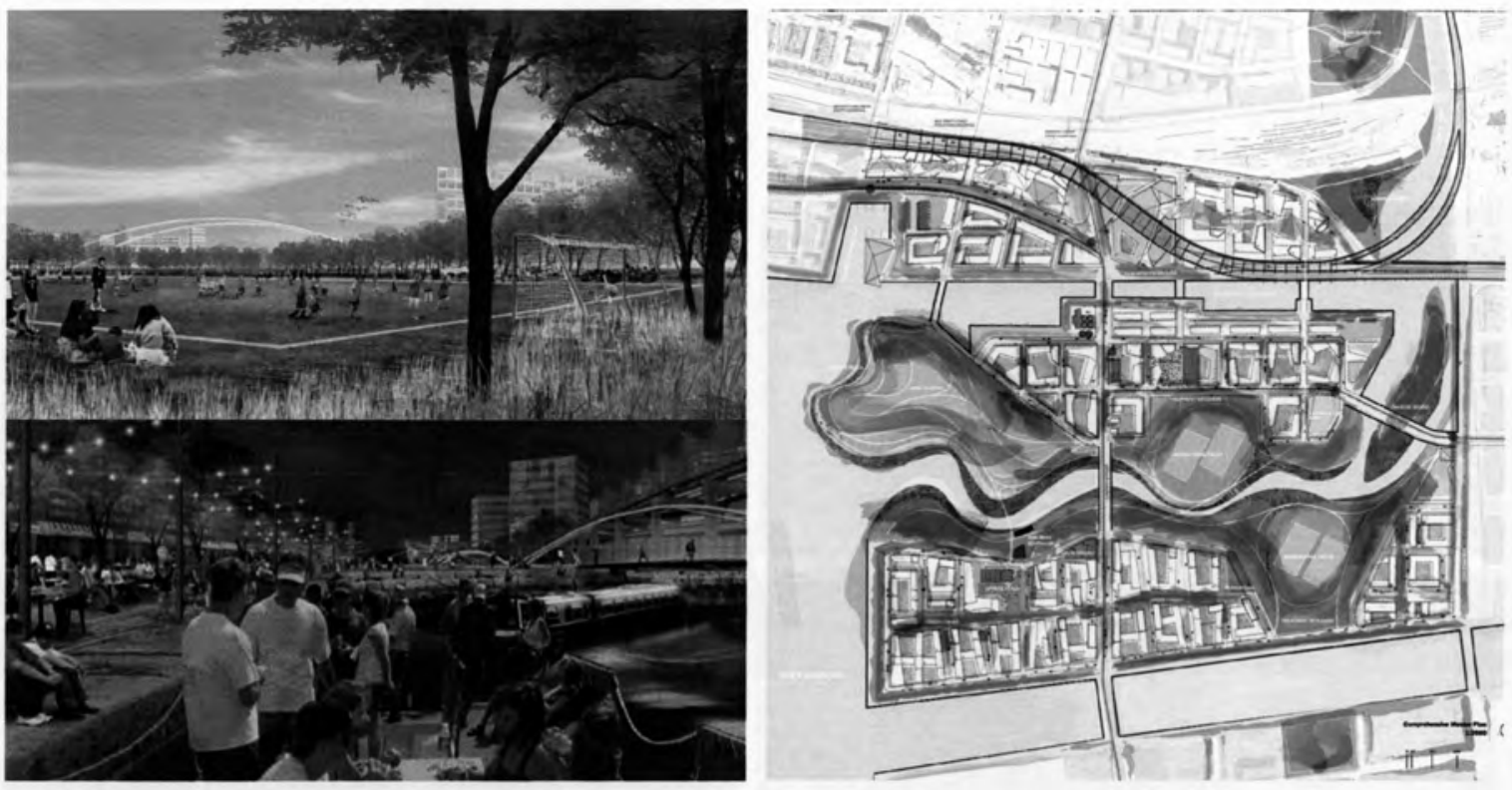

The Lower Donlands Park Toronto- MVVA Team Image Credit: Waterfront Toronto
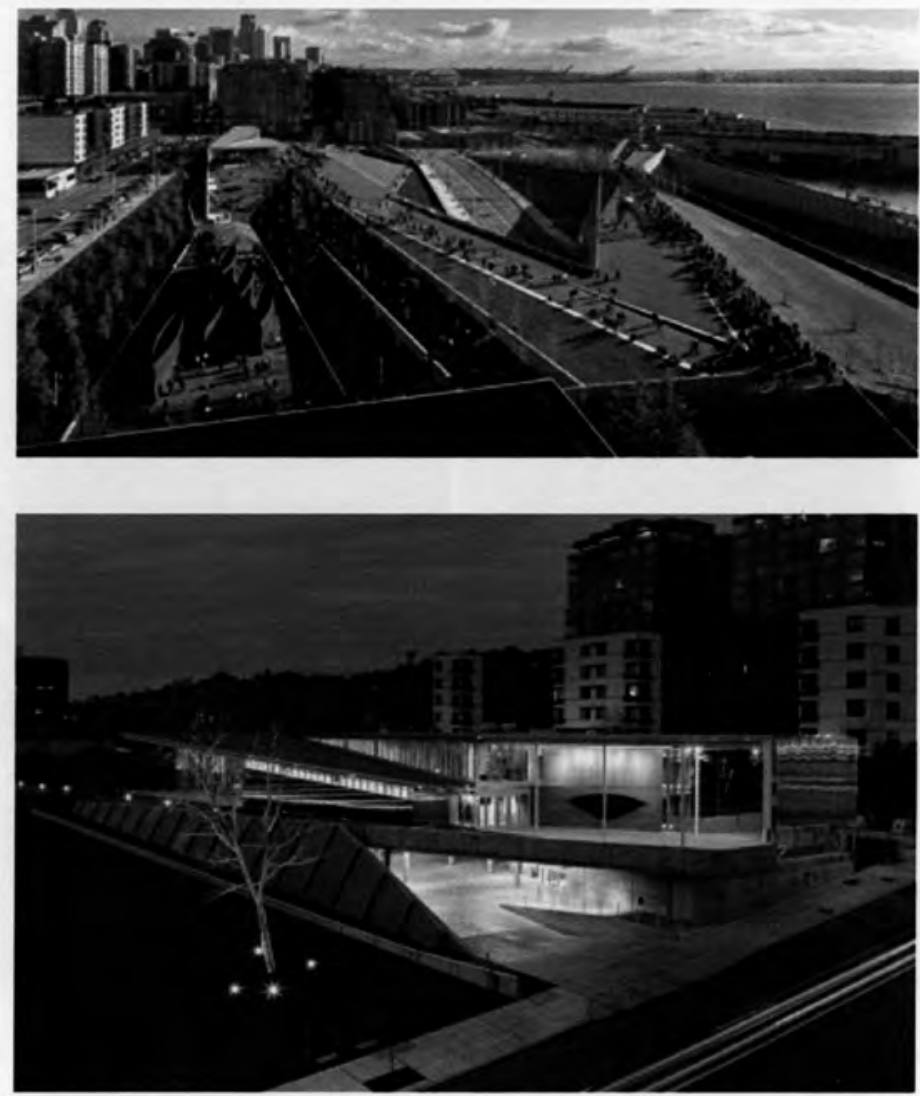

Art Museum and Olympic Sculpture Park Seattle- Weiss/Manfredi Image Credit: Weissmanfredi 


\section{Appendix 6}

Design Project: Programmatic Analysis

\begin{tabular}{|l|l|}
\hline Wellness Centre Programming \\
\hline \multicolumn{2}{|l|}{} \\
\hline Room & Area \\
\hline
\end{tabular}

\begin{tabular}{|l|l|}
\hline Common Areas & \\
\hline Reception Gatekeeper & $40 \mathrm{~m} 2$ \\
Information Kiosks & $40 \mathrm{~m} 2$ \\
Market/Café seating & $44 \mathrm{~m} 2$ \\
Community Kitchen & $80 \mathrm{~m} 2$ \\
Multi Functional Room & $80 \mathrm{~m} 2$ \\
\hline
\end{tabular}

\begin{tabular}{|l|l|}
\hline Recreational Pool Programs & \\
\hline Reception and Control & $57 \mathrm{~m} 2$ \\
Locker Rooms (Apodyterium) & $184 \mathrm{~m} 2$ \\
Office Manager & $12 \mathrm{~m} 2$ \\
Staff Room & $46 \mathrm{~m} 2$ \\
Main Recreational Pool & $488 \mathrm{~m} 2$ \\
First Aid & $11 \mathrm{~m} 2$ \\
\hline
\end{tabular}

\begin{tabular}{|c|l|}
\hline Clinical Programs & \\
\hline Exam Rooms & \\
Medical Exam Room & $12 \mathrm{~m} 2$ \\
Medical Exam Room & $12 \mathrm{~m} 2$ \\
Medical Exam Room & $12 \mathrm{~m} 2$ \\
Massage Therapy Room & $12 \mathrm{~m} 2$ \\
Chiropractor & $12 \mathrm{~m} 2$ \\
Psychologist & $12 \mathrm{~m} 2$ \\
Dietician & $12 \mathrm{~m} 2$ \\
Naturopath/Accupuncture & $12 \mathrm{~m} 2$ \\
Massage Therapy Room & $12 \mathrm{~m} 2$ \\
Environmental Therapy Rooms & $14 \mathrm{~m} 2$ \\
Pharmacy & $26 \mathrm{~m} 2$ \\
Satelitte Workstations x 3 & $3 \mathrm{~m} 2$ \\
Clinical Lab & $14 \mathrm{~m} 2$ \\
Office (Manger) & $12 \mathrm{~m} 2$ \\
Office (Rehab Director) & $12 \mathrm{~m} 2$ \\
Staff Room & $21 \mathrm{~m} 2$ \\
\hline
\end{tabular}

\begin{tabular}{|c|c|c|}
\hline \multicolumn{3}{|l|}{ SPA Programs } \\
\hline \multicolumn{2}{|c|}{ Warm Room (Tepidarium A) } & $95 \mathrm{~m} 2$ \\
\hline \multicolumn{2}{|c|}{ Hot Air Room (Tepidarium B) } & $36 \mathrm{~m} 2$ \\
\hline \multicolumn{2}{|c|}{ Steam Bath 01 (Laconicum) } & $33 \mathrm{~m} 2$ \\
\hline \multicolumn{2}{|c|}{ Steam Bath 02 (Sauna) } & $25 \mathrm{~m} 2$ \\
\hline \multicolumn{2}{|c|}{ Warm Baths (Calidarium) } & $76 \mathrm{~m}^{2}$ \\
\hline \multicolumn{2}{|c|}{$\begin{array}{l}\text { Hot Baths (Calidarium) } \\
\text { Cold Bath (Frigidarium) }\end{array}$} & $70 \mathrm{~m} 2$ \\
\hline \multirow{2}{*}{\multicolumn{3}{|c|}{$\begin{array}{l}\text { Cold Bath (rnigidarium) } \\
\text { Massage Rooms }\end{array}$}} \\
\hline & & \\
\hline & A (Standard) & $14 \mathrm{~m} 2$ \\
\hline & B (Standard) & $14 \mathrm{~m} 2$ \\
\hline & C (Soap \& Brush massage) & $14 \mathrm{~m} 2$ \\
\hline & (Underwater Massage) & $14 \mathrm{~m} 2$ \\
\hline Laundry room & & $14 \mathrm{~m} 2$ \\
\hline Laundry Storage & & $6 \mathrm{~m} 2$ \\
\hline Public Showers & & $6 m 2$ \\
\hline Hot Tea Bar & & $22 \mathrm{~m} 2$ \\
\hline Rest Areas & & $54 \mathrm{~m} 2$ \\
\hline Services & & $134 \mathrm{~m} 2$ \\
\hline
\end{tabular}

\begin{tabular}{|l|l|}
\hline Fitness Programs & \\
\hline Weight Room/Cardio Training & $190 \mathrm{~m} 2$ \\
Aerobic/Physiotherapy Studio/Dance Studio & $80 \mathrm{~m} 2$ \\
Locker rooms/showers/washrooms & $132 \mathrm{~m} 2$ \\
Fitness Testing Office & $12 \mathrm{~m} 2$ \\
Fitness Testing Office & $12 \mathrm{~m} 2$ \\
Juice Bar & $21 \mathrm{~m} 2$ \\
\hline
\end{tabular}


Analysis of physical facility requirements

Project: Wellness Centre 2009

Name of Space: Gatekeeper/Waiting and Reception

Department Community Edu Net Assignable Sq. m

Space Description

Purpose: An area of welcome and direction for users and patrons

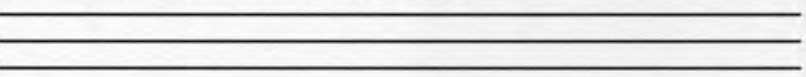

Space Relationships:

a. General - How should this space relate to general areas in the building? eg: offices, teaching areas, loading dock, storage areas.

Located as the intial point of interaction upon entering the facility; adjacent to the informat

b. Specific - Is this space part of a suite?

Yes $\square \quad$ № $\square$

If yes, then describe its relationships to other spaces in the su

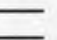

Activity Description:

$\rightarrow \quad$ The area is envisioned as an comprehensive welcoming, consultation and further Gr education post regarding the services provided within the community centre; a Activity type: (insert $x$ in applicable box)

$\begin{array}{lll}\text { 1. Admin Office } & \square & \text { 6. Education } \\ \text { 2. Private Office } & \text { 7. Therapy } \\ \text { 3. Staff Room } & \text { 8. Information } \\ \text { 4. Medical Clinic } & \square & \text { 9. Common Area } \\ \text { 5. Fitness } & \square & \text { 10.Other Specify: }\end{array}$

\section{Users: (insert number in appropriate boxes)}

$\begin{array}{llll}\text { Staff } & \text { Fitness Users } \\ \text { Patient } & \text { Therapy Users } \\ \text { Students } & \text { Avg. hours per week usage } \\ \text { Community } & & \end{array}$

Space Components
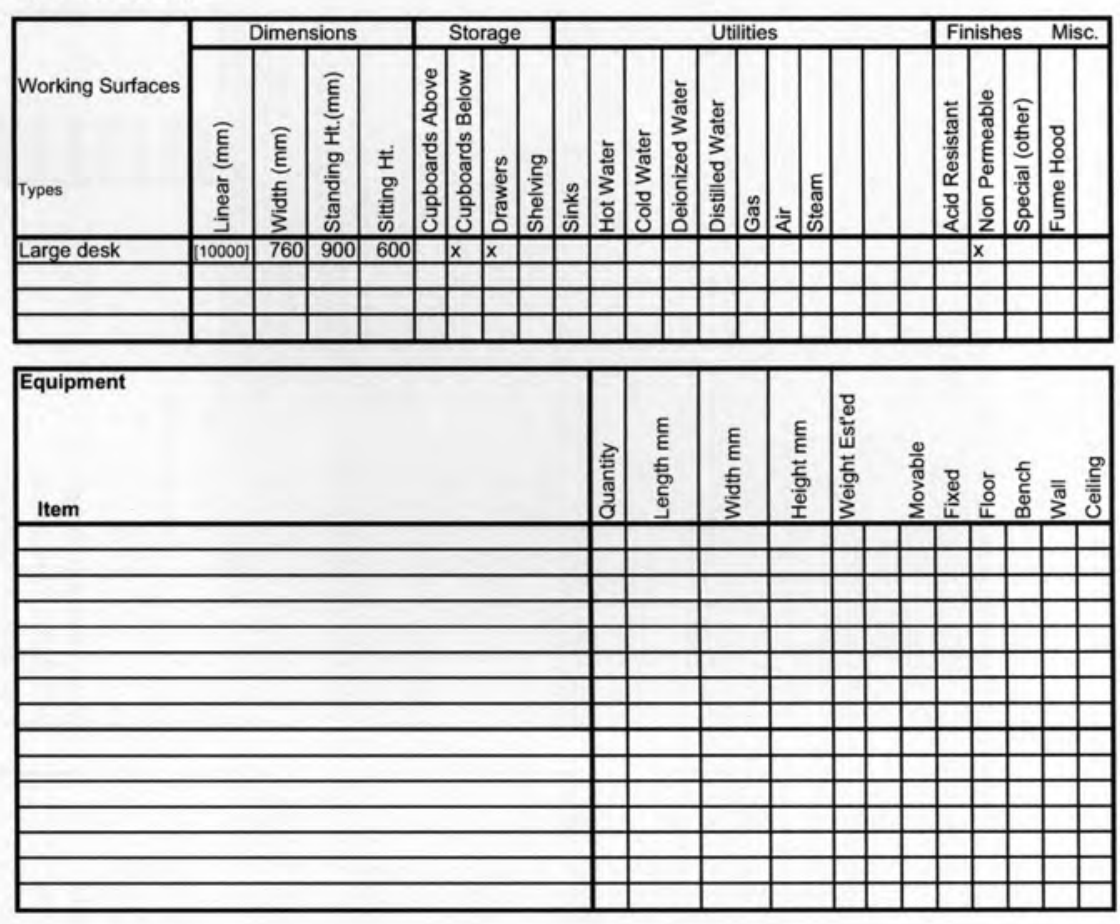

\begin{tabular}{ll|l|}
\hline $\begin{array}{l}\text { Communications: } \\
\text { (place Xin boxif required) } \\
\text { Telephone }\end{array}$ & $\begin{array}{l}\text { Audio Visual } \\
\text { The following audio visual aids will be used: } \\
\text { P.A System }\end{array}$ \\
Cable TV & $\begin{array}{l}\text { Television } \\
\text { Projector } \\
\text { Internet Connection }\end{array}$ \\
Other Specify: & Oound Reinforcement \\
\hline
\end{tabular}




\begin{tabular}{|ll|}
\hline Furnishings: & (place x in box if required) \\
\hline Storage Cabinets & $\square$ Info Board $\square \quad$ Shelving $\square$ \\
Fixed: (List) & 2 digital reference interfaces \\
Movable: (List) & Lounge chairs and table for up to 10 patrons \\
&
\end{tabular}

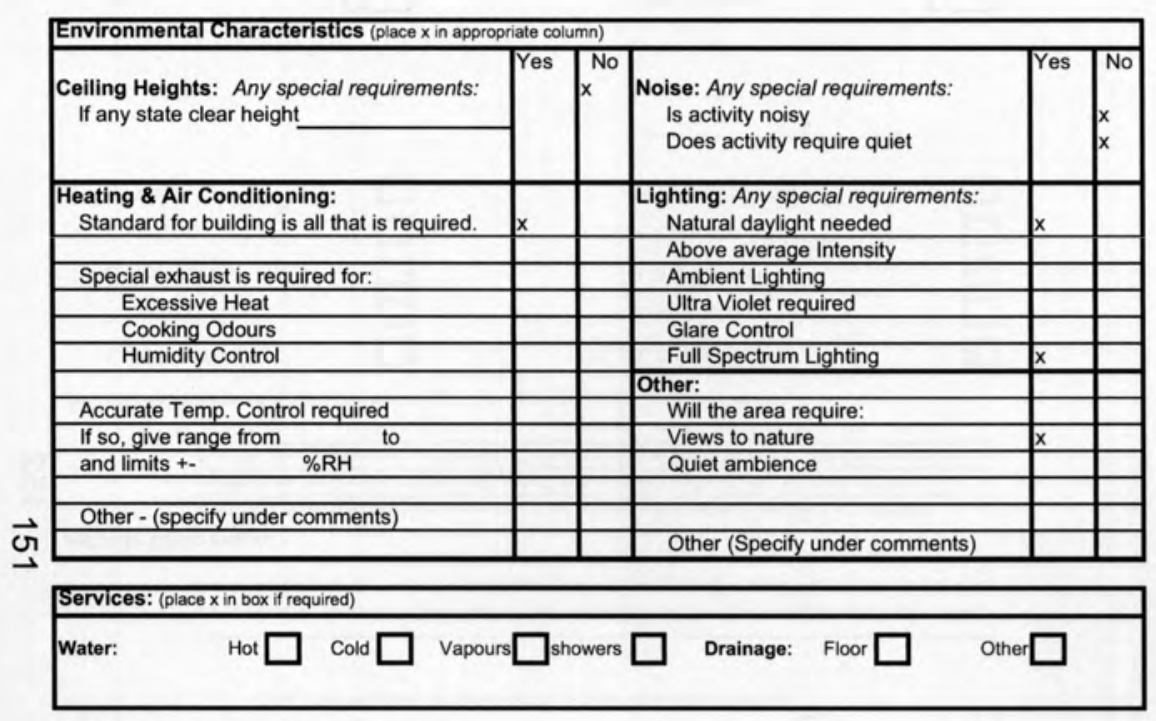

Additional Requirements or Comments:

$\longrightarrow$

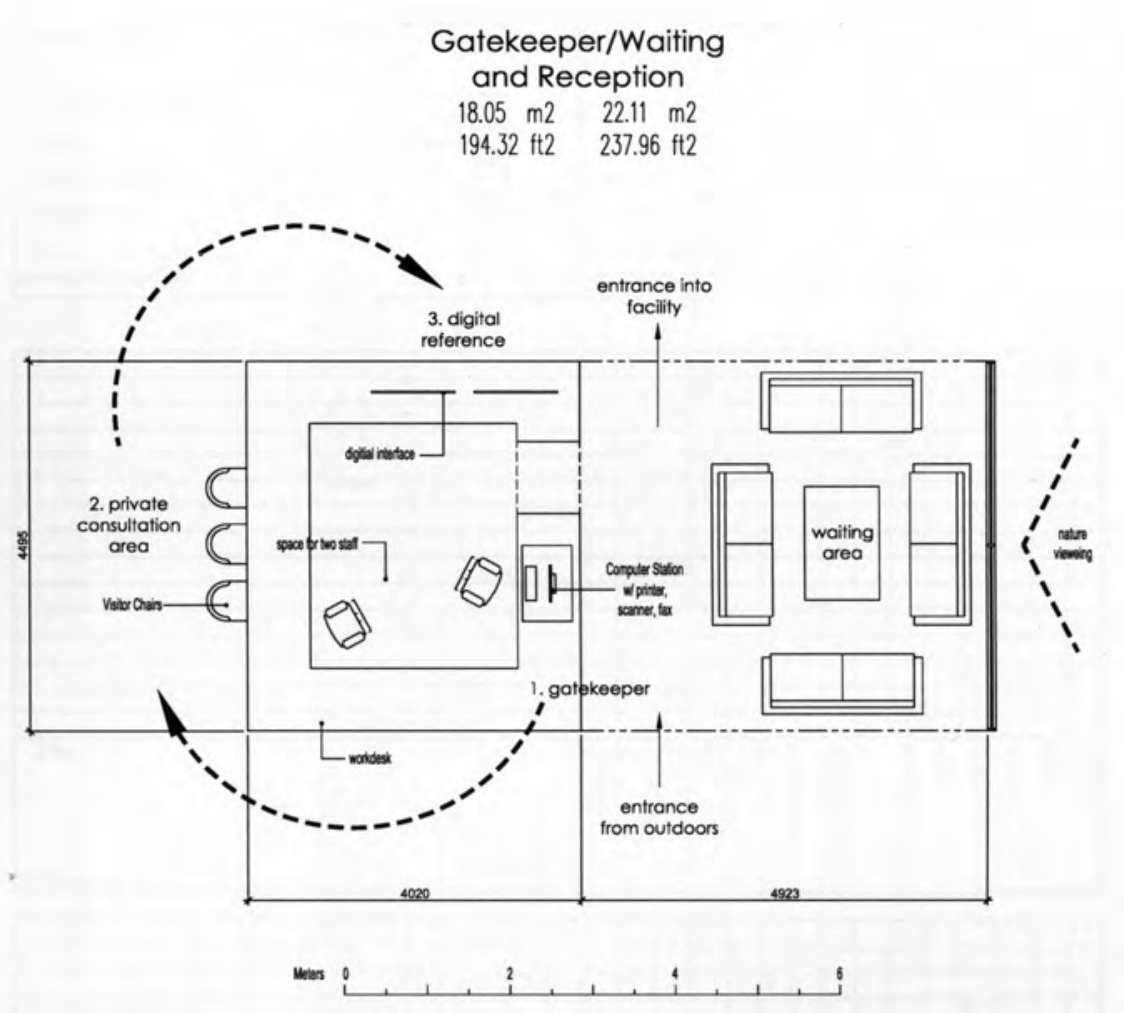




\section{Analysis of physical facility requirements}

Project: Wellness Centre 2009

Name of Space: Information Kiosk Department $\frac{\text { Community Edu. }}{40}$
Net Assignable Sq. m

\section{Space Description}

Purpose: An area dedicated to providing community knowledge regarding health services, alternative therapies and methods of disease prevention

ships:

Space Relationships:
a. General - How should this space relate to general areas in the building? eg: offices, teaching areas, loading dock, storage areas.

Located close to the entrance area of the facility, and ajacent to the gatekeeper and its combined services

b. Specific - Is this space part of a suite?

Yes $\square$

If yes, then describe its relationships to other spaces in the suite

No

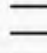

\section{Activity Description:}

$\vec{N}$

A space that offers digital resources, including internet resources and medical databases for those community members interested in taking control of their own health- the gatekeeper will help patrons logging onto resources with further guidance

Activity type: (insert $x$ in applicable box)

1. Admin Office
2. Private Office
3. Staff Room
4. Medical Clinic
5. Fitness

6. Education
7. Therapy
8. Information
9. Common Area
10.Other Specify:

Users: (insert number in appropriate boxes)

$\begin{array}{llll}\text { Staff } & \text { Fitness Users } \\ \text { Patient } & \text { Therapy Users } \\ \text { Students } & & \\ \text { Community } & \text { Avg. hours per week usage } & \end{array}$

Space Components
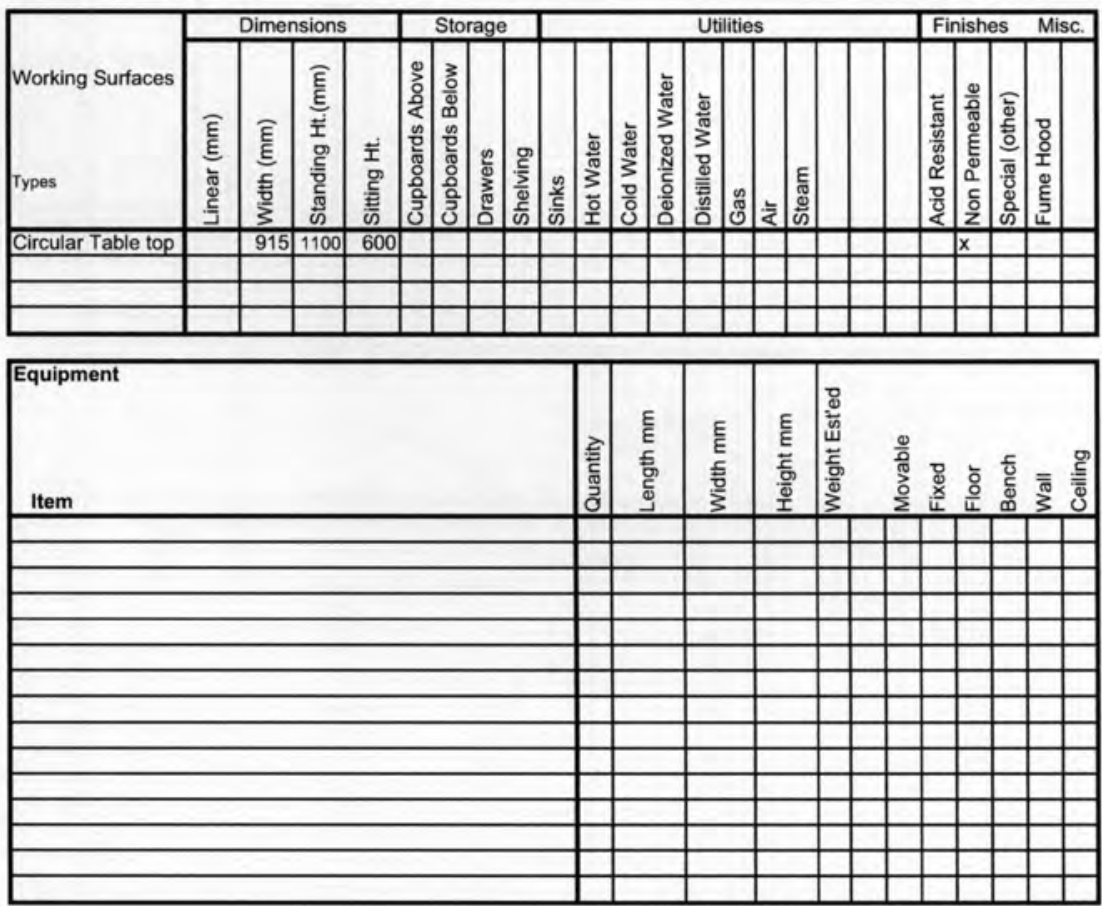

\begin{tabular}{l|l|l}
\hline $\begin{array}{l}\text { Communications: } \\
\text { (place } X \text { in box If required) } \\
\text { Telephone }\end{array}$ & $\begin{array}{l}\text { Audio Visual } \\
\text { The following audio visual aids will be used: } \\
\text { P.A System }\end{array}$ \\
Cable TV & Television \\
Internet Connection & Projector \\
Other Specify: & Sound Reinforcement \\
\hline
\end{tabular}




\begin{tabular}{|ll|}
\hline Furnishings: & (place x in box if required) \\
\hline Storage Cabinets & $\square$ info Board $\square \quad$ Shelving $\square \quad$ Computer Station $\square$ \\
Fixed: (List) & 8 fixed internet stations @ 3 computers each (24 computers total) \\
\cline { 2 - 2 } & \\
\hline Movable: (List) &
\end{tabular}

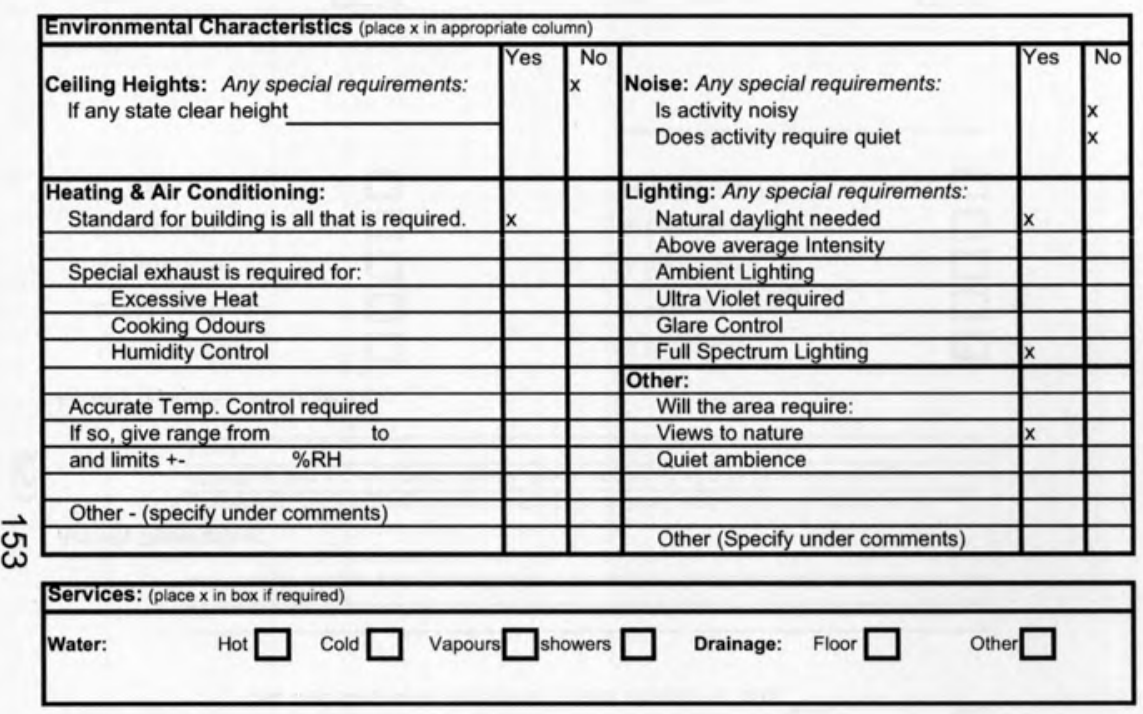

Additional Requirements or Comments:

this space should be adjacent to the café market
Information Kiosks

$40.50 \mathrm{~m} 2$

$435.94 \mathrm{ft} 2$

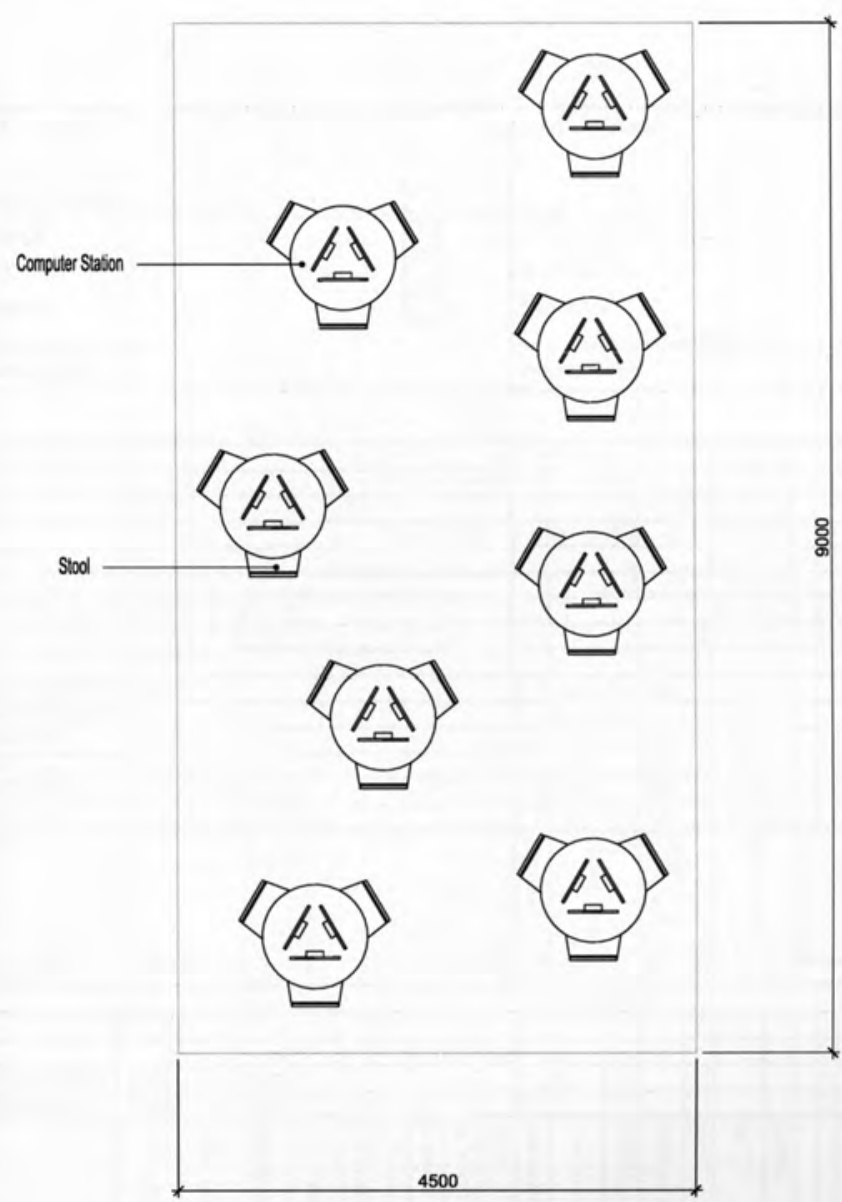

Meiers

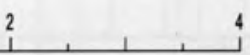




\section{Analysis of physical facility requirements}

Project: Wellness Centre 2009

Name of Space: Community Kitchen Classroom

Department Community Edu. Net Assignable Sq. m

\section{Space Description}

Purpose: To provide an area for community nutritional education

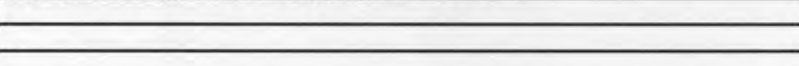

\section{Space Relationships:}

a. General - How should this space relate to general areas in the building? eg: offices, teaching areas, loading dock, storage areas.

Located close to the entrance and majority of the community programming

Located ose

$\begin{array}{ll}\text { b. Specific - Is this space part of a suite? } & \text { If yes, then describe its relationships to other spaces in the suite } \\ \end{array}$

Activity Description:

$\vec{C} \quad$ Community cooking classes will teach both children and adults the importance $\vec{\perp} \quad$ of both eating locally grown organic foods and ways of prepearing nutritional

Activity type: (insert $x$ in applicable box)

1. Admin Office
2. Private Office
3. Staff Room
4. Medical Clinic
5. Fitness

6. Education

7. Therapy

8. Information

9. Common Area

10.Other Specify:

区

$\square$

$\square$

Users: (insert number in appropriate boxes)

$\begin{array}{llll}\text { Staff } & \text { Fitness Users } \\ \text { Patient } & \text { Therapy Users } \\ \text { Students } & & \\ \text { Community } & \text { Avg. hours per week usage }\end{array}$

Space Components
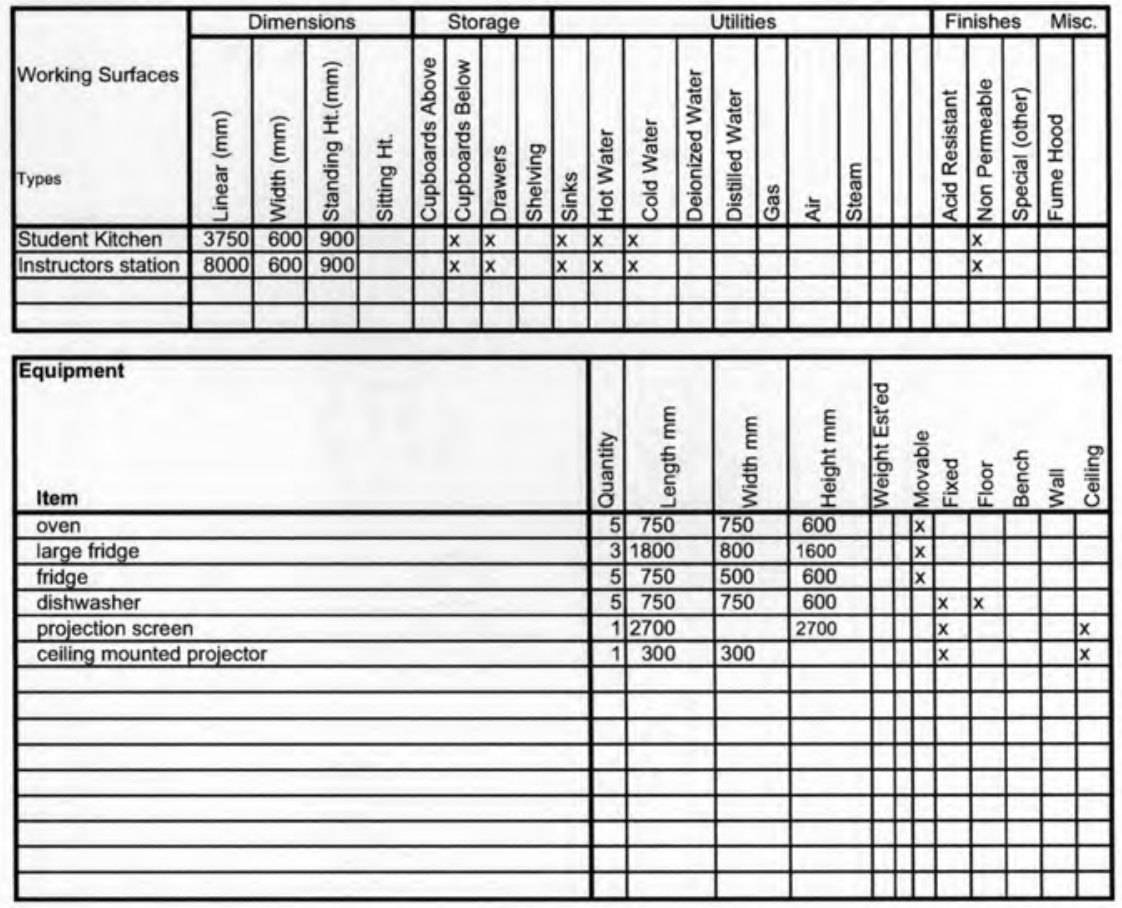

\begin{tabular}{ll|l|}
\hline $\begin{array}{l}\text { Communications: } \\
\text { (place X in box if required) } \\
\text { Telephone }\end{array}$ & $\begin{array}{l}\text { Audio Visual } \\
\text { The following audio visual aids will be used: } \\
\text { P.A System }\end{array}$ \\
Cable TV & Television \\
Internet Connection & Projector \\
Other Specify: & Sound Reinforcement \\
\hline
\end{tabular}




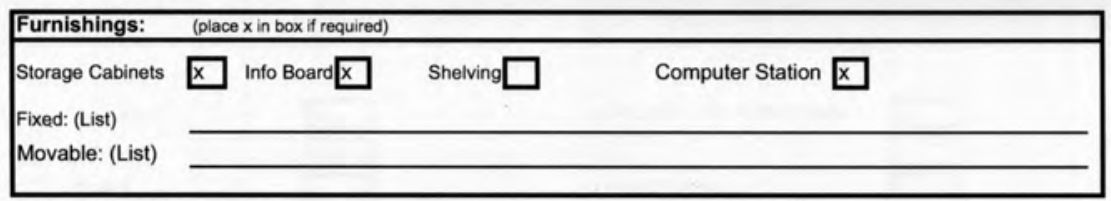

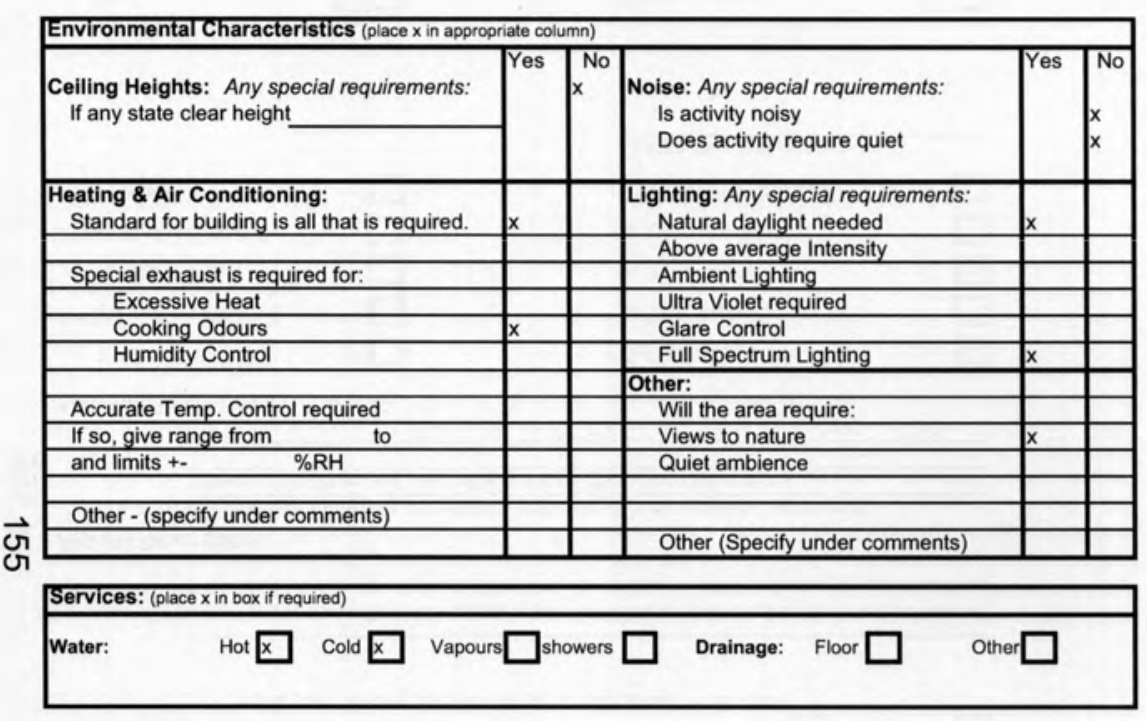

Additional Requirements or Comments:

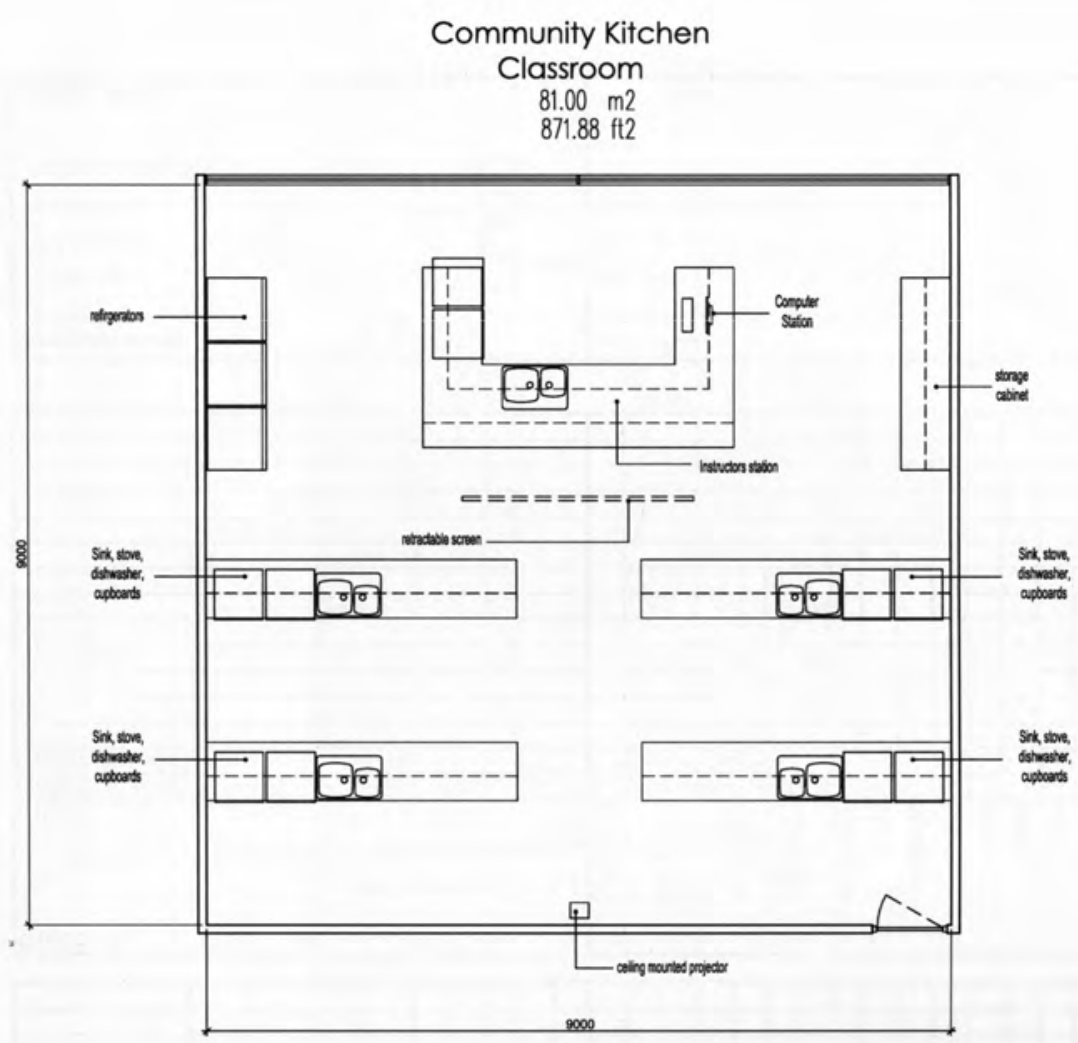




\section{Analysis of physical facility requirements}

Project: Wellness Centre 2009

Name of Space: Mulit-Functional Room

Department Community Edu Net Assignable Sq. m

\section{Space Description}

Purpose: To provide a multi-functional space for activities that involve community gahtering

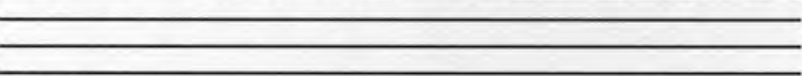

Space Relationships:

a. General - How should this space relate to general areas in the building? eg: offices, teaching areas, loading dock, storage areas.

Located close to the entrance and majority of the community programming

b. Specific - Is this space part of a suite?
If yes, then describe its relationships to other spaces in the suite
If yes, then descibe its relationships to other speces in the Yes

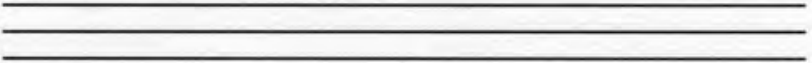

Activity Description:

a space that facilitates community activities- this can include community classes, meetings and presentations from the community at large

Activity type: (insert $x$ in applicable box)

$\begin{array}{lll}\text { 1. Admin Office } & \square & \text { 6. Education } \\ \text { 2. Private Office } & \text { 7. Therapy } \\ \text { 3. Staff Room } & \text { 8. Information } \\ \text { 4. Medical Clinic } & \square & \text { 9. Common Area } \\ \text { 5. Fitness } & \square & \text { 10.Other Specify: } \\ & \text { Community gathering }\end{array}$

Users:

(insert number in appropriate boxes)

$\begin{array}{lll}\text { Staff } & \text { Fitness Users } \\ \text { Patient } & \text { Therapy Users } \\ \text { Students } & \text { Avg. hours per week usage } \\ \text { Community } & \end{array}$

Space Components
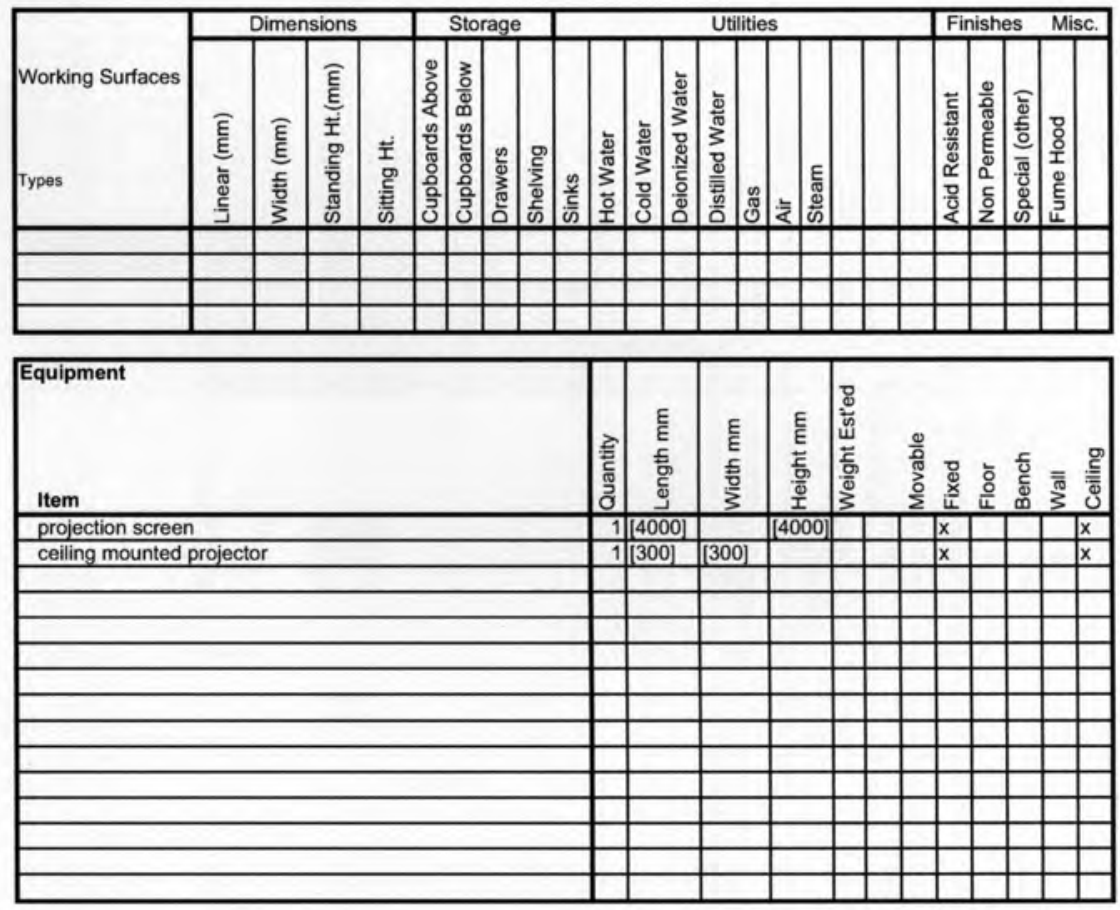

\begin{tabular}{ll|l|}
\hline $\begin{array}{l}\text { Communications: } \\
\text { (place X in box if required) } \\
\text { Telephone }\end{array}$ & $\begin{array}{l}\text { Audio Visual } \\
\text { The following audio visual aids will be used: } \\
\text { P.A System }\end{array}$ \\
Cable TV & $\begin{array}{l}\text { Television } \\
\text { Projector } \\
\text { Internet Connection }\end{array}$ \\
Other Specity: & Sound Reinforcement \\
\hline
\end{tabular}




\begin{tabular}{|ll|}
\hline Furnishings: $\quad$ (place x in box if required) \\
\hline Storage Cabinets \\
Fixed: (List) \\
Movable: (List)
\end{tabular}

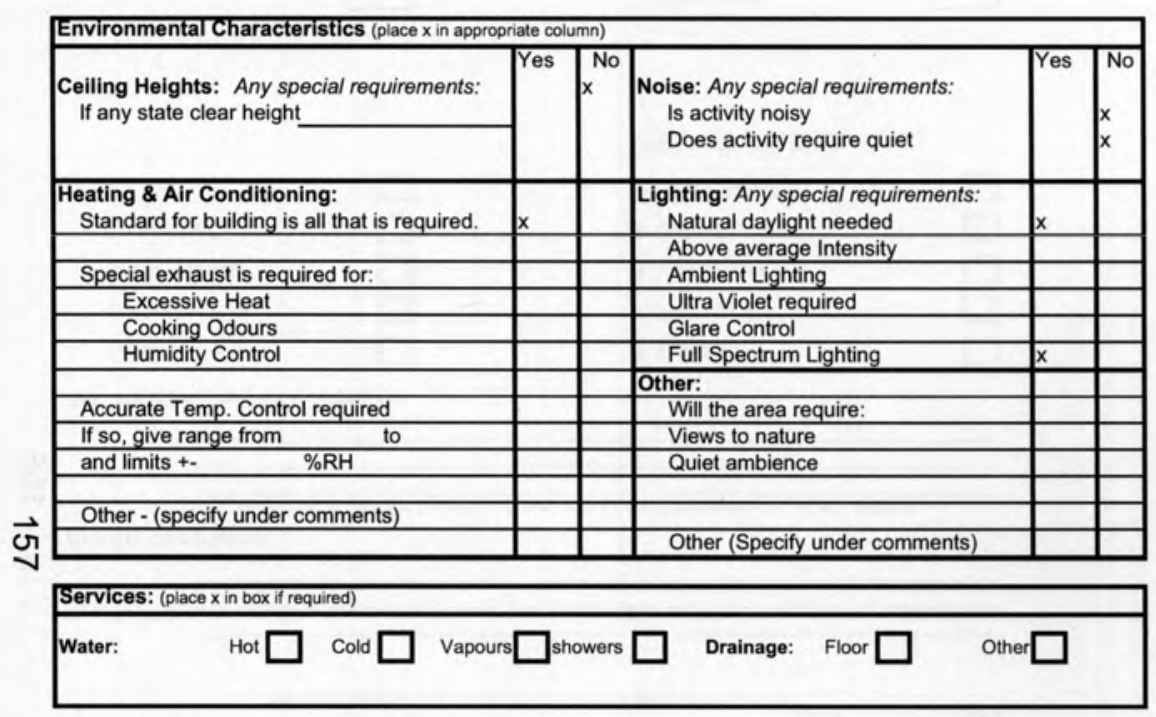

Additional Requirements or Comments:

$\overline{ }$

Multi-Functional Room

$81.00 \mathrm{~m} 2$

$871.88 \mathrm{ft} 2$

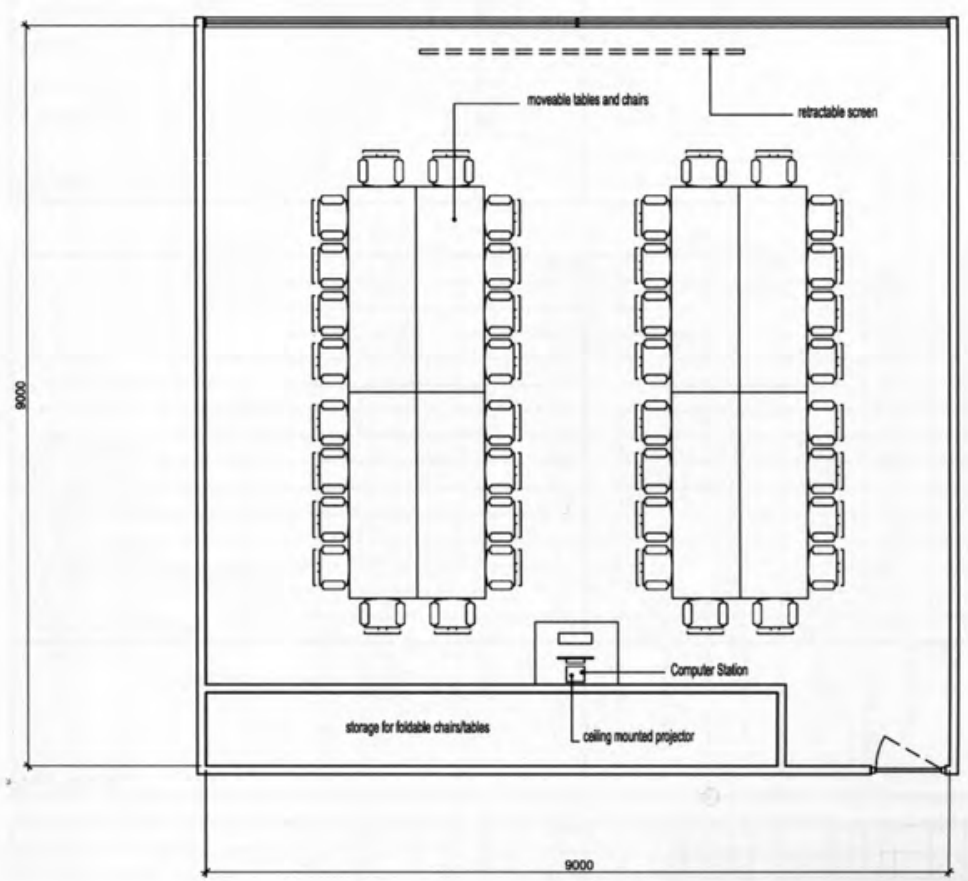

men 


\section{Analysis of physical facility requirements}

Project: Wellness Centre 2009

Name of Space: Market café

Department Community Edu Net Assignable Sq. m

\section{Space Description}

Purpose: To allow for a healthy community food source and social space

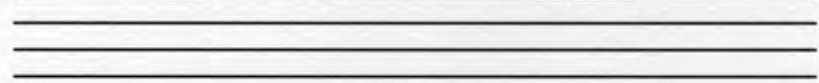

Space Relationships:

a. General - How should this space relate to general areas in the building? eg: offices, teaching areas, loading dock, storage areas.

Located close to the entrance and majority of the community programming

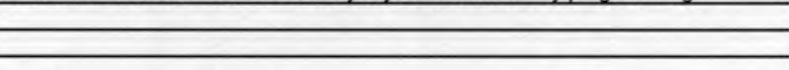

b. Specific - Is this space part of a suite?

Yes $\square \quad$ № $\square$

If yes, then describe its relationships to other spaces in the sult

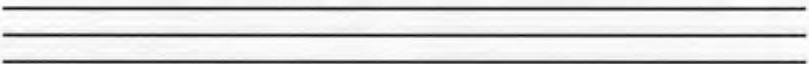

Activity Description:

ज्ञ

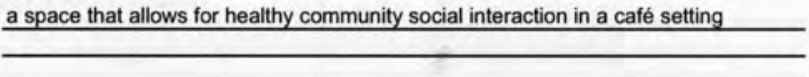

Activity type: (insert $\mathrm{x}$ in applicable box)

$\begin{array}{lll}\text { 1. Admin Office } & \text { 6. Education } \\ \text { 2. Private Office } & \text { 7. Therapy } \\ \text { 3. Staff Room } & \text { 8. Information } \\ \text { 4. Medical Clinic } & \text { 9. Common Area } \\ \text { 5. Fitness } & \square & \text { 10.Other Specify: }\end{array}$

Users: (insert number in appropriate boxes)

$\begin{array}{lll}\text { Staff } & \text { Fitness Users } \\ \text { Patient } & \text { Therapy Users } \\ \text { Students } & \text { Avg. hours per week usage } \\ \text { Community } & & \end{array}$

Space Components
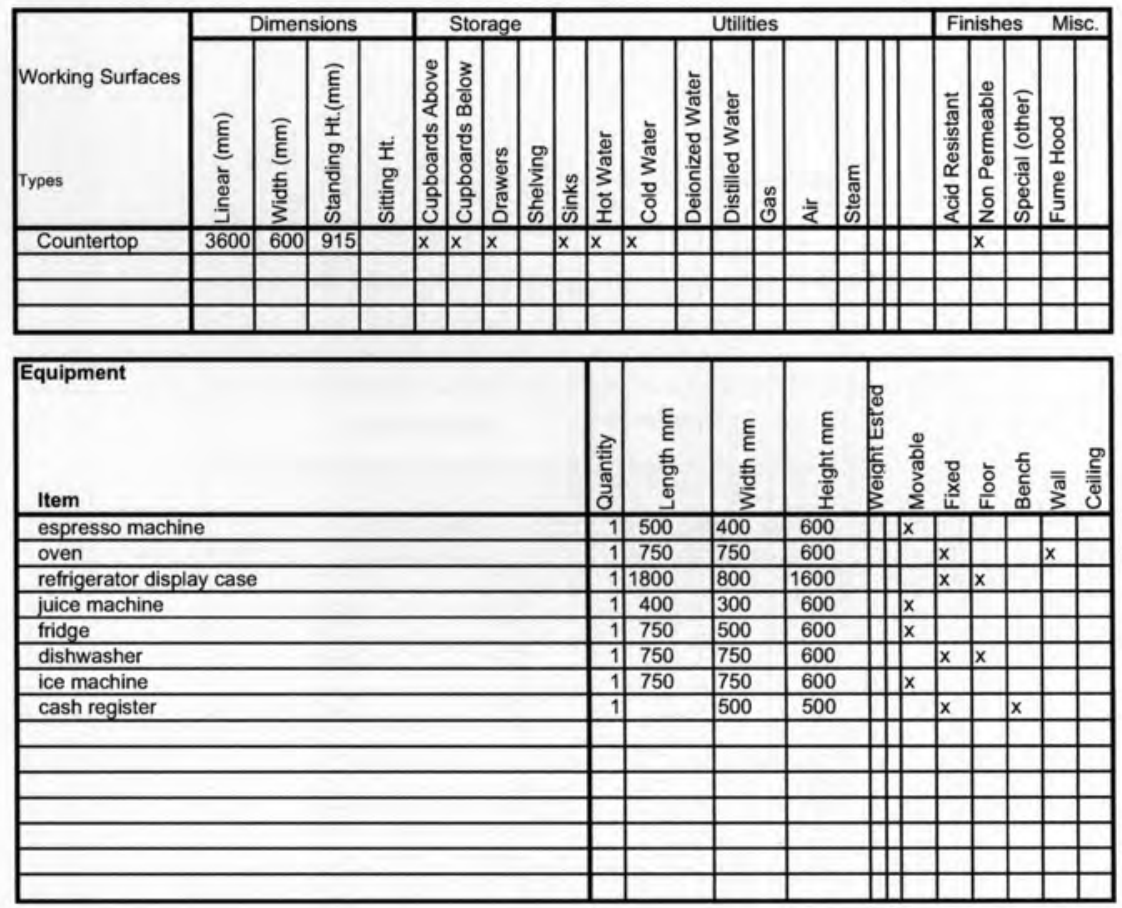

\begin{tabular}{ll|l|}
\hline $\begin{array}{l}\text { Communications: } \\
\text { (place X in box if required) } \\
\text { Telephone }\end{array}$ & $\begin{array}{l}\text { Audio Visual } \\
\text { The following audio visual aids will be used: } \\
\text { P.A System }\end{array}$ \\
$\begin{array}{l}\text { Cable TV } \\
\text { Internet Connection }\end{array}$ & $\begin{array}{l}\text { Television } \\
\text { Projector } \\
\text { Other Specify: Digital Wi-fi Zone }\end{array}$ & Sound Reinforcement \\
\hline
\end{tabular}




\begin{tabular}{|ll|}
\hline Furnishings: & (place x in boxif required) \\
\hline Storage Cabinets & $\square$ Info Board $\square \quad$ Shelving \\
Fixed: (List) & \\
Movable: (List) & 16 moveable chairs; 8 moveable tables \\
\hline
\end{tabular}

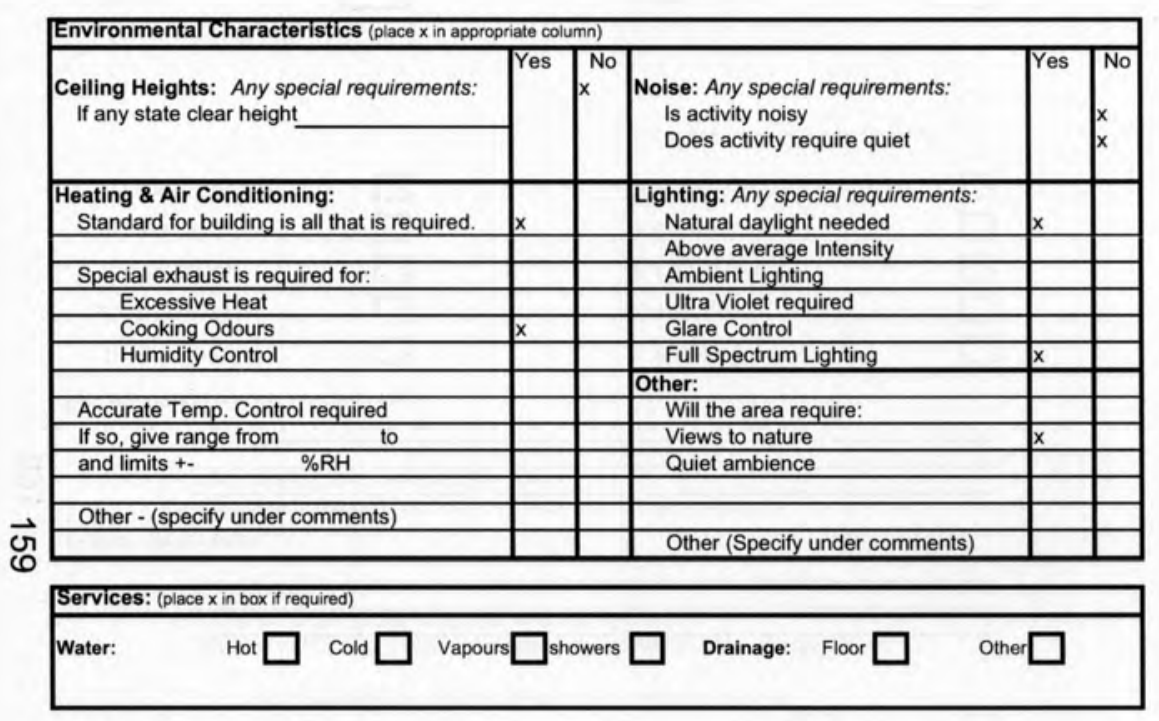

Additional Requirements or Comments:

Space will have a back storage area of $8 \mathrm{~m} 2$

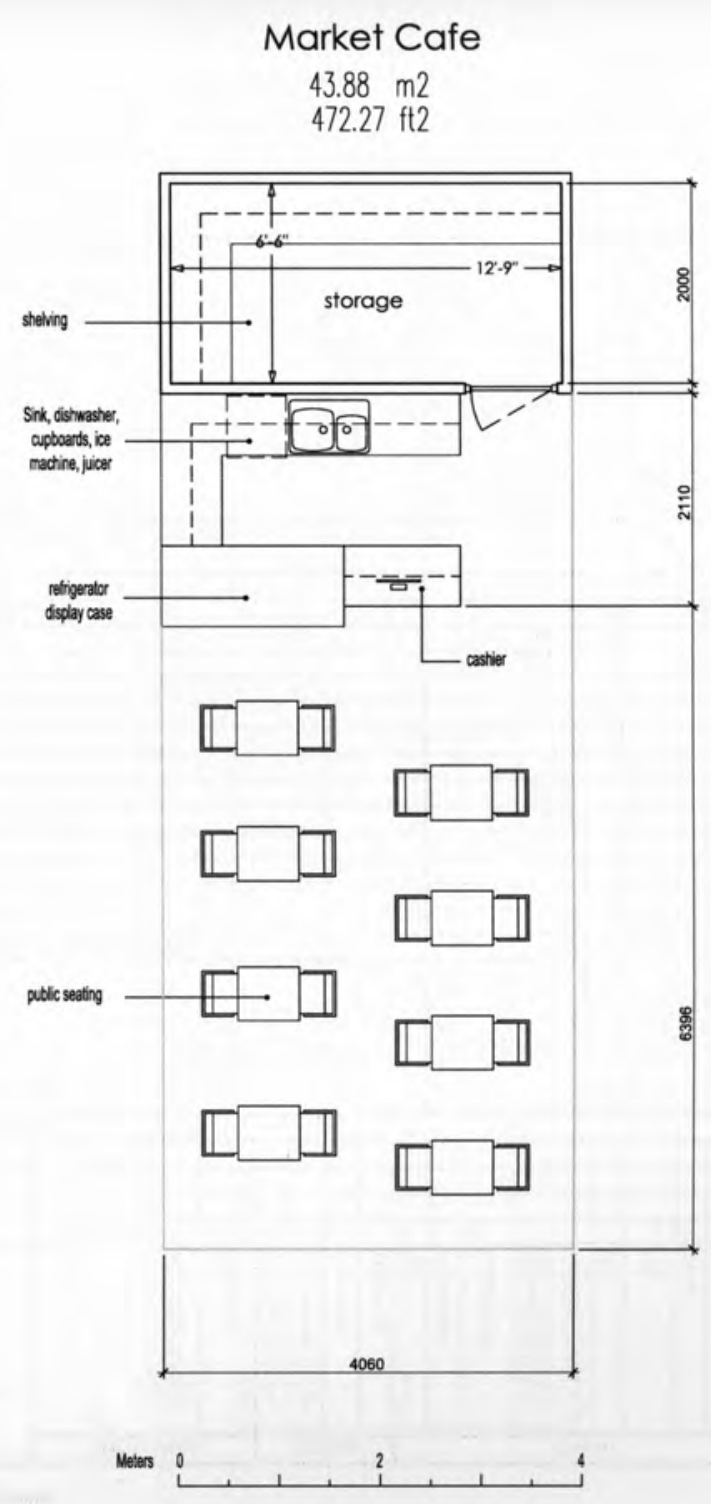




\section{Analysis of physical facility requirements}

Project: Wellness Centre 2009

Name of Space: Medical Examination Room

Department Clinical Wing

\section{Space Description}

Purpose: To provide area for examination of sick individuals, and individuals interested in alernative therpaies

Space Relationships:

a. General - How should this space relate to general areas in the building? eg: offices, teaching areas, loading dock, storage areas.

Proximity to main entrance; supported by satellite computer server workstations Yes $区$ № $\square$ If yes, then describe its relationships to other spaces in the suite Medical rooms shall be clustered together; access to other specialists can occur (as in the 'family health team' model of care)

\section{Activity Description:}

$\vec{\circ}$

consultation with practitioner to discuss patients level of health, or necessity of futher counseling

Activity type: (insert $x$ in applicable box)

1. Admin Office
2. Private Office
3. Staff Room
4. Medical Clinic
5. Fitness

Users: (insert number in appropriate boxes)

$\begin{array}{llll}\text { Staff } & \text { Fitness Users } \\ \text { Patient } & \text { Therapy Users } \\ \text { Students } & & \\ \text { Community } & \text { Avg. hours per week usage }\end{array}$

7. Therapy
8. Information
9. Common Area
10.Other Specify:

Space Components
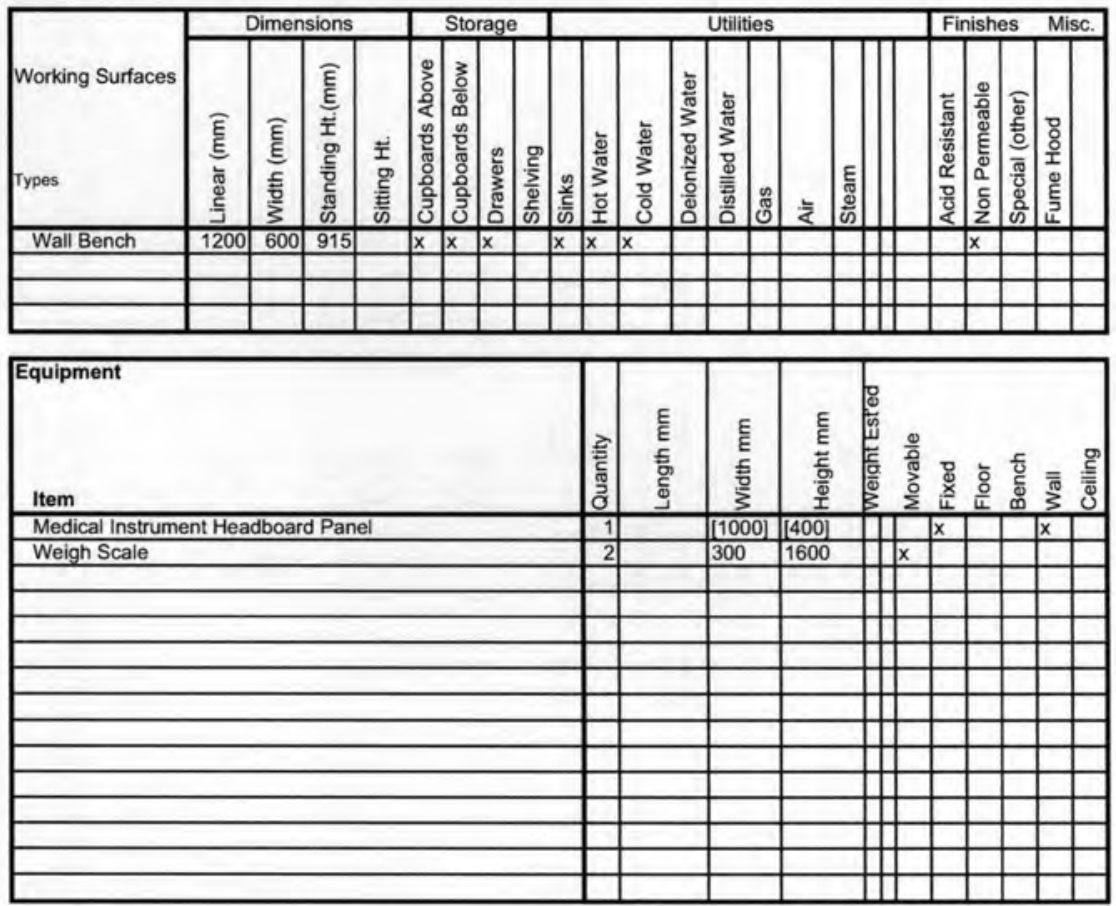

\begin{tabular}{ll|l|}
\hline $\begin{array}{l}\text { Communications: } \\
\text { (place X in box f frequired) } \\
\text { Telephone }\end{array}$ & $\begin{array}{l}\text { Audio Visual } \\
\text { The following audio visual aids will be used: } \\
\text { P.A System }\end{array}$ \\
Cable TV & Television \\
Internet Connection & Projector \\
Other Specify: & Sound Reinforcement \\
\hline
\end{tabular}




\begin{tabular}{|ll|}
\hline Furnishings: & (place x in boxif required) \\
\hline Storage Cabinets & $\square$ Info Board $\square \quad$ Shelving $\square$ \\
Fixed: (List) & Medical Exam bed \\
Movable: (List) & chairs $\times 2$ \\
\hline
\end{tabular}

\begin{tabular}{|c|c|c|c|c|c|}
\hline \multicolumn{6}{|c|}{ EnvironmentalCharacteristics (place $x$ in appropriate column) } \\
\hline $\begin{array}{l}\text { Ceiling Heights: Any special requirements: } \\
\text { If any state clear height }\end{array}$ & Tes & $x^{\text {No }}$ & $\begin{array}{l}\text { Noise: Any special requirements: } \\
\text { Is activity noisy } \\
\text { Does activity require quiet }\end{array}$ & Yes & $\overline{\text { No }}$ \\
\hline \multirow[t]{2}{*}{$\begin{array}{l}\text { Heating \& Air Conditioning: } \\
\text { Standard for building is all that is required. }\end{array}$} & $\mathrm{x}$ & & $\begin{array}{l}\text { Lighting: Any special requirements: } \\
\text { Natural daylight needed }\end{array}$ & $x$ & \\
\hline & & & Above average Intensity & & \\
\hline Special exhaust is required for: & & & Ambient Lighting & & \\
\hline Excessive Heat & & & Ultra Violet required & & \\
\hline Cooking Odours & & & Glare Control & & \\
\hline \multirow[t]{2}{*}{ Humidity Control } & & & Full Spectrum Lighting & $\mathrm{x}$ & \\
\hline & & & Other: & & \\
\hline Accurate Temp. Control required & & & Will the area require: & & \\
\hline \multirow{2}{*}{$\begin{array}{lll}\text { If so, give range from } & \text { to } \\
\text { and limits +- } & \% R H\end{array}$} & & & Views to nature & $x$ & \\
\hline & & & Quiet ambience & & \\
\hline \multirow{2}{*}{ Other - (specify under comments) } & & & & & \\
\hline & & & & & \\
\hline & & & Other (Specify under comments) & & \\
\hline \multicolumn{6}{|l|}{ Services: (place $x$ in box if required) } \\
\hline \multicolumn{3}{|l|}{ Water: } & Drainage: & & \\
\hline
\end{tabular}

Additional Requirements or Comments:

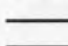

\section{Medical Exam Room}

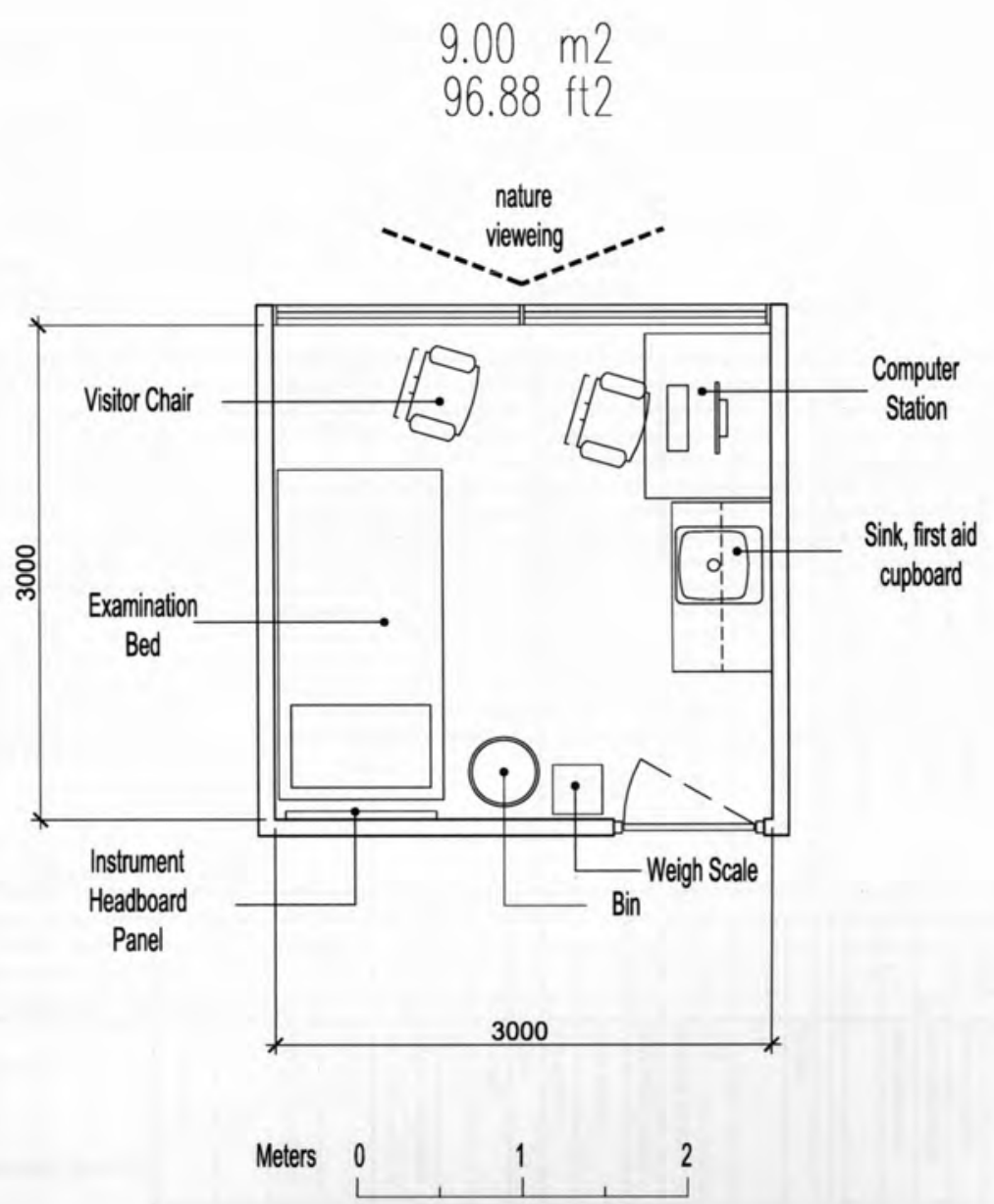




\section{Analysis of physical facility requirements}

Project: Wellness Centre 2009 Name of Space: Integrative Medical Examination Room

Department Clinical Wing Net Assignable Sq. m

\section{Space Description}

Purpose: To provide area for examination of sick individuals, and individuals interested in alernative therpaies

\section{Space Relationships:}

a. General - How should this space relate to general areas in the building? eg: offices, teaching areas, loading dock, storage areas.

Proximity to main entrance; supported by communal computer server workstations Pranterar Yes $\square$ № $\square$ If yes, then describe its relationships to other spaces in the suite Medical rooms shall be clustered together; access to other specialists can occur (as in the family health team' model of care)

Activity Description:

$\overrightarrow{\text { N }}$

consultation with practitioner to discuss patients level of health, or necessity of futher counseling: physical remediation

Activity type: (insert x in applicable box)

1. Admin Office

2. Private Office

$\square$

3. Staff Room

4. Medical Clinic

$\square$

$\square$

5. Fitness

6. Education

7. Therapy

8. Information

9. Common Area

10.Other Specify:

$\square$
$\square$
$\square$

Users:

(insert number in appropriate boxes)

Staff

Patient

Students

Community

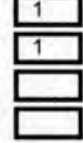

Fitness Users

Therapy Users

Avg. hours per week usage
Space Components
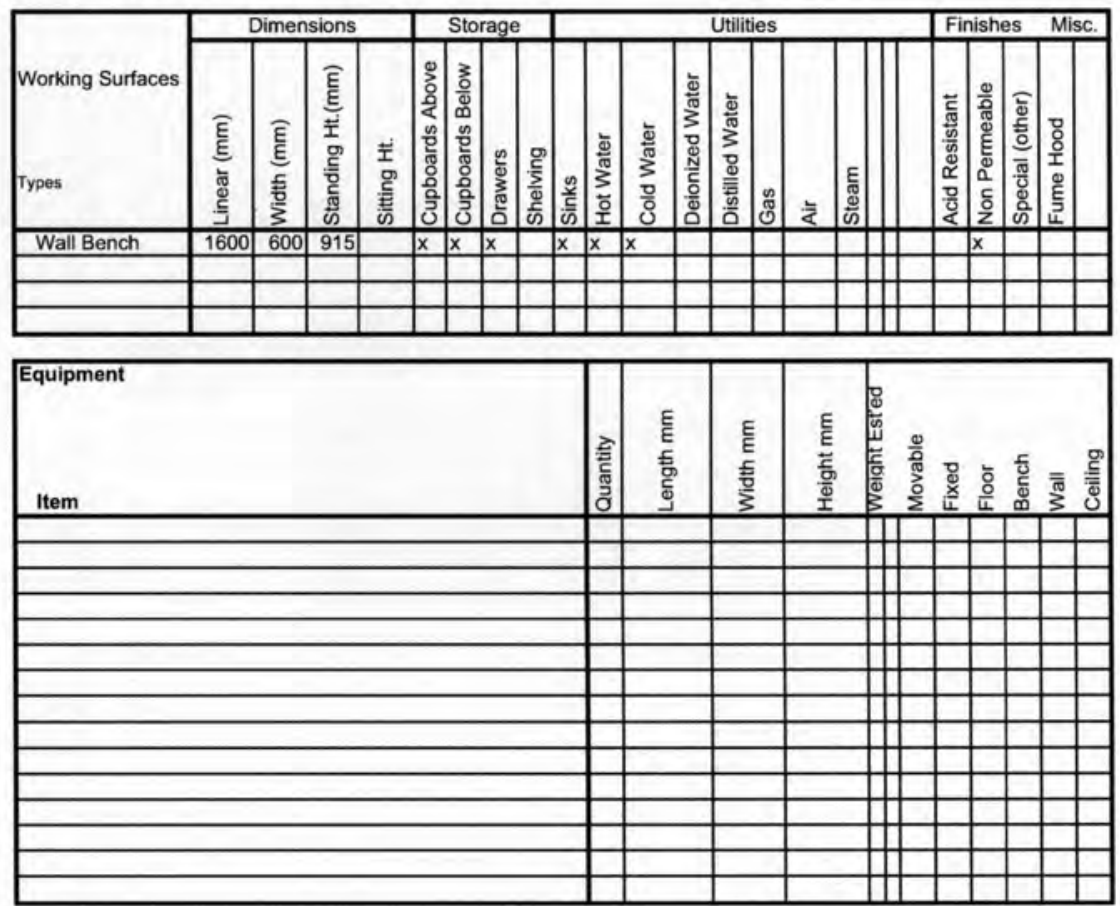

\begin{tabular}{|l|l|}
\hline $\begin{array}{l}\text { Communications: } \\
\text { (place X in box if required) } \\
\text { Telephone }\end{array}$ & $\begin{array}{l}\text { Audio Visual } \\
\text { The following audio visual aids will be used: } \\
\text { P.A System }\end{array}$ \\
Cable TV & Television \\
Internet Connection & Projector \\
Other Specify: & Sound Reinforcement \\
\hline
\end{tabular}




\begin{tabular}{|ll|}
\hline Furnishings: & (place x in box if required) \\
\hline Storage Cabinets & $\square$ info Board $\square \quad$ Shelving $\square \quad$ Computer Station $\square$ \\
Fixed: (List) & Exam bed, Chiropractors bed or massage table \\
& \\
\cline { 2 - 2 }
\end{tabular}

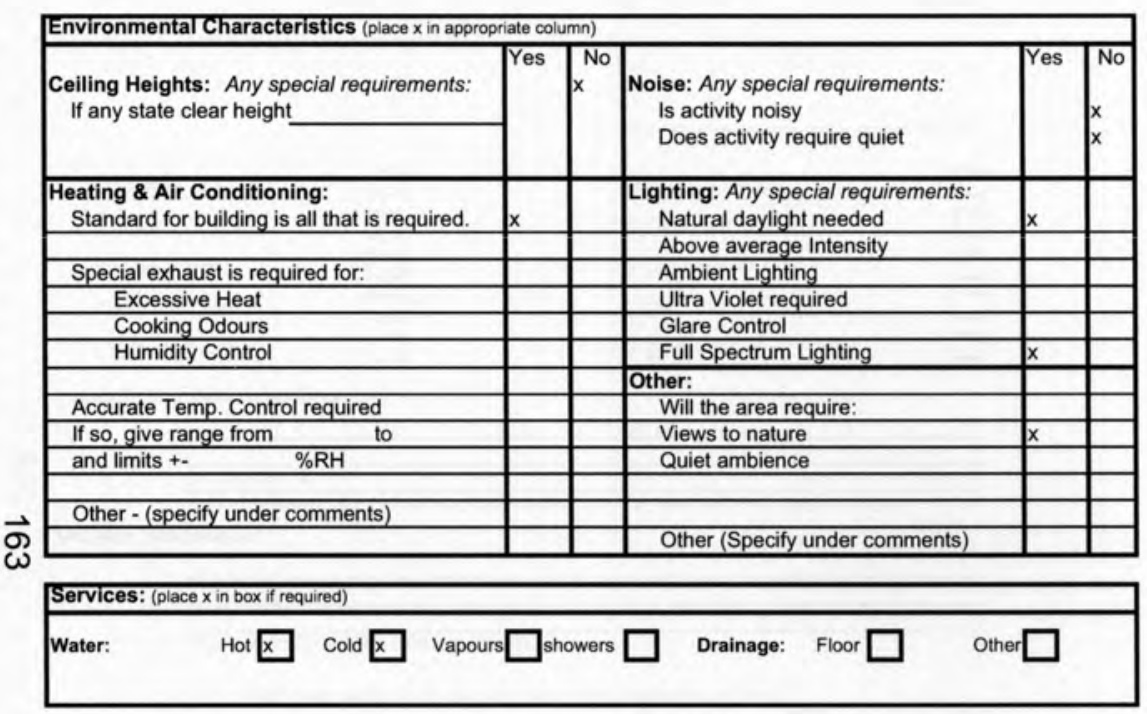

Additional Requirements or Comments:

Practitioners will utilise the communal satellite computer workstations to access medical server

\section{Integrative Medical Exam Room}

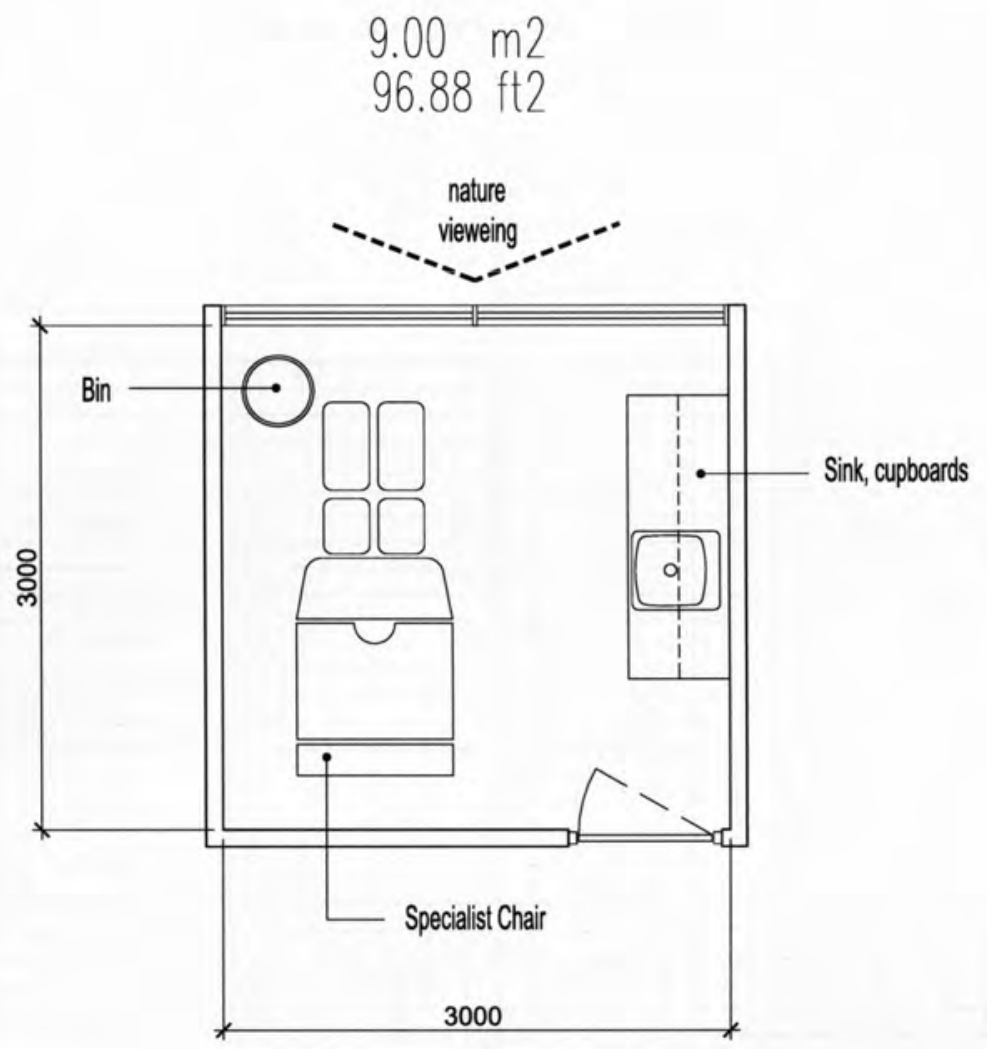

Meters

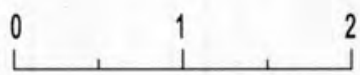




\section{Analysis of physical facility requirements}

Project: Wellness Centre 2009

Name of Space: Clinical Lab

Department Clinical wing

Net Assignable Sq. m

\section{Space Descriptio}

Purpose: To provide an area where clinical blood testing may occur

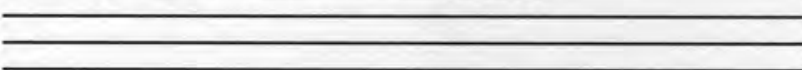

Space Relationships:

a. General - How should this space relate to general areas in the building? eg: offices, leaching areas, loading dock, storage areas.

close to medical examination rooms

- Is this space part of a suite?

b. Specific - Is this space part of a suite?

No $\square$

close to medical examination rooms

$\overline{\overline{ }} \overline{ }$

\section{Activity Description:}

$\vec{\triangleright}$

a lab for clinicians to conduct patient blood tests, analysis and interpretation
Activity type: (insert x in applicable box)
1. Admin Office
$\begin{array}{ll}\text { 2. Private Office } \\ \text { 3. Staff Room }\end{array}$
$\begin{array}{ll}\text { 4. Medical Clinic } & \text { 6. Education } \\ \text { 5. Fitness } & \text { 7. Therapy }\end{array}$

Users: (insert number in appropriate boxes)

$\begin{array}{lll}\text { Staff } & \text { Fitness Users } \\ \text { Patient } & \text { Therapy Users } \\ \text { Students } & & \\ \text { Community } & \text { Avg. hours per week usage }\end{array}$

Space Components
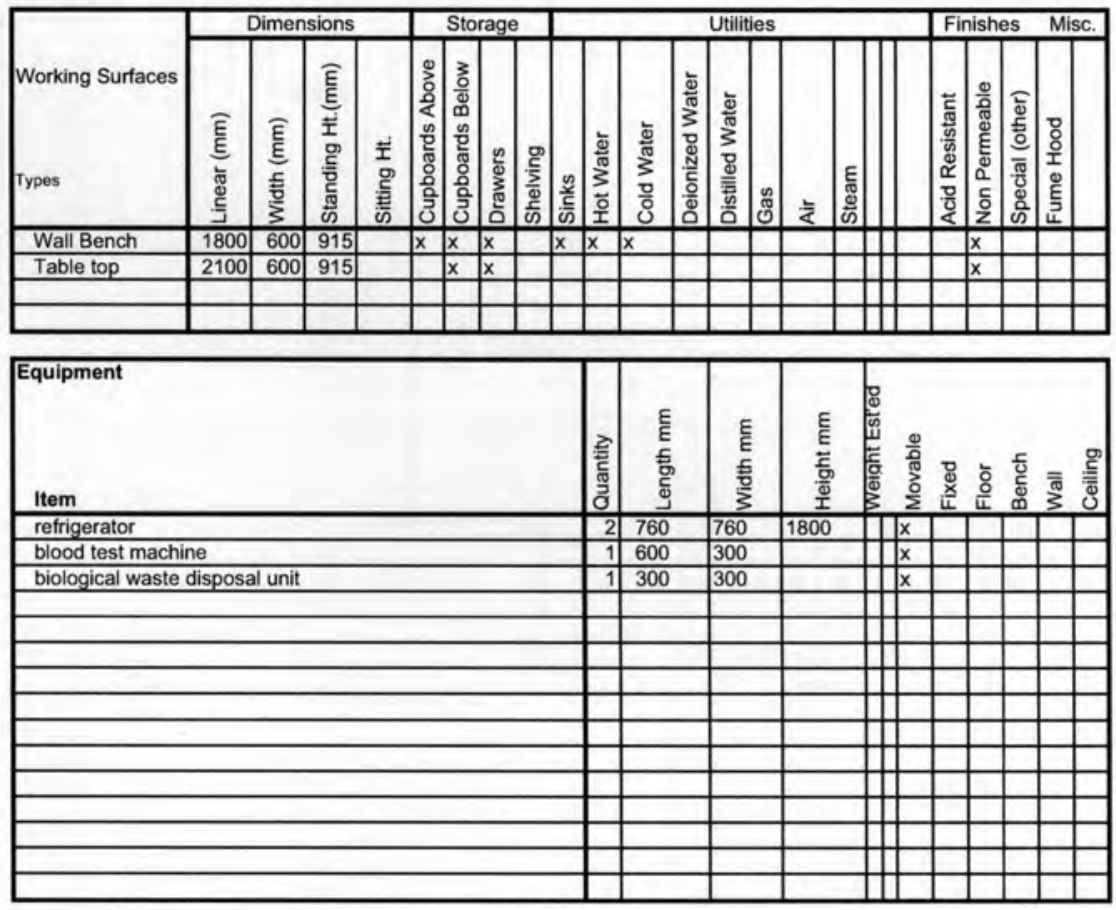

\begin{tabular}{ll|l|}
\hline $\begin{array}{l}\text { Communications: } \\
\text { (place X in box if required) } \\
\text { Telephone }\end{array}$ & $\begin{array}{l}\text { Audio Visual } \\
\text { The following audio visual aids will be used: } \\
\text { P.A System } \\
\text { Cable TV }\end{array}$ \\
Internet Connection & Television \\
Projector \\
Other Specify:
\end{tabular}




\begin{tabular}{|ll|}
\hline Furnishings: $\quad$ (place x in boxif required) \\
\hline Storage Cabinets \\
Fixed: (List) \\
Movable: (List)
\end{tabular}

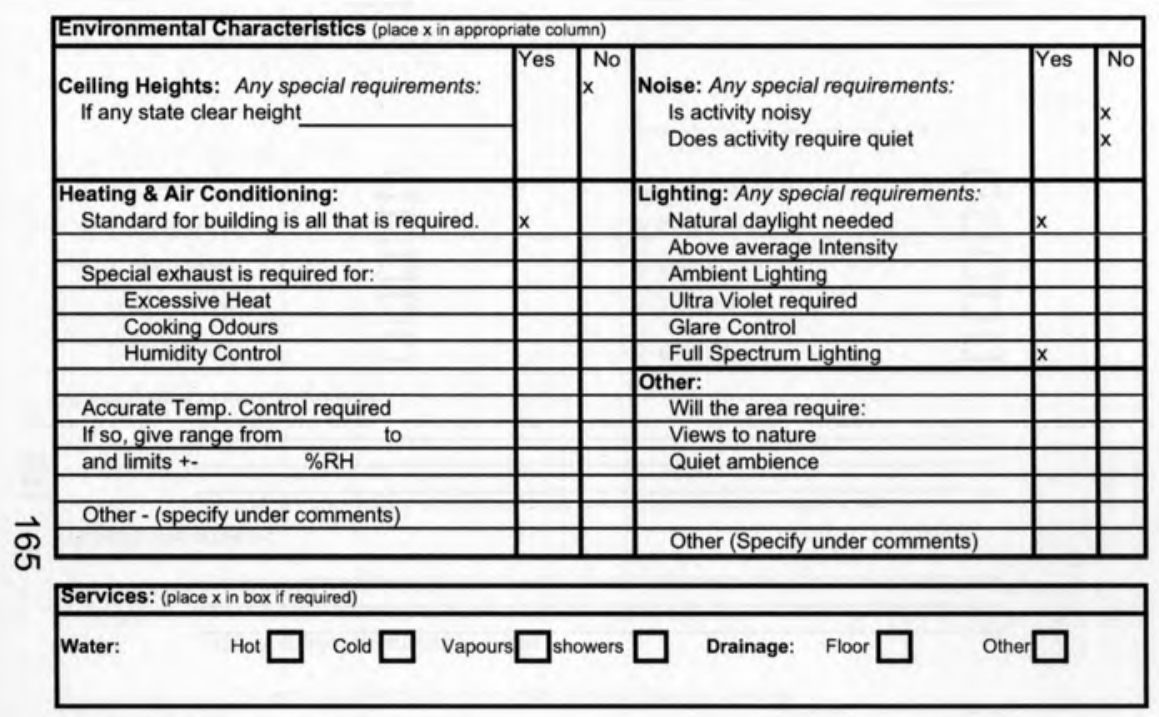

Additional Requirements or Comments:

$\overline{ }$

\section{Clinical Lab}

$13.50 \mathrm{~m} 2$

$145.31 \mathrm{ft} 2$

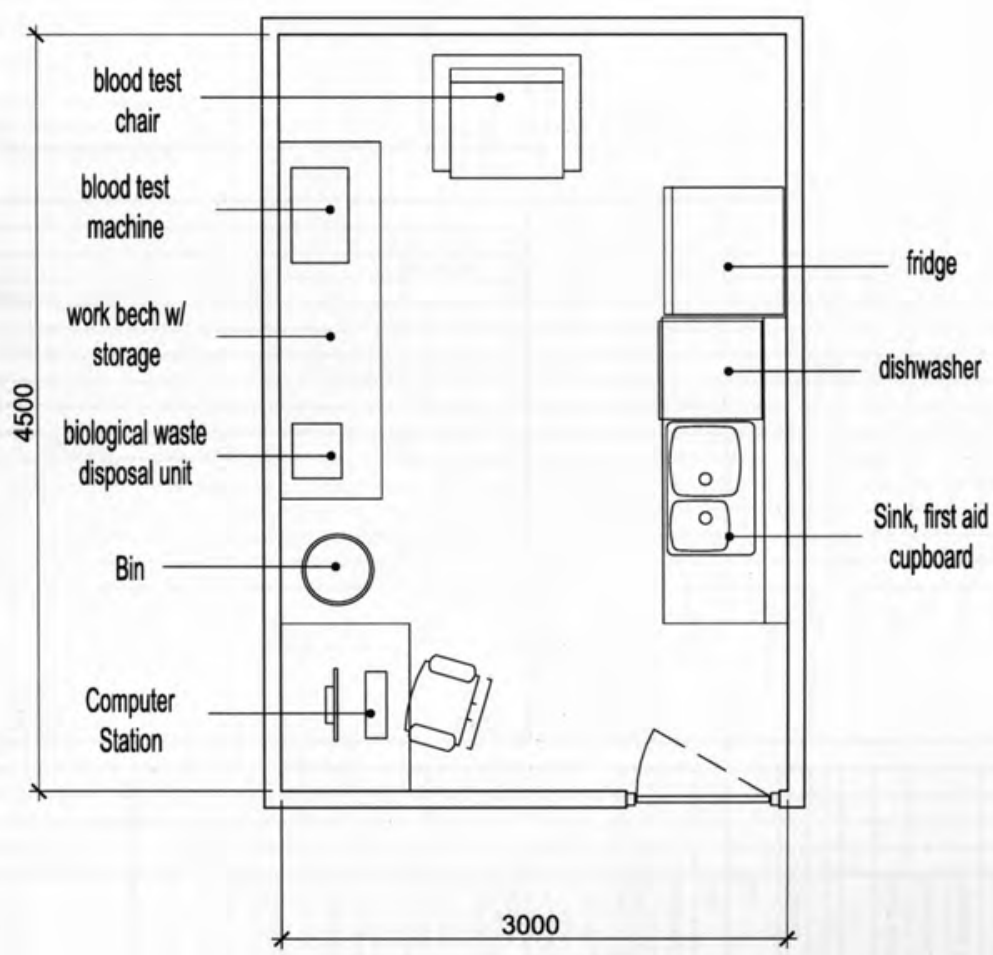

Meters $0 \quad 1 \quad 2$ 
Analysis of physical facility requirements

Project: Wellness Centre 2009

Name of Space: Office (manager)

Department Clinical wing

Net Assignable Sq. m

Space Description

Purpose: To provide an area for the clinical office manager

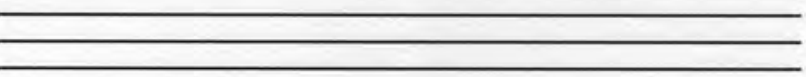

Space Relationships:

a. General - How should this space relate to general areas in the building? eg: offices, teaching areas, loading dock, storage areas.

close to medical examination rooms

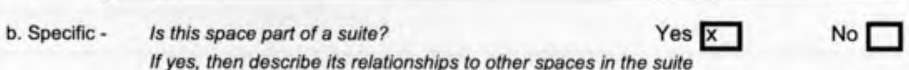

ships to other spaces in the suite close to medical examination rooms

Activity Description:

$\vec{\sigma}$

an area for the manger to supervise the successful operation of the clinic

Activity type: (insert $x$ in applicable box)

1. Admin Office

2. Private Office

3. Staff Room

4. Medical Clinic

5. Fitness

$\square$
$\square$
$\square$
$\square$

6. Education

7. Therapy

8. Information

9. Common Area

10.Other Specify:

$\square$
$\square$
$\square$
$\square$

Users: (insert number in appropriate boxes)

$\begin{array}{lll}\text { Staff } & \text { Fitness Users } \\ \text { Patient } & \text { Therapy Users } \\ \text { Students } & \end{array}$

Space Components
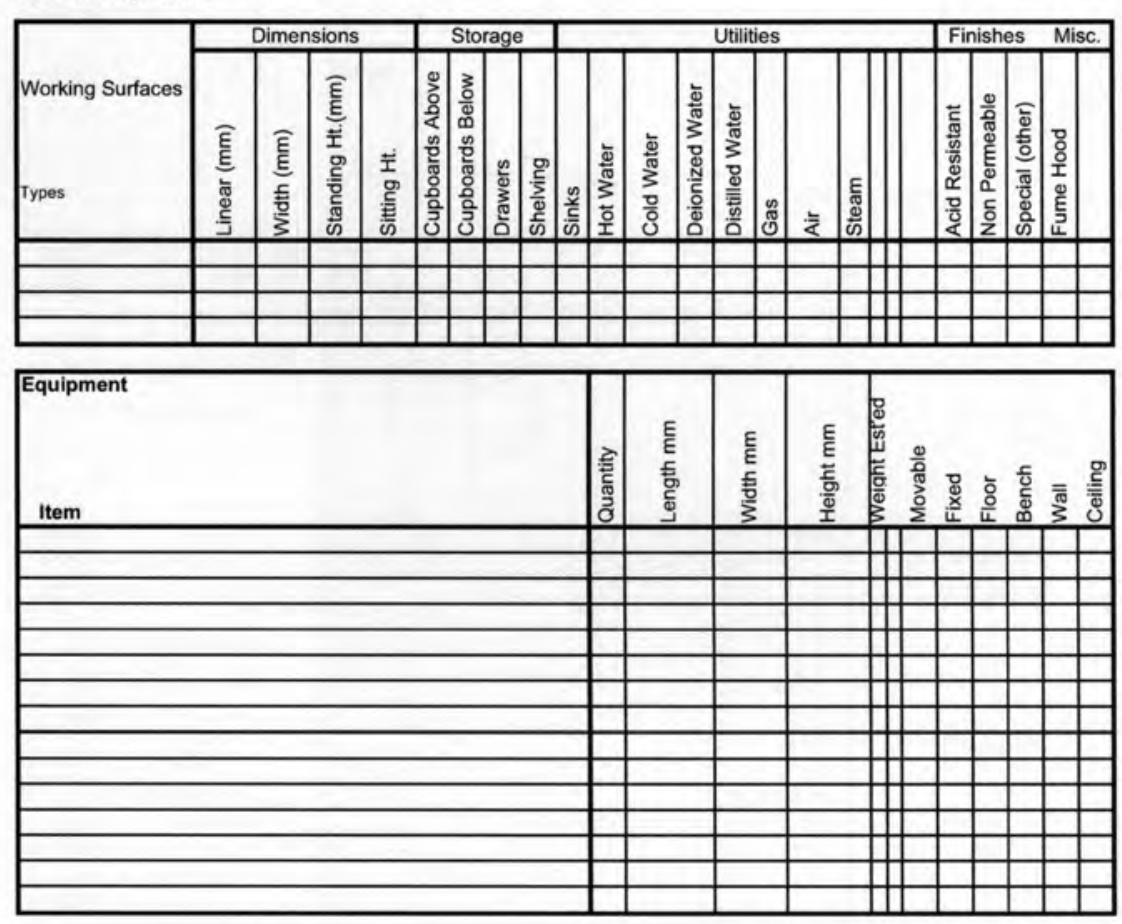

\begin{tabular}{ll|l|}
\hline $\begin{array}{l}\text { Communications: } \\
\text { (place X in box if required) } \\
\text { Telephone }\end{array}$ & $\begin{array}{l}\text { Audio Visual } \\
\text { The following audio visual aids will be used: } \\
\text { P.A System }\end{array}$ \\
$\begin{array}{l}\text { Cable TV } \\
\text { Internet Connection }\end{array}$ & $\begin{array}{l}\text { Television } \\
\text { Projector } \\
\text { Sound Reinforcement }\end{array}$ \\
Other Specify: & Other Specify: \\
\hline
\end{tabular}


Furnishings: (place $x$ in box if required)

Storage Cabinets $\square$ Info Board $\square \quad$ Shelving $\square \quad$ Computer Station $\square$

Fixed: (List)

Movable: (List) filing cabinets, computer chair and visitor chair

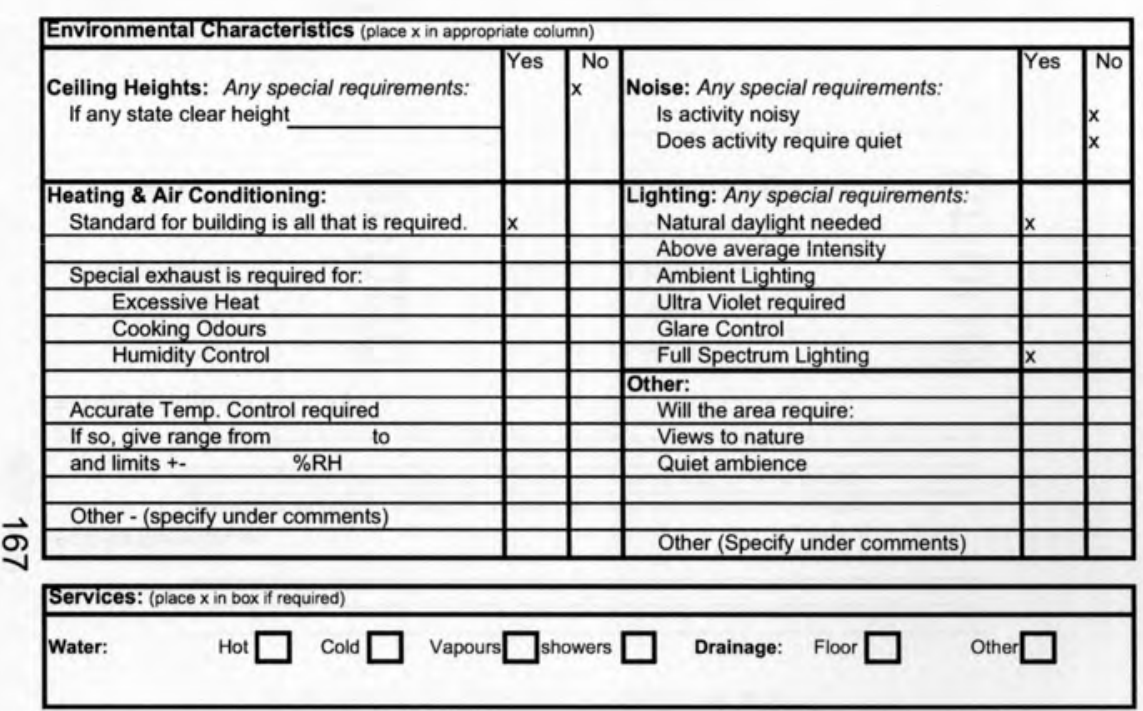

Additional Requirements or Comments:

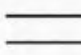

\section{Office \\ (office manager)}
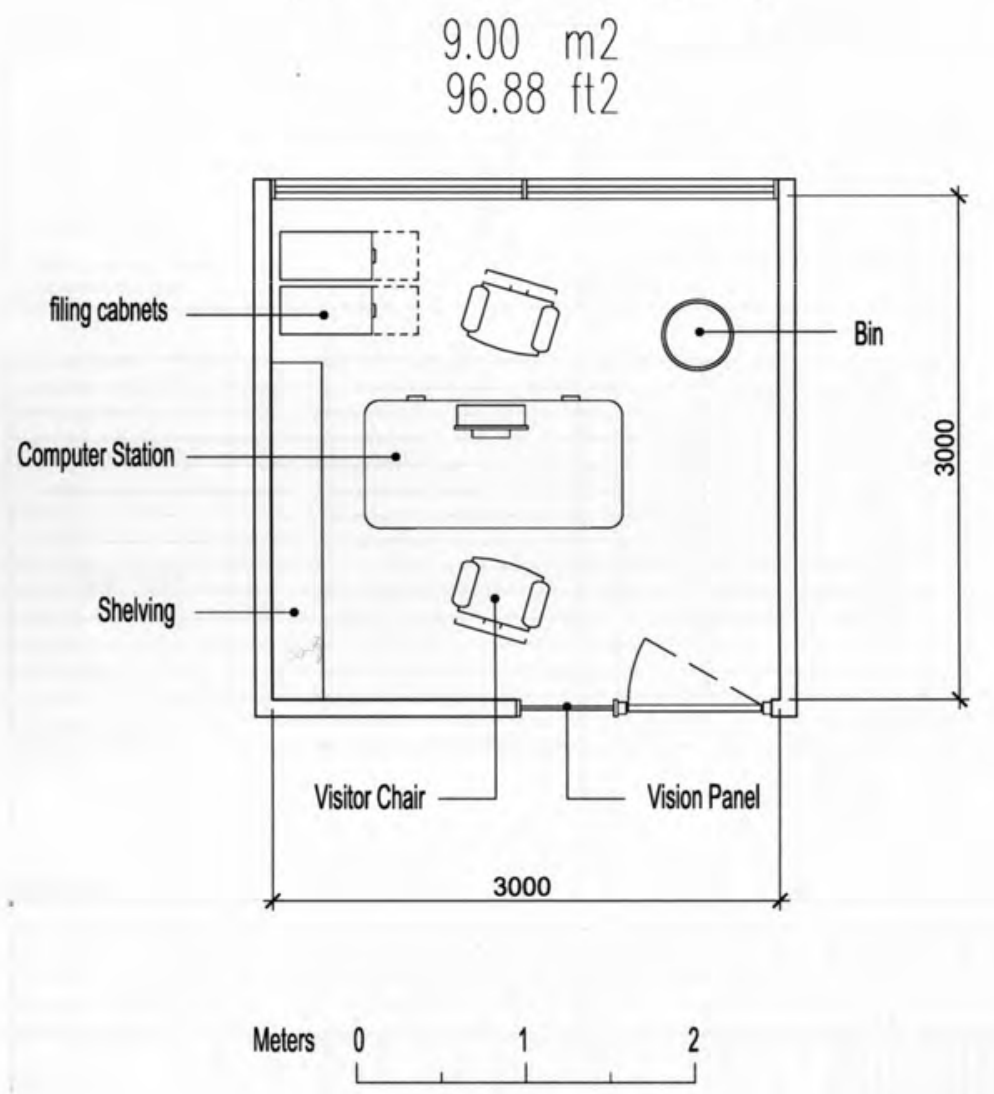


\section{Analysis of physical facility requirements}

Project: Wellness Centre 2009

Name of Space: Nutritionist Office

Department Fitness Wing Net Assignable Sq. m

\section{Space Description}

Purpose:

An area dedicated for nutritionist consultation

\section{Space Relationships:}

a. General - How should this space relate to general areas in the building? eg: offices, teaching areas, loading dock, storage areas.

within the medical clinic b. Specific - Is this space part of a suite?
If yes, then describe its relationships to other spaces in the suite

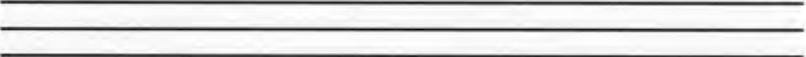

Activity Description:

$\overrightarrow{\quad \text { individuals interested in getting asessed for their personal levels of physical }}$

$\vec{\sigma} \quad$ fitness regarding nutritional information may consult with the nutritionist

\section{Activity type: (insert $\mathrm{x}$ in applicable box)}

$\begin{array}{llll}\text { 1. Admin Office } & \square & \text { 6. Education } & \\ \text { 2. Private Office } & \square & \text { 7. Therapy } & \\ \text { 3. Staff Room } & \square & \text { 8. Information } & \\ \text { 4. Medical Clinic } & \square & \text { 9. Common Area } & \square \\ \text { 5. Fitness } & \square & \text { 10.Other Specify: }\end{array}$

Users:

(insert number in appropriate boxes)

$\begin{array}{lll}\text { Staff } & \text { Fitness Users } \\ \text { Patient } & \text { Therapy Users } \\ \text { Students } & \end{array}$

Space Components
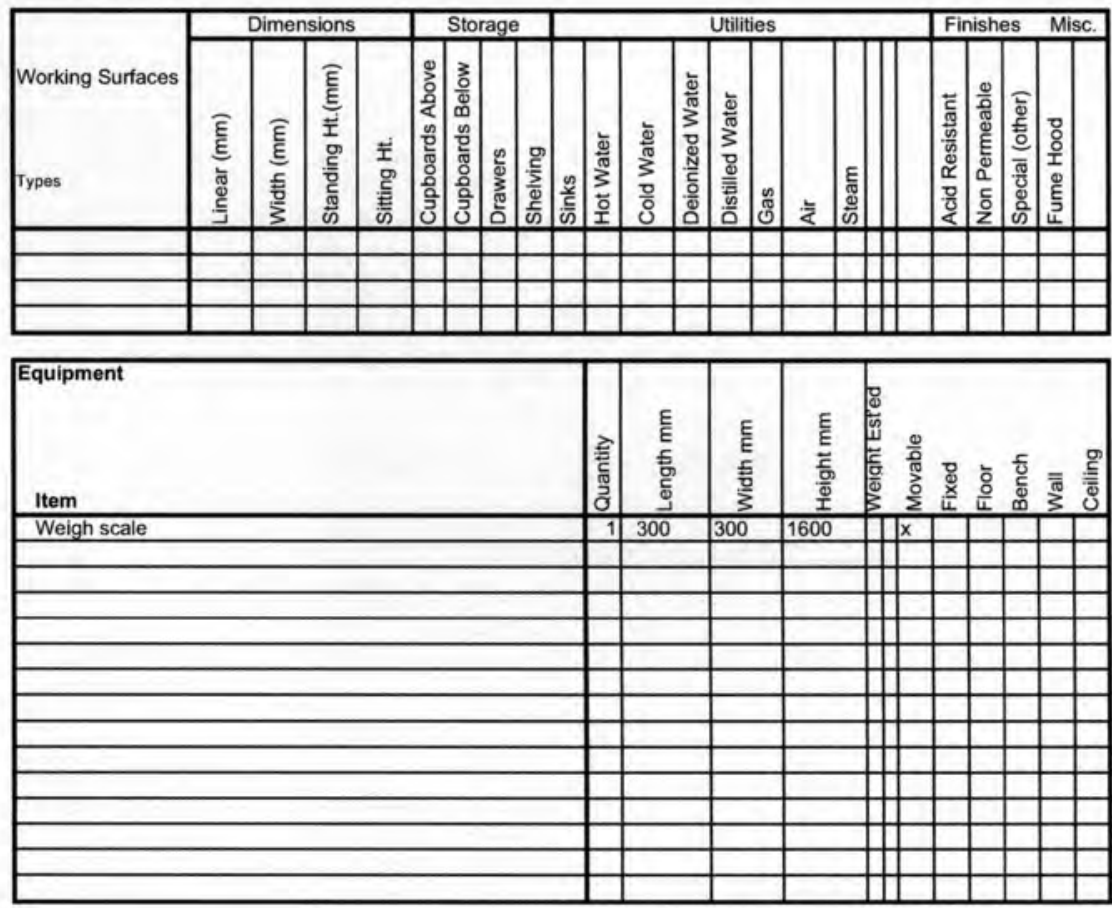

\begin{tabular}{ll|l|}
\hline $\begin{array}{l}\text { Communications: } \\
\text { (place X in box If required) } \\
\text { Telephone }\end{array}$ & $\begin{array}{l}\text { Audio Visual } \\
\text { The following audio visual aids will be used: } \\
\text { P.A System }\end{array}$ \\
Cable TV & Television \\
Internet Connection & Projector \\
Other Specify: & Sound Reinforcement \\
\hline
\end{tabular}




\begin{tabular}{|lll|}
\hline Furnishings: & (place xin box if required) \\
\hline Storage Cabinets & & \\
Fixed: (List) & Info Board & \\
Movable: (List) & visitors chair & \\
\hline
\end{tabular}

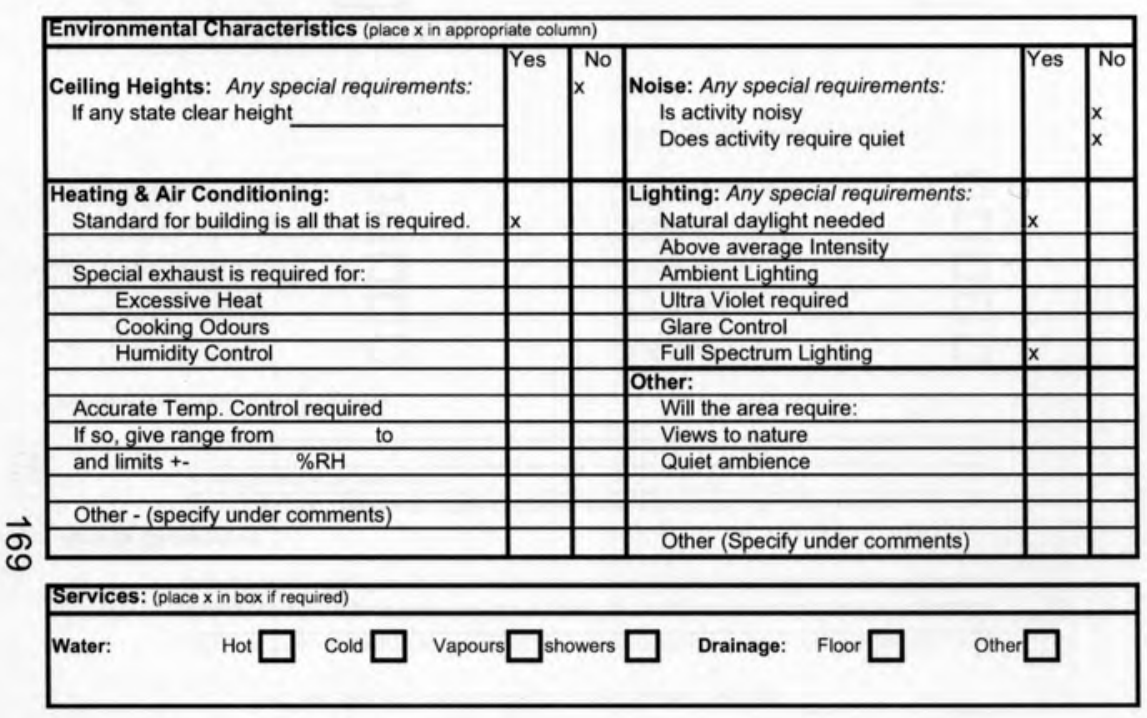

Additional Requirements or Comments:



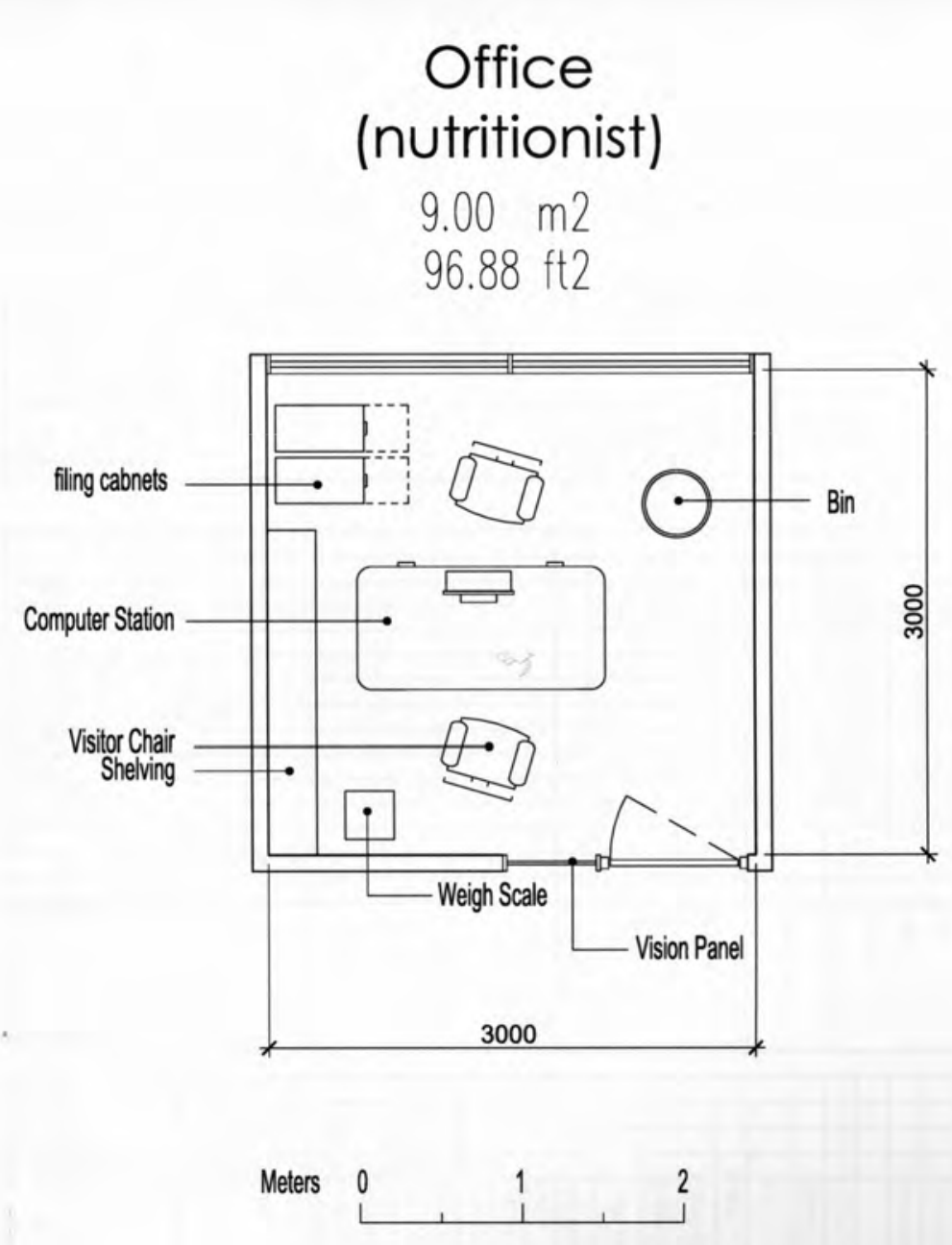


Analysis of physical facility requirements

Project: Wellness Centre 2009

Name of Space: Satellite workstations

Department Community Net Assignable Sq. m 3

Space Description

Purpose: To provide an area for the rehab director

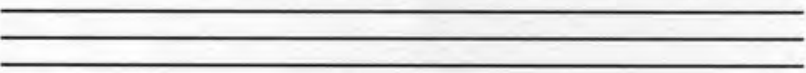

Space Relationships:

a. General - How should this space relate to general areas in the building? eg: offices, teaching areas, loading dock, storage areas.

close to medical examination rooms

b. Specific- Is this space part of a suite? Yes $\square$

close to medical examination rooms, but in the common areas for quick access

\section{Activity Description:}

To provide an area for the clinical staff to access the database that carries

$\overrightarrow{0}$

Activity type: (insert $x$ in applicable box)

$\begin{array}{lll}\text { 1. Admin Office } & \\ \text { 2. Private Office } & \square & \text { 6. Education } \\ \text { 3. Staff Room } & \text { 7. Therapy } \\ \text { 4. Medical Clinic } & \text { 8. Information } \\ \text { 5. Fitness } & \square & \text { 9. Common Area }\end{array}$

Users: (insert number in appropriate boxes)

$\begin{array}{lll}\text { Staff } & \begin{array}{l}\text { Fitness Users } \\ \text { Therapy Users } \\ \text { Students }\end{array} \\ \text { Community } & \text { Avg. hours per week usage }\end{array}$

Space Components
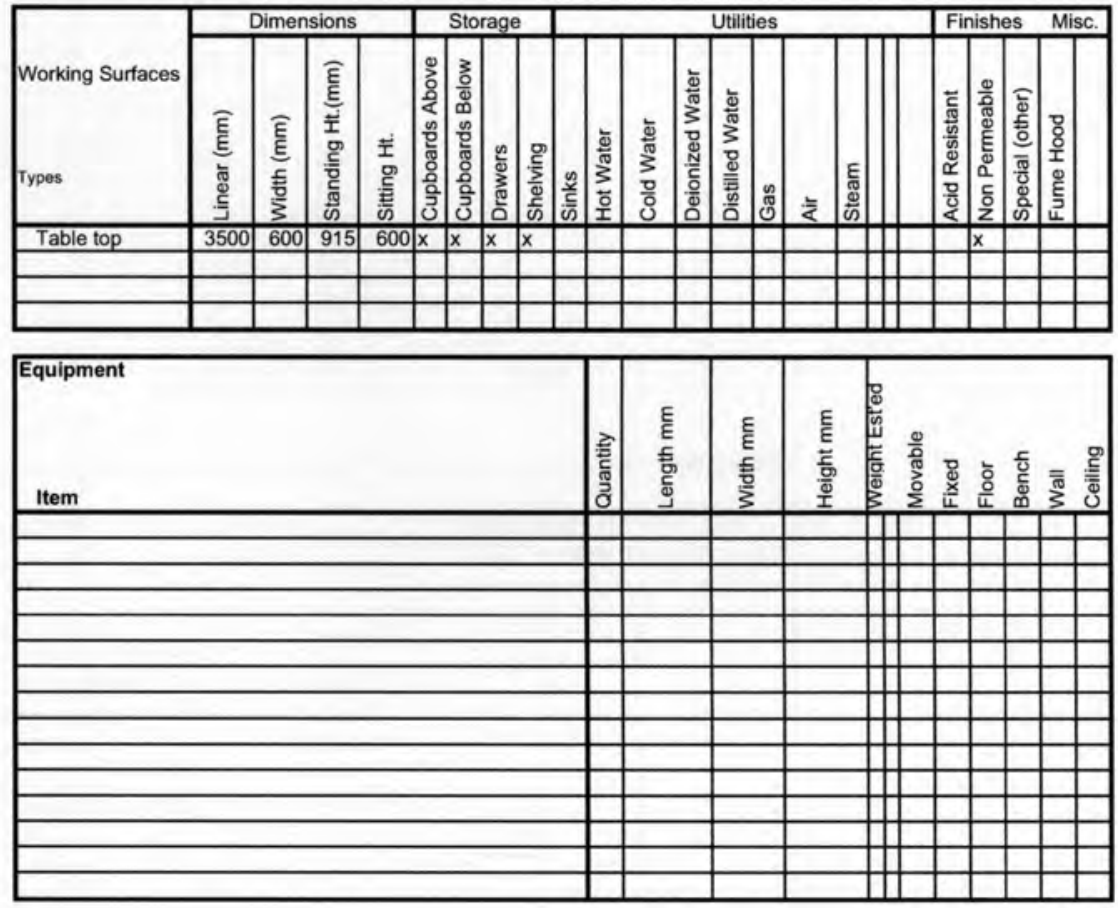

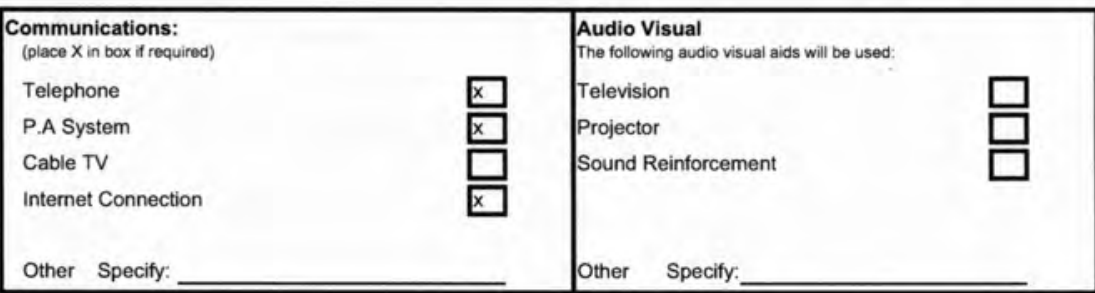




\begin{tabular}{|llll|}
\hline Furnishings: & (place xin boxif required) & & \\
\hline Storage Cabinets & $\square$ into Board $\square$ & Shelving $\square$ & Computer Station $\square$ \\
Fixed: (List) & & \\
Movable: (List) & standing stool & \\
\hline
\end{tabular}

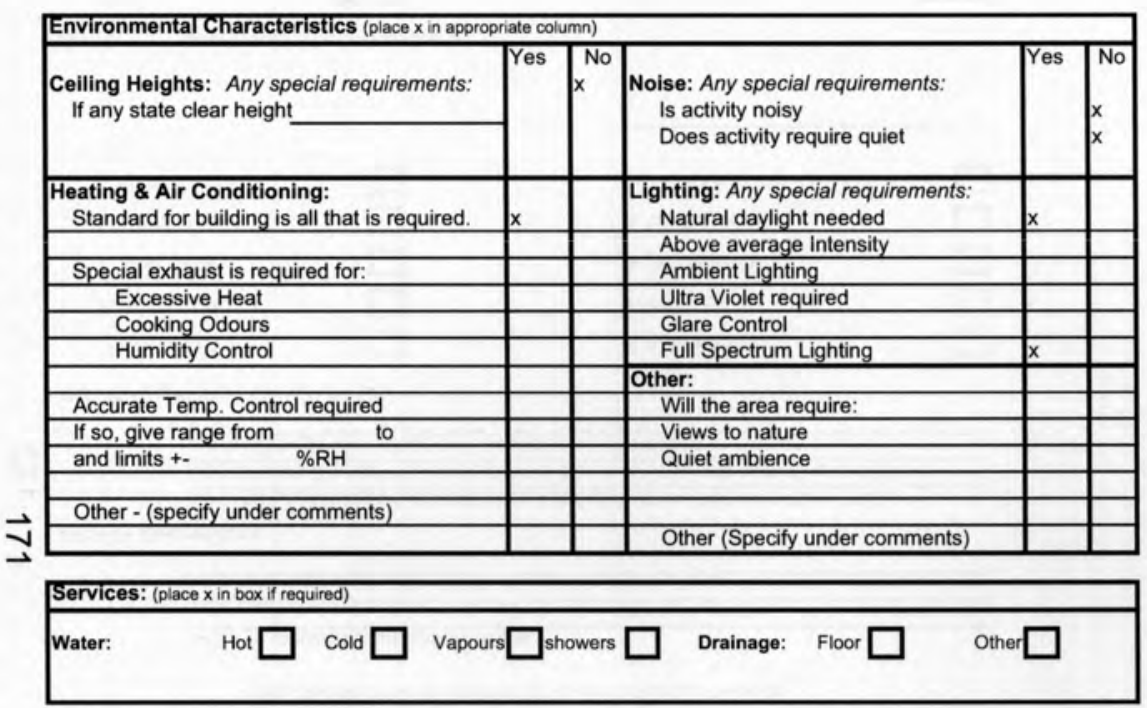

Additional Requirements or Comments:

This space is intended for quick in-and-out access for clinical staff to look up

confidential patient charts, and additional medical information

\section{Satellite Workstation}

$3.00 \mathrm{~m} 2$

$32.29 \mathrm{ft} 2$

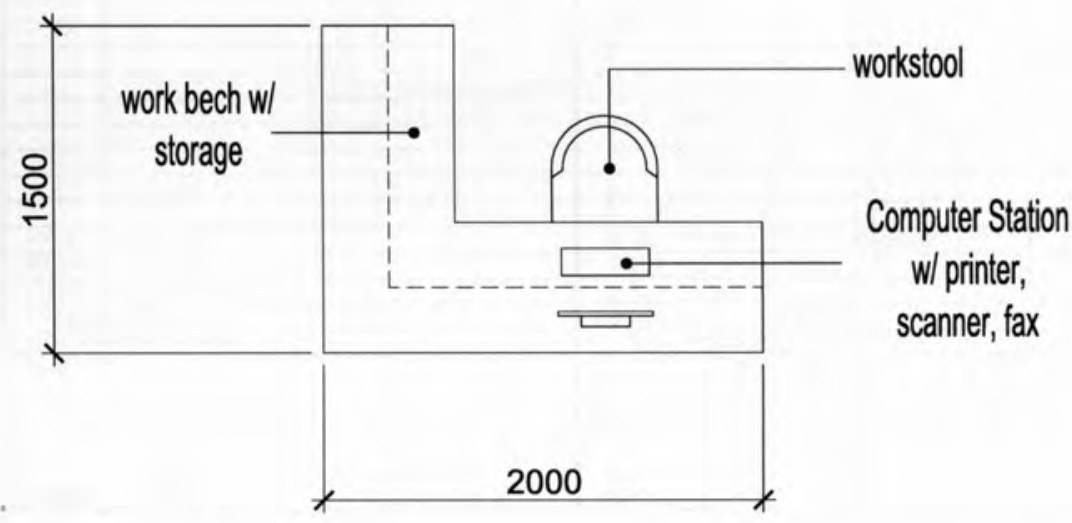

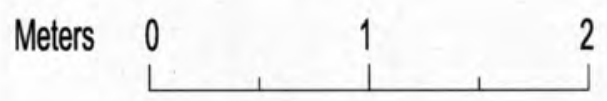




\section{Analysis of physical facility requirements}

Project: Wellness Centre 2009 Name of Space: Environmental Therapy Room

Department Clinical Wing

\section{Space Description}

Purpose: To provide an area that offers natural/artificial therpaies for patient betterment

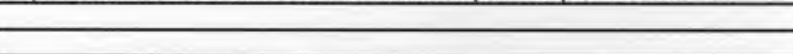

Space Relationships:

a. General - How should this space relate to general areas in the building? eg: offices, teaching areas, loading dock, storage areas.

close to medical examination rooms

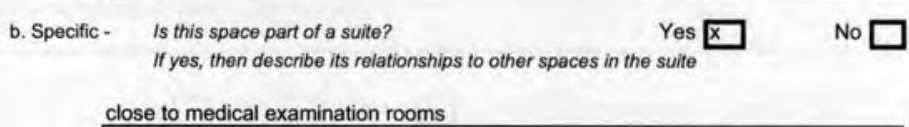
close to medical examination rooms

Activity Description:

$\begin{array}{ll}\vec{N} & \frac{\text { undergo psychological rehabilitation through a space that uses the calming }}{\text { phenomena of nature to relax patients-this is done in both natural and artificial }} \\ \text { ways, through the interior and exterior environment }\end{array}$

Activity type: (insert $\mathrm{x}$ in applicable box)

1. Admin Office

2. Private Office

3. Staff Room

4. Medical Clinic

$\square$

$\square$

$\square$

5. Fitness

$\square$

$\square$

Users:

(insert number in appropriate boxes)

Staff

Patient

Students

Community

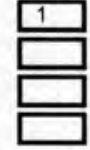

6. Education

7. Therapy

8. Information

9. Common Are

10.Other Specify:

$\square$

$\mathrm{x}$

$\square$

$\square$

,

Therapy Users

Avg. hours per week usage
Space Components
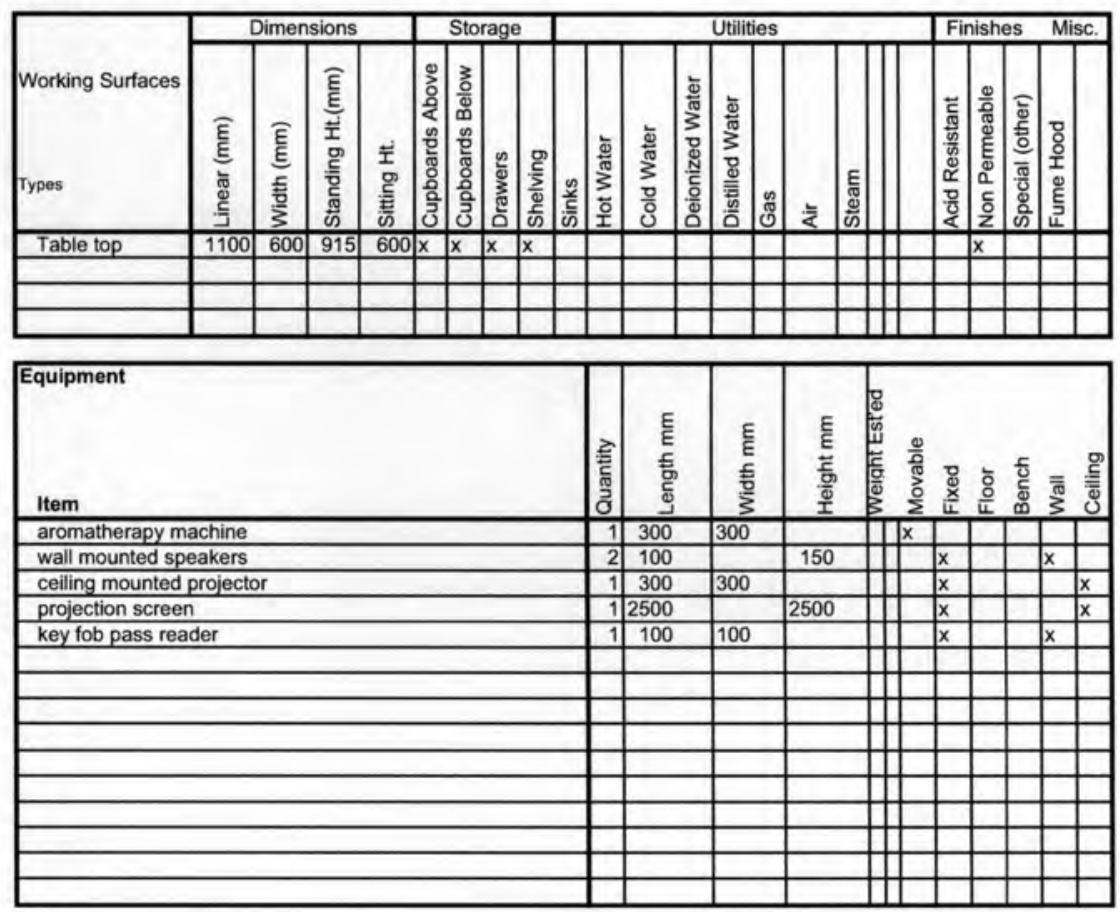

\begin{tabular}{ll|l|}
\hline $\begin{array}{l}\text { Communications: } \\
\text { (place Xin box if required) } \\
\text { Telephone }\end{array}$ & $\begin{array}{l}\text { Audio Visual } \\
\text { The following audio visual aids will be used: } \\
\text { P.A System }\end{array}$ \\
Cable TV \\
Internet Connection
\end{tabular}




\begin{tabular}{|ll|}
\hline Furnishings: & (place x in boxif required) \\
\hline Storage Cabinets & $\square$ info Board $\square \quad$ Shelving $\square$ \\
Fixed: (List) & outdoor planter w/ native shrubbery \\
Movable: (List) & lounger, indoor sofa, and outdoor chair \\
\hline
\end{tabular}

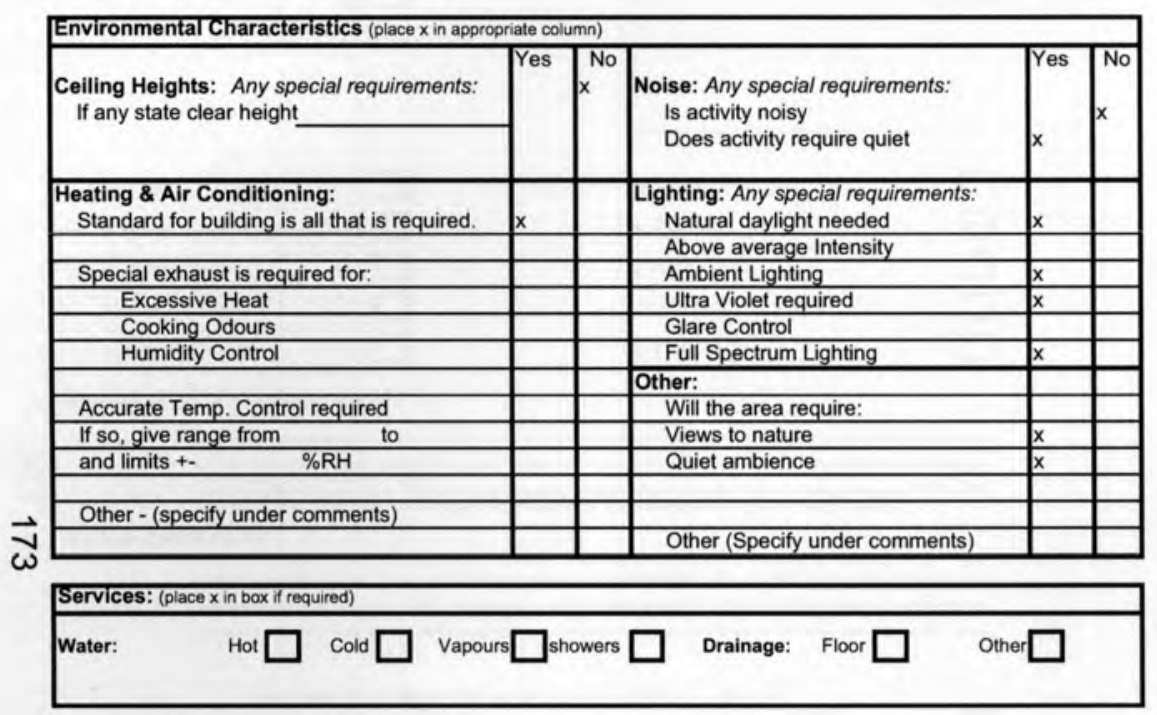

Additional Requirements or Comments:

this area will be accessed through prescription only; rooms will need to be booked ahead of time and be accessed through an electronic key fob reader

\section{Environmental Therapy Room}

$14.16 \mathrm{~m} 2$

$152.42 \mathrm{ft} 2$

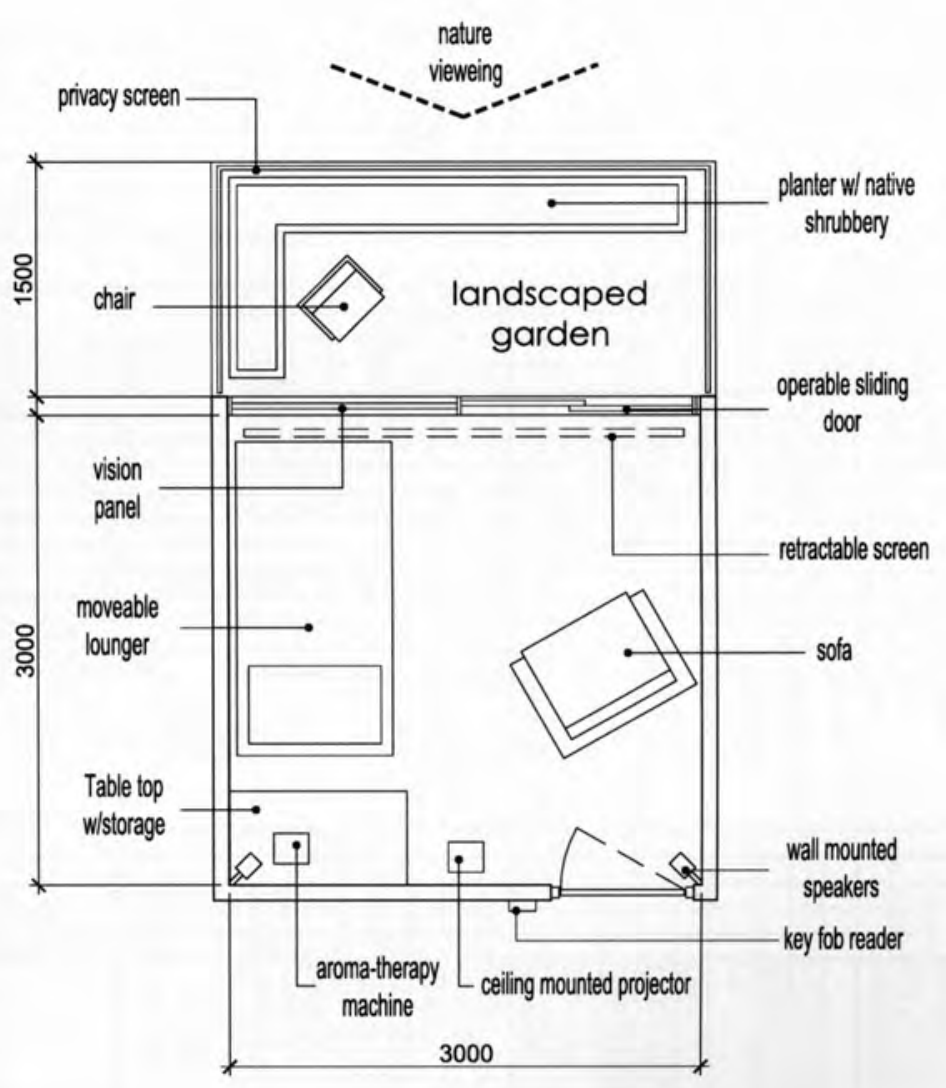

Meters 0

2 


\section{Analysis of physical facility requirements}

Project: Wellness Centre 2009

Name of Space: Pharmacy

Department Clinical Wing

Space Description

Purpose: An area that provides clinical medical drug prescriptions to walk in clinic

\section{Space Relationships:}

a. General - How should this space relate to general areas in the building? eg: offices, teaching areas, loading dock, storage areas.

this space should be close to the entry, adjacent to the waiting area and close to the medical exam rooms

b. Specific - Is this space part of a suite? Yes $\square \quad$ No $\square$ If yes, then describe its relationships to other spaces in the suite

Activity Description:

$\vec{I}$

N

patients will undergo consultation witht the pharmacist and wait while their prescription is being filled

Activity type: (insert $x$ in applicable box)

1. Admin Office

2. Private Office

3. Staff Room

4. Medical Clinic

$\square$

5. Fitness

$\square$

$\square$

$\square$

$\square$

Users:

(insert number in appropriate boxes)

$\begin{array}{lll}\text { Staff } & \text { Fitness Users } \\ \text { Patient } & \text { Therapy Users } \\ \text { Students } & \text { Avg. hours per week usage } \\ \text { Community } & & \end{array}$

7. Therapy

$\square$

8. Information

9. Common Area

$\square$

10. Other Specify

$\square$

Space Components
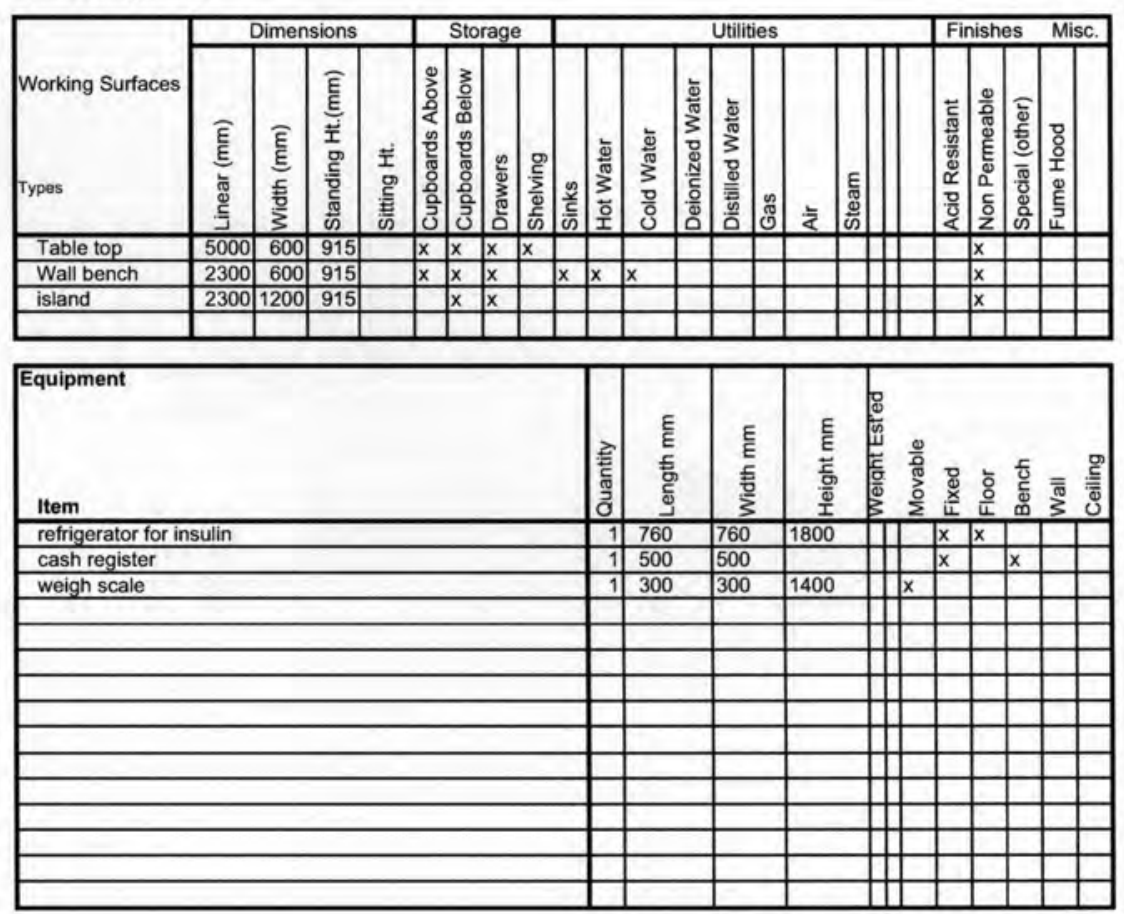

Communications:
(place Xin box if required)
Telephone
P.A System
Cable TV
Internet Connection
Other Specify:

\begin{tabular}{ll}
\hline \\
$\square$ \\
\hline$\square$
\end{tabular}

udio Visua

e following audio visual aids will be used

Television

rojector

ound Reinforcement

$\square$
$\square$ 


\begin{tabular}{|ll|}
\hline Furnishings: & (place x in boxif required) \\
\hline Storage Cabinets & $\square$ Info Board $\square$ Shelving \\
Fixed: (List) & \\
Movable: (List) & 2 consultation chairs (one of which will contain blood pressure test machine) \\
\hline
\end{tabular}

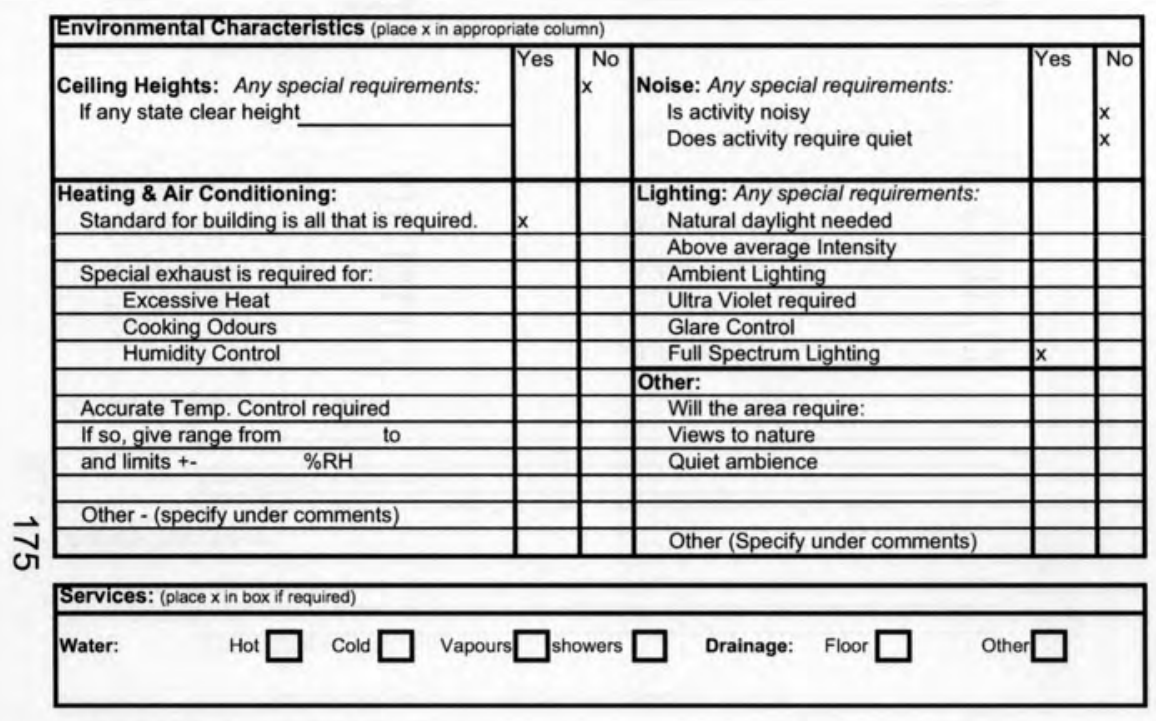

Additional Requirements or Comments:

pharmacy will have a space for medicine dispersal, patient consultation, backroom storage and over the counter transactions

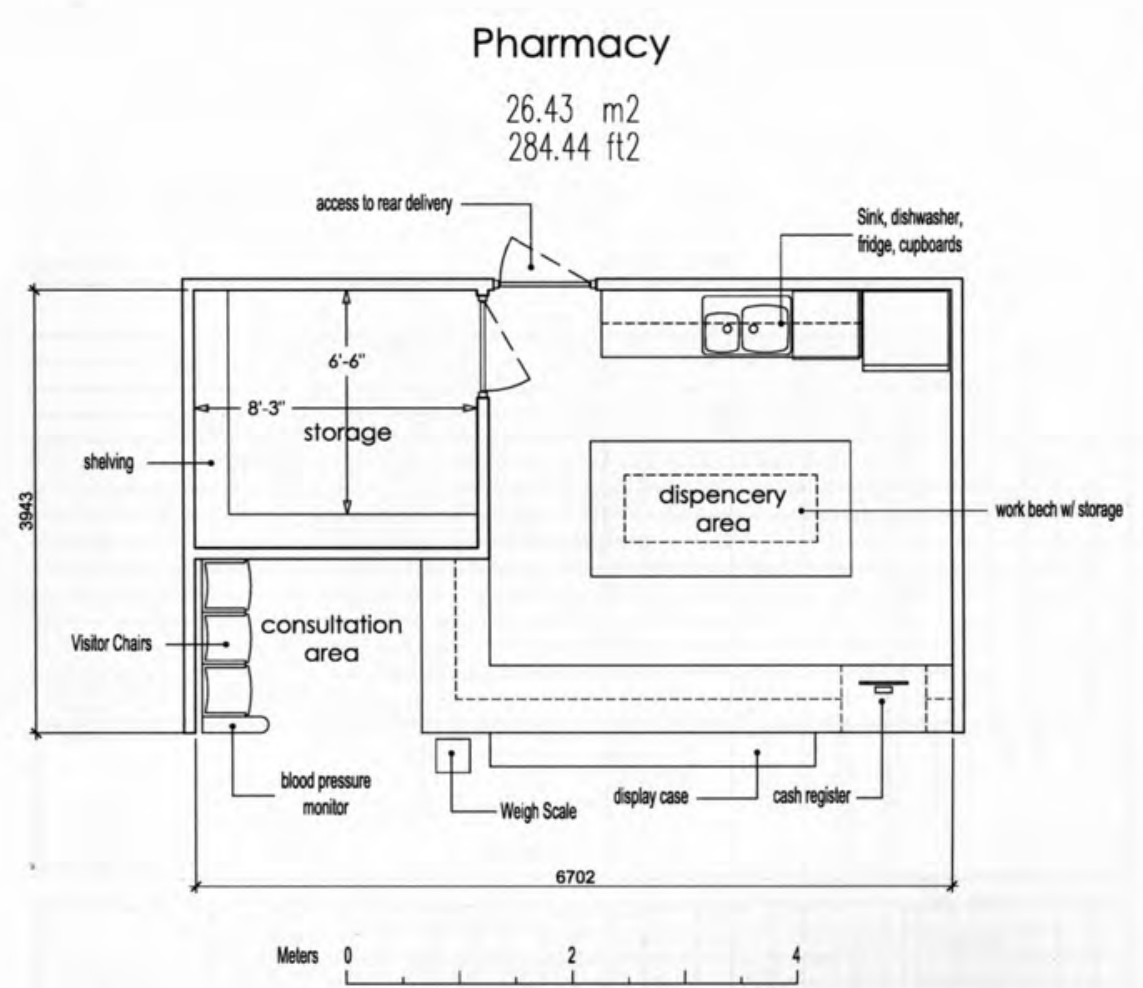




\section{Analysis of physical facility requirements}

Project: Wellness Centre 2009 Name of Space: Staffroom

Department Clinical Wing Net Assignable Sq. m

\section{Space Description}

Purpose: An area provided for medical staff and other building staff to relax, enjoy meals and have informal meetings

Space Relationships:

a. General - How should this space relate to general areas in the building? eg: offices, leaching areas, loading dock, storage areas.

this space should be located in the back of house, out of the way from community spaces $\begin{array}{ll}\text { b. Specific - Is this space part of a suite? } & \text { Yes } \\ \text { If yes, then describe its relationships to other spaces in the suite }\end{array}$ a part of the medical clinic

Activity Description:

$\vec{a} \quad$ a space for staff to have lunch that extends the collaborative nature of the गे family health team mode

Activity type: (insert $x$ in applicable box)

1. Admin Office

2. Private Office

3. Staff Room

4. Medical Clinic

5. Fitness

Users:

(insert number in appropriate boxes)

Staff

Patient

Students

Community $\square$

$\square$

$\square$

$\square$

$\square$

6. Education

7. Therapy

8. Information

9. Common Area

10.Other Specify:

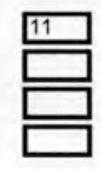

Fitness Users

Therapy Users

Avg. hours per week usage
Space Components
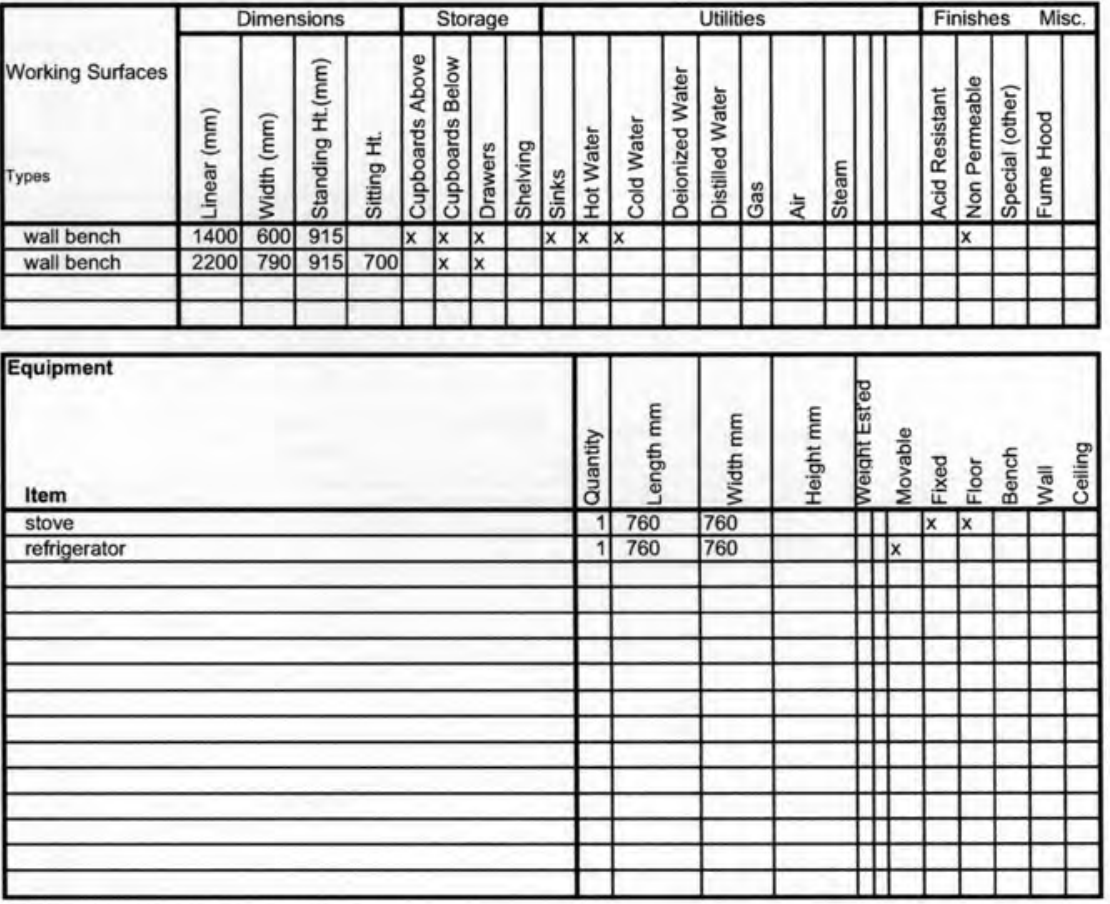

Communications:
(olace $X$ in box if requirt

Telephone

P.A System

Cable TV

Internet Connection

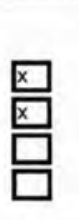

Audio Visual

The following audio visual aids will be used:

Television

Projector

Sound Reinforcement

$\square$

Other Specify:

Other Specify: 


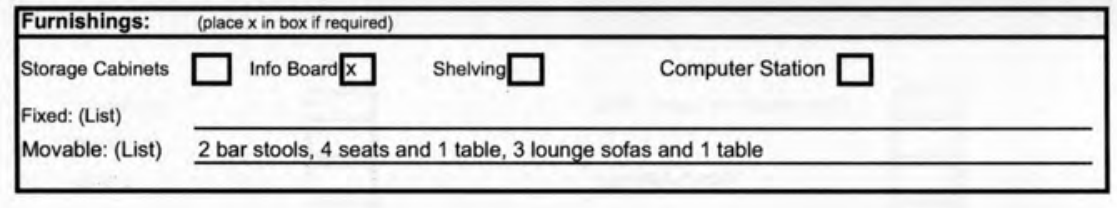

\begin{tabular}{|c|c|c|c|c|c|}
\hline $\begin{array}{l}\text { Ceiling Heights: Any special requirements: } \\
\text { If any state clear height }\end{array}$ & \begin{tabular}{|l|} 
Yes \\
\end{tabular} & $x^{\text {No }}$ & $\begin{array}{l}\text { Noise: Any special requirements: } \\
\text { Is activity noisy } \\
\text { Does activity require quiet }\end{array}$ & Yes & $\begin{array}{l}\text { No } \\
x \\
x\end{array}$ \\
\hline $\begin{array}{l}\text { Heating \& Air Conditioning: } \\
\text { Standard for building is all that is required. }\end{array}$ & $x$ & & $\begin{array}{l}\text { Lighting: Any special requirements: } \\
\text { Natural daylight needed }\end{array}$ & & \\
\hline & & & Above average Intensity & & \\
\hline Special exhaust is required for: & & & Ambient Lighting & & \\
\hline Excessive Heat & & & Ultra Violet required & & \\
\hline Cooking Odours & $x$ & & Glare Control & & \\
\hline Humidity Control & & & Full Spectrum Lighting & $x$ & \\
\hline & & & Other: & & \\
\hline Accurate Temp. Control required & & & Will the area require: & & \\
\hline If so, give range from & & & Views to nature & & \\
\hline and limits +- $\quad \%$ RH & & & Quiet ambience & & \\
\hline & & & & & \\
\hline Other - (specify under comments) & & & & & \\
\hline & & & Other (Specify under comments) & & \\
\hline
\end{tabular}

\begin{abstract}
\begin{tabular}{l} 
Services: (place x in box if required) \\
\hline Water: $\quad$ Hot $\square$ Cold $\square$ Vapours $\square$ showers $\square$ Drainage: Floor $\square$ Other $\square$
\end{tabular}
\end{abstract}
Additional Requirements or Comments:

\section{Staff Room}

$21.69 \mathrm{~m} 2$

$233.44 \mathrm{ft} 2$

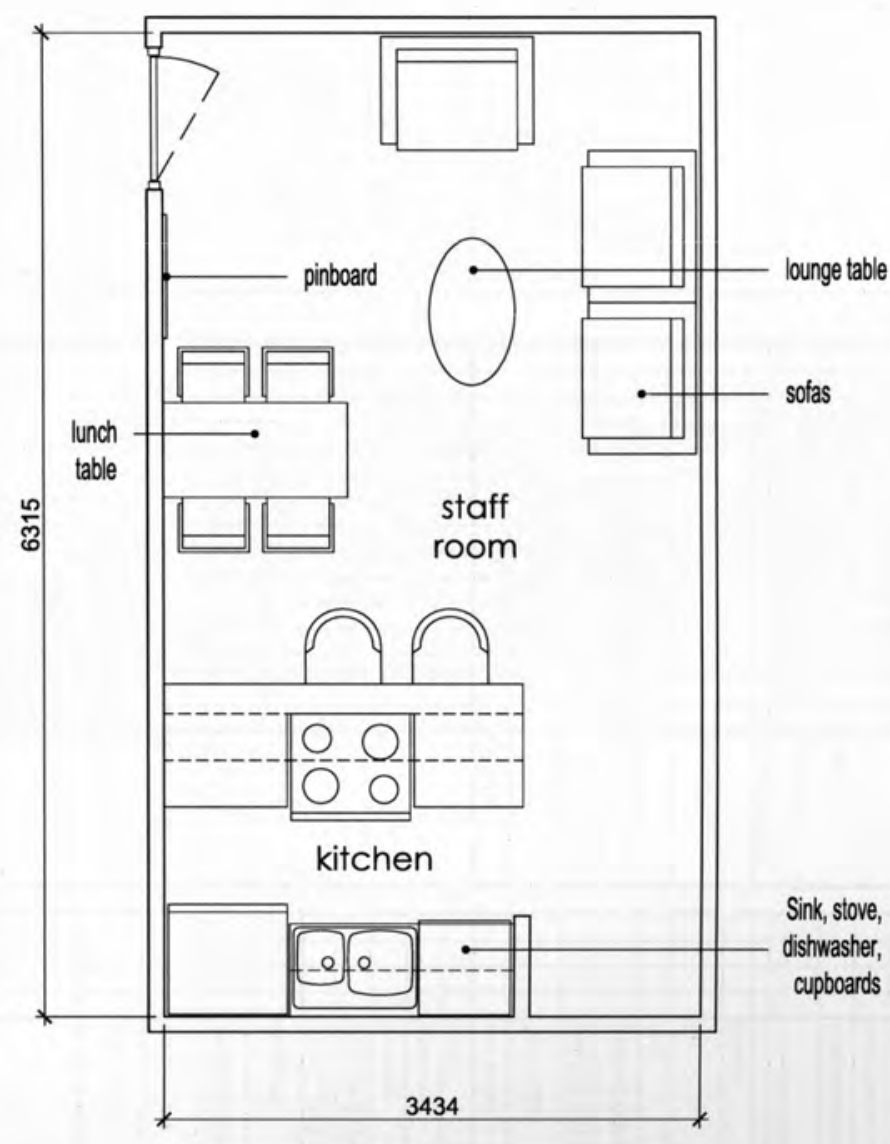

Meters 0 2 


\section{Analysis of physical facility requirements}

Project: Wellness Centre 2009

Name of Space: Weight Room/Cardio Trainin

Space Description

Purpose:

An area for community fitness

\section{Space Relationships:}

a. General - How should this space relate to general areas in the building? eg: offices, teaching areas, loading dock, storage areas.

close to the aerobics/physiotherapy room to take advantage of a communal locker room scenario

b. Specific - Is this space part of a suite?

Yes $\square \quad$ No $\square$

If yes, then describe its relationships to other spaces in the suite
Department Fitness Wing Net Assignable Sq. m [190]
-

\section{Activity Description:} $\overrightarrow{\text { individuals interested in weight training or cardio vascular conditiong may }}$ Activity type: (insert x in applicable box)

1. Admin Office
2. Private Office
3. Staff Room
4. Medical Clinic
5. Fitness

6. Education

7. Therapy

8. Information

9. Common Area

10.Other Specify:

$\square$
$\square$
$\square$
$\square$

Users: $\quad$ (insert number in appropriate boxes)

$\begin{array}{lll}\text { Staff } & \text { Fitness Users } \\ \text { Patient } & \text { Therapy Users } \\ \text { Students } & \text { Avg. hours per week usage } \\ \text { Community } & \end{array}$

Space Components
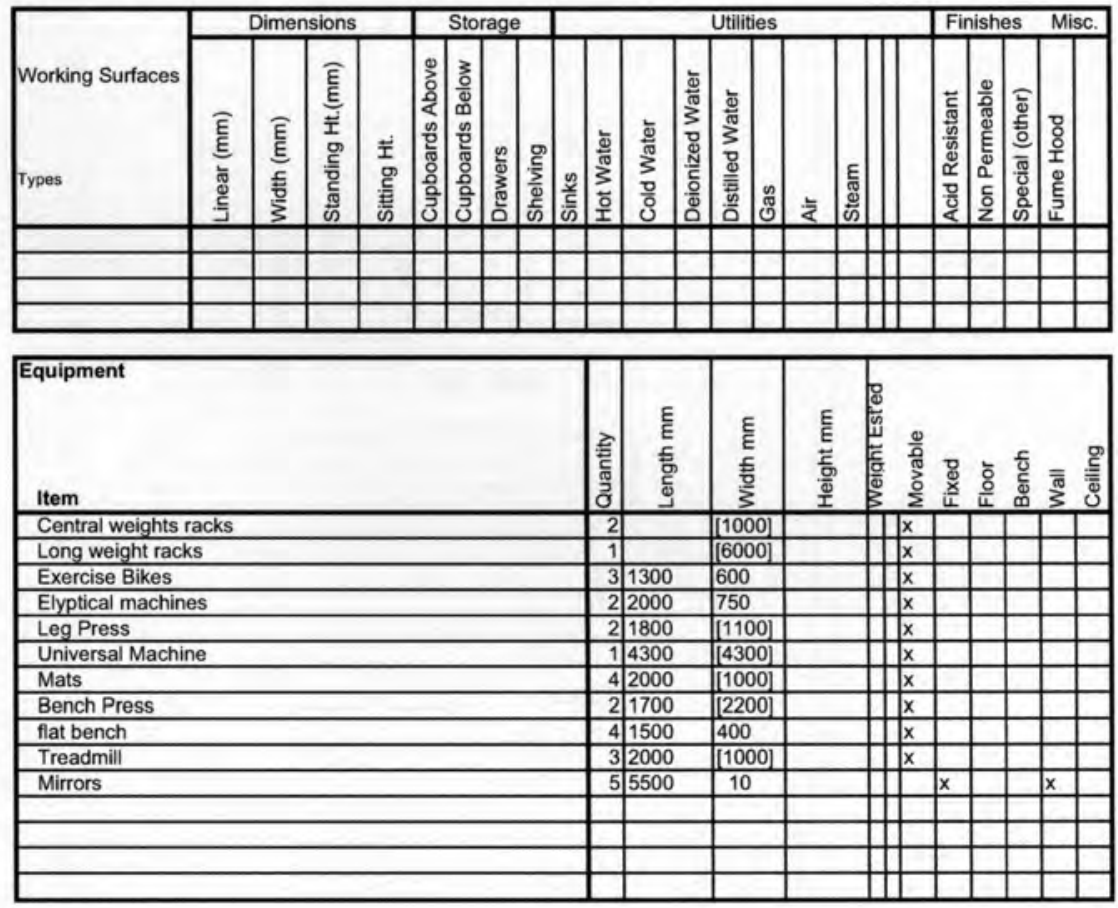

\begin{tabular}{ll|l|}
\hline $\begin{array}{l}\text { Communications: } \\
\text { (place X in box if required) }\end{array}$ & $\begin{array}{l}\text { Audio Visual } \\
\text { The following audio visual aids will be used: } \\
\text { Telephone }\end{array}$ \\
P.A System & Television \\
Cable TV & Projector \\
Internet Connection & Sound Reinforcement \\
Other Specify: & & Other Specify. Wall Mounted Speakers \\
\hline
\end{tabular}




\begin{tabular}{|lll|}
\hline Furnishings: $\quad$ (place x in box lif required) \\
\hline Storage Cabinets \\
Fixed: (List) \\
Movable: (List)
\end{tabular}

\begin{tabular}{|c|c|c|c|c|c|}
\hline \multicolumn{6}{|c|}{ Environmental Characteristics (place $\mathrm{x}$ in appropriate column) } \\
\hline $\begin{array}{l}\text { Ceiling Heights: Any special requirements: } \\
\text { If any state clear height }\end{array}$ & Yes & $x^{\text {No }}$ & $\begin{array}{l}\text { Noise: Any special requirements: } \\
\text { Is activity noisy } \\
\text { Does activity require quiet }\end{array}$ & Yes & $\overline{\mathrm{No}}$ \\
\hline \multirow[t]{2}{*}{\begin{tabular}{|l} 
Heating \& Air Conditioning: \\
Standard for building is all that is required.
\end{tabular}} & $x$ & & $\begin{array}{l}\text { Lighting: Any special requirements: } \\
\text { Natural daylight needed }\end{array}$ & $x$ & \\
\hline & & & Above average Intensity & & \\
\hline Special exhaust is required for: & & & Ambient Lighting & & \\
\hline Excessive Heat & $x$ & & Ultra Violet required & & \\
\hline Cooking Odours & & & Glare Control & & \\
\hline \multirow[t]{2}{*}{ Humidity Control } & & & Full Spectrum Lighting & $x$ & \\
\hline & & & Other: & & \\
\hline Accurate Temp. Control required & & & Will the area require: & & \\
\hline \multirow{2}{*}{$\begin{array}{ll}\text { If so, give range from } & \text { to } \\
\text { and limits +- } & \% R H \\
\end{array}$} & & & Views to nature & $x$ & \\
\hline & & & Quiet ambience & & \\
\hline & & & & & \\
\hline \multirow[t]{2}{*}{ Other - (specify under comments) } & & & & & \\
\hline & & & Other (Specify under comments) & & \\
\hline \multicolumn{6}{|l|}{ ervices: (place $x$ in box if required) } \\
\hline \multicolumn{3}{|l|}{ Water: } & Drainage: Floor [ & 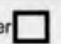 & \\
\hline
\end{tabular}

\section{Additional Requirements or Comments:}

This space will be act as a community weightroom/cardio room that will need membership

to utilise, however those that are prescribed fitness by the medical staff may use the facility as well

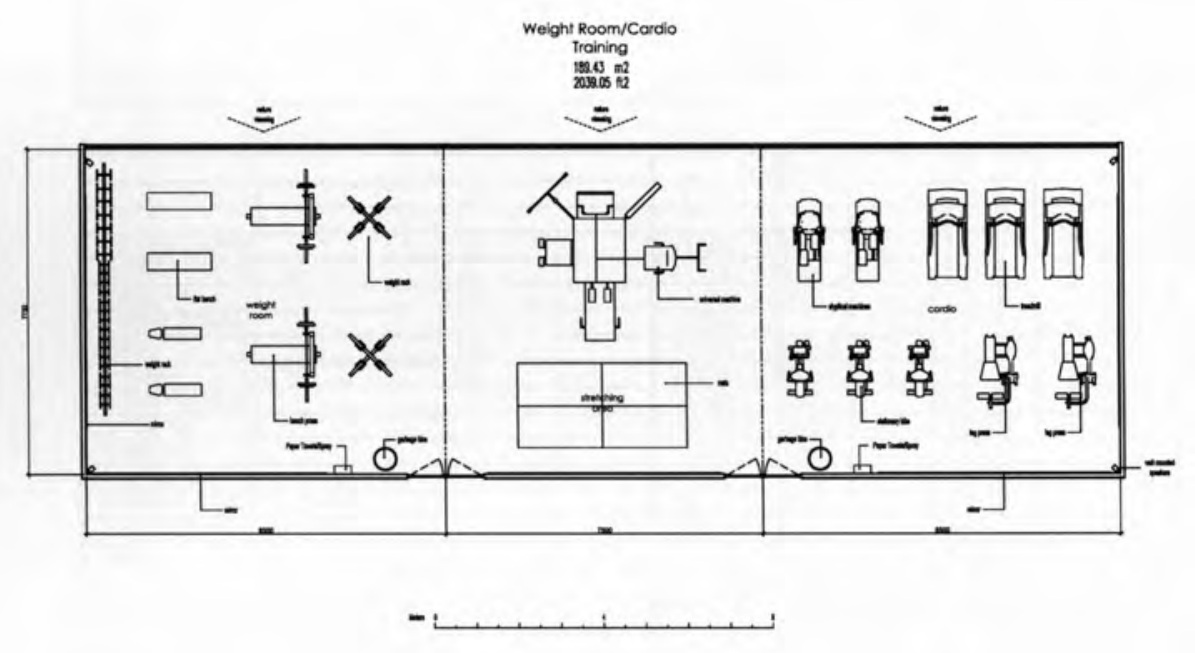




\section{Analysis of physical facility requirements}

Project: Wellness Centre 2009

Name of Space: Physiotherapy Studio

Department Fitness Wing Net Assignable Sq. m

\section{Space Description}

Purpose: To provide an area where patients may undergo physical rehabilitation

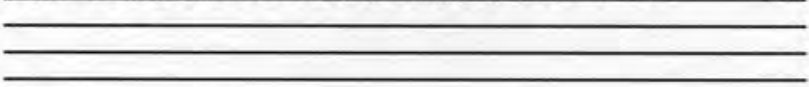

Space Relationships:

a. General - How should this space relate to general areas in the building? eg: offices, teaching areas, loading dock, storage areas.

close to other fitness rooms to take advantage of communal locker rooms

\section{cre} b. Specific - Is this space part of a suite?
If yes, then describe its relationships to other spaces in the suite

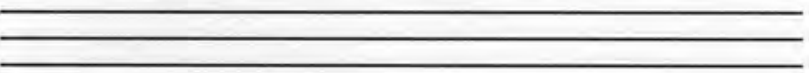

\section{Activity Description:}

$\vec{\circ}$

undergo physical rehabilitation- cardiopulminary, geriatric therapy, orthapedic

Activity type: (insert $x$ in applicable box)

1. Admin Office

2. Private Office

3. Staff Room

4. Medical Clinic

5. Fitness

$\square$

$\square$

$\square$

$\square$

区

Users:

(insert number in appropriate boxes)

$\begin{array}{lll}\text { Staff } & \text { Fitness Users } \\ \text { Patient } & \text { Therapy Users } \\ \text { Students } & \end{array}$

7. Therapy

$\square$

8. Information

9. Common Area

10.Other Specify:
Space Components
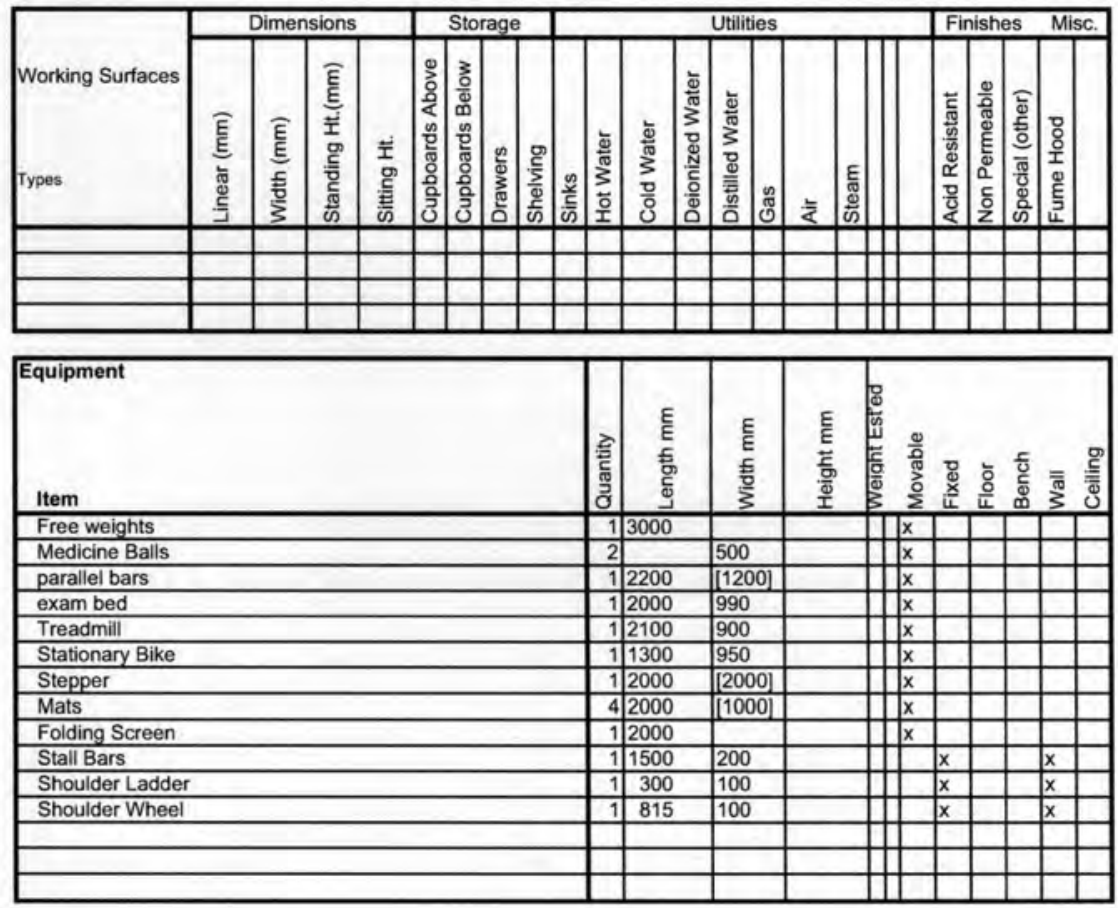

$\square$

Audio Visual

Communications:

The following audio visual aids will be used

Telephone

P.A System

Cable TV

Television

Projector

Internet Connection

Sound Reinforcement

Other Specify:

Other Specify: 


\begin{tabular}{|lll|}
\hline Furnishings: $\quad$ (place x in box if required) \\
\hline Storage Cabinets \\
Fixed: (List) \\
Movable: (List)
\end{tabular}

\begin{tabular}{|c|c|c|c|c|c|}
\hline \multicolumn{6}{|c|}{ Environmental Characteristics (place $x$ in appropriate column) } \\
\hline $\begin{array}{l}\text { Ceiling Heights: Any special requirements: } \\
\text { If any state clear height }\end{array}$ & Yes & $x^{\text {No }}$ & $\begin{array}{l}\text { Noise: Any special requirements: } \\
\text { Is activity noisy }\end{array}$ & $\begin{array}{l}\text { Yes } \\
x\end{array}$ & No \\
\hline \multirow[t]{2}{*}{$\begin{array}{l}\text { Heating \& Air Conditioning: } \\
\text { Standard for building is all that is required. }\end{array}$} & $\mathrm{x}$ & & $\begin{array}{l}\text { Lighting: Any special requirements: } \\
\text { Natural daylight needed }\end{array}$ & $x$ & \\
\hline & & & Above average Intensity & & \\
\hline Special exhaust is required for: & & & Ambient Lighting & & \\
\hline Excessive Heat & $x$ & & Ultra Violet required & & \\
\hline Cooking Odours & & & Glare Control & & \\
\hline \multirow[t]{2}{*}{ Humidity Control } & & & Full Spectrum Lighting & $x$ & \\
\hline & & & Other: & & \\
\hline Accurate Temp. Control required & & & Will the area require: & & \\
\hline \multirow{2}{*}{$\begin{array}{ll}\text { If so, give range from } & \text { to } \\
\text { and limits +- } & \% R H\end{array}$} & & & Views to nature & $x$ & \\
\hline & & & Quiet ambience & & \\
\hline \multirow{2}{*}{\begin{tabular}{|l|} 
Other - (specify under comments) \\
\end{tabular}} & & & & & \\
\hline & & & & & \\
\hline & & & Other (Specify under comments) & & \\
\hline \multicolumn{6}{|l|}{ Services: (place $x$ in box if required) } \\
\hline \multicolumn{3}{|l|}{ Water: } & Drainage: & & \\
\hline
\end{tabular}

Additional Requirements or Comments:

This space will have dual functions: during daytime operations this will be a dedicated

physiotherapy space-at night the space will become an aerobics studio for community

yoga and tai chi classes
Physiotherapy Studio

$81.00 \mathrm{~m} 2$

$871.88 \mathrm{ft} 2$

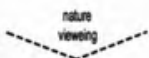

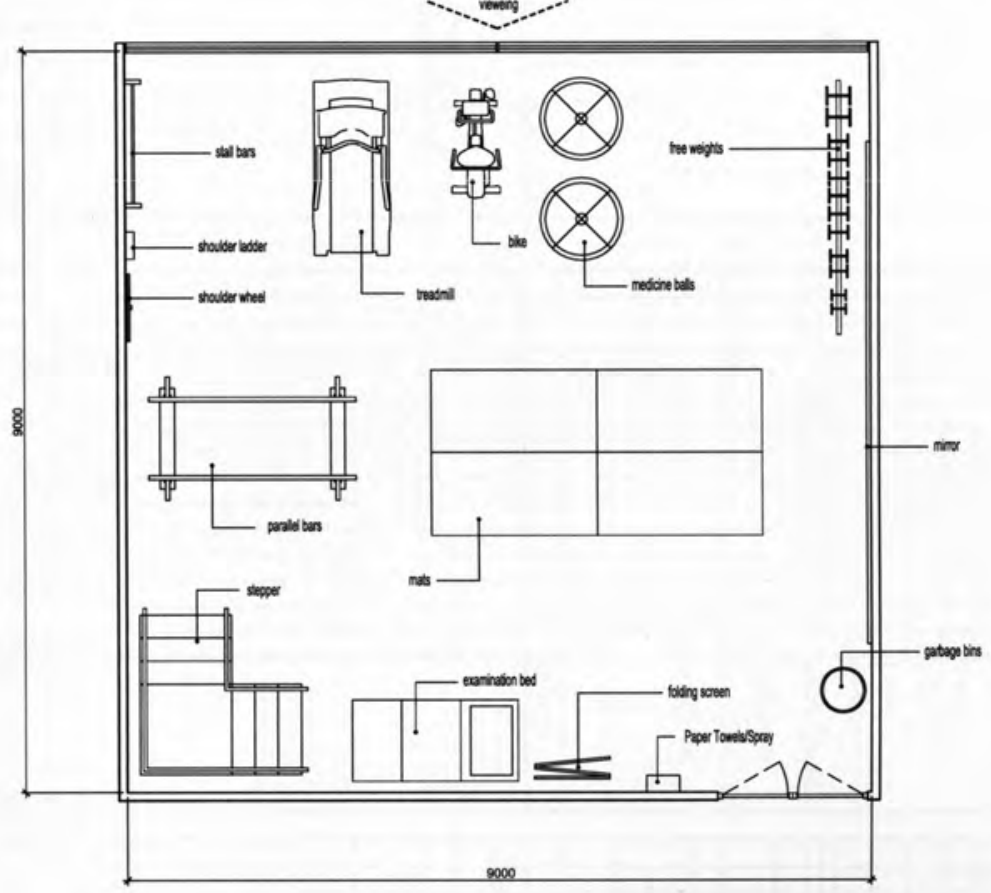

0 


\section{Analysis of physical facility requirements}

Project: Wellness Centre 2009

Name of Space: Fitness Office

Department Fitness Wing

Net Assignable Sq. m

\section{Space Description}

Purpose: An area dedicated for fitness trainers to offer fitness services

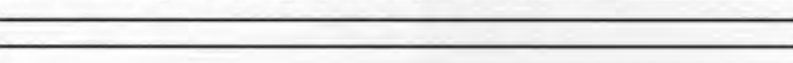

Space Relationships:

a. General - How should this space relate to general areas in the building? eg: offices, teaching areas, loeding dock storage arees.

close to the aerobics/physiotherapy room and the weight/cardio room

Is this space part of a suite?

If yes, then describe its relationships to other spaces in the sulte

$\bar{\square}$

\section{Activity Description:}

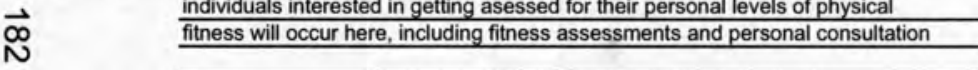
Activity type: (insert $x$ in applicable box)

1. Admin Office
2. Private Office
3. Staff Room
4. Medical Clinic
5. Fitness

6. Education

7. Therapy

8. Information

9. Common Area

10.Other Specify:

$\square$

$\square$

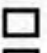

$\square$

Users: (insert number in appropriate boxes)

$\begin{array}{llll}\text { Staff } & \text { Fitness Users } \\ \text { Patient } & \text { Therapy Users } \\ \text { Students } & \text { Avg. hours per week usage } \\ \text { Community } & & \end{array}$

Space Components
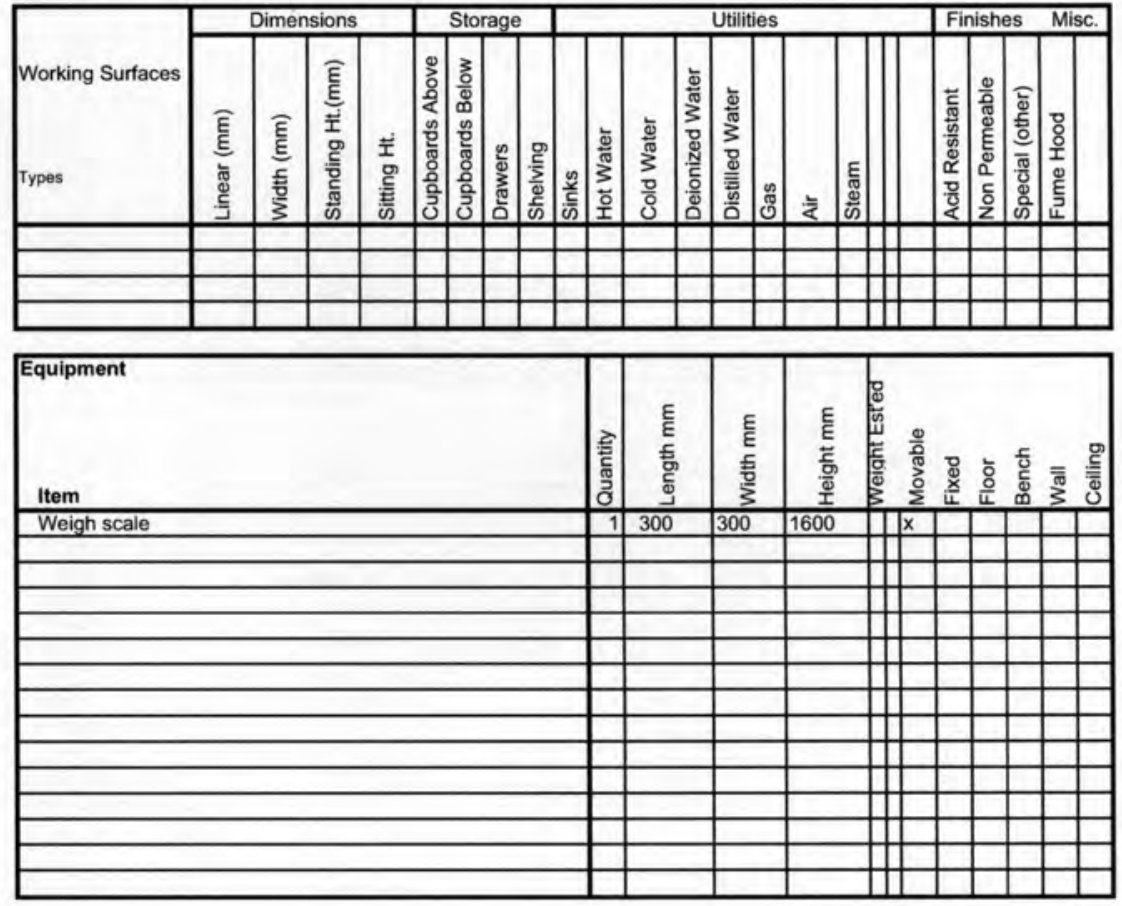

\begin{tabular}{|l|l|}
\hline $\begin{array}{l}\text { Communications: } \\
\text { (place X in box if fequired) } \\
\text { Telephone }\end{array}$ & $\begin{array}{l}\text { Audio Visual } \\
\text { The following audio visual aids will be used: } \\
\text { P.A System }\end{array}$ \\
Cable TV & Television \\
Internet Connection & Projector \\
Other Specify: & Sound Reinforcement \\
\hline
\end{tabular}




\begin{tabular}{|c|c|c|c|}
\hline \multirow{3}{*}{$\begin{array}{l}\text { Storage Cabinets } \\
\text { Fixed: (List) } \\
\text { Movable: (List) }\end{array}$} & 网 & Shelving & \\
\hline & & & \\
\hline & visitors chair & & \\
\hline
\end{tabular}

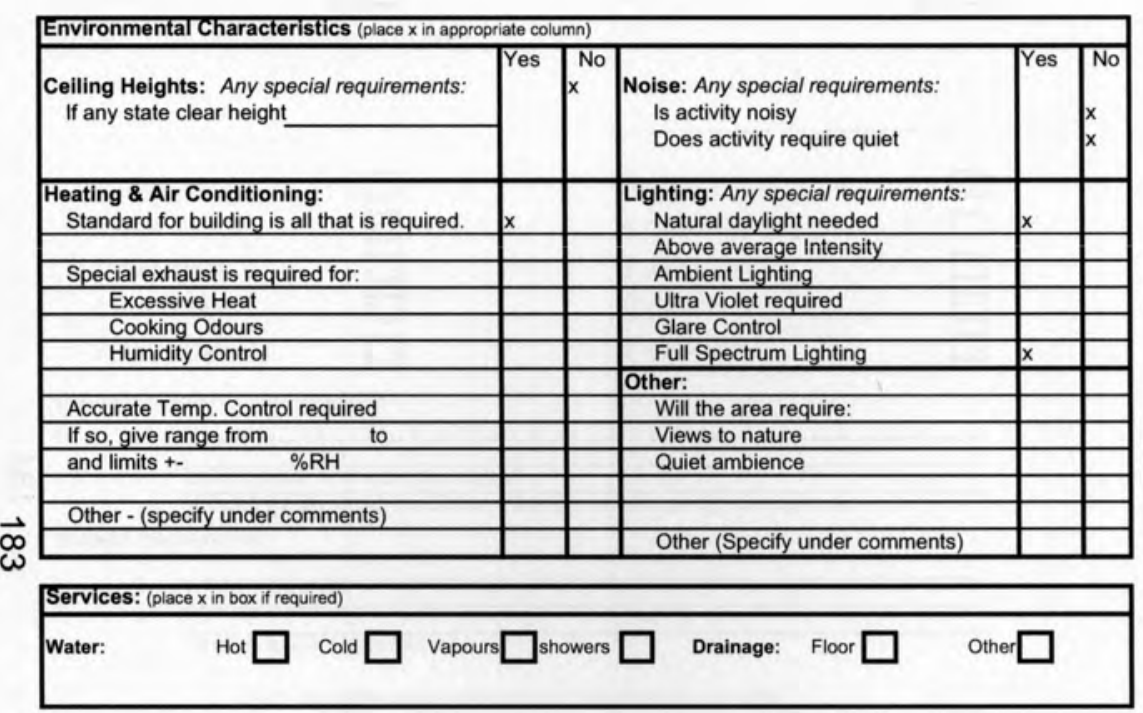

Additional Requirements or Comments:
Office

(fitness testing)

$9.00 \mathrm{~m} 2$

$96.88 \mathrm{ft} 2$

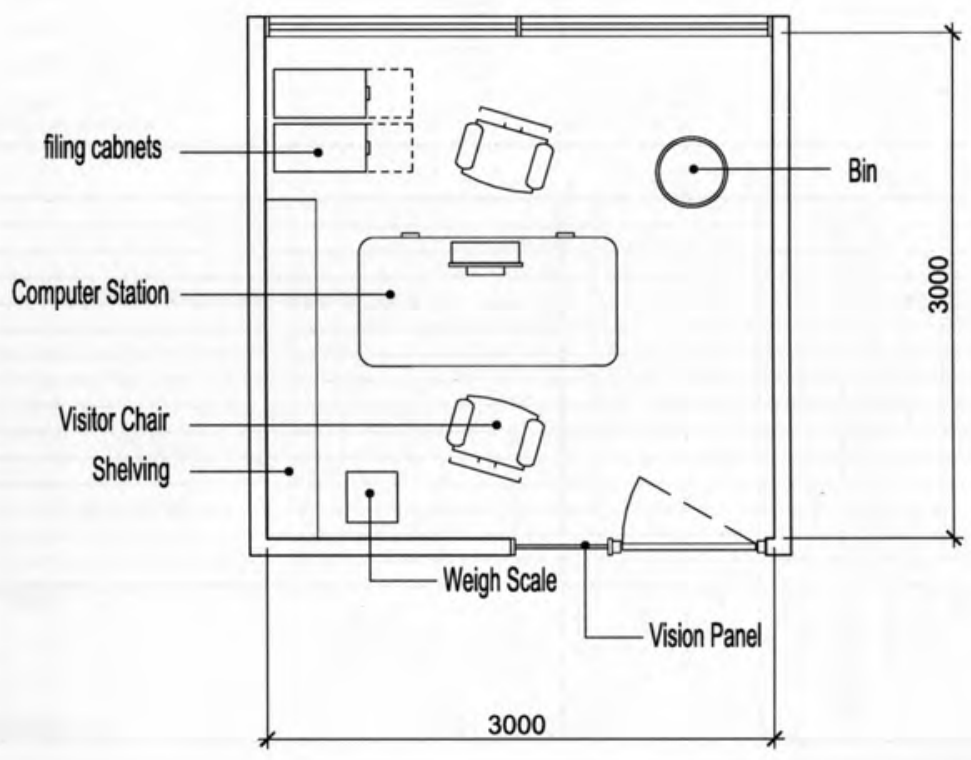

Meters 0

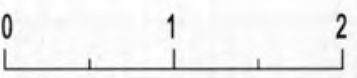




\section{Analysis of physical facility requirements}

Project: Wellness Centre 2009

Name of Space: Office (rehab director)

Department Clinical Wing

Net Assignable Sq. m

\section{Space Description}

Purpose:

To provide an area for the rehab director

\section{Space Relationships:}

a. General - How should this space relate to general areas in the building? eg: offices, leaching areas, loading dock, storage areas.

close to medical examination rooms

\begin{tabular}{llll}
\hline Is this space part of a suite? & \\
\hline
\end{tabular}

b. Specific - Is this space part of a suite?

If yes, then describe its relationships to other spaces in the suit

close to physiotherapy room

\section{Activity Description:}

$\vec{\infty} \quad$ an area for the rehab director to supervise the successful operation of

$\stackrel{\infty}{+}$

Activity type: (insert x in applicable box)

1. Admin Office

2. Private Office

$\square$

3. Staff Room

4. Medical Clinic

$\square$

$\square$

$\square$

5. Fitness

$\square$

6. Education

7. Therapy

8. Information

9. Common Area

10.Other Specify:

$\square$
$\square$
$\square$
$\square$

Users: $\quad$ (insert number in appropriate boxes)

$\begin{array}{lll}\text { Staff } & \text { Fitness Users } \\ \text { Patient } & \text { Therapy Users } \\ \text { Students } & \text { Avg. hours per week usage } \\ \text { Community } & \end{array}$

Space Components
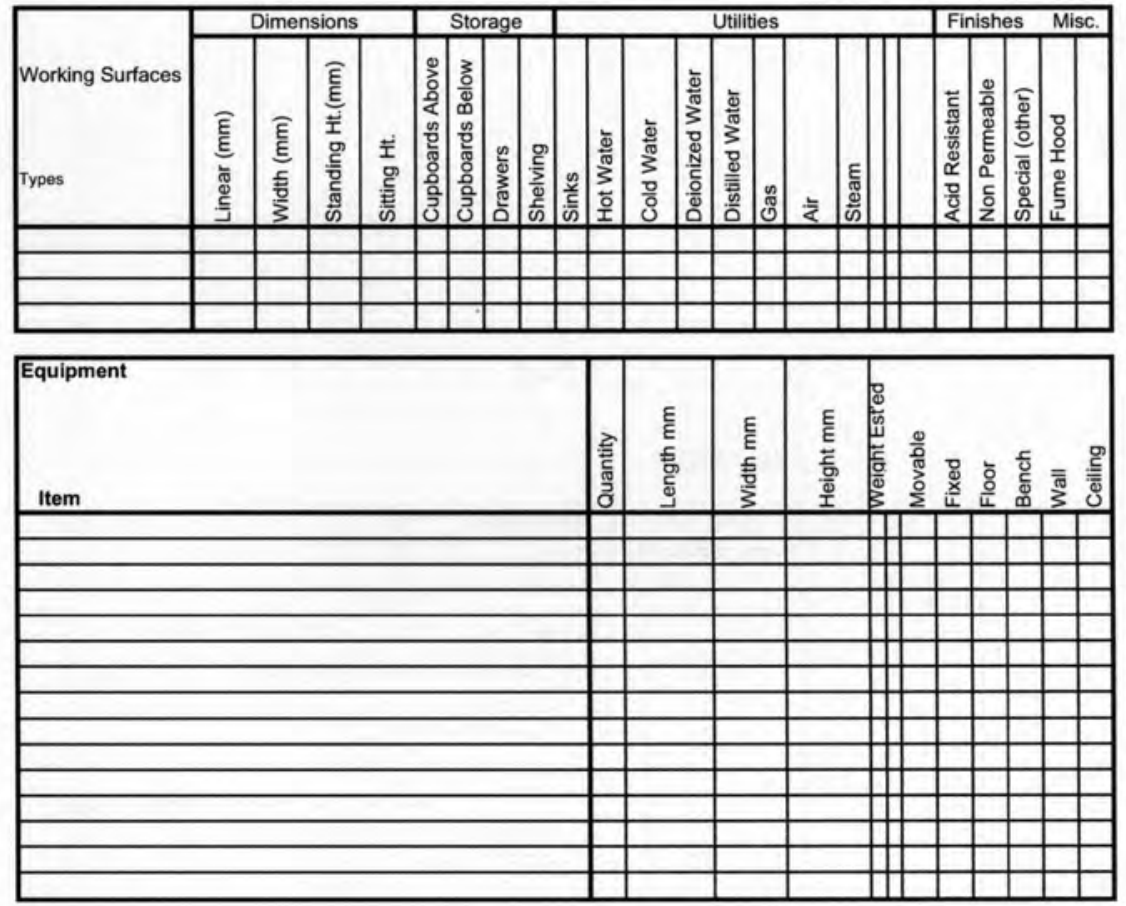

\begin{tabular}{|l|l|}
\hline $\begin{array}{l}\text { Communications: } \\
\text { (place X in box if required) } \\
\text { Telephone }\end{array}$ & $\begin{array}{l}\text { Audio Visual } \\
\text { The following audio visual aids will be used: } \\
\text { P.A System }\end{array}$ \\
Cable TV & Television \\
Internet Connection & Projector \\
Other Specify: & Sound Reinforcement \\
\hline
\end{tabular}




\begin{tabular}{ll|}
\hline Furnishings: & (place x in box if required) \\
\hline Storage Cabinets & $\square$ Info Board $\square \quad$ Shelving $\square$ \\
Fixed: (List) & \\
Movable: (List) & filing cabinets, computer chair and visitor chair \\
\hline
\end{tabular}

\begin{tabular}{|c|c|c|c|c|c|}
\hline $\begin{array}{l}\text { Ceiling Heights: Any special requirements: } \\
\text { If any state clear height }\end{array}$ & Yes & $x^{\text {No }}$ & $\begin{array}{l}\text { Noise: Any special requirements: } \\
\text { Is activity noisy }\end{array}$ & Yes & No \\
\hline \multirow[t]{2}{*}{\begin{tabular}{|l|} 
Heating \& Air Conditioning: \\
Standard for building is all that is required.
\end{tabular}} & $x$ & & $\begin{array}{l}\text { Lighting: Any special requirements: } \\
\text { Natural daylight needed }\end{array}$ & $x$ & \\
\hline & & & Above average Intensity & & \\
\hline Special exhaust is required for: & & & Ambient Lighting & & \\
\hline Excessive Heat & & & Ultra Violet required & & \\
\hline Cooking Odours & & & Glare Control & & \\
\hline \multirow[t]{2}{*}{ Humidity Control } & & & Full Spectrum Lighting & $x$ & \\
\hline & & & Other: & & \\
\hline Accurate Temp. Control required & & & Will the area require: & & \\
\hline If so, give range from to & & & Views to nature & & \\
\hline and limits +- & & & Quiet ambience & & \\
\hline \multirow{3}{*}{ Other - (specify under comments) } & & & & & \\
\hline & & & & & \\
\hline & & & Other (Specify under comments) & & \\
\hline
\end{tabular}

\section{Services: (place $x$ in box if required)}

Water:

Hot $\square$ Cold $\square$ Vapours $\square$ showers $\square$

Drainage: Floor $\square$

Other $\square$

or Comments:

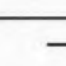

\section{Office \\ (re-hab director)}

$9.00 \mathrm{~m} 2$

$96.88 \mathrm{ft} 2$

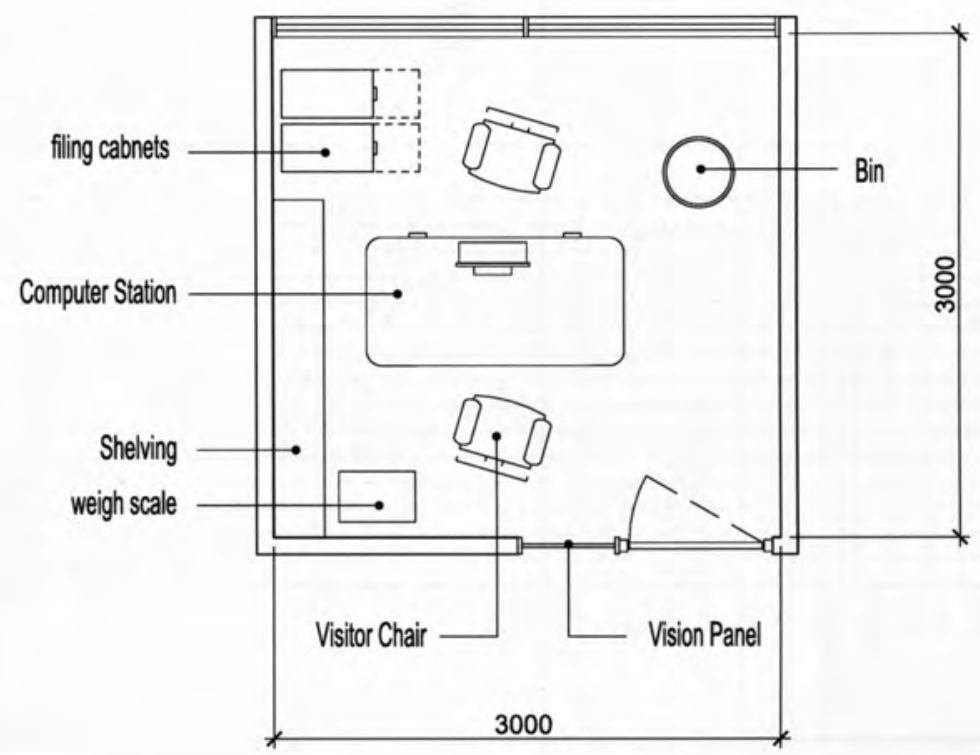

Meters $0,1,2$ 


\section{Analysis of physical facility requirements}

Project: Wellness Centre 2009

Name of Space: Juice Bar

\section{Space Description}

Purpose: To allow for a nutritional source of replenishment after physical exercise

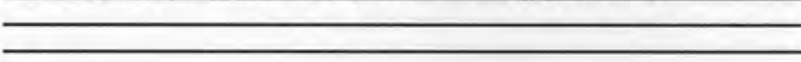

Space Relationships:

a. General- - How should this space relate to general areas in the building? eg: offices, teaching areas, loading dock, storage areas.

Located adjacent to the fitness rooms

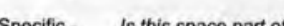

Yes $\square$

No $\square$
Space Components
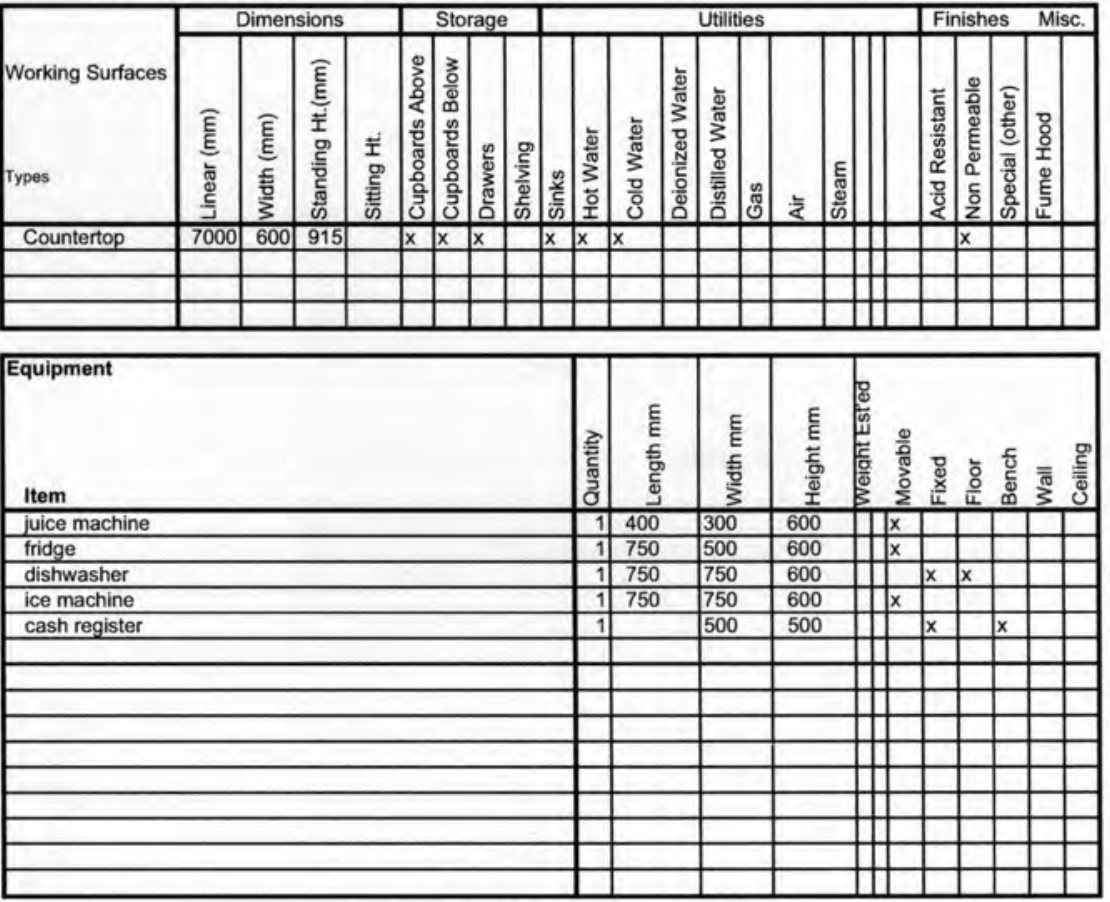

6. Education

$\square$

$\begin{array}{lll}\text { 1. Admin Office } & \square & \text { 6. Education } \\ \text { 2. Private Office } & \text { 7. Therapy } \\ \text { 3. Staff Room } & \text { 8. Information } \\ \text { 4. Medical Clinic } & \square & \text { 9. Common Area } \\ \text { 5. Fitness } & \square & \text { 10.Other Specify: }\end{array}$

Community gathering

Communications:

place $X$ in box

Telephone

P.A System

Cable TV

Internet Connection

Other Specify

\begin{tabular}{l|l} 
Audio Visual \\
The following audio visual aids will be used: \\
Television \\
Projector \\
Sound Reinforcement
\end{tabular}

$\begin{array}{lll}\begin{array}{l}\text { Staff } \\ \text { Patient }\end{array} & \text { Fitness Users } \\ \text { Students } & \text { Therapy Users } \\ \text { Community } & \text { Avg. hours per week usage }\end{array}$


Furnishings: (place $\mathrm{x}$ in box if required)

Storage Cabinets $\square$ Info Board $\square \quad$ Shelving $\square \quad$ Computer Station $\square$

Fixed: (List)

Movable: (List) 7 moveable bar stools

\begin{tabular}{|c|c|c|c|c|c|}
\hline \multicolumn{6}{|c|}{ Environmental Characteristics (place $\mathrm{x}$ in appropriate column) } \\
\hline $\begin{array}{l}\text { Ceiling Heights: Any special requirements: } \\
\text { If any state clear height }\end{array}$ & Yes & $x^{\text {No }}$ & $\begin{array}{l}\text { Noise: Any special requirements: } \\
\text { Is activity noisy }\end{array}$ & Yes & $\begin{array}{l}\text { No } \\
x \\
x\end{array}$ \\
\hline $\begin{array}{l}\text { Heating \& Air Conditioning: } \\
\text { Standard for building is all that is required. }\end{array}$ & $x$ & & $\begin{array}{l}\text { Lighting: Any special requirements: } \\
\text { Natural daylight needed }\end{array}$ & $x$ & \\
\hline & & & Above average intensity & & \\
\hline Special exhaust is required for: & & & Ambient Lighting & & \\
\hline Excessive Heat & & & Ultra Violet required & & \\
\hline Cooking Odours & & & Glare Control & & \\
\hline Humidity Control & & & Full Spectrum Lighting & $x$ & \\
\hline & & & Other: & & \\
\hline Accurate Temp. Control required & & & Will the area require: & & \\
\hline If so, give range from & & & Views to nature & & \\
\hline $\begin{array}{ll}\text { and limits }+- & \% \mathrm{RH} \\
\end{array}$ & & & Quiet ambience & & \\
\hline & & & & & \\
\hline Other - (specify under comments) & & & & & \\
\hline
\end{tabular}

\section{Services: (place $x$ in box if required)}

Water: $\quad$ Hot $\square$ Cold $\square$ Vapours $\square$ showers $\square$ Drainage: Floor $\square$ Other $\square$

Additional Requirements or Comments:

Space will have a back storage area of $8 \mathrm{~m} 2$

\section{Fitness Juice} Bar

$21.56 \mathrm{~m} 2$

$232.07 \mathrm{ft} 2$

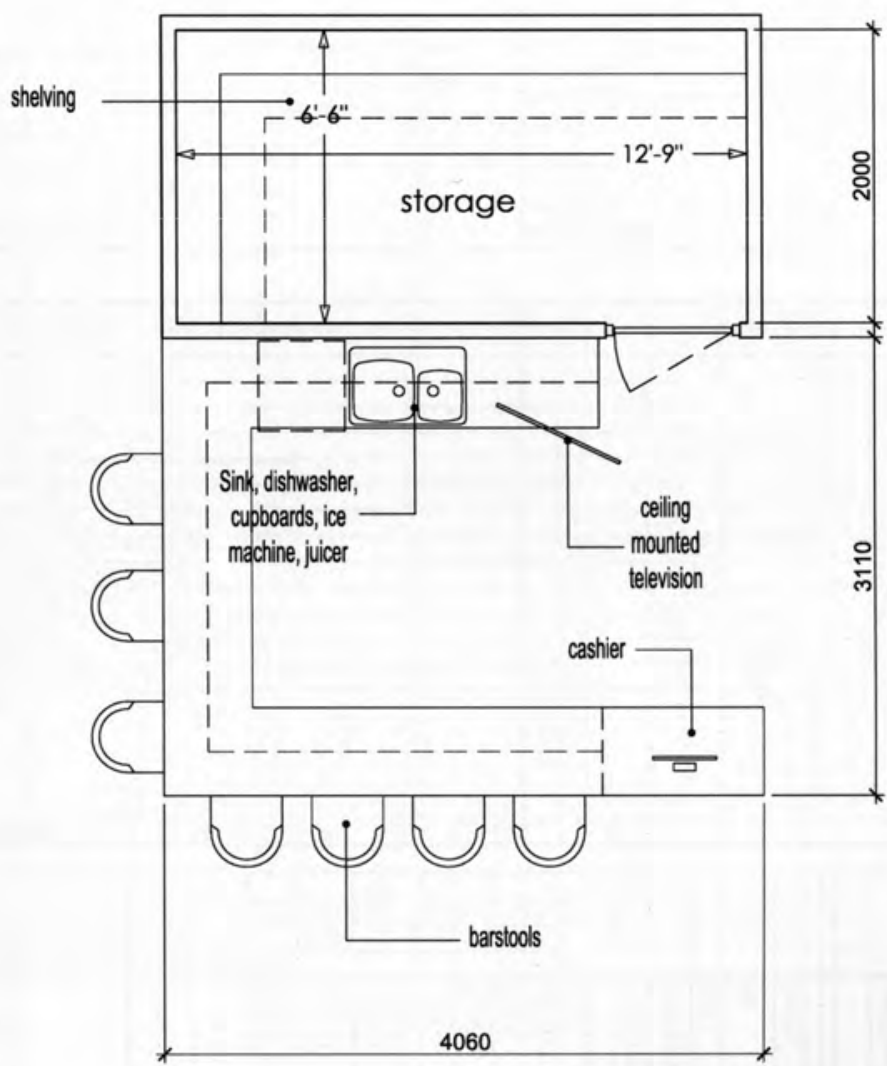

Meters

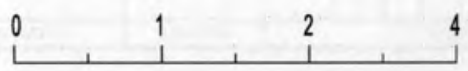




\section{Analysis of physical facility requirements}

Project: Wellness Centre 2009

Name of Space: Lockers/Showers (Both sexes)

\section{Space Description}

Purpose: To allow an area for fitness users to shower/change

\section{Space Relationships:}

How should this space relate to general areas in the building? eg: offices. eaching areas, loading dock, storage areas.

Located adjacent to the fitness rooms

\section{-}

b. Specific - Is this space part of a suite?

If yes, then describe its relationships to other spaces in the suit

No $\square$

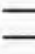

\section{Activity Description:}

$\vec{\infty}$

showering, changing and washing up

\section{Activity type: (insert $x$ in applicable box)}

1. Admin Office
2. Private Office
3. Staff Room
4. Medical Clinic
5. Fitness

6. Education

7. Therapy

8. Information

9. Common Area

10.Other Specify:

$\square$
$\square$
$\square$

Users: (insert number in appropriate boxes)

$\begin{array}{lll}\begin{array}{l}\text { Staff } \\ \text { Patient } \\ \text { Students } \\ \text { Community }\end{array} & \begin{array}{l}\text { Fitness Users } \\ \text { Therapy Users }\end{array} \\ \text { Avg. hours per week usage }\end{array}$

Space Components
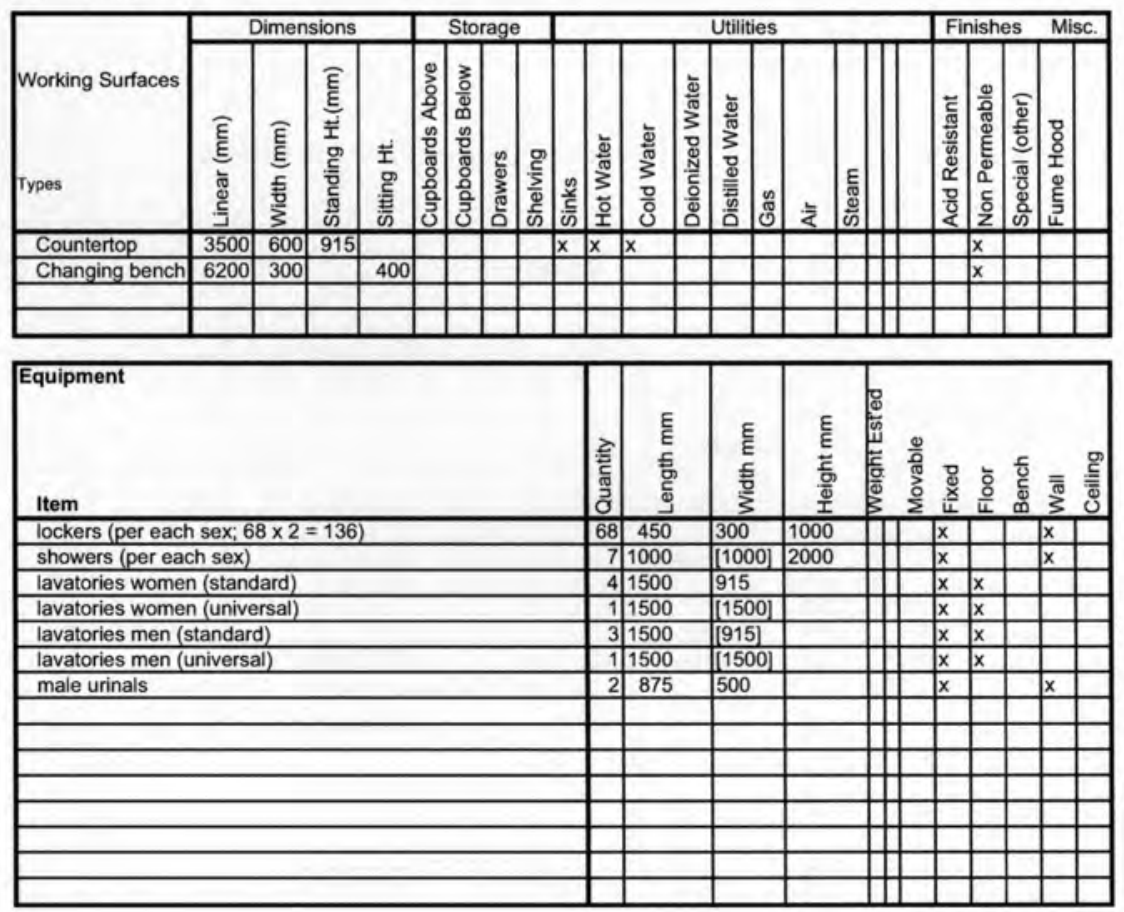

\begin{tabular}{|l|l|}
\hline $\begin{array}{l}\text { Communications: } \\
\text { (place } X \text { in box if required) } \\
\text { Telephone }\end{array}$ & $\begin{array}{l}\text { Audio Visual } \\
\text { The following audio visual aids will be used: } \\
\text { P.A System } \\
\text { Cable TV }\end{array}$ \\
Internet Connection & Television \\
Other Specify: & Pojector \\
\hline
\end{tabular}


Furnishings: (place $x$ in box if required)

\section{Storage Cabinets}

Fixed: (List)

Movable: (List) laundry basket

\begin{tabular}{|c|c|c|c|c|c|}
\hline \multicolumn{6}{|c|}{ Environmentalcharacteristics (place $x$ in appropriate column) } \\
\hline $\begin{array}{l}\text { Ceiling Heights: Any special requirements: } \\
\text { If any state clear height }\end{array}$ & Yes & $x^{\text {No }}$ & $\begin{array}{l}\text { Noise: Any special requirements: } \\
\text { Is activity noisy } \\
\text { Does activity require quiet }\end{array}$ & Tes & $\begin{array}{l}\text { No } \\
x \\
x\end{array}$ \\
\hline \multirow[t]{2}{*}{$\begin{array}{l}\text { Heating \& Air Conditioning: } \\
\text { Standard for building is all that is required. }\end{array}$} & $x$ & & $\begin{array}{l}\text { Lighting: Any special requirements: } \\
\text { Natural daylight needed }\end{array}$ & & \\
\hline & & & Above average Intensity & & \\
\hline Special exhaust is required for: & & & Ambient Lighting & & \\
\hline Excessive Heat & $x$ & & Ultra Violet required & & \\
\hline Cooking Odours & & & Glare Control & & \\
\hline \multirow[t]{2}{*}{ Humidity Control } & $\bar{x}$ & & Full Spectrum Lighting & $x$ & \\
\hline & & & Other: & & \\
\hline Accurate Temp. Control required & & & Will the area require: & & \\
\hline If so, give range from to & & & Views to nature & & \\
\hline \multicolumn{2}{|l|}{ and limits +- $\quad \% R H$} & & Quiet ambience & & \\
\hline \\
\hline \multicolumn{2}{|l|}{ Other - (specify under comments) } & & Other (Specify under comments) & & \\
\hline \multicolumn{6}{|l|}{ Services: (place $x$ in box if required) } \\
\hline \multicolumn{3}{|l|}{ Water: } & Drainage: & & \\
\hline
\end{tabular}

Additional Requirements or Comments:

space will contain a janitors closet; space will contain 4 sinks;
Locker Rooms/showers/wc

$66.33 \mathrm{~m} 2 \quad 66.33 \mathrm{~m} 2$

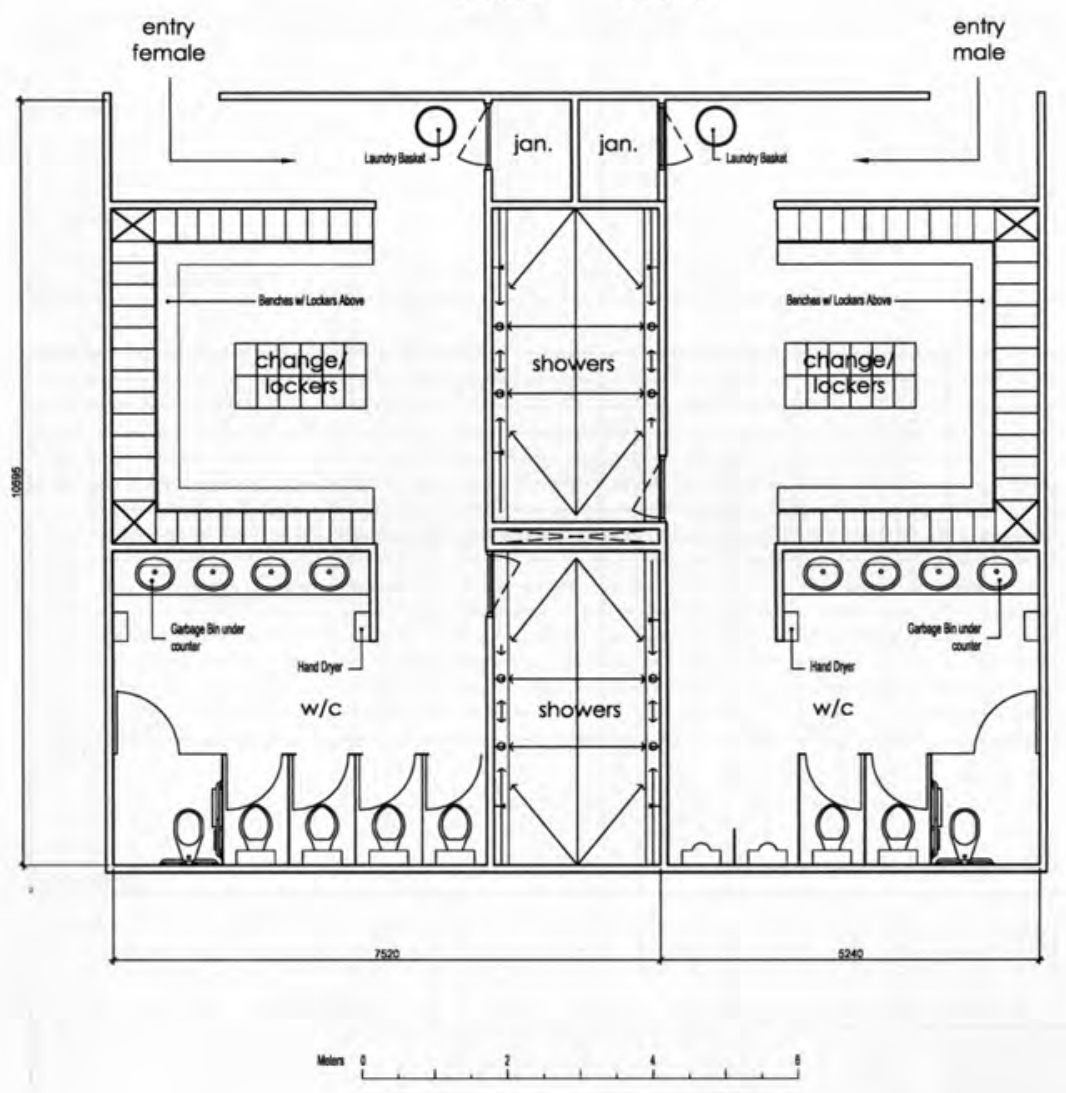




\section{Analysis of physical facility requirements}

Project: Wellness Centre 2009

Name of Space: Laundry storage

Department Fitness Wing Net Assignable Sq. $m$. 6

\section{Space Description}

Purpose: An area for the storage of towel linens for fitness users

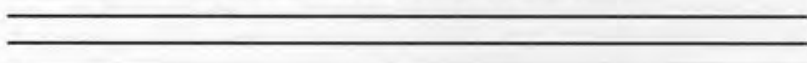

Space Relationships:

a. General - How should this space relate to general areas in the building? eg: offices, teaching areas, loading dock, storage areas.

Located with proximity to the fitness rooms b. Specific - Is this space part of a suite?
If yes, then describe its relationships to other spaces in the suite

\section{Activity Description:}

\begin{tabular}{ll}
$\overrightarrow{8}$ & \multicolumn{2}{l}{ Janitors will move clean towels from main laundry to this temporary storage } \\
\hline of both clean and dirty towels \\
Activity type: (insert x in applicable box) \\
1. Admin Office \\
2. Private Office \\
3. Staff Room \\
4. Medical Clinic
\end{tabular}

Users: (insert number in appropriate boxes)

$\begin{array}{lll}\text { Staff } & \text { Fitness Users } \\ \text { Patient } & \text { Therapy Users } \\ \text { Students } & \text { Avg. hours per week usage } \\ \text { Community } & \end{array}$

Space Components
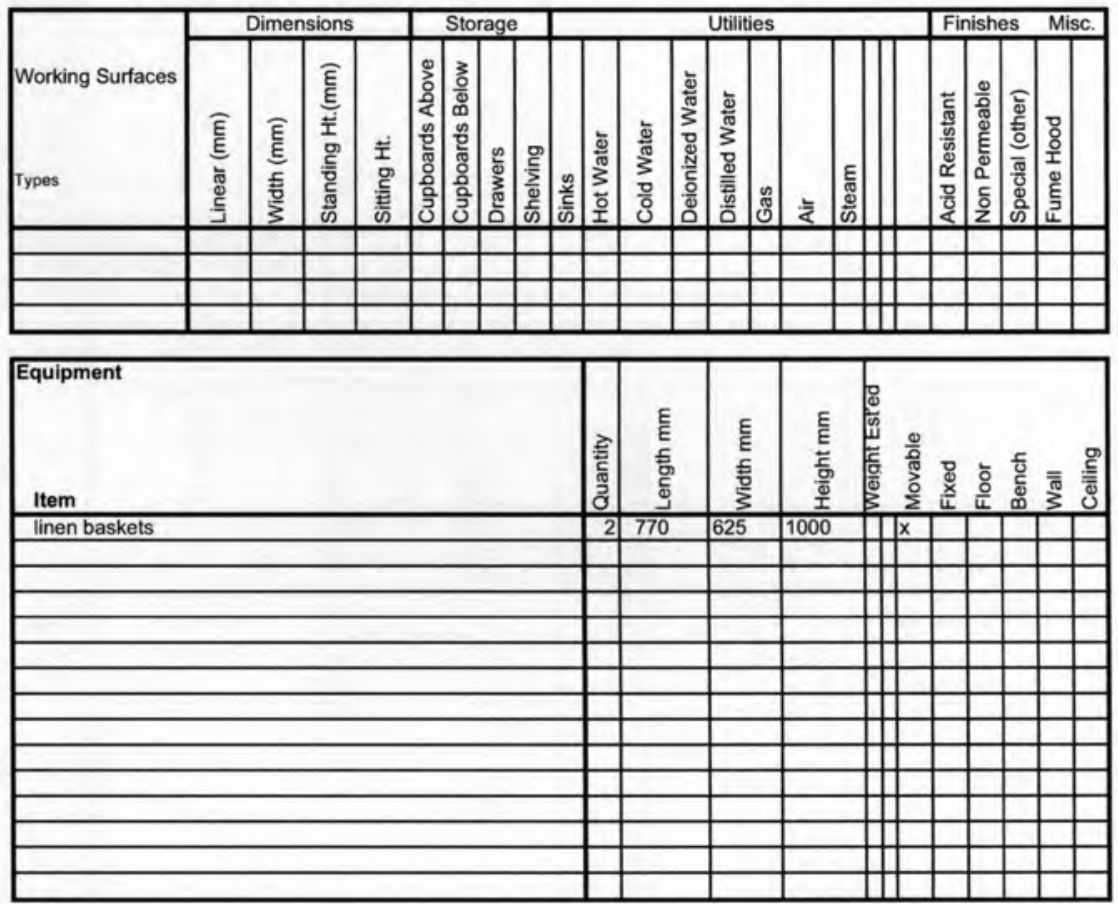

\begin{tabular}{|l|l|}
\hline $\begin{array}{l}\text { Communications: } \\
\text { (place X in box if required) } \\
\text { Telephone }\end{array}$ & $\begin{array}{l}\text { Audio Visual } \\
\text { The following audio visual aids wall be used: } \\
\text { P.A System } \\
\text { Cable TV }\end{array}$ \\
Internet Connection & Television \\
Other Specify: & Projector \\
\hline
\end{tabular}




\section{Analysis of physical facility requirements}

Project: Wellness Centre 2009

Name of Space: Reception and Control

Department Public Swim Net Assignable Sq. m

\section{Space Description}

Purpose:

An area of welcome and direction for users and patrons

Space Relationships:

a. General - How should this space relate to general areas in the building? eg: offices, teaching areas, loading dock, storage arees.

Located as the intial point of interaction upon entering the swim facility; adjacent to the locker rooms

b. Specific - Is this space part of a sute? Yes $\square \quad$ No $\square$ If yes, then describe its relationships to other spaces in the suite

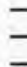

Activity Description: $\overrightarrow{0} \quad$ The area is envisioned as an comprehensive welcoming, and further direction

$\vec{N}$

Activity type: (insert $x$ in applicable box)

$\begin{array}{lll}\text { 1. Admin Office } & \square & \text { 6. Education } \\ \text { 2. Private Office } & \text { 7. Therapy } \\ \text { 3. Staff Room } & \square & \text { 8. Information } \\ \text { 4. Medical Clinic } & \square & \text { 9. Common Area } \\ \text { 5. Fitness } & \square & \text { 10.Other Specify: }\end{array}$

Users: (insert number in appropriate boxes)

Staff

Patient

Students

Community
Fitness Users

Therapy Users

Avg. hours per week usage
Space Components
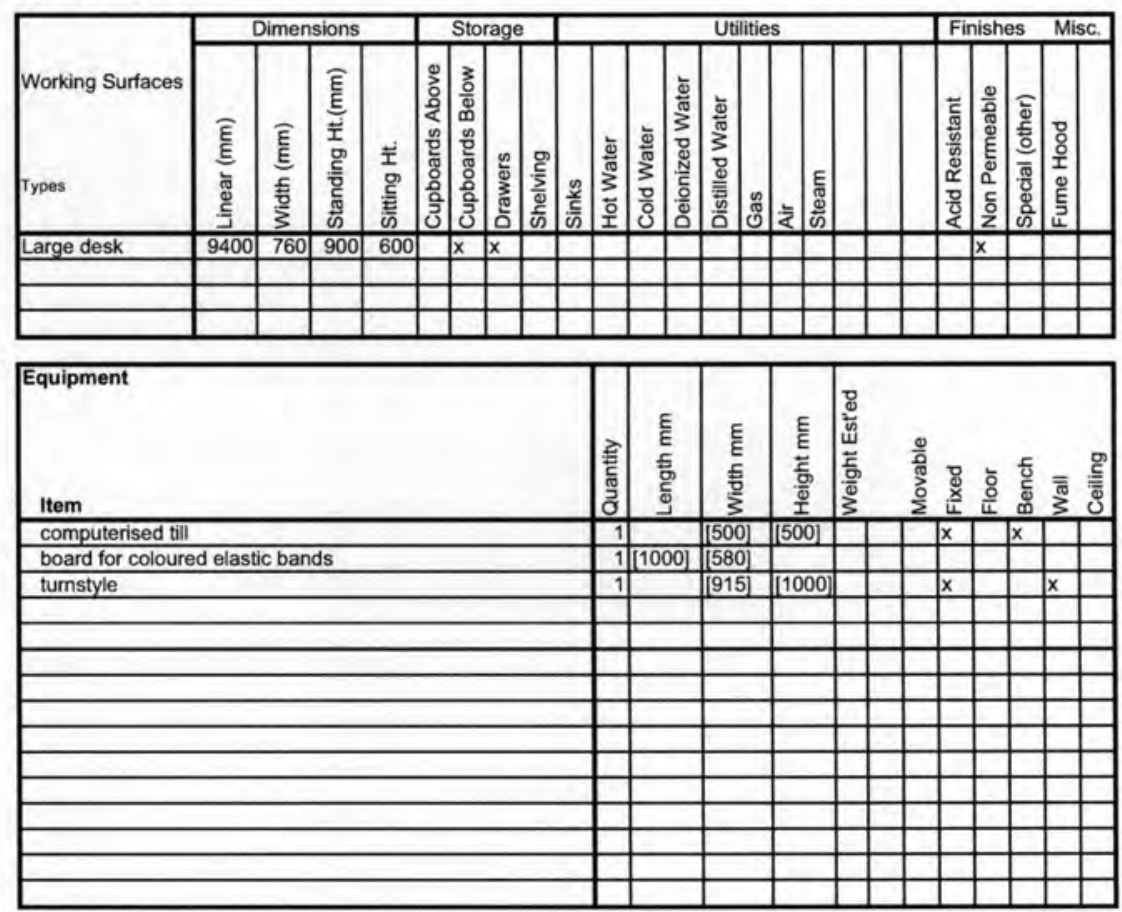

\begin{tabular}{ll|l|}
\hline $\begin{array}{l}\text { Communications: } \\
\text { (place X in box if required) } \\
\text { Telephone }\end{array}$ & $\begin{array}{l}\text { Audio Visual } \\
\text { The following audio visual ads will be used: } \\
\text { P.A System }\end{array}$ \\
Cable TV & $\begin{array}{l}\text { Television } \\
\text { Internet Connection }\end{array}$ \\
Projector \\
Sound Reinforcement
\end{tabular}




\begin{tabular}{|lll|}
\hline Furnishings: & (place x in box if required) \\
\hline Storage Cabinets & $\square$ Info Board $\square$ & \\
Fixed: (List) & & \\
Movable: (List) & Shelving $\square$ \\
\hline
\end{tabular}

\begin{tabular}{|c|c|c|c|c|c|}
\hline \multicolumn{6}{|c|}{ Environmental Characteristics (place $\mathrm{x}$ in appropriate column) } \\
\hline $\begin{array}{l}\text { Ceiling Heights: Any special requirements: } \\
\text { If any state clear height }\end{array}$ & Tyes & $x^{\text {No }}$ & $\begin{array}{l}\text { Noise: Any special requirements: } \\
\text { Is activity noisy } \\
\text { Does activity require quiet }\end{array}$ & Yes & $\begin{array}{l}\text { No } \\
x \\
x\end{array}$ \\
\hline \multirow[t]{2}{*}{$\begin{array}{l}\text { Heating \& Air Conditioning: } \\
\text { Standard for building is all that is required. }\end{array}$} & $x$ & & $\begin{array}{l}\text { Lighting: Any special requirements: } \\
\text { Natural daylight needed }\end{array}$ & $x$ & \\
\hline & & & Above average Intensity & & \\
\hline Special exhaust is required for: & & & Ambient Lighting & & \\
\hline Excessive Heat & & & Ultra Violet required & & \\
\hline Cooking Odours & & & Glare Control & & \\
\hline \multirow[t]{2}{*}{ Humidity Control } & $x$ & & Full Spectrum Lighting & $x$ & \\
\hline & & & Other: & & \\
\hline Accurate Temp. Control required & & & Will the area require: & & \\
\hline If so, give range from to & & & Views to nature & $x$ & \\
\hline and limits +- $\quad \% R H$ & & & Quiet ambience & & \\
\hline \multirow[b]{2}{*}{ Other - (specify under comments) } & & & & & \\
\hline & & & & & \\
\hline Other - (speciry under comments) & & & Other (Specify under comments) & & \\
\hline \multicolumn{6}{|l|}{ Services: (place $x$ in box if required) } \\
\hline \multicolumn{3}{|l|}{ Water: } & Floor $\square$ & & \\
\hline
\end{tabular}

Additional Requirements or Comments:

Receptionist will give patrons coloured bands, towels and locker keys; receptionist will control the amount of individuals entering the facility

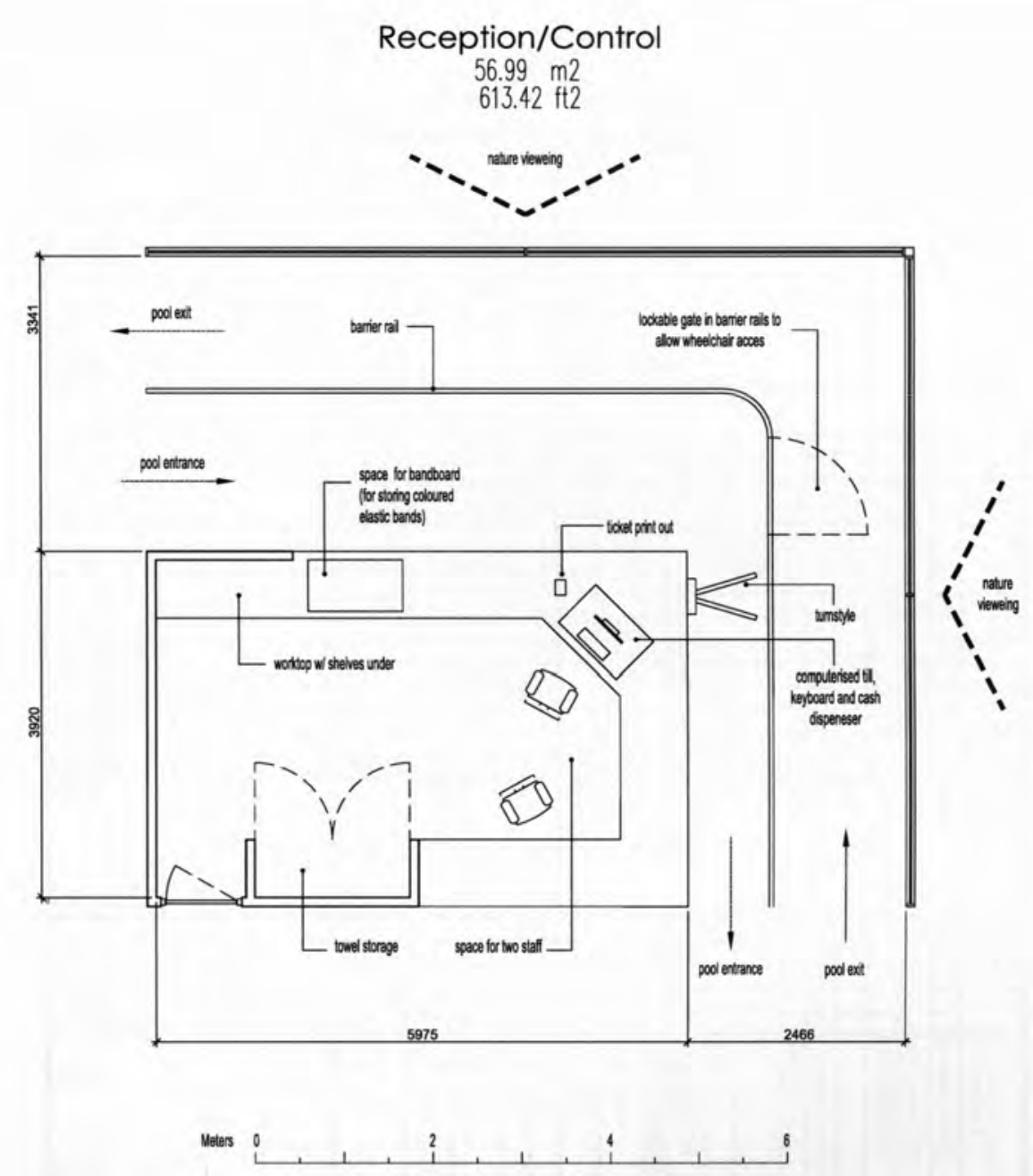




\section{Analysis of physical facility requirements}

Project: Wellness Centre 2009

Name of Space: Lockers/Showers (Both sexes)

Department Public Swim

Space Description

Purpose: To allow an area for fitness users to shower/change

\section{Space Relationships:}

a. General - How should this space relate to general areas in the building? eg. offices

Located adjacent to the reception control

b. Specific - Is this space part of a suite?
If yes, then describe its relations

Yes $\square \quad$ № $\square$

If yes, then describe its relationships to other spaces in the suite

Activity Description

$\vec{\oplus}$

$\overrightarrow{0}$

Activity type: (insert $x$ in applicable box)

$\begin{array}{lll}\text { 1. Admin Office } & \square & \text { 6. Education } \\ \text { 2. Private Office } & \square & \text { 7. Therapy } \\ \text { 3. Staff Room } & \text { 8. Information } \\ \text { 4. Medical Clinic } & \square & \text { 9. Common Area } \\ \text { 5. Fitness } & \square & \text { 10.Other Specify: } \\ & \text { Public Swim }\end{array}$

Users: (insert number in appropriate boxes)

Staff

Patient

Students

Community

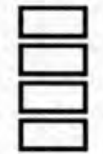

Fitness Users

Therapy Users

Swim users

Avg. hours per week usage
Space Components
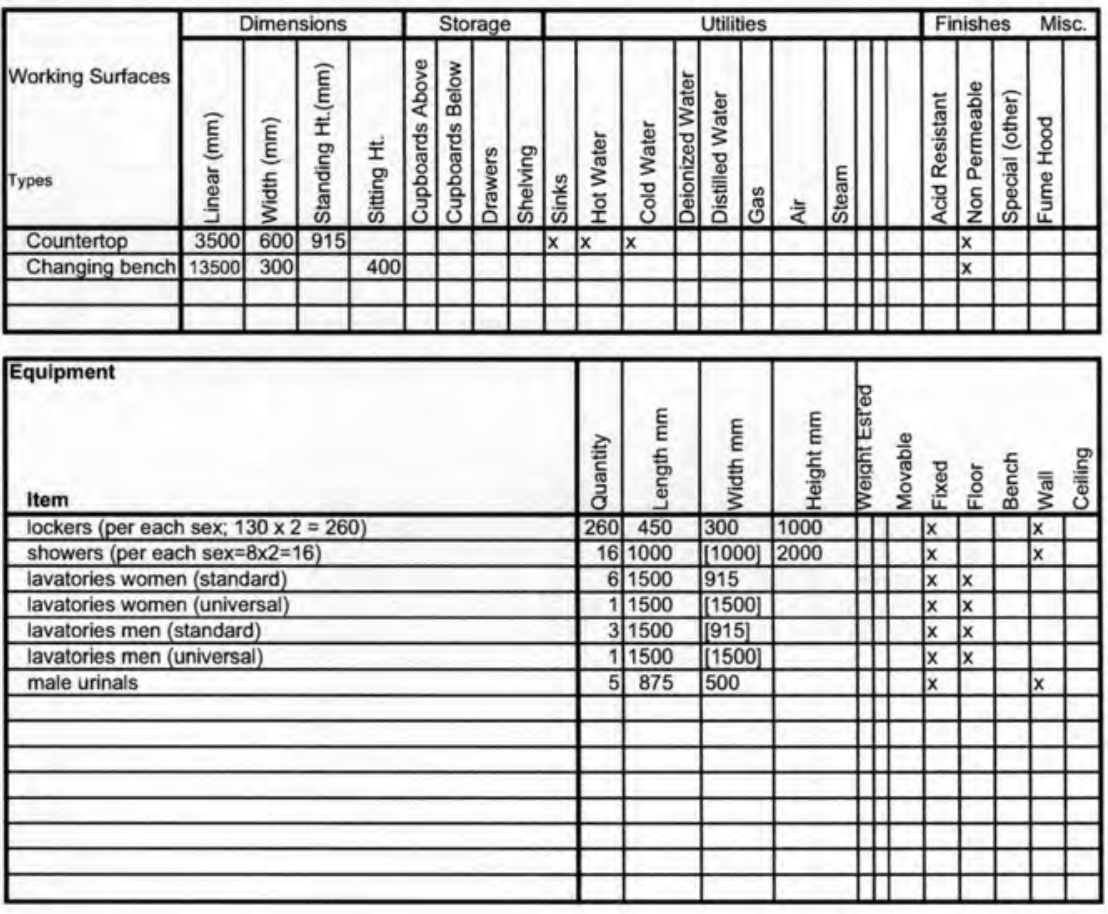

\begin{tabular}{|l|l|}
\hline $\begin{array}{l}\text { Communications: } \\
\text { (place X in box if required) } \\
\text { Telephone }\end{array}$ & $\begin{array}{l}\text { Audio Visual } \\
\text { The tollowing audio visual aids wall be used: } \\
\text { P.A System } \\
\text { Cable TV } \\
\text { Internet Connection }\end{array}$ \\
Other Specify: & $\begin{array}{l}\text { Television } \\
\text { Projector } \\
\text { Sound Reinforcement }\end{array}$ \\
\hline
\end{tabular}




\begin{tabular}{|lll|}
\hline Furnishings: & (place x in box If required) \\
\hline Storage Cabinets & $\square$ Info Board $\square$ \\
Fixed: (List) & & \\
Movable: (List) & laundry basket \\
\hline
\end{tabular}

\begin{tabular}{|c|c|c|c|c|c|}
\hline \multicolumn{6}{|c|}{ EnvironmentalCharacteristics (place $x$ in appropriate column) } \\
\hline $\begin{array}{l}\text { Ceiling Heights: Any special requirements: } \\
\text { If any state clear height }\end{array}$ & Tyes & $x^{\text {No }}$ & $\begin{array}{l}\text { Noise: Any special requirements: } \\
\text { Is activity noisy }\end{array}$ & Tyes & $\int_{x}^{\text {No }}$ \\
\hline \multirow[t]{2}{*}{$\begin{array}{l}\text { Heating \& Air Conditioning: } \\
\text { Standard for building is all that is required. }\end{array}$} & $x$ & & $\begin{array}{l}\text { Lighting: Any special requirements: } \\
\text { Natural daylight needed }\end{array}$ & & \\
\hline & & & Above average Intensity & & \\
\hline Special exhaust is required for: & & & Ambient Lighting & & \\
\hline Excessive Heat & $x$ & & Ultra Violet required & & \\
\hline Cooking Odours & & & Glare Control & & \\
\hline \multirow[t]{2}{*}{ Humidity Control } & $x$ & & Full Spectrum Lighting & $x$ & \\
\hline & & & Other: & & \\
\hline \multirow{2}{*}{\multicolumn{2}{|c|}{$\begin{array}{l}\text { Accurate Temp. Control required } \\
\text { If so, give range from } 22^{\circ} \mathrm{C} \text { to } 24^{\circ} \mathrm{C}\end{array}$}} & & Will the area require: & & \\
\hline & & & Views to nature & & \\
\hline \multicolumn{2}{|l|}{ and limits +- $\quad \%$ RH } & & Quiet ambience & & \\
\hline \multirow{2}{*}{\multicolumn{2}{|c|}{ Other - (specify under comments) }} & & & & \\
\hline & & & & & \\
\hline & & & Other (Specify under comments) & & \\
\hline \multicolumn{6}{|l|}{ Services: (place $x$ in box if required) } \\
\hline \multicolumn{3}{|l|}{ Water: } & Drainage: Floor $\square$ & $\square$ & \\
\hline
\end{tabular}

Additional Requirements or Comments:

space will contain a janitors closet; space will contain 4 sinks:

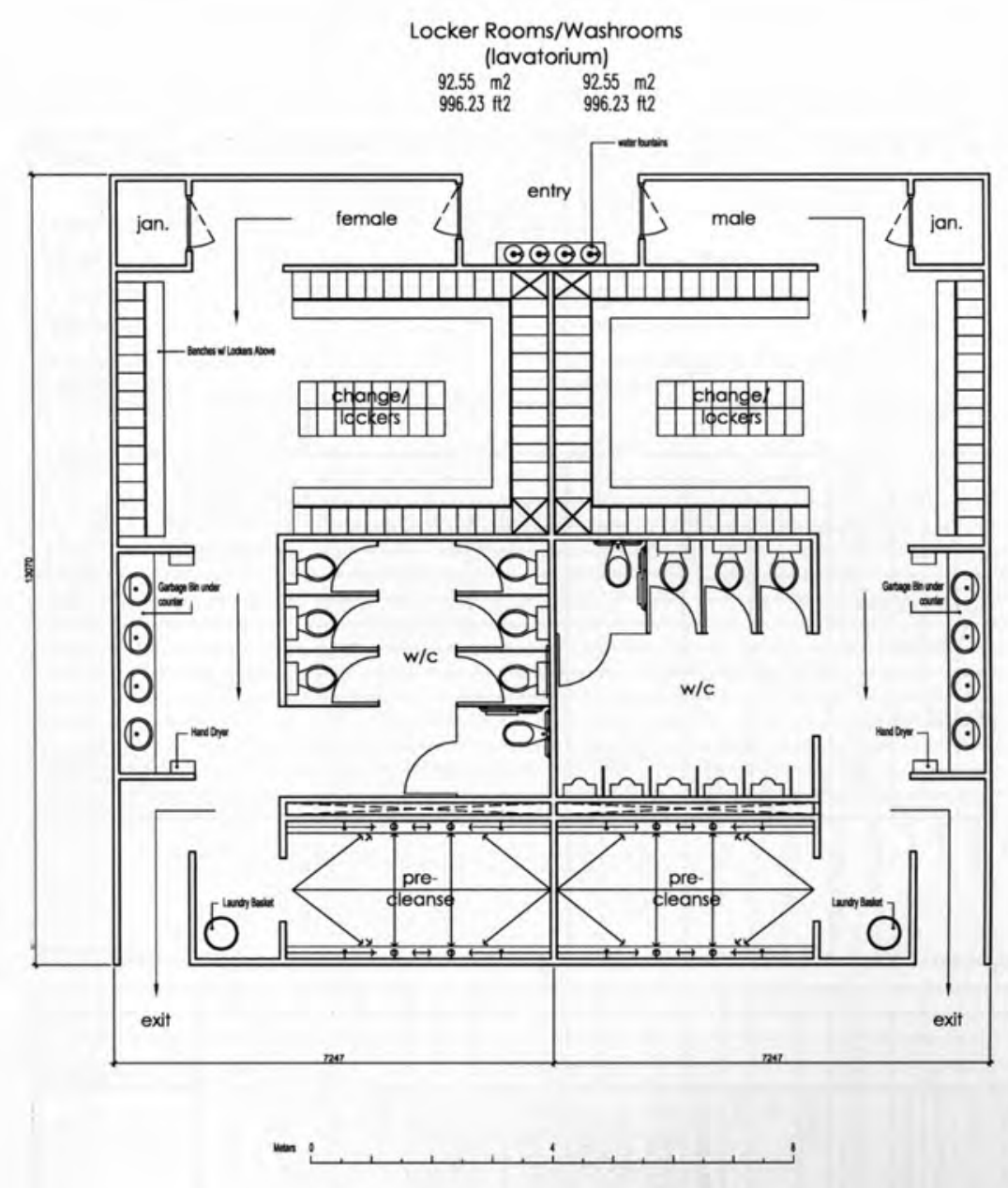


Analysis of physical facility requirements

Project: Wellness Centre 2009

Department Public Swim

Name of Space: First Aid

Net Assignable Sq. m

Space Description

Purpose: To provide an area for first aid treatment

Space Relationships:

a. General - How should this space relate to general areas in the building? eg: offices, teaching areas, loading dock, storage areas.

close to the main swimming poo

b. Specific - Is this space part of a suite?
If yes, then describe its relationships to other spaces in the suite

Activity Description:

$\vec{\varphi} \quad$ a space for certified individuals to conduct medical treatment in case of

Activity type: (insert $x$ in applicable box)

1. Admin Office

2. Private Office

$\square$

3. Staff Room

4. Medical Clinic

$\square$

6. Education

7. Therapy

8. Information

9. Common Area

10. Other Specify:

public swim

Users: $\quad$ (insert number in appropriate boxes)

$\begin{array}{lll}\text { Staff } & \text { Fitness Users } \\ \text { Patient } & \text { Therapy Users } \\ \text { Students } & \text { Avg. hours per week usage } \\ \text { Community } & \end{array}$

Space Components
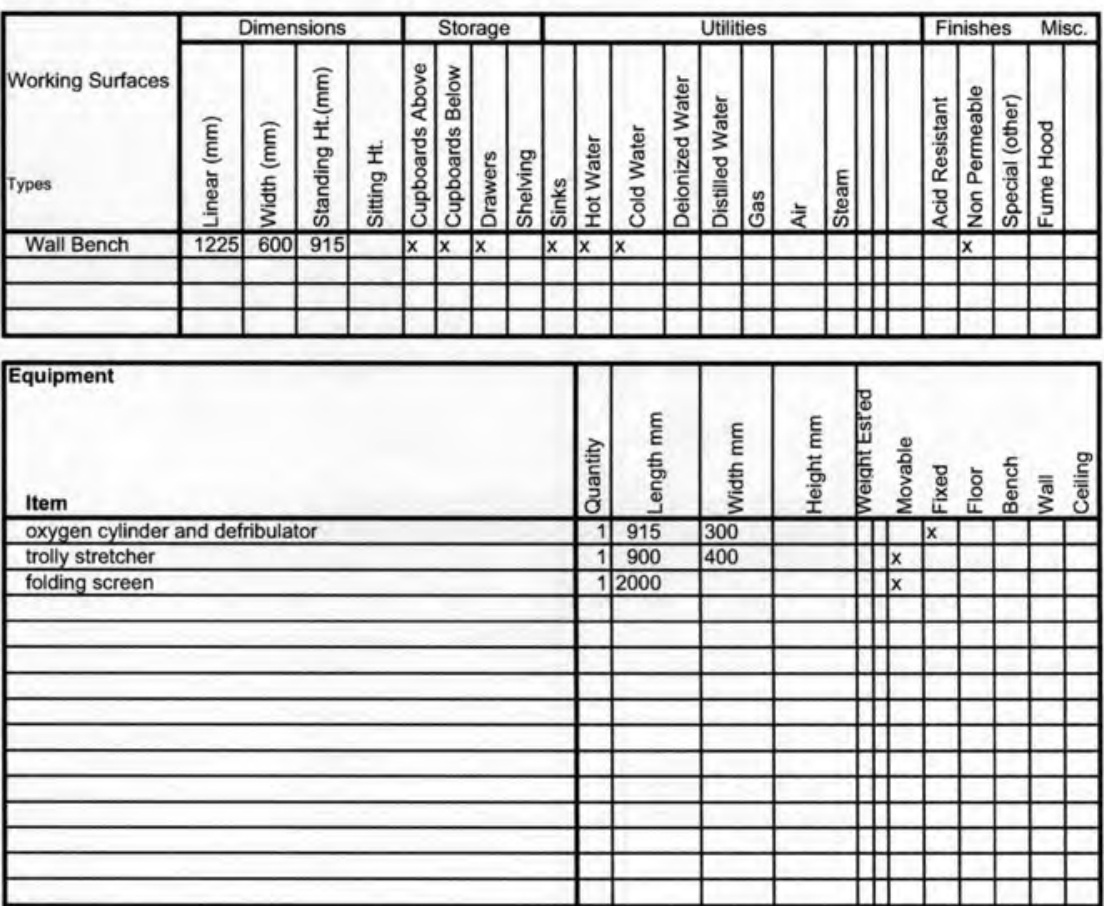

\begin{tabular}{ll|l|}
\hline $\begin{array}{l}\text { Communications: } \\
\text { (placo X in box if required) } \\
\text { Telephone }\end{array}$ & $\begin{array}{l}\text { Audio Visual } \\
\text { The following audio visual aids will be used: } \\
\text { P.A System } \\
\text { Cable TV }\end{array}$ \\
Internet Connection & Television \\
Projector \\
Other Specify:
\end{tabular}




\begin{tabular}{|ll|}
\hline Furnishings: & (place xin boxif required) \\
\hline Storage Cabinets & $\square$ Info Board $\square \quad$ Shelving $\square$ \\
Fixed: (List) & \\
Movable: (List) & 2 staff chairs, \\
\hline
\end{tabular}

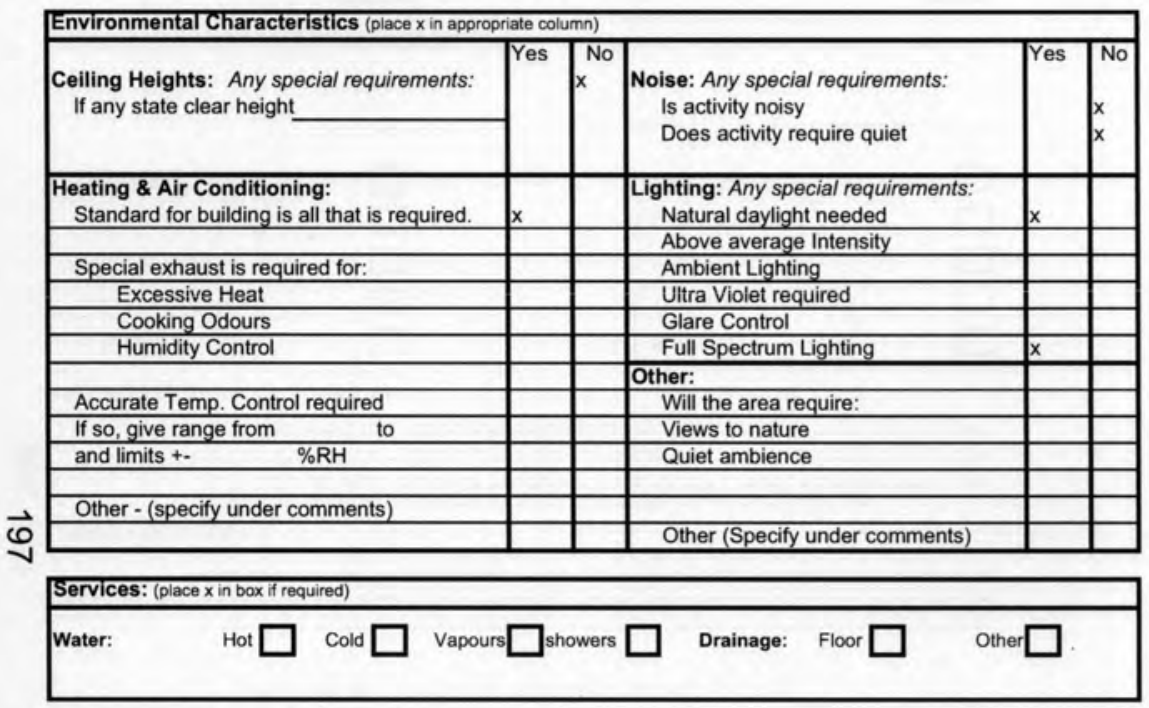

Additional Requirements or Comments:



\section{First Aid}

$10.50 \mathrm{~m} 2$

$113.02 \mathrm{ft} 2$

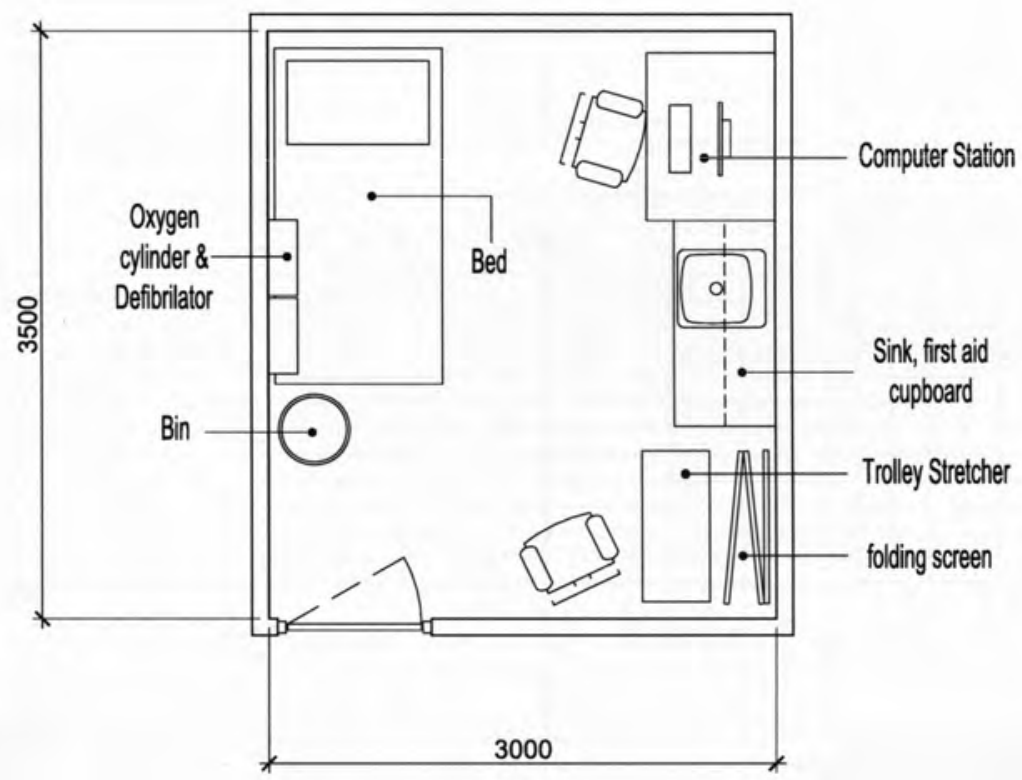

Meters

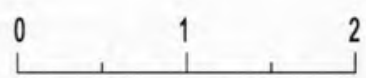




\section{Analysis of physical facility requirements}

Project: Wellness Centre 2009

Name of Space: Office (manager)

Department Public swim Net Assignable Sq. m

Space Description

Purpose: To provide an area for the public swim office manager

\section{Space Relationships:}

. General - How should this space relate to general areas in the building? eg: offices, teaching areas, loading dock, storage areas.

close to medical main swimming pool

b. Specific - Is this space part of a suite?
If yes, then describe its relationships to other spaces in the suite

Activity Description:

$\vec{\oplus}$

an area for the manger to supervise the successful operation of the pool

Activity type: (insert $x$ in applicable box)

$\begin{array}{lll}\text { 1. Admin Office } & \square & \text { 6. Education } \\ \text { 2. Private Office } & \square & \text { 7. Therapy } \\ \text { 3. Staff Room } & \square & \text { 8. Information } \\ \text { 4. Medical Clinic } & \square & \text { 9. Common Area } \\ \text { 5. Fitness } & \square & \text { 10.Other Specify: } \\ & & \text { Public Swim }\end{array}$

Users: (insert number in appropriate boxes)

$\begin{array}{lll}\text { Staff } & \text { Fitness Users } \\ \text { Patient } & \text { Therapy Users } \\ \text { Students } & \end{array}$

Space Components
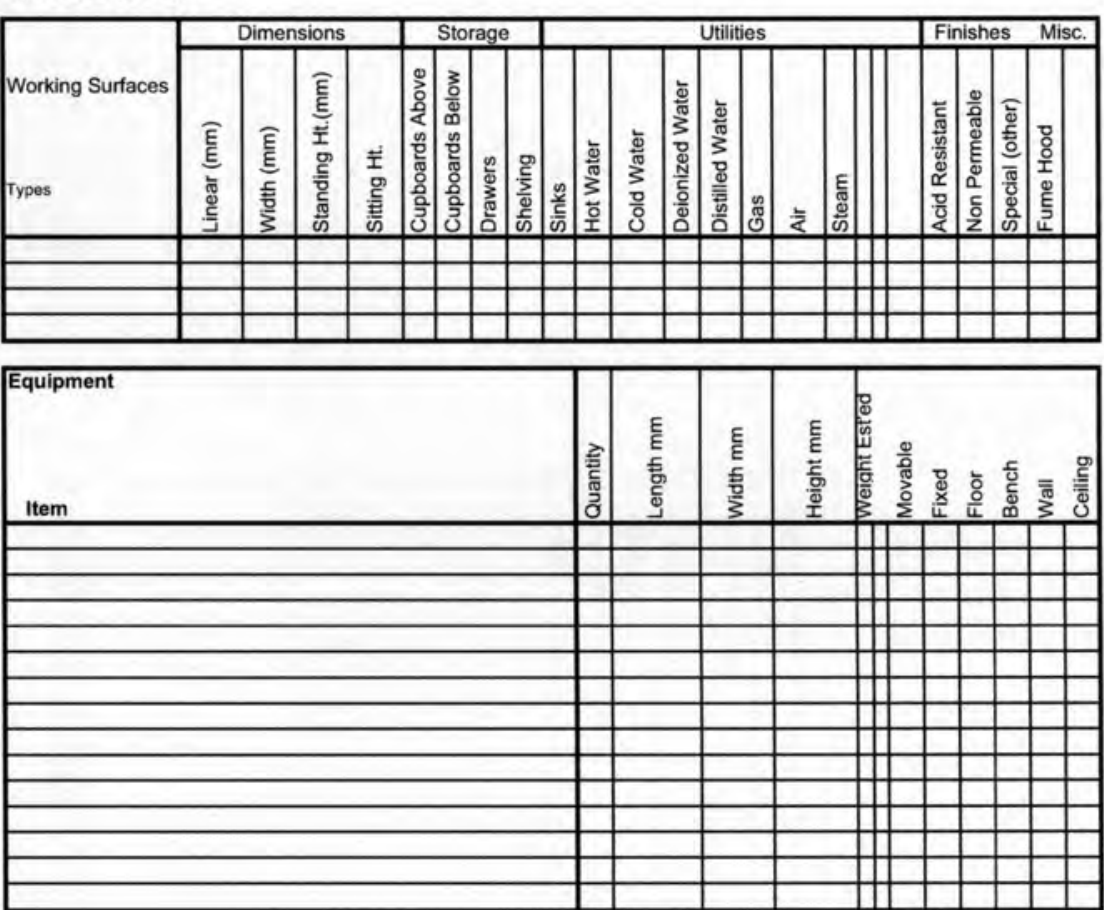

\begin{tabular}{|l|l|}
\hline $\begin{array}{l}\text { Communications: } \\
\text { (place } X \text { in box if required) }\end{array}$ & $\begin{array}{l}\text { Audio Visual } \\
\text { The following audio visual aids will be used: } \\
\text { Telephone }\end{array}$ \\
$\begin{array}{l}\text { P.A System } \\
\text { Cable TV } \\
\text { Internet Connection }\end{array}$ & $\begin{array}{l}\text { Television } \\
\text { Projector } \\
\text { Sound Reinforcement }\end{array}$ \\
Other Specify: & Other Specify: \\
\hline
\end{tabular}




\begin{tabular}{|ll|}
\hline Furnishings: & (place xin boxif required) \\
\hline Storage Cabinets & $\square$ info Board $\square \quad$ Shelving $\square$ \\
Fixed: (List) & \\
Movable: (List) & filing cabinets, computer chair and visitor chair \\
\hline
\end{tabular}

\begin{tabular}{|c|c|c|c|c|c|}
\hline \multicolumn{6}{|c|}{ Environmental Characteristics (place $x$ in appropriate column) } \\
\hline $\begin{array}{l}\text { Ceiling Heights: Any special requirements: } \\
\text { If any state clear height }\end{array}$ & Yes & $x^{\text {No }}$ & $\begin{array}{l}\text { Noise: Any special requirements: } \\
\text { Is activity noisy } \\
\text { Does activity require quiet }\end{array}$ & Yes & $\begin{array}{l}\text { No } \\
x \\
x\end{array}$ \\
\hline \multirow[t]{2}{*}{$\begin{array}{l}\text { Heating \& Air Conditioning: } \\
\text { Standard for building is all that is required. }\end{array}$} & $\mathrm{x}$ & & $\begin{array}{l}\text { Lighting: Any special requirements: } \\
\text { Natural daylight needed }\end{array}$ & $\mathrm{x}$ & \\
\hline & & & Above average Intensity & & \\
\hline Special exhaust is required for: & & & Ambient Lighting & & \\
\hline Excessive Heat & & & Ultra Violet required & & \\
\hline Cooking Odours & & & Glare Control & & \\
\hline \multirow[t]{2}{*}{ Humidity Control } & & & Full Spectrum Lighting & $x$ & \\
\hline & & & Other: & & \\
\hline Accurate Temp. Control required & & & Will the area require: & & \\
\hline \multirow{2}{*}{$\begin{array}{ll}\text { If so, give range from } & \text { to } \\
\text { and limits +- } & \% R H \\
\end{array}$} & & & Views to nature & & \\
\hline & & & Quiet ambience & & \\
\hline & & & & & \\
\hline \multirow[t]{2}{*}{ Other - (specify under comments) } & & & & & \\
\hline & & & Other (Specify under comments) & & \\
\hline \multicolumn{6}{|l|}{ Services: (place $x$ in box if required) } \\
\hline \multicolumn{3}{|l|}{ Water: } & Drainage: & $\square$ & \\
\hline
\end{tabular}

Additional Requirements or Comments:

\section{Office \\ (office manager)}

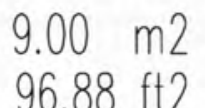

$96.88 \mathrm{ft} 2$
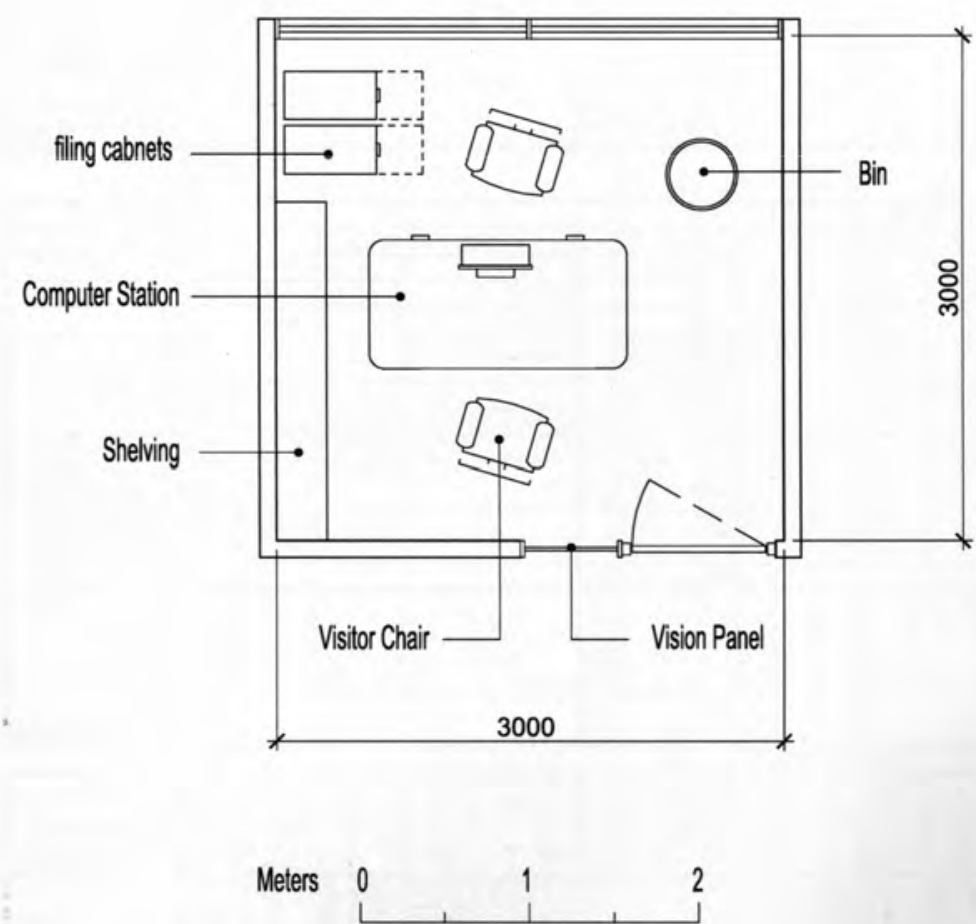
Analysis of physical facility requirements

Project: Wellness Centre 2009

Department Public Swim

Project: of Space: Staffroom

Net Assignable Sq. m

Space Description

Purpose: An area provided for swim staff and other building staff to change, relax, enjoy meals and have informal meetings

Space Relationships:

a. General - How should this space relate to general areas in the building? eg: offices, teaching areas, loading dock, storage areas.

this space should be located in the back of house, out of the way from community spaces

b. Specific - Is this space part of a suite? $\quad$ Yes $\square \quad$ № $\square$ yes, then describe its relationships to other spaces in the suito

$\longrightarrow+2$

Activity Description:

응

a space for staff to have lunch, change for shifts and have informal meetings

Activity type: (insert $x$ in applcable box)

$\begin{array}{lll}\text { 1. Admin Office } & \text { 6. Education } \\ \text { 2. Private Office } & \square & \text { 7. Therapy } \\ \text { 3. Staff Room } & \text { 8. Information } \\ \text { 4. Medical Clinic } & \square & \text { 9. Common Area } \\ \text { 5. Fitness } & \square & \text { 10.Other Specify: }\end{array}$

Users: $\quad$ (insert number in appropriate boxes)

$\begin{array}{lll}\begin{array}{l}\text { Staff } \\ \text { Patient } \\ \text { Students } \\ \text { Community }\end{array} & \begin{array}{l}\text { Fitness Users } \\ \text { Therapy Users }\end{array}\end{array}$

Space Components
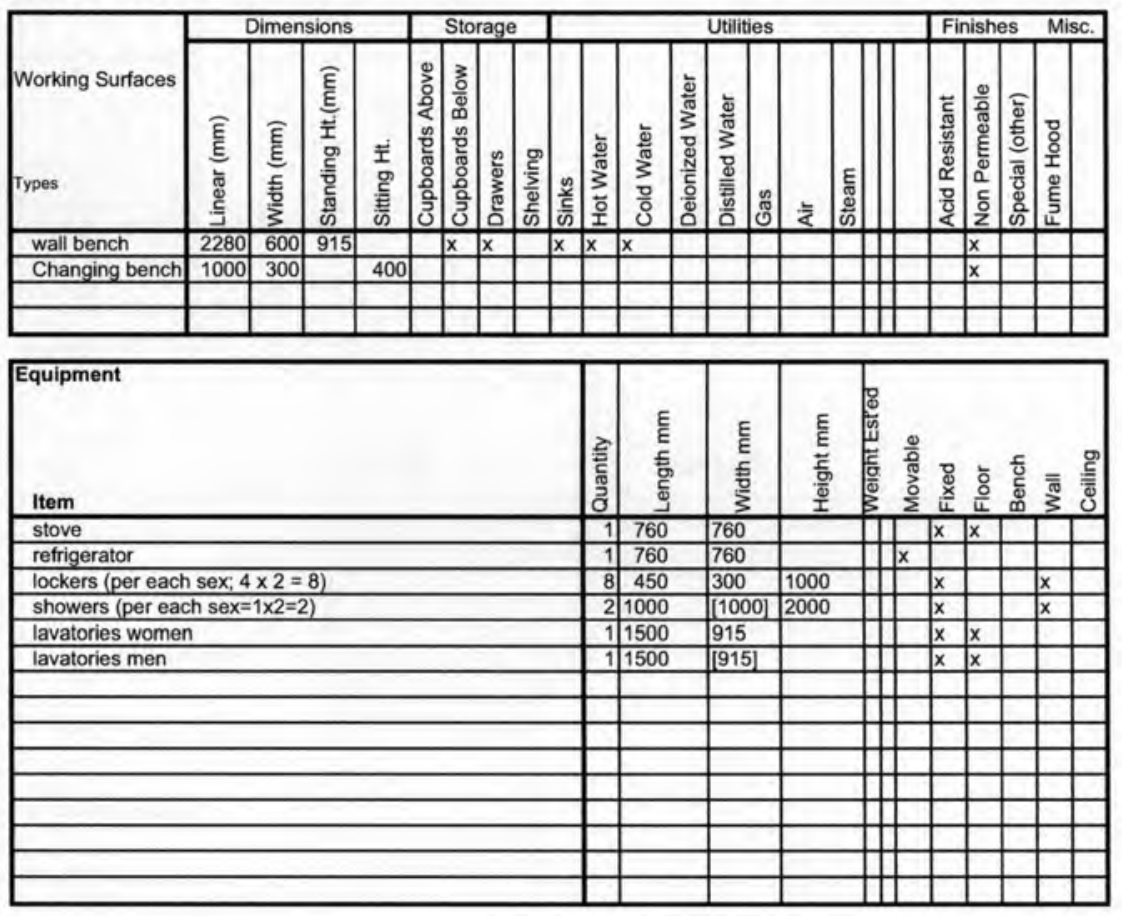

\begin{tabular}{ll|l|}
\hline $\begin{array}{l}\text { Communications: } \\
\text { (place X in box f frequired) } \\
\text { Telephone }\end{array}$ & $\begin{array}{l}\text { Audio Visual } \\
\text { The following audio visual aids will be used: } \\
\text { P.A System }\end{array}$ \\
Cable TV & Television \\
Internet Connection & Projector \\
Other Sound Reinforcement & \\
\hline
\end{tabular}




\begin{tabular}{|ll|}
\hline Furnishings: $\quad$ (place x in box if required) \\
\hline Storage Cabinets \\
Fixed: (List) \\
Movable: (List) & Info Board $\square \quad$ Shelving $\square$ \\
\cline { 2 - 2 }
\end{tabular}

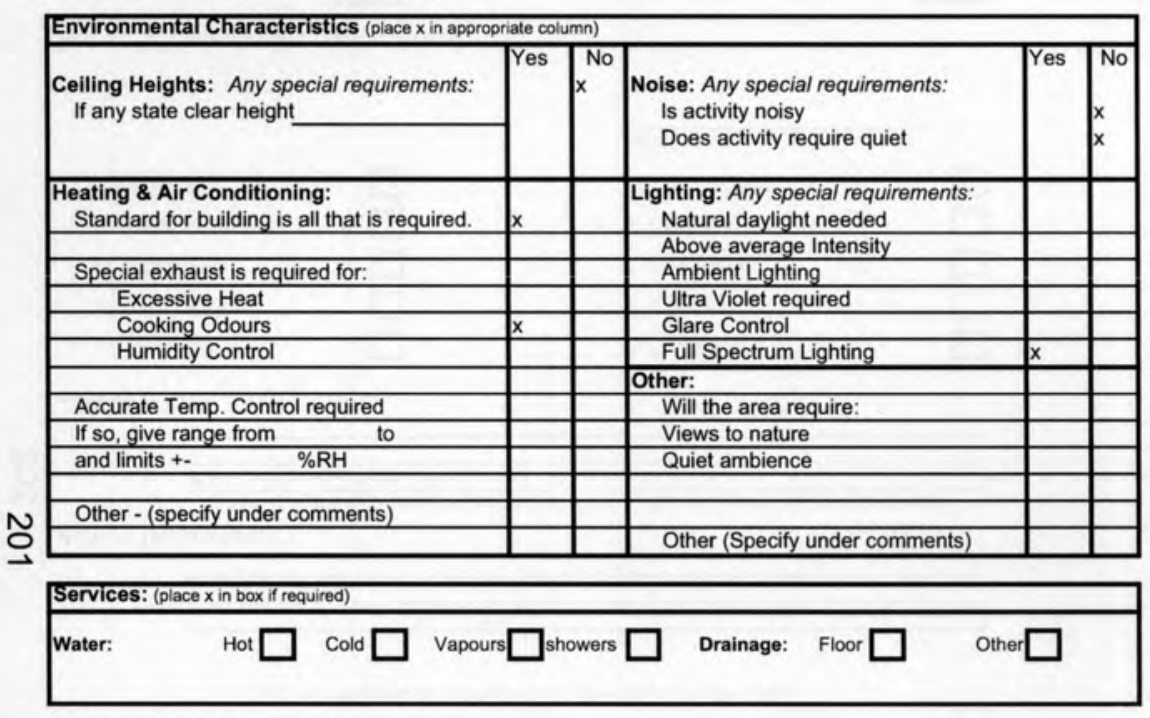

Additional Requirements or Comments:

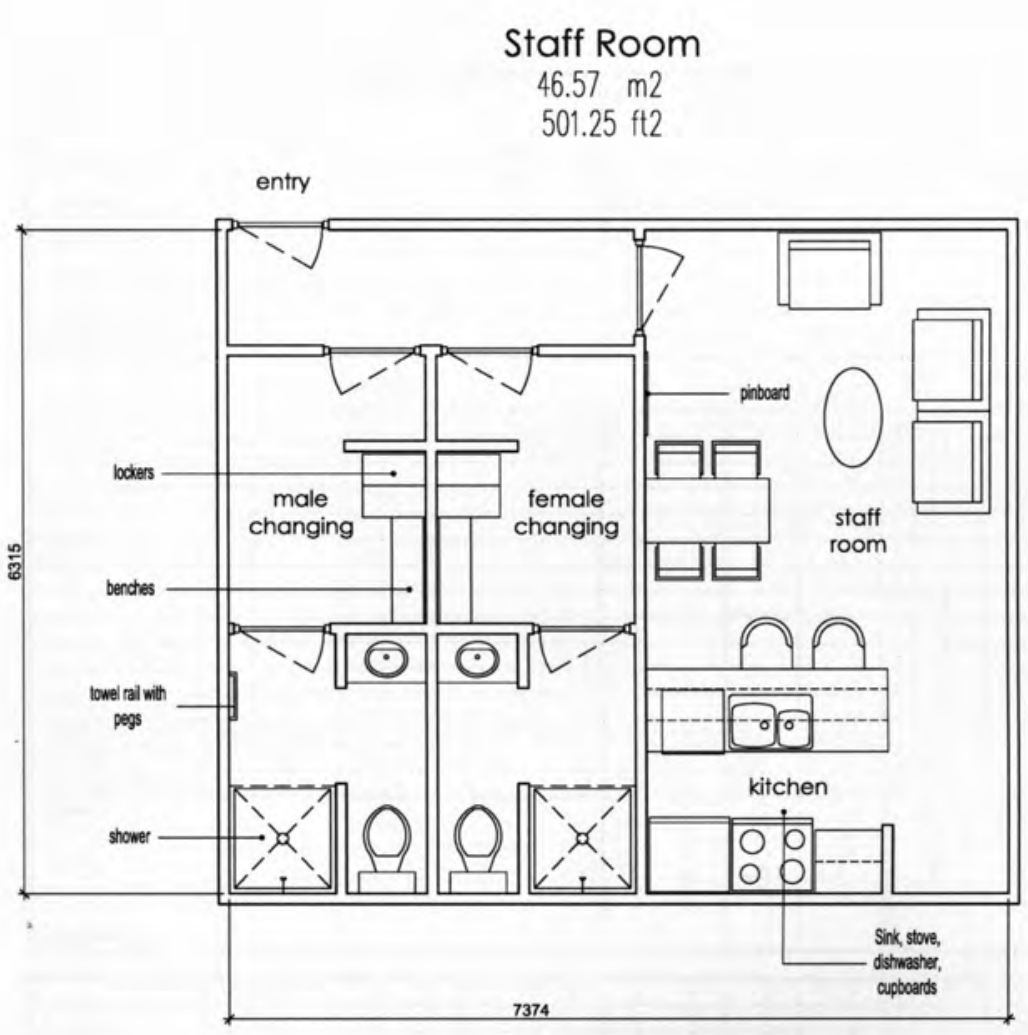

Neters
$6.57 \mathrm{~m} 2$

$501.25 \mathrm{ft2}$

$+2$ 


\section{Analysis of physical facility requirements}

Project: Wellness Centre 2009 Name of Space: Laundry Room

Department Public Swim

\section{Space Description}

Purpose: An area dedicated to the cleaning and storage of towels for the public swim

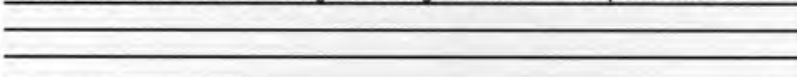

\section{Space Relationships:}

a. General - How should this space relate to general areas in the building? eg: offices, teaching areas, loading dock, storage areas.

Located with proximity to the front desk b. Specific - Is this space part of a suite?
If yes, then describe its relationships to other spaces in the suite

\section{Activity Description:}

N Janitors will undergo cleaning towles, and move them to both the front desk and

N

Activity type: (insert x in applicable box)

1. Admin Office
2. Private Office
3. Staff Room
4. Medical Clinic
5. Fitness

6. Education

7. Therapy

8. Information

9. Common Area

10.Other Specify:

services

$\square$

Users: (insert number in appropriate boxes)

$\begin{array}{lll}\text { Staff } & \text { Fitness Users } \\ \text { Patient } & \text { Therapy Users } \\ \text { Students } & \text { Avg. hours per week usage } \\ \text { Community } & \end{array}$

Space Components
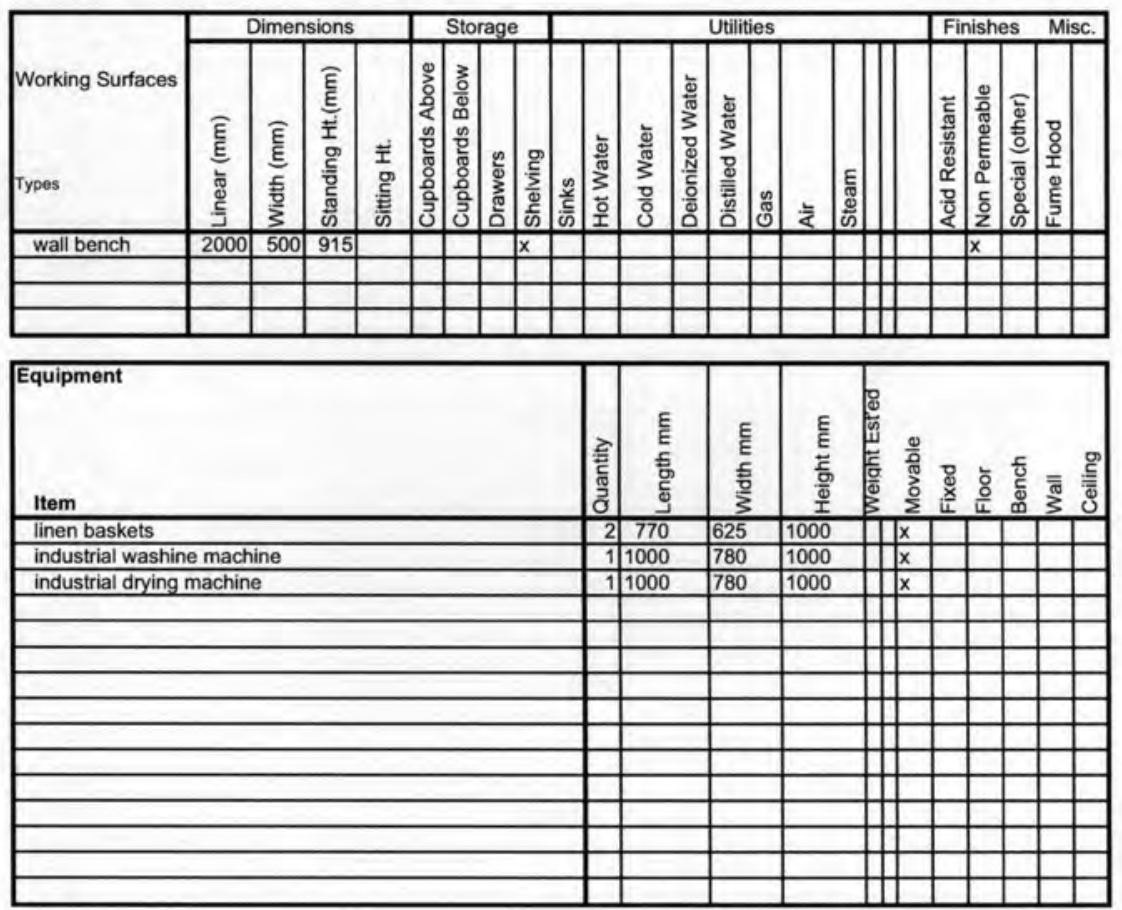

\begin{tabular}{ll|l|}
\hline $\begin{array}{l}\text { Communications: } \\
\text { (place X in box if required) } \\
\text { Telephone }\end{array}$ & $\begin{array}{l}\text { Audio Visual } \\
\text { The following audio visual aids will be used. } \\
\text { P.A System } \\
\text { Cable TV } \\
\text { Internet Connection }\end{array}$ \\
Other Specify: & Television \\
Projector \\
Sound Reinforcement
\end{tabular}




\begin{tabular}{|lll|}
\hline Furnishings: & (place x in boxif required) \\
\hline Storage Cabinets & $\square$ Info Board $\square \quad$ Shelving $\square$ \\
\hline Fixed: (List) \\
Movable: (List)
\end{tabular}

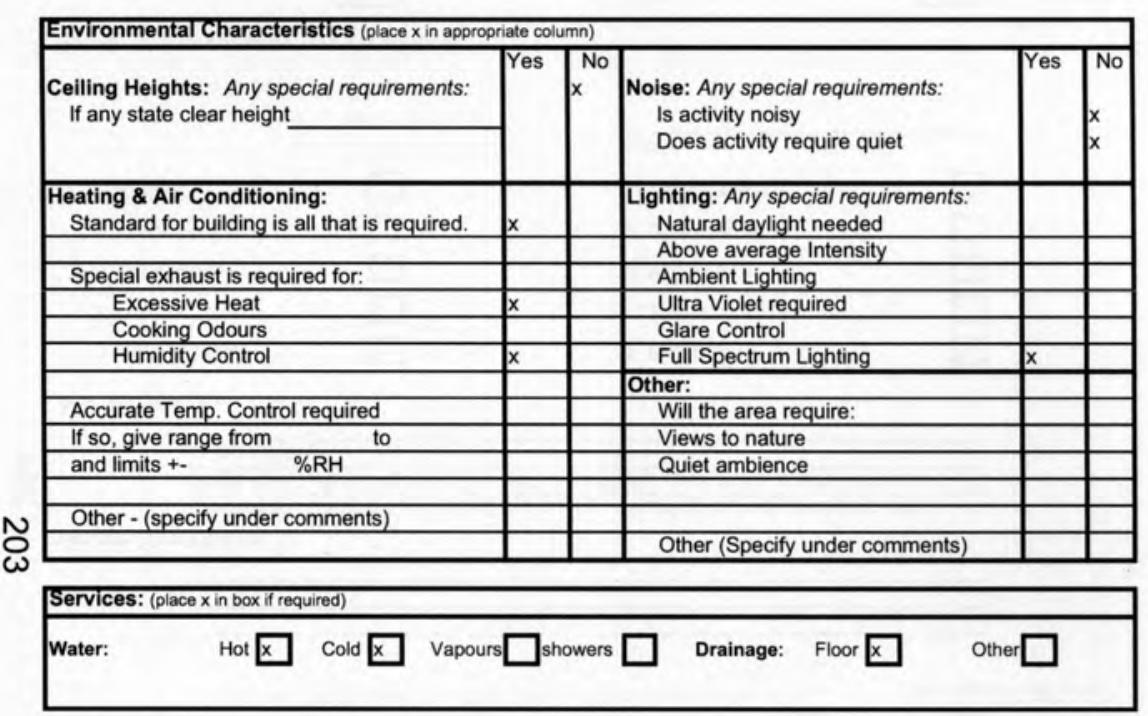

Additional Requirements or Comments:

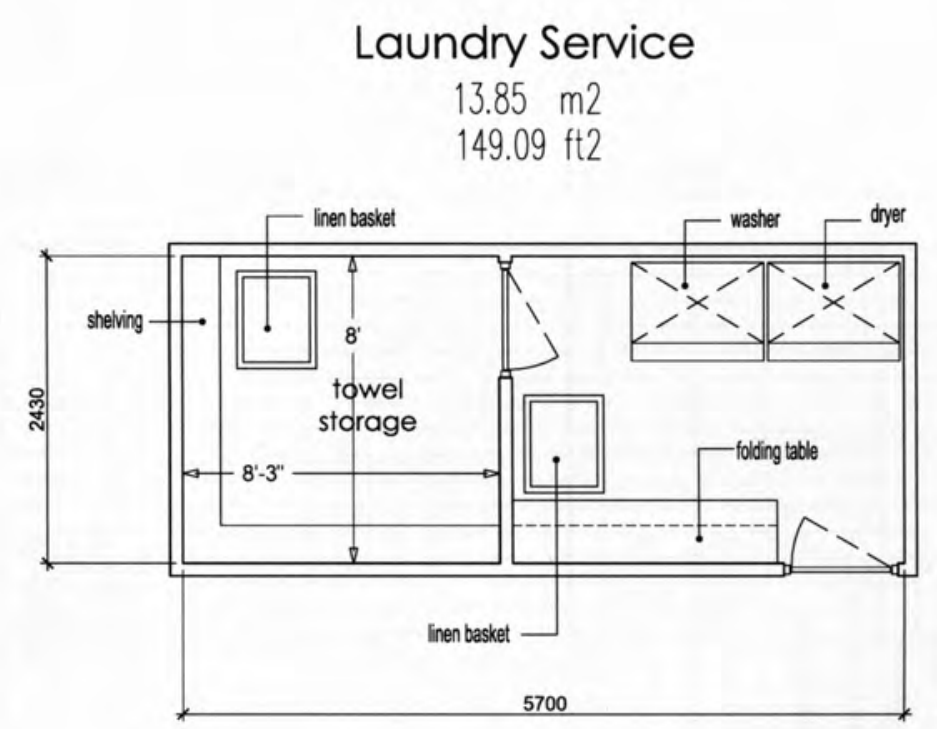

Meters 0

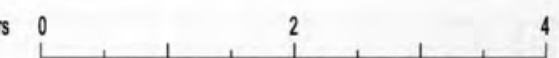




\section{Analysis of physical facility requirements}

Project: Wellness Centre 2009

Name of Space: Recreational Pool

Space Description

Purpose: An area dedicated to community swimming

Department Public Swim Net Assignable Sq. m [488] $\operatorname{pool}(251 \mathrm{~m})+\operatorname{deck}(237 \mathrm{~m})=488 \mathrm{~m} 2$

\section{Space Relationships:}

a. General - How should this space relate to general areas in the building? eg: offices,

teaching areas, loading dock, storage areas.

Located as the centre piece of the swim facility; adjacent to lockers and front desk

b. Specific - Is this space part of a suite?
If yes, then describe its relationships to other spaces in the suite

No

$\longrightarrow$

Activity Description:

C $\quad$ Community will undergo recreational public swimming; instructed classes will

$\stackrel{8}{+}$ range from toddlers to adults; as well, aquatherapy and water sports will occur

Activity type: (insert x in applicable box)

$\begin{array}{llll}\text { 1. Admin Office } & \square & \text { 6. Education } \\ \text { 2. Private Office } & \square & \text { 7. Therapy } \\ \text { 3. Staff Room } & \square & \text { 8. Information } \\ \text { 4. Medical Clinic } & \square & \text { 9. Common Area } \\ \text { 5. Fitness } & \square & \text { 10.Other Specify: } \\ & \text { public swim }\end{array}$

Users: (insert number in appropriate boxes)

$\begin{array}{lll}\text { Staff } & \text { Fitness Users } \\ \text { Patient } & \text { Therapy Users } \\ \text { Students } & \text { Avg. hours per week usage } \\ \text { Community } & \end{array}$

Space Components
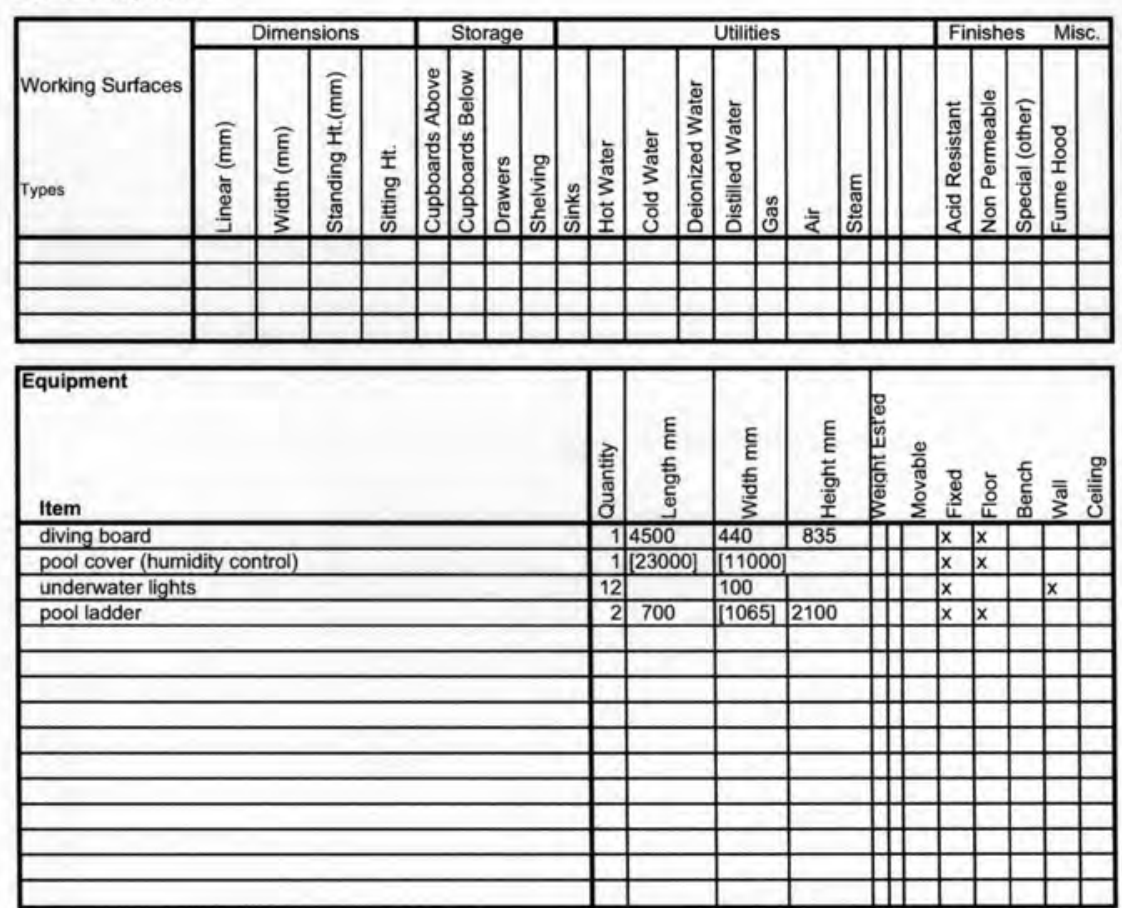

\begin{tabular}{|c|c|}
\hline $\begin{array}{l}\text { Communications: } \\
\text { (place } \mathrm{X} \text { in box if required) }\end{array}$ & $\begin{array}{l}\text { Audio Visual } \\
\text { The following audio visual aids will be used }\end{array}$ \\
\hline Telephone & Television \\
\hline P.A System & Projector \\
\hline Cable TV & Sound Reinforcement \\
\hline Internet Connection & \\
\hline Other Specify: & Specify: \\
\hline
\end{tabular}




\begin{tabular}{|ll|}
\hline Furnishings: & (place x in boxif required) \\
\hline Storage Cabinets & $\square$ Info Board $\square \quad$ Shelving $\square$ \\
Fixed: (List) \\
Movable: (List)
\end{tabular}

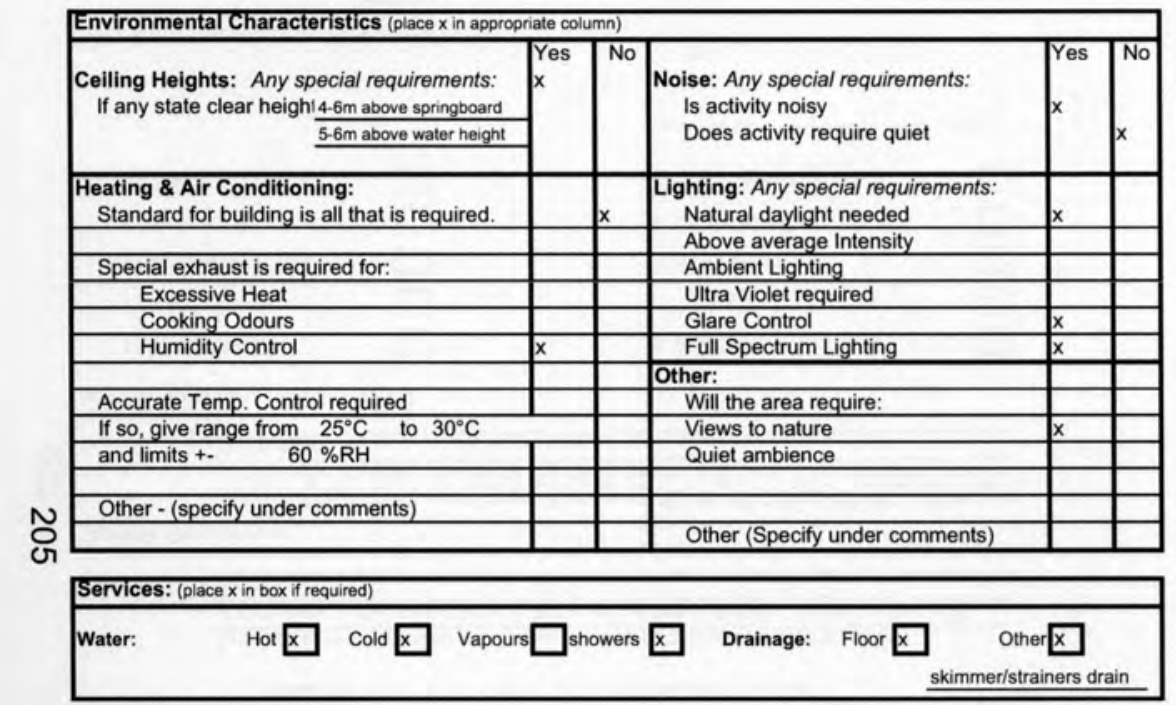

Additional Requirements or Comments:

pool water shall be $27^{\circ} \mathrm{C}$; the ventilation rate shall be based on TUS recommendation of 10 litres $/ \mathrm{s}$ per sq.m of total pool hall area (ie area of pool plus 'wet' surrounds)
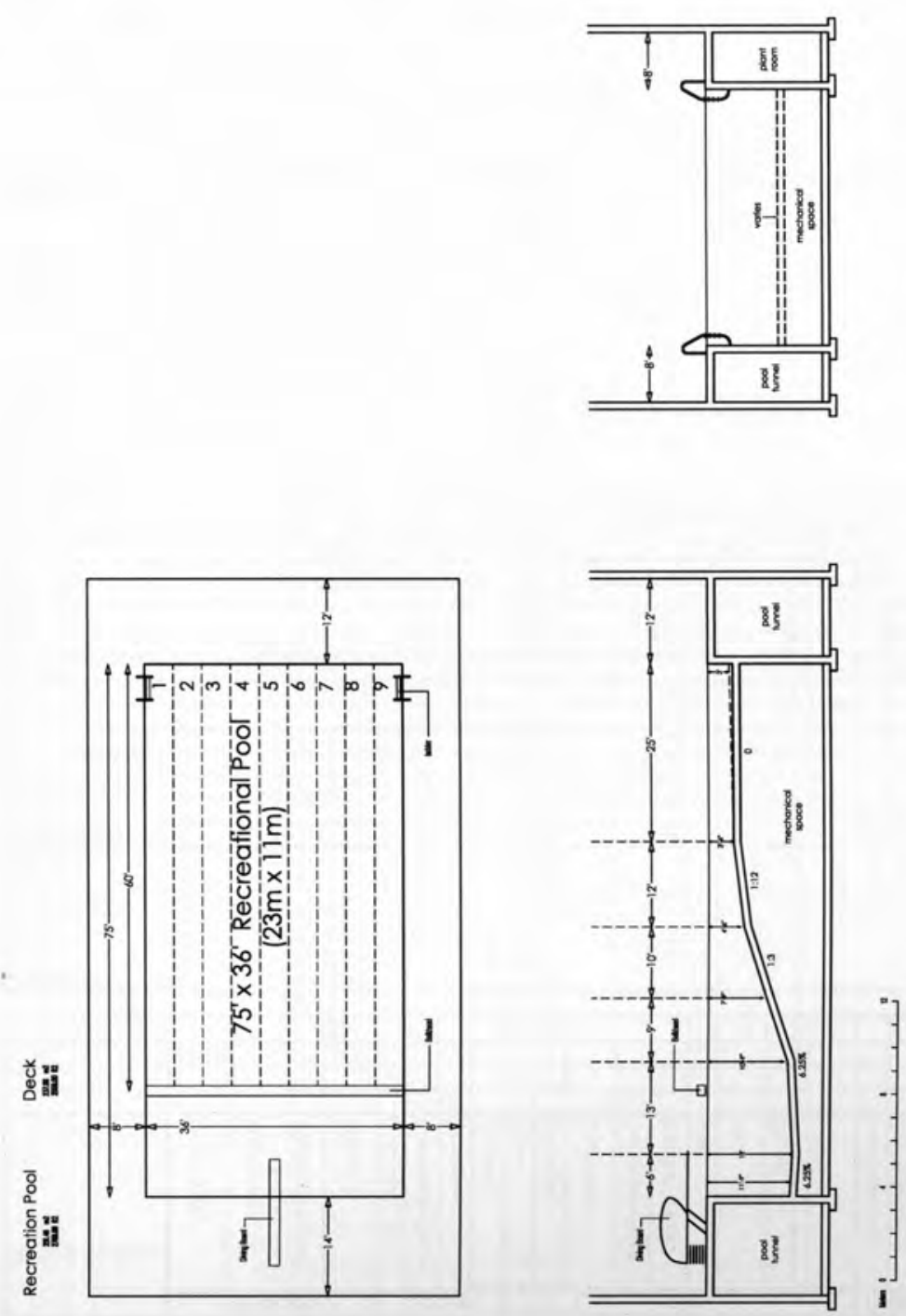
Analysis of physical facility requirements

Project: Wellness Centre 2009 Name of Space: Warm Air Room

Department Therapeutic baths

Space Description

Purpose: To provide a space for community relaxation, socializing, and physical therapy within a serene bathing atmosphere

Space Relationships:

a. General - How should this space relate to general areas in the building? eg: offices, teaching areas, loading dock, storage areas.

\section{ב \\ b. Specific - Is this space part of a suite?

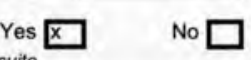 \\ If yes, then describe its relationships to other spaces in the suite}

Located as the first room within a series that comprises the entire bathing experienced; procession through this space comes after the change rooms and

Activity Description:

N Turkish style relaxing warm room where aromatic oils will diffuse into the atmosphere to help relax and calm the mind; muscle relaxation will come from thermally massive to help relax and calm the mind; muscle relaxation will come from thermally massive
tile loungers

Activity type: (insert $x$ in applicable box)

$\begin{array}{lll}\text { 1. Admin Office } & \square & \text { 6. Education } \\ \text { 2. Private Office } & \square & \text { 7. Therapy } \\ \text { 3. Staff Room } & \square & \text { 8. Information } \\ \text { 4. Medical Clinic } & \square & \text { 9. Common Area } \\ \text { 5. Fitness } & \square & \text { 10.Other Specify: }\end{array}$

\section{Users: $\quad$ (insert number in appropriate boxes)}

$\begin{array}{lll}\begin{array}{l}\text { Staff } \\ \text { Patient } \\ \text { Students } \\ \text { Community }\end{array} & \begin{array}{l}\text { Fitness Users } \\ \text { Therapy Users }\end{array}\end{array}$

Space Components
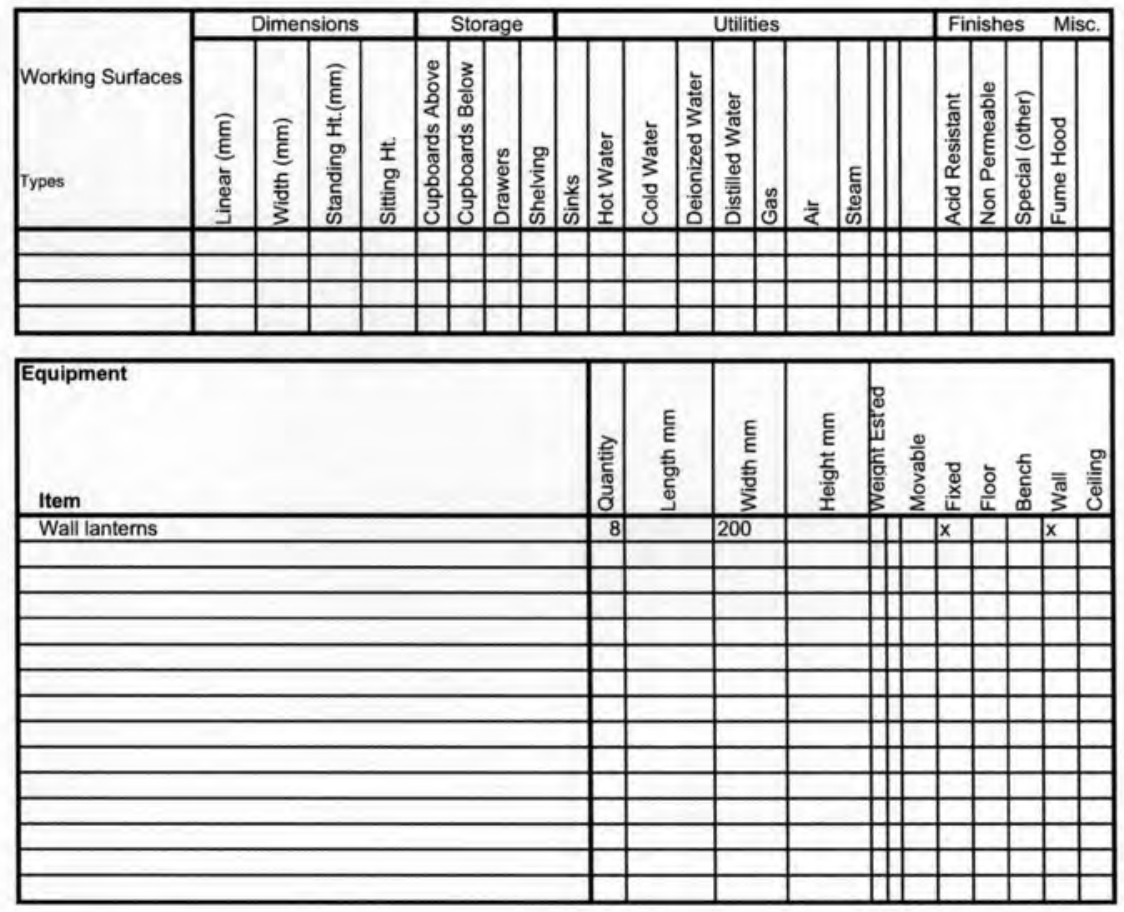

\begin{tabular}{l|l|}
\hline $\begin{array}{l}\text { Communications: } \\
\text { (place Xin boxif frequired) } \\
\text { Telephone }\end{array}$ & $\begin{array}{l}\text { Audio Visual } \\
\text { The following audio visual aids will be used: } \\
\text { P.A System }\end{array}$ \\
Cable TV \\
Internet Connection
\end{tabular}




\begin{tabular}{|ll|}
\hline Furnishings: & (place x in boxif required) \\
\hline Storage Cabinets & $\square$ Info Board $\square \quad$ Shelving $\square \quad$ Computer Station $\square$ \\
Fixed: (List) & tile loungers, wash basins, water mister/oil bowl, fireplace \\
\cline { 2 - 2 } & \\
\cline { 2 - 2 } &
\end{tabular}

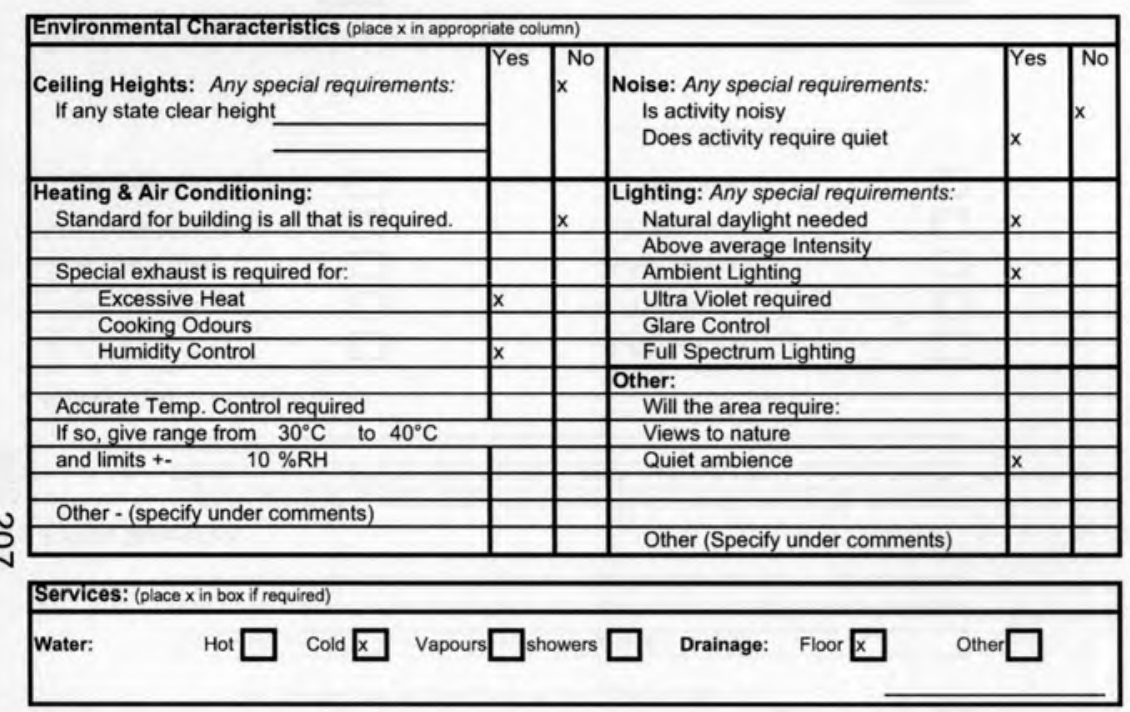

\section{Additional Requirements or Comments:}

Approximate amount of time spent is 15 minutes:
Warm Air Room

(Tepidarium)

$94.50 \mathrm{~m} 2$

$1017.21 \mathrm{ft} 2$

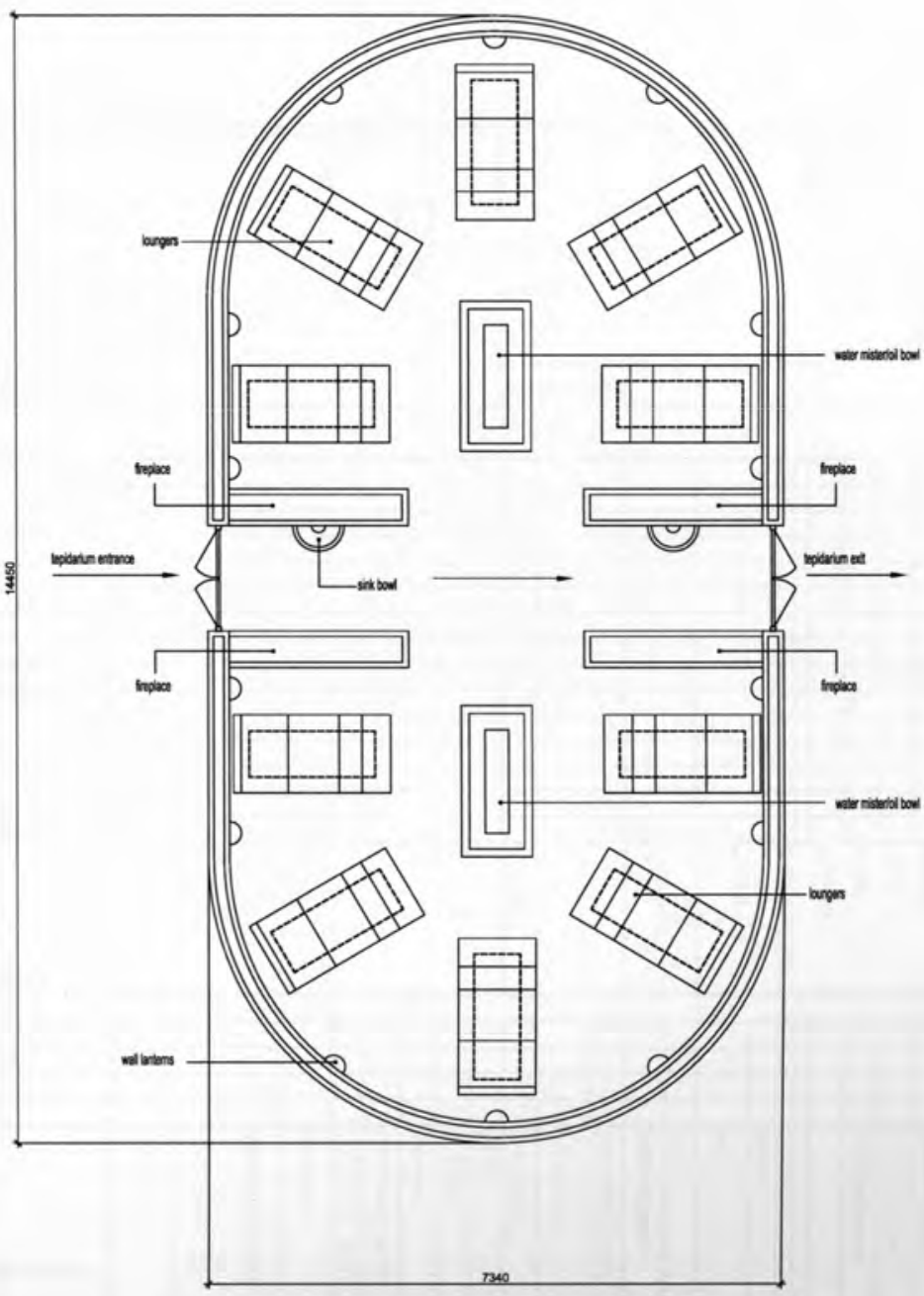

o 
Project: Wellness Centre 2009

Name of Space: Hot Air Room

Department Therapeutic baths Net Assignable Sq. m 36

Space Description

Purpose: To provide a space for community relaxation, socializing, and physical therapy within a serene bathing atmosphere

Space Relationships:

a. General - How should this space relate to general areas in the building? eg: offices, teaching areas, loading dock, storage areas.

b. Specific - Is this space part of a suite? Yes $\mathrm{X}$ № $\square$ If yes, then describe its relationships to other spaces in the suit Procession through this space comes after the warm air room

\section{Activity Description}

$N \quad$ Turkish style relaxing warm room where aromatic oils will diffuse into the atmosphere to help relax and calm the mind; muscle relaxation will come from thermally massive tile loungers

Activity type: (insert $x$ in applicable box)

1. Admin Office

2. Private Office

$\square$
$\square$
$\square$

6. Education

7. Therapy

8. Information

4. Medical Clinir

9. Common Area

10. Other Specify:

$\square$
$\square$
$\square$
$\square$

Users: (insert number in appropriate boxes)

$\begin{array}{lll}\begin{array}{l}\text { Staff } \\ \text { Patient } \\ \text { Students } \\ \text { Community }\end{array} & \begin{array}{l}\text { Fitness Users } \\ \text { Therapy Users }\end{array} \\ \text { Avg. hours per week usage }\end{array}$

Space Components
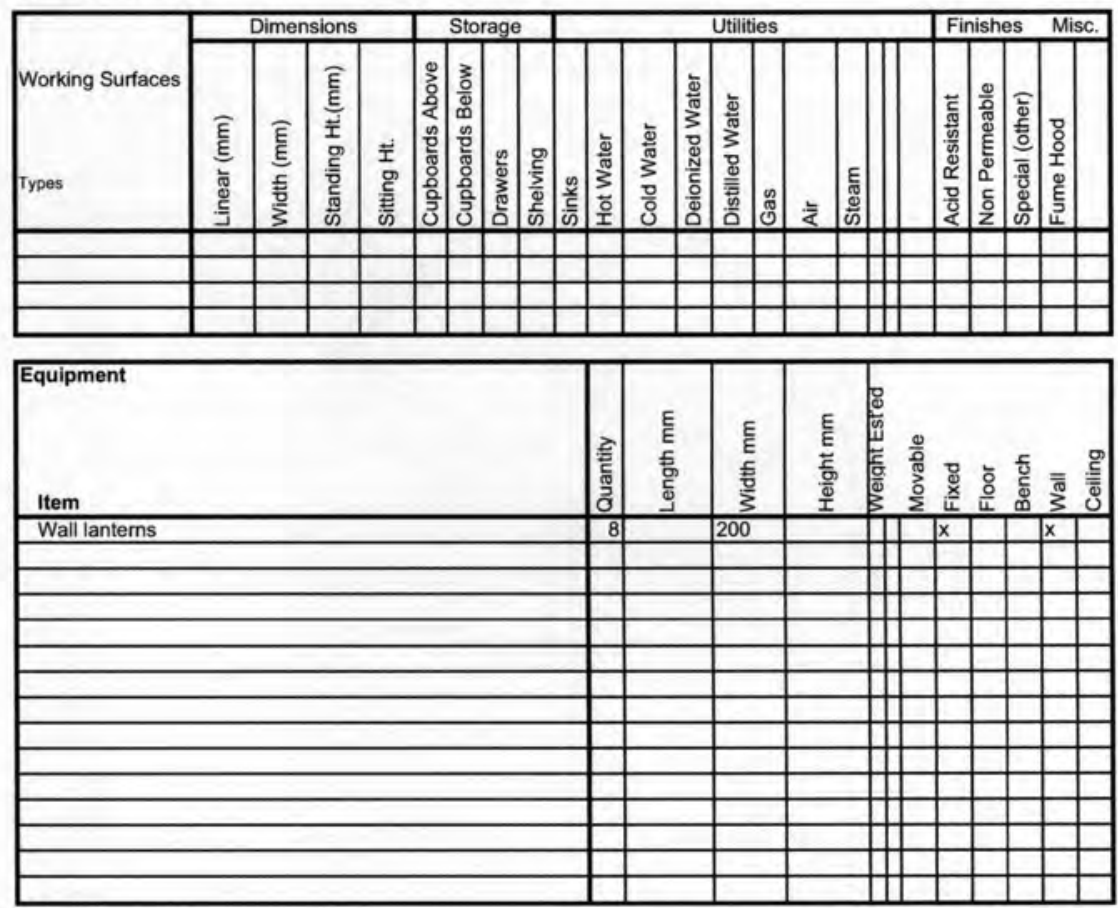

Communications:

Telephone

Telephone

Cable TV

Internet Connection

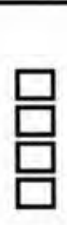

Audio Visual

The following audio visual aids will be used

Television

Projector

Sound Reinforcement

$\square$

Other Specify:

Other Specify: backround white noise 


\begin{tabular}{|ll|}
\hline Furnishings: & (place xin boxif required) \\
\hline Storage Cabinets & $\square$ Info Board $\square \quad$ Shelving $\square \quad$ Computer Station $\square$ \\
Fixed: (List) & tile loungers, wash basins, water misterloil bowl, \\
\\
Movable: (List)
\end{tabular}

\begin{tabular}{|c|c|c|c|c|c|}
\hline \multicolumn{6}{|l|}{ nvironmental Characteristics } \\
\hline $\begin{array}{l}\text { Ceilling Heights: Any special requirements: } \\
\text { If any state clear hight. }\end{array}$ & Yes & No & $\begin{array}{l}\text { Noise: Any special requirements: } \\
\text { Is activity noisy }\end{array}$ & Tes & \\
\hline $\begin{array}{l}\text { Heating \& Air Conditioning: } \\
\text { Standard for building is all that is required. }\end{array}$ & & $x$ & $\begin{array}{l}\text { Lighting: Any special requirements: } \\
\text { Natural daylight needed }\end{array}$ & $x$ & \\
\hline & & & Above average Intensity & & \\
\hline Special exhaust is required for: & & & & $x$ & \\
\hline Excessive Heat & $x$ & & Ultra Violet required & & \\
\hline & & & Control & & \\
\hline Humidity Control & $x$ & & Full Spectrum Lighting & & \\
\hline np. Control required & & & Pther: & & \\
\hline If so, give range from $40^{\circ} \mathrm{C}$ to $50^{\circ} \mathrm{C}$ & & & Views to nature & & \\
\hline and limits +- & & & Quiet ambience & $f^{x}$ & \\
\hline Other - (specify under comments) & & & & & \\
\hline & & & Other (Specify under comments) & & \\
\hline
\end{tabular}

\section{Services: (place xin box if required) \\ Water: $\quad$ Hot $\square$ Cold $\square$ Vapours $\square$ showers $\square$ Drainage: Floor $\square$ Other $\square$}

Additional Requirements or Comments:

Approximate amount of time spent here is 5 minutes:
Hot Air Room

(Tepidarium)

$35.78 \mathrm{~m} 2$

$385.12 \mathrm{ft} 2$

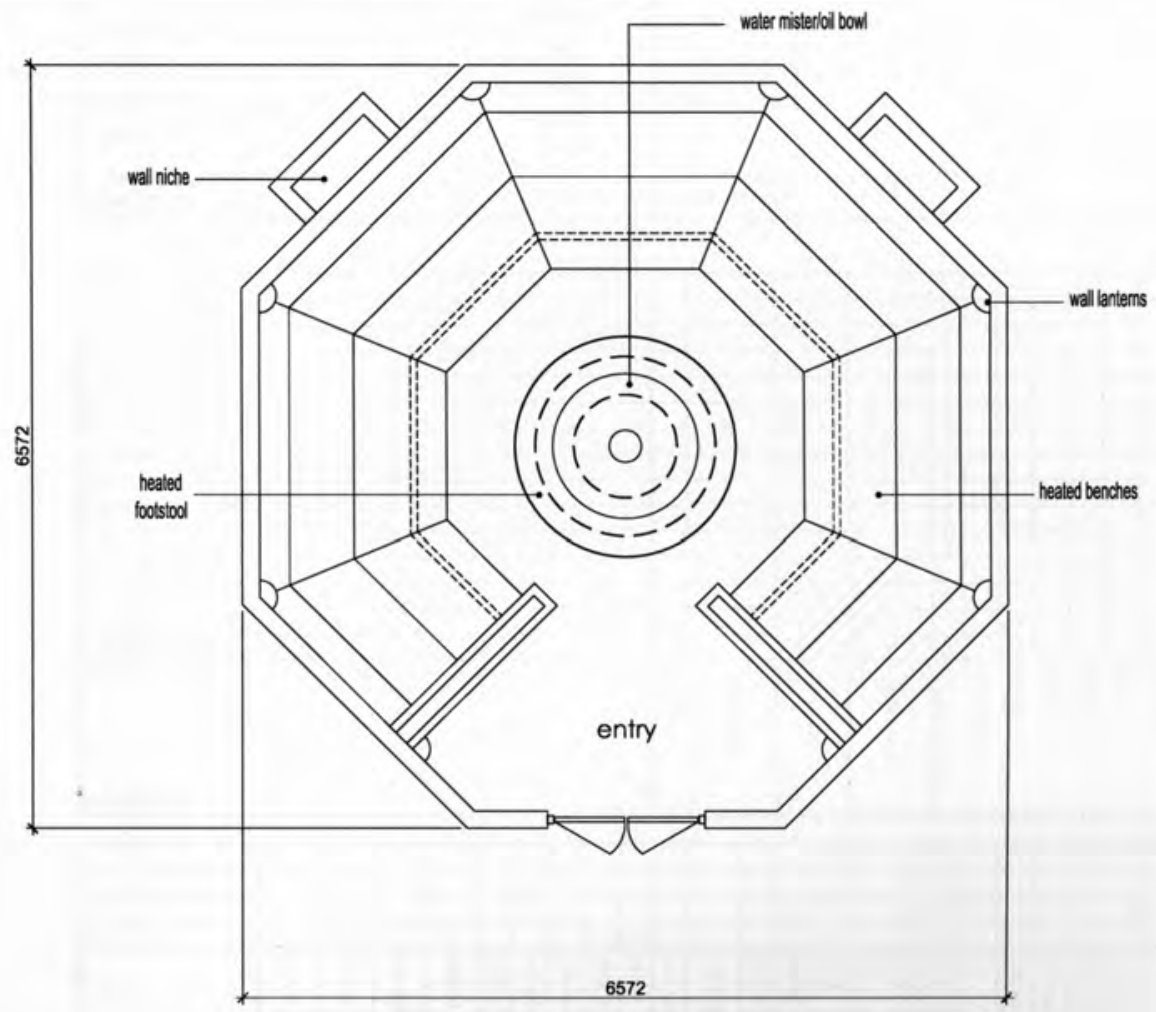

Neters 0 


\section{Analysis of physical facility requirements}

Project: Wellness Centre 2009

Name of Space: Dry Steam Room (Finnish sauna)

Department Therapeutic baths Net Assignable Sq. m 25

Space Description

Purpose: To provide a space for community relaxation, socializing, and physical therapy within a serene bathing atmosphere

Space Relationships:

a. General - How should this space relate to general areas in the building? eg: offices, teaching areas, loading dock, storage areas.

b. Specific -

Is this space part of a suite? Yes $\square \quad$ No $\square$ If yes, then describe its relationships to other spaces in the suite Procession through this space comes after the hot air room

Activity Description

N Finnish style relaxing steam room where water poured over hot rocks will diffuse into the atmosphere to help relax and calm the body \& mind; muscle relaxation will come from the fact that the bather is resting on warm cedar planks

Activity type: (insert $x$ in applicable box)

$\begin{array}{lll}\text { 1. Admin Office } & \square & \text { 6. Education } \\ \text { 2. Private Office } & \square & \text { 7. Therapy } \\ \text { 3. Staff Room } & \text { 8. Information } \\ \text { 4. Medical Clinic } & \square & \text { 9. Common Area } \\ \text { 5. Fitness } & \square & \text { 10.Other Specify: }\end{array}$

Users: (insert number in appropriate boxes)

Staff

Patient

Students

Community
Fitness Users

Therapy Users

Avg. hours per week usage
Space Components
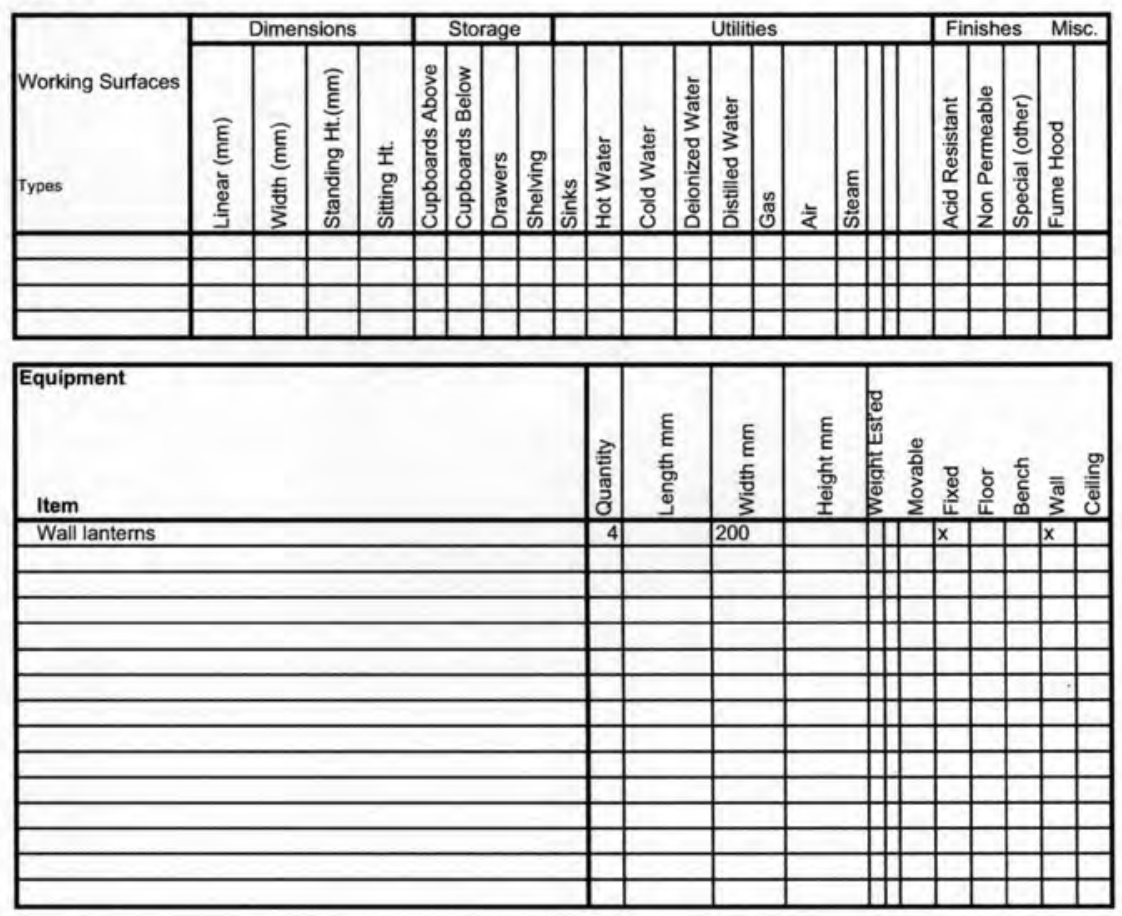

\begin{tabular}{ll|l|}
\hline $\begin{array}{l}\text { Communications: } \\
\text { (place X in boxif required) } \\
\text { Telephone }\end{array}$ & $\begin{array}{l}\text { Audio Visual } \\
\text { The following audio visual aids wil be used: } \\
\text { P.A System }\end{array}$ \\
Cable TV & $\begin{array}{l}\text { Television } \\
\text { Projector } \\
\text { Internet Connection }\end{array}$ \\
Sound Reinforcement & Other Specify: \\
\hline
\end{tabular}




\begin{tabular}{|ll|}
\hline Furnishings: $\quad$ (place x in box if required) \\
\hline Storage Cabinets \\
Fixed: (List) & info Board $\square \quad$ Shelving $\square \quad$ Computer Station $\square$ \\
Movable: (List) & \\
\cline { 2 - 2 } & \\
\hline
\end{tabular}

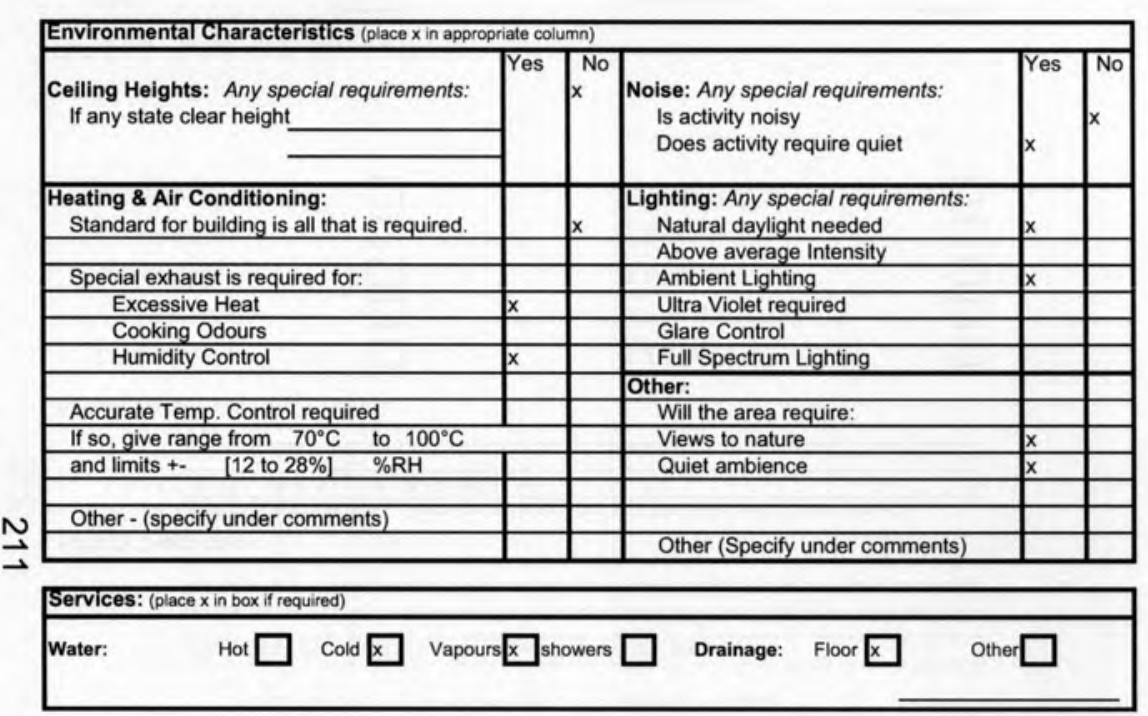

Additional Requirements or Comments:

Approximate amount of time spent here is 5 minutes:

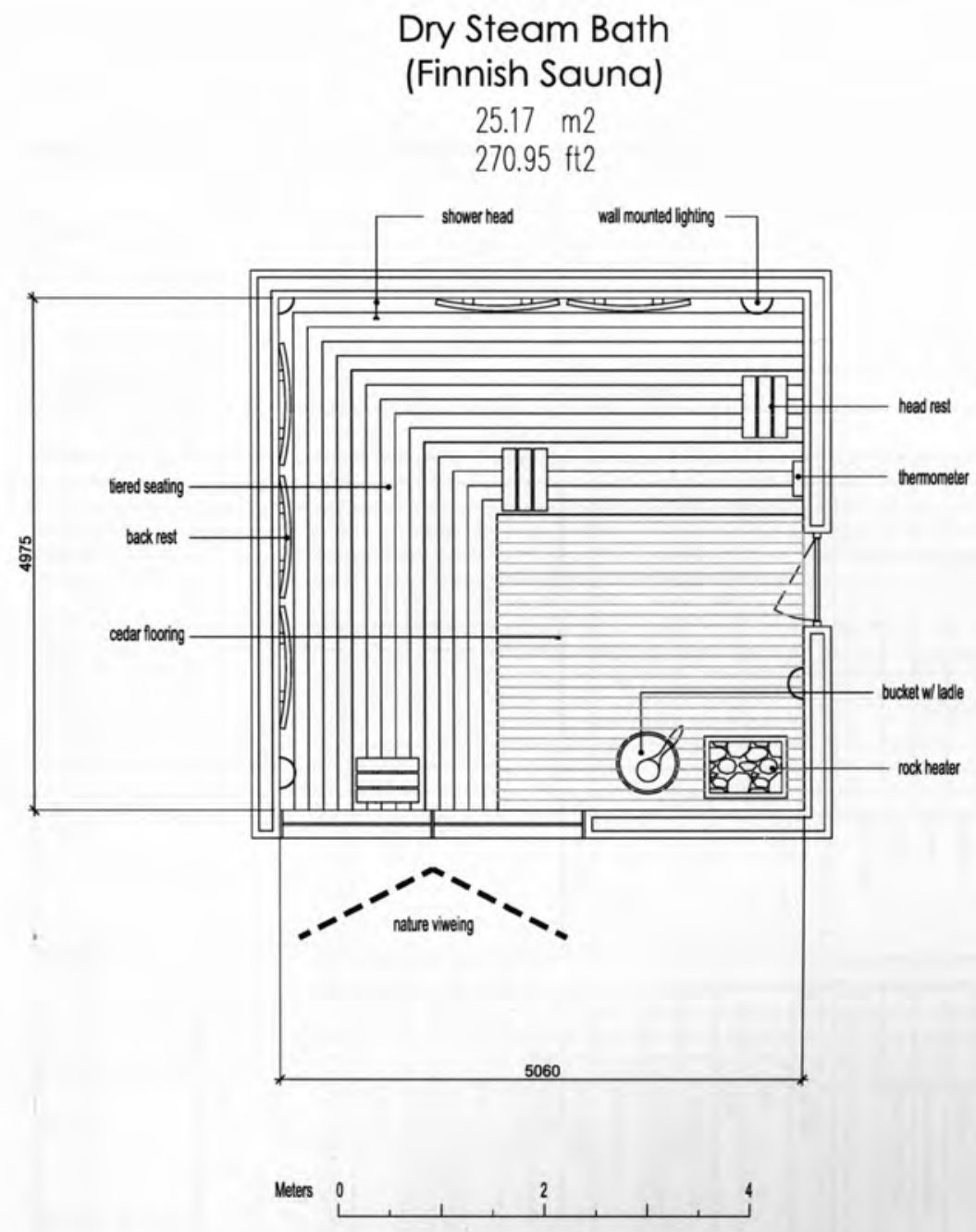




\section{Analysis of physical facility requirements}

Project: Wellness Centre 2009

Name of Space: Wet Steam Room (Hamam sauna)

Department Therapeutic baths Net Assignable Sq. m 33

\section{Space Descriptio}

Purpose: To provide a space for community relaxation, socializing, and physical therapy within a serene bathing atmosphere

Space Relationships:

a. General - How should this space relate to general areas in the building? eg: offices teaching areas, loading dock, storage areas.

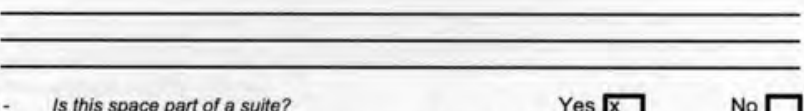

b. Specific - Is this space part of a suite?

Yes $\square \quad$ No $\square$

If yes, then describe its relationships to other spaces in the suite

Procession through this space comes after the dry steam room

\section{Activity Description:}

$\stackrel{N}{N}$

Activity type: (insert $x$ in applicable box)

$\begin{array}{lll}\text { 1. Admin Office } & \square & \text { 6. Education } \\ \text { 2. Private Office } & \square & \text { 7. Therapy } \\ \text { 3. Staff Room } & \text { 8. Information } \\ \text { 4. Medical Clinic } & \square & \text { 9. Common Area } \\ \text { 5. Fitness } & \square & \text { 10.Other Specify: }\end{array}$

Users: (insert number in appropriate boxes)

Staff

Patient

Students

Community

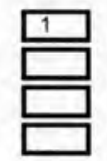

\section{Fitness Users}

Therapy Users

Avg. hours per week usage
Space Components
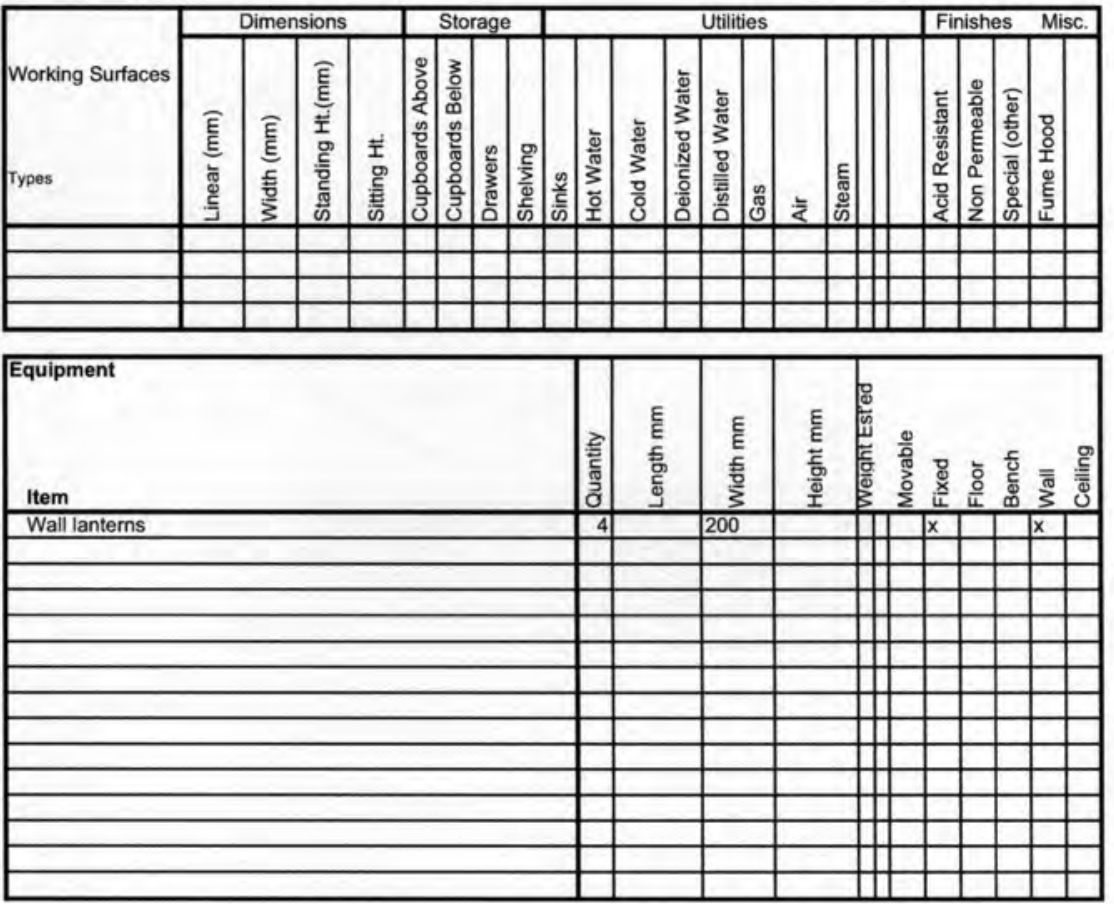

$\begin{aligned} & \text { Communications: } \\ & \text { (place X in box if required) } \\ & \text { Telephone } \\ & \text { P.A System } \\ & \text { Cable TV } \\ & \text { Internet Connection }\end{aligned}$
Other Specify:




\begin{tabular}{|ll|}
\hline Furnishings: & (place x in boxif required) \\
\hline Storage Cabinets & $\square$ info Board $\square \quad$ Shelving $\square \quad$ Computer Station $\square$ \\
Fixed: (List) & tiered stone seating, cooling fountains, shower heads \\
Movable: (List) & \\
\cline { 2 - 2 } & \\
\hline
\end{tabular}

\begin{tabular}{|c|c|c|c|c|c|}
\hline \multicolumn{6}{|c|}{ Environmental Characteristics (place $x$ in appropriate column) } \\
\hline $\begin{array}{l}\text { Ceiling Heights: Any special requirements: } \\
\text { If any state clear height }\end{array}$ & Yes & $x^{\text {No }}$ & $\begin{array}{l}\text { Noise: Any special requirements: } \\
\text { Is activity noisy }\end{array}$ & Yes & No \\
\hline \multirow[t]{2}{*}{$\begin{array}{l}\text { Heating \& Air Conditioning: } \\
\text { Standard for building is all that is required. }\end{array}$} & & $x$ & $\begin{array}{l}\text { Lighting: Any special requirements: } \\
\text { Natural daylight needed }\end{array}$ & $x$ & \\
\hline & & & Above average Intensity & & \\
\hline Special exhaust is required for: & & & Ambient Lighting & $x$ & \\
\hline \multirow{2}{*}{$\begin{array}{l}\text { Excessive Heat } \\
\text { Cooking Odours }\end{array}$} & $x$ & & Ultra Violet required & & \\
\hline & & & Glare Control & & \\
\hline Humidity Control & $x$ & & Full Spectrum Lighting & & \\
\hline \multirow[b]{2}{*}{ Accurate Temp. Control required } & & & Other: & & \\
\hline & & & Will the area require: & & \\
\hline \multicolumn{2}{|l|}{ If so, give range from $40^{\circ} \mathrm{C}$ to $45^{\circ} \mathrm{C}$} & & Views to nature & & \\
\hline \multicolumn{2}{|l|}{ and limits +- $100 \%$ RH } & & Quiet ambience & $x$ & \\
\hline \multirow{2}{*}{ Other - (specify under comments) } & & & & & \\
\hline & & & Other (Specify under comments) & & \\
\hline
\end{tabular}

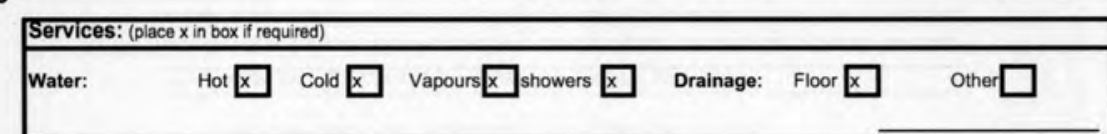

Additional Requirements or Comments:

Approximate amount of time spent here is 10 minutes:

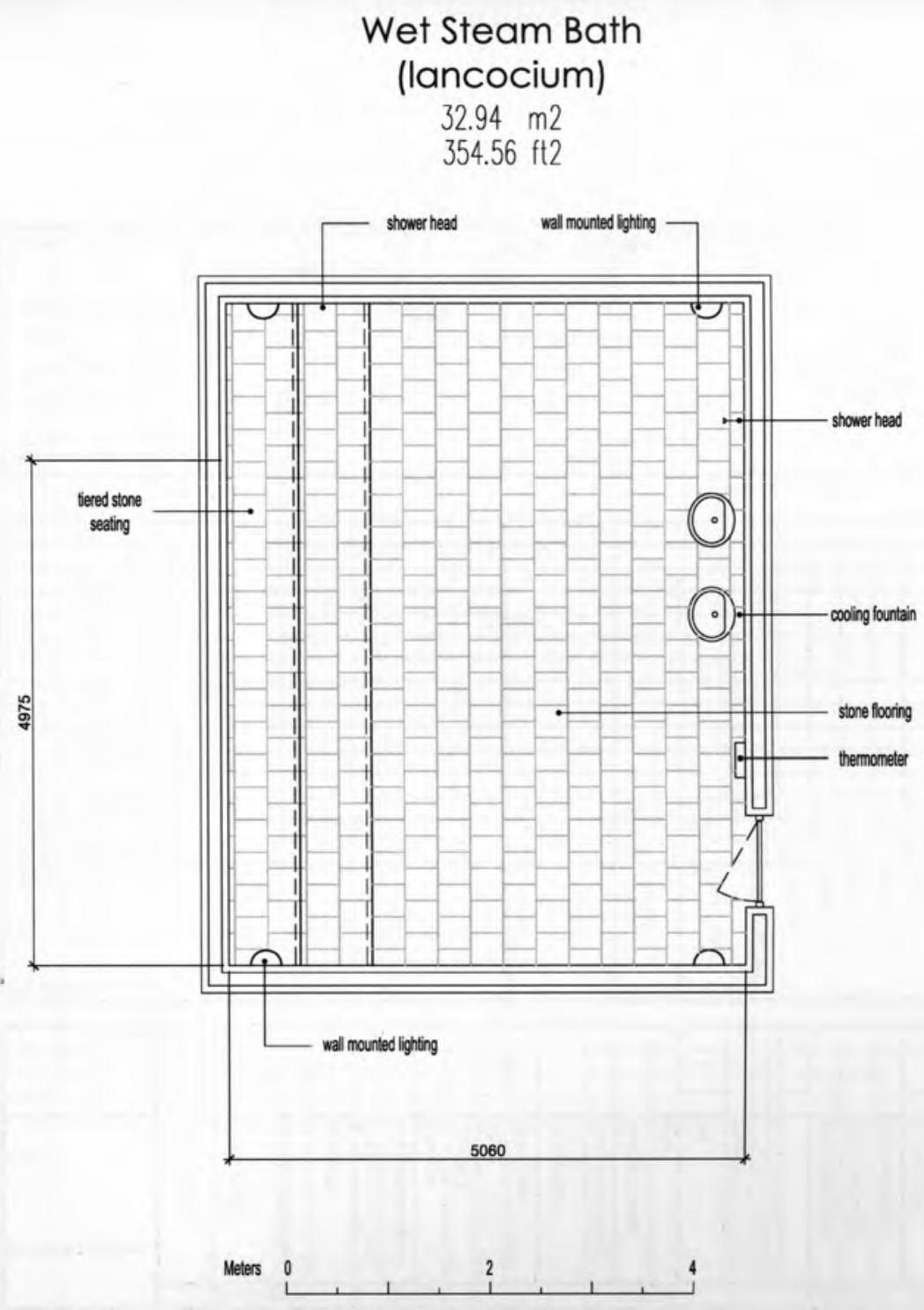




\section{Analysis of physical facility requirements}

Project: Wellness Centre 2009 Name of Space: Warm Thermal Bath

Department Therapeutic baths Net Assignable Sq. m. 76

\section{Space Description}

Purpose: To provide a space for community relaxation, socializing, and physical therapy within a serene bathing atmospher

Space Relationships:

a. General- - How should this space relate to general areas in the building? eg: offices, teaching areas, loading dock, storage areas.

b. Specific - Is this space part of a suite? Yes $\square \quad$ No $\square$ If yes, then describe its relationships to other spaces in the suite Procession through this space comes after the wet steam room

\section{Activity Description}

Bathers enjoy the therapeutic qualities of the warm waters; tiered seating allows
for a place to congregate and socialise
Activity type: (insert x in applicable box)
$\begin{array}{ll}\text { 1. Admin Office } \\ \text { 2. Private Office } \\ \text { 3. Staff Room } \\ \text { 4. Medical Clinic }\end{array}$
$\begin{array}{ll}\text { 5. Fitness } & \text { 6. Education }\end{array}$

Users: (insert number in approprate boxes)

$\begin{array}{ll}\begin{array}{l}\text { Staff } \\ \text { Patient } \\ \text { Students } \\ \text { Community }\end{array} & \text { Fitness Users } \\ \text { Therapy Users }\end{array}$

Space Components
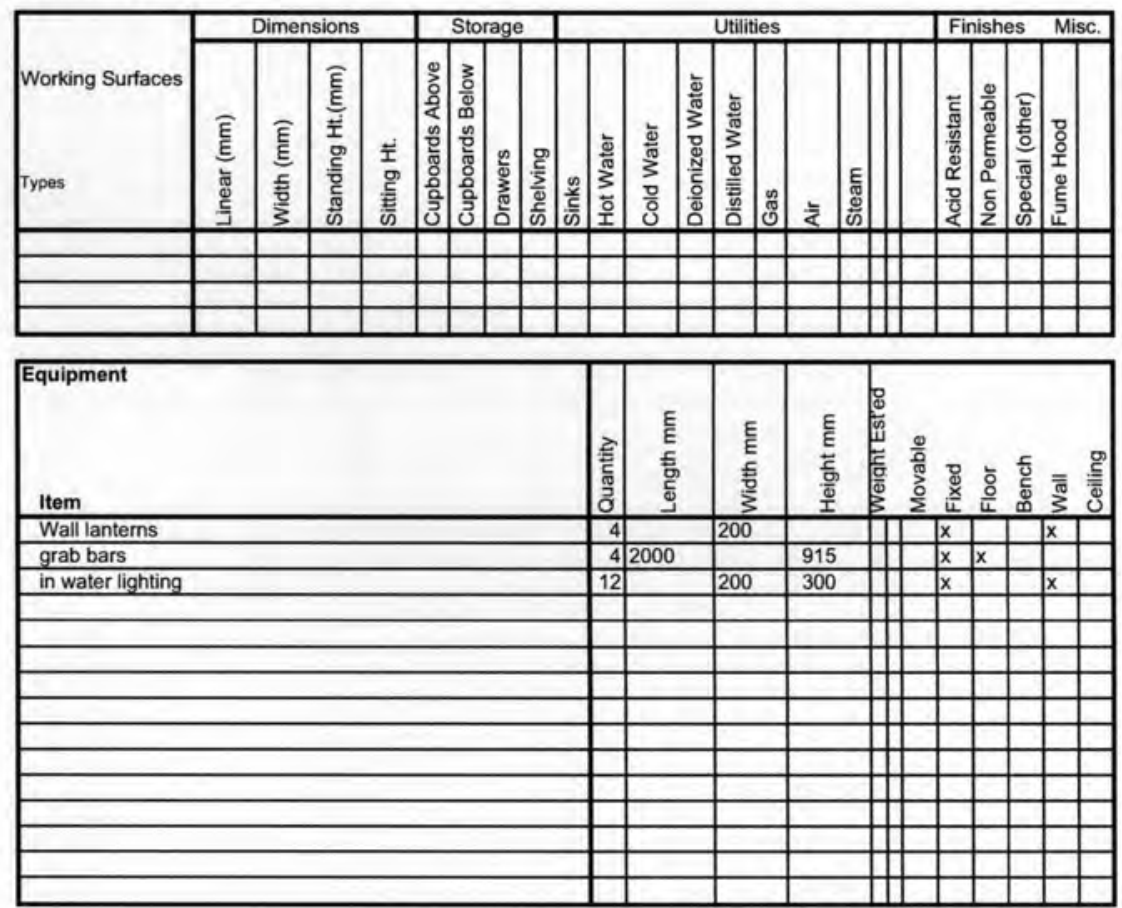

\begin{tabular}{ll|l|}
\hline $\begin{array}{l}\text { Communications: } \\
\text { (place X In boxif required) } \\
\text { Telephone }\end{array}$ & $\begin{array}{l}\text { Audio Visual } \\
\text { The following audio visual aids will be used: } \\
\text { P.A System }\end{array}$ \\
Cable TV & Television \\
Internet Connection & Projector \\
Sound Reinforcement & \\
Other Specify: & Other Specify. white backround noise \\
\hline
\end{tabular}




\begin{tabular}{|ll|}
\hline Furnishings: $\quad$ (place x in box if required) \\
\hline Storage Cabinets \\
Fixed: (List) \\
Movable: (List) & Info Board $\square \quad$ Shelving $\square$ \\
\cline { 2 - 2 } & \\
\end{tabular}

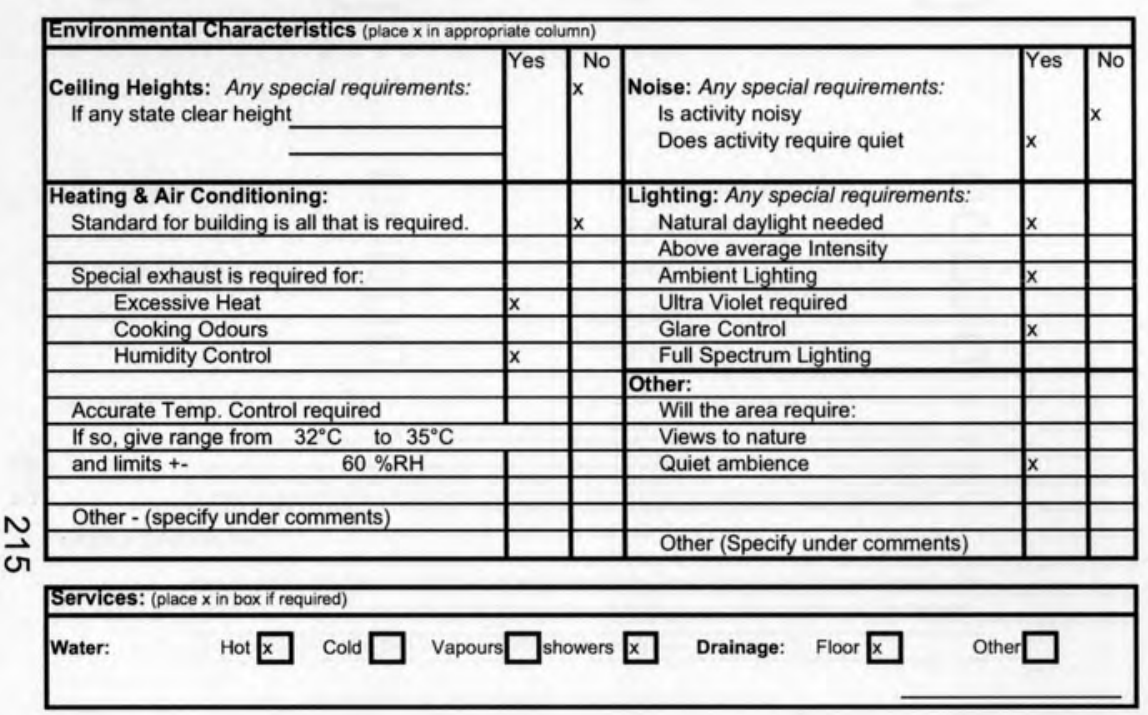

\section{Additional Requirements or Comments:}

Approximate amount of time spent here is 10 minutes;

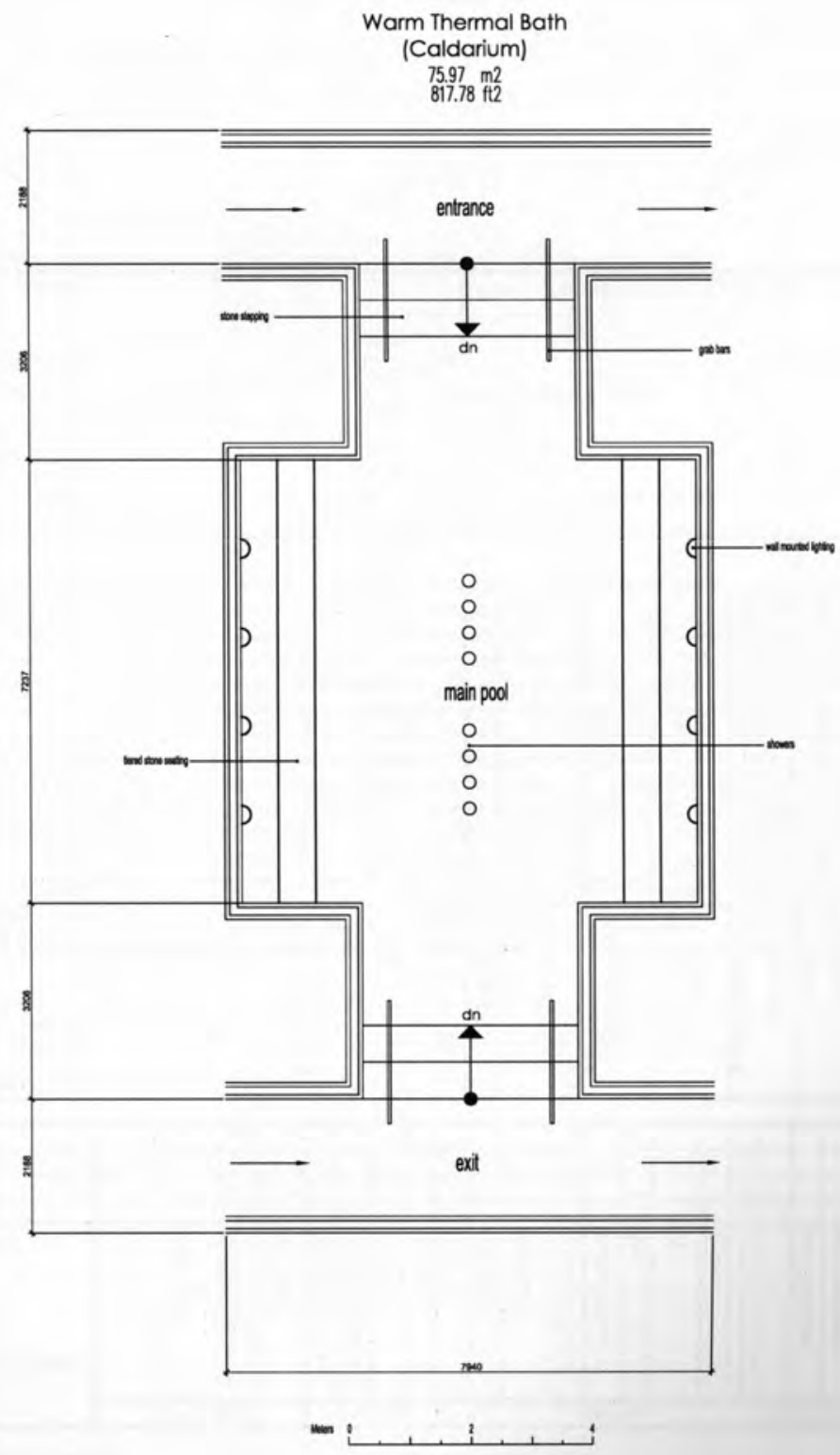




\section{Analysis of physical facility requirements}

Project: Wellness Centre 2009

Name of Space: Hot Thermal Bath

Department Therapeutic baths Net Assignabie Sq. m 70

\section{Space Description}

Purpose: To provide a space for community relaxation, socializing, and physical therapy within a serention within a serene bathing atmosphere

Space Relationships:

a. General - How should this space relate to general areas in the building? eg: offices, teaching areas, loading dock, storage areas.

b. Specific - Is this space part of a suite? Yes $区$ № $\square$ Procession through this space comes after the warm thermal bath

\section{Activity Description:}

Bathers enjoy the therapeutic qualities of the hot waters; tiered seating allows

O

Activity type: (insert $x$ in applicable box)

$\begin{array}{lll}\text { 1. Admin Office } & \square & \text { 6. Education } \\ \text { 2. Private Office } & \square & \text { 7. Therapy } \\ \text { 3. Staff Room } & \text { 8. Information } \\ \text { 4. Medical Clinic } & \square & \text { 9. Common Area } \\ \text { 5. Fitness } & \square & \text { 10.Other Specify: }\end{array}$

Users: (insert number in appropriate boxes)

Staff

Patient

Students

Community

\section{Fitness Users}

Therapy Users

Avg. hours per week usage
Space Components
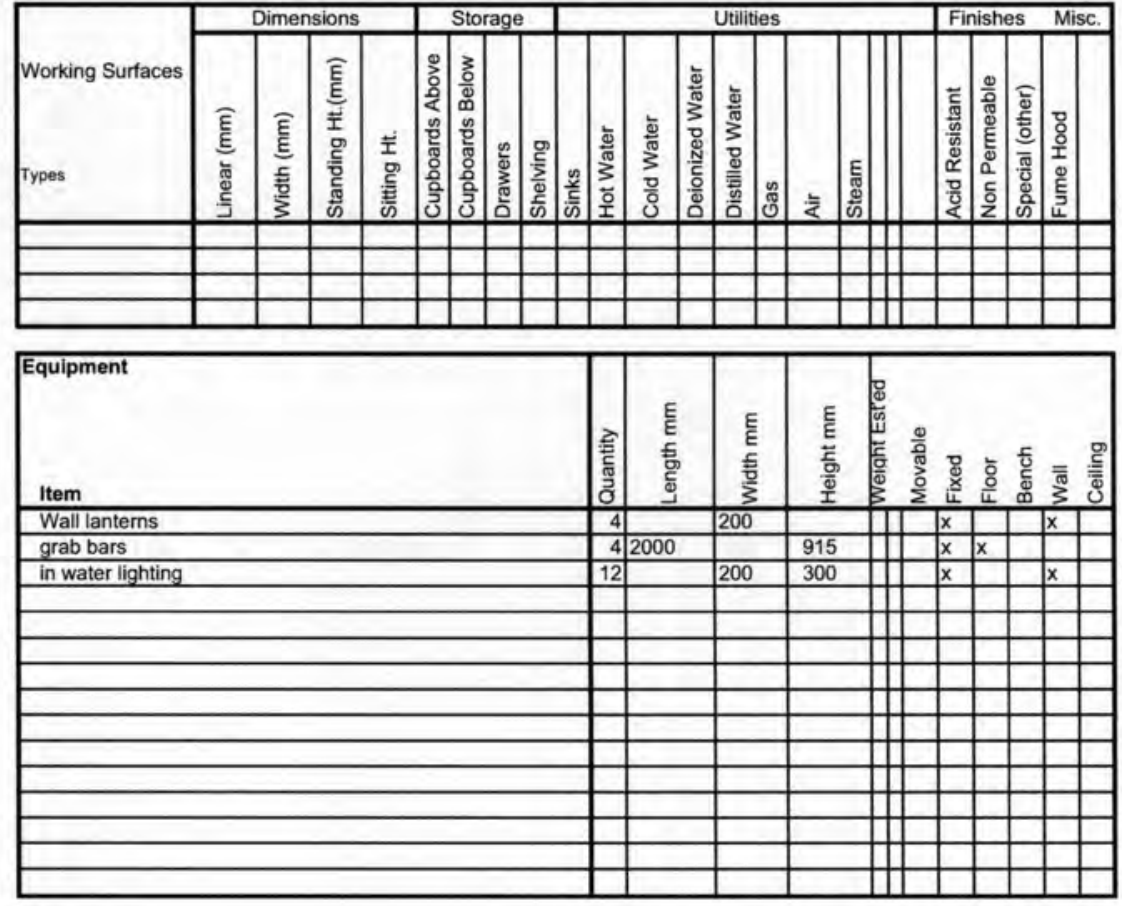

Communications:

(place $X$ in box if required)

Telephone

P.A System

Cable TV

Internet Connection

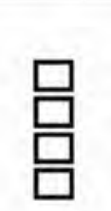

Audio Visual

The following audio visual aids wall be used

Television

Projector

Sound Reinforcement

Other Specify

Other Specify: white backround nois

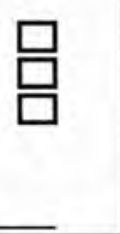




\begin{tabular}{|ll|}
\hline Furnishings: $\quad$ (place x in boxif required) \\
\hline Storage Cabinets \\
Fixed: (List) \\
Movable: (List) & info Board $\square$ Shelving $\square$ \\
\cline { 2 - 2 } & \\
\end{tabular}

\begin{tabular}{|c|c|c|c|c|c|}
\hline \multicolumn{6}{|c|}{ Environmental Characteristics (place $x$ in appropriate column) } \\
\hline $\begin{array}{l}\text { Ceiling Heights: Any special requirements: } \\
\text { If any state clear height }\end{array}$ & Yes & $x^{\text {No }}$ & $\begin{array}{l}\text { Noise: Any special requirements: } \\
\text { Is activity noisy }\end{array}$ & Tyes & $\begin{array}{l}\text { No } \\
x\end{array}$ \\
\hline $\begin{array}{l}\text { Heating \& Air Conditioning: } \\
\text { Standard for building is all that is required. }\end{array}$ & & $x$ & $\begin{array}{l}\text { Lighting: Any special requirements: } \\
\text { Natural daylight needed }\end{array}$ & $\mathrm{x}$ & \\
\hline & & & Above average Intensity & & \\
\hline Special exhaust is required for: & & & Ambient Lighting & $x$ & \\
\hline Excessive Heat & $x$ & & Ultra Violet required & & \\
\hline Cooking Odours & & & Glare Control & $x$ & \\
\hline Humidity Control & $x$ & & Full Spectrum Lighting & & \\
\hline & & & Other: & & \\
\hline Accurate Temp. Control required & & & Will the area require: & & \\
\hline If so, give range from $40^{\circ} \mathrm{C}$ to $45^{\circ} \mathrm{C}$ & & & Views to nature & & \\
\hline and limits +- & & & Quiet ambience & $x$ & \\
\hline & & & & & \\
\hline Other - (specify under comments) & & & & & \\
\hline & & & Other (Specify under comments) & & \\
\hline Services: (place $x$ in box if required) & & & & & \\
\hline Hot $\square$ Cold $\square$ & & & Drainage: Floor $\square$ & & \\
\hline
\end{tabular}

Additional Requirements or Comments:

Approximate amount of time spent here is 10 minutes
Hot Thermal Bath

(Caldarium)

$70.04 \mathrm{~m} 2$

$753.89 \mathrm{ft} 2$

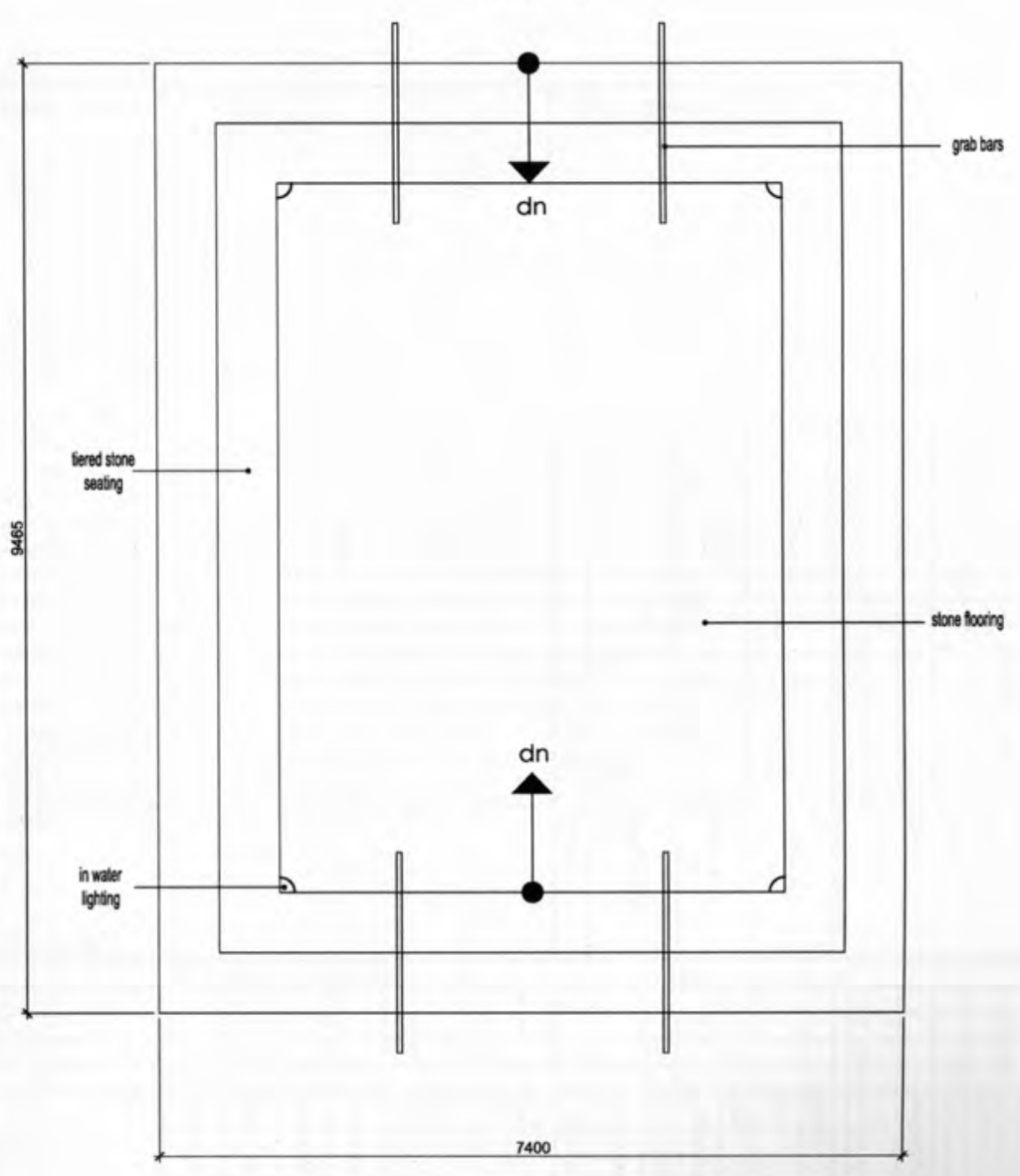

Neters 


\section{Analysis of physical facility requirements}

Project: Wellness Centre 2009

Name of Space: Cold Thermal Bath

Department Therapeutic baths Net Assignable Sq. m 91

Space Description

Purpose: To provide a space for community relaxation, socializing, and physical therapy within a serene bathing atmosphere

Space Relationships:

a. General - How should this space relate to general areas in the building? eg: offices teaching areas, loading dock, storage areas.

$$
\text { b. Specific - }
$$

Is this space part of a suite? Yes $\times$

No $\square$

Procession through this space comes after the hot thermal bath

Activity Description:

Bathers enjoy the therapeutic qualities of cold waters; bathers take an invigorating $\overrightarrow{\text { plunge into the cold waters to finish the exfoliation process }}$

Activity type: (insert $x$ in applicable box)

$\begin{array}{lll}\text { 1. Admin Office } & \square & \text { 6. Education } \\ \text { 2. Private Office } & \text { 7. Therapy } \\ \text { 3. Staff Room } & \text { 8. Information } \\ \text { 4. Medical Clinic } & \square & \text { 9. Common Area } \\ \text { 5. Fitness } & \square & \text { 10.Other Specify: }\end{array}$

\section{Users: (insert number in appropriate boxes)}

$\begin{array}{lll}\begin{array}{l}\text { Staff } \\ \text { Patient } \\ \text { Students } \\ \text { Community }\end{array} & \begin{array}{l}\text { Fitness Users } \\ \text { Therapy Users }\end{array} \\ \text { Avg. hours per week usage }\end{array}$

Space Components
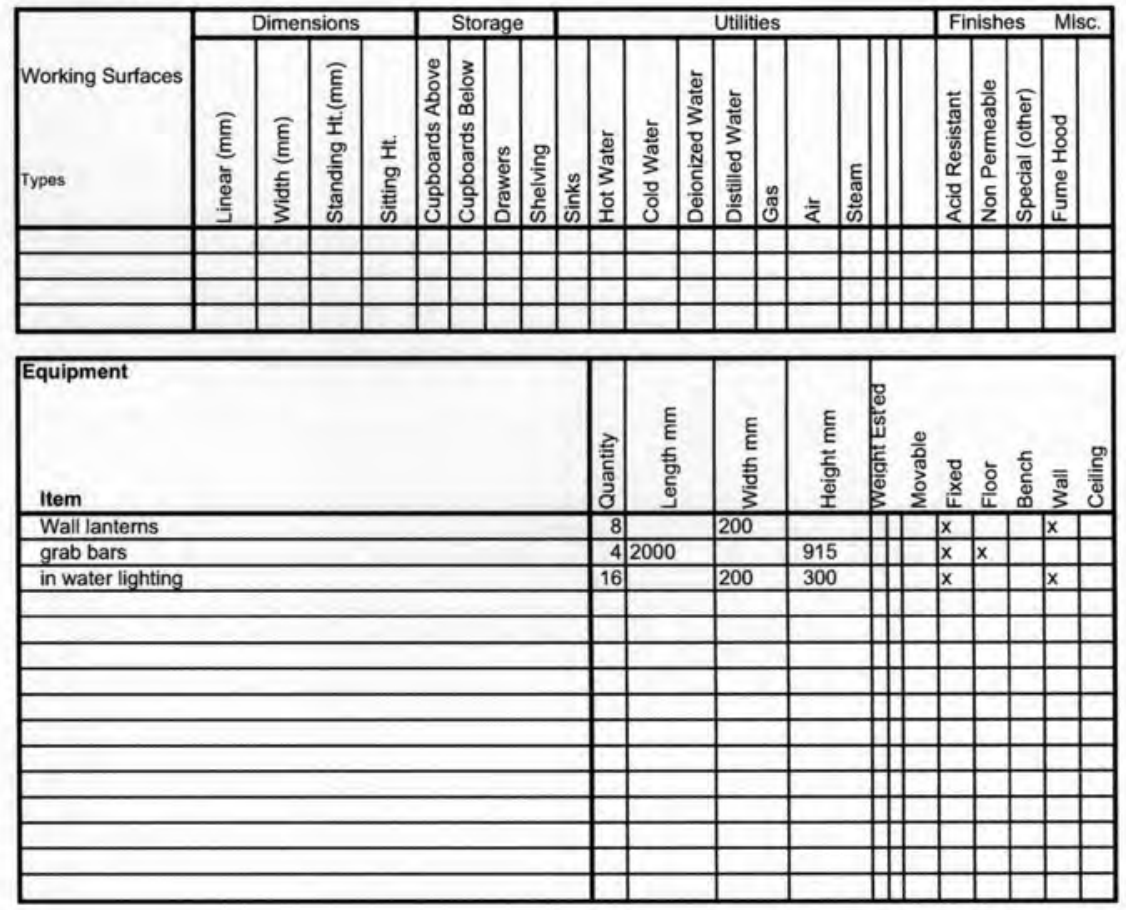

\begin{tabular}{|l|l|}
\hline $\begin{array}{l}\text { Communications: } \\
\text { (place X in box if roquired) } \\
\text { Telephone }\end{array}$ & $\begin{array}{l}\text { Audio Visual } \\
\text { The following audio visual aids will be used: } \\
\text { P.A System } \\
\text { Cable TV } \\
\text { Internet Connection }\end{array}$ \\
Other Specify: & $\begin{array}{l}\text { Television } \\
\text { Projector } \\
\text { Sound Reinforcement }\end{array}$ \\
\hline
\end{tabular}




\begin{tabular}{|ll|}
\hline Furnishings: & (place x in box If required) \\
\hline Storage Cabinets & $\square$ Info Board $\square$ \\
Fixed: (List) & tiered stone stepping \\
Movable: (List) & \\
\cline { 2 - 2 } & \\
\hline
\end{tabular}

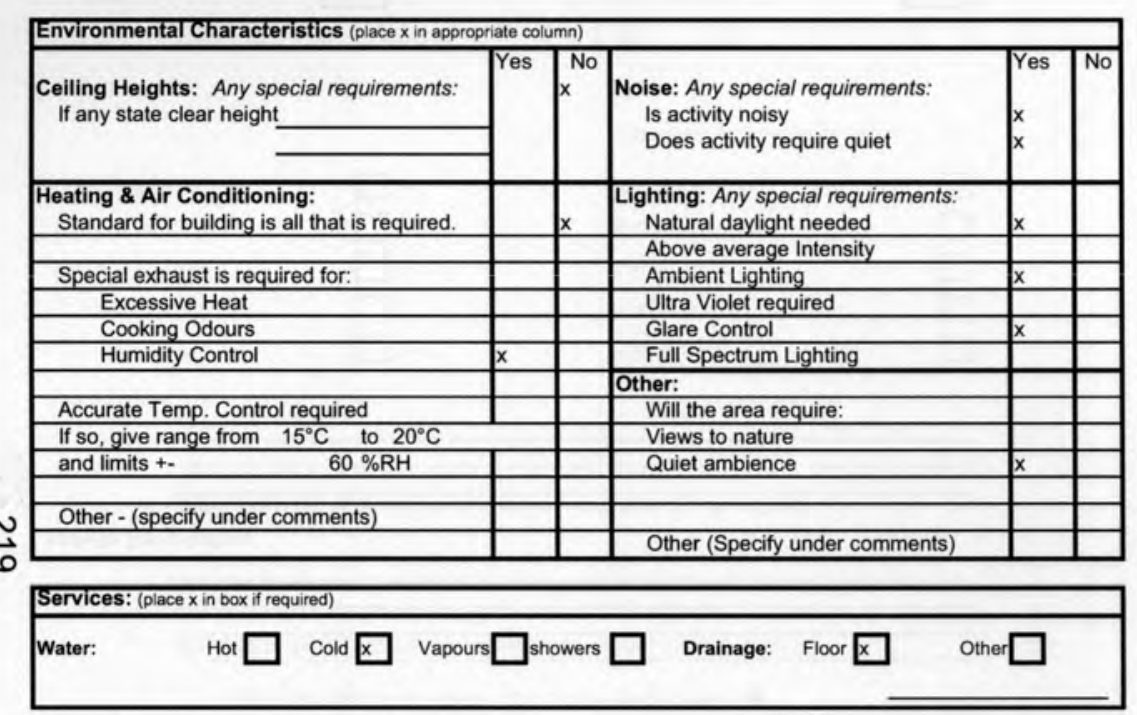

Additional Requirements or Comments:

Approximate amount of time spent here is 1 minutes;

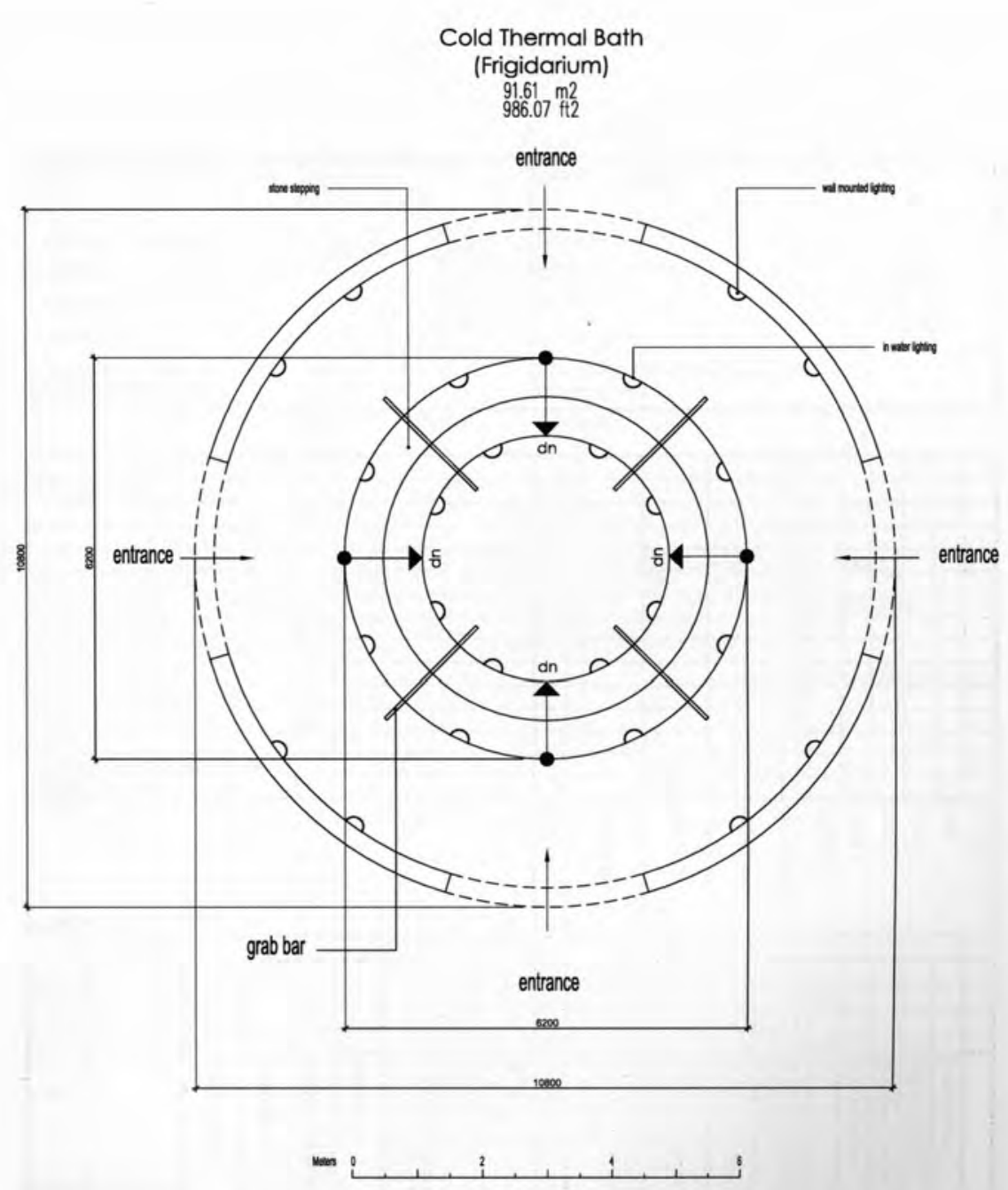


Analysis of physical facility requirements

Project: Wellness Centre 2009

Name of Space: Public Showers

Department Therapeutic baths Net Assignable Sq. m $\frac{6}{6}$

Space Description

Purpose: To provide a space for community to shower off after the entire bathing experience

Space Relationships:

General - How should this space relate to general areas in the building? eg: offices, teaching areas, loading dock, storage areas.

\section{(1)}

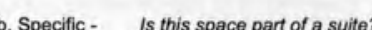
Yes No $\square$ If yes, then describe its relationships to other spaces in the suite Procession through this space comes after the cold thermal bath

\section{Activity Description}

bathers use the open ended showers then move to dry off and rest
Activity type: (insert x in applicable box)
$\begin{array}{ll}\text { 1. Admin Office } & \text { 6. Education } \\ \text { 2. Private Office } & \text { 7. Therapy } \\ \text { 3. Staff Room } & \text { 8. Information } \\ \text { 4. Medical Clinic } & \text { 9. Common Area } \\ \text { 5. Fitness } & \text { 10.Other Specify: }\end{array}$

Users: (insert number in appropriate boxes)

$\begin{array}{lll}\text { Staff } & \text { Fitness Users } \\ \text { Patient } & \text { Therapy Users } \\ \text { Students } & \end{array}$

Space Components
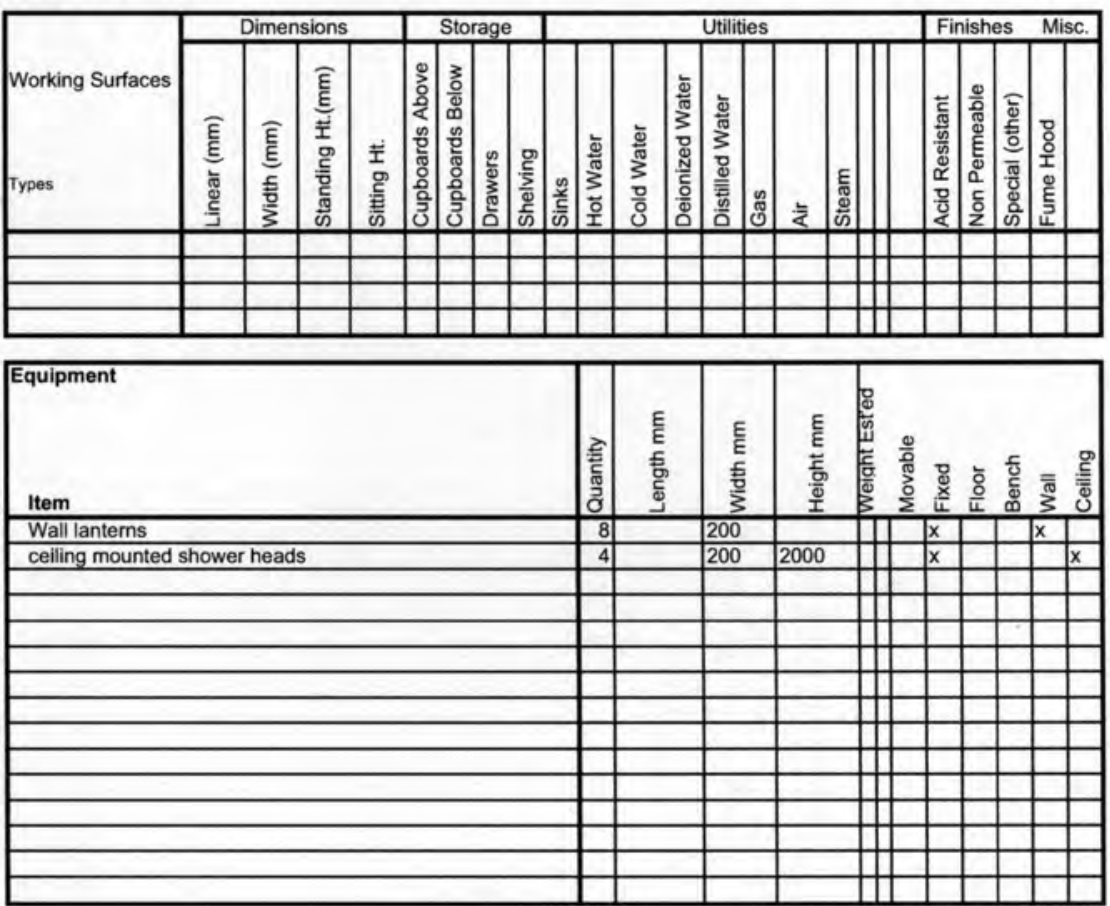

\begin{tabular}{ll|l}
\hline $\begin{array}{l}\text { Communications: } \\
\text { (place X in box ff required) } \\
\text { Telephone }\end{array}$ & $\begin{array}{l}\text { Audio Visual } \\
\text { The following audio visual aids will be used: } \\
\text { P.A System }\end{array}$ \\
Cable TV & Television \\
Internet Connection & Projector \\
Other Specify: & Sound Reinforcement \\
\hline
\end{tabular}




\begin{tabular}{|ll|}
\hline Furnishings: $\quad$ (place x in boxif required) \\
\hline Storage Cabinets \\
Fixed: (List) \\
Movable: (List)
\end{tabular}

\begin{tabular}{|c|c|c|c|c|c|}
\hline \multicolumn{6}{|l|}{ nvironmental Characteristics (place $x$ in appro } \\
\hline $\begin{array}{l}\text { Ceiling Heights: Any special requirements: } \\
\text { If any state clear height }\end{array}$ & Yes & $x^{\text {No }}$ & $\begin{array}{l}\text { Noise: Any special requirements: } \\
\text { Is activity noisy } \\
\text { Does activity require quiet }\end{array}$ & Yes & $\begin{array}{l}\text { No } \\
x \\
x \\
x\end{array}$ \\
\hline $\begin{array}{l}\text { Heating \& Air Conditioning: } \\
\text { Standard for building is all that is required. }\end{array}$ & & $x$ & $\begin{array}{l}\text { Lighting: Any special requirements: } \\
\text { Natural daylight needed }\end{array}$ & & \\
\hline & & & Above average Intensity & & \\
\hline Special exhaust is required for: & & & Ambient Lighting & $x$ & \\
\hline Excessive Heat & & & Ultra Violet required & & \\
\hline Cooking Odours & & & Glare Control & & \\
\hline Humidity Control & $\bar{x}$ & & Full Spectrum Lighting & & \\
\hline & & & Other: & & \\
\hline Accurate Temp. Control required & & & Will the area require: & & \\
\hline If so, give range from $18^{\circ} \mathrm{C}$ to $20^{\circ} \mathrm{C}$ & & & Views to nature & & \\
\hline and limits +- & & & Quiet ambience & $x$ & \\
\hline & & & & & \\
\hline Other - (specify under comments) & & & & & \\
\hline & & & Other (Specify under comments) & & \\
\hline
\end{tabular}

Services: (place $x$ in box if required)

Water: $\quad$ Hot $\square$ Cold $\square$ Vapours $\square$ showers $\square$ Drainage: Floor $\square$ Other $\square$

Additional Requirements or Comments:

Approximate amount of time spent here is 5 minutes;
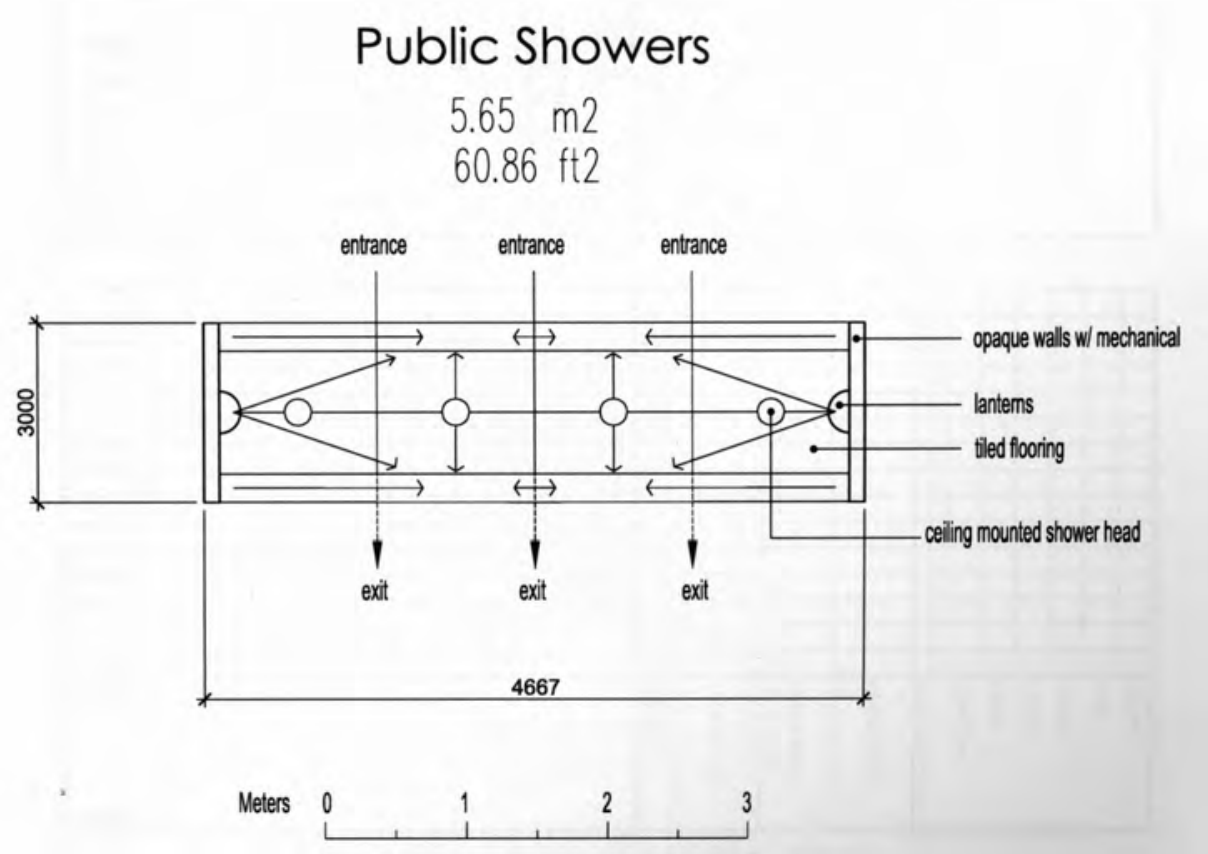


\section{Analysis of physical facility requirements}

Project: Wellness Centre 2009

Name of Space: Dry off \& Rest Area

Department Therapeutic baths Net Assignable Sq. m 54 $6 \mathrm{~m} 2 \times 9=54$

\section{Space Description}

Purpose: To provide a space for dry off and rest after the entire bathing experience

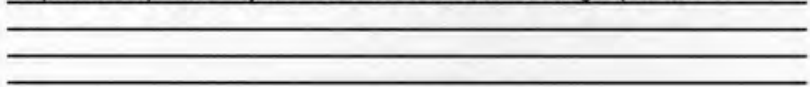

Space Relationships:

a. General - How should this space relate to general areas in the building? eg: offices, teaching areas, loading dock, storage areas.

b. Specific -

Is this space part of a suite?

If yes, then describe its relationships to other spaces in the suito

Procession through this space comes after the showers

Activity Description

bathers will lay down and relax: methods of relaxation include music therapy views N

Activity type: (insert $x$ in applicable box)

$\begin{array}{lll}\text { 1. Admin Office } & \square & \text { 6. Education } \\ \text { 2. Private Office } & \text { 7. Therapy } \\ \text { 3. Staff Room } & \text { 8. Information } \\ \text { 4. Medical Clinic } & \square & \text { 9. Common Area } \\ \text { 5. Fitness } & \square & \text { 10.Other Specify: }\end{array}$

Users: (insert number in appropriate boxes)

Staff

Patient

Students

Community
Fitness Users

Therapy Users

Avg. hours per week usage
Space Components
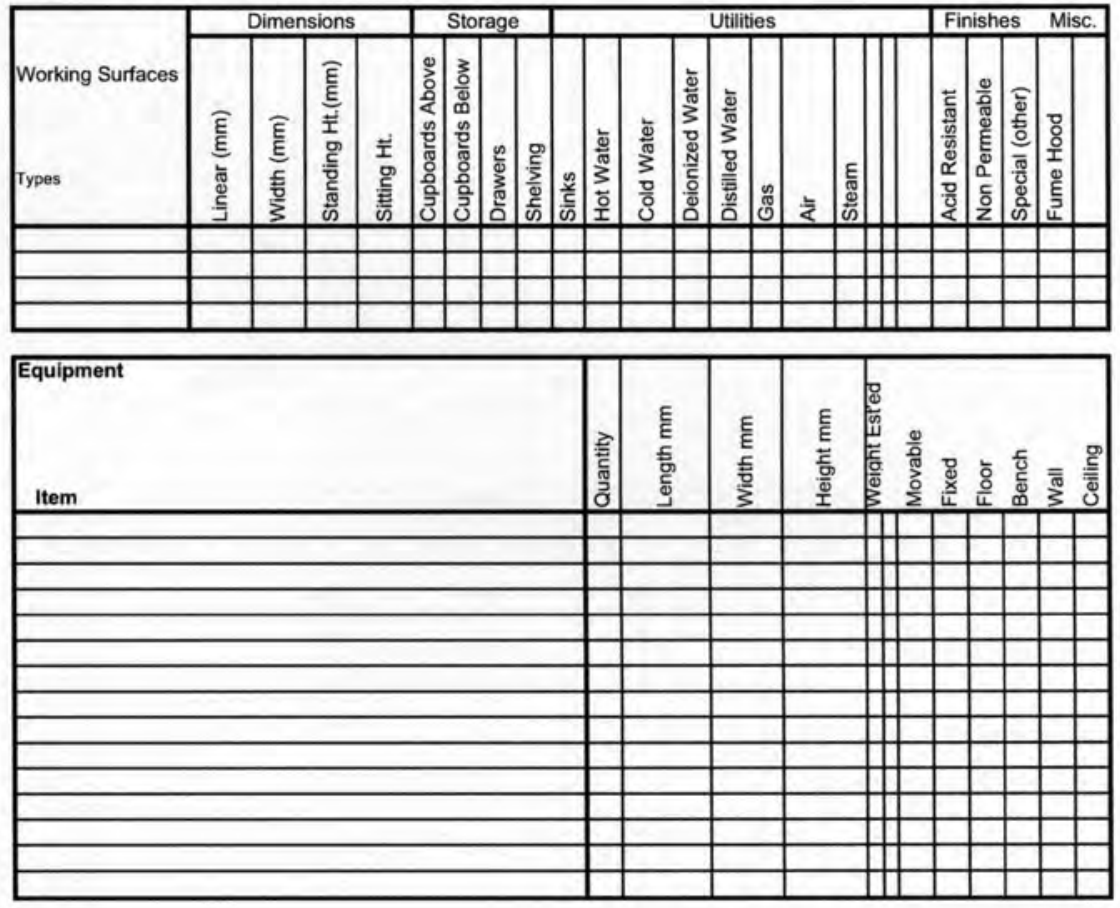

\begin{tabular}{ll|l}
\hline $\begin{array}{l}\text { Communications: } \\
\text { (place X in box if frequired) } \\
\text { Telephone }\end{array}$ & $\begin{array}{l}\text { Audio Visual } \\
\text { The following audio visual aids will be used: } \\
\text { P.A System }\end{array}$ \\
Cable TV \\
Internet Connection
\end{tabular}




\begin{tabular}{|ll|}
\hline Furnishings: & (place x in boxif required) \\
\hline Storage Cabinets & $\square$ Info Board $\square$ \\
Fixed: (List) & privacy screen \\
Movable: (List) & lounger, table top unit, task light \\
\hline
\end{tabular}

\begin{tabular}{|c|c|c|c|c|c|}
\hline \multicolumn{6}{|c|}{ Environmental Characteristics (place $x$ in appropriate column) } \\
\hline $\begin{array}{l}\text { Ceiling Heights: Any special requirements: } \\
\text { If any state clear height }\end{array}$ & Yes & $x^{\text {No }}$ & $\begin{array}{l}\text { Noise: Any special requirements: } \\
\text { Is activity noisy } \\
\text { Does activity require quiet }\end{array}$ & Yes & $\begin{array}{l}x \\
x\end{array}$ \\
\hline \multirow[t]{2}{*}{$\begin{array}{l}\text { Heating \& Air Conditioning: } \\
\text { Standard for building is all that is required. }\end{array}$} & & $x$ & $\begin{array}{l}\text { Lighting: Any special requirements: } \\
\text { Natural daylight needed }\end{array}$ & $x$ & \\
\hline & & & Above average Intensity & & \\
\hline Special exhaust is required for: & & & Ambient Lighting & & \\
\hline Excessive Heat & & & Ultra Violet required & & \\
\hline \multirow{2}{*}{$\begin{array}{l}\text { Cooking Odours } \\
\text { Humidity Control }\end{array}$} & & & Glare Control & & \\
\hline & $x$ & & Full Spectrum Lighting & $x$ & \\
\hline Humidity Control & & & Other: & & \\
\hline Accurate Temp. Control required & & & Will the area require: & & \\
\hline \multicolumn{2}{|l|}{ If so give range from } & & Views to nature & $x$ & \\
\hline \multicolumn{2}{|l|}{ and limits +- $\quad \%$ RH } & & Quiet ambience & $x$ & \\
\hline \multirow{2}{*}{\multicolumn{2}{|c|}{ Other - (specify under comments) }} & & & & \\
\hline & & & & & \\
\hline \multicolumn{2}{|l|}{ Other - (specify under comments) } & & Other (Specify under comments) & & \\
\hline \multicolumn{6}{|l|}{ Services: (place $x$ in box if required) } \\
\hline \multicolumn{3}{|l|}{ Water: } & Drainage: $\quad$ Floor $\square$ & $\square$ & \\
\hline
\end{tabular}

Additional Requirements or Comments:

Approximate amount of time spent here is 30 minutes;

\section{Dry-Off \& Rest Area}
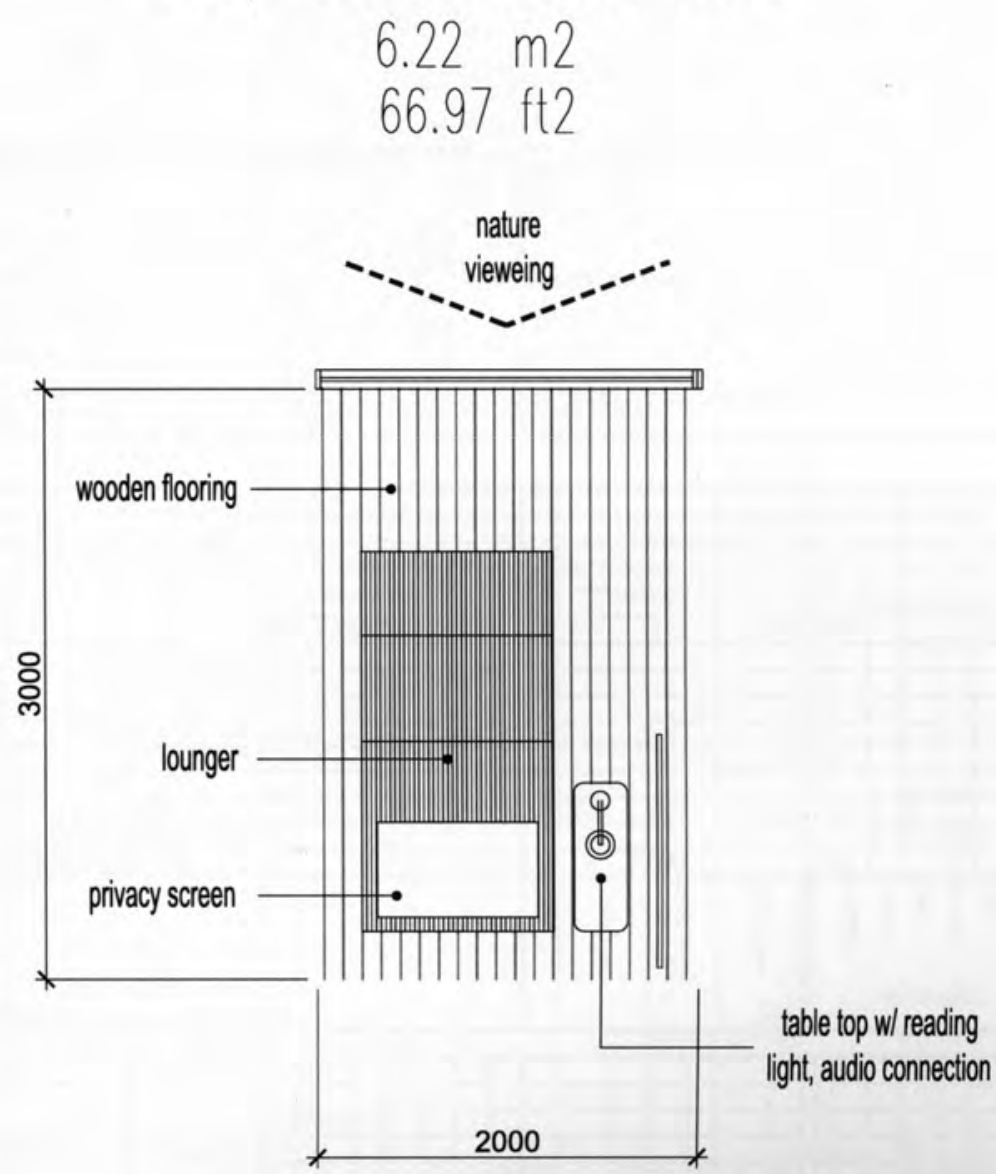

Meters

2 


\section{Analysis of physical facility requirements}

Project: Wellness Centre 2009

Name of Space: Massage Room

Department Therapeutic baths $14 \mathrm{~m} \times 4=56 \mathrm{~m}$

\section{Space Description}

Purpose: To provide therapeutic massage services for the community

Space Relationships:

a. General - How should this space relate to general areas in the building? eg: offices, teaching areas, loading dock, storage areas.

b. Specific -

Is this space part of a suite?

If yes, then describe its relationships to other spaces in the suite

№ $\square$

Massages can take place at any time during the spa experience

\section{Activity Description:}

N

bathers will lay down and relax; staff will undergo several methods of massage including physical touch as well as water massages

Activity type: (insert $x$ in applicable box)

$\begin{array}{lll}\text { 1. Admin Office } & \square & \text { 6. Education } \\ \text { 2. Private Office } & \square & \text { 7. Therapy } \\ \text { 3. Staff Room } & \text { 8. Information } \\ \text { 4. Medical Clinic } & \square & \text { 9. Common Area } \\ \text { 5. Fitness } & \square & \text { 10.Other Specify: }\end{array}$

\section{Users: (insert number in appropriate boxes)}

$\begin{array}{lll}\text { Staff } & \text { Fitness Users } \\ \text { Patient } & \text { Therapy Users } \\ \text { Students } & \text { Avg. hours per week usage } \\ \text { Community } & \end{array}$

Space Components
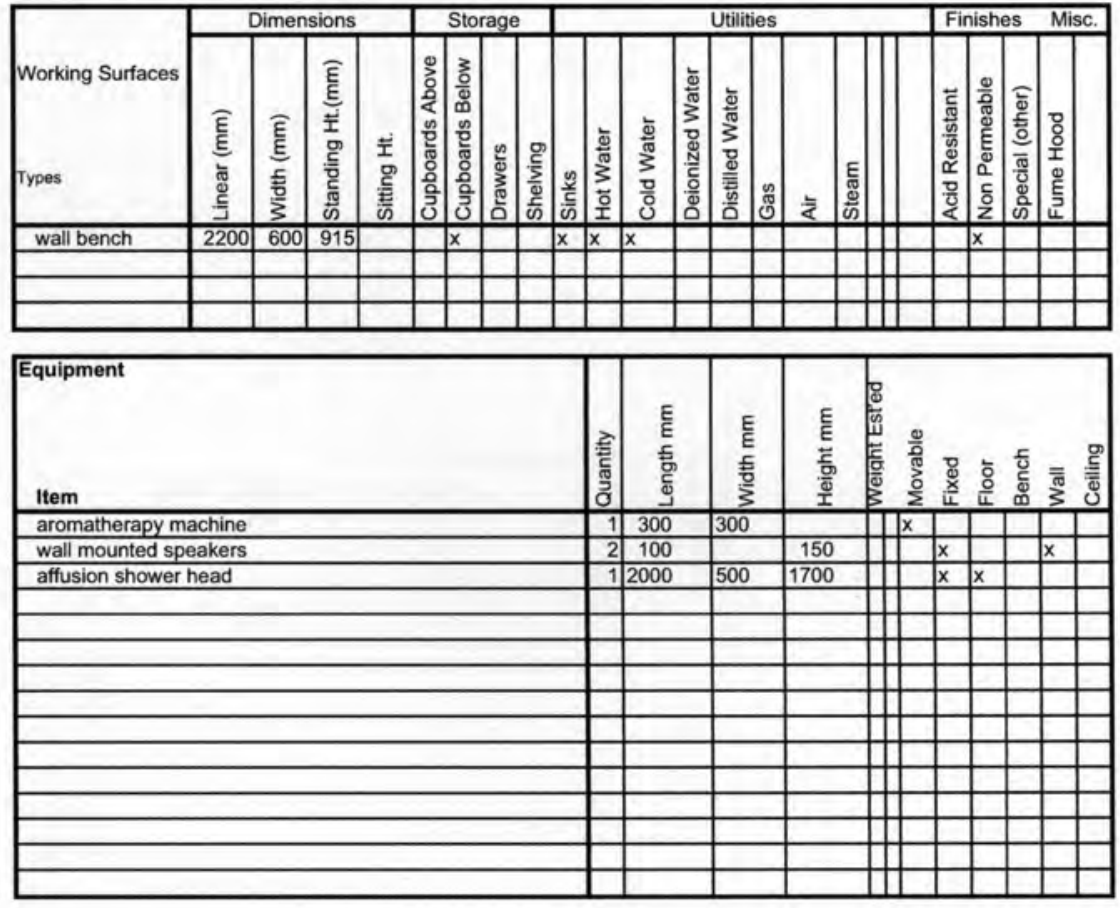

\begin{tabular}{l|l|l}
\hline $\begin{array}{l}\text { Communications: } \\
\text { (place Xin boxiff frequired) } \\
\text { Telephone }\end{array}$ & $\begin{array}{l}\text { Audio Visual } \\
\text { The following audio visual aids will be used: } \\
\text { P.A System } \\
\text { Cable TV } \\
\text { Internet Connection }\end{array}$ \\
Other Specify: & Television \\
Projector \\
Sound Reinforcement
\end{tabular}




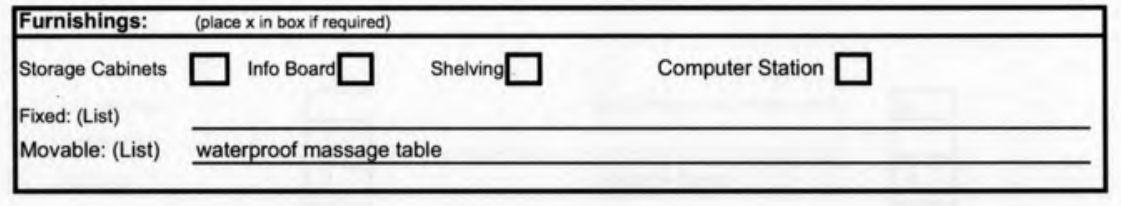

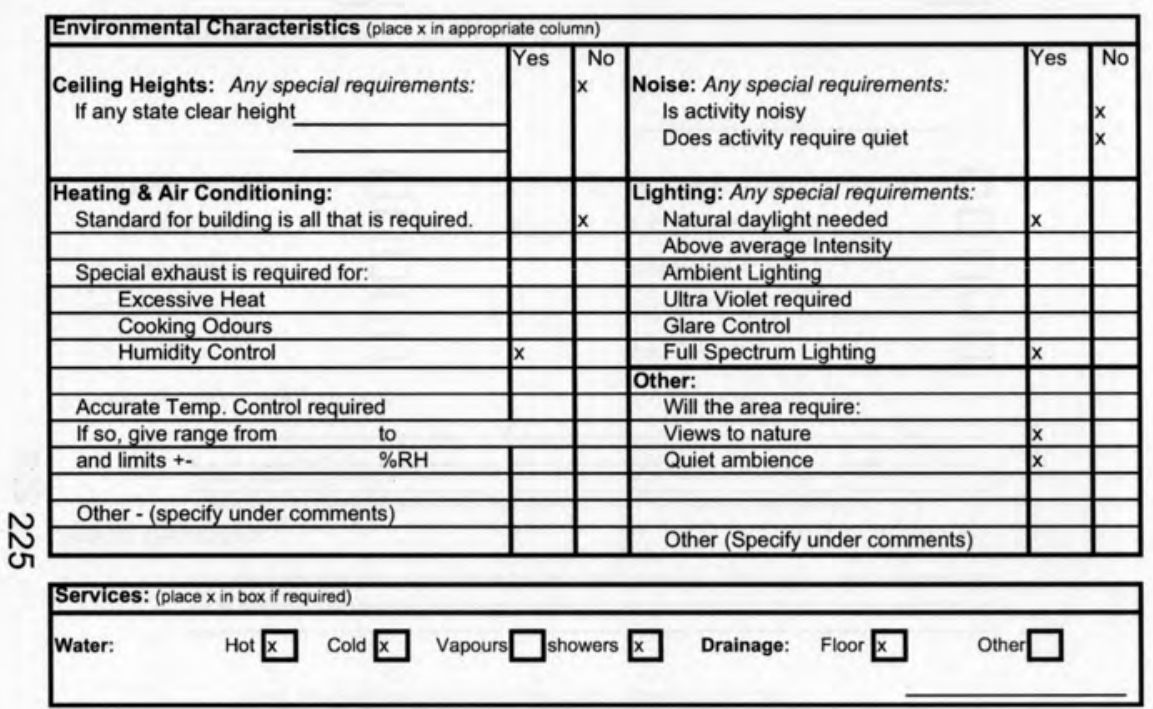

Additional Requirements or Comments:

Approximate amount of time spent here is 15 minutes:

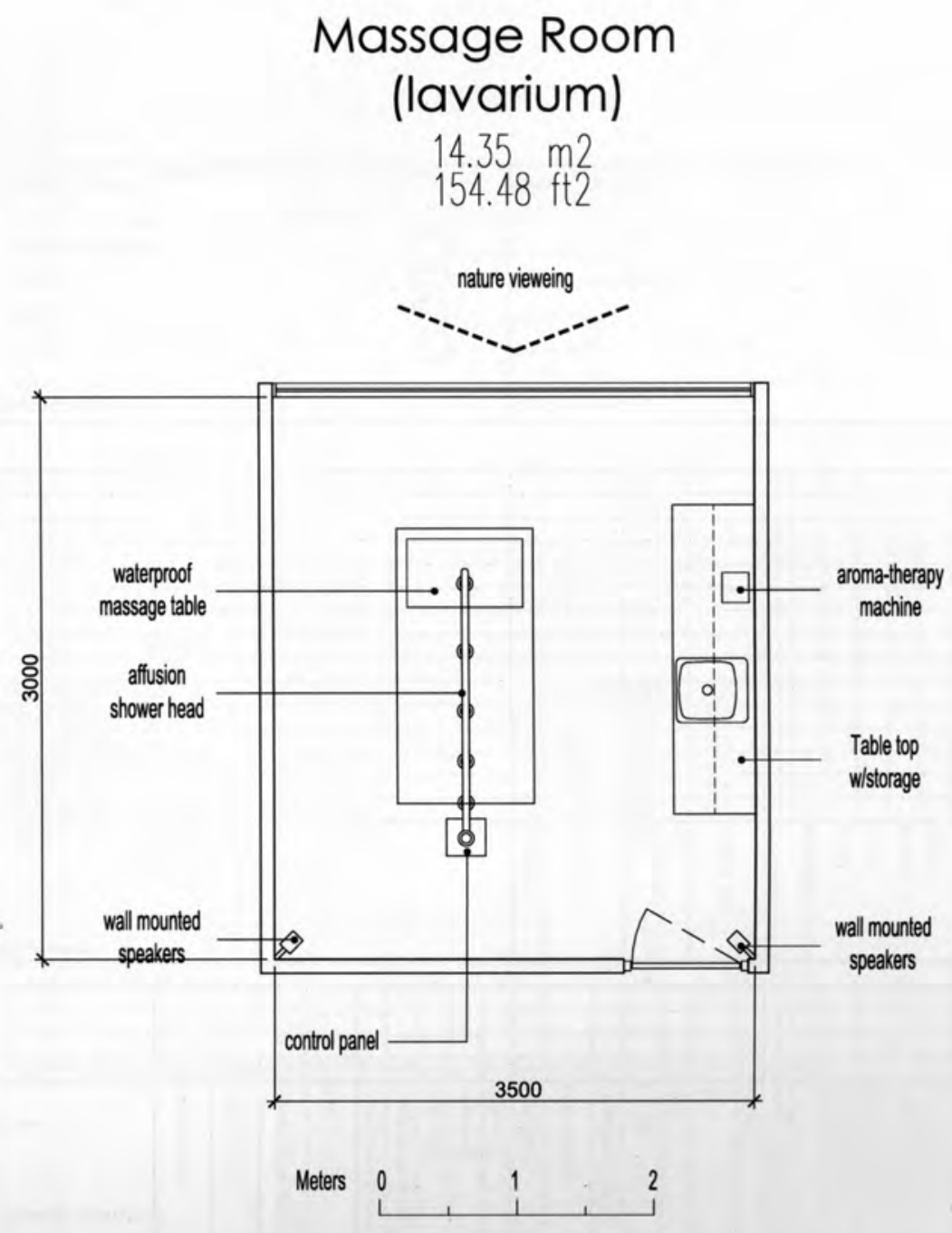




\section{Analysis of physical facility requirements}

Project: Wellness Centre 2009

Name of Space: Hot Tea Bar

Space Description

Purpose:
To allow for replenishment after therapeutic spa experience

Space Relationships:

a. General - How should this space relate to general areas in the building? eg: offices,

teaching areas, loading dock, storage areas.

Located adjacent to the rest areas

b. Specific - Is this space part of a suite?
If yes, then describe its relationships to other spaces in the suite

\section{Activity Description}

N a space that extends the feeling of the rest area, but also facilitates for

N

Activity type: (insert $\mathrm{x}$ in applicable box)

$\begin{array}{llll}\text { 1. Admin Office } & \square & \text { 6. Education } & \\ \text { 2. Private Office } & \square & \text { 7. Therapy } & \\ \text { 3. Staff Room } & \square & \text { 8. Information } & \\ \text { 4. Medical Clinic } & \square & \text { 9. Common Area } \\ \text { 5. Fitness } & \square & \text { 10.Other Specify: }\end{array}$

Users:

(insert number in appropriate boxes)

Staff

Patient

Students

Community
Fitness Users

Therapy Users

Avg. hours per week usage
Space Components
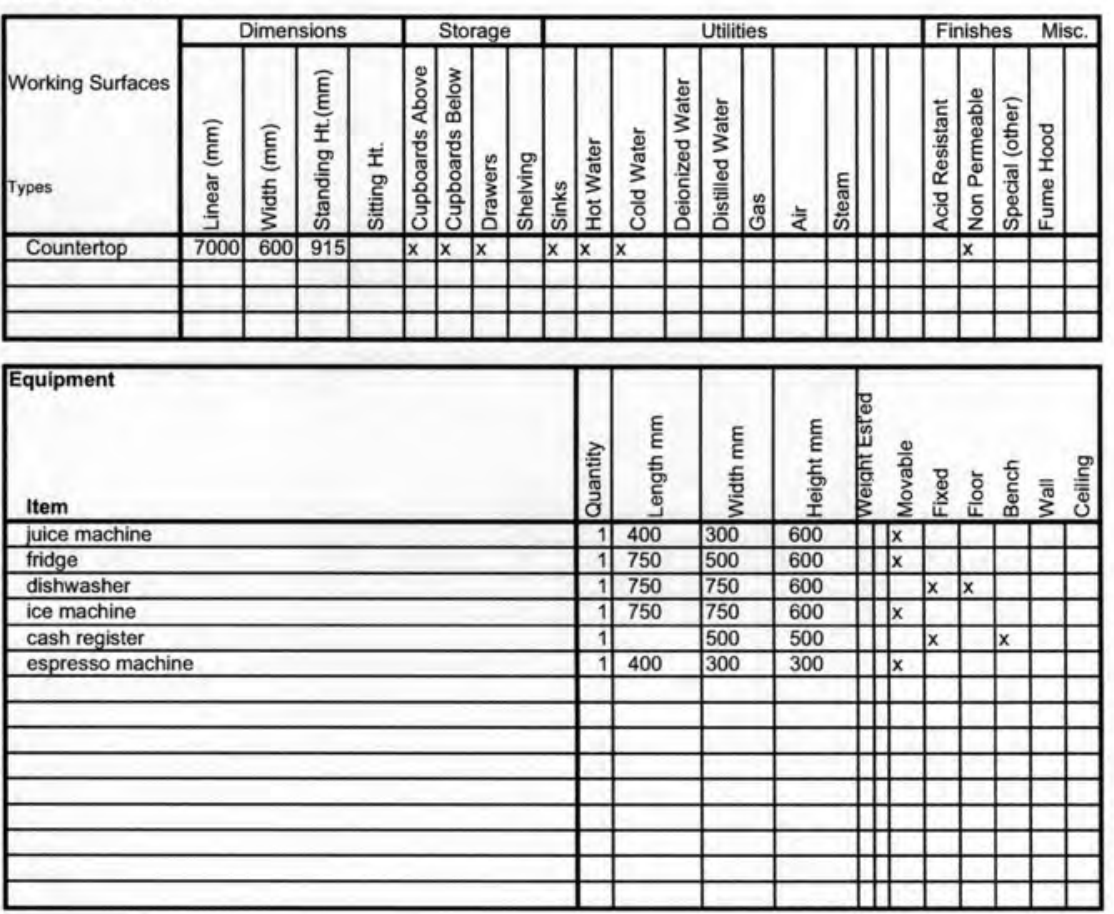

\begin{tabular}{ll|l|}
\hline $\begin{array}{l}\text { Communications: } \\
\text { (place } X \text { in box } \text { f frequired) } \\
\text { Telephone }\end{array}$ & $\begin{array}{l}\text { Audio Visual } \\
\text { The following audio visual aids will be used: } \\
\text { P.A System }\end{array}$ \\
Cable TV & Television \\
Internet Connection & Projector \\
Sound Reinforcement & \\
\hline
\end{tabular}




\begin{tabular}{|ll|}
\hline Furnishings: & (place $\mathrm{x}$ in box if required) \\
\hline Storage Cabinets & $\square$ Info Board $\square \quad$ Shelving \\
Fixed: (List) & \\
Movable: (List) & 3 moveable bar stools, 3 lounge chairs, 3 tables w/ audio connection \\
\cline { 2 - 2 }
\end{tabular}

\begin{tabular}{|c|c|c|c|c|c|}
\hline \multicolumn{6}{|c|}{ Environmental Characteristics (place $\mathrm{x}$ in appropriate column) } \\
\hline $\begin{array}{l}\text { Ceiling Heights: Any special requirements: } \\
\text { If any state clear height }\end{array}$ & Yes & $x^{\text {No }}$ & $\begin{array}{l}\text { Noise: Any special requirements: } \\
\text { Is activity noisy }\end{array}$ & Tyes & No \\
\hline \multirow[t]{2}{*}{$\begin{array}{l}\text { Heating \& Air Conditioning: } \\
\text { Standard for building is all that is required. }\end{array}$} & $\mathrm{x}$ & & $\begin{array}{l}\text { Lighting: Any special requirements: } \\
\text { Natural daylight needed }\end{array}$ & $x$ & \\
\hline & & & Above average Intensity & & \\
\hline Special exhaust is required for: & & & Ambient Lighting & & \\
\hline Excessive Heat & & & Ultra Violet required & & \\
\hline Cooking Odours & & & Glare Control & & \\
\hline \multirow[t]{2}{*}{ Humidity Control } & & & Full Spectrum Lighting & $x$ & \\
\hline & & & Other: & & \\
\hline Accurate Temp. Control required & & & Will the area require: & & \\
\hline \multirow{2}{*}{$\begin{array}{ll}\text { If so, give range from } & \text { to } \\
\text { and limits +- } & \% \mathrm{RH}\end{array}$} & & & Views to nature & $x$ & \\
\hline & & & Quiet ambience & $x$ & \\
\hline \multirow{3}{*}{ Other - (specify under comments) } & & & & & \\
\hline & & & & & \\
\hline & & & Other (Specify under comments) & & \\
\hline
\end{tabular}

Services: (place $x$ in box if required)

Water: $\quad$ Hot $\square$ Cold $\square$ Vapours $\square$ showers $\square$ Drainage: Floor $\square \quad$ Other $\square$

Additional Requirements or Comments:

Approximate amount of time spent here is 15 minutes;

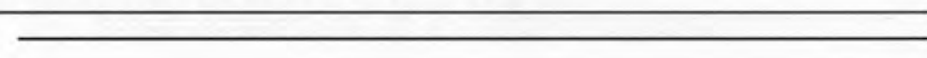

\section{Hot Tea Bar}

$21.56 \mathrm{~m} 2$

$232.07 \mathrm{ft} 2$
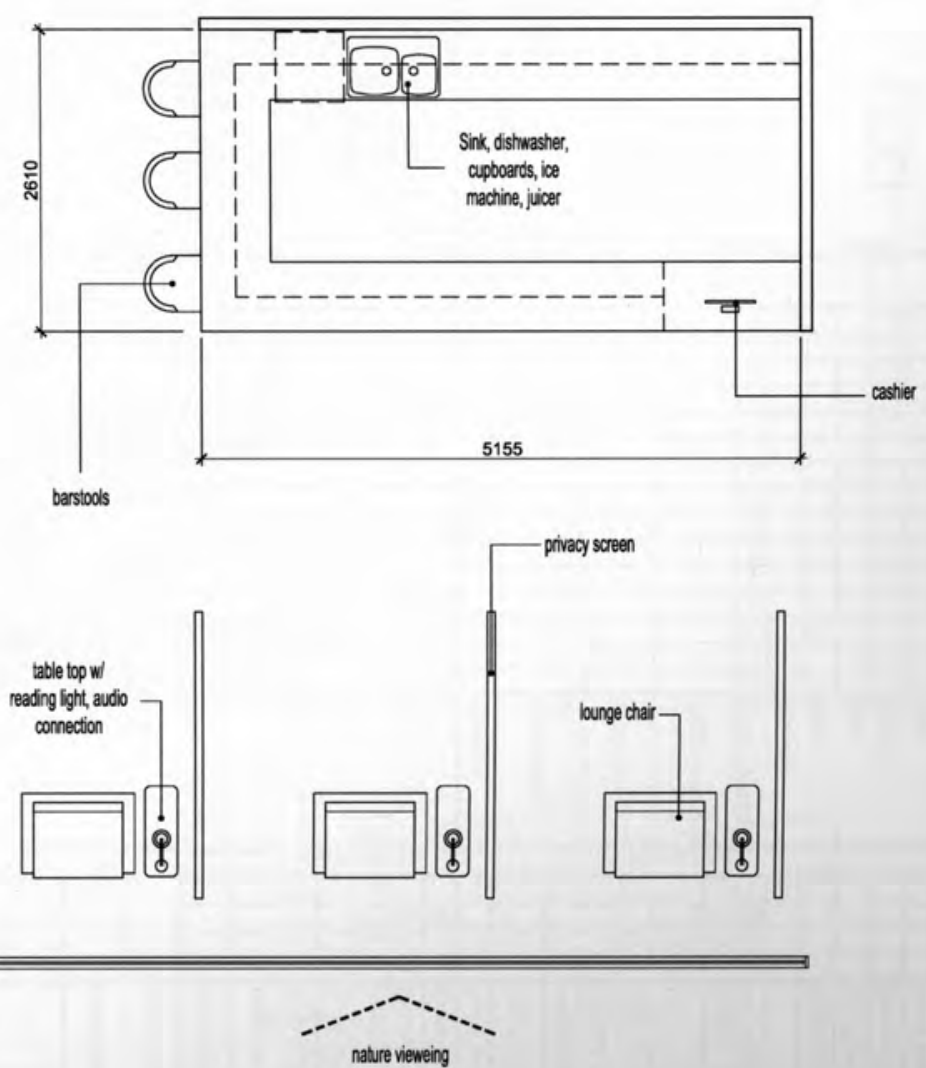

Meters

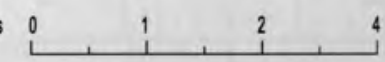




\section{Analysis of physical facility requirements}

Project: Wellness Centre 2009

Name of Space: Services

\section{Space Description}

Purpose: An area dedicated to the proper servicing of the community centre with respect to HVAC and water treatmen

Space Relationships:

a. General - How should this space relate to general areas in the bullding? eg: offices, teaching areas, loading dock storage areas.

Located in the back of house and out of the way of the community b. Specific - Is this space part of a suite?
If yes, then describe its relationships to other spaces in the suite

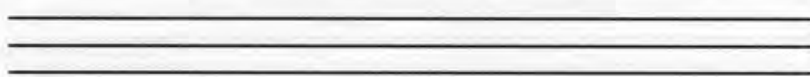

Activity Description: Mechanical equipment shall be monitored and thus must be accessible to

N

Activity type: (insert $x$ in applicable box)
1. Admin Office

2. Private Office

3. Staff Room

4. Medical Clinic

5. Fitness

$\square$
$\square$
$\square$
$\square$

6. Education

7. Therapy

8. Information

9. Common Area

10.Other Specify:

Services

\section{Users: (insert number in appropriate boxes)}

Staff

Patient

Students

Community

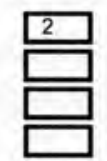

\section{Fitness Users}

Therapy Users

Avg. hours per week usage
Space Components
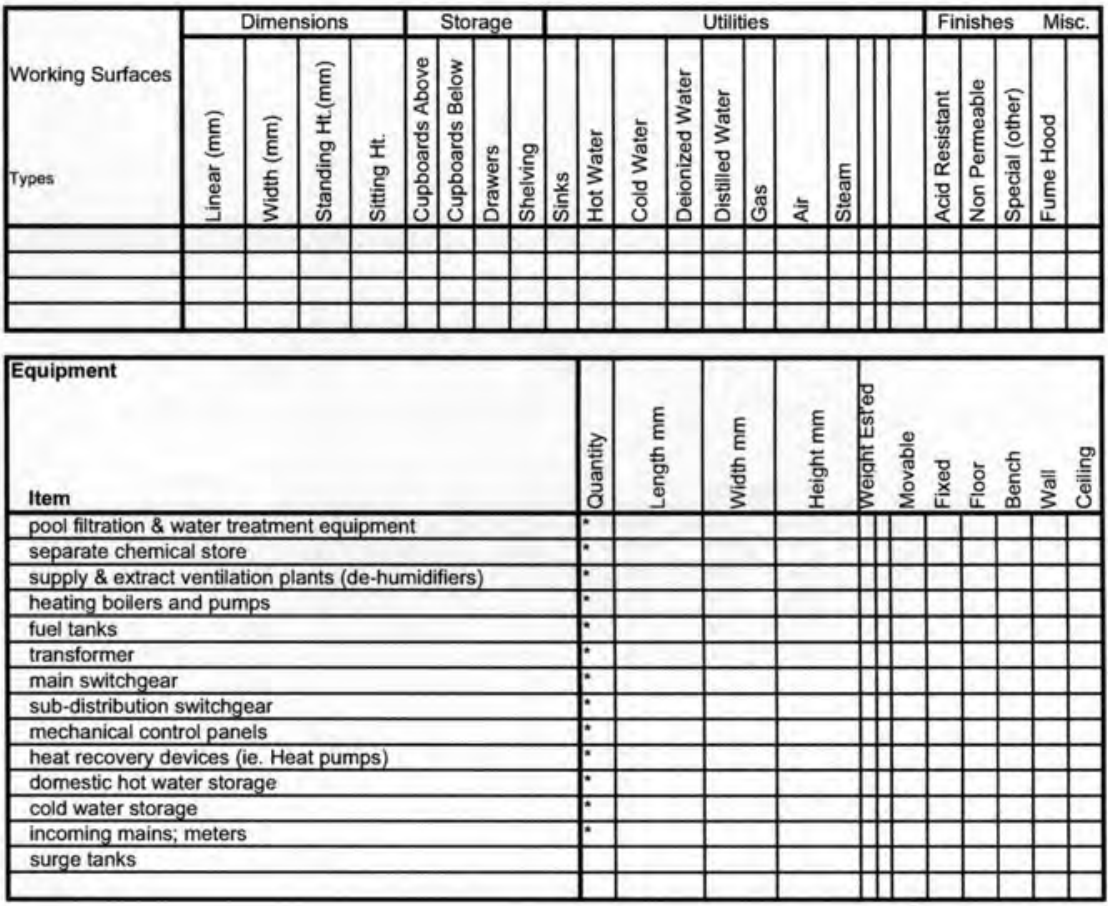

\begin{tabular}{|l|l|}
\hline $\begin{array}{l}\text { Communications: } \\
\text { (place X in box if required) }\end{array}$ & $\begin{array}{l}\text { Audio Visual } \\
\text { The following audio visual aids will be used: } \\
\text { Telephone }\end{array}$ \\
P.A System & Television \\
Cable TV & Projector \\
Internet Connection & Sound Reinforcement \\
Other Specify: & Other Specify: \\
\hline
\end{tabular}




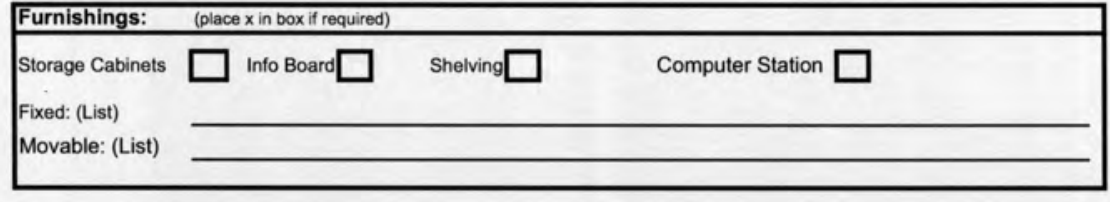

్ㅡㅇ

\begin{tabular}{|c|c|c|c|c|c|}
\hline \multicolumn{6}{|c|}{ Environmental Characteristics (place $x$ in appropriate column) } \\
\hline $\begin{array}{l}\text { Ceiling Heights: Any special requirements: } \\
\text { If any state clear height }\end{array}$ & $\begin{array}{l}\text { Yes } \\
x\end{array}$ & No & $\begin{array}{l}\text { Noise: Any special requirements: } \\
\text { Is activity noisy }\end{array}$ & $\begin{array}{l}\text { Yes } \\
x\end{array}$ & No \\
\hline \multirow[t]{2}{*}{$\begin{array}{l}\text { Heating \& Air Conditioning: } \\
\text { Standard for building is all that is required. }\end{array}$} & $\mathrm{x}$ & & $\begin{array}{l}\text { Lighting: Any special requirements: } \\
\text { Natural daylight needed }\end{array}$ & & \\
\hline & & & Above average Intensity & $x$ & \\
\hline Special exhaust is required for: & & & Ambient Lighting & & \\
\hline Excessive Heat & $x$ & & Ultra Violet required & & \\
\hline Cooking Odours & & & Glare Control & & \\
\hline \multirow[t]{2}{*}{ Humidity Control } & $x$ & & Full Spectrum Lighting & & \\
\hline & & & Other: & & \\
\hline Accurate Temp. Control required & & & Will the area require: & & \\
\hline If so, give range from to & & & Views to nature & & \\
\hline and limits +- & & & Quiet ambience & & \\
\hline \multicolumn{6}{|l|}{ Other - (specify under comments) } \\
\hline & & & Other (Specify under comments) & & \\
\hline
\end{tabular}

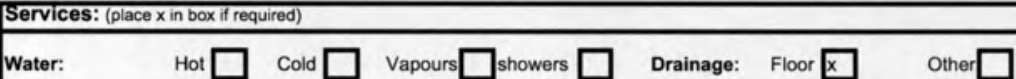

Additional Requirements or Comments:

* mechanical service sizing/loads has not been specifically calculated; general sizing

standards have been implimented for pools of similar size and use
Services

$1347 \mathrm{~m} 2$

15061 th

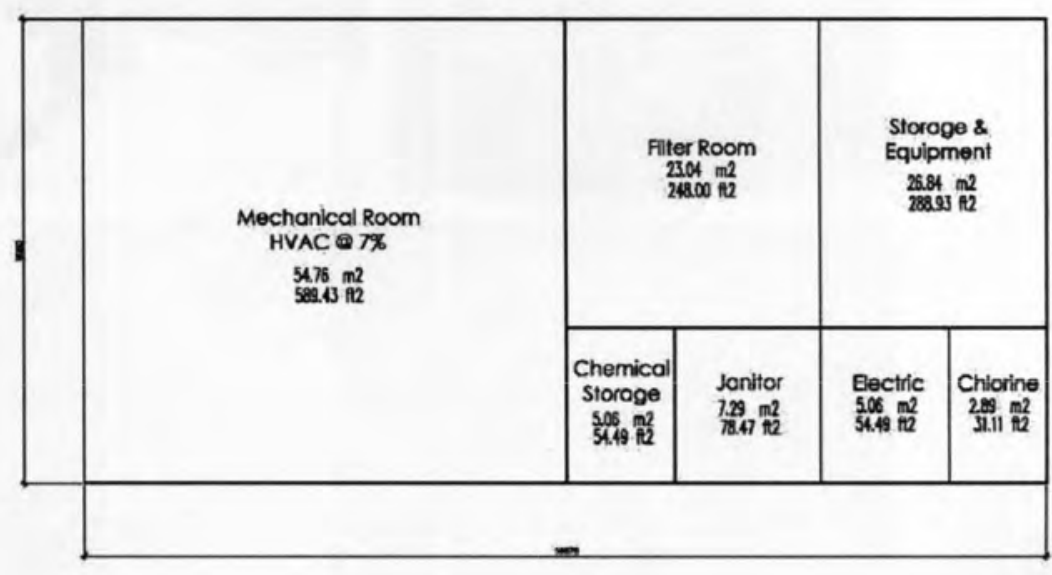

$-1, \ldots, 1, \ldots+\ldots$,

Pool water Flow (Multiple Pools)

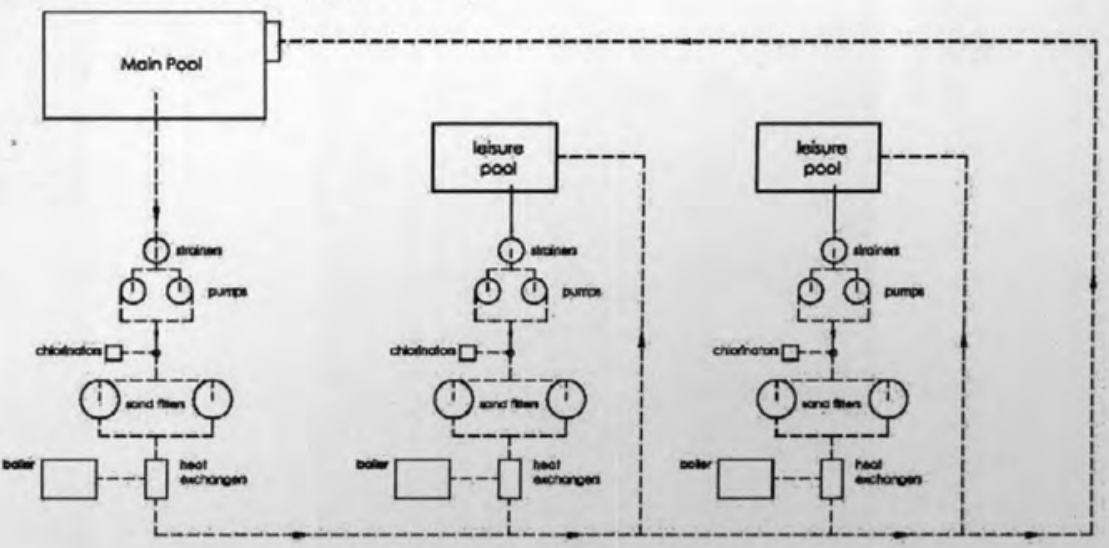



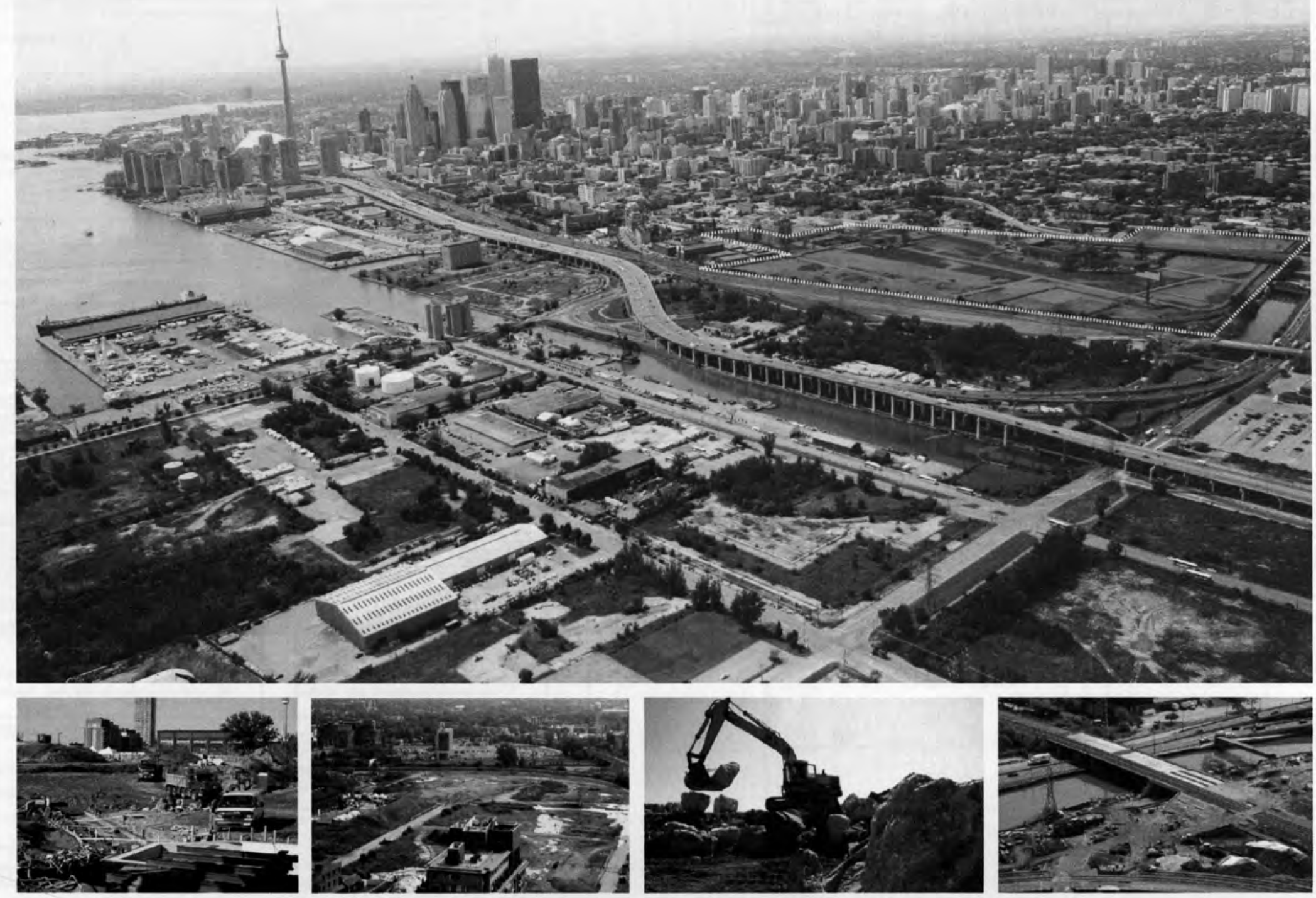


\section{Appendix 8}

West Donlands Infrastructure Development

Image Credit: Ontario Realty Corporation

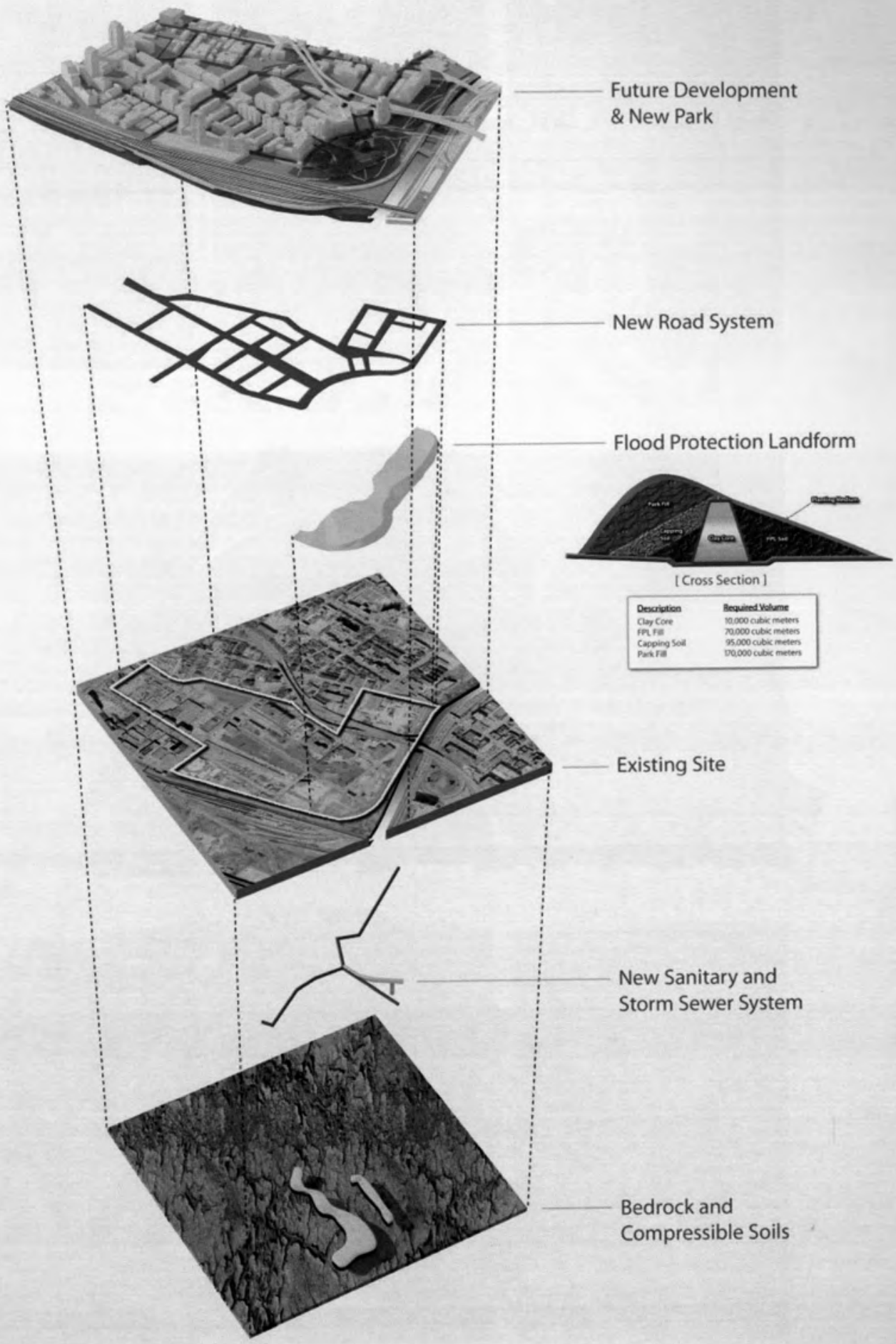

Westi Don Lands 


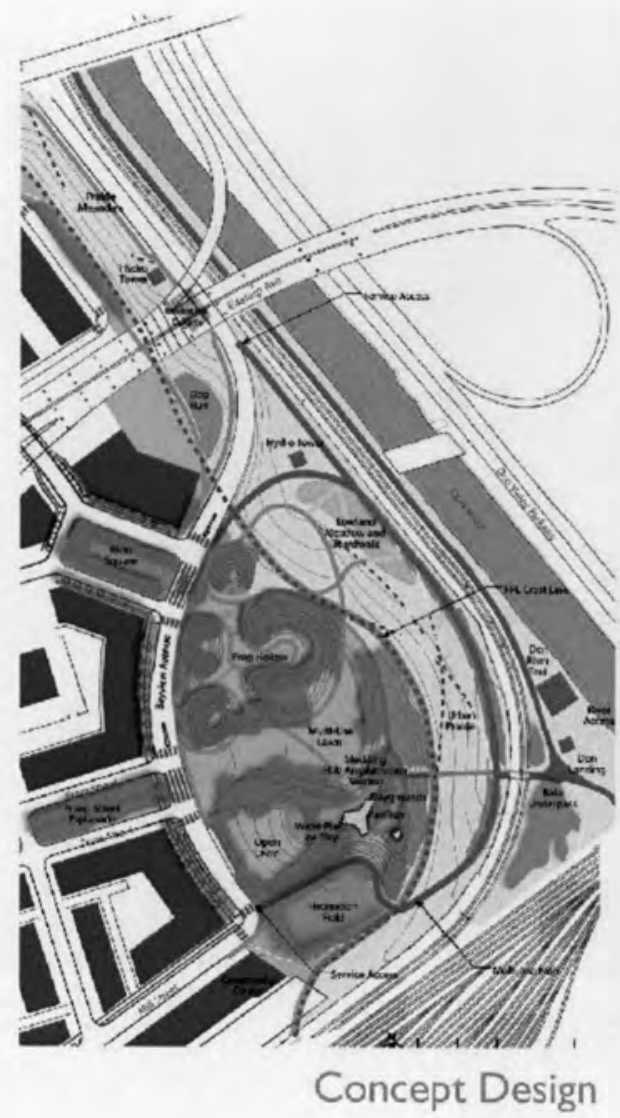

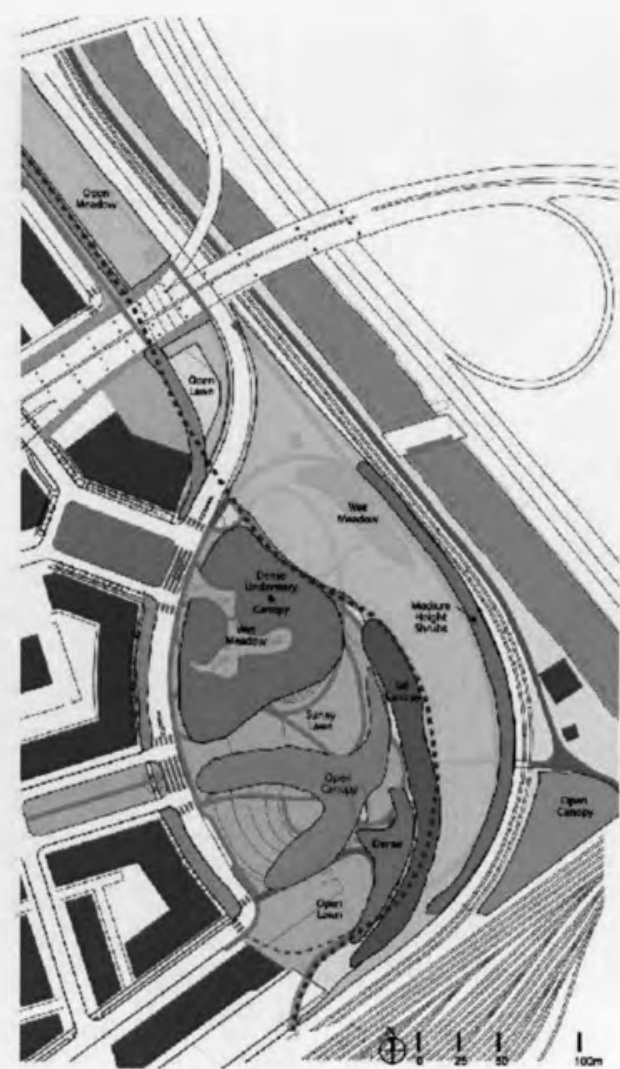

Planting Design Concept

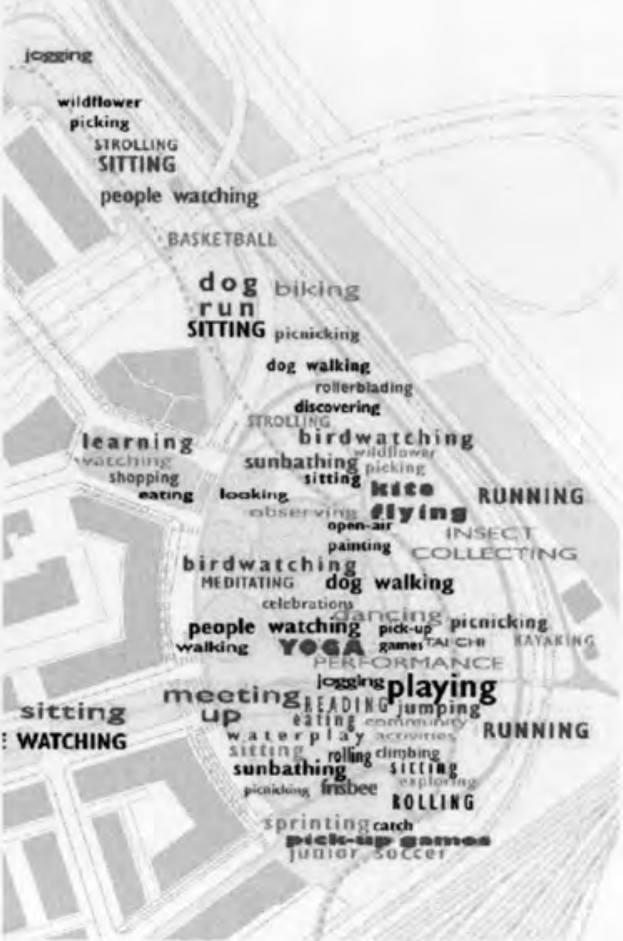

Seasonal park Elements

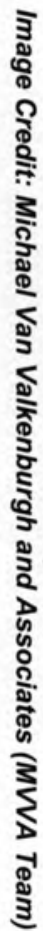

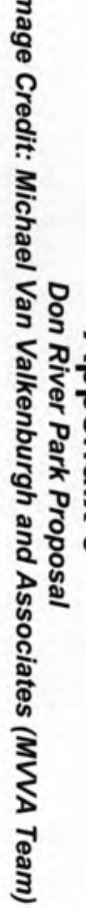
ป⿻ำ

응

인

줄 을

ปัํำ 
Appendix 10

Steven Holl Architects: District Energy Heating/Cooling Plant Image Credits: Waterfront Toronto
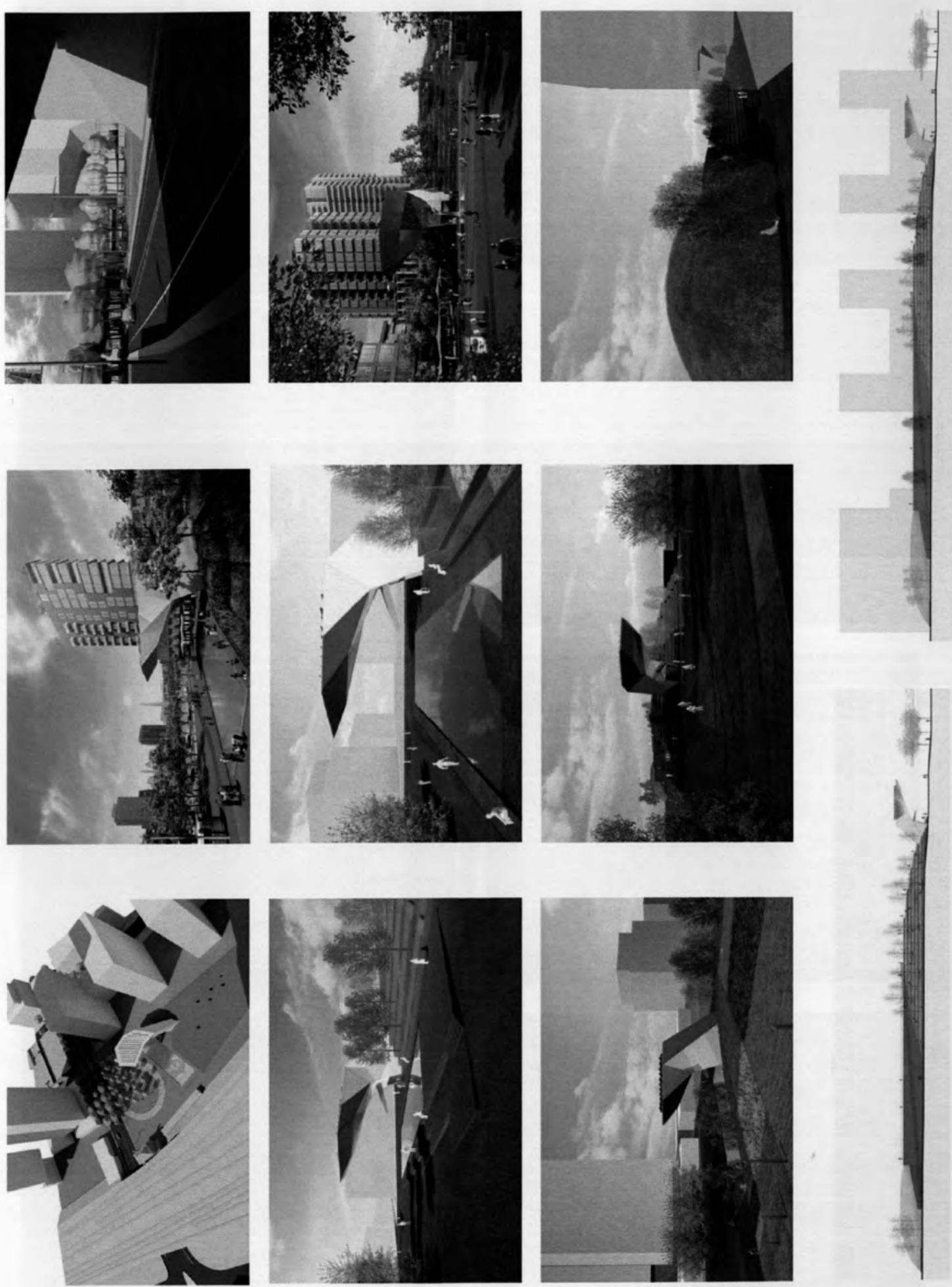


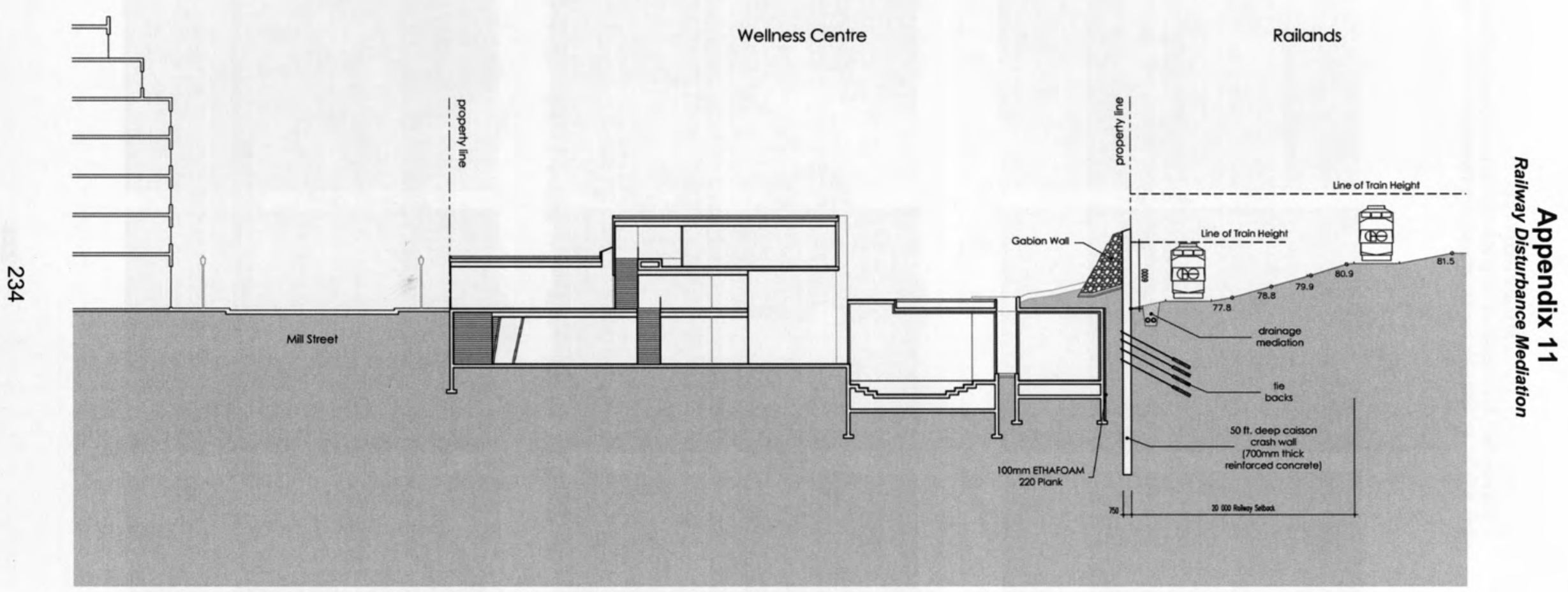




\section{Appendix 12}

Physical modeling exercises were used to achieve a porous building mass that would make large connections with the external physical environment
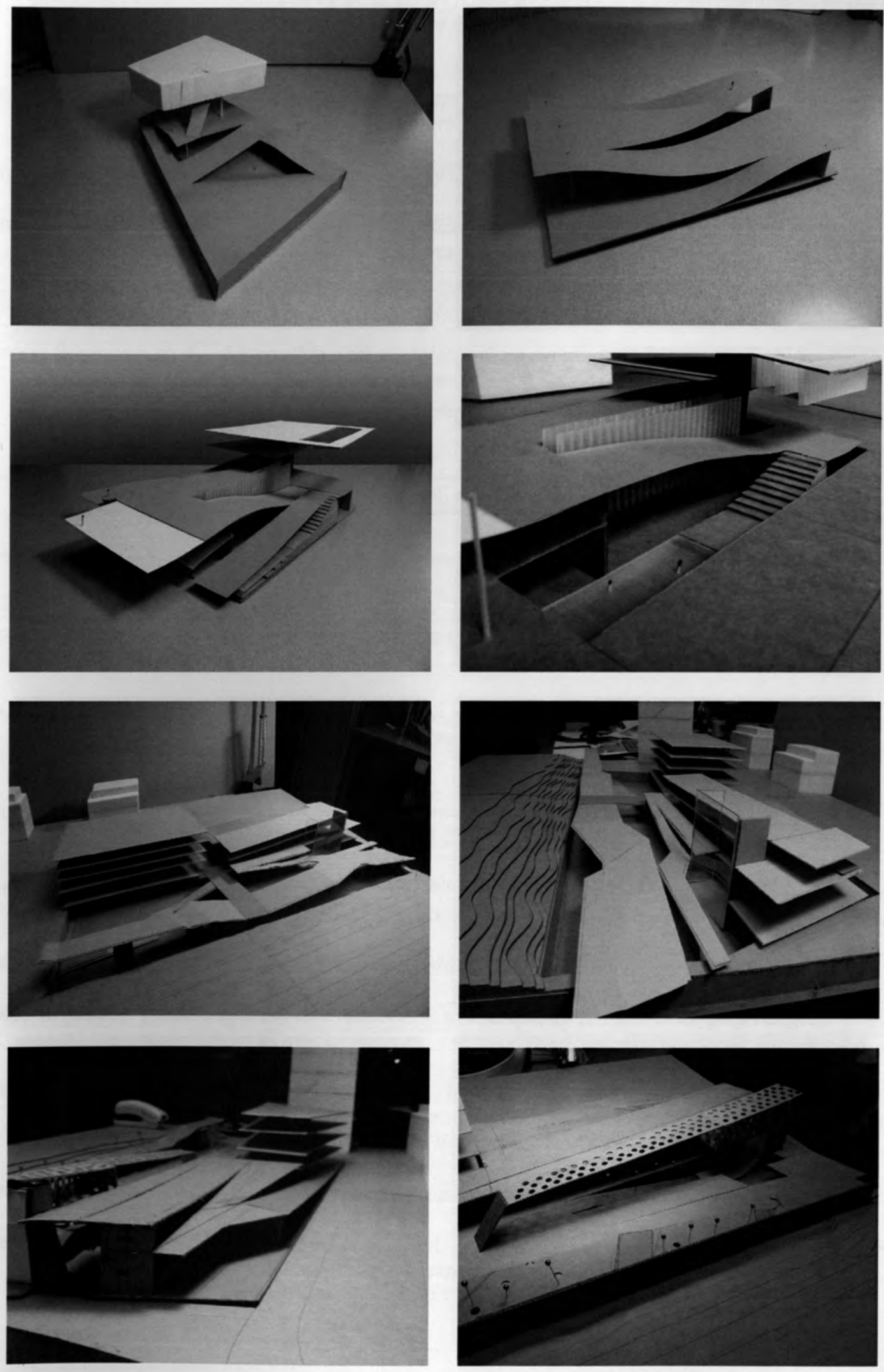
Appendix 13

Physical lighting studies were conducted in order to achieve varying levels of porous daylight penetration into spaces
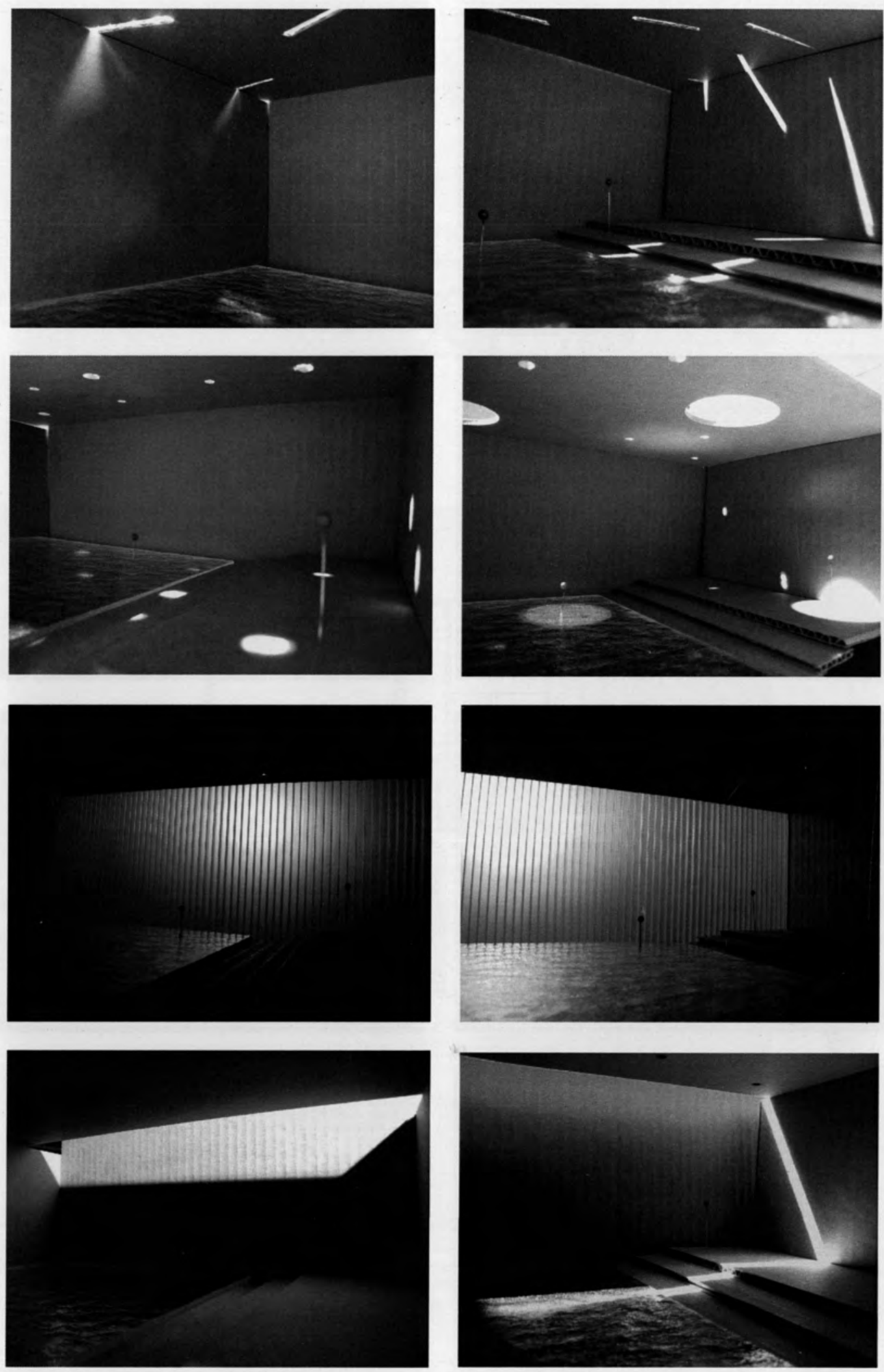


\section{Reference List}

Addington, M., Kienz, N., \& Intrachooto, S. (2001). Mat buildings and the environment. In Sarkis, H. (2001). CASE: Le Corbusier's Venice Hospital and the mat building revival. Munich: Prestel Verlag.

Ashnen + Allen (2008). Greening the patient experience. Retrieved September 27, 2008, from http://www.iidexneocon.com/2008/images/uploads/IIDEX___Green_Patient_Room.pdf

Bergdolt, K. (1999). Wellbeing: A cultural history of healthy living. Cambridge: Polity Press.

Berry, L., Parker, D., Coile, R., \& Hamilton, D. (2004). The business case for better buildings [Electronic Version]. Healthcare and Financial Management, 58, 76-86.

Bivins, R. (2007). Alternative medicine: A history. New York: Oxford University Press.

Canada Business. (n.d.). Alternative and complementary health care business. Retrieved November 22, 2008, from http://www.canadabusiness.ca/servlet/ ContentServer?cid=1099919424295\&pagename=CBSC_FE\%2Fdisplay\&lang=en\&c=Gui deHowto

Canada Green Building Council. (2004). LEED green building rating system. Ottawa, Ontario, Canada: Canada Green Building Council.

Canadian Press. (2007). One in six reports diagnosis of depression. Retrieved Februaury 21, 2008, from Ctv.ca.

Capra, F. (1996). A new scientific understanding of living systems: The web of life. New York: Anchor Books.

CBC News. (2007, July 17). Baby boomers swell ranks of retirement-aged Canadians. CBC News. Retrieved September 12, 2008, from http://www.cbc.ca/canada/story/2007/07/ $17 /$ census-canada.html

Colucci, G. (2008). Apotex Centre, Baycrest Centre for geriatric care project brief. Toronto, Ontario, Canada: A.J. Diamond, Donald Schmitt and Company.

Crenlin, J., Connor, J., \& Andersen, R. (1997). Alternative healthcare in Canada: Nineteenth-and twentieth- century perspectives. Toronto, Ontario, Canada: Canadian Scholars' Press.

Cruse, A. (2004). Roman medicine. Gloucestershire: Tempus Publishing.

Davis, M. (2008). Financing hospital investment. In Prasad, S. (2008). Changing hospital architecture. London: RIBA Publishing.

Dunai, A., Novak, M., Chung, S.A., Kayumov, L., Keszei, A., Levitan, R., \& Shapiro, C.M. (2007) Diet and physical activity: Moderate exercise and bright light treatment in overweight and obese individuals [Electronic Version]. Obesity, 15(7), 1749-1757.

Farr, D. (2008). Sustainable urbanism: Urban design with nature. Hoboken, NJ: Wiley Press.

Felepchuk, L. (2008). Canada's fattest and fittest. Sympatico MSN: Health and Fitness. Retrieved October 07,2008 , from http://healthandfitness.sympatico.msn.ca/

Canadas+fattest+and+fittest/DietFitness/Canadas+fattest+and+fittest.htm?isfa=1 
Franklin, M. (2000) Handled with care. [Electronic Version]. Canadian Architect. 11, 30-35.

Guite, H., \& Toy, S. (2008). Social and physical factors for building happiness. In Wernick, J. (2008). Building happiness: Architecture to make you smile. London: Black Dog Publishing.

Hamilton, K. (2004). Four levels of evidence based practice. Retrieved September 12, 2008 from http://www.aia.org/nwsltr print.cfm?pagename=aiaj a 20041201 fourlevels

Hamilton, K., \& Watkins, D. (2009). Evidence based design for multiple building types. Hoboken, $\mathrm{NJ}$ : John Wiley \& Sons Press.

Hinkle, L.E., \& Loring, W. (1977). The effect of the man-made environment on health and behaviour. Atlanta: Department of Health, Education, and Welfare.

Hobday, R. (2006). The light revolution: Health, architecture and the sun. Edinburgh: Findhorn Press.

Jinks, M., Lassey, M., \& Lassey, W. (1997). Health care systems around the world: Characteristics, issues, reforms. New York, NJ: Prentice-Hall.

Joseph, A. (2006). The impact of light on outcomes in healthcare settings. Retrieved October 21 , 2008 from http://www.healthdesign.org/research/reports/documents/ CHD_Issue_Paper2.pdf

Joseph, A. (2006). The impact of the environment on infections in healthcare facilities. Retrieved November 2, 2008 from http://www.healthdesign.org/research/reports/documents/ CHD_Issue_Paper1_001.pdf

Joseph, A., \& Ulrich, R. (2007). Sound control for improved outcomes in healthcare settings. Retrieved October 17, 2008 from http://www.healthdesign.org/research/reports/ documents/CHD_Issue_Paper4.pdf

Joye, Y. (2007). Architectural lessons from environmental psychology: The case of biophilic architecture [Electronic Version]. Review of General Psychology, 11(4), 305-328.

King, H. (2001). Greek and Roman medicine. London: Bristol Classical Press.

Lister, N.M. (2008). Placing food. In J. Knechtel, (2008). Food. Cambridge: MIT Press.

Low, J. (2004). Using alternative therapies: A qualitative analysis. Toronto, Ontario, Canada: Canadian Scholars' Press.

Marberry, S.O. (1995). Innovations in healthcare design. New York: Van Nostrand Reinhold.

Marcus, C., \& Barnes, M. (1995). Gardens in healthcare facilities: Uses, therapeutic benefits, and design recommendations. Los Angeles: The Centre for Health Design.

Martel, L., \& Caron, E. (2006). Portrait of the Canadian population in 2006, by age and sex, 2006 census. Statistics Canada: Census. Retrieved September 24, 2008, from http://www12.statcan.ca/english/census06/analysis/agesex/pdf/ 97-551-XIE2006001.pdf

McDonough, W., \& Braungart, M. (2002). Cradle to Cradle: remaking the way we make things. New York: North Point press. 
Medical Dictionary. (2008). Preventative Medicine. Retrieved October 20, 2008, from http:// medical-dictionary.thefreedictionary.com/preventive+medicine

Mobach, M. (2004). Evidence-based design [Electronic Version]. Pharm World Sci, 26, 245.

Muir, M. (2003). Gestalt art therapy for people with dementia. Retrieved November 12, 2008 from http://www.designandhealth.com/edu_res/ Margaret\%20Muir\%20WCDH2003.pdf

Naess, A. (1989). Ecology, Community and Lifestyle: Outline of an Ecosophy Cambridge: Cambridge University Press.

Nield, L. (2008). Postscript: Re-inventing the hospital. In Prasad, S. (2008). Changing hospital architecture. London: RIBA Publishing.

Oldenburg, R. (1989). The great good place: Cafes, coffee shops, bookstores, bars, hair salons, and other hangouts at the heart of a community. Philadelphia: Da Capo.

Pallasmaa, J. (2005). The eyes of the skin: Architecture and the senses. Chichester: WileyAcademy.

Prasad, S. (2008). Changing hospital architecture. London: RIBA Publishing.

Purves, G. (2002). Healthy living centres: A guide to primary health care design. Oxford: The Architectural Press.

Rapp, B. (2000). The arts in hospital and care as culture. Retrieved November 11, 2008 from http://www.designandhealth.com/edu_res/Birgitta\%20Rapp,\%20WCDH2000.pdf

Rogers, J.L. (2005). Current trends in pediatric hospital design: Are they right for your organization? Karlsberger Knowledge, 1. Retrieved October, 06, 2008, from http:// www.karlsberger.com/downloads/CurrentTrendsInPedHospDesign.pdf

Sadler, B. (2006). The business case for building better hospitals [Electronic Version]. Trustee, $59,35-36$.

Sarkis, H. (2001). CASE: Le Corbusier's Venice hospital and the Mat building revival. Munich: Prestel Verlag.

Schweitzer, M., Gilpin, L., \& Frampton, S. (2004). Healing spaces: Elements of environmental design that make an impact on health [Electronic Version]. The Journal of Alternative and Complimentary Medicine, 10, 71-83.

Sharr, A. (2007). Heidegger for architects. London: Routledge.

Shields, M., \& Tjepkema, M. (2006). Regional differences in obesity [Electronic Version]. Statistics Canada: Health Reports, 17(3), 61-67.

Stow, D. (2008). Transformation in healthcare architecture: from the hospital to a healthcare organism. In Prasad, S. (2008). Changing hospital architecture. London: RIBA Publishing.

The Centre for Health Design (2008). The Centre for Health Design. Retrieved September 27 , 2008, from http://www.healthdesign.org/ 
The International Academy for Design and Health (2008). The International Academy for Design and Health. Retrieved September 27, 2008, from http://www.designandhealth.com/

The World Fact Book. (2008). Japan. CIA- The World Fact book. Retrieved October 17, 2008, from https://www.cia.gov/library/publications/the-world-factbook/print/ja.html

Tossell, I. The Globe and Mail. (2008). One of the life-science capitals of the world. Retrieved October 22, 2008, from the ProQuest database.

Ulrich, R.S. (1984). View through a window may influence recovery from surgery [Electronic Version]. Science, 224(4647), 420-421.

Ulrich, R.S. (2000). Effects of healthcare environmental design on medical outcomes. Retrieved October 06, 2008 from http://www.designandhealth.com/edu_res/

Roger\%20S.\%20Ulrich\%20p49.pdf

Wernick, J. (2008). Building happiness: Architecture to make you smile. London: Black Dog Publishing.

Williams, M. (1991). Design for therapeutic outcomes. In Marberry, S.O. (1995). Innovations in healthcare design. New York: Van Nostrand Reinhold.

Zeidler, E.H. (1974). Healing the hospital; McMaster Health Science Centre: Its conception and evolution. Toronto: Zeidler Partnership.

Zeidler, E.H. (2003). Holistic healthcare buildings. Retrieved October 29, 2008 from http:// www.designandhealth.com/edu_res/EB\%20Zeidler\%20WCDH2003.pdf

Zimring, C., \& Bosch, S. (2008). Building the evidence base for evidence based design: Editors' introduction. [Electronic Version]. Environment and Behaviour, 40, 147-150.

Zumthor, P. (1998). Thinking architecture. Basel: Birkhauser.

Zumthor, P. (2007). Peter Zumthor: Therme Vals. Zurich: Scheidegger \& Spiess. 